OPTIMISATION DU TAUX DE COMPENSATION SÉRIE D'UNE LONGUE LIGNE DE DISTRIBUTION

CAS DE L'HYDRO-QUEBEC : ABITIBI TEMINSCAMINGUE

\author{
ÉTUDIANT: \\ EDER ERNESTO FERNANDEZ RAMIREZ
}

DIRECTEUR :

RENÉ WAMKEUE, Ing. PhD.

CO-DIRECTEUR:

NAHI KANDIL, Ing. PhD.

COLLABORATEUR INDUSTRIELLE:

JACQUES EAST, Ing. Hydro-Québec 


\section{Bibliothèque}

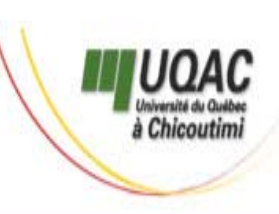

\section{Mise en garde/Advice}

Afin de rendre accessible au plus Motivated by a desire to make the grand nombre le résultat des results of its graduate students' travaux de recherche menés par ses research accessible to all, and in étudiants gradués et dans l'esprit des accordance with the rules règles qui régissent le dépôt et la governing the acceptation and diffusion des mémoires et thèses diffusion of dissertations and produits dans cette Institution, theses in this Institution, the l'Université du Québec à Université du Québec à Chicoutimi (UQAC) est fière de Chicoutimi (UQAC) is proud to rendre accessible une version make a complete version of this complète et gratuite de cette œuvre. work available at no cost to the reader.

L'auteur conserve néanmoins la The author retains ownership of the propriété du droit d'auteur qui copyright of this dissertation or protège ce mémoire ou cette thèse. thesis. Neither the dissertation or Ni le mémoire ou la thèse ni des thesis, nor substantial extracts from extraits substantiels de ceux-ci ne it, may be printed or otherwise peuvent être imprimés ou autrement reproduced without the author's reproduits sans son autorisation. permission. 


\section{TABLE DES MATIÈRES}

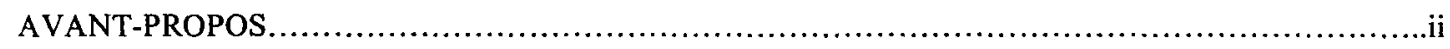

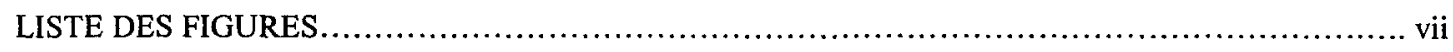

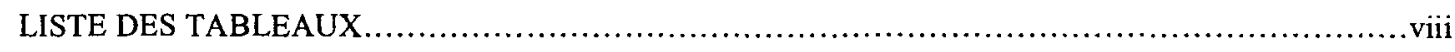

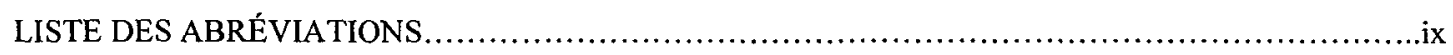

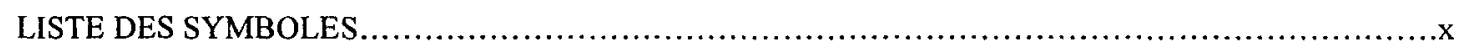

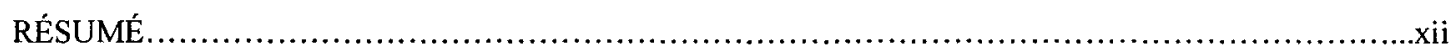

CHAPITRE I. CONCEPTS IMPORTANTS DANS LA CONCEPTION DU PROJET ................................

1.1 L'étude de stabilité des réseaux électriques comme un critère de base dans le développement du

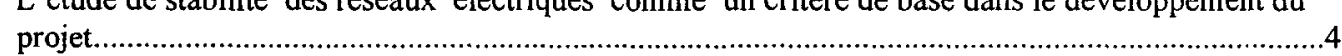

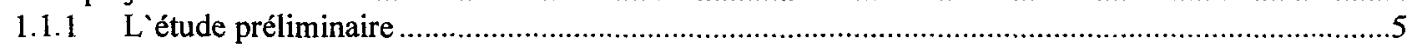

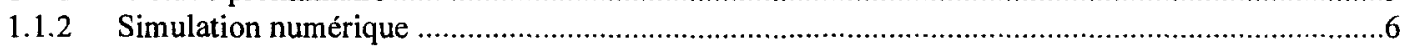

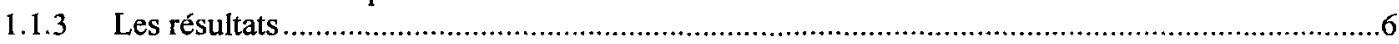

1.2 Description des solutions envisagées face à la problématique exposée dans le projet .....................7

1.3 Planification du projet comme une méthode d'optimisation basée sur le concept de stabilité du réseau électrique.

\section{CHAPITRE II. DÉVELOPEMENT DE LA MÉTHODE D'OPTIMISATION PAR L'ÉVALUATION DES} CONTRAINTES DE STABILITÉ

2.1 Méthode d'optimisation appliquée dans le projet ............................................................. 13

2.2 Identification de la fonction objective et les contraintes de stabilité ......................................14

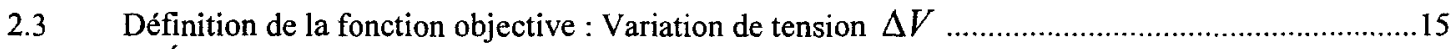

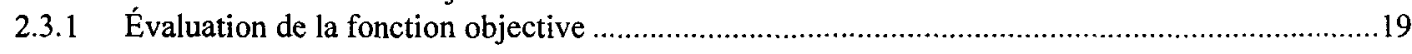

$2.4 \quad$ Contraintes de stabilité de la méthode d'optimisation.........................................................21

2.4.1 Contrainte de stabilité No 1 : Taux de compensation série .................................................21

2.4.2 Contrainte de stabilité No 2 : Transfert maximal de puissance .............................................22

2.4.3 Contrainte de stabilité No 3 : Oscillations de la vitesse des moteurs........................................29

2.4.4 Contrainte de stabilité No 4 : Puissance de court-circuit. .......................................................32

2.4.5 Contrainte de stabilité N. 5 : Phénomène de papillotement..................................................43

2.4.6 Contrainte de stabilité No 6 : Pertes de puissance active et réactive ......................................54

2.4.7 Contrainte de stabilité No 7. Résonance sous-synchrone (SSR) ..........................................57 


\section{CHAPITE III. METHODE ITERATIVE DE CALCUL DU TAUX DE COMPENSATION SÉRIE ET DE}

LA POSITION OPTIMALE .....................................................................................66

3.1 Étape $1:$ Identification et définition des contraintes de stabilité et de la fonction objective .............67

3.1.1 Condition initiale de la réactance capacitive du condensateur série ........................................68

3.1.2 Condition initiale de la position du banc de condensateurs série ...........................................68

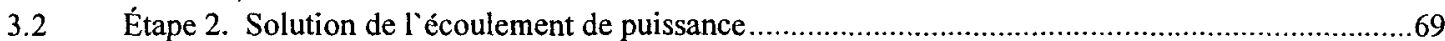

3.3 Étape 3. Évaluation des contraintes de stabilité et de la fonction objective ...............................70

3.4 Condition 1. Boucle de variation de la réactance capacitive de la compensation série sur la ligne de

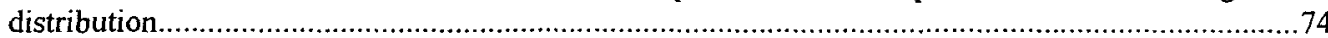

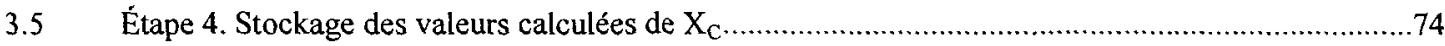

3.6 Condition 2. Boucle de variation de la position de la réactance capacitive sur la ligne de

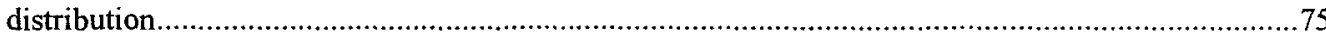

3.6.1 Étape 5. Résultats de la méthode d’optimisation et simulation des tests typiques.....................75

3.7 Comparaison de calcul de la position optimal de Xc avec la méthode basée sous des conditions de tension en amont et en aval du condensateur série....

CHAPITRE IV. SIMULATIONS ET RÉSULTATS

4.1 Présentation du modèle de simulation du réseau de distribution et des essais à simuler ................86

4.1.1 Modèle de simulation numérique Matlab/Simulink du réseau de distribution radial ...................86

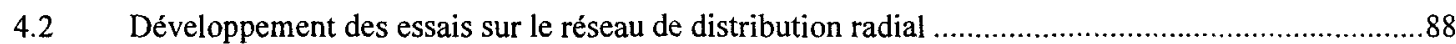

4.2.1 Essai 1. Enclenchement de la ligne........................................................................8.

4.2.2 Essai 2. Performance du réseau de distribution radial en régime transitoire à $t=0.5 \mathrm{sec} \ldots \ldots \ldots \ldots . .95$

4.2.3 Essai 3. Performance du réseau de distribution radial en régime permanent $t=3 \mathrm{sec} . \ldots \ldots \ldots \ldots . . . .97$

4.2.4 Essai 4. Performance des groupes moteurs en régime permanent à $t=3 \mathrm{sec} \ldots \ldots \ldots \ldots . . . .99$

4.2.5 Essai 5 (a). Perturbation du réseau de distribution radial en présence du court-circuit

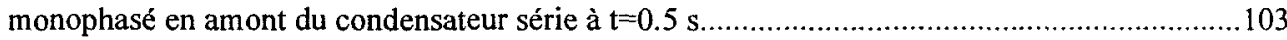

4.2.6 Essai 5 (b). Perturbation du réseau de distribution radial en présence d un court circuit

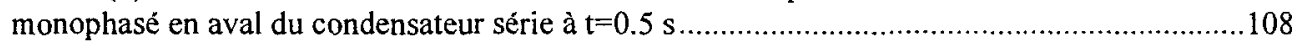

4.2.7 Essai 6. Perturbation du réseau de distribution radial en présence d'un court circuit

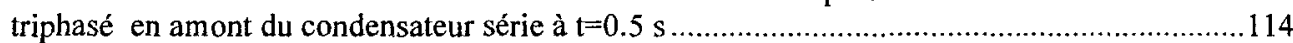

4.2.8 Essai 6. Perturbation du réseau de distribution radial en présence d’un court-circuit

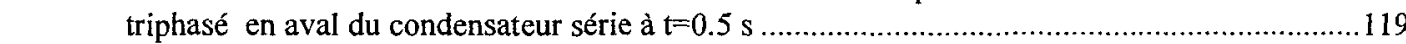

4.2.9 Essai 7. Performance du réseau de distribution radial due à la variation brusque de charge dans le groupe moteur $\mathrm{M} 2$ à $\mathrm{t}=0.6 \mathrm{sec}$.

4.2.10 Essai 8. Démarrage à vide des moteurs asynchrones......................................................129 


\begin{abstract}
AVANT-PROPOS
La réalisation de ce projet n'aurait sans doute pas été possible sans l'aide et le soutien des personnes suivantes:

J'adresse tout d'abord mes remerciements au Professeur René Wamkeue qui m'a encouragé à entreprendre et à mener à terme cette thèse, pour son appui moral et financier et la confiance dès le début en mes capacités. Au professeur Nahi Kandil pour son appui moral et financier et son encadrement dans le domaine de la stabilité des réseaux électriques. Trouvez ici chers professeurs, l'expression de mon admiration et de ma reconnaissance.
\end{abstract}

Je tiens à remercier le Gouvernement de la Colombie et du Ministère de l'Éducation du Québec qui m'ont fourni la bourse m'ayant permís de réaliser mes études de maîtrise en ingénierie.

Je remercie Monsieur ingénieur Jacques East de Hydro-Québec qui a notablement contribué à la réalisation ce projet de maîtrise.

Je remercie le personnel du département des sciences appliquées pour son accueil chaleureux.

La réalisation de mon projet n'aurait pas été possible sans le soutien moral et affectif des membres de ma famille dans les moments les plus difficiles. Je remercie mes parents pour leur appui et leur dévotion durant toutes ces années. En terminant, je voudrais remercier Rossana pour son appui constant, son amour et sa compréhension en tous ces moments d'efforts malgré la distance qui nous a séparés. 


\section{TABLEAU DES FIGURES}

Figure 1 : Diagramme du réseau de distribution radial de Hydro-Québec

Figure 2: Condition de profil de tension sans la compensation série (a) Circuit équivalent du réseau de distribution radial et (b) diagramme vectoriel de variation de tension.

Figure 3 : Condition de tension avec compensation série (a) Circuit équivalent du réseau de distribution radial. (b) diagramme vectoriel de variation de tension..................................................... 18

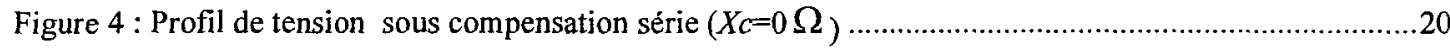

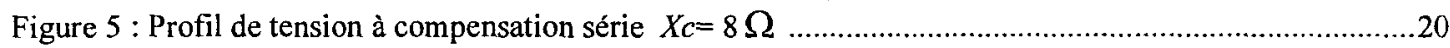

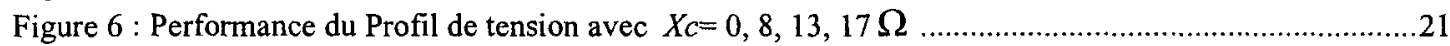

Figure 7 : (a) Diagramme monophasé du réseau électrique de distribution radial (a)non compensé (b) compensé

Figure 8: Effet de la compensation série sur le transfert de puissance. .............................................25

Figure 9 : Diagramme monophasé du réseau radial analysé pour développer le critère de transfert de puissance en considérant la résistance de la ligne de transport ...............................................26

Figure 10 : Performance de transfert maximal de puissance aux différents taux de compensation série........28

Figure 11 : Performance de la puissance active par rapport à la longueur de la ligne.............................28

Figure 12: Diagramme monophasé du réseau radial avec défaut en aval du condensateur série ....................33

Figure 13 : Transfert de puissance par rapport à l'angle sous des conditions de court circuit.......................33

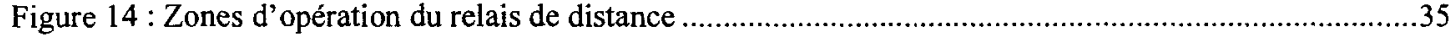

Figure 15 : Performance de la réactance équivalente de la ligne par rapport à la position du condensateur série.

Figure 16 : Performance de la réactance équivalente de la ligne par rapport à la position optimale du

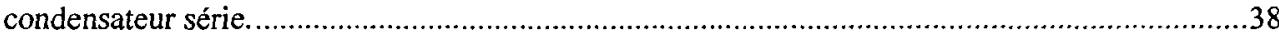

Figure 17 : Performance de la réactance équivalente de la ligne par rapport à la position optimale du

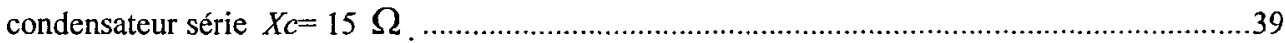

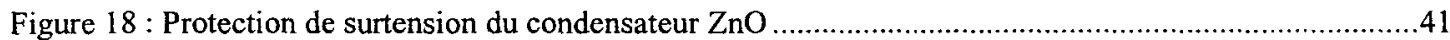

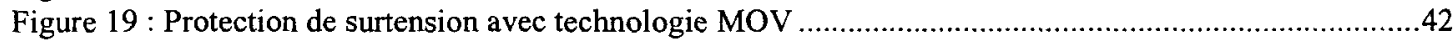

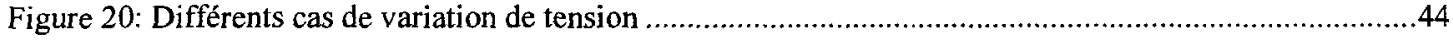

Figure 21 : Modèle électrique et diagramme vectoriel du réseau électrique. ..........................................44

Figure 22 : Seuil de perception de l'œil de l'abonné aux fluctuations de tension. ......................................48

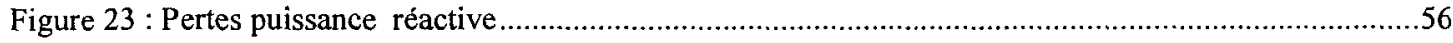

Figure $24:$ Pertes de puissance active dans la ligne de distribution .......................................................56

Figure $25:$ Résultats de l'étude de réponse en fréquence à la fin de la ligne. $X c=8 \Omega \ldots \ldots \ldots \ldots \ldots \ldots \ldots \ldots . . .59$

Figure $26:$ Performance amplifiée de l'étude de réponse en fréquence à la fin de la ligne. $X c=8 \Omega$..........59

Figure 27 : Performance de l'étude de réponse en fréquence à la fin de la ligne $X c=15 \Omega$......................60

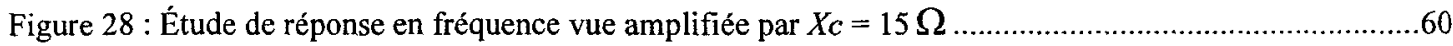

Figure 29 : Schéma du condensateur série en parallèle avec une résistance contrôlée par le thyristor. Configuration développée par N.G Hingorani.

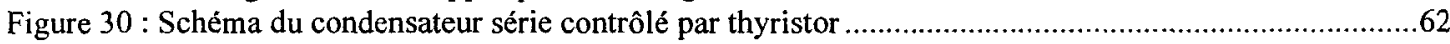

Figure 31 : Schéma du condensateur série avec équipement antirésonance ........................................63

Figure 32 : Performance de la demande maximale de puissance des clients residentiels et industriels par rapport au système de protection antirésonance du condensateur série ..............................65

Figure 33 : Diagramme du profil de tension avec différentes positions du banc de condensateurs série sur

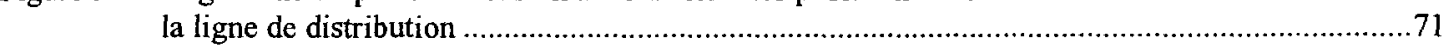

Figure 34 : Région standard d'opération du taux de compensation série optimale. ..................................77

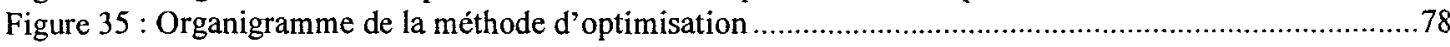

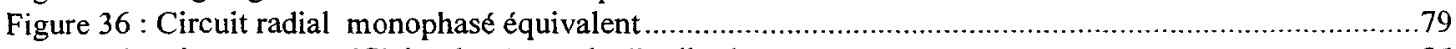

Figure 37 : Diagramme unifilaire du réseau de distribution à compenser ..............................................86

Figure 38 : Diagramme équivalent du réseau de distribution en Power System Blockset...........................87 
Figure 39 : Modèle numérique Matlab/Simulink du bloc des groupes moteurs M1, M2, M3 et M4 ...........87

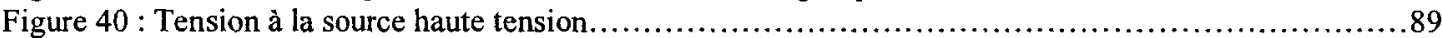

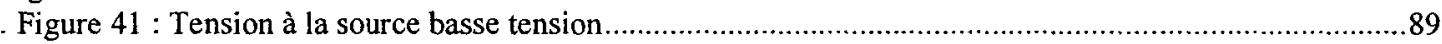

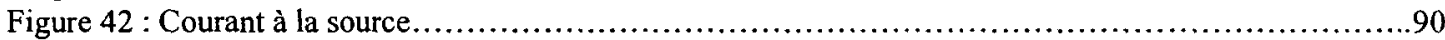

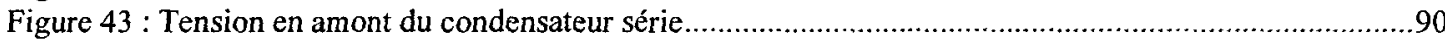

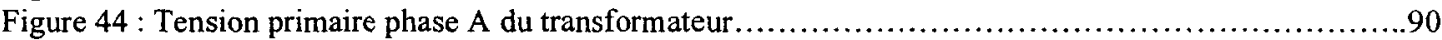

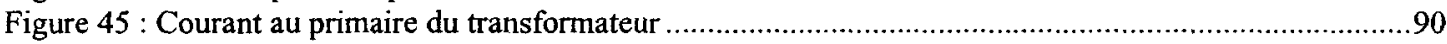

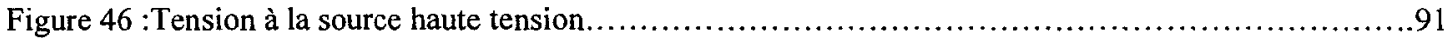

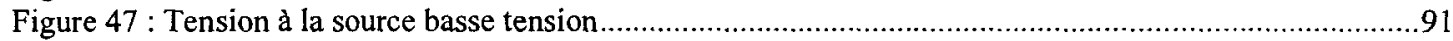

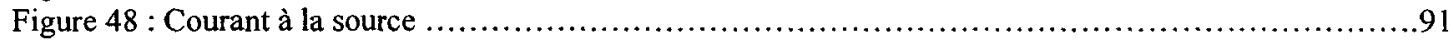

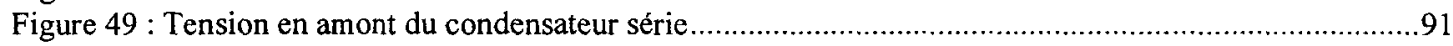

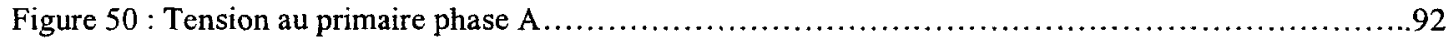

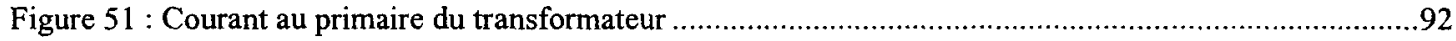

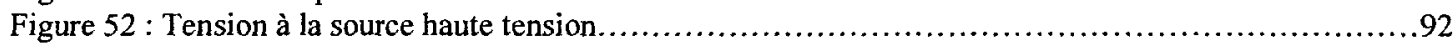

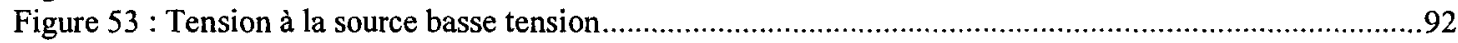

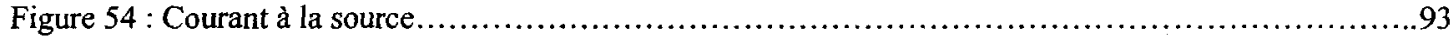

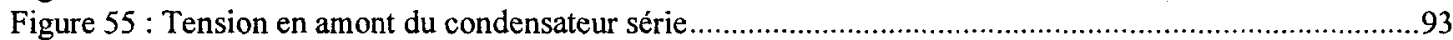

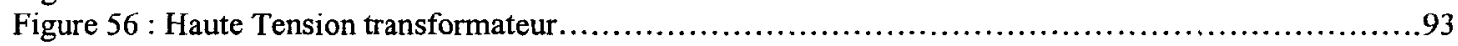

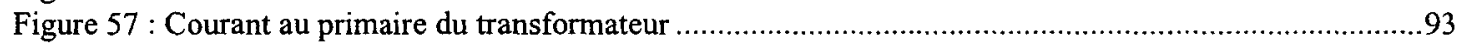

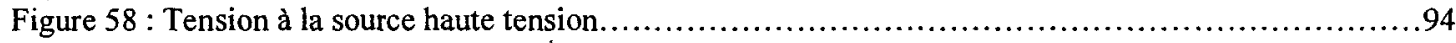

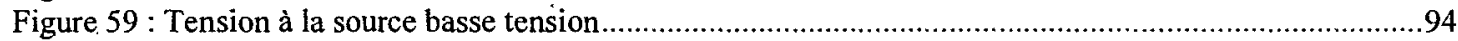

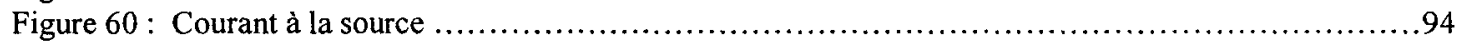

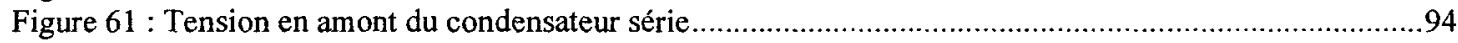

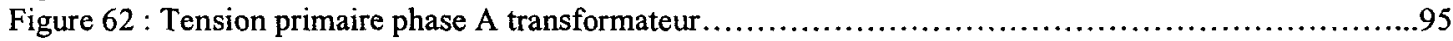

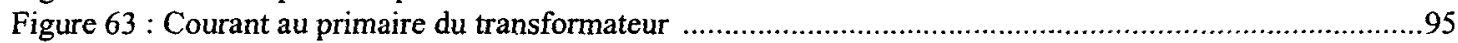

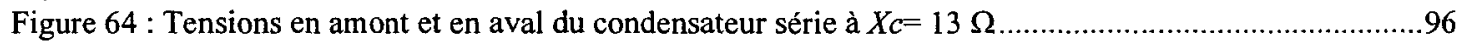

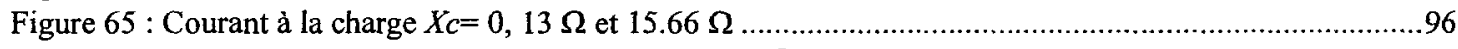

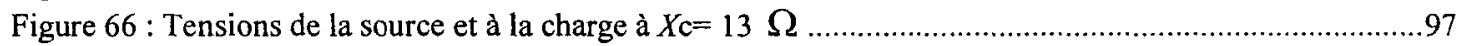

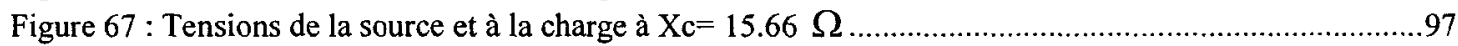

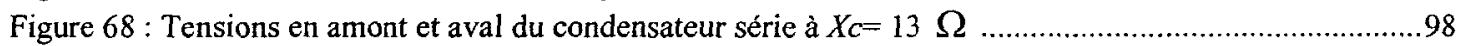

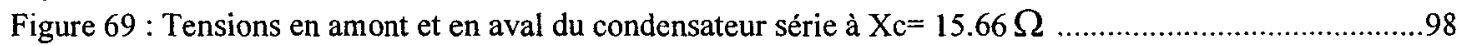

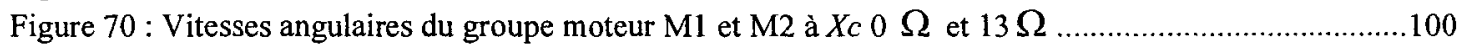

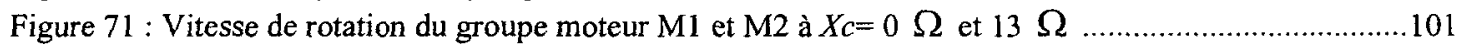

Figure 72 : Vitesse de rotation du groupe moteur M1 et M2 à $X c=15.66 \Omega \ldots \ldots \ldots \ldots \ldots \ldots \ldots \ldots \ldots \ldots \ldots \ldots 1$

Figure 73 : Modèle numérique du réseau de distribution radial avec le bloc d'un court-circuit monophasé en amont du condensateur série ............................................................... 103

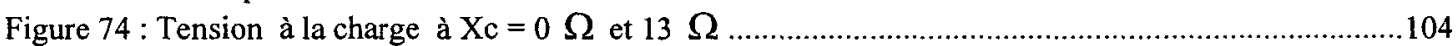

Figure 75 : Tensions en amont et en aval du condensateur série à $X c=0$ et $13 \Omega$ lors d'un court-circuit en amont du condensateur.

Figure $76:$ Courant de court-circuit du groupe moteur M1 ........................................................105

Figure 77 : Courant de court-circuit du groupe moteur M2 .........................................................105

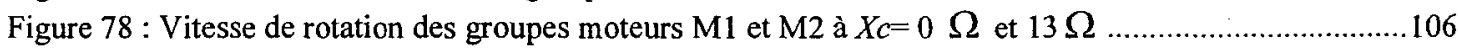

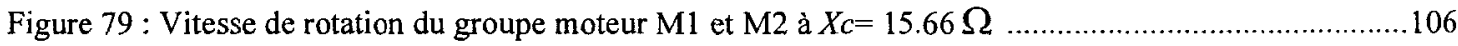

Figure $80:$ Couple à la sortie du groupe moteur M1 et M2 ................................................................107

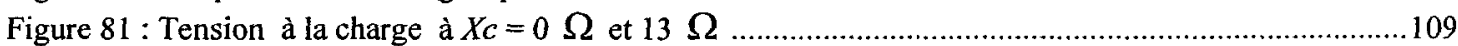

Figure 82 : Tensions en amont et en aval du condensateur série à $X c=13 \Omega$ lors court circuit en amont du condensateur

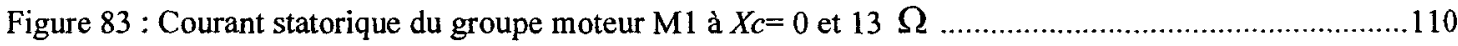

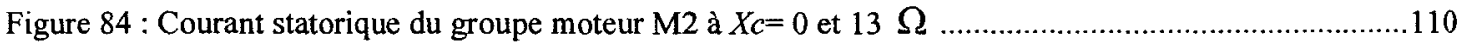

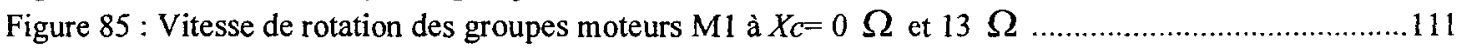

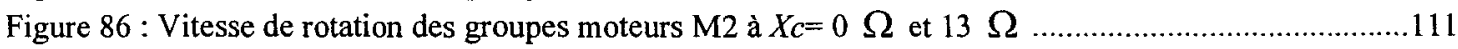

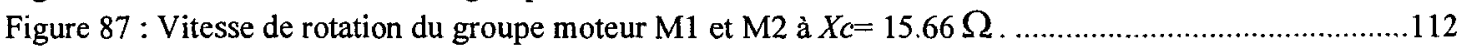

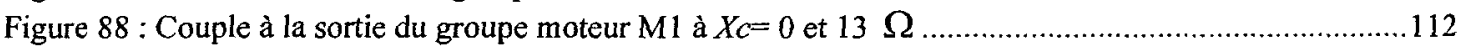




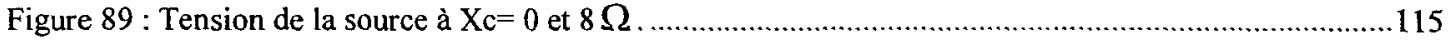

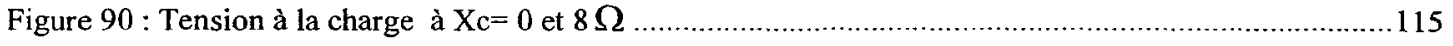

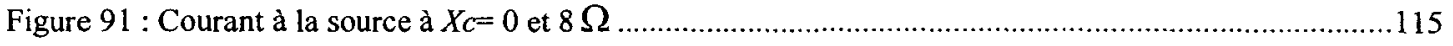

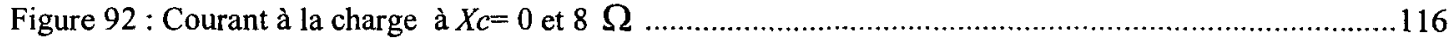

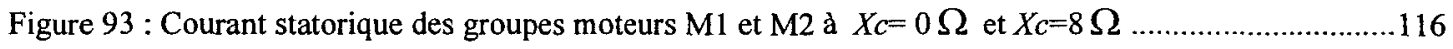

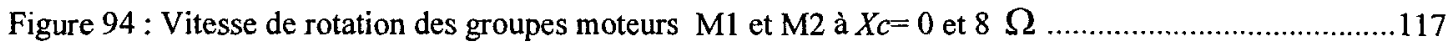

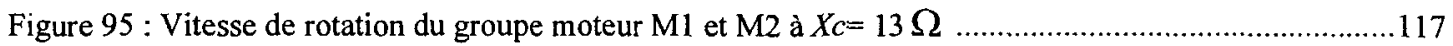

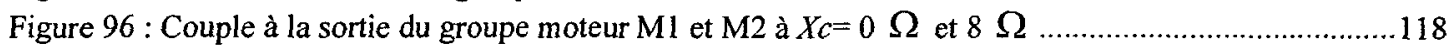

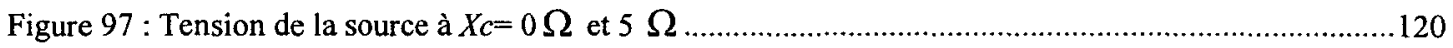

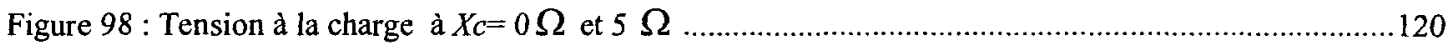

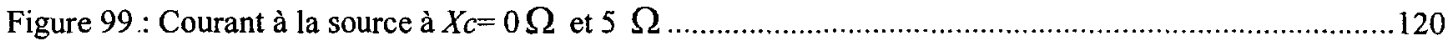

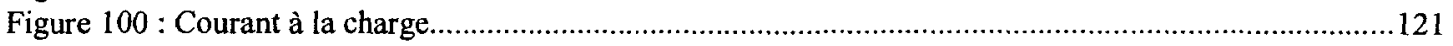

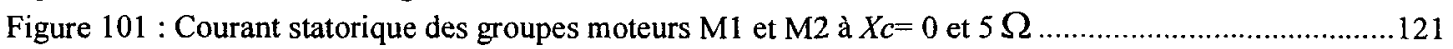

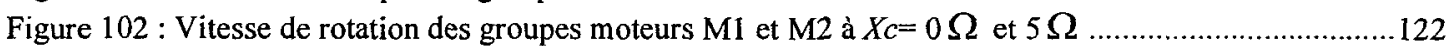

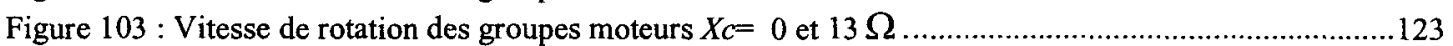

Figure 104 : Couple à la sortie des groupes moteurs M1 et M2 à $X c=0 \Omega$ et $5 \Omega \ldots \ldots \ldots \ldots \ldots \ldots \ldots \ldots \ldots \ldots . . . . . . . . . . . . . .123$

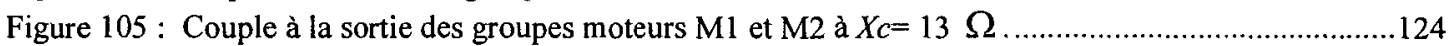

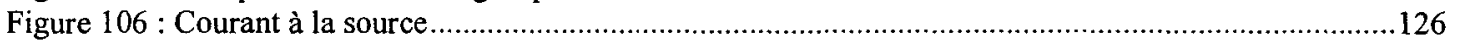

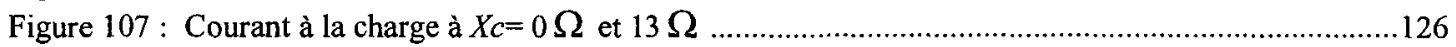

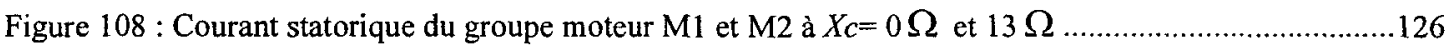

Figure $109:$ Vitesse de rotation du groupe moteur M1 et M2 ......................................................127

Figure $110:$ Couple à la sortie du groupe moteur Ml .....................................................................128

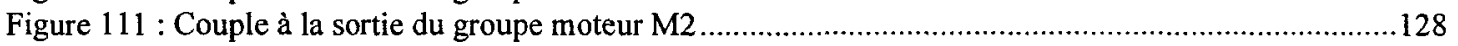

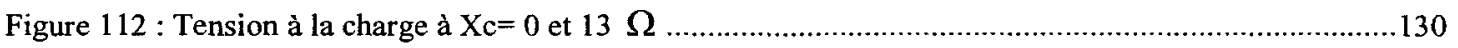

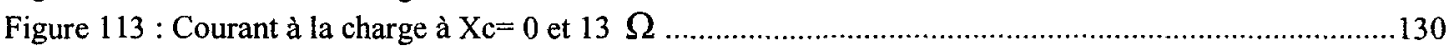

Figure 114 : Comparaison de la vitesse de rotation du groupe moteur M1 et M2 à Xc=0 et $13 \Omega \ldots \ldots \ldots . .131$

Figure $115:$ Comparaison du couple à la sortie du groupe moteur M1 et M2 à Xc= 0 et $13 \Omega \ldots \ldots \ldots \ldots \ldots . .131$ 


\section{LISTE DES TABLEAUX}

Tableau 1 Résultats de l'évaluation de la fonction objective à différents taux de compensation taux de compensation série.

Tableau 2 Intervalle du Taux de compensation série........................................................................ 22

Tableau 3 Résultats de transfert de puissance aux différents taux de compensation série...............................2 27

Tableau 4 Tangente de résistance et la réactance équivalente de la ligne par rapport au taux de compensation série..

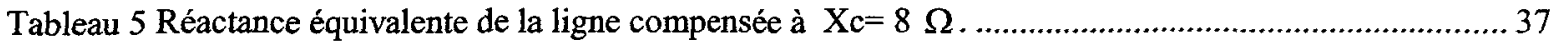

Tableau 6 Réactance équivalente de la ligne de distribution compensée à $\mathrm{Xc}=13 \Omega$. Position optimale du condensateur série $x=35 \mathrm{Kms}$.

Tableau 7 Réactance équivalente de la ligne de distribution compensée à $\mathrm{Xc}=15 \Omega$. Position optimale du condensateur série $x=32 \mathrm{Kms}$

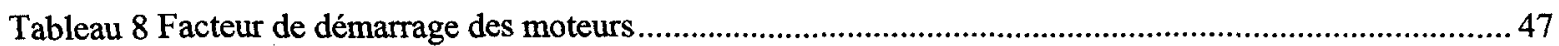

Tableau 9 Fréquence du phénomène de papillotement percept par les clients résidentiels ............................. 50

Tableau 10 Puissance de court circuit du réseau aux différents taux de compensation .................................5 52

Tableau 11 Pertes de puissance active et réactive ............................................................................................ 55

Tableau 12 Résultats de fréquences de résonance du réseau électrique. .....................................................58

Tableau 13 Résultats de fréquences de résonance du réseau électrique ......................................................60

Tableau 14 Réactance équivalente de la ligne par rapport à la position du banc des condensateur série ......... 73

Tableau 15 Résultats de la méthode d'optimisation ................................................................................. 76

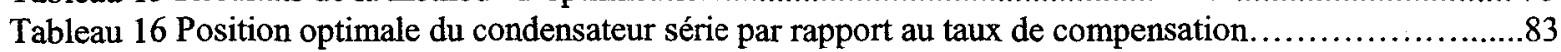

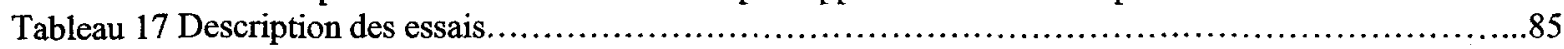

Tableau 18 Grandeurs mesurées pour la ligne sans compensation série..................................................... 98

Tableau 19 Grandeurs mesurées pour la ligne avec compensation série de $21.1 \%\left(X_{c}=5 \Omega\right)$..................... 99

Tableau 20 Grandeurs mesurées pour la ligne avec compensation série de $54.85 \%\left(\mathrm{X}_{\mathrm{c}}=13 \Omega\right)$................... 99

Tableau 21 Grandeurs mesurées pour la ligne avec compensation série de $66 \%\left(X_{c}=15.66 \Omega\right)$.................. 99

Tableau 22 Tensions phase $« \mathrm{a}, \mathrm{b}, \mathrm{c} »$ des groupes de moteurs .............................................................. 102

Tableau 23 Courants phase « $\mathrm{a}, \mathrm{b}, \mathrm{c} »$ des groupes de moteurs .............................................................. 102

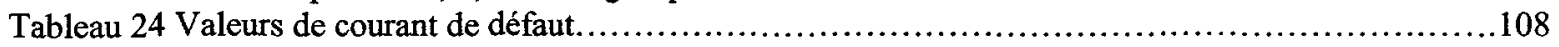

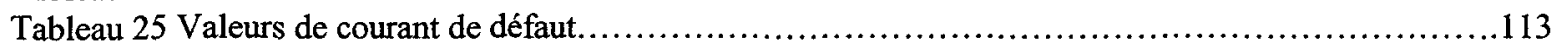

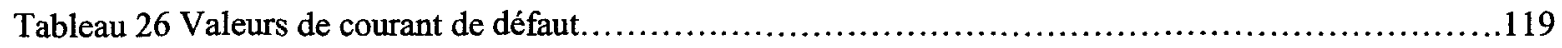

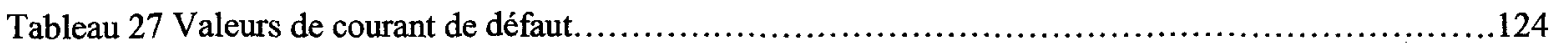




\title{
LISTE DES ABREVIATIONS
}

\author{
CA Courant alternatif \\ CC Courant continu \\ FACTS Flexible Alternating Current Transmission System \\ IEEE Institute of Electrical and Electronics Engineers \\ MW Mega Watts \\ MVA Mega-Volt Ampere \\ MVar Mega-Volt Ampere Réactif \\ SVC Compensateur statique \\ UPS Alimentation de sécurité \\ MOV Mosfet Oxide Varistor \\ ANSI American national Standards Institute \\ CAN Canadian Association of Normalisation \\ RC's Résistance antirésonance \\ SSR Subsynchronous Resonance \\ MINICAP Type du banc de condensateur série installé au réseau radial
}




\section{LISTE DES SYMBOLES}

\begin{tabular}{|c|c|}
\hline SYMBOL & SIGNIFICAT \\
\hline$V_{1}$ & Tension à la source \\
\hline$V_{2}$ & Tension à la charge \\
\hline$V_{3}$ & Tension en aval des terminaux du condensateur série \\
\hline$\Delta V$ & Variation de tension: $V_{1}-V_{2}$ \\
\hline$V_{1}^{\prime}$ & Tension en arrière de la réactance transitoire d'alternateur \\
\hline$V_{2}^{\prime}$ & Tension en arrière de la réactance transitoire des moteurs \\
\hline$\delta$ & Angle entre $V_{1}$ et $V_{2}$ \\
\hline$\delta_{0,1,2, n}$ & Angles entre la tension $V_{1}^{\prime}$ ' et $V_{2}^{\prime}$ \\
\hline$\theta_{r}$ & Angle de facteur de puissance \\
\hline$\tau$ & Taux de compensation série \\
\hline$P$ & Puissance active \\
\hline$Q$ & Puissance réactive \\
\hline$P_{\text {elec }}$ & Puissance électrique \\
\hline$P_{m e c}$ & Puissance mécanique \\
\hline$P_{\max }$ & Transfert maximal de puissance \\
\hline$P_{O}$ & Puissance d'opération du réseau. \\
\hline$K p$ & Marge de stabilité en régime permanent \\
\hline$P_{\text {perte }}$ & Perte de puissance active \\
\hline$Q_{\text {perte }}$ & Perte de puissance réactive \\
\hline$I$ & Courant dans la ligne \\
\hline$R$ & Résistance de la ligne \\
\hline$X_{L}$ & Réactance de la ligne \\
\hline$X c$ & Capacitance du condensateur série \\
\hline$X$ & Réactance équivalente de la ligne \\
\hline $\mathrm{Xs}$ & Réactance transitoire d'altemateur \\
\hline $\mathrm{Xm}$ & Réactance transitoire de moteur \\
\hline $\mathrm{w}$ & Position du banc de condensateurs série \\
\hline$R_{t l}$ & Résistance du transformateur N. $1\left(\mathrm{~T}_{1}\right)$ \\
\hline$R_{t 2}$ & Résistance du transformateur N. $2\left(\mathrm{~T}_{2}\right)$ \\
\hline$X_{t 1}$ & Réactance du transformateur N. 1 \\
\hline$X_{t 2}$ & Réactance du transformateur N. 2 \\
\hline
\end{tabular}




\begin{tabular}{ll}
$R_{m}$ & Résistance du circuit magnétique de T1 \\
$Y_{m}$ & Admittance magnétique de T1 \\
$Z s$ & Impédance équivalente de la source \\
$Z_{L}$ & Impédance de séquence positive de la ligne de distribution \\
$Z_{0}$ & Impédance de séquence homopolaire de la ligne de distribution \\
$C_{1,2}$ & Capacitances équivalentes de la ligne \\
$P F$ & Facteur de puissance \\
$O P$ & Puissance d'opération \\
$I d$ & Courant démarrage \\
$\eta$ & Rendement du moteur \\
$\beta$ & Coefficient démarrage \\
$K V A_{\text {appel }}$ & Puissance d'appel des moteurs \\
$K V A_{c c}$ & Puissance de court circuit du réseau électrique \\
$\alpha$ & Paramètre de mesure du papillotement \\
$\mathrm{g}[\mathrm{f}(\mathrm{i})]$ & Fréquence de papillotement \\
$\mathrm{Z}_{120}$ & Impédance de séquence positive \\
$\mathrm{Z}_{120}$ & Impédance de séquence négative \\
$\mathrm{E}_{120}$ & Tension de séquence positive \\
$\mathrm{E}_{120}$ & Tension de séquence négative \\
$\mathrm{Ea}$ & Tension obtenue à côté du relai distance position A \\
$\mathrm{Eb}$ & Tension obtenue à côté du relai distance position B \\
$\mathrm{Ia}$ & Courant mesuré par le relai A \\
$\mathrm{lb}$ & Courant mesuré par le relai B \\
$\mathrm{Rab}$ & Résistance mesurée de relai A au relai B \\
$\mathrm{Rba}$ & Résistance mesurée de relai A au relai B \\
$\mathrm{Rbc}$ & Résistance mesurée de relai B au relai C \\
$\mathrm{Rcb}$ & Résistance mesurée de relai C au relai B \\
$w_{o}$ & Fréquence naturelle du système \\
$w_{e}$ & Fréquence de courants sous-synchrones. \\
$\mathrm{h} 1$ & Contrainte taux maximal de la compensation série. \\
$\mathrm{h} 2$ & Contrainte transfert maximal de puissance. \\
$\mathrm{h} 3$ & Contrainte oscillations de vitesse des moteurs. \\
$\mathrm{h} 4$ & Contrainte Inversion du courant de court circuit. \\
$\mathrm{h} 5$ & Contrainte Phénomène de papillotement. \\
$\mathrm{h} 6$ & Contrainte Pertes de puissance réactive. \\
$\mathrm{h} 7$ & Contrainte perte de puissance réactive \\
$\mathrm{h} 8$ & Contrainte résonance sous-synchrone \\
$\mathrm{Pinst}$ & Papillotement instantané \\
$\mathrm{Pst}$ & Papillotement courte durée \\
$\mathrm{Plt}$ & Papillotement longue durée \\
$\mathrm{t}_{1}$ & Temps 1 distorsion de tension (papillotement instantané) \\
$\mathrm{t}_{2}$ & Temps 2 distorsion de tension (papillotement instantané) \\
$\mathrm{t}$ & Temps 3 distorsion de tension (papillotement instantané) \\
& \\
\hline
\end{tabular}




\section{RÉSUME}

Ce mémoire de maitrise traite de l'application de la technique de compensation série. La recherche vise le développement d'une méthode d'optimisation pour calculer le taux optimal de compensation série afin d'améliorer la performance du réseau de distribution radial. La méthode défini comme fonction objective la variation de tension sur la ligne de distribution qui devra être minimisée ainsi que des contraintes de stabilité à respecter. Ces contraintes sont basées sur le concept de stabilité en régime transitoire et permanent, elles sont: le transfert maximal de la puissance, les pertes de puissance réactive, l'oscillation de la vitesse des moteurs, le phénomène de papillotement ou clignotement, l'inversion du courant de court circuit et la résonance sous-synchrone (SSR). Toutes ces contraintes sont formulées sous forme d'équations et inéquations algébriques.

La mise en ouvre numérique et les simulations sont obtenues à l'aide du logiciel Matlab/Symspowersym Les performances de la topologie du réseau de distribution à étudier sont évaluées par de nombreuses simulations. 


\begin{abstract}
This research work concems an application of series compensation technique for long distance distribution network. Research aims at the development of an optimization method to compute the suitable degree of series compensation in order to improve performances of the radial distribution network. This method defines as the objective function the voltage variation on the distribution line which will be minimized subject to stability constraints to respect. The constraints are based on the concept of transient and steady state stabilities; those constraints are: maximum power transfer, losses of reactive power, oscillations of the motor speed, the phenomenon of flicker, the short circuit current inversion and sub-synchronous resonance SSR. All components of the optimization method are formulated as algebraic equations and inequations.
\end{abstract}

The numerical implementation of the work is carried out using the Matlab/Sys powersystems software. Performances of the distribution network topology are studied by several simulation scenarios. 


\section{INTRODUCTION}

Au cours des dernières années, la croissance constante de la population a forcé les compagnies d'électricité à entreprendre des nouveaux projets énergétiques pour fournir un service de bonne qualité aux utilisateurs résidentiels et industriels. Mais ces projets ont exigé de grands investissements dans la recherche de nouvelles solutions qui permettraient de maintenir la stabilité des réseaux électriques à travers les technologies dont les objectifs viseront à améliorer des variables tels que le transfert de la puissance aux niveaux exigés, le maintien des valeurs de tension acceptables ainsi que le facteur de puissance approprié entre autres variables importants.

En Amérique du Nord, le réseau de distribution d'Hydro-Québec de la région de l'Abitibi-Téminscamingue (Nord du Québec) possède de longues lignes de distribution de longueur située entre $20 \mathrm{~km}$ à $60 \mathrm{~km}$ à cause de l'ampleur du territoire. Ces longues lignes alimentent de grandes industries locales comme des mines et des scieries situées loin du poste de distribution et plusieurs collectivités locales connectées sur les mêmes lignes. Pendant les heures de fonctionnement à pleine charge, ces grosses usines présentent des variations rapides de la charge comme, par exemple, le démarrage de gros moteurs asynchrones et synchrones, l'entraînement des broyeurs, des treuils, etc. Ces variations produisent une chute considérable de tension au point de raccordement. Les tensions deviennent plus basses que les tensions admissibles, selon la norme de tensions recommandée pour les réseaux à courant alternatif de l'association canadienne de normalisation, CAN3-C235-83 (voir app. B, p. 174 à 175). À cause de la diminution de tension, les équipements ne fonctionnent pas d'une manière satisfaisante sous des conditions qui assurent la stabilité du réseau. Cette situation est néfaste autant pour l'industrie concernée que pour les clients résidentiels connectés à la même barre. À cette baisse de tension s'ajoute un contenu harmonique créé par des charges non-lineaire tels 
que les convertisseurs de puissance, les alimentations stabilisées et les ordinateurs dont les effets combinés détériorent la qualité de l'onde électrique.

Le but de ce projet consiste à développer une méthode d'optimisation basée sous des contraintes de stabilité du réseau où la fonction objective à minimiser est la variation de la tension aux barres connectées à la ligne de transport. La valeur optimale à déterminer est le taux de compensation série. L'originalité de notre recherche réside dans la planification de la méthode d'optimisation qu' intègre une formulation analytique des contraintes de stabilité et un développement des simulations pour valider le processus d’optimisation. Ce procédé considère les différents phénomènes qui affectent les composantes du réseau électrique. Les contraintes utilisées dans cette étude devront considérer tous les paramètres techniques liés aux phénomènes présentés au niveau des alternateurs, des transformateurs, de la ligne de transport et de la charge.

Dans le chapitre 1, nous analyserons les concepts les plus importants pour développer le projet, la stratégie à utiliser pour faire l'étude des différents grandeurs ainsi que les phénomènes qui participeront dans le projet.

Dans le chapitre 2, nous développerons une formulation mathématique des composantes de la méthode d'optimisation qui seront postérieurement évaluées afin d'assurer que le réseau fonctionne dans des conditions de stabilité.

Dans le chapitre 3 , nous expliquerons la méthode de calcul itérative du taux de compensation et de la position qui nous permet de maintenir la stabilité dans le réseau.

Dans le chapitre 4, les résultats analytiquement calculés de la méthode d'optimisation permettront d'effectuer des essais pour observer la performance du réseau électrique lors des perturbations. 


\section{CHAPITRE I}

\section{L'ÉTAT DE L'ART DANS LA COMPENSATION DES RESEAUX DE DISTRIBUTION}

L'énergie électrique étant très difficilement stockable, il doit $\mathrm{y}$ avoir en permanence un équilibre entre la production et la consommation. L'industrie qui utilise des machines toumantes caractérisées par leurs inerties mécaniques et/ou électriques, demande aux opérateurs des réseaux électriques de maintenir un équilibre afin de garantir une tension relativement constante. Normalement, face à une variation de puissance, le système électrique, après quelques oscillations, retrouve sa stabilité. Dans certains cas, le régime oscillatoire peut diverger, c'est pour ça que plusieurs études sont nécessaires afin de garantir la stabilité du réseau électrique. Le concept de stabilité devient comme l'élément primordial pour étudier le réseau électrique, en particulier, le cas d'un réseau industriel qui comprend plusieurs groupes moteurs, charges résidentielles et une installation du banc de condensateurs série. Cette étude permettra de vérifier l'état de stabilité ou instabilité, les causes et les effets induits par l'ajout de cette technologie dans le réseau ainsi que d'établir quelles sont les précautions à prendre. 


\subsection{L'étude de stabilité des réseaux électriques comme un critère de base dans le développement du projet}

Un système de puissance se trouve dans une condition d'opération de stabilité si toutes les quantités physiques qui sont mesurées et qui décrivent la condition d'opération du système sont constantes pour buts d'analyse. Lorsque le système se trouve dans une condition stable et qu'il se produit un changement soudain ou une séquence de changements dans les grandeurs du système, on dit que le système présente une perturbation de sa condition de stabilité. Ces perturbations peuvent être grandes et petites selon leur origine. Une grande perturbation se produit lorsque les équations non linéaires qui décrivent la dynamique du système de puissance ne pourront pas se modéliser comme des équations linéaires afin de développer une analyse de stabilité. Les défauts dans les systèmes de transport de l'énergie, les changements brusques de charge, les pertes d'unités génératrices et les manœuvres dans des lignes sont des exemples de grandes perturbations (Grainger et Stevenson, 1997).

Si le système de puissance fonctionne sous une condition de stabilité stable et se produit un changement qui peut être analysé de manière appropriée à travers ses équations dynamiques algébriques linéaires, on dira que cette perturbation devient petite. Un exemple d'une perturbation petite peut être un changement dans le gain d'un régulateur de tension dans le système d'excitation d'un groupe alternateur.

Les réseaux électriques sont dits stables si, depuis la présence d'une perturbation, ils reviennent sous les mêmes conditions d'opération de stabilité en régime permanent. Cependant, si après une grande perturbation et si le réseau fonctionne dans une condition différente d'opération, on dira qu'il se trouve dans une condition transitoirement stable (Glouver et Sarma, 1994).

Généralement, les études de stabilité en régime permanent sont moins étendues dans ses buts que les études de stabilité transitoire et impliquent fréquemment une seule 
machine qui opère et reliée à une barre infinie ou quelques machines qui présentent des petites perturbations. Ainsi, les études de stabilité en régime permanent examinent la stabilité du système sous des variations incrémentielles dans les grandeurs ou sous des conditions d'opération autour du point d'équilibre. Alors, les équations différentielles non linéaires et algébriques du système sont remplacées par un ensemble d'équations linéaires qui se résolvent à travers des méthodes d'analyse linéaire afin de déterminer si le système est stable (Grainger et Stevenson, 1997).

D'ailleurs, la finalité de l'étude de stabilité proposée dans le projet sera de déterminer si les variables des machines perturbées (d'alternateur et des groupes moteurs), en incluant la compensation série, reviennent au point d'opération stable avec une vitesse constante. Une étude de stabilité suit une certaine logique et contient plusieurs étapes qui seront considérées comme le plan à suivre pour développer le projet. Les étapes d une étude de stabilité peuvent se classifier comme suit :

\subsubsection{L'étude préliminaire}

Comme la précision des résultats dépend directement des caractéristiques du réseau de distribution, l'étude commencera par chercher les valeurs numériques exactes des composantes du réseau. Ensuite la modélisation consiste à décrire quantitativement les lois physiques qui régissent le fonctionnement des éléments du réseau.

Dans le projet, on considérera les différents phénomènes qui se présentent et on inclura les critères techniques établis par les normes d'opération des réseaux électriques. Le calcul des conditions initiales commence avec des écoulement de puissance qui est déterminé par l'ordinateur dont le programme calcule des grandeurs comme des tensions aux noeuds, des courants et des puissances dans les branches, les sources, les charges et les machines. 


\subsubsection{Simulation numérique}

La topologie et les composantes du réseau peuvent changer selon différents types de perturbations comme des courts-circuits, pertes partielles d'alimentation (lignes, transformateurs, générateurs), démarrages de gros moteurs et incidences de phénomènes très communs perçues dans les réseaux électriques. Aujourd'hui, plusieurs logiciels permettent d'analyser la performance du réseau. Ils developpent numériquement les systèmes d'équation qui représentent le comportement des machines et des composantes du réseau. Toutes les charges et les alternateurs contribuent au fonctionnement de l'ensemble et le problème devient très grand. Pour cette raison, il convient de simplifier les composantes dans un seul élément équivalent qui permettra de représenter un groupe de moteurs en un moteur équivalent ayant un comportement identique.

D' ailleurs, différents scénarios sont expérimentés pour traiter diverses perturbations simulées afin de trouver les résultats et démontrer l'efficacité de l'étude de stabilité.

\subsubsection{Les résultats}

Les résultats se traduisent par des courbes d'évolution dans le temps tels que les tensions et courants sur les différentes barres, le condensateur, la ligne de transport ainsi que les puissances transitées, les variables des mesures des machines (courant, vitesse, couple mécanique), les régulations de tension et des entraînements mécaniques. Ils concernent donc le fonctionnement du système électrique en régime perturbé et ils permettent la vérification de la stabilité, la capacité de secours possible lors défaut, la confirmation du type de protection à installer et le réglage des régulations. 


\subsection{Description des solutions envisagées face à la problématique exposée dans le projet}

Les ingénieurs et les chercheurs ont développé des dispositifs capables de diminuer des perturbations présentées dans les réseaux électriques. Ces dispositifs utilisés par l'industrie sont les dispositifs FAITS, (FACTS, Flexible AC Transmission System), UPS (Uninterruptive Power Supplies), les régulateurs de tension et les différentes techniques de compensation de l'énergie. Ces techniques deviennent comme des solutions acceptables permettant non seulement de supporter les changements brusques de la charge mais également d’améliorer le profil de tension. Ces solutions comprennent aussi l'utilisation de l'électronique de puissance à haute amplitude de courant et de tension (Coonick, Green et Jaimoukha, 1999). D'abord, les dispositifs FAITS (FACTS), systèmes flexibles de transmission à courant continu, qui sont capables de commander les trois paramètres les plus importants dans le réseau électrique tels que les grandeurs de tension, l’angle de phase entre les tensions et l'impédance de la ligne. Ces dispositifs augmentent la capacité de transfert de puissance existante et ainsi améliorent la couverture des clients. De plus, ils permettent d'opérer le réseau sous des conditions de stabilité avec un impact minimal sur l'environnement. La construction des projets des nouvelles lignes de transport peut être évitée grâce à ces dispositifs en réduisant les coûts d'opération (Coonick, Green et Jaimoukha, 1999). Deuxièmement, l'utilisation de dispositifs comme les UPS (Alimentation de sécurité) pourront maintenir la puissance secondaire ou de secours en cas de perte de la puissance de service. Ces dispositifs protègent les systèmes électriques et électroniques, les contrôleurs de processus et les données, lorsque survient une chute de tension ou une perte totale de l'énergie. Cependant, il y a quelques désavantages car ils sont installés dans les charges locales ou clients résidentiels dont la couverture de protection est indépendante pour chaque équipement. Il est important de mentionner qu'il existe d'autres solutions que les entreprises de l'énergie pourraient utiliser. II s'agit de l'installation des régulateurs de tension à différents points de raccordement sur une ligne de distribution au point où survient une chute de tension. Ces dispositifs permettent de soutenir la tension aux 
niveaux acceptables. Cependant, le temps de réponse n'est pas adéquat car les régulateurs utilisent plusieurs minutes pour élever les tensions à des niveaux adéquats. Ce temps de réponse affecte la performance du réseau en injectant des oscillations qui produiront le papillotement de tension, un phénomène très commun dans les réseaux de distribution.

Plusieurs techniques de compensation du réseau ont été utilisées. Cela comprend la technique de compensation shunt qui intervient sur la tension à chaque barre, la technique de compensation série qui intervient l'impédance équivalente de la ligne de distribution et la technique utilisant le compensateur statique (Marceau, 1993). La technique de compensation shunt consiste en un groupe de condensateurs en parallèle avec les inductances shunt équivalentes de la ligne de transport. Ce schéma permet d'injecter la puissance en KVars demandées pour le système au point de raccordement du banc des condensateurs. Un condensateur shunt aura le même effet qu'un compensateur synchrone surexcité. Le but de cette compensation est de maintenir la tension constante au bout de la ligne indépendamment des variations de la charge (Johnson, 1994). La compensation série consiste en un groupe de condensateurs en série avec l'inductance équivalente de la ligne de transport. Ce schéma diminue l'impédance de la ligne, il réduit la dégradation de la tension et augmente la capacité de transport de la puissance (Miske 2001). Contrairement à la compensation shunt, la technique de compensation série change instantanément avec les variations de la charge ce qui crée un effet de dépendance. Voila un facteur important à considérer dans l'étude de stabilité d'un réseau de distribution compensé (Omata et Uemura, 1999).

De manière générale, il faut signaler que la compensation, avec l'utilisation des condensateurs shunt et série, corrige la tension en augmentant le facteur de puissance à la charge. Les paramètres comme l'amplitude de la charge, le facteur de puissance et la réactance de la ligne sont des données demandées par les deux techniques de compensation. L'application du condensateur série diffère du condensateur shunt au 
niveau de l'installation puisque la grandeur de la puissance réactive utilisée dans la compensation shunt est plus grande par rapport à la compensation série. Normalement, dans les applications industrielles, la valeur en KVars de la compensation série est d'un quart à un demi de la compensation shunt en permettant ainsi la réduction des coûts de l'installation (Westinghouse, 1976). Les perturbations produites par des installations compensées dans le réseau électrique sont devenues une contrainte très importante et une constante préoccupation due aux phénomènes de résonance produits par ces perturbations. De récentes études développées par Hydro-Québec ajoutent à la technique de compensation série, la technique de compensateur statique (SVC) dont l'objectif est d'injecter de la puissance réactive à la barre qui présente la chute de tension la plus basse. Mais cette technique élève les coûts d'opération due au fait que les composantes sont très cher ainsi que leur maintenance (Larsen et Gerin-Lajoie, 1990).

Selon les alternatives mentionnées précédemment, la compensation série est la solution la plus appropriée et la plus sollicitée par les entreprises de transport de l'énergie puisqu'elle présente un meilleur rapport d'efficacité vs coût d'opération. En termes de stabilité, cette technologie présente plusieurs avantages tels que, l'élévation du transfert de puissance, le temps de réponse grâce à l'effet instantané sur les profils de tension en évitant le phénomène de papillotement et essentiellement à la sensibilité aux variations de la charge. Ces caractéristiques sont des facteurs très importants particulièrement dans les régions où les lignes de distribution sont longues et les clients résidentiels dispersés et où, en plus, des clients industriels exigent une plus grande demande de puissance du réseau.

\subsection{Planification du projet comme une méthode d'optimisation basée sur le concept de stabilité du réseau électrique}

Comme on l'a vu, le concept de stabilité est un concept prioritaire à maintenir dans les réseaux électriques lors des perturbations. Plusieurs grandeurs sont affectées lorsque 
elles apparaissent. Les phénomènes les plus critiques peuvent être évalués et quantifiés au moyen d'une formulation mathématique. Une fois l'équation obtenue, il faut procéder à identifier les paramètres affectés par ces perturbations et les sélectionner comme les contraintes de stabilité qui seront les critères de l'optimisation.

D'ailleurs, la problématique exposée dans le projet indique que la variation de tension dans un réseau devient comme un problème très important dans le concept de stabilité du réseau de distribution. Cela nous permet de décrire cette situation causant l'instabilité du réseau comme un facteur perturbateur à minimiser.

Ayant choisi la compensation série comme la solution envisagée, on procède à développer une méthode d'optimisation en considérant les effets positifs que cette technologie pourrait produire dans la performance du réseau à la figure 1 , ainsi que des perturbations à éviter.

Les contraintes de stabilité à analyser pour développer la méthode d'optimisation sont entre autres :

- Les variations de tension à l'intérieur de la plage recommandée par la norme de tension admissibles, CAN3-C235-83 (voir app. B, p. 150 à 151).

- Les limites acceptables de la compensation série afin d'éviter la surcompensation de la ligne de transport.

- La performance du transfert maximal de la puissance et l'amélioration du facteur de puissance de la ligne de transport.

- L'effet de la position du condensateur série sur la ligne de transport.

- Les niveaux de puissance de court circuit minimal acceptable pour éviter le phénomène de papillotement.

- La performance du condensateur série par rapport aux différentes protections électriques sur le condensateur série. 
- Les pertes de puissance active et réactive dans le réseau électrique.

- Étude de réponse en fréquence pour identifier les fréquences de résonance sous-synchrone et hyper-synchrone.

Voici les essais à réaliser pour valider les résultats de la méthode d'optimisation :

- Mise sous tension de la ligne: On examinera le courant d'envahissement des transformateurs raccordés à la ligne.

- Déclenchement de la ligne de transport sans défaut.

- Défauts triphasés en aval et en amont du condensateur série.

- Démarrage des gros moteurs asynchrones.

- Variation brusque de la charge mécanique des moteurs.

- Analyse de réponse en fréquence pour identifier le phénomène de résonance sous-synchrone lors du démarrage des moteurs asynchrones et proposer une solution pour les éviter. 
Transformateur en parallele Delta-Y $120 \mathrm{kV} / 26.4 \mathrm{kV}, 13.5 / 18 / 22.5 \mathrm{MVA}$ $\mathrm{R}_{\mathrm{t} 1}=0.0185, \mathrm{X}_{\mathrm{tl}}=0.345$, $\mathrm{Rm}=1150 \mathrm{pu}, \mathrm{Ym}=700 \mathrm{pu}$ at $\mathrm{V}_{1}=1.05 \mathrm{pu}$

Charge R, L: IMVA

Transformateur $\mathrm{Y}-\mathrm{Y}$ $25 \mathrm{kV} / 600 \mathrm{~V}, 833.33 \mathrm{KVA}$ $\mathrm{R}_{\mathrm{t} 2}=0.002 \mathrm{pu} \mathrm{X} \mathrm{X}_{\mathrm{t} 2}=0.08 \mathrm{pu}$

4 Groupes moteurs

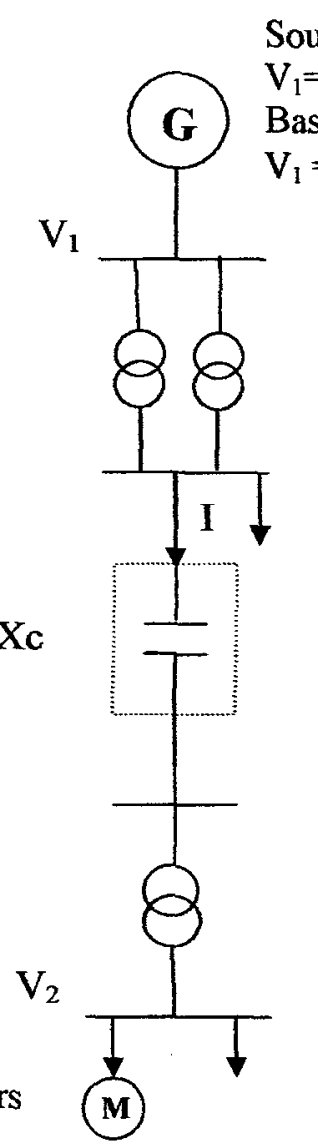

Source equivalente Thevenin Hydro-Quebec $\mathrm{V}_{1}=1.05 \mathrm{pu}, \mathrm{Z}=0.03+\mathrm{j} 0.2 \mathrm{pu}$

Base : 100MVA, $120 \mathrm{kV}$ $\mathrm{V}_{\mathrm{l}}=123 \mathrm{kV}$ to $126 \mathrm{kV}$

Ligne de distribution de $60 \mathrm{kms}$ $25 \mathrm{kV}$, type $477 \mathrm{MCM}$ AL avec neutre $\mathrm{Zl}=0.123+\mathrm{j} 0.395 \mathrm{ohm} / \mathrm{km}$ $\mathrm{Z} 0=0.431+\mathrm{j} 1.240 \mathrm{ohm} / \mathrm{km}$ $\mathrm{Cl}=30.567 \mathrm{nF}, \mathrm{CO}=11.003 \mathrm{nF}$

Condensateur protégé par Schema NGH

Charge R, L: 1MVA

Figure 1 Diagramme du réseau de distribution radial de Hydro-Québec 


\section{CHAPITRE II}

\section{DÉVELOPEMENT DE LA MÉTHODE D'OPTIMISATION PAR L'ÉVALUATION DES CONTRAINTES DE STABILTTÉ}

Dans ce chapitre, nous développons une étude préliminaire des composantes de la méthode d'optimisation dont le but de trouver la valeur optimale du taux de compensation série et sa position. On identifie et définit la fonction objective et les contraintes. Selon le mandat du projet, la fonction objective à minimiser sera la variation de tension sur la ligne de distribution. Les grandeurs électriques, les phénomènes et les normes techniques d'opération du réseau seront les contraintes de stabilité. Comme la méthode est itérative, on utilise une solution initiale de l'écoulement de puissance qui est appliquée au réseau afin d'obtenir leurs grandeurs pour évaluer la fonction objective et les contraintes de stabilité.

\subsection{Méthode d'optimisation appliquée dans le projet}

La théorie de l'optimisation est un cadre mathématique permettant d'interpréter et de résoudre dans les mêmes termes un grand nombre de problèmes d'analyse numérique. On part de la préexistence d'un modèle mathématique et d'un critère à optimiser. Un modèle mathématique est le premier pas vers une analyse quantitative et qualitative d'un système. Un modèle contient généralement des variables dites d'état et des variables dites de commande. Ces variables sont liées entre elles par des relations, dites contraintes, statiques ou dynamiques (Culioli, 1994). Le critère qu'on cherche à 
optimiser est une grandeur sur laquelle les variables de commande peuvent influer. La méthode d'optimisation proposée devra fournir de la solution optimale pour les modèles mathématiques correspondants. Si le développement de l'optimisation a été bien fait, cette solution optimale permettra d'améliorer le comportement du système en question, mais aussi de mieux le connaître. On s'attachera, dans l'application de l'optimisation, à ne pas découpler l'aspect modélisation de l'aspect optimisation. Dans la pratique, il est important de comprendre que l'on ne dispose que d'une information locale sur la fonction à minimiser. Cette information peut être un ensemble de valeurs de la fonction des points choisis par l'utilisateur. On peut aussi disposer de valeurs de la dérivée de cette fonction ou d'approximations de cette dérivée (différences finies ou valeurs exactes entachées d'un bruit de mesure ou de calcul), ou d'indications sur le comportement à l'infini de la fonction. Il s'agit d'élaborer une stratégie intelligente pour choisir en quels points on désire plus d'information (Culioli, 1994). D'abord, on doit définir la fonction $f$ comme une fonction numérique définie sur un intervalle $[a, b]$ et la variable $X$ comme l'argument de son minimum sur cet intervalle.

$$
\min f(X) \text { sous } h(X)
$$

On définie $h$ comme des contraintes qui permettront d'évaluer la fonction objective dans son intervalle. Ses contraintes sont formulées comme des égalités (2-2), inégalités (2-3) et des valeurs nulles (2-4).

$$
\begin{gathered}
h(X)=p \\
h(X) \leq 0 \text { ou } h(X) \geq 0 \\
h(X)=0
\end{gathered}
$$

\subsection{Identification de la fonction objective et les contraintes de stabilité}

Dans ce projet, nous proposons le développement d'une méthode d'optimisation en considérant les paramètres électriques, les perturbations qui conduisent à l'instabilité, les 
normes et critères techniques qui régissent l'opération des réseaux de distribution de l'énergie afin de maintenir leur stabilité. Tous ces éléments seront les variables d'entrée et les critères d'optimisation. D'abord, on doit identifier et déterminer la fonction objective et des contraintes que nous avons définies comme les contraintes de stabilité puisque celles-ci constituent la base des critères d'opération pour lesquels le réseau est stable.

Le but du projet est de trouver la valeur optimale du taux de compensation série. Cette valeur devra réduire la variation de tension sur une ligne de distribution de longue distance. Selon le mandat du projet, la variation de tension, expression (2-5) doit être minimisée. Cette expression inclura la variable qui représente la compensation série dont la valeur influera les contraintes de stabilité du réseau de distribution.

$$
\operatorname{Min} \Delta V=f(X c)
$$

En ce qui concerne les contraintes de stabilité, elles sont identifiées et définies comme suit:

- Degré de compensation série, hl.

- Transfert maximal de puissance, $\mathrm{h} 2$.

- Oscillation de vitesse de rotation, h3.

- Inversion du courant de court circuit, h4.

- Phénomène de papillotement, h5.

- Pertes de puissance réactivé, h6.

- Résonance sous-synchrone, h7.

\subsection{Définition de la fonction objective : Variation de tension $\Delta V$}

D'abord, la réduction de la variation de tension est considérée comme l'objectif le plus important de la compensation série. L'ajout de cette compensation consiste à 
installer un condensateur qui se comporte comme un régulateur de tension en produisant une élévation instantanée et continue à partir du positionnement du condensateur.

Par contre, la réduction de variation de tension est liée à la performance des paramètres qui déterminent la stabilité du réseau tels que le facteur de puissance, le courant de la charge, le taux de compensation série, la puissance active et réactive consommée dans le système (Miske, 2001). Pour les caractéristiques antérieures, on considère l'équation qui exprime la réduction de la variation de tension comme fonction objective à minimiser dans la méthode d'optimisation.

La réduction de variation de tension peut être définie par rapport aux deux conditions de performance de tension: Profil de tension sans compensation et avec compensation série (Miske, 2001). Pour la première condition de profil de tension sans compensation, le réseau de distribution radial est représenté à la figure 2 (a) et le diagramme vectoriel, figure 2 (b).

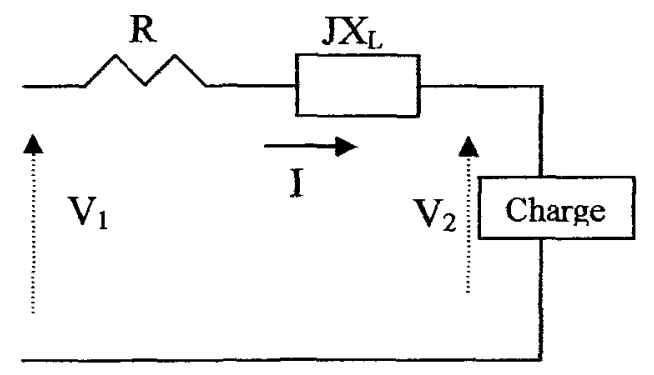

(a)

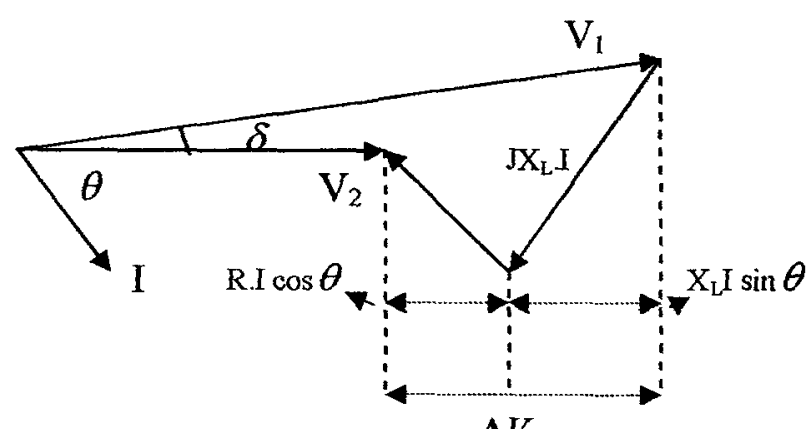

(b)
$\Delta V$

Figure 2 : Condition de profil de tension sans la compensation série (a) Circuit équivalent du réseau de distribution radial et (b) diagramme vectoriel de variation de tension.

Selon le diagramme vectoriel ci- dessus, la variation de tension par phase peut s'exprimer de manière approximative en fonction de la puissance active et réactive (Miske, 2001): 
Puissance active: $\quad \mathrm{P}_{\mathrm{R}}=\mathrm{V}_{2} \cdot \mathrm{I} \cdot \cos \theta$

Puissance réactive : $\mathrm{Q}_{\mathrm{R}}=\mathrm{V}_{2} \cdot \mathrm{I} \cdot \sin \theta$

À partir des puissances active et réactive, on obtient l'équation suivante qui représente la variation de tension à la charge :

$$
\Delta V \equiv V_{1}-V_{2} \cong R \cdot I \cdot \cos \theta+X_{L} \cdot I \sin \theta
$$

La puissance apparente à la charge en fonction de la tension et du courant s'écrit comme (2-9) :

$$
S_{R}=V_{2 . I}
$$

L'angle entre les tensions à l'extrémité de la ligne en fonction de la puissance active et réactive devient :

$$
\begin{gathered}
\cos \theta=\frac{P_{R}}{S_{R}} \\
\sin \theta=\frac{Q_{R}}{S_{R}}
\end{gathered}
$$

On ajoute les équations (2-9, 2-10 et 2-11) dans l'équation (2-8) pour arriver à l'équation (2-12). Cette expression donne la valeur approximative de la variation de tension sur la ligne :

$$
\Delta V=\mathrm{V}_{1}-\mathrm{V}_{2}=\frac{P_{R} \cdot R}{V_{2}}+\frac{Q_{R} \cdot X_{L}}{V_{2}}
$$

En résolvant l'équation (2-13), on obtient l'équation qui exprime la première condition de tension :

$$
\Delta V \approx \frac{P_{R} \cdot R+Q_{R} \cdot X_{L}}{V_{2}}
$$

Pour la deuxième condition de profil de tension avec la compensation série, il faut ajouter la réactance capacitive en série avec la réactance de la ligne (Miske, 2001). La 


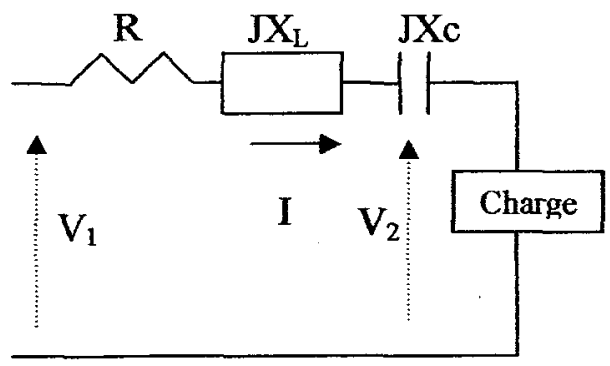

(a)

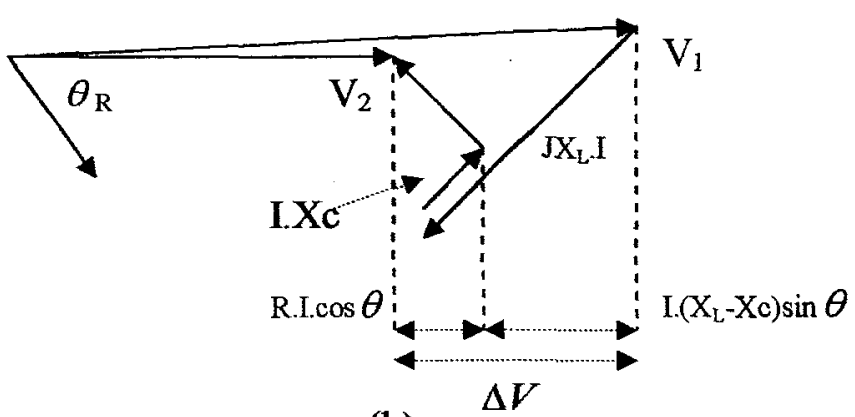

(b)

Figure 3 : Condition de tension avec compensation série (a) Circuit équivalent du réseau de distribution radial. (b) diagramme vectoriel de variation de tension.

Comme dans la figure 2 , une réduction importante de variation de tension est produite lorsqu'il s'ajoute à la réactance inductive de la ligne, la valeur de la réactance du condensateur série.

L'équation (2-14) contient la valeur de la réactance du condensateur série et des contraintes de stabilité du réseau indiqué antérieurement dans la formulation de la méthode d’optimisation proposée avec les inégalités ou contraintes de stabilité (Miske, 2001).

$$
\Delta V \cong \frac{P_{R} \cdot R+Q_{R} \cdot\left(X_{L}-X c\right)}{V_{2}}
$$

$P_{R}$ : Puissance active absorbée par la charge. $\quad V_{2}$ : Tension à la fin de la ligne $\mathrm{Q}_{\mathrm{R}}$ : Puissance réactive absorbée par la charge. $\mathrm{R}$ : Résistance totale de la ligne de transport. $\mathrm{X}_{\mathrm{L}}$ : Réactance totale de la ligne de transport $\mathrm{Xc}$ : Réactance capacitive compensation série.

\subsection{1 Évaluation de la fonction objective}

L'évaluation de la fonction objective est développée au moyen d'une solution initiale d'écoulement de puissance appliquée dans un modèle typique du réseau de 
distribution radial. Ce modèle devient comme le réseau qu'on étudiera pour développer le projet (voir app. A, p. 141 à 149). Les intervalles d'opération du taux de compensation série sont définis à partir de la norme canadienne de tensions recommandées par les réseaux à courant alternatif de 0 à $50 \mathrm{kV}$. La norme canadienne de normalisation (voir app. B, p. 150 à 151) stipule que les limites recommandées pour les variations de tension à courant alternatif doivent être entre 540 et $625 \mathrm{~V}$. Le tableau 1 montre les valeurs de tension obtenues de la solution initiale de l'écoulement de puissance.

\section{Tableau 1}

Résultats évaluation de la fonction objective à différentes taux de compensation série

\begin{tabular}{|c|c|c|c|c|c|}
\hline $\mathrm{Xc}(\Omega)$ & $\tau(\%)=\frac{X_{C}}{X_{L}}$ & $\begin{array}{c}\text { V2 }(25 \mathrm{kV}) \\
\text { Primaire } \\
\text { transformateur }\end{array}$ & $\begin{array}{c}\mathrm{V} 2(600 \mathrm{~V}) \\
\text { Secondaire } \\
\text { transformateur }\end{array}$ & $\begin{array}{c}\text { V2 (p.u.) } \\
\text { Secondaire } \\
\text { transformateur }\end{array}$ & $\Delta \mathrm{V}(\mathrm{V})$ \\
\hline 0 & 0 & 24.06 & 577.59 & 0.9626 & 940 \\
\hline 8 & 33.76 & 24.24 & 581.89 & 0.9698 & 760 \\
\hline 9 & 37.97 & 24.26 & 582.43 & 0.9707 & 740 \\
\hline 10 & 42.19 & 24.29 & 582.96 & 0.9716 & 710 \\
\hline 11 & 46.41 & 24.31 & 583.5 & 0.9725 & 690 \\
\hline 12 & 50.63 & 24.33 & 584.03 & 0.9734 & 670 \\
\hline 13 & 54.85 & 24.35 & 584.56 & 0.9743 & 650 \\
\hline 14 & 59.07 & 24.37 & 585.09 & 0.9752 & 630 \\
\hline 15 & 63.3 & 24.4 & 585.62 & 0.976 & 600 \\
\hline 16 & 67.51 & 24.42 & 586.15 & 0.9769 & 580 \\
\hline 17 & 71.73 & 24.44 & 586.68 & 0.977 & 560 \\
\hline
\end{tabular}

Par contre, la performance du condensateur série peuvant être observées en développant une étude du profil de tension par rapport à la position dans la ligne (Miske,2001). L'étude est développée avec des valeurs de réactance capacitive de $X c=0,8,13$, et $15 \Omega$. Cette étude nous aidera à identifier les effets de la variation de tension produite par l'ajout du condensateur série. 


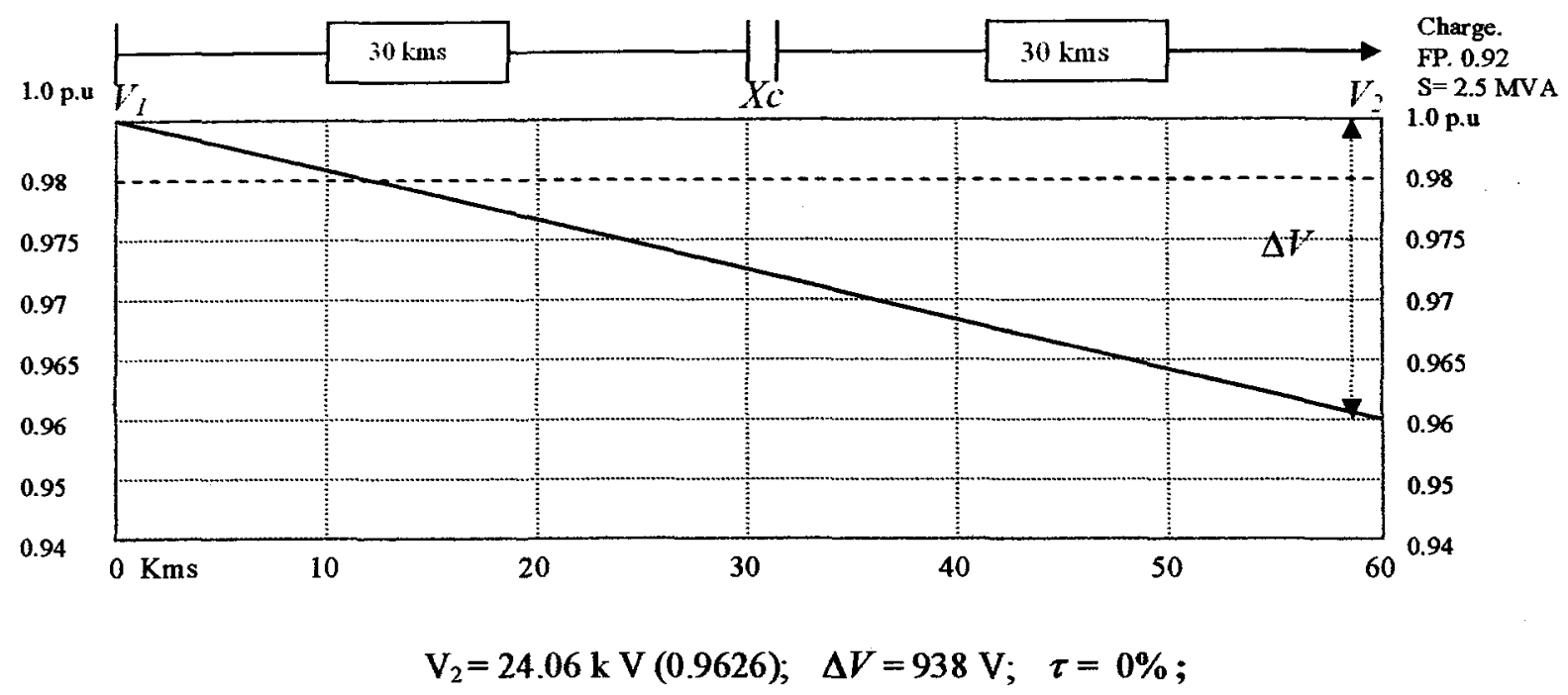

Figure 4 : Profil de tension sous compensation série $(X c=0 \Omega)$

La figure 4 montre la valeur de tension du réseau radial sans la compensation série ainsi que la tension $V_{2}=24.04 \mathrm{kV}(0.9626$ p.u). À partir de $X c=8 \Omega$, le réseau présente une réduction de la variation de tension de la charge en provoquant une élévation instantanée du profil de tension en aval du condensateur série, figure 5.

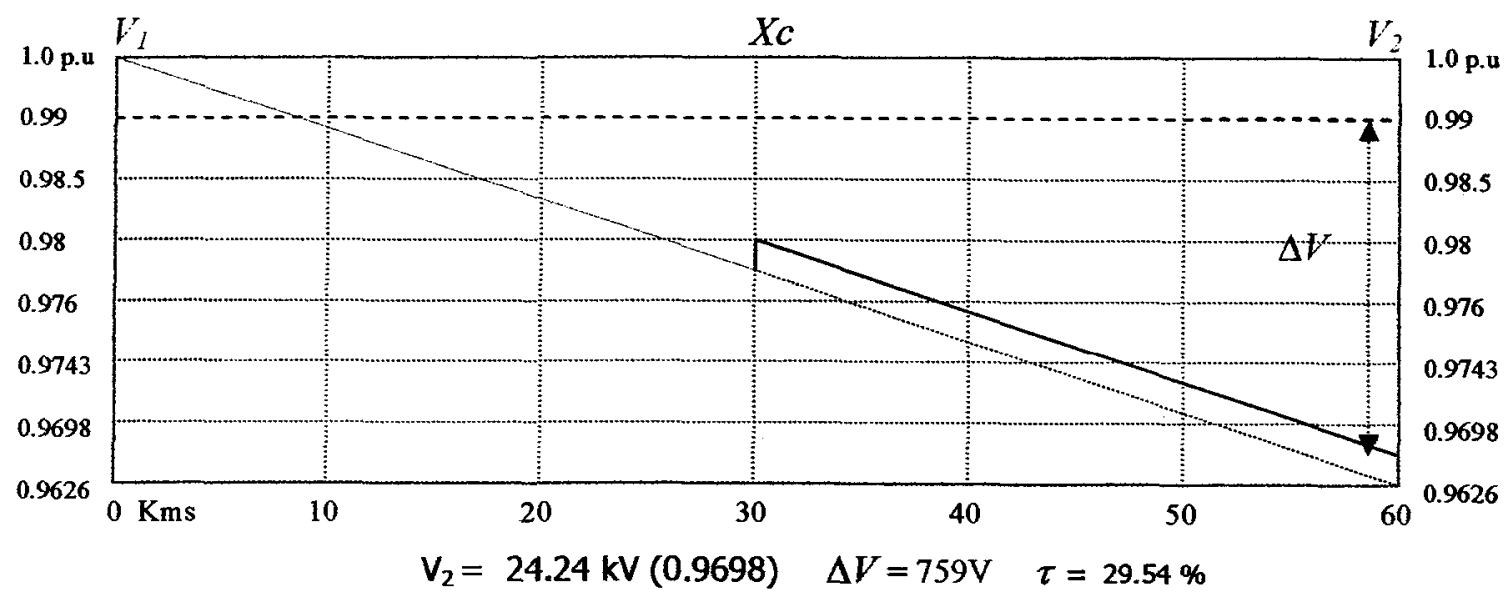

Figure 5 : Profil de tension à compensation série $X c=8 \Omega$ 
L'ajout de la réactance série, à partir de $X c=8 \Omega$, génère un effet positif sur le profil de tension à la fin de la ligne en réduisant la variation de tension à $\Delta V=759 \mathrm{~V}$, figure 6.

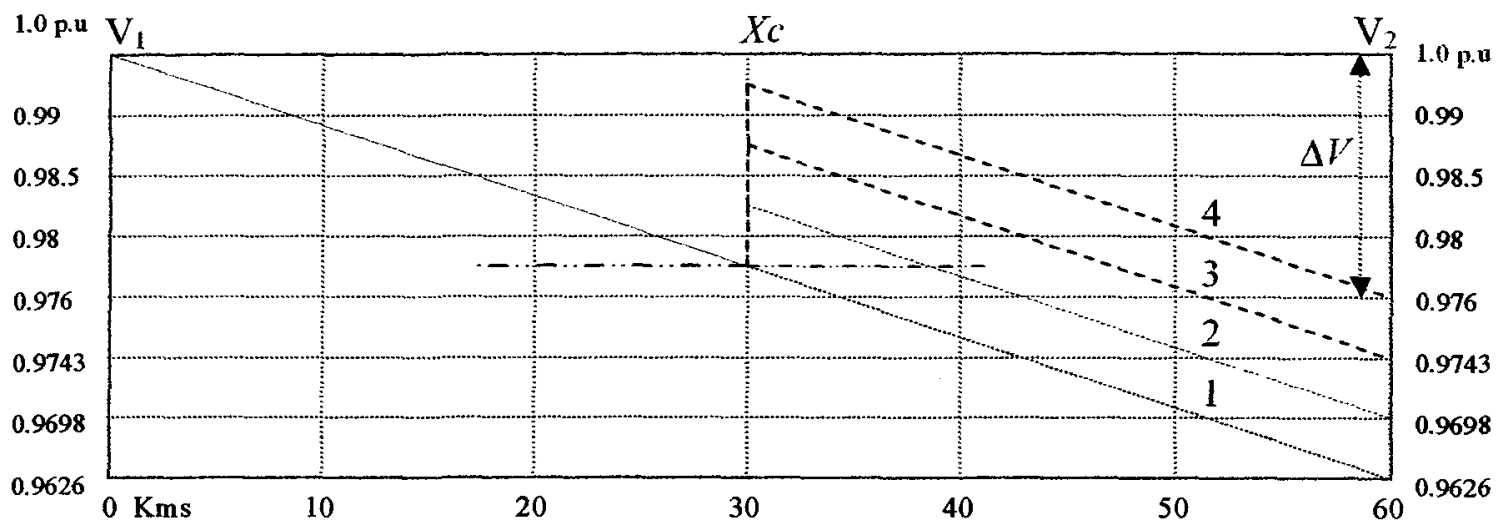

$1 \mathrm{~V}_{2}=24.06 \mathrm{kV}(0.9357) \quad \Delta V=940 \mathrm{~V} \tau=0 \%$ sans compensation série

$2 \mathrm{~V}_{2}=25.24 \mathrm{kV}(0.9502) \quad \Delta V=760 \mathrm{~V} \quad \tau=33.76 \% \quad X c=8 \Omega$

$3 \mathrm{~V}_{2}=25.35 \mathrm{kV}(0.9579) \quad \Delta V=650 \mathrm{~V} \quad \tau=54.85 \% \quad X c=13 \Omega$

$4 \mathrm{~V}_{2}=25.4 \mathrm{kV}(0.9537) \quad \Delta V=600 \mathrm{~V} \quad \tau=63.3 \% \quad X c=15 \Omega$

Figure 6 : Performance du Profil de tension avec $X c=0,8,13,17 \Omega$

En conclusion, la présence de la compensation série affecte instantanément les conditions de tension en aval du condensateur série. L'accroissement de $X c$ permet de réduire $\Delta V$ sur la ligne en augmentant le profil de tension à partir des valeurs supérieures à 0.96 p.u.

\subsection{Contraintes de stabilité de la méthode d'optimisation}

\subsubsection{Contrainte de stabilité No 1 : Taux de compensation série}

La compensation série peut s'exprimer selon l'équation (2-15) comme le rapport entre la valeur de la réactance capacitive de la compensation série et la réactance inductive de la ligne de transport. 


$$
\tau=\frac{X_{C}}{X_{L}}
$$

La compensation série est habituellement utilisée pour des valeurs plus petites que $100 \%$. Une valeur du taux de compensation plus grande, c'est-à-dire $X c \geq X_{L}$, est considérée comme surcompensation, les réseaux électriques sont compensés jusqu'à des valeurs d'environ 70\% selon certaines normes (Christi et Luttzelberger.1991).

Le tableau 2 montre les valeurs du taux de compensation par rapport à la valeur de réactance capacitive ajoutée à la ligne. Le critère par lequel nous pouvons déterminer la valeur minimale du taux de compensation dépend des valeurs acceptables de tension au point d'utilisation (voir app. B, p. 150 à 151).

Par la ligne de distribution du réseau à étudier, la valeur de la réactance est calculée par l'équation (2-16) comme suit :

$$
X=R+j X_{L}=[0.134+\mathrm{j} 0.395 \Omega / \mathrm{km}] .60 \mathrm{kms}=8.04+\mathrm{j} 23.7 \Omega
$$

Tableau 2

Valeurs du Taux de compensation série.

\begin{tabular}{|c|c|c|c|c|c|c|c|c|c|}
\hline$X_{C}(\Omega)$ & 8 & 9 & 10 & 11 & 12 & 13 & 14 & 15 & 16 \\
\hline$\tau(\%)$ & 33.76 & 37.97 & 42.19 & 46.41 & 50.63 & 54.85 & 59.07 & 63.3 & 67.51 \\
\hline
\end{tabular}

\subsubsection{Contrainte de stabilité No 2 : Transfert maximal de puissance}

Premièrement, on définit le transfert de puissance comme la capacité du réseau à transmettre la puissance active produite par la source ou par d'autres alternateurs et charges branchées à la ligne de transport. Ce paramètre est déterminé par l'amplitude des tensions à chaque côté de la ligne, l'angle entre les vecteurs de tensions et l'impédance équivalente du système entre deux barres (Westinghouse, 1976). 
Le transfert de puissance est l'un des plus importants paramètres en terme de stabilité des réseaux électriques. Ce paramètre peut être amélioré à travers la méthode de compensation série. Le but de cette méthode est de maximiser la puissance d'un point d'opération en régime permanent suivi d'une perturbation à un autre point sous les mêmes conditions de stabilité sans perdre le synchronisme des altemateurs branchés au réseau. La transmission de puissance augmente la capacité de chargement du système pour supporter plus de charge en améliorant les profils de tension et le facteur de puissance (Duncan Glover J., 1994).

\subsubsection{Performance de transfert de puissance par rapport à la stabilité transitoire et la stabilité en régime permanent.}

Un paramètre considéré important dans la détermination du transfert de puissance est l'angle entre les vecteurs de tension. Cet angle définit la performance de la puissance transportée et permet de contrôler la stabilité transitoire et la stabilité en régime permanent. D'abord, l'étude de la stabilité transitoire du système de puissance nous permet de savoir si les altemateurs arrivent à la fréquence synchrone $(60 \mathrm{~Hz})$, deuxièmement, d'obtenir les nouveaux angles de puissance qui pourraient varier pendant des perturbations ou contingences comme les pertes de génération, des opérations de déclenchement de la ligne de transport, des défauts de court-circuit et des changements brusques de charge. Lors de ces contingences, la stabilité transitoire peut être identifiée typiquement pendant les premières secondes où la puissance de sortie mécanique et la tension de l'alternateur seront assumées comme constantes. La participation d'un groupe turbine-alternateur, l'excitation du réseau, la manœuvre du transformateur et le contrôle de l'écoulement de puissance peuvent stabiliser ou déstabiliser, pendant quelques minutes, le réseau lorsqu'une perturbation se produira. Le transfert maximal de puissance est obtenu sous des conditions idéales de stabilité en régime permanent lorsque l'angle entre les tensions est plus bas que $90^{\circ}$. Cependant, dans des conditions de stabilité, des réseaux électriques avec des alternateurs en synchronisme, peuvent présenter des perturbations qui limiteraient l'angle entre $30^{\circ}$ et $45^{\circ}$ degré (Westinghouse, 
1976). L'équation (2-17) détermine d'une manière générale (en négligeant la résistance de la ligne) le transfert de puissance selon la figure 7(a):

$$
P=\frac{V_{1} V_{2}}{X_{L}} \sin \delta
$$

La figure 7 (a) montre un réseau électrique initialement non compensé. On suppose que le réseau fonctionne en régime permanent. La puissance électrique est égale à la puissance mécanique $P_{\text {elec }}=P_{m e c}$, et l'angle $\delta_{0}=\delta$. Le transfert de puissance démarre lorsque le rotor de l'alternateur accélère aux valeurs plus grandes que $P_{\text {mec }}$ et ce transfert augmentera par rapport à l'élévation de l'angle de puissance et le rotor décélérera en produisant une élévation maximale de l'angle de puissance qui arrive à une valeur maximale de $\delta=\delta_{1}$, figure 7 (Oliveira, Gardos et Fonseca, 1991).
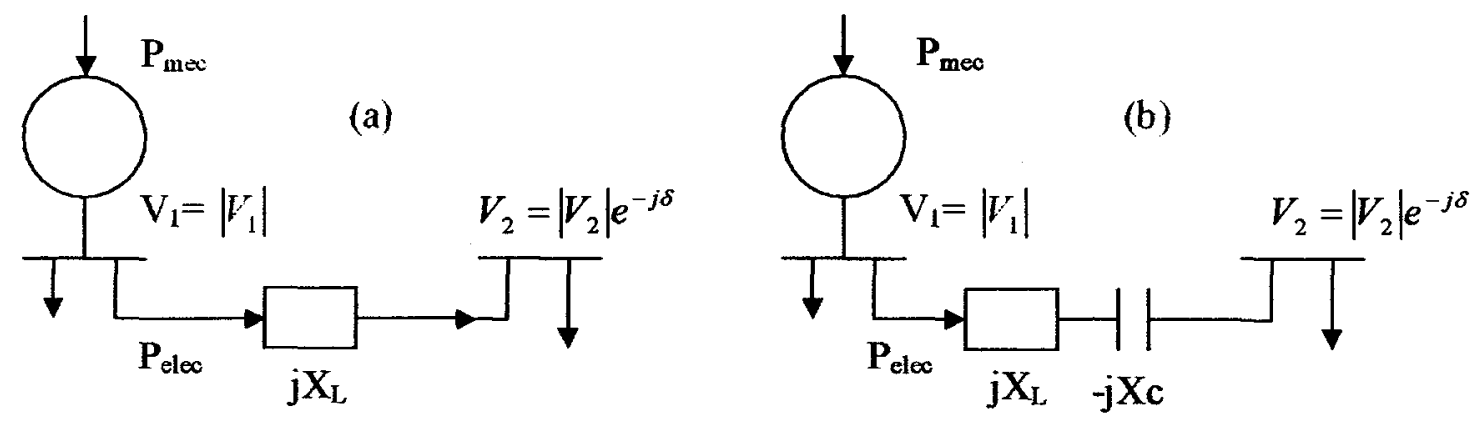

Figure 7 : Diagramme monophasé du réseau électrique de distribution radial (a)non compensé (b) compensé

Les pertes mécaniques et électriques dans le système stabilisent l'angle $\delta$ à un point d'opération en régime permanent $\delta_{1}$. Si l'angle $\delta$ dépasse $\delta_{2}$, figure 8 , la puissance électrique devient plus grande que la puissance mécanique et le rotor accélérera de nouveau en provoquant un accroissement de $\delta$, et par conséquent la perte de la stabilité (Oliveira, Gardos et Fonseca, 1991). 


\subsubsection{Effet de la compensation série sur le transfert maximal de puissance}

La compensation série aide à transférer la puissance sans changer les paramètres du réseau. Ce type de compensation consiste à ajouter une réactance capacitive à la réactance équivalente de la ligne de transport en réduisant sa grandeur. L'effet de cette réduction augmente les limites de transfert de puissance à des valeurs plus grandes que celle de la puissance d'opération. Le transfert de puissance du réseau est déterminé par l'équation (2-18).

$$
P=\frac{V_{1} V_{2}}{X_{L}-X_{C}} \sin \delta
$$

La figure 7 (b) montre la configuration du réseau électrique avec un condensateur série. L'effet instantané du condensateur augmente l'amplitude du courant due à la réduction de la réactance équivalente en provoquant un accroissement du transfert de puissance. La figure 8 montre l'effet sur l'angle entre les vecteurs de tension lorsqu'on augmente les valeurs de la réactance capacitive. (Wang, Dai et Tck Ooi, 1991).

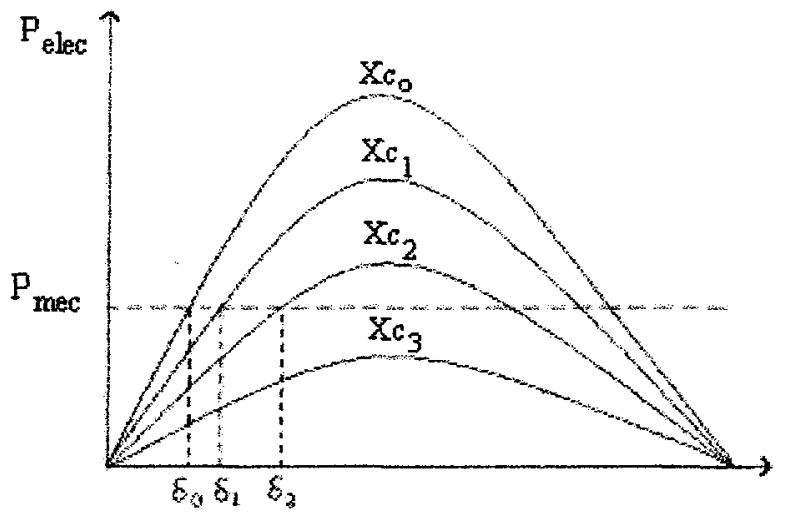

$X c: \quad X c_{3}<X c_{2}<X c_{1}<X c_{0} \quad \delta: \delta_{0}<\delta_{1}<\delta_{2}$

Figure 8 : Effet de la compensation série sur le transfert de puissance.

Pour la réactance capacitive $X c_{3}$, le réseau ne peut pas transmettre la puissance due au fait que la puissance électrique est plus petite que la puissance mécanique. Cela entraînerait l'instabilité du réseau. La compensation série, à partir de $X c_{O}$ jusqu'à $X c_{2}$, maintient le réseau dans les limites de stabilité considérant que $\boldsymbol{P}_{\text {elec }} \geq \boldsymbol{P}_{\text {mec }}$. 
Généralement, pour une compensation série de $50 \%$, la réactance équivalente se réduit à la moitié de sa grandeur, et le transfert de puissance double sa grandeur (Christi et Luttzelberger. 1991).

\subsubsection{Formulation du critère de transfert maximal de puissance du réseau électrique avec la compensation série considérant la résistance de la ligne de transport}

Le critère de transfert maximal de puissance est basé sur le développement d'une formulation qui concède de calculer la valeur de la puissance active aux différents niveaux de compensation en tenant compte des paramètres tels que le facteur de puissance, la résistance de la ligne, la réactance équivalente de la ligne et la tension à la charge. Cette formule mathématique est caractérisée par la précision et la sensibilité dues à la dépendance aux variations des paramètres précédemment décrits. II existe plusieurs méthodes pour formuler le transfert maximal de puissance (en négligeant la résistance du conducteur). On propose une formulation pour déterminer ce transfert maximal de puissance en considérant la résistance de la ligne. D'abord, le transit de puissance avec une contrainte sur la puissance réactive est défini selon les caractéristiques et les paramètres du réseau radial à la figure 9 .

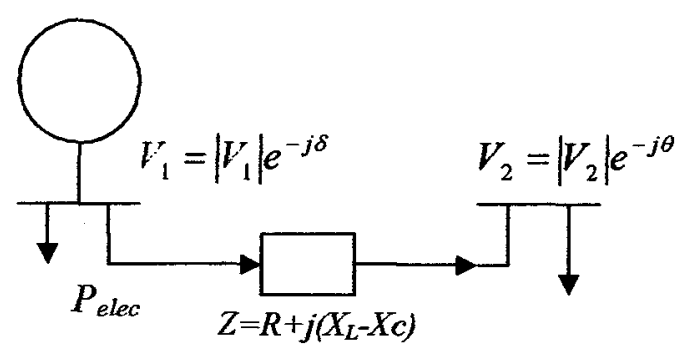

Tension barre alternateur : $V_{1} \angle \delta$ Tension à la charge : $\quad V_{2} \angle \theta$ Impédance totale de la ligne : $Z=R+j\left(X_{L}-X c\right)$

Figure 9 : Diagramme monophasé du réseau radial analysé pour développer le critère de transfert de puissance en considérant la résistance de la ligne de transport 
Selon la figure 9, la puissance active, équation (2-19) et la puissance réactive (220) deviennent ainsi:

$$
\begin{gathered}
P=\frac{V_{1} V_{2}}{Z} \cos (\delta-\theta)-\frac{V_{2}^{2}}{Z} \cos \delta \\
Q=\frac{V_{1} V_{2}}{Z} \sin (\delta-\theta)-\frac{V_{2}^{2}}{Z} \sin \delta
\end{gathered}
$$

En utilisant la formulation utilisée dans cette contrainte (voir app. C, p. 152 à 157), on obtient l'équation pour calculer le transfert maximal de puissance $P_{\max }(2-21)$ en fonction des tensions, l'angle de vecteur de tension de $V_{1}$ et $V_{2}$, le facteur de puissance à la charge et l'impédance équivalente de la ligne comme suit :

$$
P_{\max }=\frac{-V_{2}^{2} \cos (\delta-\theta) \cos x \pm \cos x \sqrt{V_{1}^{2} V_{2}^{2}-V_{2}^{4} \sin ^{2}(\delta-x)}}{Z}
$$

À partir de cette formulation, on détermine le transfert maximal de puissance aux différents taux de compensation série. Les paramètres de tension et des angles seront obtenus de la solution de l'écoulement de puissance initiale. Les résultats obtenus sont montrés dans le tableau 3 , et la courbe de la performance du transfert maximal de puissance sera montrée à la figure 10 .

\section{Tableau 3}

Résultats de transfert de puissance aux différents taux de compensation série

\begin{tabular}{|c|c|c|}
\hline $\mathrm{XC}$ & $\tau(\%)$ & $\begin{array}{c}\text { Transf. Max } \\
\text { Puissance (MW) }\end{array}$ \\
\hline 8 & 33.76 & 3.9 \\
\hline 10 & 42.19 & 3.98 \\
\hline 12 & 50.63 & 4.05 \\
\hline 13 & 54.85 & 4.08 \\
\hline 14 & 59.07 & 4.12 \\
\hline 16 & 67.51 & 4.18 \\
\hline 17 & 71.73 & 4.2 \\
\hline
\end{tabular}




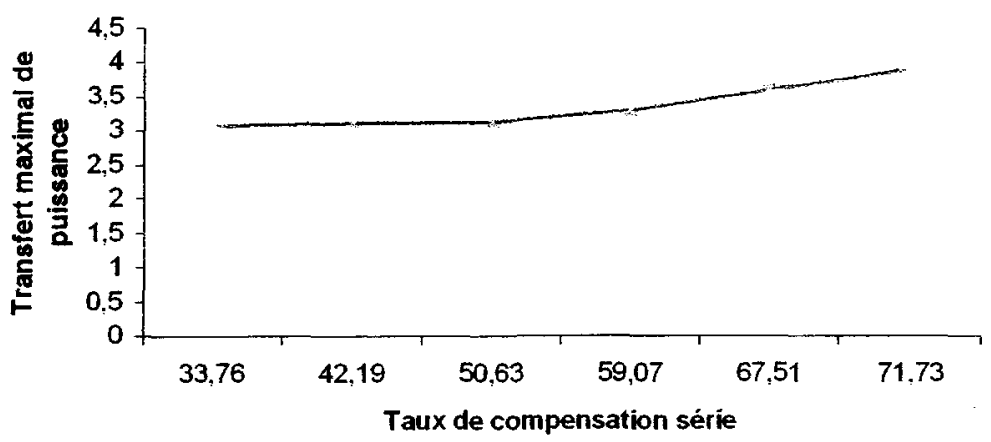

Figure 10 : Performance de transfert maximal de puissance aux différents taux de compensation série.

D'ailleurs, la performance de la puissance peut s'observer lorsque l'on calcule la valeur de la réactance $X_{L}$ qui varie par rapport à l'mplitude de la ligne de distribution selon la distance de transfert de puissance (Zheng et Gao, 2002). Les résultats obtenus, figure 11, montrent que pour le réseau sous des conditions initiales.

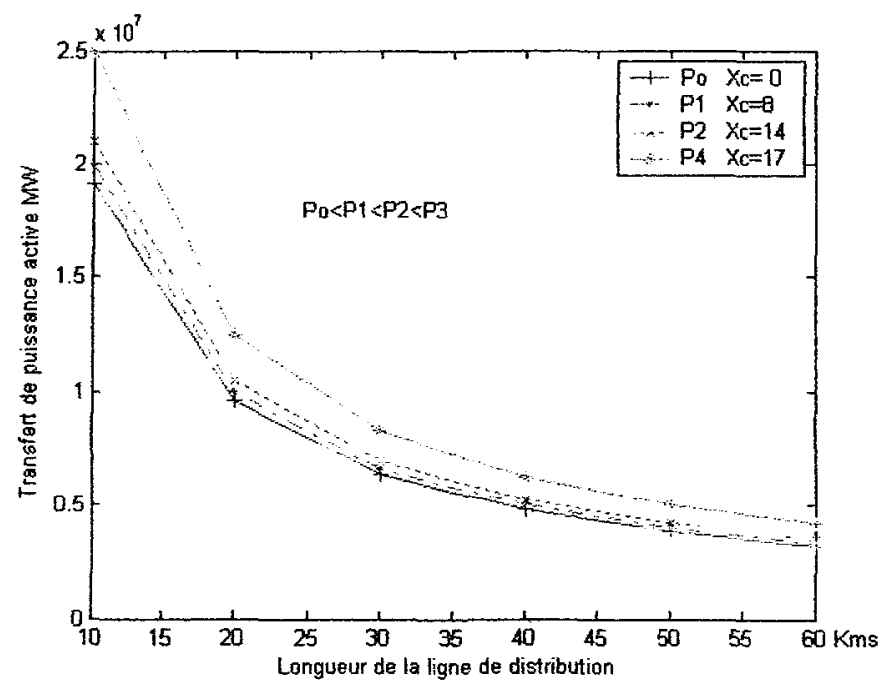

Po : Transfert de puissance active sans compensation série. Po: $2.3 \mathrm{MW}$

$P_{I}:$ Transfert de puissance active à $X c=8 \Omega \quad \tau=33.76 \% \quad P_{l}: 3.9 \mathrm{MW}$

$\begin{array}{llll}P_{2}: \text { Transfert de puissance active à } X c=13 \Omega & \tau=54.85 \% & P_{2}: 4.08 \mathrm{MW}\end{array}$

$P_{3}:$ Transfert de puissance active à $X c=15 \Omega \tau=63.3 \% \quad P_{3}: 4.16 \mathrm{MW}$

Figure 11 : Performance de la puissance active par rapport à la longueur de la ligne. 
La puissance active initiale d'opération sera $P o=2.3 \mathrm{MW}$. Lorsque la valeur de la réactance capacitive de la compensation série augmente, la courbe de performance de puissance montre un accroissement sur la ligne de distribution. Trois cas sont développés pour démontrer cette caractéristique. Réactance capacitive de $X c=8,13$ et $15 \Omega$ et des valeurs de transfert de puissance de $P_{1}=3.9 \mathrm{MW}, P_{2}=4.08 \mathrm{MW}$ et $P_{3}=$ $4.16 \mathrm{MW}$ respectivement.

\subsubsection{Contrainte de stabilité No 3 : Oscillations de la vitesse des moteurs}

Le phénomène se produit lorsque la vitesse du rotor présente des oscillations de vitesse pendant le démarrage ou en opération normale. Ces oscillations sont sinusoïdales et peuvent osciller légèrement par-dessus ou par-dessous la vitesse synchrone en produisant une accélération et décélération des moteurs (Truong, 2004). De même manière, il existe des manœuvres habituelles dans les réseaux électriques comme le déclenchement de circuits électriques, des variations de charge ou de l'excitation des moteurs entre autres, qui pouvaient produire ces oscillations et la présence du phénomène de papillotement de tension dans certains cas. (Westinghouse, 1976).

Plusieurs études montrent que le phénomène est aussi provoqué par le chargement du condensateur série et par le transfert de puissance active et réactive dans la ligne de distribution. Toutefois, il existe un paramètre utilisé pour mesurer cet échange de I'énergie. Le paramètre est considéré comme le rapport $\mathrm{R} / \mathrm{X}$ ou aussi appelé comme le facteur de dissipation. Ce facteur se définit comme le rapport entre la dissipation de l'énergie causée par l'échauffement de la résistance totale de la ligne et la capacité d'accumulation de l'énergie de la réactance totale de la ligne. Ce phénomène est aussi causé grâce à l'écoulement de puissance active qui est capable de compenser la variation de tension occasionnée par la composante résistive de la ligne de distribution (Twining et Holmes, 1998).

Cependant, l'excessif échauffement du matériel du condensateur série engendre des pertes dans la résistance provoquant aussi des amplitudes du courant trop hautes. 
Celles-ci créent des oscillations de fréquence plus grandes que la fréquence naturelle en affectant les composants intemes du condensateur série (Power Systems handbook, 1999). Le paramètre pour mesurer le phénomène d'oscillations de vitesse est appelé comme le facteur de dissipation (Power Systems handbook, 1999). Ce facteur est défini à partir de la tangente de l'angle de l'impédance totale de la ligne (2-22) :

$$
\tan \delta=\frac{R}{X_{L}-X_{C}}
$$

Ce facteur varie entre 0 et 1 et il montre des amplitudes très élevées, surtout pour les aires rurales dont les lignes de distribution se caractérisent par leurs longues distances. Pour développer cette contrainte, on définit la valeur de la résistance et la réactance de la ligne ainsi que la réactance capacitive du condensateur série. Les valeurs sont montrées dans l'équation (2-23):

$$
j X=\mathrm{j}\left(X_{L}-X c\right)=(23.7-X c) \mathrm{j}, R=8.04 \Omega
$$

Ensuite, on calcule la valeur du facteur de dissipation par rapport aux différents taux de compensation série. Voir table 4.

\section{Tableau 4}

Tangente de résistance et la réactance équivalente de la ligne par rapport au taux de compensation série.

\begin{tabular}{|c|c|c|c|}
\hline$X_{C}$ & $T$ & $\begin{array}{c}\text { Réactance } \\
\text { équivalente } X_{\mathrm{L}}-X_{c}\end{array}$ & $\mathrm{R} / \mathrm{X}$ \\
\hline 8 & 33.76 & 15.7 & 0.51 \\
\hline 10 & 42.19 & 13.7 & 0.59 \\
\hline 12 & 50.63 & 11.7 & 0.69 \\
\hline 14 & 59.07 & 9.7 & 0.83 \\
\hline 15 & 63.29 & 8.7 & 0.92 \\
\hline 15.66 (max) & 66.08 & 8.04 & 1.00 \\
\hline 16 & 67.51 & 7.7 & 1.04 \\
\hline 17 & 71.73 & 6.7 & 1.20 \\
\hline
\end{tabular}


Le critère pour analyser le facteur de dissipation nous indique que le phénomène d'oscillations de vitesse de rotor peut être évité en considérant l'équation (2-24) (Westinghouse, 1976).

$$
0 \leq \frac{R}{X_{L}-X_{C}} \leq 1 \quad \text { Si, } \mathrm{Xc}<\mathrm{X}_{\mathrm{I}} \text {, et } \mathrm{Xc} \leq \mathrm{X}_{\mathrm{L}}-\mathrm{R}
$$

Selon cette formulation, par un facteur de dissipation de 1, la valeur maximale de la réactance capacitive devient $X c=15.66 \Omega$, à un taux de compensation d'environ $66 \%$, voir tableau 4. Jusqu'à ce point, il est possible de maintenir la stabilité du réseau pendant une période normale d'opération en évitant l'échauffement dans l'installation du condensateur série et des courants élevés dans la ligne qui pourraient provoquer le phénomène précédemment décrit.

\subsubsection{Rapport entre le démarrage de moteurs et le facteur de dissipation $R / X$}

Dans le cas des machines synchrones, leur fonctionnement exige l'égalité des vitesses de rotation du rotor et du champ toumant statorique. En régime permanent, le couple électromagnétique et le couple extérieur de l'organe accouplé à la machine synchrone s'équilibrent exactement. Cependant, les perturbations de la marche synchrone apparaissent dès que cet équilibre est rompu. Ces perturbations sont provoquées par des fluctuations de l'état du réseau comme la variation du courant d'excitation, enclenchement et déclenchement des consommateurs entraînant des variations de tension et l'échauffement produit pour du courant élevé dans le condensateur série.

Pour une ligne de distribution excessivement compensée, le phénomène des oscillations de vitesse se produit si les moteurs démarrent pendant des périodes où la charge est faible. Ces oscillations sont évitées si l'angle de facteur de puissance de la charge (après le démarrage du moteur) est égal ou plus grand que l'angle d'impédance du circuit (avec le condensateur série). (Westinghouse, 1976). Pour les machines 
asynchrones, leur démarrage s'accompagne d'une dissipation d'énergie sous forme de chaleur dans les enroulements statorique et rotorique susceptibles de provoquer des échauffements dangereux surtout dans le rotor à cause des densités de courant très élevées. Le démarrage de la machine asynchrone peut se classifier en démarrage court dont le temps de démarrage est plus petit (10 secondes) et le démarrage long conduisant à un temps de démarrage de 60 à 100 secondes. L'appel de courant de ces démarrages est de l'ordre de 4 à 6 fois le courant nominal en provoquant des chutes de tension souvent inacceptables. Pour cette raison, les utilisateurs réduisent le courant de démarrage par des dispositifs annexes tout en assurant une mise en vitesse adéquate du groupe. L'étude du critère de la contrainte de facteur de dissipation $\mathrm{R} / \mathrm{X}$ permet d'éviter des courants trop élevés qui produisent des échauffements excessifs dans le condensateur série et dans les machines électriques branchées à la ligne de transport(Chatelain, 1989).

\subsubsection{Contrainte de stabilité No 4 : Puissance de court-circuit.}

Dans l'opération normale des systèmes de puissance, des perturbations dans les réseaux électriques réduisent les limites de stabilité transitoire dues aux changements brusques de la charge, des défauts et du déclenchement de lignes. Ces perturbations ont incité les entreprises de transport de l'énergie à installer des dispositifs comme le condensateur série. Celui-ci réduit ces perturbations en assurant une opération acceptable du réseau dans les limites de stabilité transitoire. Toutefois, il existe un aspect de sécurité très important à considérer, il s'agit de la protection du condensateur série. Ce dispositif sur la ligne augmente les niveaux du courant de court-circuit en comparant avec une ligne non compensée. Ces valeurs du courant dépendront de la localisation du condensateur et des caractéristiques de paramètres électriques du réseau (Oliveira, Gardos et Fonseca, 1991). La figure 12 nous permet d'étudier la performance d'un réseau électrique en régime transitoire sous différentes conditions du défaut. 


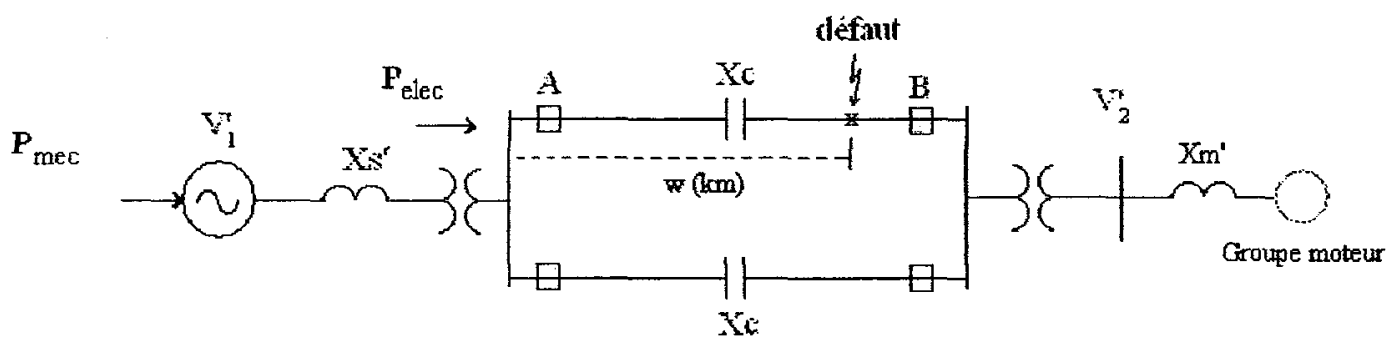

$V_{1}^{\prime}$ : Tension source.

$\mathrm{V}_{2}^{\prime}$ : Tension à la charge.

A, B : Disjoncteurs

$\mathrm{Xs}^{\prime}$ : Réactance sous transitoire de l'alternateur. w : Localisation défaut.

$P_{\text {mec }}$ : Puissance mécanique de l'alternateur $\quad P_{\text {elec }}$ : Puissance électrique

Figure 12 : Diagramme monophasé du réseau radial avec défaut en aval du condensateur série

D'abord, l'équation générale de la puissance pendant la période transitoire est définie comme l'expression (2-25):

$$
P=\frac{V_{1}^{\prime} V_{2}^{\prime}}{X^{\prime}} \sin \delta
$$

Il est important de souligner que la valeur de $X^{\prime}$ varie selon les conditions de défaut tel que défaut triphasé, phase-phase à la terre, et phase terre. En ce qui concerne le défaut triphasé, il réduit le chargement de la ligne aux valeurs proches à la limite de la stabilité. Ces caractéristiques sont montrés à la figure 13.

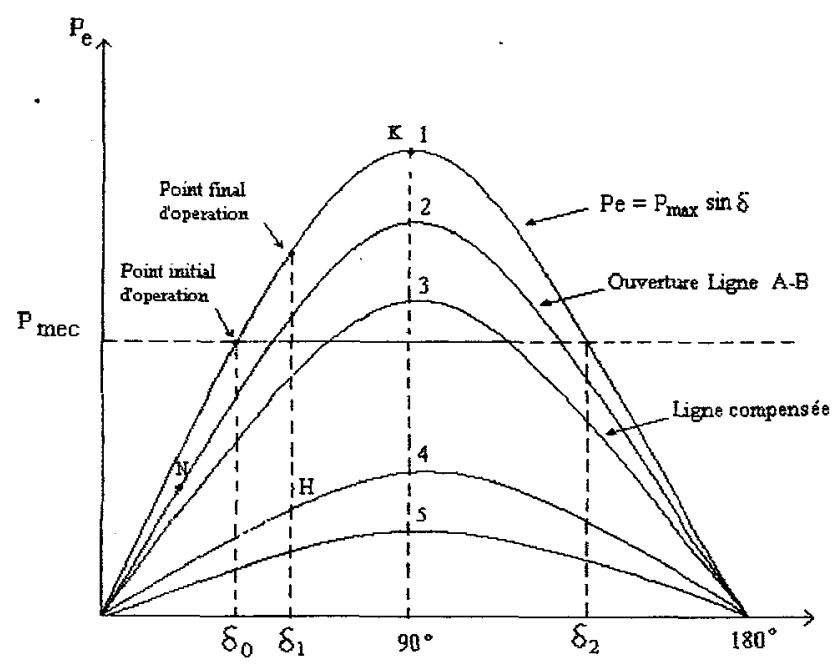

Figure 13 : Transfert de puissance par rapport à l'angle sous des conditions de court circuit. 
D'abord, on suppose que le réseau se trouve initialement en régime permanent à $P_{\text {elec }}=P_{m e c}$ et l'angle entre les tensions $\mathrm{V}_{1}$ (en arrière de la réactance transitoire d'alternateur $\mathrm{Xs}^{\prime}$ ) et la tension $\mathrm{V}^{\prime}$ ' (en arrière de la réactance transitoire des moteurs $\mathrm{Xm}^{\prime}$ ) est égal à $\delta_{0}=\delta$. Lorsqu'un court circuit se produit, le transfert de puissance diminue grâce à l'ouverture des protections $\mathbf{A}$ et $\mathbf{B}$, courbe 2 . Ici, la ligne en parallèle maintient la stabilité du réseau ainsi que le transfert de puissance pendant le défaut dus à la manoeuvre d'ouverture de protections $\mathbf{A}$ et $\mathbf{B}$ en permettant d'éteindre le courant défaut. Ensuite, le transfert de puissance augmente à une valeur de puissance plus grande que la puissance mécanique jusqu'au point $\mathrm{K}$. Ici, la puissance électrique est très élevée et le rotor d'alternateur réduit sa vitesse au point $N$. Dans ce point d'opération, le régulateur de tension et la résistance du système réduisent l'oscillation de la puissance, et le rotor accélère de nouveau jusqu'au point final d'opération où la puissance électrique est égale à la puissance mécanique $P_{\text {elec }}=P_{m e c}$ (Westinghouse, 1976). Par un défaut triphasé, la grandeur du transfert de puissance diminue plus que celle obtenue par le défaut ligne terre due au fait que la réactance équivalente $X^{\prime}$ du système considère les impédances des trois phases en augmentant sa grandeur. Le comportement est le même que pour la courbe 2 où le rotor accélère et l'angle $\delta$ s'élève jusqu'à une valeur maximale $\delta_{1}$ au point $\mathrm{H}$, mais la puissance électrique $P_{\text {ele }}$ n'est pas suffisante et plus petite que la puissance mécanique $P_{m e c}$ en conduisant le réseau hors des limites de stabilité.

Pour une ligne de transport compensée, courbe 3 , le dispositif de protection $\mathrm{du}$ condensateur débranche instantanément ce dispositif pendant des conditions de défaut. L'antérieure manœuvre provoque que la grandeur de la puissance électrique $P_{\text {elec }}$ soit plus grande que la puissance mécanique $P_{m e c}$ car l'angle de puissance a des valeurs plus élevées que $\delta_{0}$. Ensuite, le rotor décélérera et l'angle de puissance deviendra à une valeur maximale de $\delta=\delta_{1}$ afin de maintenir la stabilité du réseau (Oliveira, Gardos et Fonseca, 1991). 


\subsubsection{1 Éléments de protection de la ligne de transport avec la compensation série.}

Ayant défini la configuration du condensateur, on procède à la description des types de protections électriques de la ligne compensée. Elles constituent les relais à distance. Ces éléments répondent aux amplitudes élevées de tension et de courant. Il existe trois types de relais à distance: le relais d'impédance, d'admittance et de réactance. Le relais à distance plus utilisé dans les lignes de transport est le relais de réactance, il s'agit d'une protection non directionnelle utilisée pour protéger la ligne indépendante de la résistance. Il est habituellement utilisé dans une ligne de transport où la résistance défaut a la même magnitude que la longueur de la ligne (Grigsby, 2001).

La configuration de ces relais à distance peut se déterminer à partir de la figure 14 . Afin d'expliquer la performance des relais, on divise la ligne de transport en trois zones d'opération. Selon la figure 14, le relais de distance foumit la protection instantanée de $80 \%$ à $90 \%$ de la section de ligne protégée, et la protection de temps de retard est de 15 à 30 cycles en aval de la ligne plus la protection de secours au-dessus de la section de la ligne adjacente à 30 cycles délai.

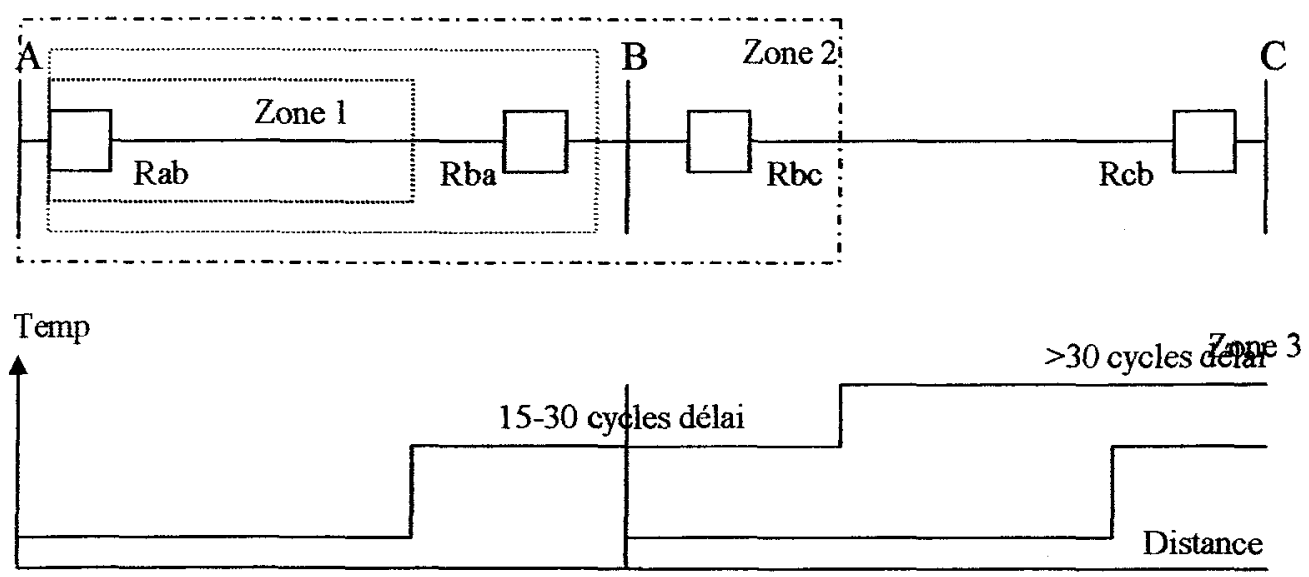

Figure $14:$ Zones d'opération du relais de distance

En ce qui concerne le branchement, les relais sont souvent branchés aux tensions et courants en configuration delta. Cette configuration mesure la tension et le courant entre 
deux phases. La valeur de $Z_{1}$ est l'impédance de séquence positive entre la localisation du relais et défaut. Cette valeur est exprimée dans l'équation (2-26) par rapport aux tensions mesurées dans le relais de protection A et B (Grisgby, 2001):

$$
\mathrm{Z}_{1}=\frac{E a-E b}{I a-I b}
$$

\section{a) Phénomène d'inversion d'amplitude de tension et du courant dans les relais à distance.}

Les relais à distance ne garantissent pas la sécurité du condensateur à cause de la présence des phénomènes d'inversion de tension et du courant. Le phénomène d'inversion de tension n'est pas considéré dans notre étude parce qu'il apparaît seulement dans lignes de transport en parallèle. Le phénomène d'inversion du courant apparait lorsque le relais à distance perçoit un défaut en direction inverse. La condition pour ce phénomène est $\mathrm{Xc}>\mathrm{X}_{\mathrm{L}}$ au point de défaut. Dans cette position la réactance équivalente devient négative, elle produit un courant capacitive en provoquant des niveaux de puissance de court-circuit très élevés (Novosel, Phadke, Saha et Lindahl, 1997).

En considérant le diagramme du réseau radial de la figure 12 , le courant de

courtcircuit s'exprime selon l'équation (2-30) (Novosel, Phadke, Saha et Lindahl, 1997):

$$
I_{f}=\frac{V_{1}}{X_{S}+X t+l \cdot X_{L}-X c}
$$

L'objectif de l'étude de cette contrainte est de trouver la position optimale du condensateur pour laquelle le réseau évite le phénomène d'inversion du courant lorsqu'un défaut apparaît dans le réseau. Pour observer la performance de la réactance équivalente de la ligne lors d'un défaut, on analyse trois cas différents de compensation série. Les valeurs choisies seront obtenues par rapport aux résultats de la contrainte de position optimale du condensateur série. 
Cas 1. Taux de compensation série $33.76 \%(X c=8 \Omega)$ à $1=45 \mathrm{Km}$

Le condensateur est branché à $45 \mathrm{Km}$ de la source. Pour une valeur de $\mathrm{Xc}=8 \Omega$, la réactance équivalente $X$ est inductive et plus grande que zéro. II n'y a aucune présence du phénomène d'inversion du courant, figure 15. Dans le cas de court-circuit en amont du condensateur, le courant défaut $I_{f}$ sera limité que par la résistance et la réactance équivalente de la ligne. Le tableau 5 montre les valeurs obtenues de la réactance équivalente de la ligne $\mathrm{X}\left(X_{L}-X c\right)$ aux différentes positions du condensateur.

Tableau 5

Réactance équivalente de la ligne compensée à $\mathrm{Xc}=8 \Omega$

\begin{tabular}{|c|c|c|}
\hline Longueur ligne & Réactance inductive $\mathrm{X}_{\mathrm{L}}(\Omega)$ & Réactance totale $\mathrm{X}(\Omega)$ \\
\hline 10 & 3.95 & 3.95 \\
\hline 20 & 7.9 & 7.9 \\
\hline 30 & 12.06 & 12.06 \\
\hline 40 & 15.8 & 15.8 \\
\hline 45 & 17.8 & 9.8 \\
\hline 50 & 19.75 & 11.75 \\
\hline 60 & 23.7 & 15.7 \\
\hline
\end{tabular}

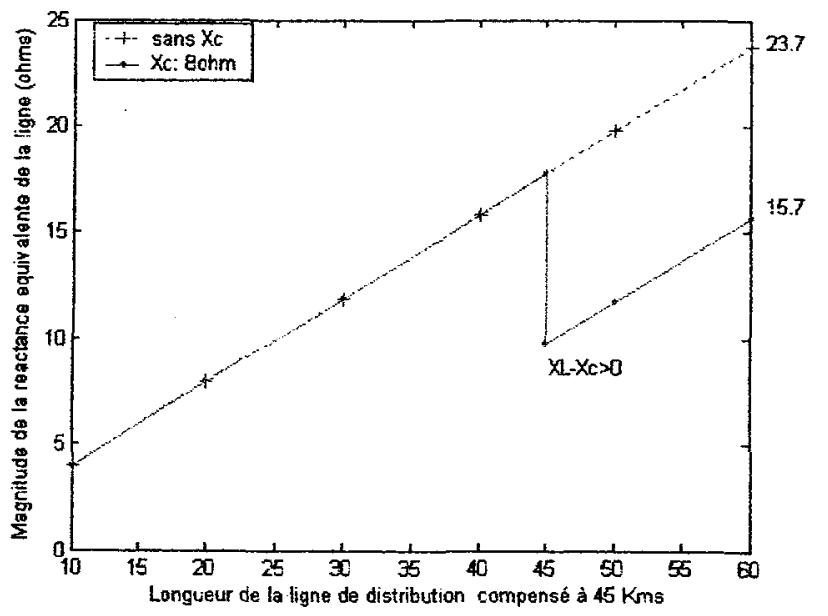

Figure 15 : Performance de la réactance équivalente de la ligne par rapport à la position du condensateur série. 


\section{Cas 2. Taux de compensation série de $54.85 \%(X \mathrm{X}=13 \Omega)$ à $E 35 \mathrm{Km}$}

Le condensateur série est branché à $35 \mathrm{Km}$ de la source. La réactance équivalente $X$ est encore inductive et plus grande que zéro, mais lamplitude de cette réactance devient très petite pour limiter la courant de défaut $I_{f}$. La valeur de $X=2.6 \Omega$. Les résultats sont observés dans le tableau 6 et la performance de la réactance équivalente dans la figure 16.

\section{Tableau 6}

Réactance équivalente de la ligne de distribution compensée à Xc=13 $\Omega$. Position optimale du condensateur série $\mathrm{x}=35 \mathrm{Km}$.

\begin{tabular}{|c|c|c|}
\hline Longueur ligne & Réactance inductive $\mathrm{XL}(\Omega)$ & Réactance totale $\mathrm{X}(\Omega)$ \\
\hline 10 & 3.95 & 3.95 \\
\hline 20 & 7.9 & 7.9 \\
\hline 35 & 13.83 & 13.83 \\
\hline 35 & $\mathbf{1 3 . 8 3}$ & $\mathbf{0 . 8 3}$ \\
\hline 40 & 15.8 & 2.8 \\
\hline 50 & 19.75 & 6.75 \\
\hline 60 & 23.7 & 10.7 \\
\hline
\end{tabular}

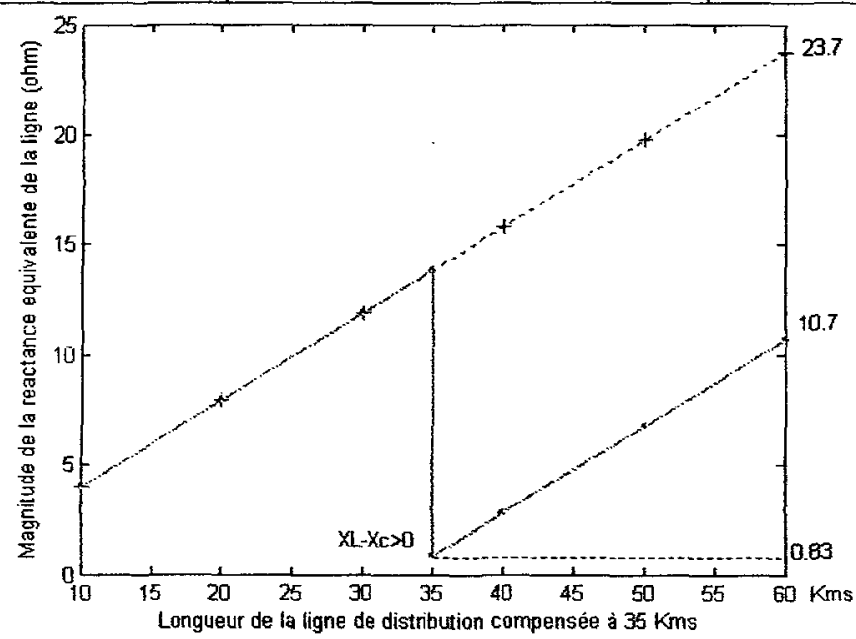

Figure 16: Performance de la réactance équivalente de la ligne par rapport à la position optimale du condensateur série. 


\section{Cas 3. Taux de compensation série de $63.3 \%(\mathrm{Xc}=15 \Omega)$ à $\ell=35 \mathrm{Kms}$}

Dans ce cas, le condensateur série est branché à $35 \mathrm{Km}$ de la source. La réactance équivalente $X$ est capacitive parce que la valeur de la réactance capacitive est plus grande que la réactance inductive $X_{L}$ de la ligne, voir figure 17. Si un court-circuit se produit, le courant défaut sera seulement limité par la résistance. Il faut éviter que la valeur de $\mathrm{X}_{\mathrm{C}}$ se trouve sous la condition suivante (2-28):

$$
X c \geq X s+l . X_{L}
$$

La variable $X s$ est la réactance équivalente de la source, $\mathrm{X}_{\mathrm{L}}$ comme la réactance de la ligne et « $\zeta »$ le point défaut. Les valeurs de réactance sont montrées dans le tableau 7 .

\section{Tableau 7}

Réactance équivalente de la ligne de distribution compensée à $X c=15 \Omega$. Position optimale du condensateur série $l=32 \mathrm{Km}$

\begin{tabular}{|c|c|c|}
\hline Longueur ligne & Réactance inductive XL $(\Omega)$ & Réactance totale $X(\Omega)$ \\
\hline & 3.95 & 3.95 \\
\hline 30 & 7.9 & 7.9 \\
\hline 32 & 12.64 & 11.85 \\
\hline 40 & 12.64 & -2.36 \\
\hline 50 & 15.8 & 0.8 \\
\hline 60 & 19.75 & 4.75 \\
\hline
\end{tabular}

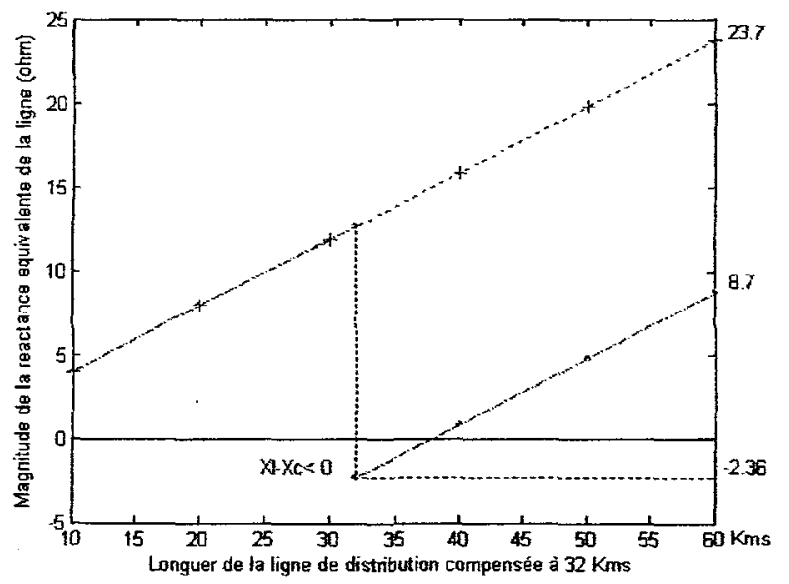

Figure 17 : Performance de la réactance équivalente de la ligne par rapport à la position optimale du condensateur série $X c=15 \Omega$. 
En conclusion, la position du condensateur série est importante en termes de l'étude de courant de court-circuit, mais ce paramètre est indépendant de la chute de tension à la charge et des contraintes de stabilité comme la puissance active et réactive (Miske, 2001).

\subsubsection{Types de protection électrique du condensateur série}

Dans les installations typiques de condensateurs série, il existe plusieurs techniques utilisées pour protéger les condensateurs sous conditions de court-circuit. Les tensions et courants lors des conditions de défaut se trouvent à des niveaux plus grands que les valeurs d'opération normale. Habituellement, les bancs de condensateurs série peuvent supporter $200 \%$ de leur valeur de tension d'opération sans affecter le diélectrique. Pour cette raison, il faut utiliser des condensateurs de rangs de courant continue équivalent à $50 \%$ du courant maximal qui l'écoule pendant le défaut, ou à travers l'utilisation d'un dispositif qui limitera la surtension aux terminaux du condensateur. Les bancs de condensateurs série sont branchés lors du démarrage des moteurs. Dans ce cas, le rang de protection de courant du condensateur devra être au moins de $67 \%$ plus grand que le courant d'opération des moteurs qui sont reliés en parallèle à d'autres charges. Actuellement, les lignes de transport compensées utilisent les protections électriques avec la technologie $\mathrm{ZnO}$ (Zinc oxide resistor) (figure 18) et MOV (Metal Oxide Varistor), figure 19. En ce qui concerne le type de protection $\mathrm{ZnO}$, il présente un système de coupure (by-pass) qui permet d'isoler le condensateur. Ce processus de coupure est limité par la durée du défaut en permettant que le condensateur compense le service pendant le court-circuit et pouvoir maintenir la stabilité transitoire du réseau. En plus, l'utilisation de la technologie $\mathrm{ZnO}$ permet une réinsertion instantanée du condensateur série après que le courant de court circuit est éteint. La réinsertion de extinction de l'arc requière seulement de 1 à 1.5 cycles de la fréquence fondamental. Le temps de réinsertion se trouve dans le range de 300 à $\mathbf{4 0 0}$ millisecondes pour éviter les surtensions due à la réinsertion. 


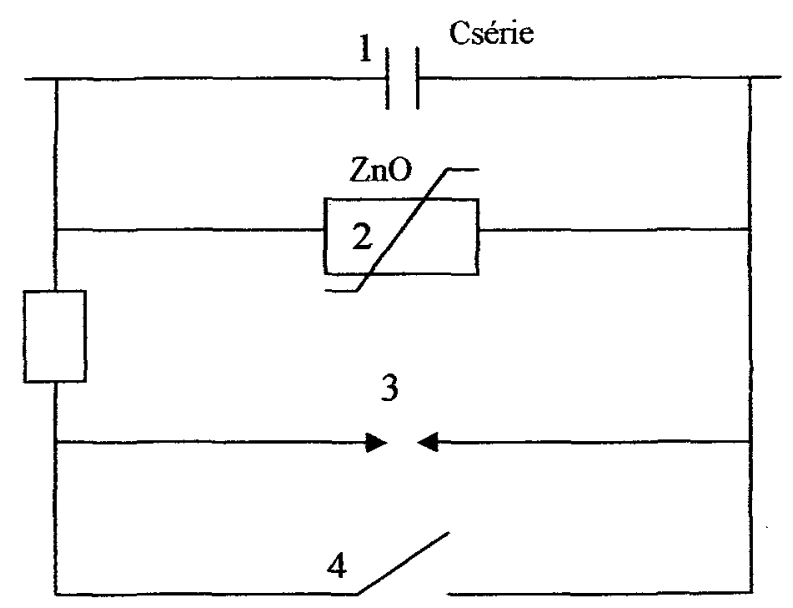

Figure 18 : Protection de surtension du condensateur $\mathrm{ZnO}$

Composantes de protection $\mathrm{ZnO}$ (figure 17) :
1. Condensateur série
3.Extintion de l'arc électrique
2. Varistor $\mathrm{ZnO}$
4.Disjoncteur

Par contre, la technologie de protection de surtension avec MOV est composée de varistors de haute énergie, d'un disjoncteur de découplage et de l'inductance de décharge. Le varistor de haute énergie contient des disques d'oxyde de métal en série et en parallèle dans une couverture de porcelaine. Les disques se composent des éléments d'oxyde de Zinc et d'autres métaux choisis. Le partage égal d'énergie est assuré parmi les disques par des méthodes particulièrement développées de mesure et d'essai.

Les composantes physiques de disques permettent une prolongation de l'efficacité ainsi que des caractéristiques tension-courant fortement non linéaires.

Le contact shunt est automatiquement fermé si la tension aux terminaux du condensateur série ne détecte plus les conditions défaut sur la ligne. Ce contact est automatiquement ouvert une fois le disjoncteur accomplie l'opération prévue et les conditions de circuit reviennent à la normalité environ une minute. 
Le varistor fonctionne indépendamment à chaque phase tandis que le contact shunt opère dans les trois phases. Les réacteurs de décharge limitent la grandeur du courant dérivé du condensateur par le contact shunt, figure 18. Les dispositifs d'extinction prévoient les oscillations indésirables qui peuvent se produire par l'excitation de transformateurs et le démarrage de moteurs, figure 19.

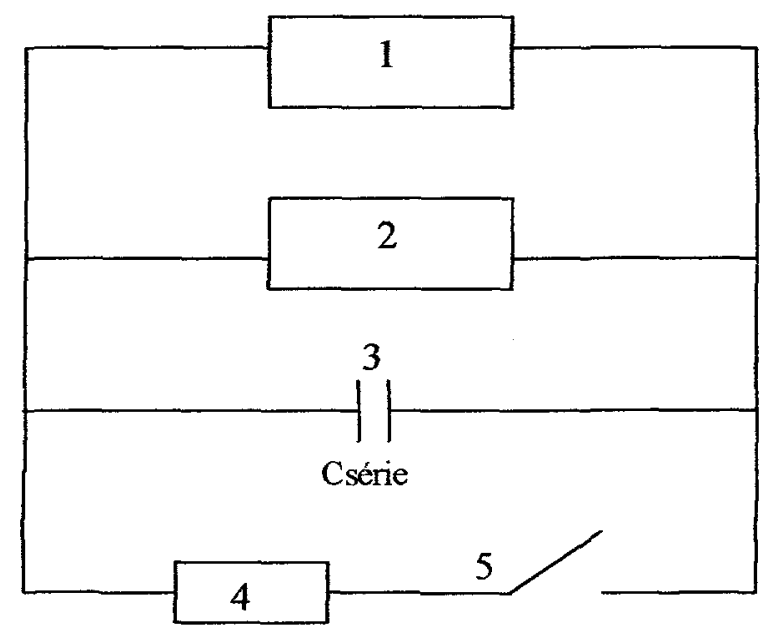

Figure 19 : Protection de surtension avec technologie MOV Composantes protection MOV ( figure 18):

1. Dispositif de coupure d'irruption, 2. Banc de varistor en série et parallèle.

3. Condensateur série 4. Inductance pure.

5. Interrupteur.

Dans les lignes de distribution rurales, les entreprises comme Hydro-Québec ont installé des protections basées sur la technologie MOV dans les installations des bancs de condensateurs série. La technologie MOV qui aura la même fonction qu'un parafoudre, combine un matériau isolé polymérique à un oxyde de métal qui permet une meilleure performance tout en protégeant le condensateur sous des conditions de surtension.

Cet équipement (voir app. D, p. 158 à 162) présente les suivantes caractéristiques: 
- Condition au niveau de normes techniques, il a été essayé par rapport à ANSI/IEEE C62.11-1987, section 8.10 et CAN/CSA C233.1-1987.

- Mode défaut évitant la défragmentation: l'équipement peut supporter des courants défaut sans la défragmentation des composantes. Essais acceptés par ANSI/IEEE C62.11-1987, section 8.10 et CAN/CSA C233.1-1987, section 7.9.

- Joint contre l'humidité : l'ensemble de métal d'oxyde est encapsulé dans une capsule polymérique pour éliminer le plus commun défaut dans les MOV, pénétration de l'humidité dans les composantes.

- Varistor de haute énergie: La technologie MOV peut absorber une grande quantité de l'énergie sans produire de défaut.

- Les parties mécaniques du dielectric robustes supportent l'arc court-circuit.

\subsubsection{Contrainte de stabilité N. 5 : Phénomène de papillotement.}

\subsubsection{Définition du papillotement.}

Le phénomène de « flicker » ou papillotement de lumière est défini comme un clignotement ou une oscillation de la lumière. Il se manifeste en basse tension et peut être ressenti visuellement par des utilisateurs de lampes alimentées par une source commune à l'éclairage et à une charge perturbatrice. Ces charges sont branchées à tout niveau de tension sur la ligne de transport.

La mise en service ou hors service des charges des utilisateurs industriels tels que les fours à arc, les soudeuses, le démarrage de moteurs, le manœuvre de batteries de condensateurs, entre autres, produit des variations de tension sur le réseau de distribution. Toutefois, ces variations sont généralement faibles, lentes et peu perceptibles et peuvent être corrigées par des systèmes de régulation de tension. La figure 20 montre des types de variation de tension pendant la présence des perturbations dans le reseau. 


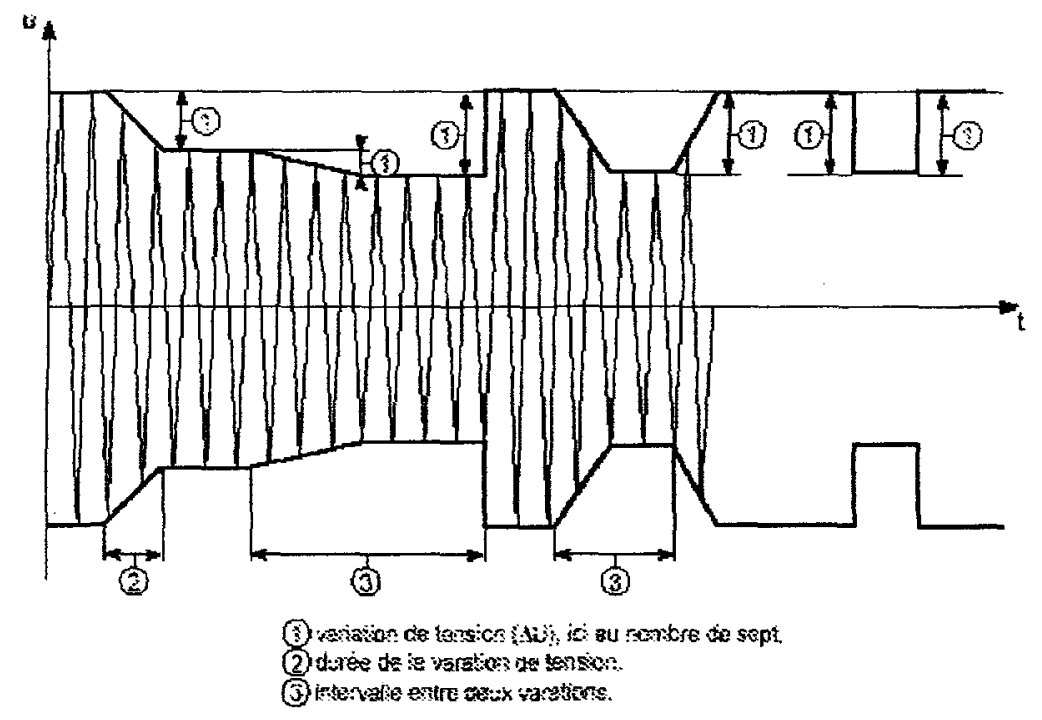

Figure 20 : Différents cas de variation de tension

Les types de variation de tension sont au nombre de 7 , chacune présente une différente durée de variation par rapport à la perturbation qui est provoquée dans ce moment. L'intervalle entre deux variations est observé lorsque la magnitude de la tension qui devient en régime permanent varie grâce à une perturbation et, quelques secondes après, revient au régime permanent. Pour développer la formulation du phénomène de papillotement, on utilisera le modèle du réseau électrique de la figure 21 (Wierda, Merlin Gerin ,1995).

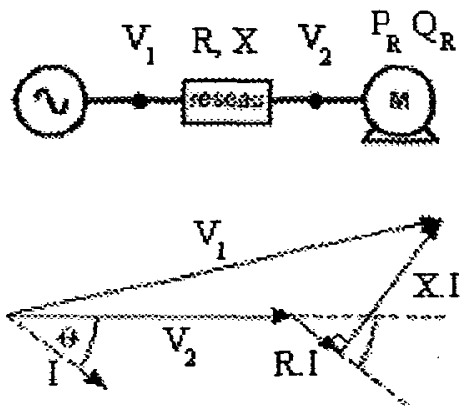

Figure 21 : Modèle électrique et diagramme vectoriel du réseau électrique. 
D'abord, on considère que l'angle entre les tensions $V_{1}$ et $V_{2}$ est faible, alors l'équation de variation de tension peut être déterminée telle que démontrée dans l'expression (2-29) :

$$
\Delta V=V I-V 2=R \cdot I \cdot \cos \theta+X \cdot I \cdot \sin \theta
$$

Les équations de puissance active et réactive (2-30) du modèle de la figure 23 peuvent se calculer comme suit :

$$
P_{R}=V_{2} \cdot I \cdot \cos \theta \text { et } Q_{R}=V_{2} \cdot I \cdot \sin \theta
$$

Ce qui donne l'expression (2-31):

$$
\Delta V=\frac{R P_{R}+X Q_{R}}{V_{2}}
$$

La valeur relative sera exprimée en multipliant l'équation de chaque côte par la tension nominale du réseau. L'expression (2-31) devient comme (2-32):

$$
\frac{\Delta V}{V_{2}}=\frac{R I \cos \theta+X_{L} I \sin \theta}{V_{2}^{2}}
$$

Alors la valeur de la variation de tension par rapport à la puissance apparente dans le système sera (2-33):

$$
\Delta V=\frac{V_{2}\left(R I \cos \theta+X_{L} \sin \theta\right)}{V_{2}^{2}}
$$

En considérant que la puissance apparente d'un élément perturbateur peut se calculer comme suit (2-34) :

$$
K V A_{\text {appelé }}=V_{2 . I}
$$

En développant l'expression (2-33) dans (2-34), on obtient l'équation (2-35) qui représente la variation de tension d'une charge perturbatrice :

$$
\Delta V \%=\frac{K V A_{\text {appete }}\left(R \cos \theta+X_{L} \sin \theta\right)}{V_{2}^{2}} * 100
$$


Dans cette étude, on a imputé le papillotement à des charges perturbatrices de moteurs asynchrones parce que le démarrage est considéré comme la principale cause qui provoque ce phénomène de papillotement sur la ligne de distribution.

\subsubsection{Perturbation du démarrage de moteurs}

L'industrie utilise principalement deux types de moteur : Le moteur à induction et le moteur synchrone. Le moteur à induction se caractérise par sa robustesse et sa maintenance facile. Mais le moteur synchrone devient plus avantageux que le moteur à induction dont le coût est élevé. Pour établir la valeur de la puissance appelée d'un moteur, on formule l'équation qui exprime cette puissance par rapport aux paramètres du moteur. En identifiant les paramètres utilisés dans la formulation, on développe alors l'équation qui détermine la puissance appelée au démarrage d'un moteur (2-36) :

$$
K V A_{\text {appele }}=\frac{O P \cdot 0.746 \cdot(I d / I n) \cdot \beta}{\eta \cdot F \cdot P .}
$$

- OP : Puissance nominal

- $\quad \eta$ : Rendement

- F.P : Facteur de puissance

- Id : Courant de démarrage.

- In : Courant en régime normal

- $\quad \beta \quad$ Facteur de démarrage.

Par contre, le moteur synchrone ne peut démarrer seul, pour cette raison, son rotor est équipé d'une cage d'écureuil qui lui permet de démarrer comme un moteur à induction. Le moteur à induction crée un appel de courant de six à huit fois supérieur au courant nominal. Pour réduire la surcharge dûe à la demande élevée du courant, les utilisateurs appliquent une tension réduite en utilisant des dispositifs tels que un 
autotransformateur, de résistances série et d'autres éléments qui sont représentés comme le facteur de démarrage des moteurs classifiés dans le tableau 8.

Tableau 8

Facteurs de démarrage des moteurs

\begin{tabular}{|l|r|} 
Méthode de démarrage & Facteur démarrage \\
\hline Branchement direct sur la ligne & 1 \\
\hline Etoile-delta & 0,33 \\
\hline Autotransformateur & \\
\hline Prise $80 \%$ & 0,68 \\
\hline Prise $65 \%$ & 0,46 \\
\hline Prise 50\% & 0,29 \\
\hline Résistance ou réactance & 0,8 \\
\hline $80 \%$ voltage & 0,65 \\
\hline $65 \%$ voltage & 0,5 \\
\hline $50 \%$ voltage & 0,25 \\
\hline avec 5 étapes de résistance &
\end{tabular}

\subsubsection{Quantification et mesure du phénomène de papillotement}

Le calcul de papillotement peut être développé par un seul élément perturbateur, mais lorsque plusieurs éléments sont en jeu, le problème devient plus complexe, dû à l'intervention du principe d'addition des fluctuations de tension. L'idée de ce principe consiste à ramener la fréquence et l'amplitude de la fluctuation des éléments perturbateurs produits à une fréquence et à une amplitude équivalente afín de les regrouper en un seul élément perturbateur. Toutefois, il existe la possibilité que ces éléments fonctionnent simultanément ou en superposition. (Hydro-Québec, 1983). Les paramètres principaux du principe de papillotement proposé sont obtenus à partir des expériences suivantes:

- La sensation de gêne comme fonction du carré de l'amplitude de la fluctuation de la tension et de la durée de celle-ci, figure 22.

- La sensibilité de l'utilisateur aux fluctuations d'éclairement qui est maximale autour de $10 \mathrm{~Hz}(8.8 \mathrm{~Hz})$, figure 22 .

- Le seuil minimal de perception à la fréquence de $8.8 \mathrm{~Hz}(0.25 \%)$ et le seuil minimal de gêne $(0.5 \%)$, figure 22 . 


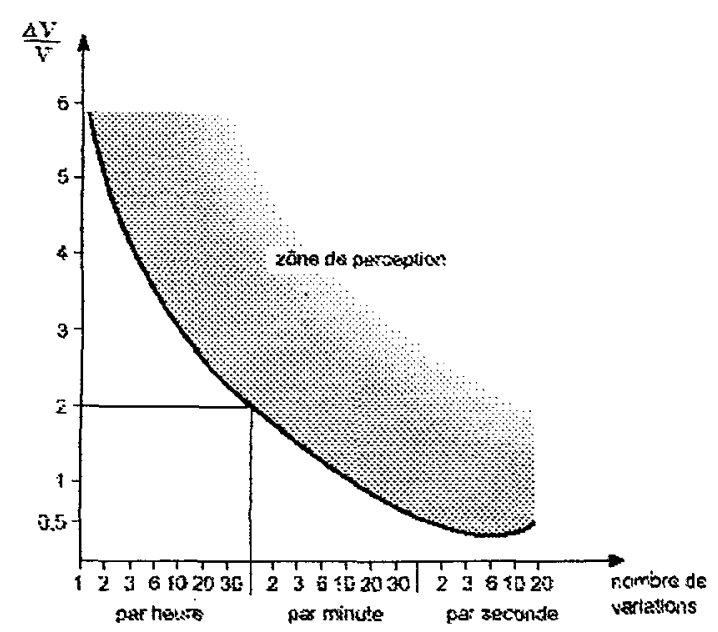

Figure 22 : Seuil de perception de l'œil de l'abonné aux fluctuations de tension.

La gêne perçue pour une fluctuation de la tension à une fréquence différente à 8.8 $\mathrm{Hz}$ et une amplitude $\mathrm{a}_{\mathrm{f}}$ est égale à la gêne perçue en une fluctuation de la tension à 8.8 Hz avec une amplitude équivalente exprimée dans l'équation (2-37):

$$
\mathrm{a} 8,8=\mathrm{g}_{\mathrm{f}} \cdot \mathrm{a}_{\mathrm{f}}
$$

Le coefficient $g_{f}$ ne dépend que de la fréquence de cette fluctuation de tension initiale $\left(g_{f} \leq 1 ; g_{8.8}=1\right)$. Alors, toute fluctuation de tension peut être exprimée en fonction de fluctuation de tension équivalente à $8.8 \mathrm{~Hz}$ (Wierda, Merlin Gerin , 1995). Ensuite, on développe la superposition de plusieurs fluctuations de tension à fréquences $f_{i}$ et amplitudes $a_{i}$ différents, équivalant à une fluctuation d'amplitude as,8 avec une fréquence de $8.8 \mathrm{~Hz}$ calculée à partir de l'équation (2-38) comme suit:

$$
\mathbf{a}_{8.8}(\mathrm{t})=\sqrt{\sum_{i} a_{8.8 i}^{2}(t)}=\sqrt{\sum_{i} a_{i}^{2} \cdot g_{f i}^{2}}
$$

Le paramètre $a_{8.8}(t)$ appelé papillotement instantané est calculé en fonction du temps. Ce paramètre a été observé pour une fluctuation de tension, fréquence, amplitude à durée déterminée (Wierda, Merlin Gerin ,1995). 
Les données principales pour l'étude de papillotement sont obtenues à partir d'une population relativement homogène constituée d'adultes occupés à lire (voir app. $E, p$. 160 à 162). La courbe A de la norme C22.1 (voir app. E, p. 163 à 165) montre la limite d'irritation qui est définie comme la mesure maximale à laquelle l'organe de la vue peut être affecté physiologiquement. La courbe $\mathrm{A}$ de l'annexe $\mathrm{E}$ montre la limite de visibilité qui est la mesure minimale correspondant à la perception visuelle du phénomène de papillotement.

Hydro-Québec a développé des normes qui expriment les limites de papillotement se manifestant sur le réseau de distribution. La norme C.22.1 représentée dans la courbe A (voir app. E, p. 163 à 165) calcule la limite maximale de papillotement sur un réseau de distribution, c'est-à-dire la limite maximale de papillotement que tout abonné peut expérimenter en tous points du réseau de distribution de moyenne et basse tension. La norme considère comme une valeur maximale l'amplitude de $0.5 \%$ à un fréquence de $8.8 \mathrm{~Hz}$. La limite de papillotement sur le réseau qui alimente des abonnés qui se trouvent dans le territoire d'un parc industriel est augmentée conformément à la courbe B. Dans ce cas, l'amplitude est de $0.6 \%$ à un fréquence de $8.8 \mathrm{~Hz}$.

La norme C.22.2 représentée dans la courbe A (voir app. E, p. 162 à 164) calcule le niveau de papillotement maximal que peut produire un abonné en tous points du réseau de moyenne tension jusqu'au poste de distribution de moyenne tension dont les effets peuvent être expérimentés par des abonnés existants. Dans ce cas, l'amplitude est de $0.3 \%$ à une fréquence de $8.8 \mathrm{~Hz}$.

La fréquence de fluctuation pour le démarrage d'un moteur est directement liée aux activités des utilisateurs. L'usage irrégulier du moteur produit des perturbations dans la tension. Un moteur qui démarre plusieurs fois en une période de temps est considéré comme la principale cause de papillotement, surtout lorsque la demande de puissance des utilisateurs branchés à la même barre est maximale. 
D'ailleurs, il existe un paramètre appelé gêne $g(f)$ qui permet de mesurer le papillotement lorsque les clients industriels démarrent des moteurs. Le paramètre $g(f)$ est défini par rapport aux différentes fréquences de papillotement observées au point d'utilisation. La classification du phénomène de papillotement s'effectue de façon statistique en consultant des clients résidentiels qui ont observé le phénomène pendant des périodes du temps. Ces observations ont été classifiées comme suit :

- Fréquence de papillotement pendant un jour.

- Fréquence de papillotement pendant une heure

- Fréquence de papillotement pendant une minute

- Fréquence de papillotement pendant une seconde.

Le tableau 9 montre les résultats obtenus par rapport aux fréquences de papillotement.

\section{Tableau 9}

Fréquence du phénomène de papillotement perçue par les clients résidentiels

\begin{tabular}{|c|c|c|c|c|c|}
\hline 1/jour & 0,043 & $15 /$ heure & 0,15 & $20 / \mathrm{min}$ & 0,328 \\
\hline 2/jour & 0,064 & $20 /$ heure & 0,157 & $30 / \mathrm{min}$ & 0,362 \\
\hline 5/jour & 0,067 & $30 /$ heure & 0,172 & $40 / \mathrm{min}$ & 0,384 \\
\hline $10 /$ jour & 0,069 & $40 /$ heure & 0,177 & $50 / \mathrm{min}$ & 0,408 \\
\hline 15 /jour & 0,072 & $50 /$ heure & 0,183 & $1 / \mathrm{sec}$ & 0,426 \\
\hline 20/jour & 0,074 & $1 / \mathrm{min}$ & 0,187 & $2 / \mathrm{sec}$ & 0,526 \\
\hline 1/heure & 0,075 & $2 / \mathrm{min}$ & 0,211 & $5 / \mathrm{sec}$ & 0,741 \\
\hline 2/heure & 0,093 & $5 / \mathrm{min}$ & 0,244 & $8 / \mathrm{sec}$ & 1 \\
\hline 5/heure & 0,121 & $10 / \mathrm{min}$ & 0,282 & $10 / \mathrm{sec}$ & 0,93 \\
\hline 10/heure & 0,141 & $15 / \mathrm{min}$ & 0,308 & $12 / \mathrm{sec}$ & 0,89 \\
\hline
\end{tabular}

Hydro-Québec a développé des mesures dans la charge branchée au réseau de distribution à étudier (voir app. A, p. 141 à 150) pendant des heures d'opération normale. Grâce aux mesures, l'identification de papillotement est possible. Les résultats et paramètres utilisés pour identifier le papillotement sont ajoutés dans le projet (voir app. F, p. 166 à 179). 


\subsubsection{Critère de sélection du taux de compensation minimal par rapport à l'étude du phénomène de papillotement.}

Le phénomène de papillotement est considéré comme un facteur perturbateur qui déstabilise le système de distribution radial. Pour cette raison, l'étude de ce phénomène devient une contrainte de la méthode d'optimisation qui permettra d'établir la valeur minimale de puissance de court-circuit par laquelle le papillotement est évité. La puissance de court circuit dépend aussi de la valeur du taux de compensation série. L'effet de la réactance capacitive de la compensation réduit la réactance totale de la ligne de transport en augmentant l'amplitude du courant de court-circuit.

Le critère technique proposé aide à trouver la valeur minimale du taux de compensation série à partir de l'analyse de puissance de court-circuit minimal développé par une scierie branché au réseau (voir app. A, p. 141 à 150).

Le développement du critère commence avec le calcul de la valeur de la puissance de court-circuit établie aux différents taux de compensation série à partir de l'équation $(2-39)$ :

$$
\mathrm{KVA}_{\mathrm{CC}}=\frac{V_{2}^{2}}{\sqrt{(R s+R t+R)^{2}+\left(X s+X_{t}+X_{L}-X c\right)^{2}}}
$$

$R s:$ Résistance de la source

$R t:$ Résistance du transformateur.

$R:$ Résistance de la ligne de transport.

$X s:$ Réactance de la source

$X c:$ Réactance capacitive du condensateur série.

$X_{L}:$ Réactance de la ligne de transport.

Le tableau 10 montre les résultats de la puissance de court-circuit calculée pour le réseau (voir app. A, p. 141 à 150) en utilisant l'équation (2-39). 
Tableau 10

Puissance de court-circuit appliquée au réseau aux différents taux de compensation.

\begin{tabular}{|c|c|c|}
\hline$X_{C}$ & $X_{C}$ (p.u.) & $M V A_{C c}$ \\
\hline 4 & 0.14 & 14.25 \\
\hline 5 & 0.18 & 14.66 \\
\hline 6 & 0.22 & 15.09 \\
\hline 7 & 0.25 & 15.43 \\
\hline 8 & 0.288 & 15.76 \\
\hline 9 & 0.324 & 16.22 \\
\hline 10 & 0.36 & 16.69 \\
\hline 11 & 0.396 & 17.19 \\
\hline 12 & 0.432 & 17.72 \\
\hline 13 & 0.468 & 18.28 \\
\hline 14 & 0.504 & 18.87 \\
\hline 15 & 0.54 & 19.49 \\
\hline 16 & 0.675 & 20.16 \\
\hline 17 & 0.717 & 20.86 \\
\hline
\end{tabular}

Maintenant on calcule la valeur de la puissance minimale de court-circuit « $\mathrm{MVA}_{\mathrm{CC}}$ " par rapport au papillotement. L'équation (2-40) montre la formulation utilisée pour calculer le paramètre $\alpha_{i}$ qui détermine la puissance d'appel par chaque moteur :

$$
\alpha_{i}=\frac{H P \cdot 0.746 W \cdot \frac{I d}{I n} \cdot \beta}{\eta \cdot F P} \cdot g[f(i)]
$$

- Facteur de puissance FP.

- Rapport entre courant de démarrage et courant nominal. $\frac{I d}{I n}$

- Type d'élément pour démarrer le moteur $\beta$.

- Rendement du moteur $\eta$

- Papillotement déterminé par le paramètre gêne $g$ [f(i)]

Ensuite, il s'effectue la somme des paramètres $\alpha$ de tous les moteurs branchés au point d'utilisation, équation (2-41). 


$$
\alpha=\sqrt{\sum \alpha_{i}^{2}}
$$

La norme de limites de papillotement sur le réseau de distribution dans la courbe A de (voir app. E, p. 163 à 165) indique que la limite maximale de papillotement sur le réseau peut s'élever dans tous les points de moyenne et basse tension du réseau de distribution à une valeur de $0.5 \%$ et à une fréquence de $8.8 \mathrm{~Hz}$. Cette limite maximale nous permet d'obtenir la relation de la puissance de court-circuit totale de la charge par rapport à sa puissance d'appel, équation $(2-42)$ :

$$
0.5 \leq \frac{\alpha .100}{K V A c c}
$$

En développant la puissance de court-circuit à gauche de l'expression (2-42), on obtient l'équation (2-43) :

$$
K V A c c \geq \frac{\alpha .100}{0.5}
$$

Après avoir obtenu la valeur de la puissance minimale de court-circuit de toute la charge, équation (2-44), on procède à la comparaison des valeurs de puissances de court-circuit obtenues précédemment dans le tableau 10 afin d'identifier la valeur minimale du condensateur série.

$$
K V A c c=\frac{V_{2}}{\sqrt{\left(R s+R t+R_{\text {ligne }}\right)^{2}+\left(X s+X_{t}+X_{L}-X c\right)^{2}}}
$$

Ce critère a été développé par une scierie dont les paramètres des groupes moteurs sont montrés dans l'annexe A. La valeur de la puissance minimale de court-circuit est de 14.6 MVA, avec un valeur de réactance capacitive du condensateur série de $5 \Omega(\tau=$ $21.1 \%$ ). Cette valeur devient comme la limite minimale de réactance pour éviter le papillotement. 


\subsubsection{Contrainte de stabilité No 6 : Pertes de puissance active et réactive}

Les réseaux électriques présentent des contingences contrôlées par des dispositifs installés sur les lignes de transport qui maintiennent les réseaux dans les limites de stabilité. Parfois, le constant écoulement de puissance charge les lignes de transport à cause de la grandeur de puissance demandée par différentes des charges branchées aux barres. Avec l'élévation de cet écoulement de puissance, les pertes de puissance réactive dans le système s'accentuent et provoquent éventuellement l'instabilité du système (Mutlu et Makran, 1998).

L'ajout de la compensation série sur la ligne permet de contrôler l'écoulement de puissance réactive afin d'améliorer tout le profil de tension en aval du condensateur. Pour cette raison, ce paramètre électrique a été choisi comme une contrainte de stabilité de la méthode d'optimisation. Cette contrainte est développée à partir d'analyse de l'équation de perte de puissance active et réactive.

D'abord, la tension résultant entre les deux barres de la ligne de transport s'exprime selon l'équation $(2-45)$

$$
V=V_{1}^{2}+V_{2}^{2}-2 \cdot V_{1} \cdot V_{2} \cdot \cos \delta
$$

La tension résultante à chaque extrême de la ligne devient comme l'expression (2-46) :

$$
V=(I . R)^{2}+(I . X)^{2}-2 \cdot(I \cdot R) \cdot(I \cdot X) \cdot \cos (90)
$$

En développant l'équation (2-46) on a l'équation $(2-47)$ :

$$
V=(I . R)^{2}+(I . X)^{2}
$$

On sait que le courant est égal à l'équation (2-48):

$$
I^{2}=\frac{V}{R^{2}+X^{2}}
$$


On ajoute la valeur du courant dans Yéquation (2-49) et on obtient:

$$
P_{\text {perte }}+j Q_{\text {perte }}=I^{2} \cdot R+I^{2} \cdot X
$$

Alors, les pertes de puissance actives et réactivés deviennent comme l'équation (2-50):

$$
P_{\text {perte }}+j Q_{\text {perte }}=V \cdot\left[\frac{R}{R^{2}+X^{2}}\right]+\mathrm{j}\left[\frac{X}{R^{2}+X^{2}}\right]
$$

Si on sélectionne seulement la partie imaginaire de l'équation (2-50), on obtiendra la performance de pertes de puissance réactive dans la ligne de transport qui est calculée à partir de l'équation (2-51):

$$
Q_{\text {perte }}=V \cdot\left[\frac{X}{R^{2}+X^{2}}\right]
$$

En utilisant les paramètres de tension obtenus avec la solution initiale développée dans le programme de l'écoulement de puissance, on obtient la valeur de perte de puissance réactive à différents taux de compensation série. Voir Tableau 11.

\section{Tableau 11}

Pertes de puissance active et réactive

\begin{tabular}{|c|c|c|c|}
\hline$X C$ & $\tau \%$ & $P_{\text {pete }}$ (p.u.) & $Q_{\text {perte (p.u.) }}$ \\
\hline 7 & 29.54 & 0.1142 & 0.0083 \\
\hline 8 & 33.76 & 0.1147 & 0.0079 \\
\hline 9 & 37.97 & 0.1152 & 0.0074 \\
\hline 10 & 42.19 & 0.1156 & 0.0069 \\
\hline 11 & 46.41 & 0.116 & 0.0064 \\
\hline 12 & 50.63 & 0.1165 & 0.006 \\
\hline 13 & 54.85 & 0.117 & 0.0055 \\
\hline 14 & 59.07 & 0.1174 & 0.005 \\
\hline 15 & 63.29 & 0.1179 & 0.0045 \\
\hline 16 & 67.51 & 0.1183 & 0.004 \\
\hline 17 & 71.73 & 0.1187 & 0.0035 \\
\hline
\end{tabular}


On peut observer la performance des pertes de puissance réactive qui diminuent lorsque le taux de compensation série est augmenté, figure 23. Cette élévation permet d'améliorer les profils de tension en aval du condensateur et, par conséquent la tension à la charge.

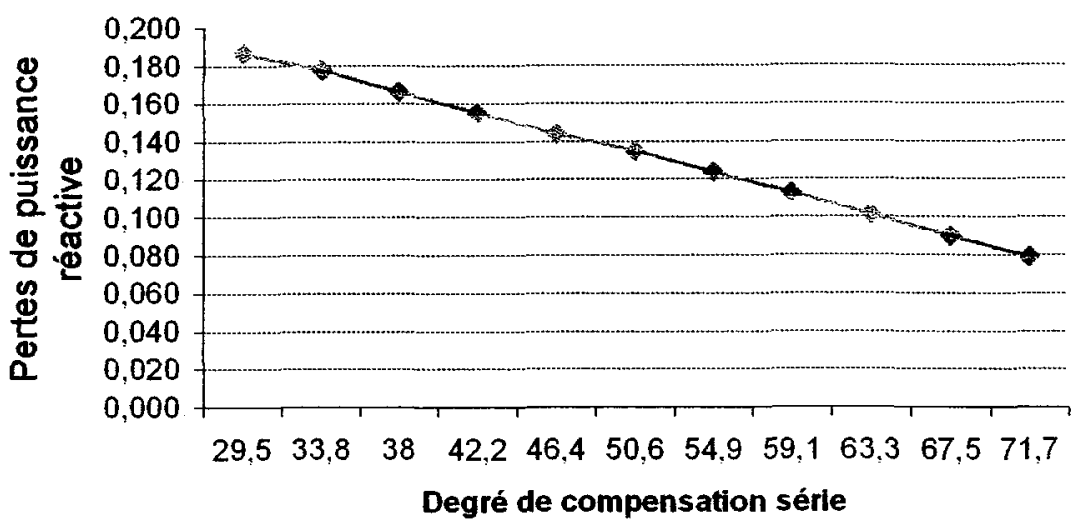

Figure 23 : Pertes puissance réactive

Par contre, les pertes de puissance active augmentent considérablement dues à la résistance de la ligne qui est toujours constante, voir tableau 11. Cette perte de puissance varie par rapport au carré de l'amplitude du courant qui augmente par l'effet de la compensation série, figure 24 .

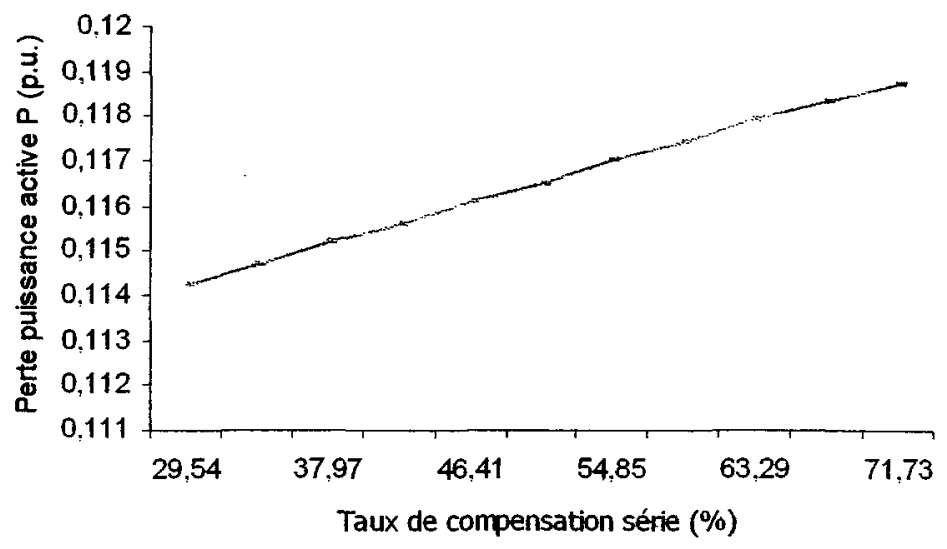

Figure 24 : Pertes de puissance active dans la ligne de distribution 


\subsubsection{Contrainte de stabilité No 7. Résonance sous-synchrone (SSR)}

\subsubsection{Définition et analyse de la résonance sous-synchrone dans le réseau électrique à configuration radiale}

La résonance sous-synchrone est considérée comme une condition d'instabilité où les composantes du système comme que la ligne de transport et le condensateur série échangent de l'énergie avec le système mécanique du réseau électrique(alternateurturbine) à une ou plus des fréquences au-dessous de la fréquence fondamental.

Le phénomène physique de l'échange de l'énergie peut être expliqué ainsi :

- Les oscillations de l'énergie entre l'énergie électrostatique de condensateurs série et l'énergie électromagnétique des inductances du réseau entraîneront les courants triphasés symétriques à une fréquence sous-synchrone.

- L'écoulement de courants dans la bobine de l'armature de l'alternateur crée une force magnétomotrice rotative qui tourne à une vitesse sous-synchrone.

- L'interaction de la force magnétomotrice rotative statorique avec la force magnétomotrice rotative de la bobine du rotor de l'alternateur développe une composante du couple électromagnétique dans l'axe de l'altemateur à une fréquence de glissement $\mathrm{w}_{o}-\mathrm{w}_{e}$, où $\mathrm{w}_{o}$ est la fréquence naturelle du système et $\mathrm{w}_{e}$ est la fréquence des courants sous-synchrones.

- Si le système mécanique à une fréquence superposée avec une fréquence de glissement $\mathrm{w}_{o}-w_{e}$, il existe la possibilité d'avoir une condition de résonance sous-synchrone électromécanique (Edris, 1991).

- La composante du couple électromagnétique, à une fréquence de glissement, fonctionne comme un accouplement afin d'échanger de l'énergie entre le système électrique et mécanique (Edris, 1991).

Ces fréquences sous-synchrones produisent la fatigue mécanique et des défauts dans le groupe altemateur/turbine en réduisant le temps de vie utile des équipements. (Youif et Dabbag, 1990). 
Pour analyser le phénomène de résonance sous-synchrone, on développe une étude de réponse en fréquence à travers le logiciel Power System Blockset. Le bloc de mesure à utiliser sera «impedance mesurement» pour mesurer l'impédance dans 3 points différents du réseau. Les résultats de l'étude de réponse en fréquence sont montrés dans la table 14 (voir app. G, p. 180 à 181).

Par rapport à l'étude de réponse en fréquence, on a choisi les valeurs du taux de compensation série de $X c=8 \Omega$ et $X c=15 \Omega$ à cause de leur grandeur d'impédance de résonance respective; ce sont les valeurs les plus petites pendant le phénomène de résonance sous-synchrone.

- Étude de réponse en fréquence au taux de compensation de $33.76 \%(X c=8 \Omega)$

L'étude de réponse en fréquence est réalisée dans le circuit équivalent monophasé du réseau (voir app. A, p. 141 à 150). Le tableau 12 démontre ces fréquences de résonance.

Tableau 12

Résultats de fréquences de résonance du réseau électrique.

\begin{tabular}{|l|c|c|}
\hline Paramètres & Résonance sous-synchrone & Résonance hypersynchrone \\
\hline Impédance & $193 \Omega$ & $1068 \Omega$ \\
\hline Fréquences & $6 \mathrm{~Hz}$ & $740 \mathrm{~Hz}$ \\
\hline
\end{tabular}

Ces fréquences sont produites par la résonance entre les inductances équivalentes de la ligne de transport et du condensateur série. Les figures 24 et 25 montrent les deux fréquences de résonance présentes dans le réseau. 

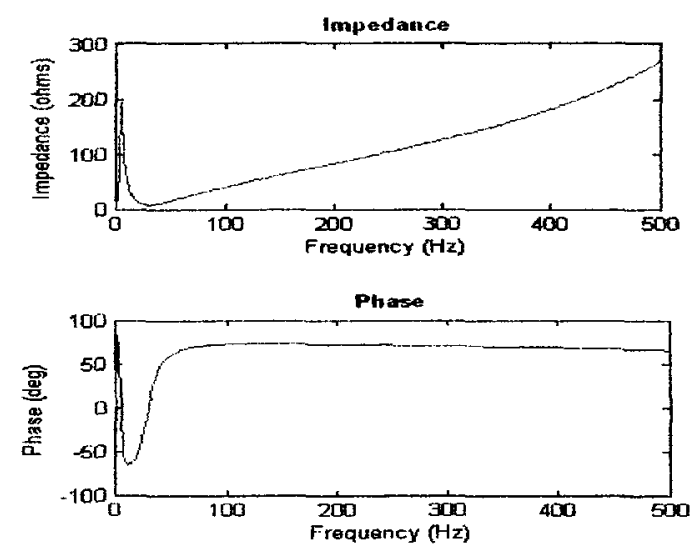

Figure 25 : Résultats de l'étude de réponse en fréquence à la fin de la ligne. $X c=8 \Omega$
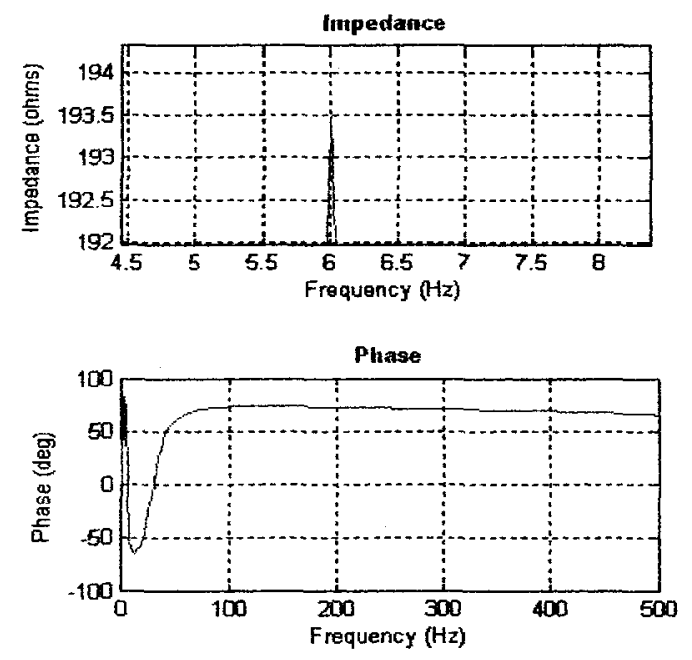

Figure 26 : Performance amplifiée de l'étude de réponse en fréquence à la fin de la ligne. $X c=8 \Omega$

\section{- Étude de réponse en fréquence à $X c=15 \Omega$}

Ce cas présent des valeurs d'impédance de résonance très hautes due à la valeur de la réactance qui entraîne un effet de résonance dans le réseau en augmentant l'impédance de résonance à $1130 \Omega$, voir le tableau 13. La performance de fréquences de résonance est observée aux les figures 26 et 27 . 


\section{Tableau 13}

Résultats de fréquences de résonance du réseau électrique

\begin{tabular}{|l|c|c|}
\hline Paramètres & Résonance sous-synchrone & Résonance hyper-synchrone \\
\hline Impédance & $225 \Omega$ & $1130 \Omega$ \\
\hline Fréquences & $8 \mathrm{~Hz}$ & $763 \mathrm{~Hz}$ \\
\hline
\end{tabular}
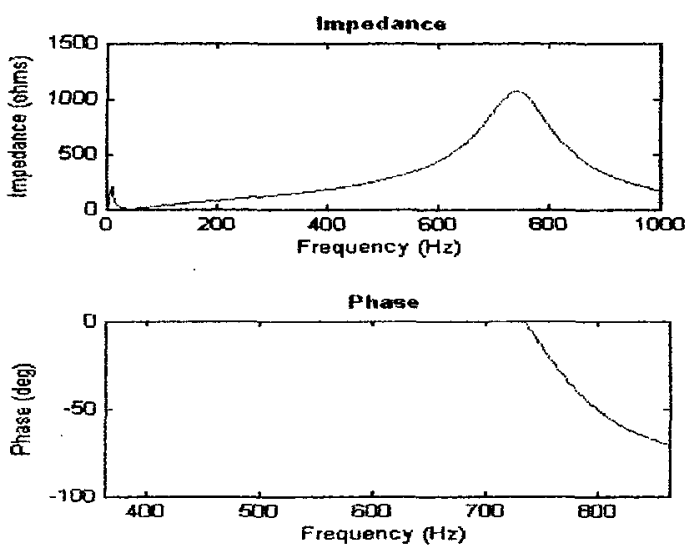

Figure 27 : Performance de l'étude de réponse en fréquence à la fin de la ligne $X c=$ $15 \Omega$
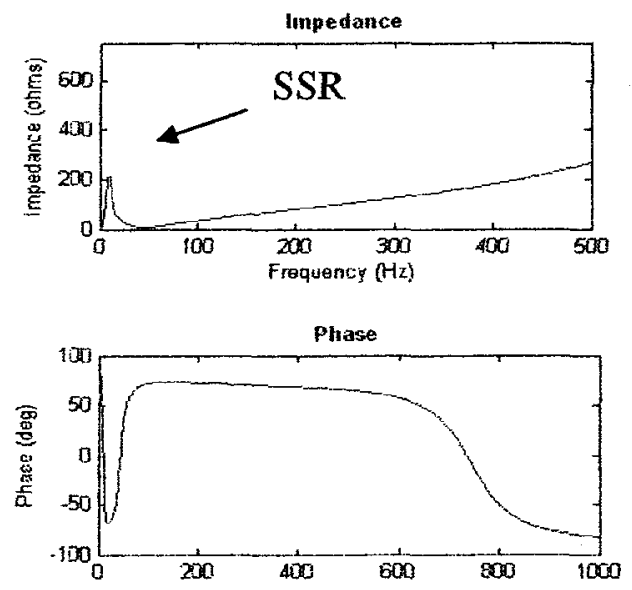

Figure 28 : Étude de réponse en fréquence vue amplifiée par $X c=15 \Omega$ 


\subsubsection{Critère de réduction de la résonance sous-synchrone.}

Le phénomène de résonance sous-synchrone peut se prévenir en recourant à différentes techniques. Le critère de réduction de la résonance sous-synchrone est développé par rapport aux trois technologies implantées par les entreprises de transport de l'énergie.

\section{- Principe de fonctionnement des équipements de protection antirésonance sous-synchrone du condensateur série.}

D'abord, pour la première technologie, l'amorçage du thyristor se produit lorsqu'il détecte une tension sous-synchrone provoquée par la résonance sous-synchrone. Cet amorçage des thyristors ajoute instantanément la résistance en parallèle du condensateur série. La configuration de la figure 29 contrôles la décharge résistive du circuit. Ce montage donne la possibilité de varier le taux de compensation série aux valeurs désirées en augmentant le transfert de puissance sans produire d'oscillations de résonance sous-synchrone.

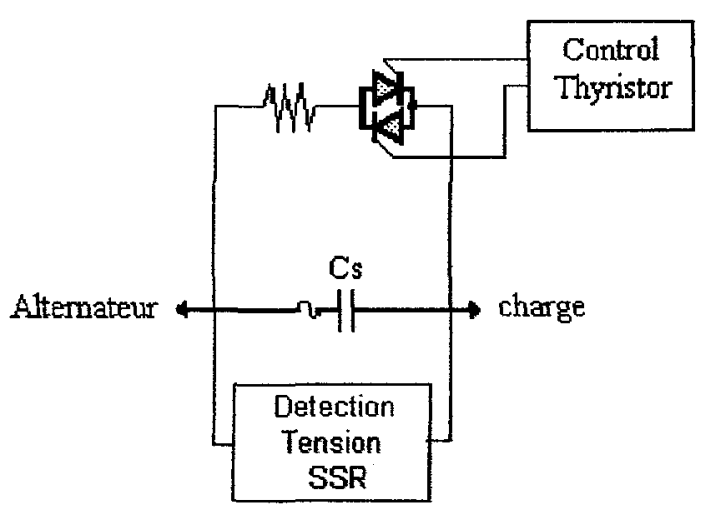

Figure 29: Schéma du condensateur série en parallèle avec une résistance contrôlée par le thyristor. Configuration développée par N.G Hingorani.

La deuxième technologie appelée la compensation série contrôlée par thyristor TCSC, figure 30 , consiste à contrôler une inductance au moyen d'un thyristor afin de 
fournir au réseau une réactance capacitive variable. L'amorçage du thyristor s'effectue lorsqu'il apparaît une tension sous-synchrone provoquées par la résonance soussynchrone. Le schéma de la figure 30 est une configuration qui améliore le transfert limité de puissance par la stabilité en régime transitoire et permanent. La technologie TCSC(Thyristor Controlled Serie Capacitor) se caractérise par un amorçage de haute vitesse et un mécanisme qui contrôle l'écoulement de puissance dans la ligne et réajuste cet écoulement pendant plusieurs contingences permettant de maintenir le réseau dans les limites admissibles de stabilité.

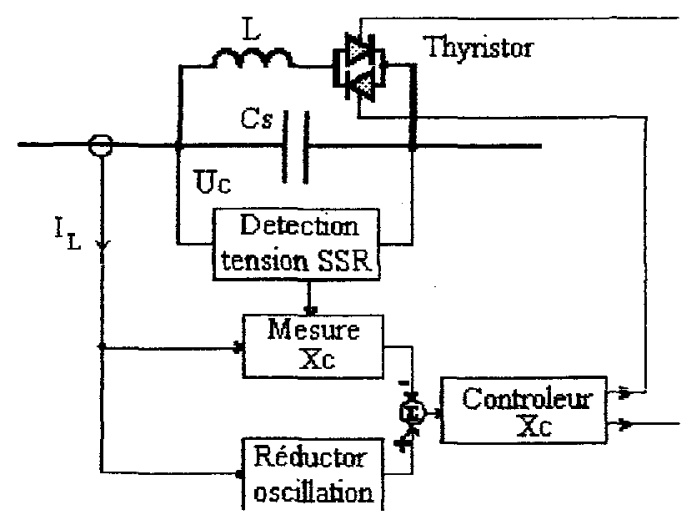

Figure 30 : Schéma du condensateur série contrôlé par thyristor

La troisième technologie connue comme MINICAP est installée comme un équipement auxiliaire pour protéger le condensateur série et d'autres éléments connectés au réseau contre la ferroresonance et des résonances produites par l'autoexcitation de moteurs à induction, figure 30 . Le détecteur de résonance est installé dans le compartiment de la commande du condensateur série. Le signal d'entrée provient des transformateurs du courant situés dans la section de haute tension du cabinet pour mesurer le courant de ligne de deux phases. Le détecteur est installé sur des cartes de microprocesseur, semblables à celles utilisées dans l'équipement de la commande destinée aux courants de ligne qui viennent de deux phases en amont du condensateur. Ces signaux du courant sont filtrés dans l'ordinateur pour les analyser et pouvoir détecter le niveau de résonance sous-synchrone. La figure 31 représente un schéma de cette troisième technologie anti-résonance. 


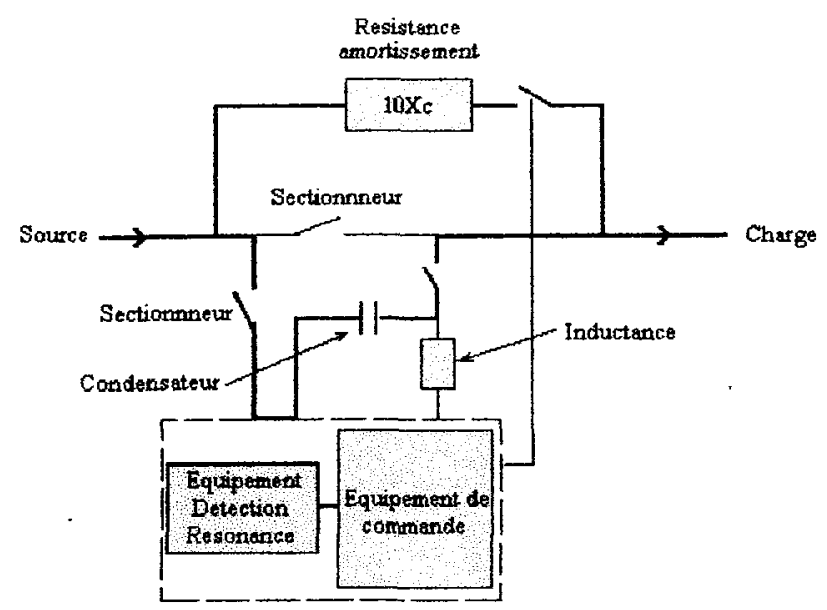

Figure 31 : Schéma du condensateur série avec équipement antirésonance

Un signal est émis lorsqu'un courant de résonance supérieur au niveau préréglé est détecté par le détecteur. Ce signal s'utilise à l'intérieur dans la commande du cabinet du condensateur série pour le commuter dans un circuit parallèle extérieur avec une résistance d'amortissement. Cette résistance parallèle peut variée entre 5 à 10 fois la réactance capacitive du condensateur série. D'ailleurs, l'ensemble du condensateur série avec d'autres composantes produisent des courants sous-harmoniques. Le détecteur de résonance contenu dans le schéma de la figure 30 permet d'amortir ces courants. Il utilise un interrupteur de contournement qui éteint ces courants à travers une résistance parallèle externe aux bornes du condensateur (Westinghouse, 1976).

\subsubsection{Effet de l'opération de démarrage des moteurs dans la demande de puissance des clients résidentiels et industriels et technique de réduction des surcharges.}

Les démarrages de moteurs sont considérés comme l'origine des perturbations expérimentées par les réseaux électriques. Le démarrage des moteurs crée un appel du courant de démarrage de six à huit fois supérieur au courant nominal. Pour réduire cette surcharge, les utilisateurs appliquent au stator une tension réduite à travers plusieurs techniques comme l'autotransformateur, la résistance série, etc (Westinghouse, 1976). 
La demande maximale de puissance des clients résidentiels se produit pendant les heures où les clients branchent les appareils chez eux. La superposition de cette demande de puissance à la demande normale de puissance des clients résidentiels produit une grande amplitude de puissance que la source ne pourrait pas transmettre. Pour cette raison, les clients industriels comme les scieries et les mines ont opté pour créer un protocole de démarrage de tous les groupes moteurs de l'industrie afin de réduire l'absorption excessive du courant de démarrage et éviter aussi la pénalisation de les entreprise de l'énergie pour la demande exagérée de transfert de puissance. Les nouvelles technologies de compensation série contiennent des configurations qui permettent de réduire la résonance sous-synchrone produite par le démarrage. Pour comprendre le fonctionnement des ces installations, on utilise la figure 32 pour montrer la performance du système antirésonance sous-synchrone par rapport à la demande maximale de puissance des clients résidentiels et industriels. L'analyse de cette performance sera expliquée en utilisant 5 scénarios différents tels que :

\section{- Scénario 1}

La demande de puissance aux heures de pleine charge de clients résidentiels branchés au point d'utilisation, $P_{\max } \mathrm{CR}$, figure 32 .

- Scénario 2

Les démarrages des moteurs des usines et des scieries, DM, demandent des valeurs de courants de 4 à 6 fois le courant nominal pour démarrer ces moteurs, figure 32 .

- Scénario 3

Le démarrage des moteurs cause des oscillations aux fréquences sous-synchrones dans la ligne de transport. Ces oscillations produisent des tensions sous-synchrones dans le condensateur série qui activeront l'amorçage du thyristor en contrôlant le déclenchement de résistances antirésonance, $\mathrm{RCs}$, afin d’éviter la résonance soussynchrone, SSR, figure 32.

- Scénario 4

Les scieries et les usines commencent les périodes d'interruption d'opérations, AO, afin d'éviter de dépasser les valeurs de demande maximale de puissance admissible 
par les normes établies à Hydro-Québec. Si une valeur de puissance de clients industriels PmaxCI est plus grande que la valeur d'opération autorisée, les clients industriels peuvent être pénalisés. Cela dépend des valeurs de puissance dépassée. Ces périodes d'arrêt d'opération évitent que la demande de puissance des clients industriels ne perturbe pas les clients résidentiels branchés à la même barre lorsque l'appel du courant de démarrage des moteurs se produit, figure 32.

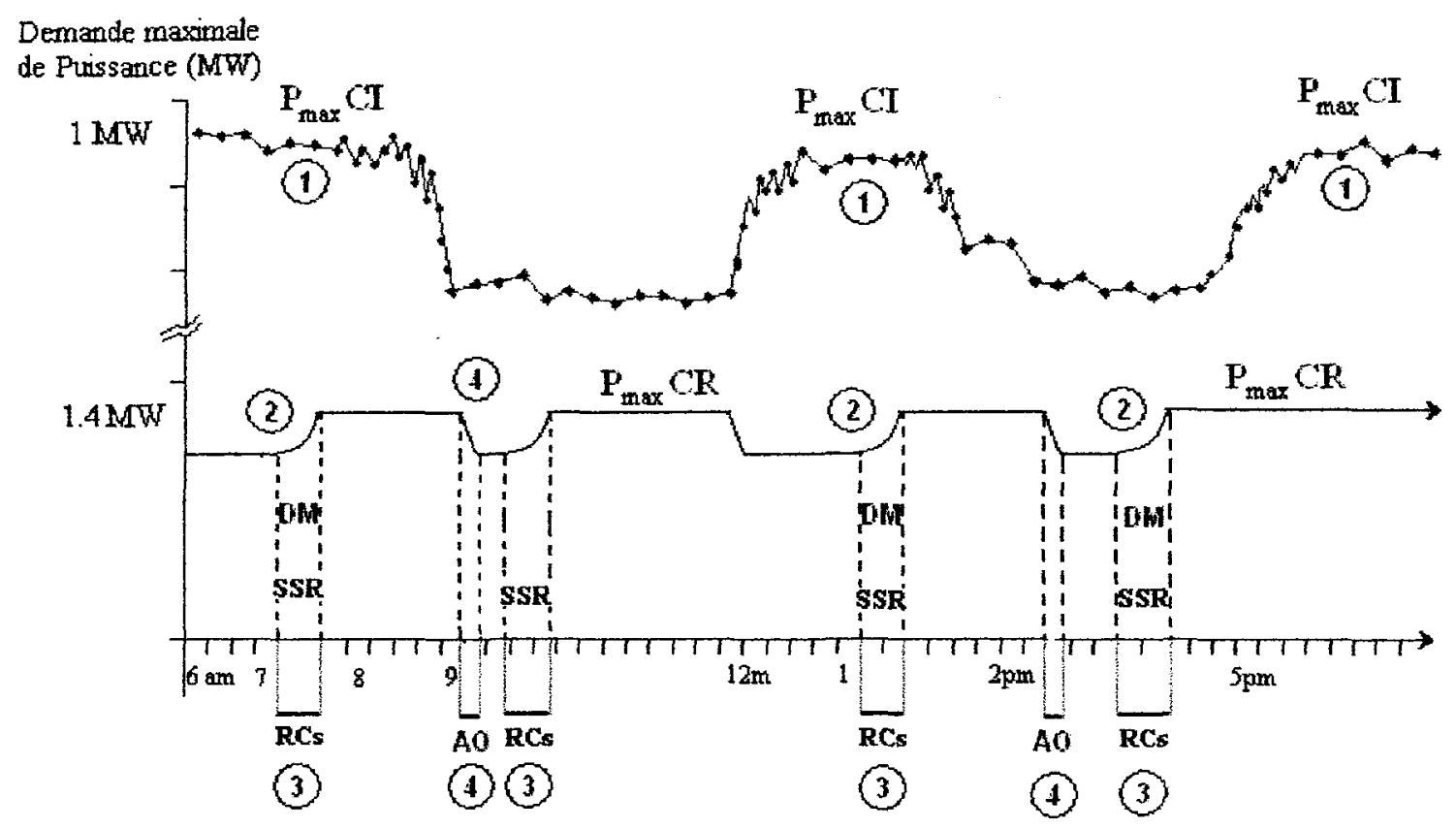

Figure 32 : Performance de la demande maximale de puissance des clients residentiels et industriels par rapport au système de protection antirésonance du condensateur série 


\section{CHAPITRE III}

\section{METHODE ITERATIVE DE CALCUL DU TAUX DE COMPENSATION SÉRIE ET DE LA POSITION OPTIMALE}

La méthode itérative est composée de 5 étapes qui tiennent compte de l'évaluation des contraintes de stabilité du réseau électrique compensé. Cette méthode comprend la construction d'une région d'opération appelée « aire optimale ». Elle a été créée à partir des limites maximales et minimales calculées pour chaque contrainte afin d'obtenir un intervalle optimal où le réseau devra être maintenu dans des conditions de stabilité.

L'optimisation se développe à partir des itérations de la réactance capacitive de la compensation série ainsi que de sa position sur la ligne de distribution. Cela nous permettra d'observer la performance des grandeurs du réseau afin de trouver la position optimale de la réactance capacitive qui conduira à minimiser la variation de tension sans produire des perturbations dans le réseau de distribution. Dans le contraintes de stabilité, on avait inclus une étude de réponse en fréquence afin d'identifier les possibles fréquences de résonance sous-synchrone produites par l'interaction entre le condensateur série et les autres éléments non-linéaires du réseau. Cependant, la topologie du réseau à étudier contient un dispositif dans l'installation du banc de condensateurs série qui le protége contre la résonance sous-synchrone à différents degrés de compensation. Pour cette raison, cette contrainte ne sera pas inclut dans l'aire optimale.

La méthode d'optimisation est implémentée dans le logiciel Matlab (voir app. H, p. 182 à 184) dont le programme permet de calculer les résultats de l'évaluation des 
contraintes et de la fonction objective. Ce programme utilise aussi le logiciel Power SimPowersystems-Simulink pour le calcul de l'écoulement de puissance fait dans le réseau de distribution à étudier.

D'ailleurs, les différentes étapes de la méthode de calcul sont expliquées dans les sections suivantes du chapitre.

\section{1 Étape 1 : Identification et définition des contraintes de stabilité et de la fonction objective}

D'abord, la fonction objective, la variation de tension (2-78), obtenue dans le chapitre II est inclue dans la région d'opération modélisée pour observer leur performance lorsque le réseau est compensé aux différents taux de compensation série, figure 36.

$$
\Delta V \cong \frac{P \cdot R+Q \cdot\left(X_{L}-X c\right)}{V_{2}}=f(X c)
$$

D'ailleurs, les contraintes de stabilité seront les éléments qui définiront les limites de la région d'opération du taux de compensation série optimal. Les contraintes définies dans la méthode d'optimisation s'expriment ainsi :

- Taux de compensation série hl

- Transfert maximal de puissance h2

- Oscillations de vitesse des moteurs h3

- Inversion du courant de court-circuit h4

- Phénomène de papillotement h5

- Pertes de puissance réactivé h6

- Résonance sous synchrone $h 7$

Ayant identifié les contraintes de stabilité, on procède l'évaluation des contraintes afin de déterminer leurs limites en utilisant la solution de l'écoulement de puissance. 


\subsubsection{Condition initiale de la réactance capacitive du condensateur série}

Les conditions initiales de la méthode d'optimisation sont posées pour démarrer la méthode d'optimisation. L'objectif de cette étape est de varier la réactance du condensateur jusqu'à ce qu'elle soit égale à la réactance inductive de la ligne. Le pas de variation de $X c$ est de $1 \Omega$ (3-79).

Selon le critère de la contrainte du taux de compensation série, la valeur minimale sera $X c=0 \Omega$ et la valeur maximale sera $X_{L}=23.7 \Omega$ (réactance inductive de la ligne de distribution). Nous devons considérer que des valeurs plus grandes que $X_{L}$, pourront produire le phénomène de surcompensation.

Dans cette étape, on définit les conditions initiales de la valeur de réactance capacitive et le pas de variation (3-52) de cette réactance comme suit :

$$
X_{C}^{0}=0,1,2, \ldots, 23.7 \Omega \quad \Delta X_{C}=1
$$

\subsubsection{Condition initiale de la position du banc de condensateurs série}

Dans cette étape, on donne une position initiale au condensateur série sur la ligne de distribution. Le paramètre « $\iota$ », qui identifie la valeur de la position, est varié par rapport à la valeur de la réactance capacitive de compensation obtenue à l'étape précédente. Cette valeur devra respecter deux conditions :

- La position du banc de condensateurs devra être entre $\mathrm{L}=0 \mathrm{~km}$ et $\mathrm{L}=60 \mathrm{~km}$ qui est la longueur de la ligne de distribution.

- La valeur de la réactance inductive de la ligne en fonction de la position « $\zeta$ » devra être plus grande que la réactance capacitive du banc de condensateurs. 


\section{2 Étape 2. Solution de l'écoulement de puissance}

Une solution de l'écoulement de puissance devient comme le point de démarrage afin d'évaluer la fonction objective et des contraintes sous de conditions de stabilité, figure 34. À l'aide du logiciel «SimPowerSystems-Simulink», il est possible d'obtenir les paramètres tels que les valeurs de tension, les angles des tensions aux barres et la puissance active et réactive transmise sur la ligne de distribution.

Les variables électriques utilisées pour évaluer les composantes de l'optimisation sont classifiées comme suit :

Les grandeurs fixes dans la méthode d'optimisation:

- Réactance inductive de la ligne de transport.

- Résistance de la ligne de transport.

- Angle de la tension à l'alternateur. Barre de référence pour l'écoulement de puissance.

- Puissance électrique des moteurs d'induction branchés à la charge HP.

- Gêne perçue par une fluctuation de tension $\mathrm{g}_{\mathrm{f}}$ obtenue dans l'étude de papillotement.

- Puissance nominale de moteurs d'induction.

- Rendement de moteur d'induction.

- Facteur de puissance de la charge branchée a but de la ligne et en parallèle avec les moteurs d'induction.

- Courant de démarrage.

- Courant nominal.

- Coefficient du type de démarrage $\beta$ de moteurs d'induction dans le scieries.

- Limite maximale de papillotement sur un réseau de distribution que tout abonné peut subir en tous points du réseau de distribution moyenne et basse tension. 
L'amplitude est de $0.5 \%$ à $8 \mathrm{~Hz}$. Ce paramètre est utilisé pour calculer la puissance de court circuit et la valeur minimale de compensation série.

Les grandeurs qui varieront dans la méthode d'optimisation deviennent comme suit :

- Tensions et les angles sur la ligne de transport et à la charge.

- Réactance capacitive du condensateur série.

- Grandeur de puissance active et réactive foumies pour le réseau électrique.

- Puissance de court circuit obtenue pour chaque variation du taux de compensation série selon l'étude de papillotement, la section 2.2 .6 , chapitre II.

- Position optimale du condensateur série qui varie par rapport au taux de compensation série.

\section{3 Étape 3. Évaluation des contraintes de stabilité et de la fonction objective}

Dans cette étape, on a obtenus tous les grandeurs pour évaluer les contraintes de stabilité, figure 36 . Les contraintes définies dans la méthode d'optimisation sont les suivantes :

- Taux de compensation série hl : Les limites maximale et minimale de cette contrainte sont considérées comme les limites initiales de la région d'opération du taux de compensation série. L'inégalité (3-53) montre que la limite minimal est $0 \%$ puisque le réseau n'est pas compensé, et la limite maximale comme 100\%, pour éviter la surcompensation du réseau.

$$
0 \leq \frac{X_{C}^{0}}{23.7} \leq 100 \% \text { avec } X_{L}=23.7 \Omega
$$


- Transfert maximal de puissance h2 : L'élévation du taux de compensation entraîne un croissance du transfert de puissance due à la réduction de la réactance équivalente de la ligne de transport. Ce transfert de puissance devra être égale ou plus grand à la puissance nominale initiale d'opération du réseau électrique. Cette contrainte h2 se définit en (3-54) comme suit :

$$
P_{O} \leq P_{\max }
$$

On effectue l'écoulement de puissance à chaque fois qu'on varie la position du banc de condensateurs dû au fait que la tension $V_{2}$ change par rapport à ce variable. Ayant obtenu chaque valeur de tension à la charge $V_{2}$, on évalue le transfert de la puissance $P_{\max }$, selon l'équation (2-81) du chapitre 2.

La figure 33 montre comme le transfert de puissance et le profil de tension sont améliorés lorsqu'on approche le banc de condensateurs série à la charge.

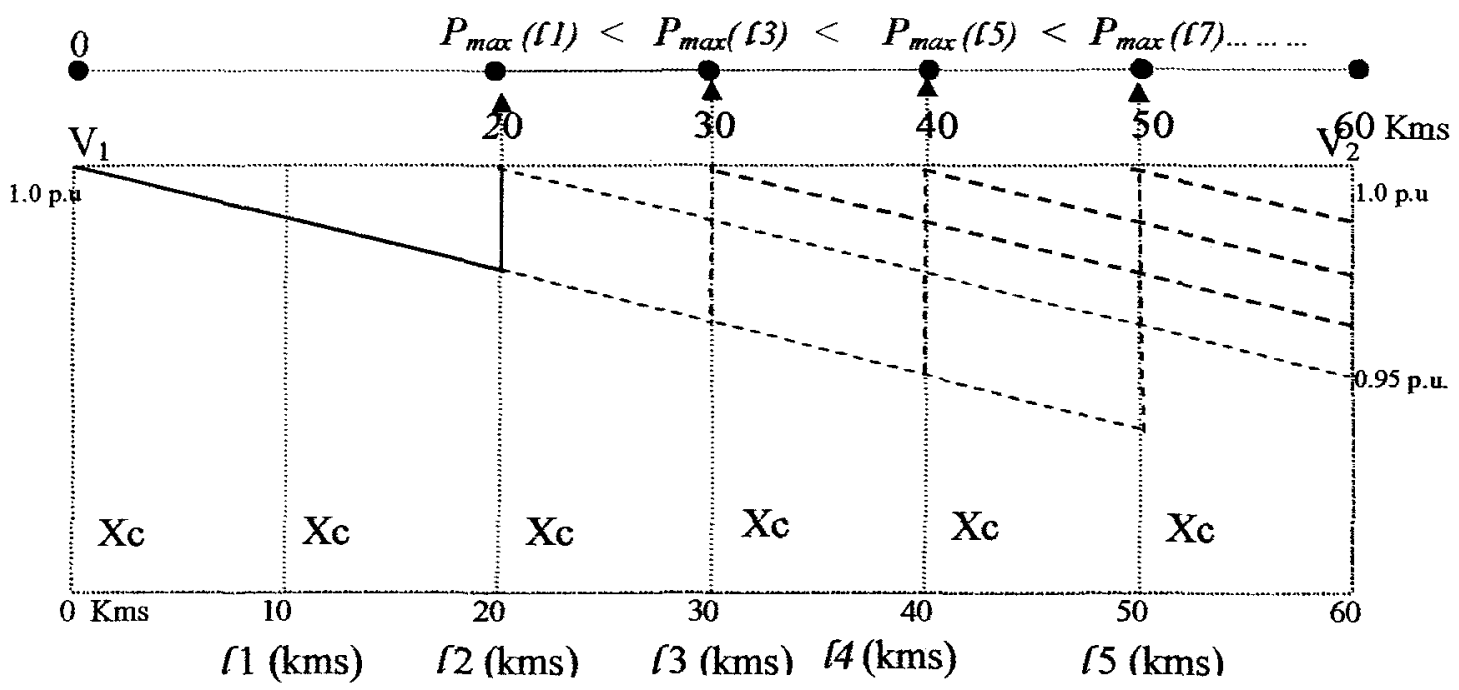

Figure 33 : Diagramme du profil de tension avec différentes positions du banc de condensateurs série sur la ligne de distribution 
- Oscillations de vitesse des moteurs h3 : La contrainte h3 sur l'oscillation de vitesse du moteur peut s'identifier avec le rapport $R / X$ qui se trouve entre 0 et 1 , expression (2-55) du chapitre II. Cette valeur réduit les effets d'échauffement au niveau du condensateur série et des moteurs en diminuant l'amplitude des courants élevés pendant leurs démarrages ou lorsque le condensateur série se trouve déclenché dans la ligne.

$$
0 \leq \frac{R}{X_{L}-X_{C}^{n}} \leq 1
$$

En considérant les valeurs de résistance et de réactance totales de la ligne, la région d'opération se réduit à $\tau=66 \%(X c=15.7 \Omega)$. Mais pendant un événement de court-circuit à côté de la charge, la réactance équivalente est négative et le condensateur pourrait s`exposer aux courants et tensions très élevés.

- Inversion du courant de court-circuit h4: Selon l'étude de cette contrainte, ce phénomène pourra être évités lorsque il se produit la condition (3-56).

$$
X_{C}^{n} \leq X_{L}^{*} \boldsymbol{l}_{n}
$$

Cette expression nous dit que la réactance inductive de la ligne, au point d'installation de la compensation, devra être plus grande que la réactance capacitive du condensateur série. Pour un taux de compensation série de $\tau=54.85 \%(X c=$ $13 \Omega$ ) à $35 \mathrm{Km}$ de la source, le réseau peut être compensé en évitant le phénomène d'inversion du courant. Toutefois, il existe des lignes de distribution dans les secteurs ruraux qui ne contiennent pas de systèmes de protection à distance. Ceci rend plus vulnérable à la ligne lorsqu'il se produit un défaut en provoquant des puissances de court-circuit très élevées. Celles-ci pourraient se reproduire si la ligne n'est pas protégée. Le tableau 15 montre les valeurs de la réactance totale de la ligne (incluant $X_{C}$ ) à différents taux de compensation. 
Tableau 14

Réactance équivalente de la ligne par rapport à la position des condensateurs série

\begin{tabular}{|c|c|c|c|c|c|c|c|c|c|c|}
\hline & \multicolumn{10}{|c|}{ Longueur (Km) } \\
\hline $\mathrm{Xc}(\Omega)$ & 10 & 15 & 20 & 25 & 30 & 35 & 40 & 45 & 50 & 55 \\
\hline 5 & 105 & 0,925 & 2,9 & 4.875 & 6,85 & 8,825 & 10,8 & 12,78 & 14,75 & 16,73 \\
\hline 7 & 306 & 1005 & 0,9 & 2.875 & 4,85 & 6,825 & 8,8 & 10,78 & 12,75 & 14,73 \\
\hline 9 & -6.65 & 309 & $1+1$ & 0.875 & 2,85 & 4,825 & 6,8 & 8,775 & 10,75 & 12,73 \\
\hline 11 & 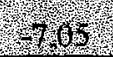 & (3) & 1 & $1+12$ & 0,85 & 2,825 & 4,8 & 6,775 & 8,75 & 10,73 \\
\hline 13 & 40.05 & 7015 & 4 & 3.125 & 115 & 0,825 & 2,8 & 4,775 & 6,75 & 8,725 \\
\hline 15 & 408 & 90175 & -7) & 125 & -5.15 & 1175 & 0,8 & 2,775 & 4,75 & 6,725 \\
\hline
\end{tabular}

*Les valeurs en gris expriment les grandeurs négatives de la réactance totale de la ligne qui pourraient provoquer l'inversion du courant de court-circuit.

- Phénomène de papillotement h5 : L'étude de papillotement permet de calculer la valeur du taux de compensation série et la puissance de court circuit minimale pour laquelle le phénomène de papillotement est réduit. En utilisant le critère de la section 2.2.6 du chapitre 2 , la valeur de la puissance de court-circuit du réseau à étudier est d'environ 14.6 MVA. Cette puissance indique que le réseau peut se compenser à partir du taux de compensation série de $\tau=21 \%(\mathrm{Xc}=5 \Omega)$.

- Pertes de puissance réactive h6 : L'ajout de la compensation série réduit des pertes de puissance réactive et améliore les profils de tension en aval du condensateur tenant compte des normes canadiennes de tension d'opération, (voir app. B, p. 150 à 151). La condition de cette contrainte s'exprime selon l'inégalité (3-57).

$$
Q_{L} \leq Q_{L o}
$$

$Q_{L}$ : Puissance réactive après l' application de la compensation série. $Q_{L O}:$ Puissance réactive initiale. 
Pour la valeur minimal de l'aire optimale, $\tau=21.1 \%$, les pertes de puissance réactive $Q_{L}$ sont d'environ 0.0083 p.u à et pour $\tau=54.85 \%$ d'environ 0.0055 p.u.

- Résonance sous-synchrone h7: le réseau de distribution à étudier contient un dispositif constitué par un thyristor qui contrôle une résistance en parallèle au condensateur série. II permet d'annuler les fréquences de résonance soussynchrones lors des démarrages des moteurs. Cette résonance apparaît avec des fréquences plus petites que la fréquence naturelle $(f=60 \mathrm{~Hz})$.

\subsection{Condition 1. Boucle de variation de la réactance capacitive de la compensation série sur la ligne de distribution}

Dans cette boucle, la méthode d'optimisation fait une comparaison des résultats obtenus de l'analyse de chaque contrainte, figure 36 . Si une valeur du taux de compensation se trouve hors des limites d'une contrainte, la boucle obligera la méthode d'optimisation d'aller au début du protocole afin de varier les conditions initiales et changer la valeur de réactance capacitive afin d'effectuer l'écoulement de puissance. Cela permettra d'évaluer nouvellement la fonction objective et les contraintes. Par contre, si la valeur du taux de compensation se trouve dans l'aire d'optimisation, il deviendra comme une valeur optimale potentielle qui sera validée à partir des simulations numériques. Le numéro maximal des interactions effectuées par la méthode arrêtera lorsque la réactance capacitive deviendra égale à la réactance inductive de la ligne. Cette étape est appelée comme la condition Nol, expression (3-58), figure 36 :

$$
X_{C}^{k+1}=X_{C}^{K}+\Delta X_{C} \text { avec } k=0 \ldots X_{L}
$$

\section{5 Étape 4. Stockage des valeurs calculées de $X_{C}$}

Dans cette étape, figure 3 , on a accumulé les valeurs de $X_{C}$ qui respectent les contraintes de stabilité. Le pas suivant sera d'évaluer ces valeurs pour différentes 
positions sur la ligne de distribution. Ici, on évalue si les valeurs de $X_{C}$ respectent les conditions des contraintes de stabilité. Après on passe à la prochaine étape de la méthode, la boucle de variation de la position.

\subsection{Condition 2. Boucle de variation de la position de la réactance capacitive sur la ligne de distribution}

En ce qui concerne la variation de la position, après avoir obtenu les valeurs de réactance capacitive dans le bloc de stockage, on procède à l'évaluation des contraintes de stabilité. Si la valeur assignée de $X c$ à $\zeta(\mathrm{km})$ ne respecte pas les conditions de stabilité, condition No2, figure 36, le protocole immédiatement change la valeur de la position de la réactance $X c(3-59)$ et redémarre l'écoulement de puissance pour évaluer les contraintes de stabilité. La variation de la position peut s'observer dans la figure 35 .

$$
\ell+\Delta l \text { avec } l \leq L
$$

\subsection{1 Étape 5. Résultats de la méthode d’optimisation et simulation des tests typiques}

L'obtention du taux de compensation série et la position optimale sont trouvées grâce à la région d'opération standard ou « l'aire optimale » qui a été déterminée à partir de la performance de la fonction objective et les limites maximales et minimales des contraintes de stabilité, figure 34 .

On constante dans l'évaluation des contraintes, que la valeur minimale de l'aire optimale du taux de compensation sera l'étude de papillotement, figure 35 . Pour le réseau de distribution de l'annexe $A$, la valeur minimale de puissance de court-circuit est égale à $K V A_{C C}=14.6$ MVA avec un taux de compensation de $\tau=21 \%(\mathrm{Xc}=5 \Omega)$, voir le tableau 16. En ce qui concerne la valeur maximale de compensation série, la contrainte sur l'inversion du courant de court-circuit et le phénomène d'oscillations de vitesse de rotation du moteur seront les éléments déterminants pour définir l'aire 
optimale, voir tableau 15. Cependant, pour le réseau étudié dans le projet, la contrainte qui assure l'obtention de la limite maximale de l'aire d'optimisation du taux de compensation est le phénomène d'oscillations de vitesse des moteurs, figure 34 . La valeur exacte de cette contrainte a été trouvée à partir des simulations numériques des groupes moteurs, voir tableau 15 .

Tableau 15

Résul tats de la méthode d'optimisation

\begin{tabular}{|c|c|c|c|c|}
\hline Contrainte & Critère & Valeur & $\mathrm{Xc}(\Omega)$ & $f(X c)=\Delta V *$ \\
\hline$\tau$ & $\mathrm{X}_{\mathrm{L}} / \mathrm{Xc}$ & $\tau \leq 100 \%$ & 23.7 & $0.58 \mathrm{kV}$ \\
\hline $\mathrm{TMP}$ & $\mathrm{Po} \leq \mathrm{Pn}$ & $2.3 \mathrm{MW} \leq \mathrm{P}_{\max }$ & 23.7 & $0.58 \mathrm{kV}$ \\
\hline $\mathrm{R} / \mathrm{X}$ & $0<\mathrm{R} / \mathrm{X}<1$ & $0<\mathrm{Xc}<15.7$ & 13 & $0.65 \mathrm{kV}$ \\
\hline $\mathrm{Icc}$ & $\mathrm{X}_{\mathrm{L}} \geq \mathrm{Xc}$ & & 13 & $0.65 \mathrm{kV}$ \\
\hline Papillotement & $K V A_{C C} \leq \frac{\alpha .100}{0.5}$ & $K V A_{C C}=14.6 M V A$ & 5 & $0.73 \mathrm{kV}$ \\
\hline
\end{tabular}

(*) Les variations de tension sont mesurées au primaire du transformateur à la charge.

On constate que la valeur du taux de compensation série optimale est $\tau=55.85 \%$ à une réactance capacitive de $X c=13 \Omega$. À cette valeur on minimise la variation de tension à $\Delta V=0.650 \mathrm{kV}$ à côté de la tension au primaire du transformateur de distribution (25 $\mathrm{kV}$ ) au point de raccordement de la charge. On remarque que cette variation de tension est plus petite que $\Delta V=0.85 \mathrm{kV}$ (sans la compensation). Alors, on peut dire que l'effet de la compensation a réussi son objectif car le profil de tension de la charge a été amélioré. Le réseau de distribution a été simulé avec cette valeur afin d'observer sa performance en régime transitoire et permanent.

En général, la compensation série améliore les profils de tension à mesure qu'on augmente le taux de compensation. On peut constater l'affirmation précédemment posée en calculant les variations de tension en aval du condensateur série et à la charge (voir app. I, p. 185 à 186). 


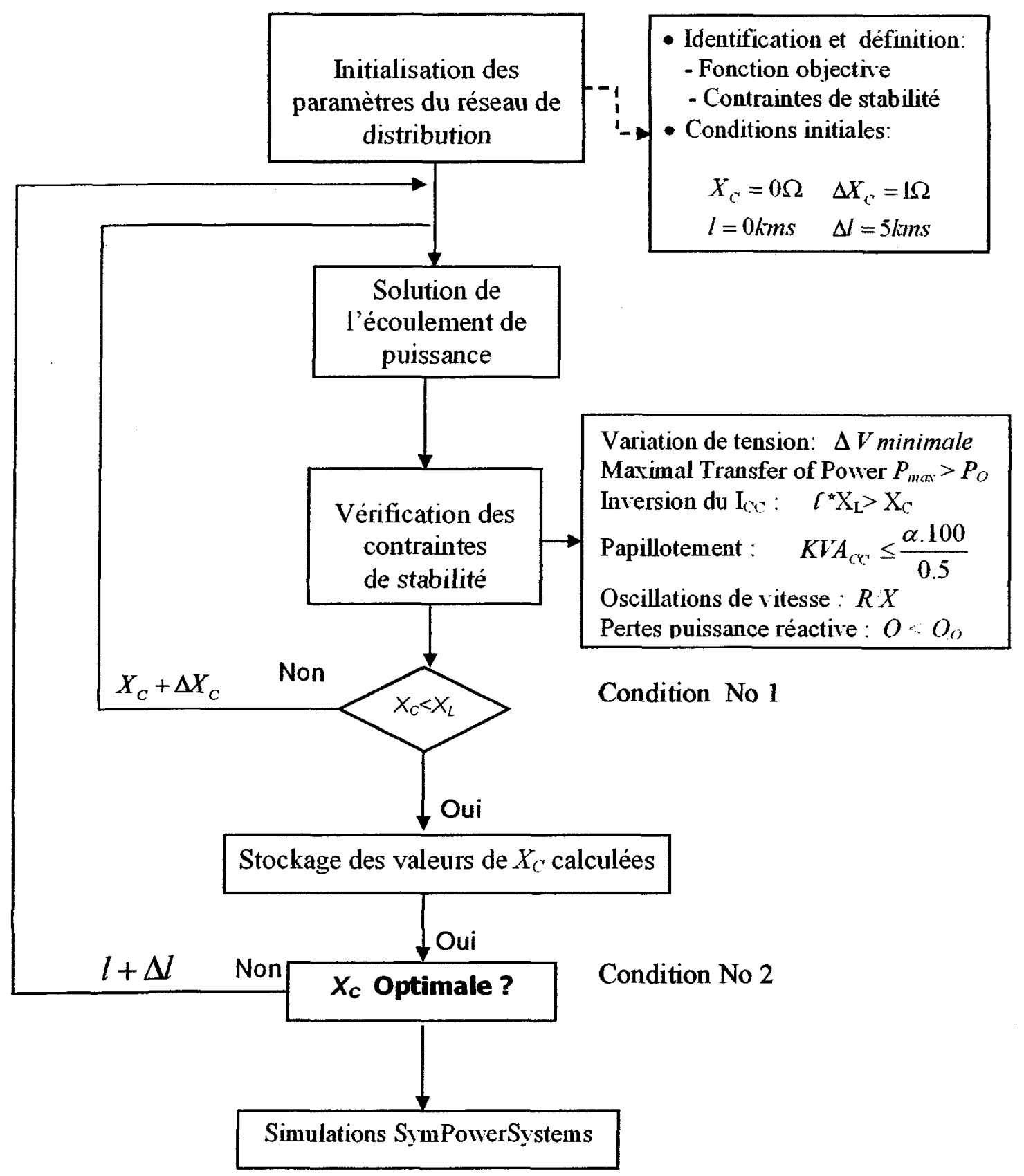

Figure 35 : Organigramme de la méthode d'optimisation 


\subsection{Comparaison de calcul de la position optimal de $\mathrm{Xc}$ avec la méthode basée sous des conditions de tension en amont et en aval du condensateur série}

La position du condensateur est considérée comme un facteur important en terme d'étude de papillotement. Si la valeur de la tension $V_{2}$ du condensateur est plus grande que le profil de tension de $V_{1}$ dans le point de raccordement, figure 36 , il est possible que cette variation de tension entraîne un papillotement élevé. D'après ce critère, on développe une formulation mathématique qui permet d'acquérir la position optimale du condensateur série à différents taux de compensation.

Le circuit équivalent du réseau de distribution radial de la figure 12 chapitre 1 , montre les tensions aux différents points d'opération les plus importants qui seront utilisés pour développer l'équation et ainsi trouver la position optimale du condensateur série.

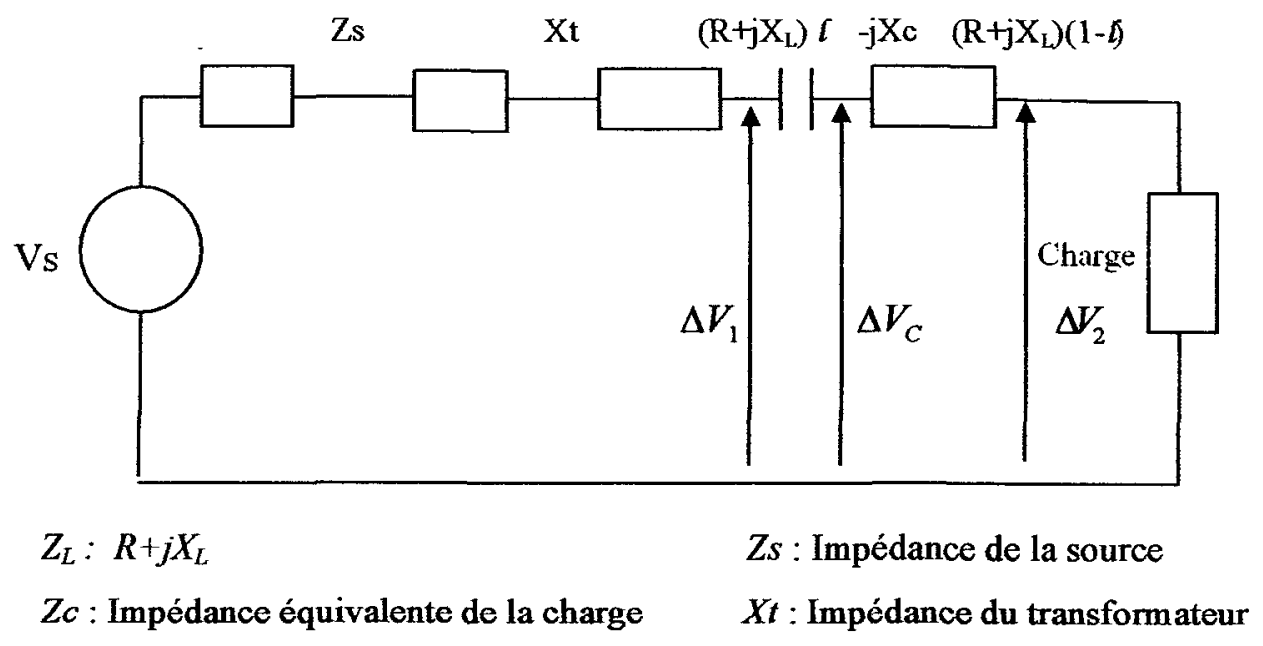

Figure 36 : Circuit radial monophasé équivalent

Selon la figure 36, les variations de tension en amont et en aval du condensateur seront développées à partir des équations suivantes:

$$
\mathrm{V}_{1}=\frac{(1-l) Z_{L}-j X c+Z c}{Z s+Z_{L}-j X c+Z c} V s
$$




$$
\mathrm{V}_{C}=\frac{(1-l) Z_{L}+Z c}{Z s+Z_{L}-j X c+Z c} V s
$$

En développant la tension à la charge par rapport à la tension de la source, l'équation obtenue devient (3-90):

$$
\mathrm{V}_{2}=\frac{Z c}{Z s+Z_{L}-j X c+Z c} V_{s}
$$

La tension en aval du condensateur est plus petite que la tension en amont à cause de l'effet instantané et continu de l'ajout du condensateur série sur la ligne. La relation de ces tensions du diagramme de la figure 13 s'exprime dans l'équation (3-91) telle que :

$$
\Delta V_{1} \% \leq \Delta V_{C} \%
$$

En remplaçant les variations de tension à côté du condensateur, on obtient l'équation $(3-64)$ :

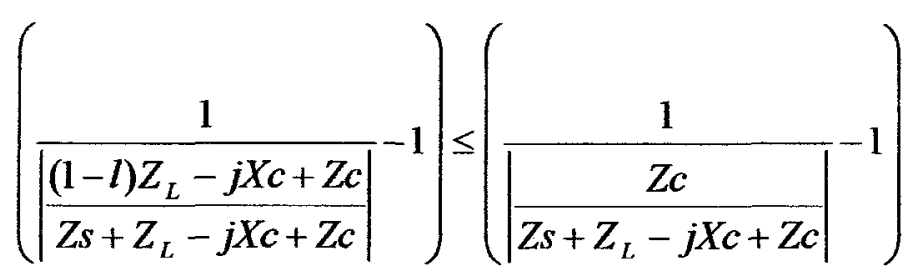

On suppose que le réseau n'est pas surcompensé, c'est-à-dire, $X c<Z_{s}+Z_{\mathrm{L}}$. Alors l'inégalité (3-65) peut s'exprimer dans l'expression (3-93) :

$$
\left|\frac{(1-l) Z_{L}-j X c+Z c}{Z s+Z_{L}-j X c+Z c}\right| \geq \mid \frac{Z c}{Z s+Z_{L}-j X c+Z c \mid}
$$

L'équation (3-66) sera exprimée en fonction de la position et de la réactance capacitive :

$$
\left|(1-l) Z_{L}-j X c+Z c\right| \geq|Z c|
$$


La même analyse sera développée pour des conditions de tension en aval du condensateur. L'équation (3-67) montre la tension à la charge par rapport à la tension de la source :

$$
V_{2}=\frac{(1-l) Z_{L}+Z c}{Z s+Z_{L}-j X c+Z c} V s
$$

Pour la condition de tension en aval du condensateur et à la charge, on arrive à l'expression (3-68):

$$
\left|\Delta V_{2} \%\right| \leq\left|\Delta V_{C} \%\right|
$$

La relation des valeurs absolues qui comprend les conditions de tension en amont et en aval du condensateur série devient l'expression (3-69):

$$
\left|\frac{1}{\left|\frac{(1-l) Z_{L}+Z c}{Z s+Z_{L}-j X c+Z c}\right|}-1\right| \leq\left|\frac{1}{\left|\frac{Z c}{Z s+Z_{L}-j X c+Z c}\right|}\right|
$$

En développant l'inégalité ci-dessus, on obtient le système (3-98),

$$
\left|\frac{(1-l) Z_{L}+Z c}{Z s+Z_{L}-j X c+Z c}\right| \geq\left|\frac{Z c}{Z s+Z_{L}-j X c+Z c \mid}\right| \Leftrightarrow 1-\frac{1}{\left|\frac{Z c}{Z s+Z_{L}-j X c+Z c \mid}\right|} \leq \frac{1}{\left|\frac{Z c}{Z s+Z_{L}-j X c+Z c}\right|}-1
$$

L'équation à gauche a été choisie pour évaluer le paramètre « $\zeta$ » qui détermine la position optimale du condensateur. Il reste l'expression de droite (3-71) :

$$
\frac{1}{\left|\frac{(1-l) Z_{L}+Z c}{Z s+Z_{L}-j X c+Z c \mid}\right|}+\frac{1}{\left|\frac{Z c}{Z s+Z_{L}-j X c+Z c \mid}\right|} \geq 2
$$


La relation obtenue sous des conditions de tension, $\Delta V_{1} \% \leq \Delta V_{C} \%$, peut être développée selon l'expression, $|a|+|b| \geq|a+b|$. Et la relation peut s'écrire comme (372) :

$$
\left|(1-l) Z_{L}-j X c\right|+|Z c| \geq|Z c|
$$

En remplaçant $Z_{L}=R+J X_{L}$, cette expression se réduit à l'équation (3-73) :

$$
(1-\Gamma)\left(\mathrm{R}+\mathrm{X}_{\mathrm{L}}\right)-\mathrm{Xc} \geq 0
$$

En divisant par $R+j X_{L}$, on obtient l'équation (3-74) :

$$
(1-\ell) \frac{R+X_{L}}{R+X_{L}}-\frac{X c}{R+X_{L}} \geq 0
$$

Alors la relation devient l'expression (3-75):

$$
(1-\ell)-\frac{X c}{R+X_{L}} \geq 0
$$

Voici la relation (3-76) qui détermine la position optimale du condensateur série par rapport au taux de compensation :

$$
\iota \leq \frac{R+X_{L}-X c}{R+X_{L}}
$$

Selon la formulation ci-dessus et le critère développé dans l'analyse de la contrainte No 3, la valeur maximale de compensation (contrainte d'oscillation de vitesse) d'environ $\mathrm{Xc}=15.66 \Omega(\tau=66 \%)$, voir tableau 16 , maintient la stabilité du réseau si le condensateur série se place à $30 \mathrm{Km}$ de la source. 
Tableau 16

Position optimale du condensateur série par rapport au taux de compensation.

\begin{tabular}{|c|c|c|c|}
\hline $\mathrm{Xc}(\Omega)$ & $\tau(\%)$ & $\begin{array}{c}\text { Réactance } \\
\text { équivalente } \\
\mathrm{X}\end{array}$ & $\begin{array}{c}\text { Position optimale } \\
\text { Condensateur } 1 \\
\text { (Kms) }\end{array}$ \\
\hline 8 & 33.76 & 15.7 & 45 \\
\hline 10 & 42.19 & 13.7 & 41 \\
\hline 12 & 50.63 & 11.7 & 37 \\
\hline 13 & 54.85 & 10.7 & 35 \\
\hline 14 & 59.07 & 9.7 & 34 \\
\hline 15 & 63.29 & 8.7 & 32 \\
\hline 15.66 & 66.07 & 8.04 & 30 \\
\hline
\end{tabular}

Selon la méthode précédemment développée, la valeur optimale pour laquelle on peut compenser le réseau doit se trouver à une distance de $l=35 \mathrm{Km}$. Cette valeur permet que la réactance équivalente soit positive. De plus, à cette position, le transfert de puissance est maximal en respectant tous les critères des contraintes de stabilité. En ce qui conceme la position optimale obtenue par la méthode d'optimisation, celle-ci nous dit que la position optimale où le condensateur série devra être installé est à environ $\boldsymbol{l}=$ $35 \mathrm{Km}$ pour éviter le phénomène d'inversion du courant de court circuit. Dans ce point la réactance totale de la ligne est de $0.83 \Omega$. Selon la méthode de condition de tension, la valeur optimale trouvée à partir de l'équation $(3$ - 76) sera de 0.5817 , alors on doit calculer le percentage par rapport à la longueur totale de la ligne de distribution. Le résultat calculé indique que le condensateur doit être installe à $34.9 \mathrm{Km}$ de la source, c'est-à-dire, à $58.17 \%$ de $60 \mathrm{Km}$.

La position optimale du banc de condensateurs est de $l=35 \mathrm{Km}$ de la source pour un taux de compensation série de $\tau=54.85 \%(\mathrm{Xc}=13 \Omega)$. Dans cette position, le réseau pourra transmettre une puissance de $2.88 \mathrm{MW}$.

Le résultat obtenu par cette seconde méthode sur la position du condensateur série est le même avec la méthode d'optimisation, ce qui montre la cohérence des méthodes développées. 


\section{CHAPITRE IV}

\section{SIMULATIONS ET RÉSULTATS}

La présentation de ces résultats a pour but de valider les résultats analytiques obtenus par la méthode d'optimisation. On développera plusieurs essais afin d'observer la performance du réseau et des machines asynchrones lorsque la ligne de distribution est compensée. L'étude de la stabilité dynamique lors des perturbations sera la base pour analyser les paramètres du réseau de distribution. Les résultats présentés sont obtenus des simulations numériques avec du logiciel SimPowerSystem-Simulink ${ }^{\text {. }}$.

La puissance totale demandée par la charge est de 2.1 MVA avec un facteur de puissance de $92 \%$. Le charge est composée d'une scierie avec 4 groupes de moteurs asynchrones, une charge branchée en amont du condensateur de 1MVA avec un FP= $90 \%$ et une autre charge de 1 MVA avec un $\mathrm{FP}=90 \%$ branchée en aval du condensateur à 60 kilomètres de la source en parallèle avec les groupes moteurs, figure. 37 .

Les essais à simuler sont choisis en considérant le mise en fonctionnement du réseau pour analyser ses performances, les démarrages de groupes moteurs et des perturbations communes produites dans les réseaux dont les effets peuvent conduire le système dans de situation d'instabilité ( voir app. J, p. 187 à 224). Le modèle Matlab/Simulink de la figure 37 est le modèle de base pour développer des essais sur le réseau. On fera des modifications sur des blocs du modèle selon l'essai correspondant. Le tableau 17 montre les différents essais proposés afin de valider les résultats obtenus de la méthode d'optimisation utilisée. 
Tableau 17

Description des essais

\begin{tabular}{|c|c|c|c|}
\hline No & Nom de l'essai & Caractéristique & Mésure \\
\hline 1 & $\begin{array}{l}\text { Déclenchement de la ligne de } \\
\text { distribution }\end{array}$ & $\begin{array}{l}\text { Examiner le déclenchement } \\
\text { de la ligne sans défaut }\end{array}$ & $\begin{array}{l}\text { - Tensions et courants } \\
\text { Transformateurs } \\
\text { Condensateur série }\end{array}$ \\
\hline 2 & $\begin{array}{l}\text { Performance du réseau de } \\
\text { distribution en régime transitoire }\end{array}$ & $\begin{array}{l}\text { Analyser la performance du } \\
\text { réseau à } t=0.5 \mathrm{sec}\end{array}$ & $\begin{array}{l}\text { Tensions et courants : } \\
\text { Condensateur série }\end{array}$ \\
\hline 3 & $\begin{array}{l}\text { Performance du réseau } \\
\text { de distribution en régime } \\
\text { permanent }\end{array}$ & $\begin{array}{l}\text { Analyser la performance du } \\
\text { réseau à } t=3 \mathrm{sec}\end{array}$ & $\begin{array}{l}\text { Tensions et courants : } \\
\text { Transformateurs } \\
\text { Condensateur série }\end{array}$ \\
\hline 4 & $\begin{array}{l}\text { Performance des moteurs } \\
\text { asynchrones en régime } \\
\text { permanent }\end{array}$ & $\begin{array}{l}\text { Analyser la performance } \\
\text { des moteurs asynchrones à } \\
\mathrm{t}=3 \mathrm{sec}\end{array}$ & $\begin{array}{l}\text { Courants de stator } \\
\text { Vitesses de rotor } \\
\text { Couples de rotor }\end{array}$ \\
\hline 5 & $\begin{array}{l}\text { Perturbation du réseau de } \\
\text { distribution radial à la présence } \\
\text { court circuit monophasé en amont } \\
\text { et en aval de } \mathrm{X}_{\mathrm{C}}\end{array}$ & $\begin{array}{l}\text { Appliquer un court circuit } \\
\text { en amont du condensateur } \\
\text { série à } t=0.5 \mathrm{sec}\end{array}$ & $\begin{array}{l}\text {-Tensions et courants: } \\
\text { Condensateur série } \\
\text { - Courant de court circuit }\end{array}$ \\
\hline 6 & $\begin{array}{l}\text { Perturbation du réseau de } \\
\text { distribution radial à la présence } \\
\text { court circuit triphasé en amont et } \\
\text { en aval de } \mathrm{X}_{\mathrm{C}}\end{array}$ & $\begin{array}{l}\text { Appliquer un court circuit } \\
\text { triphasé en amont du } \\
\text { condensateur série à t= } 0.5 \\
\text { sec }\end{array}$ & $\begin{array}{l}\text { Tensions et courants: } \\
\text { Condensateur série } \\
\text { - Courant de court circuit }\end{array}$ \\
\hline 7 & $\begin{array}{l}\text { Variation brusque de la charge } \\
\text { mécanique des moteurs. }\end{array}$ & $\begin{array}{l}\text { Dupliquer le couple } \\
\text { mécanique dans le groupe } \\
\text { moteur N.2 }\end{array}$ & $\begin{array}{l}\text {-Tensions et courants: } \\
\text { Condensateur série, } \\
\text { - Couple et vitesse, } \\
\text { - Courant stator }\end{array}$ \\
\hline 8 & $\begin{array}{l}\text { Démarrage des gros moteurs } \\
\text { asynchrones à vide. }\end{array}$ & $\begin{array}{l}\text { Faire le démarrage de gros } \\
\text { moteurs asynchrones à vide. }\end{array}$ & $\begin{array}{l}\text {-Tensions et courants } \\
\text { - Couple mécanique } \\
\text { - Vitesse angulaire } \\
\text { - Courant stator }\end{array}$ \\
\hline
\end{tabular}




\subsection{Présentation du modèle de simulation du réseau de distribution et des essais à simuler}

\subsubsection{Modèle de simulation numérique Matlab/Simulink du réseau de distribution radial}

D'abord, on a modélisé le réseau de distribution radial de la figure 37 afin d'observer les performances du réseau et l'effet de la compensation sur la ligne ainsi que le comportement des moteurs. Le modèle numérique de Matlab/Simulink de la figure 38 est le modèle équivalent du réseau à étudier.

Transformateur en parallele Delta-Y $120 \mathrm{kV} / 26.4 \mathrm{kV}, 13.5 / 18 / 22.5 \mathrm{MVA}$ $\mathrm{R}_{\mathrm{t} 1}=0.0185, \mathrm{X}_{\mathrm{t} 1}=0.345$, $\mathrm{Rm}=1150 \mathrm{pu}, \mathrm{Ym}=700 \mathrm{pu}$ at $\mathrm{V}_{1}=1.05 \mathrm{pu}$

Charge R, L: 1MVA

Transformateur Y-Y $25 \mathrm{kV} / 600 \mathrm{~V}, 833.33 \mathrm{KVA}$ $R_{\mathrm{t} 2}=0.002 \mathrm{pu} \mathrm{X} \mathrm{X}_{\mathrm{t}}=0.08 \mathrm{pu}$

4 Groupes moteurs

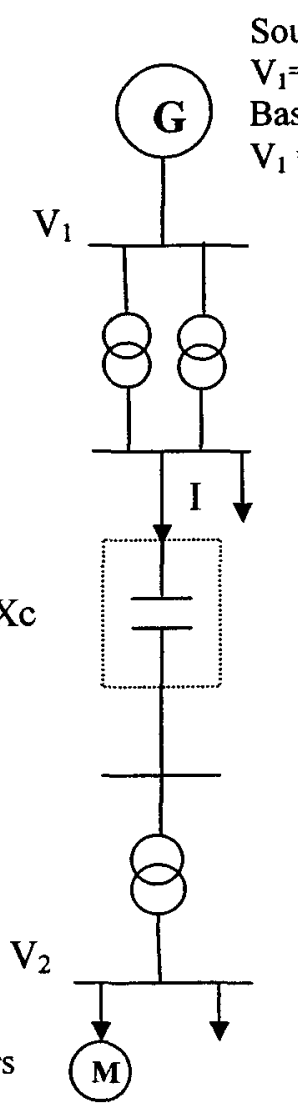

Source equivalente thevenin Hydro-Quebec $\mathrm{V}_{1}=1.05 \mathrm{pu}, \mathrm{Z}=0.03+\mathrm{j} 0.2 \mathrm{pu}$ Base :100MVA, $120 \mathrm{kV}$ $\mathrm{V}_{1}=123 \mathrm{kV}$ to $126 \mathrm{kV}$

Ligne de distribution de $60 \mathrm{kms}$ $25 \mathrm{kV}$, type $477 \mathrm{MCM}$ AL avec neutre $\mathrm{Zl}=0.123+\mathrm{j} 0.395 \mathrm{ohm} / \mathrm{km}$ $\mathrm{Z} 0=0.431+\mathrm{j} 1.240 \mathrm{ohm} / \mathrm{km}$ $\mathrm{C} 1=30.567 \mathrm{nF}, \mathrm{C} 0=11.003 \mathrm{nF}$ Condensateur protégé par NGH Scheme

Charge R, L: 1MVA

Figure 37 : Diagramme unifilaire du réseau de distribution à compenser 


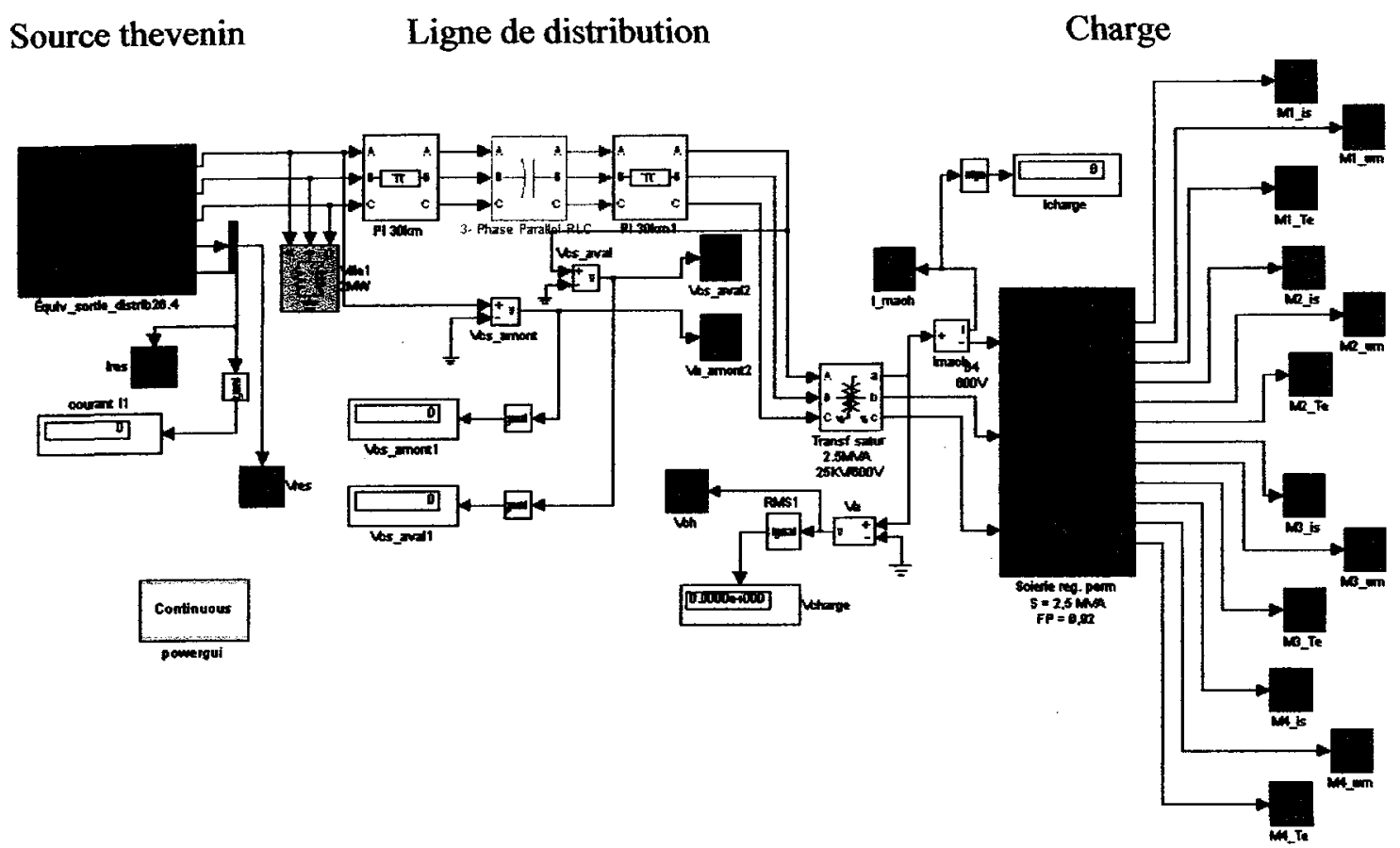

Figure 38 : Diagramme équivalent du réseau de distribution en Power System Blockset

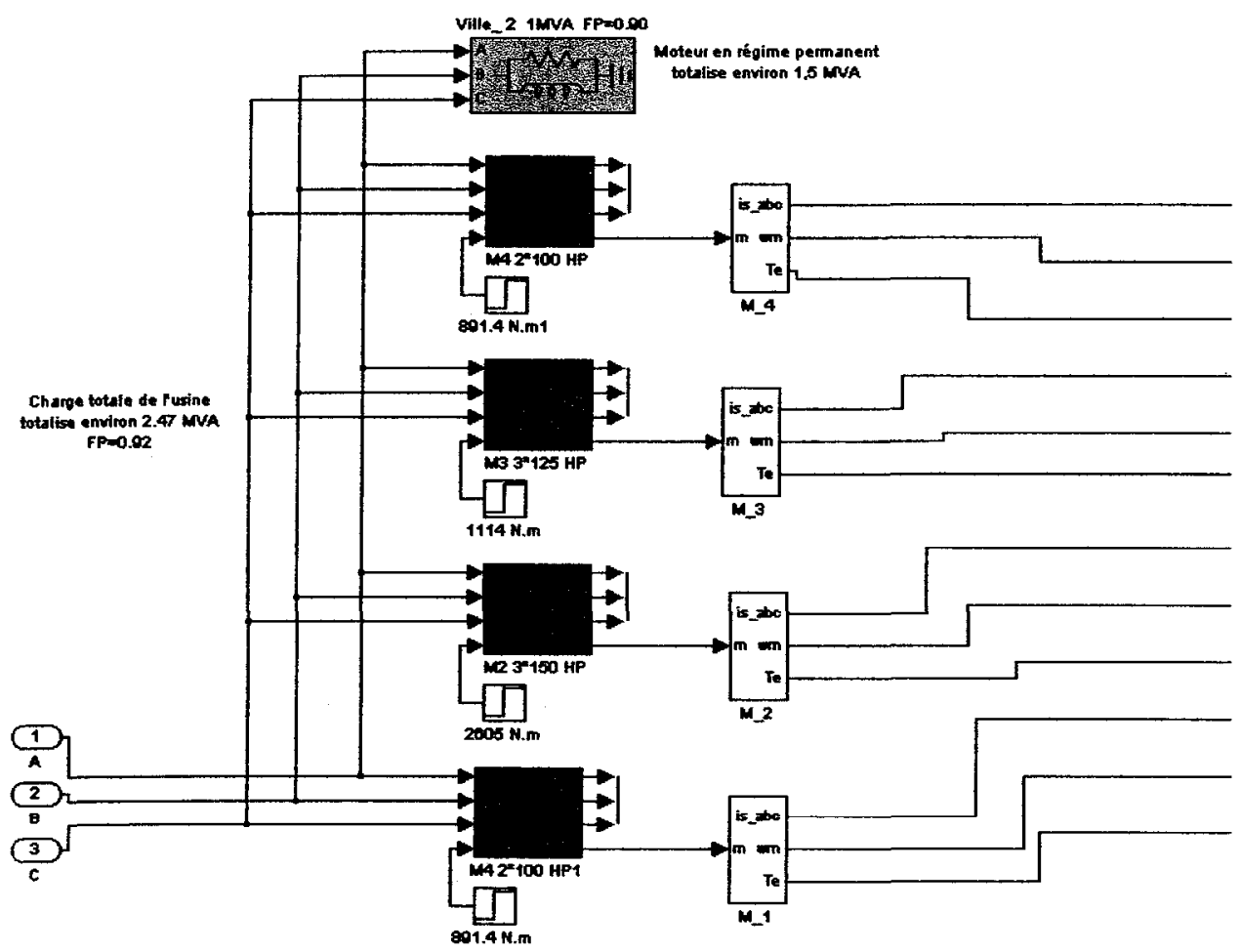

Figure 39 : Modèle numérique Matlab/Simulink du bloc des groupes moteurs M1, M2, M3 et M4. 
Le modèle numérique Matlab/Simulink de la figure 39 montre la modélisation sur le logiciel SimPowerSystems-Simulink des 4 groupes moteurs asynchrones et à la charge de 1 MVA.

Ces groupes moteurs sont branchés en parallèle avec une charge qui représente une petite ville dont leur demande de puissance est de 1 MVA avec un facteur de puissance de 0.9. Les groupes moteurs M1 (représentant les petits moteurs) et M2 (représentant les gros moteurs) seront considérés dans ce chapitre, afin de regarder l'effet de la compensation par rapport à leur puissance d'appel.

\subsection{Développement des essais sur le réseau de distribution radial}

\subsubsection{Essai 1. Enclenchement de la ligne}

Ce phénomène sera déterminé à l'aide de simulations qui permettront de savoir si la compensation série d'une ligne de distribution affecte les courants d'envahissement produits lors de l'enclenchement de transformateurs à vide sur la ligne. Ici on développera deux cas d'état du réseau. Dans le premier cas, on simule l'enclenchement de la ligne à la sortie du poste de distribution. On considère la ligne comme seule charge raccordée un transformateur à vide. Pour le deuxième cas, on simule l'enclenchement d'un transformateur sur une ligne fonctionnant en régime permanent.

L'analyse des résultats se base sous trois aspects comme suit :

- Détecter si les courants d'envahissement entraînent un phénomène d'oscillation du réseau.

- Déterminer si l'amortissement des courants d'envahissement est affecté par la compensation série sur la ligne.

- Observer l'effet de la compensation série sur les valeurs maximales des courants d'envahissement. 
Les différentes simulations sont développées considérant trois cas de compensation série du réseau. Des taux de compensation de $21 \%(X c=5 \Omega), 54.85$ $(X c=13 \Omega)$ et $66 \%(X c=15.66 \Omega)$ (voir app. J, p. 187 à 224). On simule l'enclenchement de la ligne à la sortie du poste de distribution comme seule charge raccordée au transformateur à vide au bout de la ligne. Pour chaque simulation effectuée, les résultats principaux sont présentés et les observations principales sont énoncées.

\subsubsection{Réseau de distribution radial sans compensation série}

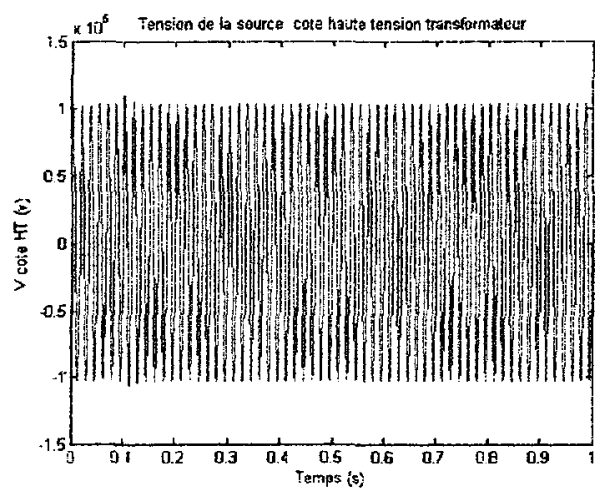

Figure 40 : Tension à la source haute tension

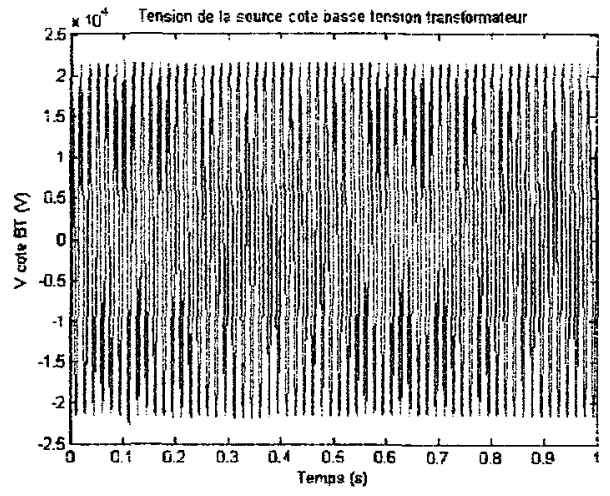

Figure 41 : Tension à la source basse tension

On peut remarquer sur les courbes ci- haut que la tension (haute tension) n'est pas affectée par l'enclenchement de la ligne lorsque elle est raccordée à un transformateur à vide. Pour ce qui concerne la tension du côté basse tension du transformateur, on remarque une élévation de l'amplitude de la tension environ $0,1 \mathrm{sec}$ après l'enclenchement. Ce phénomène est dû à la présence d'oscillations hautes fréquences produites par la manœuvre du disjoncteur, figures 40 et 41 .

Lors de l'enclenchement de la ligne on remarque à la figure 42 une amplitude du courant élevée d'environ 160A. Après l'enclenchement, le courant s'amortit et se stabilise à $t=0.7 \mathrm{sec}$. On remarque aussi la présence d'oscillations dues au manœuvre de ouverture du disjoncteur. 

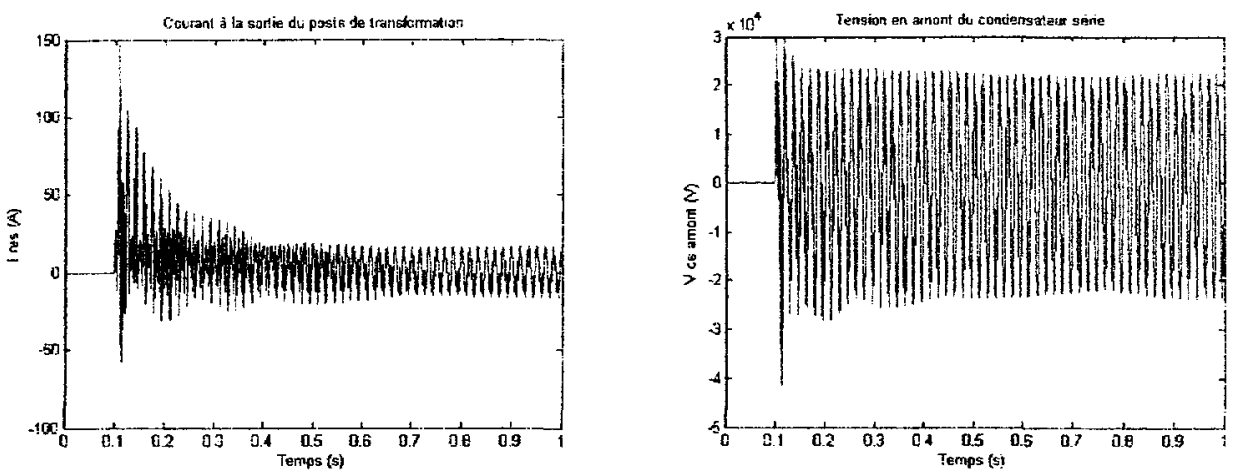

Figure 42 : Courant à la source

Figure 43 : Tension en amont du condensateur série

La tension en amont du condensateur série à la figure 43 présente une forte élévation de tension environ $41 \mathrm{kV}$ lorsque la ligne est déclenchée. Des oscillations sur la tension sont observées dues aux caractéristiques du modèle du disjoncteur. La tension au primaire du transformateur présente une surtension de - $41 \mathrm{kV}$ au moment du déclenchement de la ligne. Ensuite, la tension se stabiliser à une valeur de $25 \mathrm{kV}$ phasephase. L'amplitude négative est plus grande en oscillant entre $-22 \mathrm{kV}$ et $-26 \mathrm{kV}$, figure 44.

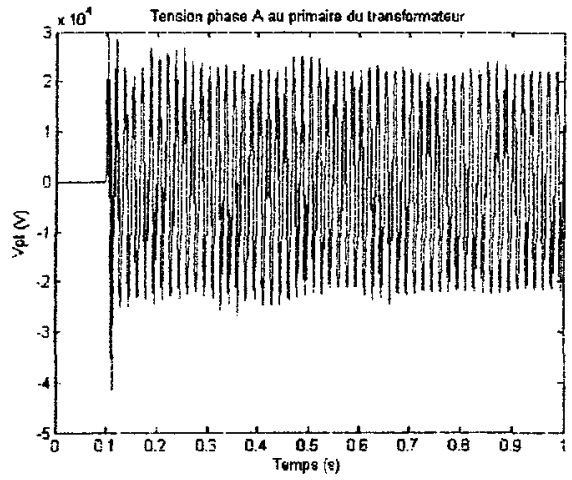

Figure 44 : Tension primaire phase A du Transformateur

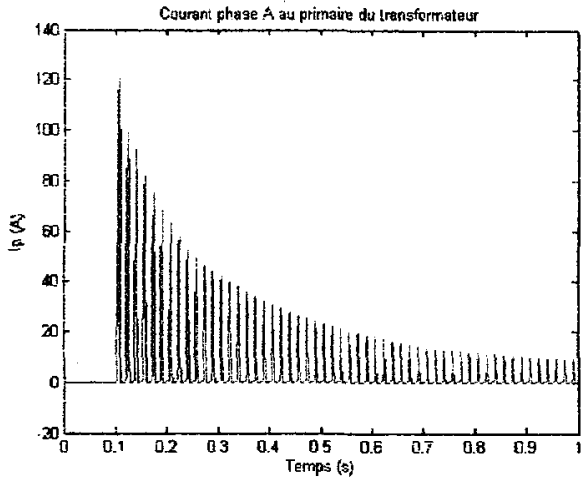

Figure 45 : Courant au primaire du Transformateur

- On observe à la figure 45 le premier pic d'amplitude très élevée du courant au primaire du transformateur d'environ 120 A lors de l'enclenchement du transformateur. Finalement ce courant s'amorti rapidement. 


\subsubsection{Réseau de distribution radial avec $\tau=21 \%(\mathrm{Xc}=5 \Omega)$}

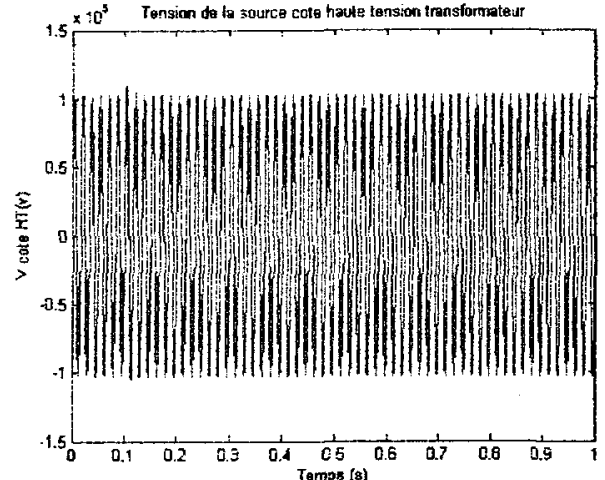

Figure 46 :Tension à la source haute tension

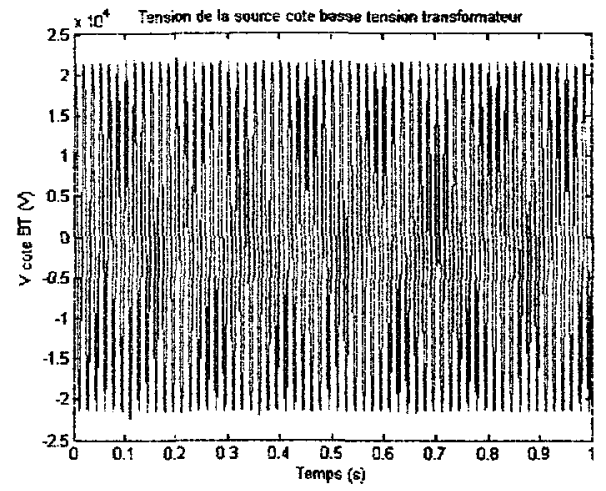

Figure 47 : Tension à la source basse tension

On peut remarquer à la figure 46 que la tension en haute tension est maintenant affectée par l'enclenchement de la ligne lorsque elle est raccordée à un transformateur à vide. On note à la figure 47 que la tension du côté basse tension du transformateur présente une petite élévation de l'amplitude de la tension lorsque l'enclenchement se produit à environ $\mathrm{t}=0.1 \mathrm{sec}$. II faut remarquer que des oscillations de hautes fréquences apparaissent lorsque le disjoncteur déclenche la ligne. La basse tension est aussi affectée par le déclenchement de la ligne en produisant un pic de tension dont le cycle négatif est plus grand que le positif.
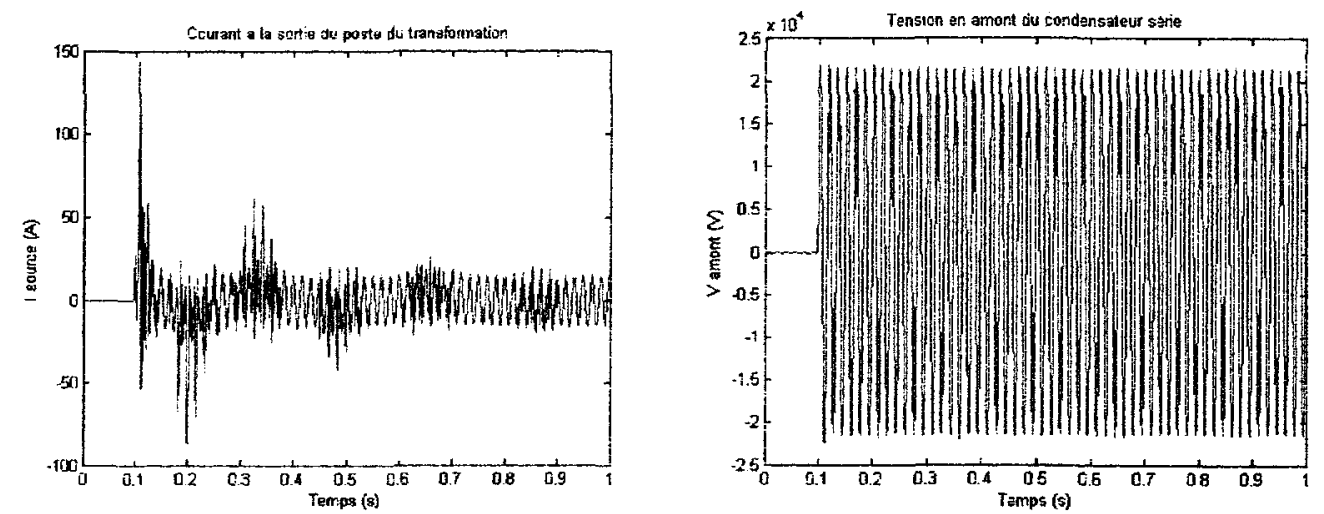

Figure 48 : Courant à la source

Figure 49 : Tension en amont du condensateur serie 

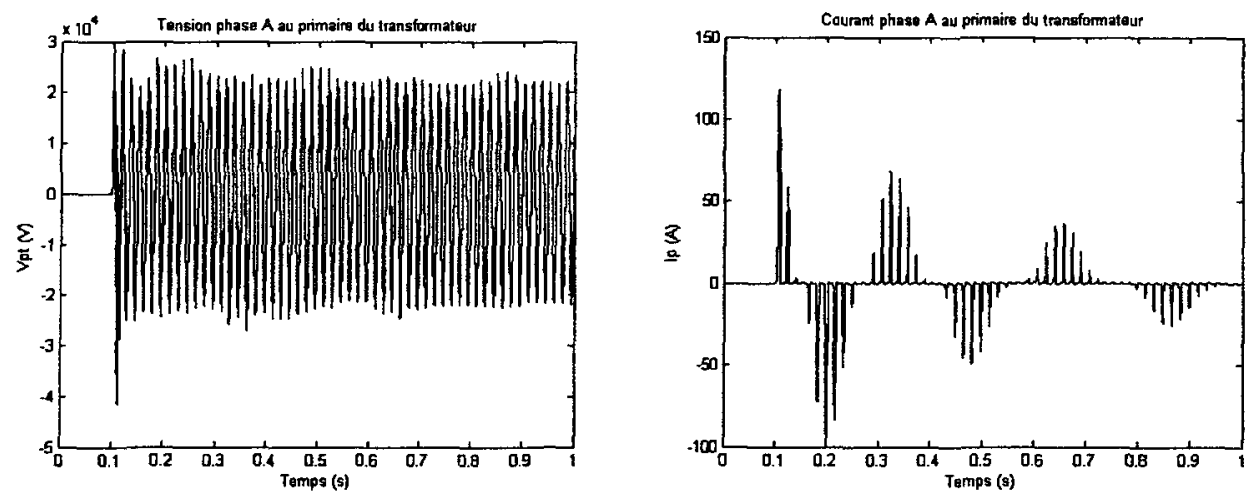

Figure 50 : Tension au primaire phase A Figure 51 : Courant au primaire du transformateur du transformateur

L'enclenchement de la ligne provoque un fort pic de tension, figure 50 et du courant au moment de l'enclenchement avec une amplitude d'environ 78A rms, figure 51. Comme dans le cas d'une compensation série de $36 \%$, on note ici que l'amplitude des pointes de courant oscille de part et d'autre de zéro à une fréquence inférieure à la fréquence fondamentale du réseau $(60 \mathrm{~Hz})$, ce qui est confirmé une fois de plus par la réponse en fréquence. Cependant, la forme de l'enveloppe des oscillations semble montrer une tendance à l'amortissement. La valeur du premier pic est plus faible que pour les deux cas précédents.

\subsubsection{Réseau de distribution radial avec $\tau=54.85 \%(X c=13 \Omega)$}
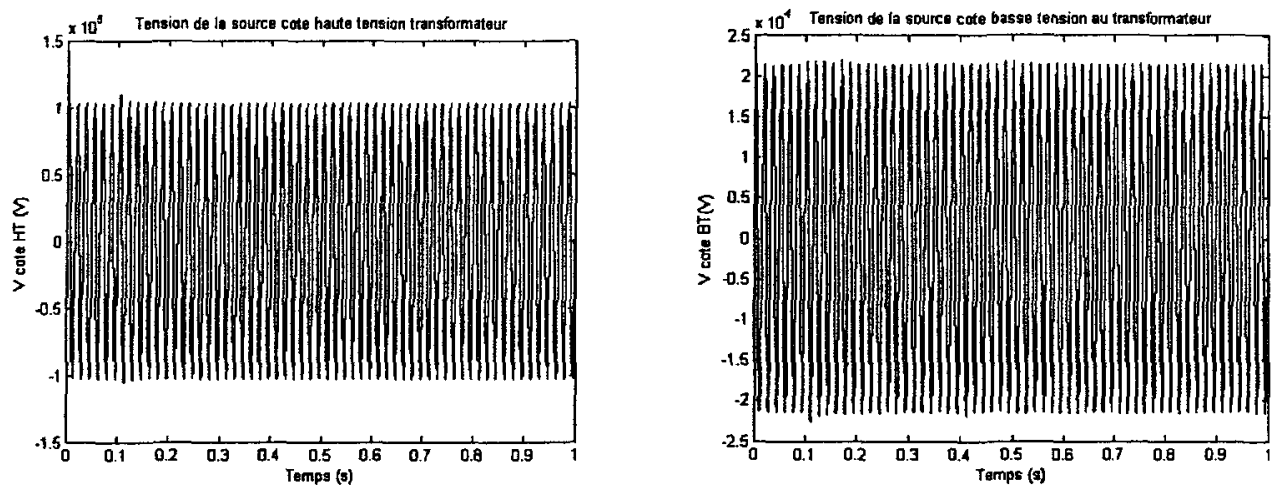

Figure 52: Tension à la source haute tension Figure 53 : Tension à la source basse tension 
On peut remarquer à la figure 52 et 53 que la tension en haute tension est affectée par l'enclenchement de la ligne lorsque elle est raccordée à un transformateur à vide. On observe que la tension du côté basse tension du transformateur présente un pic tension. La présence des oscillations de haute fréquence est due aux caractéristiques du disjoncteur.
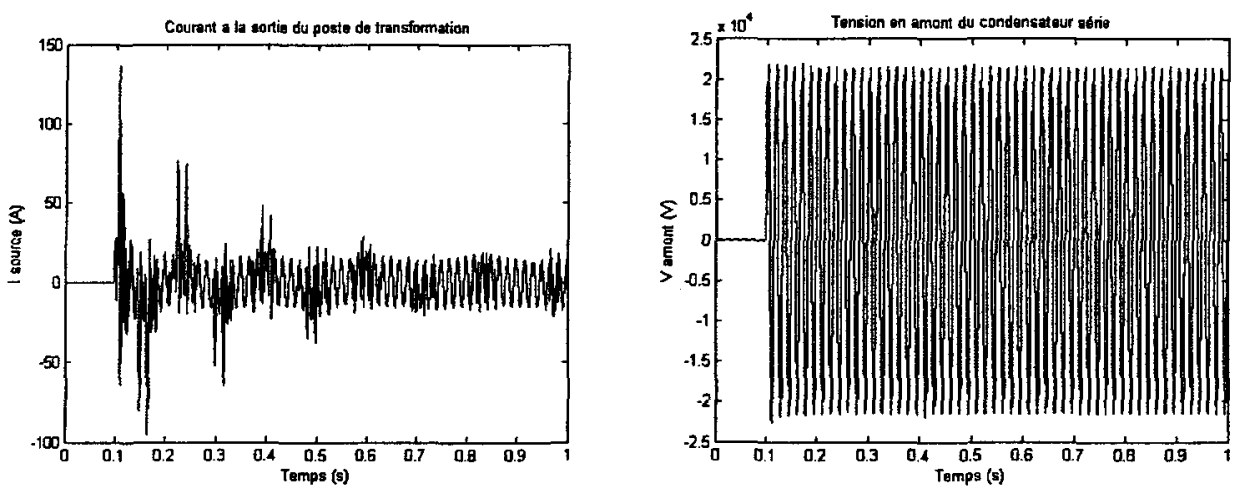

Figure 54 : Courant à la source

Figure 55 : Tension en amont du condensateur série

Lors de l'enclenchement, on remarque à la figure 54 un fort pic d'amplitude du courant environ $140 \mathrm{~A}$. On observe à la figure 55 l'effet de l'enclenchement qui produit des petits pics de tension qui sont amortis après quelques secondes. Les perturbations en partie dues au modèle du disjoncteur.
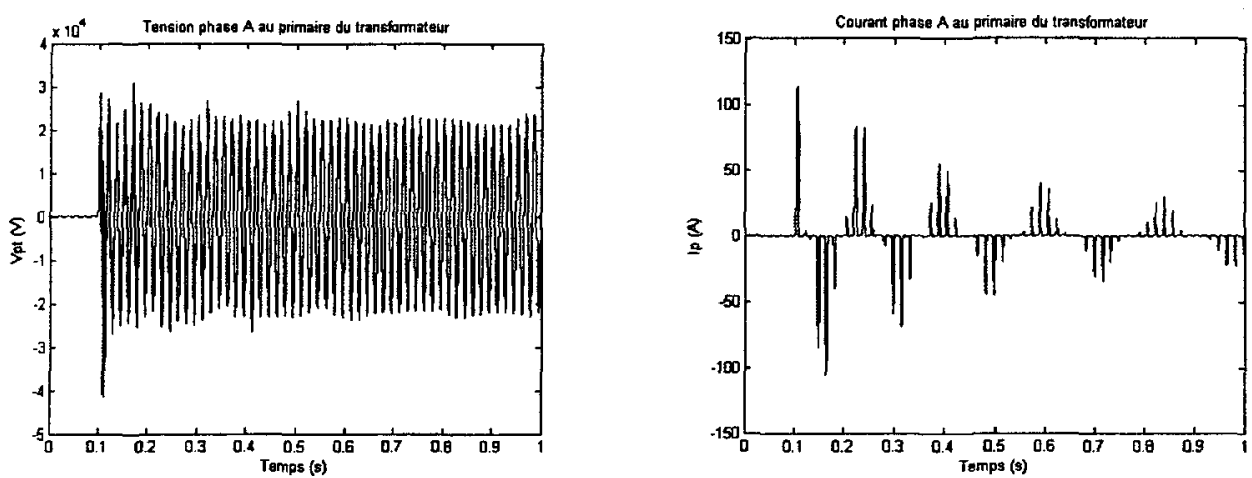

Figure 56 : Haute Tension transformateur Figure 57 : Courant au primaire du transformateur

À la figure 56 la forme de l'onde de tension au primaire du transformateur présente un pic négatif de tension environ $-40 \mathrm{kV}$ lorsque la ligne est déclenchée. L'amplitude de cette tension oscille entre environ $-25 \mathrm{kV}$ et $30 \mathrm{kV}$ phase-phase. 
Pour ce cas de compensation série à $\tau=54.85 \%$, la courant au primaire du transformateur présente un fort pic dans le premier cycle. L'amplitude du second cycle du courant est plus grande que celle obtenue dans le cas de compensation série précédente. Elle est due à la réduction de la réactance équivalente de la ligne à l'ajout du condensateur série. Les différents cycles du courant sont de fréquences plus petites que la fréquence naturelle.

\subsubsection{Réseau de distribution radial avec $\tau=66 \%(X c=15.66 \Omega)$}

On observe à la figure 58 que la tension en haute tension est encore affectée par l'enclenchement de la ligne lorsque elle est raccordée à un transformateur à vide. La tension du côté basse tension du transformateur présente un pic tension négatif ainsi que la présence d'oscillations hautes fréquences, figure 59.

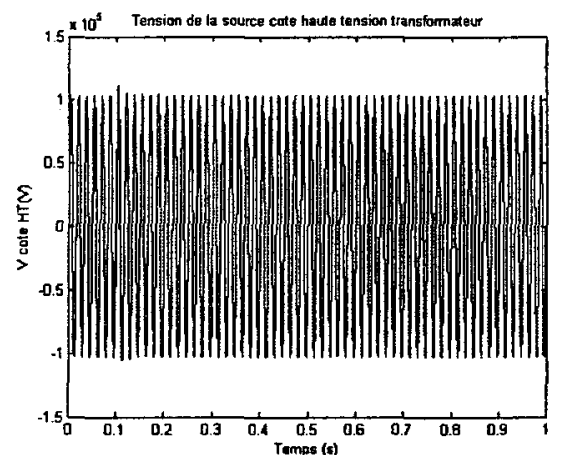

Figure 58 : Tension à la source haute tension

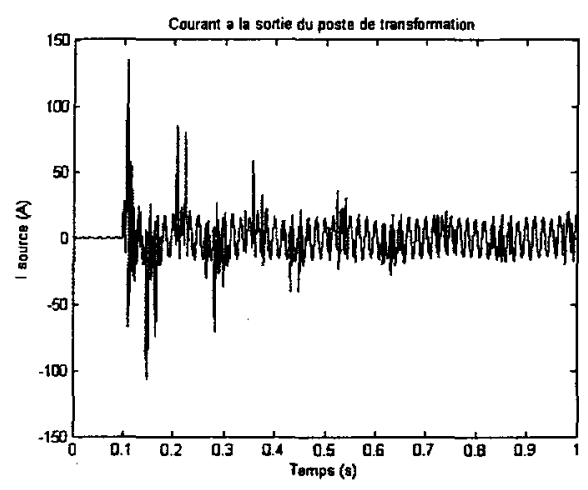

Figure 60: Courant à la source

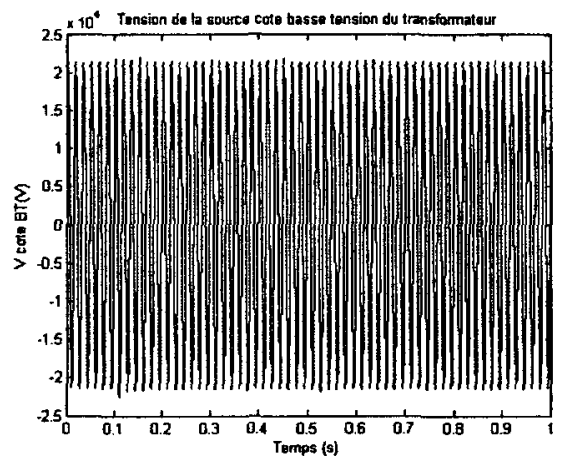

Figure 59 : Tension à la source basse tension

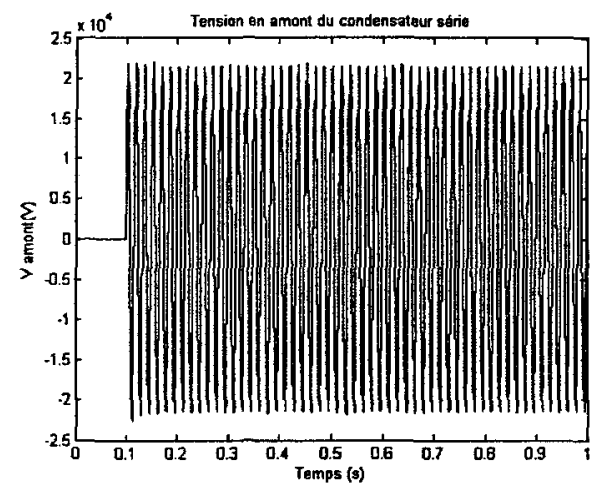

Figure 61 : Tension en amont du condensateur série 
On remarque à la figure 60 un fort pic d'amplitude du courant d'environ 140A lorsque l'enclenchement se produit. On observe que des pointes d'amplitude négatives sont encore dans la forme de l'onde du courant. La tension en amont du condensateur se trouve stable après du l'enclenchement de la ligne, figure 61.

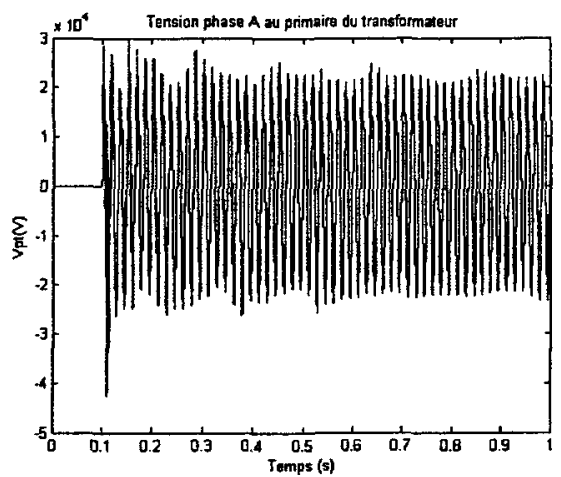

Figure 62 : Tension primaire phase $\mathrm{A}$ transformateur transformateur

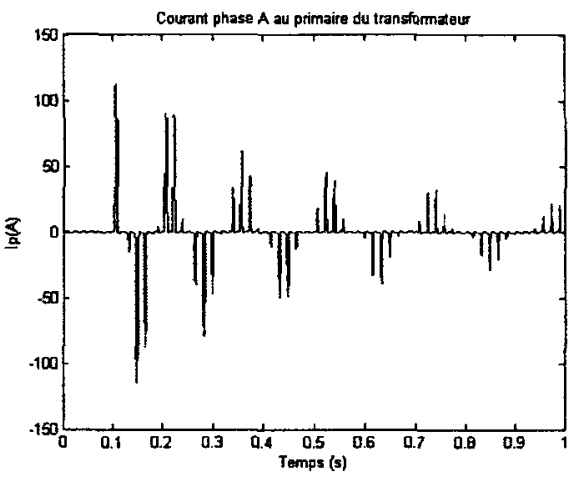

Figure 63 : Courant au primaire du transformateur

À la figure 62 la tension au primaire du transformateur présente un grand pic négatif de tension environ $-42 \mathrm{kV}$ ainsi que dans le cycle positif dont la valeur est environ $30 \mathrm{kV}$ lorsque la ligne est déclenchée. Pour ce cas de compensation série ( $\tau=66 \%$ ), le premier cycle et deuxième cycle du courant ont presque le même comportement sauf que la grandeur deviennent plu grande du fait de la réduction de la réactance équivalente de la ligne produite par l'ajoute de la compensation série. Les différents cycles du courant se trouvent au dessous de la fréquence naturelle, figure 63.

\subsubsection{Essai 2. Performance du réseau de distribution radial en régime transitoire à $\mathbf{t}=0.5 \mathrm{sec}$}

L'objectif de cet essai est de simuler le réseau de distribution radial au moyen du modèle numérique Simulink de la figure 38, afin d'analyser la performance du banc des condensateurs série sur la ligne de distribution ainsi que les groupes moteurs branchés avec une charge de 1 MVA au même point de raccordement. Le réseau sera simulé pendant $t=0.5$ secondes pour observer le comportement transitoire des moteurs lors leur démarrage. 
De même manière, la simulation permettra d'observer le comportement transitoire du condensateur série et son effet sur la tension de la ligne de distribution. La tension en aval du condensateur série présente une élévation de la tension dans les premières deux cycles figure 64 , elle se stabilise en maintenant jusqu'à la fin de la simulation à $t=0.5$ sec.
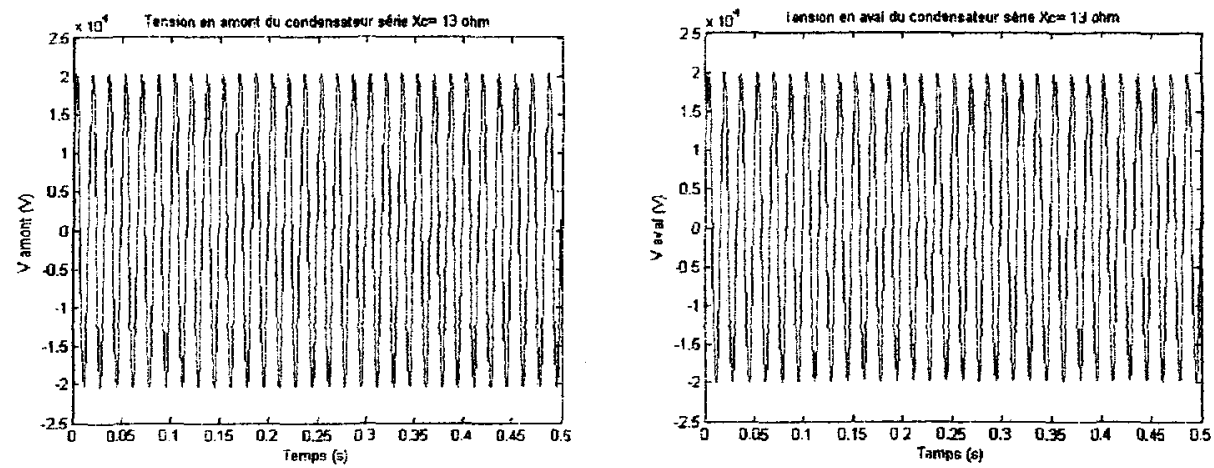

Figure 64 : Tensions en amont et en aval du condensateur série à $X c=13 \Omega$

D'ailleurs, le courant à la charge montre une performance normale en régime transitoire lorsque le réseau a été compensé. Le démarrage de la simulation nous montre l'élévation de l'amplitude du courant jusqu'à une valeur constante à environ à $t=0.4 \mathrm{sec}$. Lorsqu'on ajoute la compensation série, la forme de l'onde du courant présente des oscillations qui augmentent par rapport â la compensation, figure 65 .
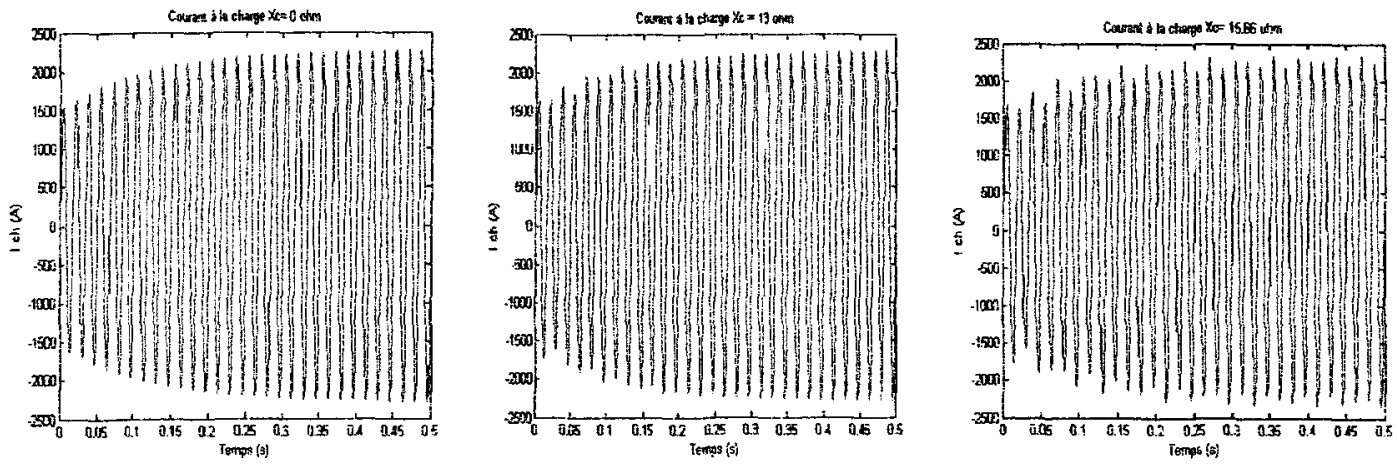

Figure 65 : Courant à la charge $X c=0,13 \Omega$ et $15.66 \Omega$

Pour $\tau=54.85(X c=13 \Omega)$ les oscillations du courant sont amorties à environ $t=$ $0.3 \mathrm{sec}$. Cependant pour des degrés de compensation série plus grands, les oscillations sont maintenues en affectant le courant à la charge, figure 65 . 


\subsubsection{Essai 3. Performance du réseau de distribution radial en régime permanent $\mathbf{t}=\mathbf{3}$ sec.}

Dans le développement de cet essai on a modélisé le réseau de distribution radial afin d'observer leur performance et l'effet de la compensation sur la ligne. Le temps de simulation sera de $t=3$ secondes pour analyser les paramètres électriques du banc de condensateurs et des moteurs branchés avec la charge. Le modèle numérique de Matlab/Simulink de la figure 63 est utilisé pour étudier la performance du réseau en régime permanent. Dans les simulations du réseau de distribution radial en régime permanent, on peut remarquer que pour un degré de compensation série à $\tau \leq 54.85 \%$, la tension de la source et en amont du condensateur ne présentent pas de changements dans sa forme excepte par la tension à la charge et en aval du condensateur série où celle-ci éprouve un bout de tension dans les premiers cycles, figures 66, 67, 68 et 69.
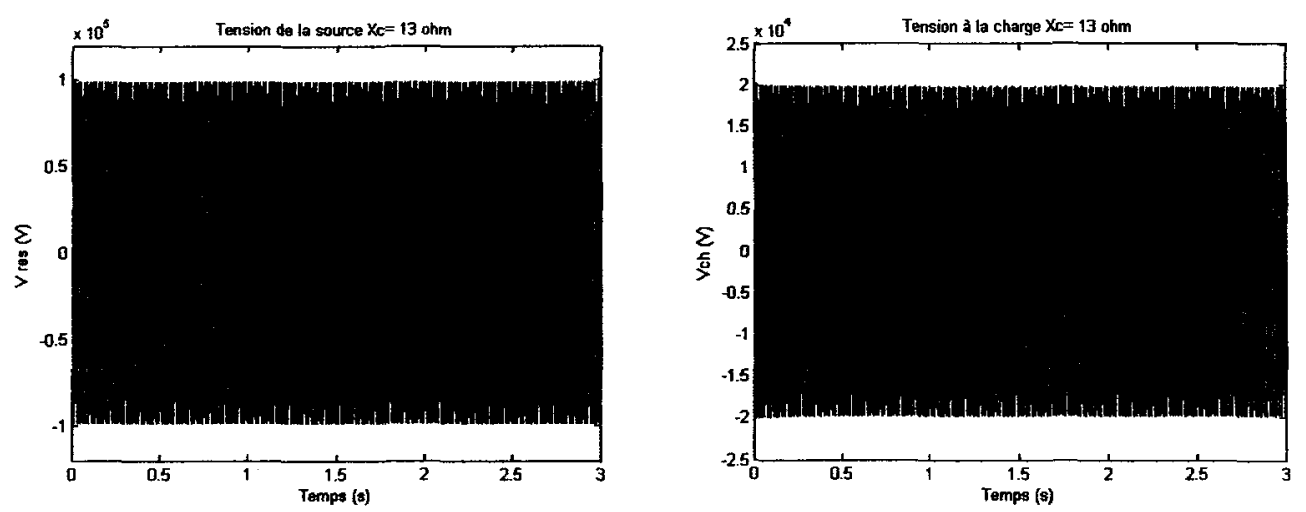

Figure 66 : Tensions de la source et à la charge à $X \mathrm{c}=13 \Omega$
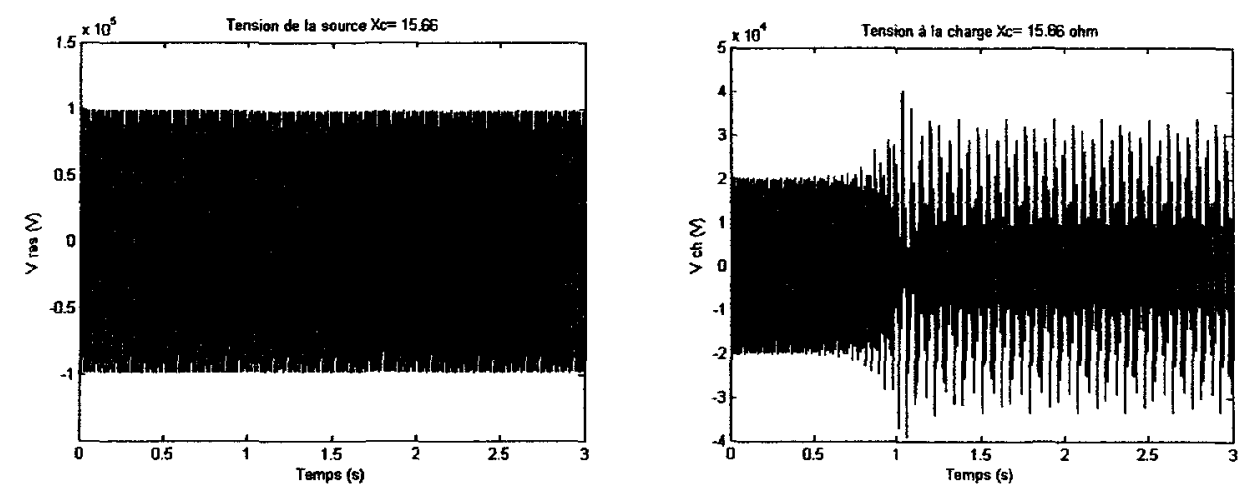

Figure 67 : Tensions de la source et à la charge à $\mathrm{Xc}=15.66 \Omega$ 
Pour les degrés de compensation série $\tau>54.85 \%$, sur la tension à la charge apparaissent des oscillations de grande amplitude qui dégradent la tension en amont et en aval du condensateur à partir des 0.7 seconds. Dans cette grandeur se produit un pic de tension qui s'amortit après quelques cycles. Cette perturbation se transmet instantanément sur la tension en aval du condensateur série, figure 69.
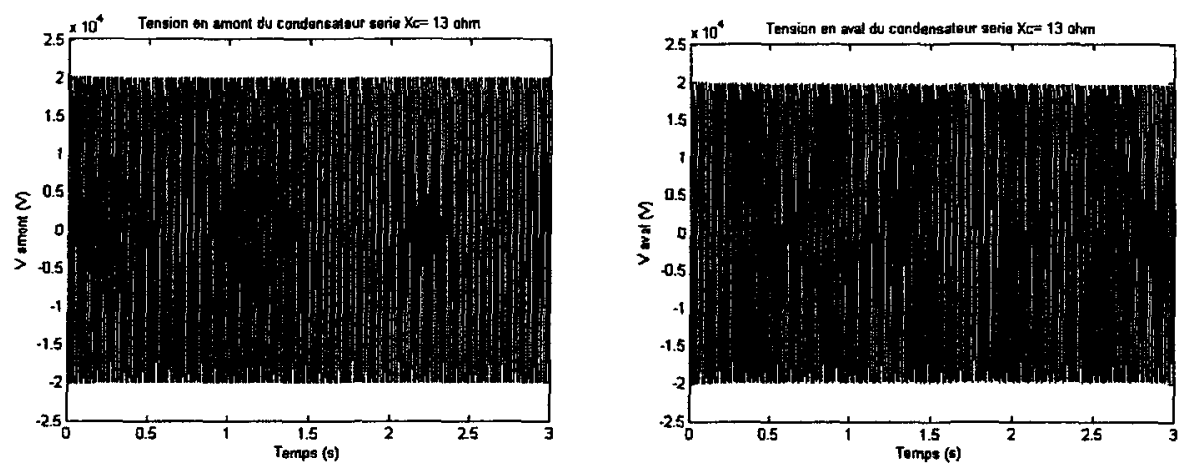

Figure 68: Tensions en amont et aval du condensateur série à $X c=13 \Omega$
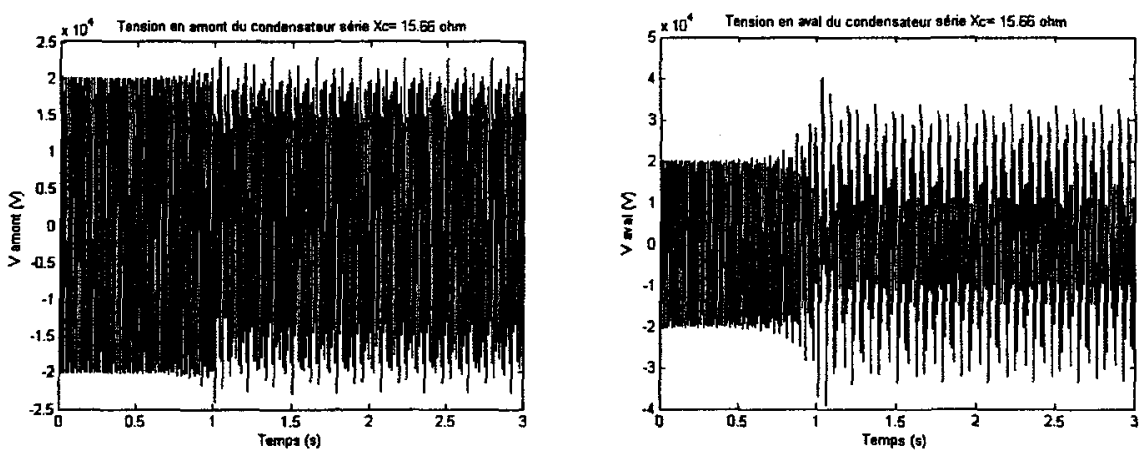

Figure 69 : Tensions en amont et en aval du condensateur série à $\mathrm{Xc}=15.66 \Omega$

Les tableaux suivants récapitulent les principales valeurs de courant et de tensions observées dans les simulations.

Tableau 18

Grandeurs mesurées pour la ligne sans compensation série

\begin{tabular}{|l|c|c|c|}
\hline \multicolumn{1}{|c|}{ Paramètres } & Reelle (rus) & Valeur p.u. & Angle (degré) \\
\hline Tension de la source & $120.96 \mathrm{kV}$ & 1.0 & 0 \\
\hline Tension à la charge & $407.38 \mathrm{~V}$ & $0.9602(600 \mathrm{~V})$ & 27.76 \\
\hline Tension amont du condensateur série & $14.42 \mathrm{kV}$ & $0.997(25 \mathrm{kV})$ & 29.39 \\
\hline Tension aval du condensateur série & $13.75 \mathrm{kV}$ & $0.9512(25 \mathrm{KV})$ & 25.57 \\
\hline Courant à la source & $62.97 \mathrm{~A}$ & $* *$ & 2.76 \\
\hline Courant à la charge & $1809 \mathrm{~A}$ & $* *$ & -62 \\
\hline
\end{tabular}


Tableau 19

Grandeurs mesurées pour la ligne avec compensation série de $21.1 \%(X c=5 \Omega)$

\begin{tabular}{|l|c|c|c|}
\hline \multicolumn{1}{|c|}{ Grandeurs } & Réelle (rms) & Valeur p.u. & Angle (degré) \\
\hline Tension de la source & $120.96 \mathrm{kV}$ & 1.0 & 0 \\
\hline Tension à la charge & $409.29 \mathrm{~V}$ & 0.9647 & 28.79 \\
\hline Tension amont du condensateur série & 14.42 & 0.9977 & 29.39 \\
\hline Tension aral du condensateur série & 13.84 & 0.9576 & 25.57 \\
\hline Courant à la source & 63.02 & $* *$ & 2.76 \\
\hline Courant à la charge & 1811 & $* *$ & -61.84 \\
\hline
\end{tabular}

Tableau 20

Grandeurs mesurées pour la ligne avec compensation série de $54.85 \%(X c=13 \Omega)$

\begin{tabular}{|l|c|c|c|}
\hline \multicolumn{1}{|c|}{ Grandeurs } & Réelle (rms) & Valeur p u. & Angle (degré) \\
\hline Tension de la source & $120.96 \mathrm{kV}$ & 1.0 & -30 \\
\hline Tension à la charge & $412.33 \mathrm{~V}$ & 0.9718 & 28.79 \\
\hline Tension amont du condensateur série & $14.42 \mathrm{kV}$ & 0.9989 & 29.39 \\
\hline Tension aval du condensateur série & $13.97 \mathrm{kV}$ & 0.9677 & 25.57 \\
\hline Courant à la source & $63.09 \mathrm{~A}$ & $* *$ & 2.76 \\
\hline Courant à la charge & $1813 \mathrm{~A}$ & $* *$ & -62 \\
\hline
\end{tabular}

Tableau 21

Grandeurs mesurées pour la ligne avec compensation série de $66 \%(X c=15.66 \Omega)$

\begin{tabular}{|l|c|c|c|}
\hline \multicolumn{1}{|c|}{ Grandeurs } & Réelle (rms) & Valeur p u. & Angle (degré) \\
\hline Tension de la source & $120.96 \mathrm{kV}$ & 1.0 & 0 \\
\hline Tension à la charge & $413.3 \mathrm{~V}$ & 0.9742 & 29 \\
\hline Tension amont du condensateur série & $14.42 \mathrm{kV}$ & 0.9989 & 29.39 \\
\hline Tension aral du condensateur série & $14.01 \mathrm{kV}$ & 0.9706 & 25.57 \\
\hline Courant à la source & $62.78 \mathrm{~A}$ & $* *$ & 2.76 \\
\hline Courant à la charge & $17.98 \mathrm{~A}$ & $* *$ & -62.01 \\
\hline
\end{tabular}

Les tensions mesurées ligne-terre. Toutes les grandeurs sont mesurées en valeur efficace. La valeur en p.u. du courant à la source se calcule avec $S=100 \mathrm{MVA}$ à $25 \mathrm{kV}$ et le courant de base est de $2309.4 \mathrm{~A}$.

\subsubsection{Essai 4. Performance des groupes moteurs en régime permanent à $t=3$ sec}

Les modèles numériques Simulink des groupes moteurs ont été simulés pendant $t=3 \mathrm{sec}$ afin d'observer leurs performances lors d'une opération normale. Le but de cet 
essai est d'identifier de possibles variations de la vitesse de rotation et le couple mécanique lorsque le réseau de distribution est compensé. On a choisi les groupes M1 et M2 car ils représentent les moteurs de grandes et petites puissances respectivement. Le groupe moteur M1 représente 2 moteurs de $100 \mathrm{HP}$ et M2 3 moteurs de $150 \mathrm{HP}$. D'abord, on a effectué des simulations pendant $t=3 \mathrm{sec}$ pour évaluer les performances des groupes moteurs telles que le courant statorique, la vitesse de rotation et le couple mécanique fourni à la sortie du groupe moteur. Les résultats des simulations montrent que pour un taux de compensation série de $\tau=54.85 \%(X c=13 \Omega)$, les groupes moteurs M1 et M2 présentent une amélioration dans la performance de la vitesse. On note qu'il n'y a pas d'oscillations dans la vitesse de rotation, figures 70 . D'ailleurs, des oscillations dans le couple mécanique des groupes moteurs M1 et M2 se produisent lorsqu'on compense le réseau à $\tau=54.85 \%(X c=13 \Omega)$, figure 71. Ces oscillations du groupe moteur M2 présentent des amplitudes les plus élevées que pour le groupe moteur M1, due au couple que doit développer le moteur d'environ 2605 N.m.
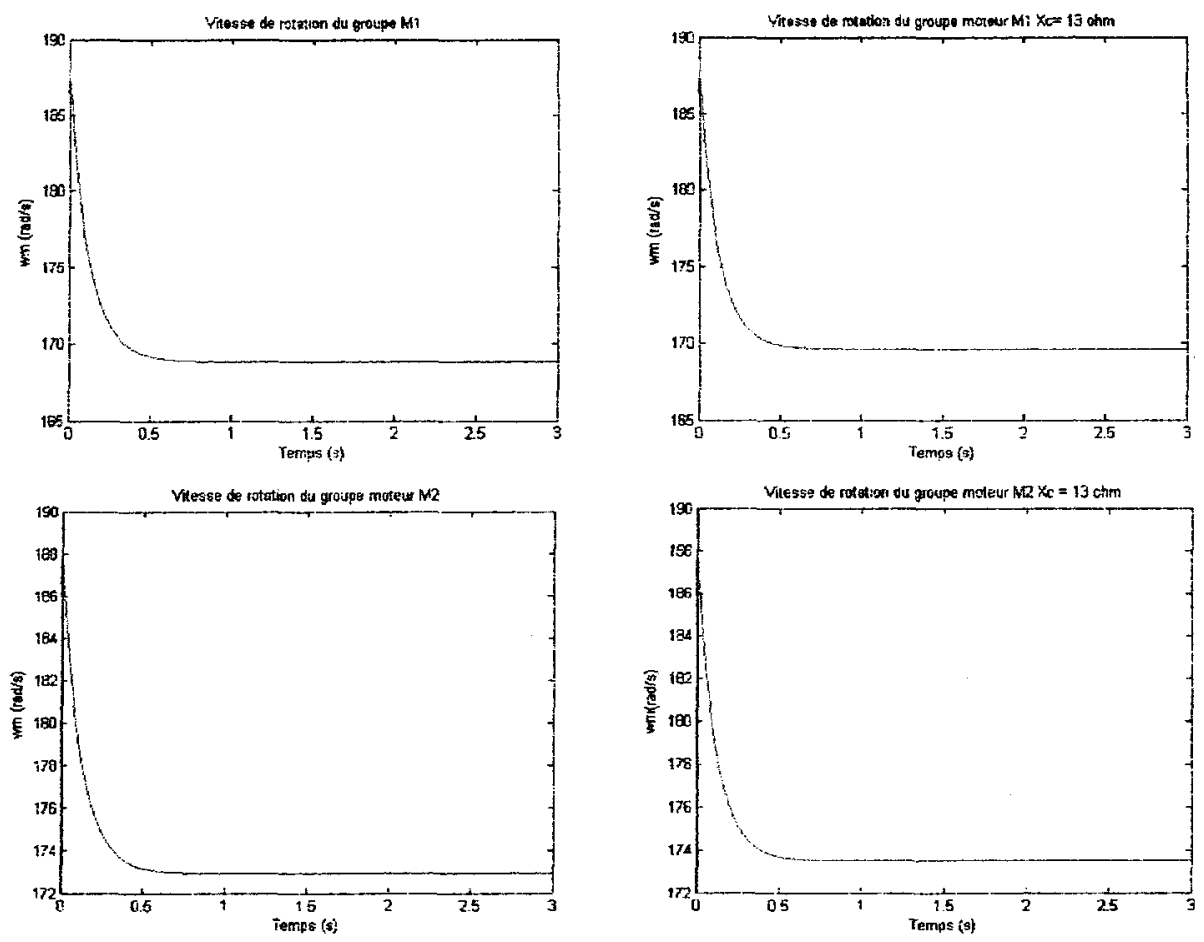

Figure 70 : Vitesses angulaires du groupe moteur $\mathrm{M} 1$ et $\mathrm{M} 2$ à $X c 0 \Omega$ et $13 \Omega$ 

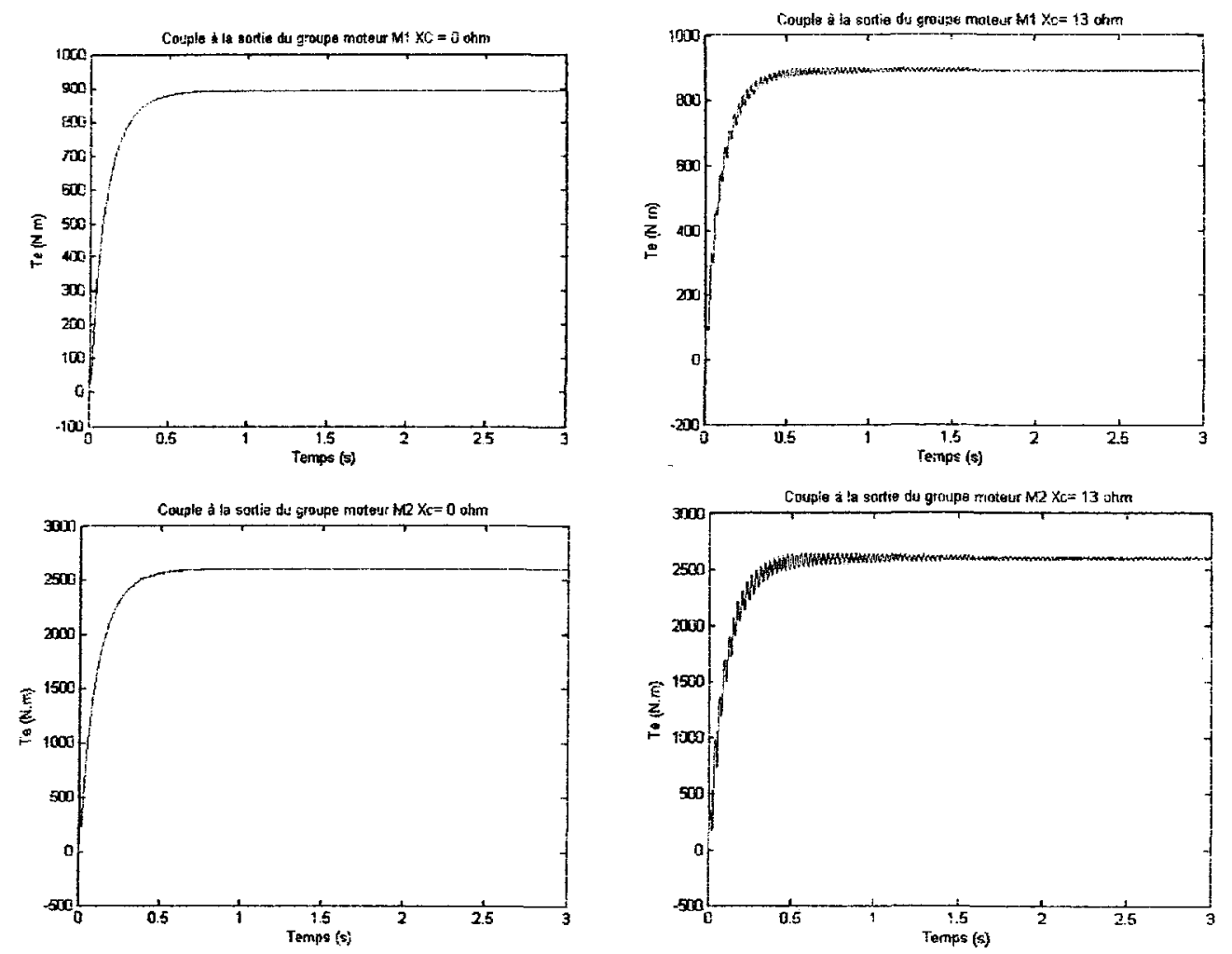

Figure 71 : Vitesse de rotation du groupe moteur $\mathrm{M} 1$ et $\mathrm{M} 2$ à $X c=0 \Omega$ et $13 \Omega$

Le cas le plus critique se trouve lorsque on compense le réseau à $\tau=66 \%$ $(\mathrm{Xc}=15.66 \Omega)$. La vitesse de rotation du groupe moteur $\mathrm{M} 1$ et $\mathrm{M} 2$ ne peut pas maintenir la stabilité en réduisant la vitesse aux valeurs très basses que la vitesse nominale, figure 80. Après $t=1 \mathrm{sec}$, il se produit des oscillations de vitesse qui se maintiennent constantes en régime permanent. Le groupe moteur M2, présente des oscillations lorsque sa vitesse tombe à une valeur inférieure à la nominal, figure 72 .
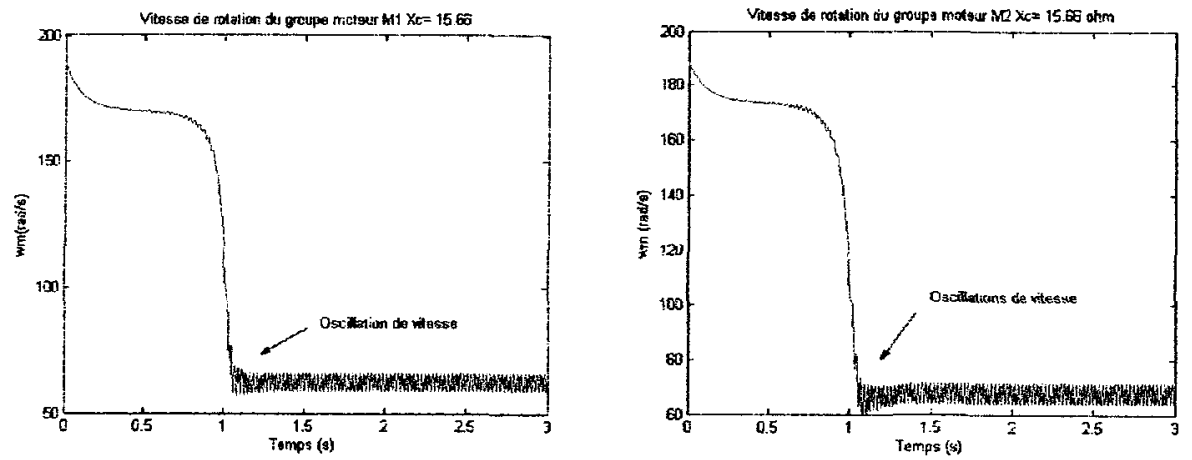

Figure 72 : Vitesse de rotation du groupe moteur M1 et $\mathrm{M} 2$ à $X c=15.66 \Omega$ 
Les grandeurs électriques en régime permanent des groupes moteurs M1, M2, M3 et M4 peuvent s'observer dans les tableaux 22 et 23.

Tableau 22

Tensions de phase $« a, b, c$, des groupes moteurs

\begin{tabular}{|c|c|c|c|c|c|c|}
\multicolumn{4}{c|}{ Groupe moteur M1 } & \multicolumn{3}{c|}{ Groupe moteur M2 } \\
\hline & Réelle & p.u. & Angle & Réelle & p.u. & Angle \\
\hline Vab & 576.12 & 0.9602 & 57.76 & 578.83 & 0.9647 & 58.16 \\
\hline Vbc & 576.12 & 0.9602 & -62.24 & 578.83 & 0.9647 & -61.84 \\
\hline Vca & 576.12 & 0.9602 & 177.76 & 578.83 & 0.9647 & 178.16 \\
\hline
\end{tabular}

Groupe moteur M3

Groupe moteur M4

\begin{tabular}{|c|c|c|c|c|c|c|}
\hline & Réelle & p.u. & Angle & Réelle & p.u. & Angle \\
\hline Vab & 583.12 & 0.9719 & 58.79 & 584.5 & 0.9742 & 59 \\
\hline Vbc & 583.12 & 0.9719 & -61.21 & 584.5 & 0.9742 & -61 \\
\hline Vea & 583.12 & 0.9719 & 178.79 & 584.5 & 0.9742 & 179 \\
\hline
\end{tabular}

Tableau 23

Courants de phase $\| \mathrm{a}, \mathrm{b}, \mathrm{c}\rangle$ des groupes moteurs Groupe moteur M1 Groupe moteur M2

\begin{tabular}{|c|c|c|c|c|c|c|}
\hline & Réelle & $\mathbf{p . u .}$ & Angle & Réelle & p.u. & Angle \\
\hline Iab & 47.845 & 0.2819 & -61.92 & 170.2 & 0.4458 & -62.01 \\
\hline Ibc & 47.845 & 0.2819 & 178.08 & 170.2 & 0.4458 & 177.99 \\
\hline Ica & 47.845 & 0.2819 & 58.08 & 170.2 & 0.4457 & 57.99 \\
\hline
\end{tabular}

Groupe moteur M3 Groupe moteur M4

\begin{tabular}{|c|c|c|c|c|c|c|}
\hline & Réelle & p.u. & Angle & Réelle & p.u. & Angle \\
\hline Iab & 59.817 & 0.1888 & -61.92 & 47.845 & 0.2819 & -61.92 \\
\hline Ibc & 59.817 & 0.1888 & 178.08 & 47.845 & 0.2819 & 178.08 \\
\hline Ica & 59.817 & 0.1888 & 58.08 & 47.845 & 0.2819 & 58.08 \\
\hline
\end{tabular}




\subsubsection{Essai 5 (a). Perturbation du réseau de distribution radial en présence du court-circuit monophasé en amont du condensateur série à $t=0.5 \mathrm{~s}$}

Dans cet essai on a simulé un court circuit monophasé en amont du condensateur série afin d'observer les effets sur les grandeurs électriques du banc de condensateurs et des moteurs branchés à la charge. Le modèle numérique utilisé pour simuler ce type de contingence habituelle dans les réseaux électriques est montré à la figure 73 .

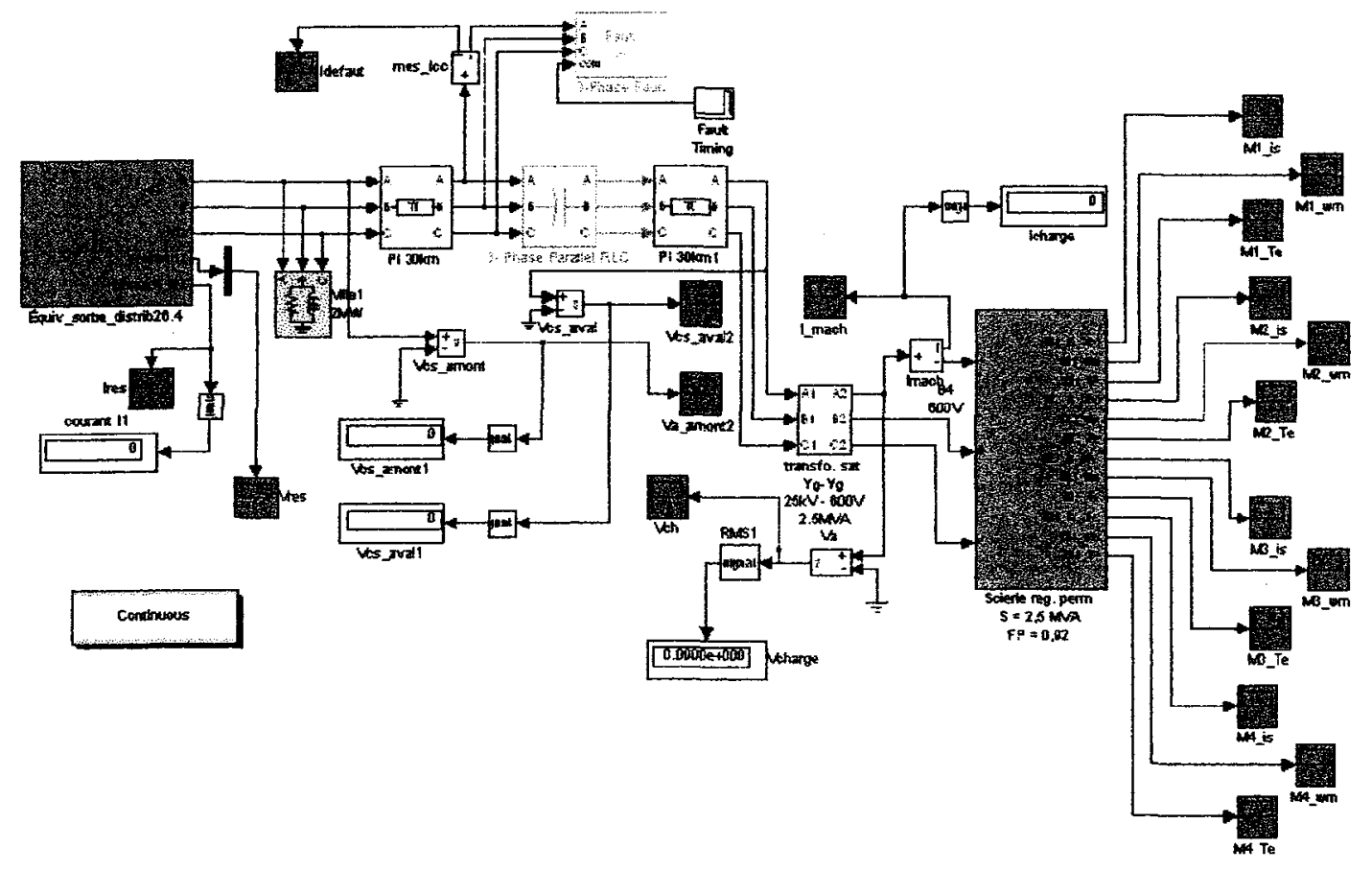

Figure 73 : Modèle numérique du réseau de distribution radial avec le bloc d'un courtcircuit monophasé en amont du condensateur série

Le court circuit monophasé produit une chute de tension à la charge pour $X c=0$ et $13 \Omega$. On a observé sur la tension à la charge à $\tau=54.85 \%(X c=13 \Omega)$ l'apparition des oscillations de grande amplitude lors de l'élimination du défaut, figure 74. Lors du réenclenchement de la ligne, de forts courants sont demandés par les groupes moteurs M1 et M2 en réduisant la tension en amont du condensateur. Une forte réduction s'observe sur la tension en aval du condensateur lorsqu'on augmente le taux de compensation, figure 75 . 

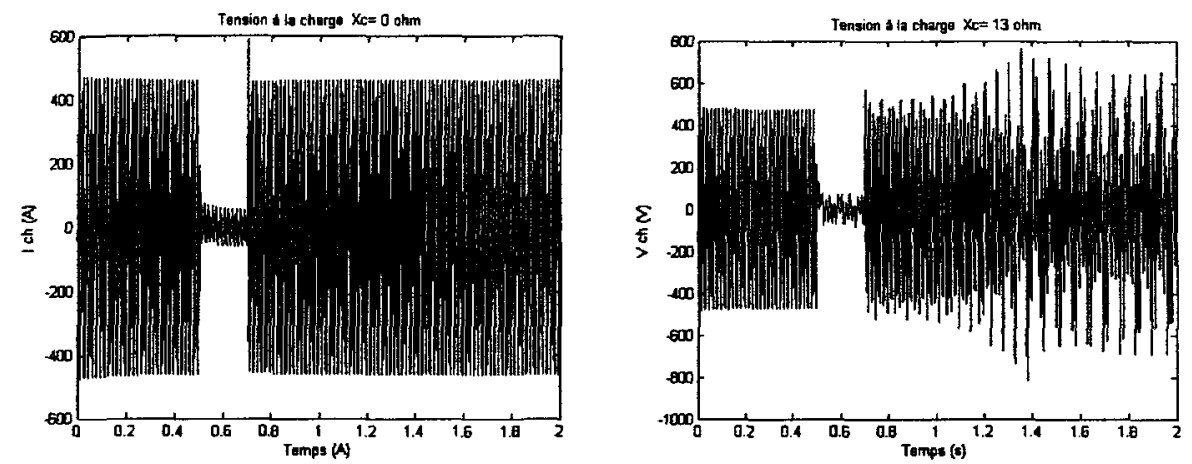

Figure 74 : Tension à la charge à Xc $=0 \Omega$ et $13 \Omega$
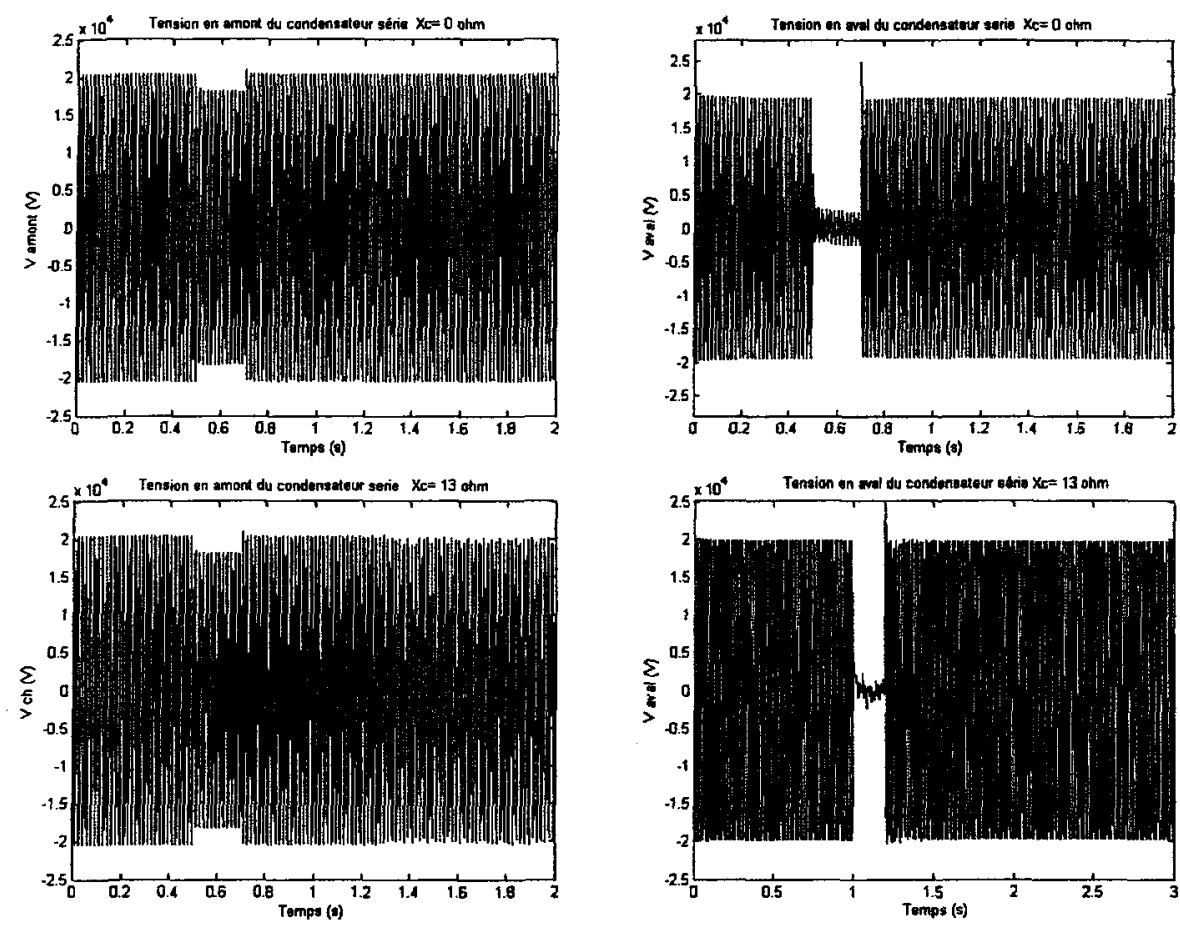

Figure 75 : Tensions en amont et en aval du condensateur série à $X c=0$ et $13 \Omega$ lors d'un court-circuit en amont du condensateur

Lors du réenclenchement de la ligne, de forts courants sont demandés par les groupes moteurs M1 et M2 en réduisant la tension à la charge et une forte réduction de vitesse de rotation lorsqu'on augmente le taux de compensation.

On note la présence des oscillations dans le courant statorique du moteur M1 et M2 lorsqu'on compense le réseau à $\tau=54.85 \%(X c=13 \Omega)$, figures 76 et 77 . L'amplitude des 
oscillations du courant est très élevée pour le groupe moteur M2 dû au fait que le groupe doit absorber beaucoup de courant pour maintenir la vitesse de rotation.
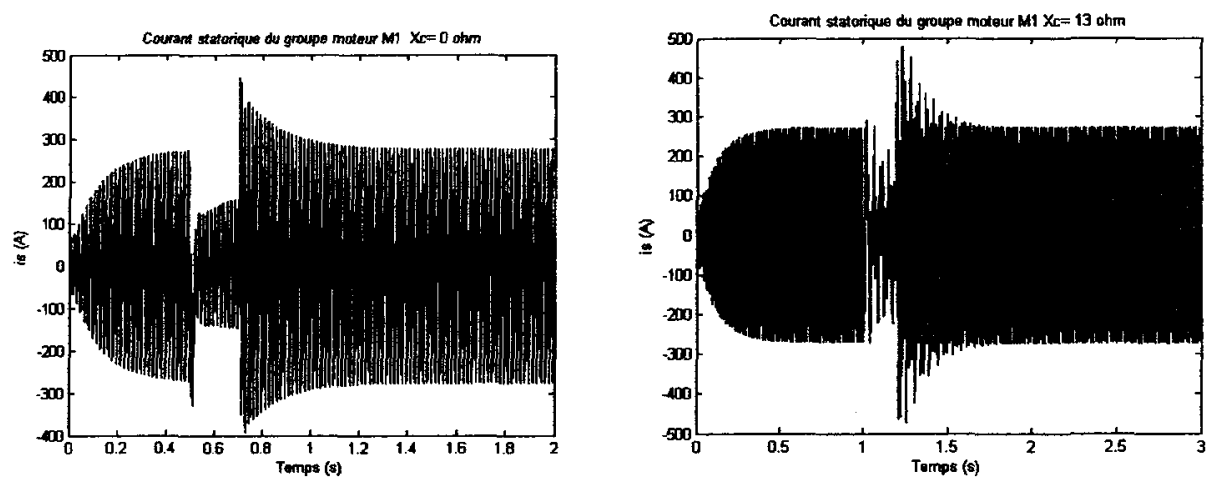

Figure 76 : Courant de court-circuit du groupe moteur M1
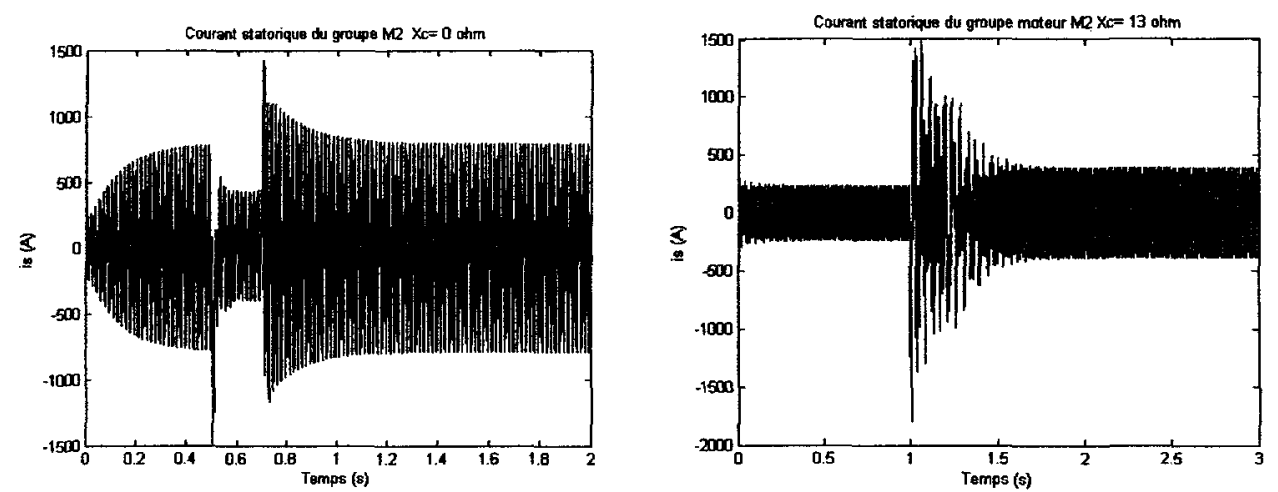

Figure 77 : Courant de court-circuit du groupe moteur M2

À la figure 78, on observe une réduction au vitesse de rotation lors du court-circuit. Après quelques cycles, le défaut est éliminé grâce renclenchement de la ligne qui permet d'élever la vitesse jusqu'à sa valeur en régime permanent. Le phénomène précédemment décrit est du à l'absorption du courant du groupe moteur afin de récupérer leur vitesse nominale. On remarque aussi la présence de petites oscillations de la vitesse de rotation du groupe moteur M2 lors du défaut mais celles-ci disparairent lorsque le système récupère la stabilité, figure 78 .

Si le réseau de distribution est compensé à $\tau=66 \%\left(X_{c}=15.66 \Omega\right)$, les deux groupes moteurs éprouvent une forte réduction lors du court-circuit. Une fois le défaut est éliminé, les groupes moteurs ne pourront pas retrouver leur vitesse nominale de 
rotation. Cette vitesse se maintiendra oscillante à un valeur plus basse que sa valeur en régime permanent. Cette contingence provoquera, par conséquence, la déstabilisation du système, figure 79 .
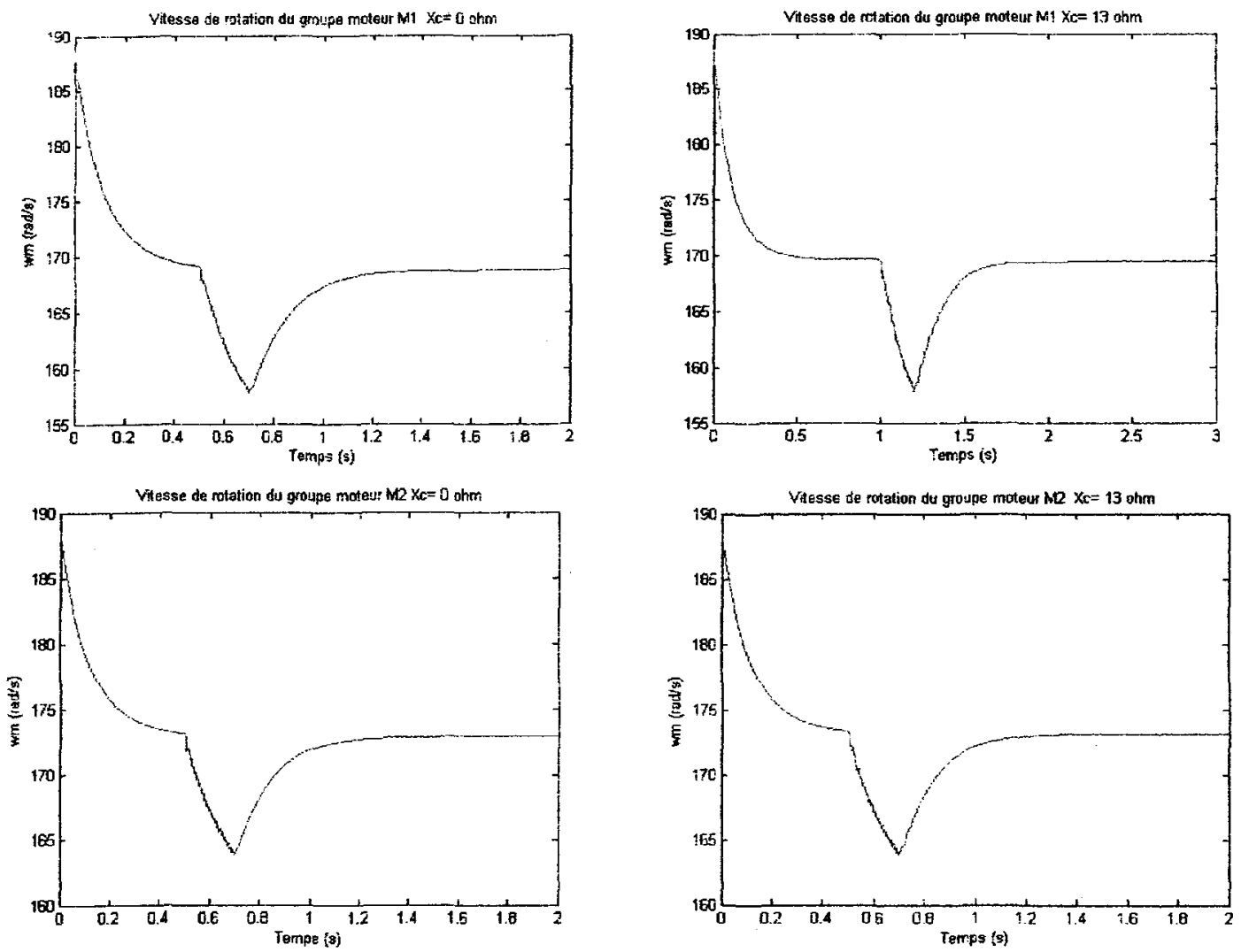

Figure 78 : Vitesse de rotation des groupes moteurs M1 et $\mathrm{M} 2$ à $X c=0 \Omega$ et $13 \Omega$
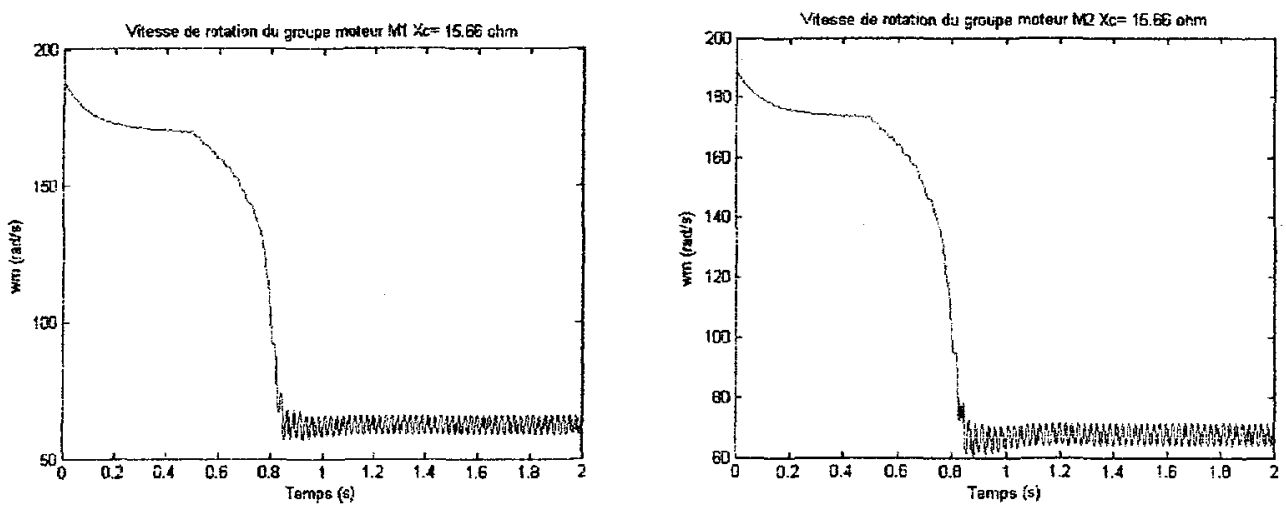

Figure 79 : Vitesse de rotation du groupe moteur M1 et M2 à $X c=15.66 \Omega$ 
En ce qui conceme le couple mécanique, les deux groupes moteurs présentent des oscillations lors d'un défaut monophasé. Ces oscillations son amorties lorsqu'il se produit le réenclenchement de la ligne de distribution. D'ailleurs, l'élévation á $\tau$ fait que l'amortissement des oscillations soit moins rapide, figure 80. Dans le groupe moteur M2, des oscillations sont plus grandes dues à la grandeur du couple mécanique, figure 80 .
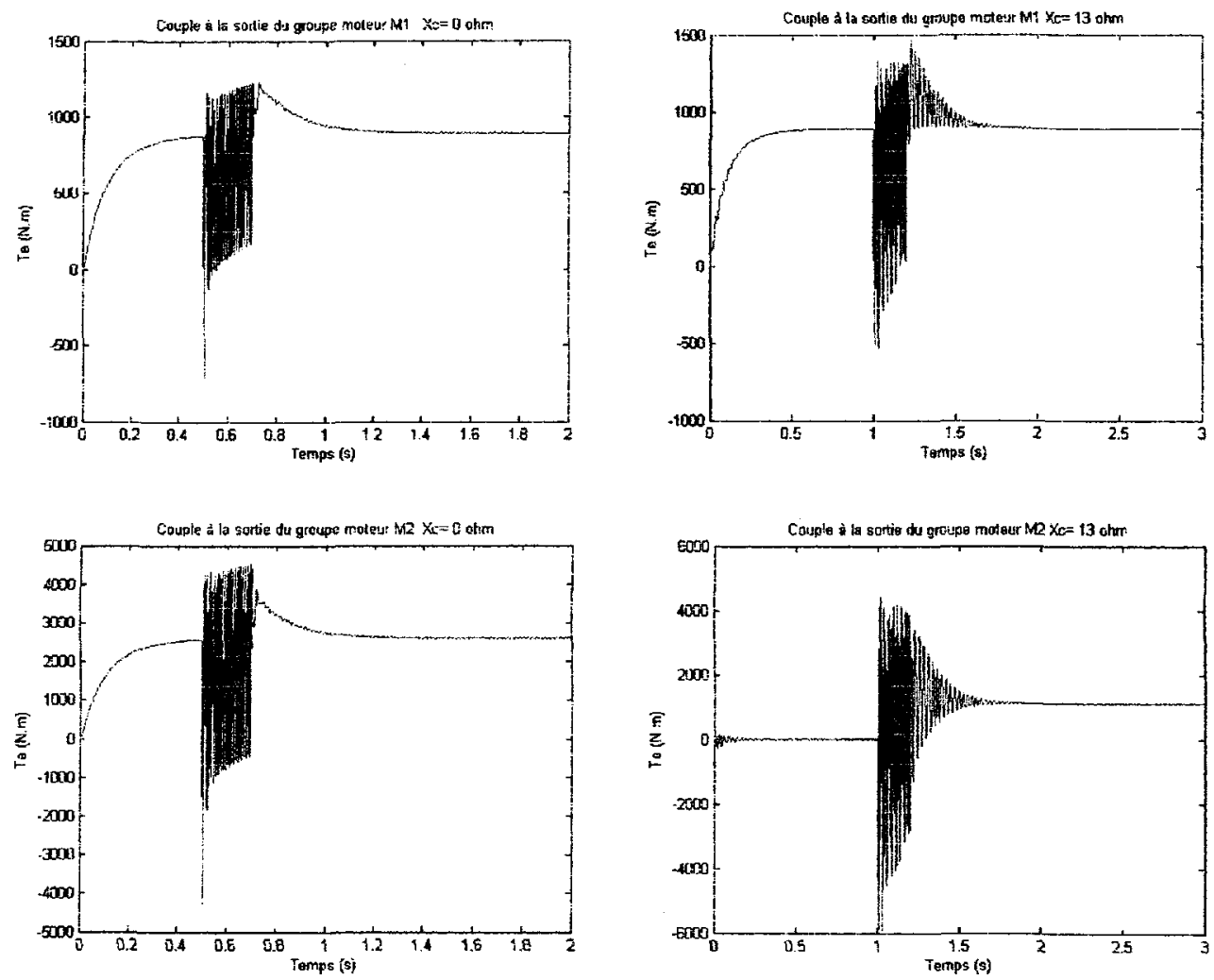

Figure 80 : Couple à la sortie du groupe moteur M1 et M2

\subsubsection{Conclusions de l'essai du réseau de distribution radial à la présence d'un court circuit monophasé en amont du condensateur série à $t=0.5 \mathrm{~s}$}

Le court circuit monophasé est considéré comme une contingence normale dans les réseaux électriques. Cette contingence a été simulée avec un défaut à la phase «a » en amont du condensateur série. Lors de cette contingence, de forts courants sont demandés par les groupes moteurs en réduisant la tension à la charge et une forte réduction de la vitesse de rotation lorsqu'on augmente le taux de compensation. Le même effet se 
produit sur le courant de court-circuit monophasé. Le tableau 24 montre les valeurs du courant de court circuit à différents degrés de compensation.

Tableau 24

Valeurs de courant de défaut

\begin{tabular}{|c|c|}
\hline Compensation série & Courant de défaut monophasé A(rms) \\
\hline Non compensé & $700 \mathrm{~A}$ \\
\hline$X_{c}=, 5 \Omega(\tau=21.1 \%)$ & $780 \mathrm{~A}$ \\
\hline$X_{C}=13 \Omega(\tau=33.76 \%)$ & $830 \mathrm{~A}$ \\
\hline$X_{C}=15.66 \Omega(\tau=54.85 \%)$ & $850 \mathrm{~A}$ \\
\hline
\end{tabular}

Lorsque le réenclenchement de la ligne est effectué, on remarque la présence d'oscillations de grande amplitude sur le courant de la source, des courants statorique des groupes moteurs ainsi que sur la tension en aval du condensateur série et à la charge respectivement. Ces oscillations apparaissent lorsque le réseau est compensé à $\tau>54.85 \Omega$. On observe la présence d'oscillations du couple des groupes moteurs avec un pic négatif lorsque le réseau est compensé à $\tau=21.1$ et $\tau=54.85 \%$. L'amortissement d'oscillations du couple mécanique est plus lent à chaque fois qu'on augmente le taux de compensation. Mais ils retrouvent leurs valeurs en régime permanent. Cela indique que le réseau se retrouve sous de conditions de stabilité. La perte de la stabilité est remarquable lorsque on compense le réseau à $\tau=66 \%\left(X_{C}=\right.$ $15.66 \Omega$ ). La vitesse des moteurs est très basse et elle ne retrouve pas sa valeur en régime permanent à cause de fortes oscillations.

\subsubsection{Essai 5 (b). Perturbation du réseau de distribution radial en présence d un court circuit monophasé en aval du condensateur série à $t=0.5 \mathrm{~s}$}

Dans cet essai on a simulé un court circuit monophasé en aval du condensateur série. À cause de la position du défaut, le condensateur produit un plus grand effet que pour celui produit dans l'essai de court-circuit en amont du condensateur série. L'objectif de cet essai est d'observer les effets sur les paramètres électriques du banc de condensateurs et des groupes moteurs. Le modèle numérique utilisé pour simuler ce type de contingence est montre à la figure 73. Dans ce défaut la tension à la charge se réduit, à cause de forts courants demandés par les groupes moteurs M1 et M2 en affectant 
la performance à la charge, figure 81. À partir du taux de compensation $\tau=54.85 \%\left(X_{C}=\right.$ $13 \Omega$ ), la tension à la charge continue en oscillant en régime permanent.
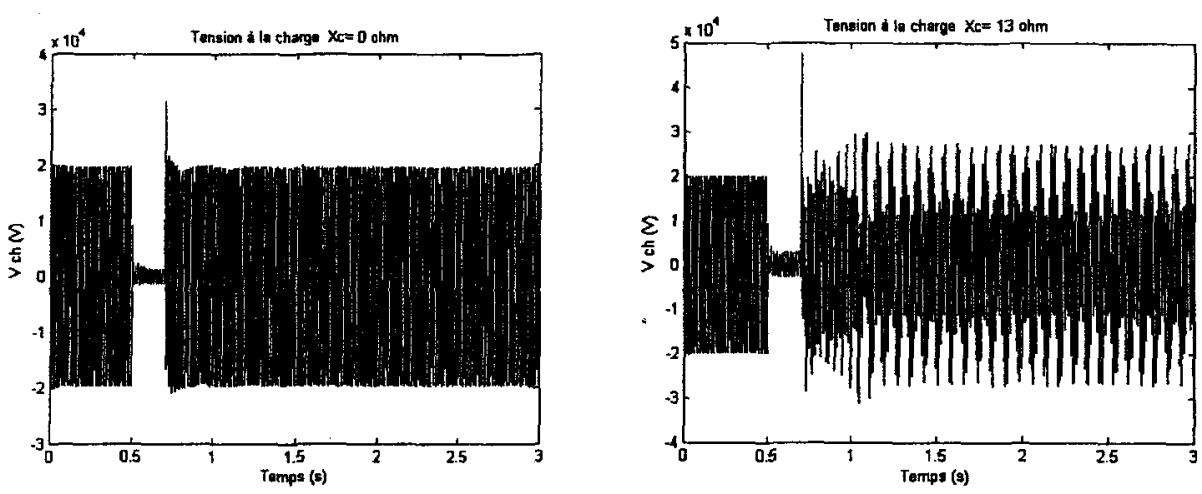

Figure $81:$ Tension à la charge à $X c=0 \Omega$ et $13 \Omega$
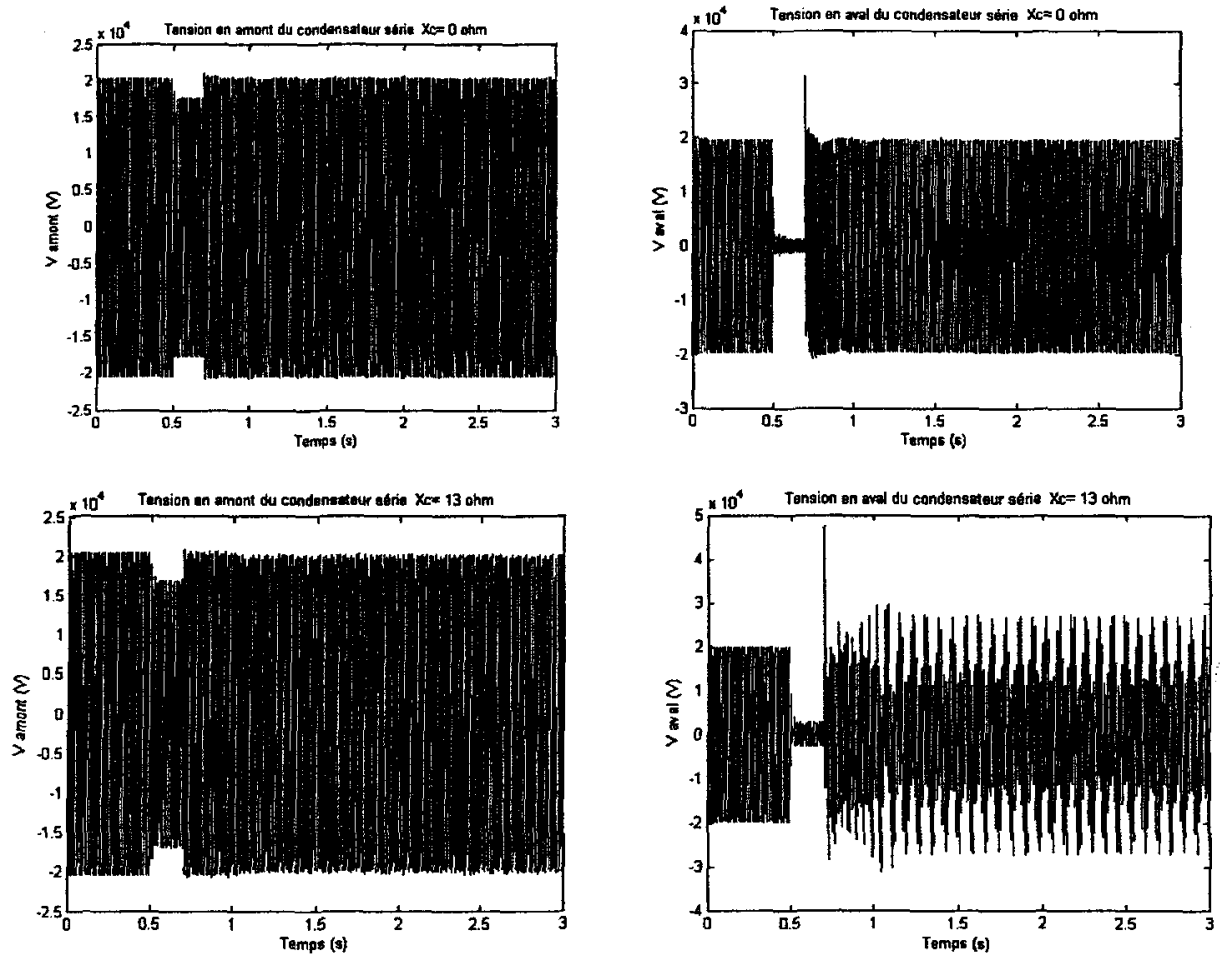

Figure 82: Tensions en amont et en aval du condensateur série à $X c=13 \Omega$ lors court circuit en amont du condensateur

D'ailleurs, la tension en amont du banc de condensateurs éprouve une réduction instantanée de sa grandeur lorsqu'on compense à $\tau=54.85 \%$. Dans ce cas, la tension en 
aval montre un petit pic de tension de quelques cycles mais, elle ne présente pas des oscillations, figure 81. Pour des taux de compensation plus grande que $\tau=54.85 \%$, la tension en aval est instable en produisant des oscillations de grande amplitude, figure 84 .

Lors du réenclenchement de la ligne de distribution, on note une forte réduction du courant statorique, figures 82 et 83 , ainsi que la vitesse de rotation des groupes moteurs à mesure qu'on augmente le taux de compensation, figure 85 et 86 .
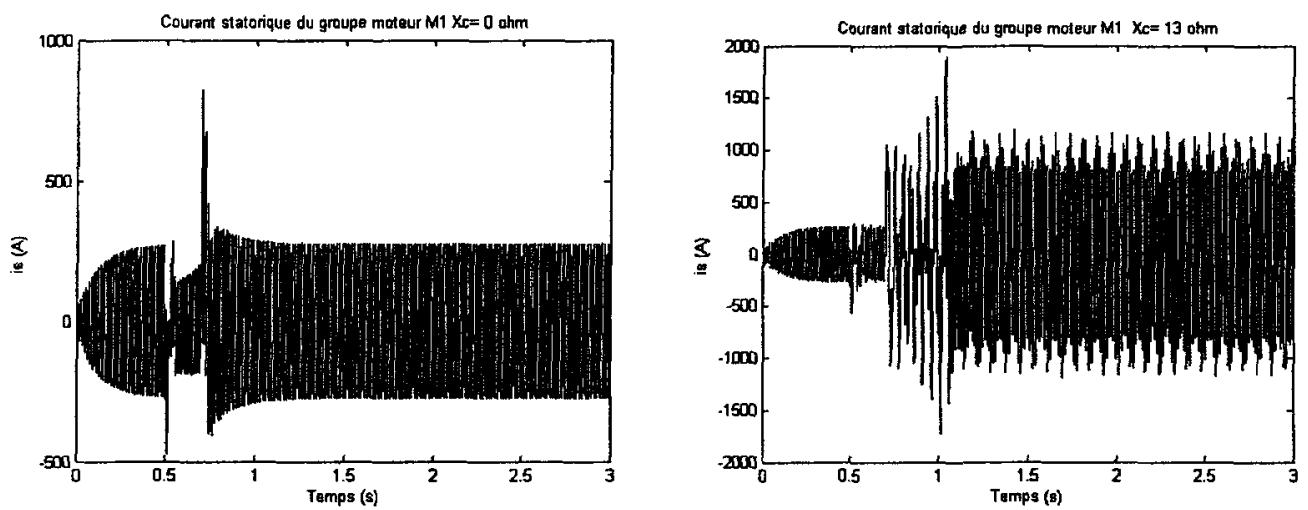

Figure 83 : Courant statorique du groupe moteur M1 à $X c=0$ et $13 \Omega$

On remarque la présence des oscillations dans le courant statorique du moteur M1 et M2 lorsque on compense le réseau à $\tau=54.85 \%(X c=13 \Omega)$. L'amplitude des oscillations du courant est très élevée pour le groupe moteur M2 dû que le groupe doit absorber beaucoup du courant pour maintenir la vitesse de rotation ainsi que la stabilité du système.
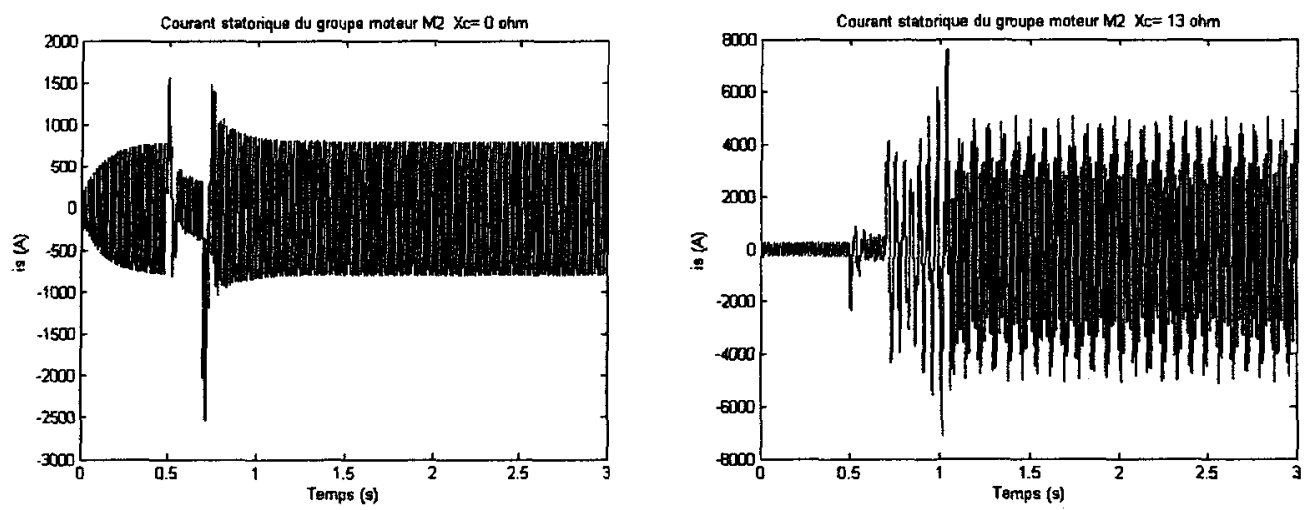

Figure 84 : Courant statorique du groupe moteur M2 à $X c=0$ et $13 \Omega$ 
Le phénomène précédemment décrit est produit grâce à l'absorption du courant du groupe moteur qui essaie de récupérer sa vitesse nominale. Lorsque le réseau est compensé à $\tau=54.85 \%(X c=13 \Omega)$, on observe la présence de petites oscillations sur la vitesse lors d'un défaut, celles-ci se maintiennent et le système ne récupère pas sa valeur en regime permanent, figure 85 et 86 .
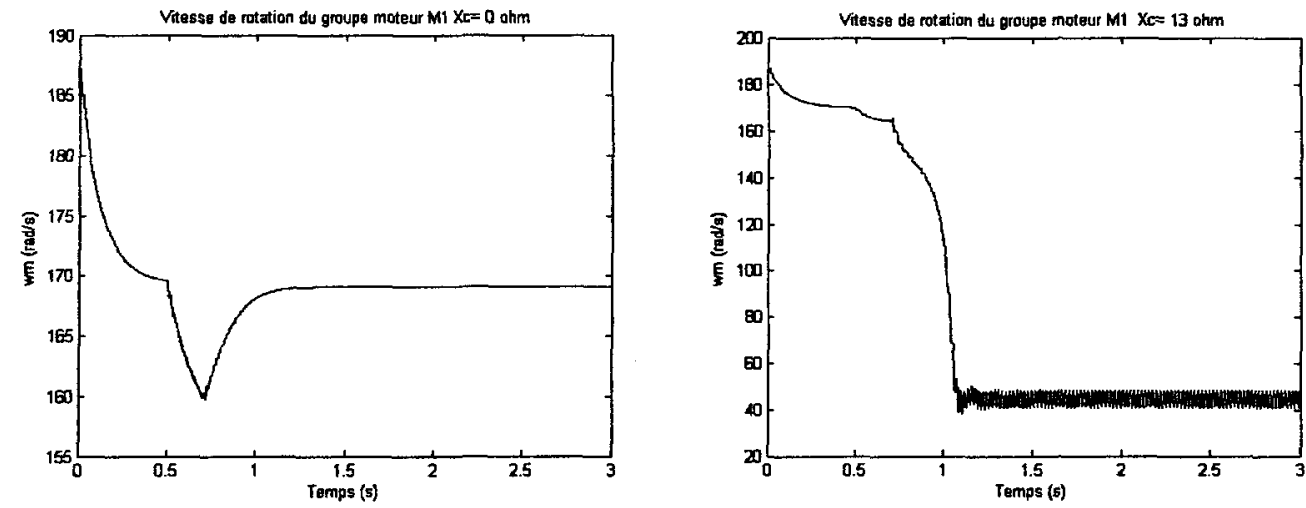

Figure 85 : Vitesse de rotation des groupes moteurs $M 1$ à $X c=0 \Omega$ et $13 \Omega$
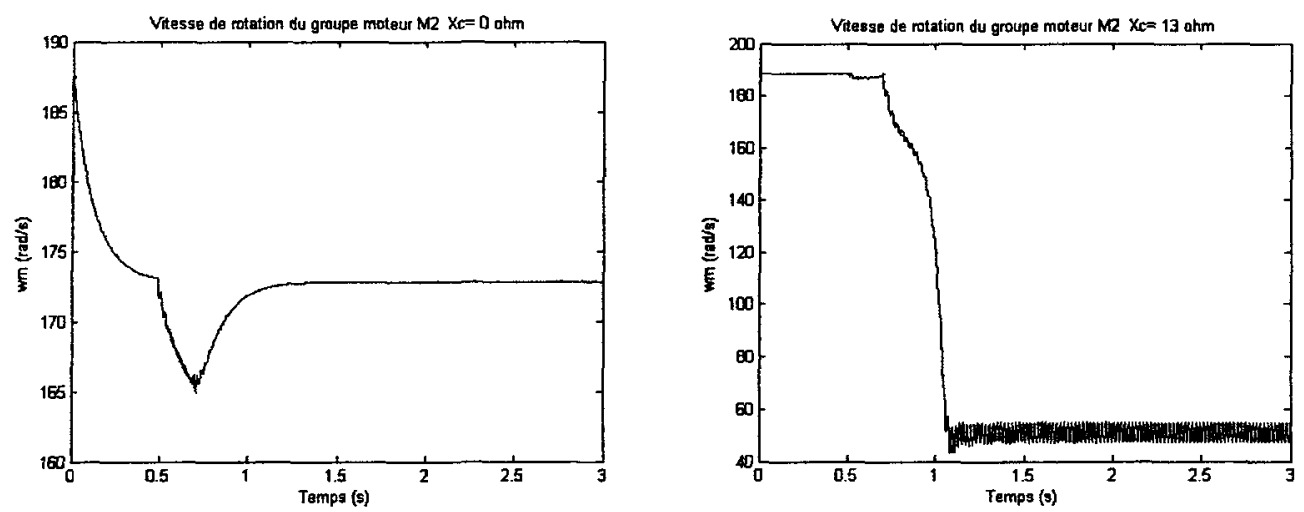

Figure 86 : Vitesse de rotation des groupes moteurs M2 à $X c=0 \Omega$ et $13 \Omega$

La vitesse de rotation des deux groupes moteurs continue en étant instable lorsqu'on augmente le taux de compensation à $\tau=66 \%(X c=15.66 \Omega)$. Les groupes moteurs éprouvent des oscillations en provoquant une réduction brusque de la vitesse et l'instabilité du système, figure 87 . 

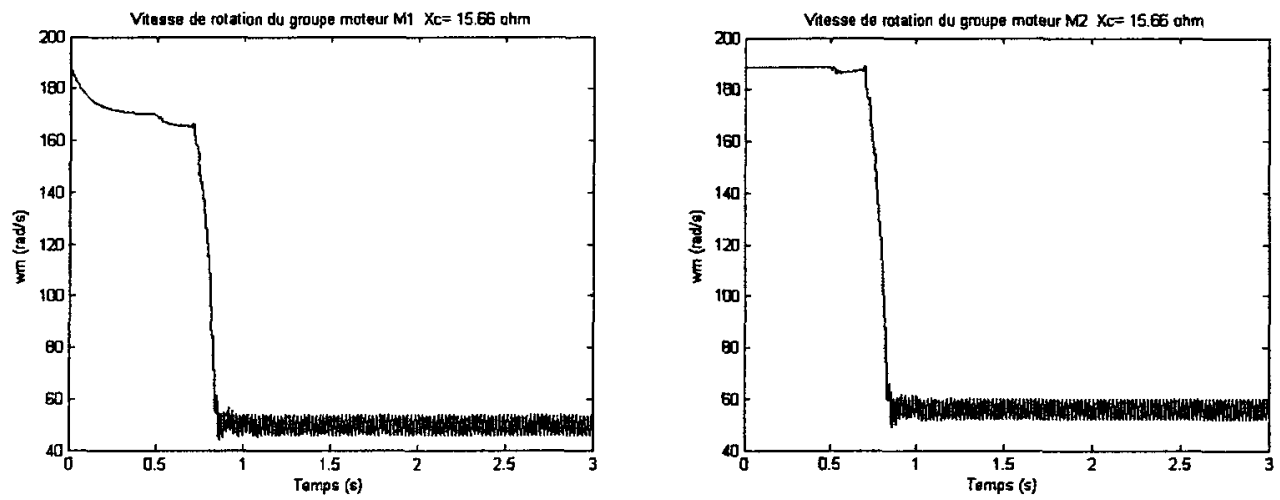

Figure 87 : Vitesse de rotation du groupe moteur M1 et M2 à $X c=15.66 \Omega$.

En ce qui concerne le couple mécanique, les deux groupes moteurs éprouvent des oscillations pendant le défaut. Ces oscillations son amorties lorsqu'il se produit le réenclenchement de la ligne de distribution. L'élévation du taux de compensation série fait que les oscillations soient moins rapides, figure 88. Le cas le plus critique se présente pour le groupe moteur M2 dont des oscillations sont plus grandes, le couple mécanique étant plus important, figure 88 .
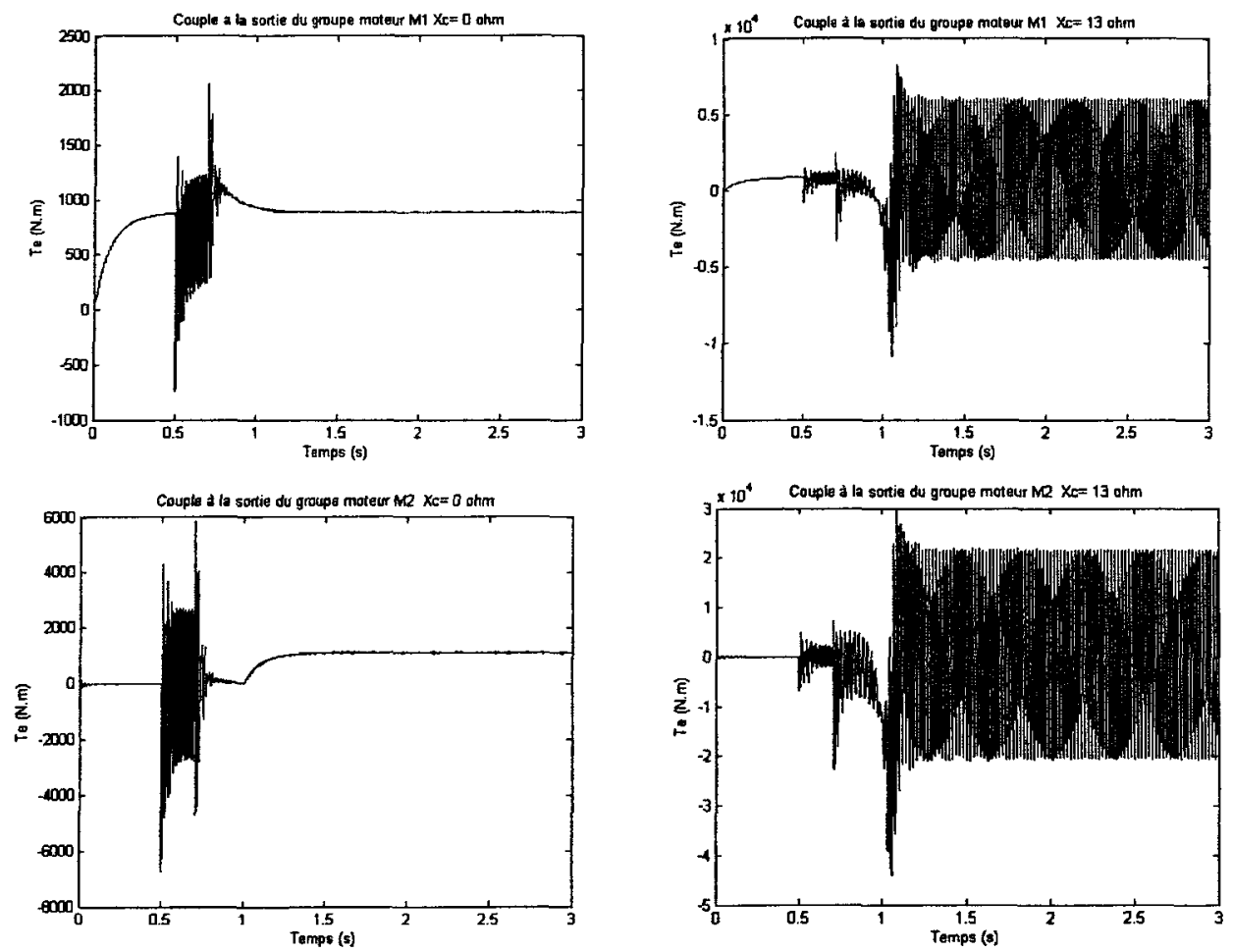

Figure 88: Couple à la sortie du groupe moteur M1 à $X c=0$ et $13 \Omega$ 


\subsubsection{Conclusions de l'essai du réseau de distribution radial en présence d'un court circuit monophasé en aval du condensateur série à $t=0.5 \mathrm{~s}$}

Cette contingence a été simulée avec un défaut à la phase «a " en aval du condensateur série. Dans ce type de contingence, la position du défaut par rapport à celle du condensateur série influera de manière importante les limites de stabilité dynamique des groupes moteurs. Lors du réenclenchement de la ligne, la courant de court-circuit produit des forts courants des groupes moteurs en produisant une réduction de la tension à la charge ainsi que la vitesse de rotation. À mesure qu'on augmente le taux de compensation, on réduit l'impédance équivalente de la ligne. Grâce à cette réduction, les courants de défaut deviennent très élevés. Les valeurs du courant de court-circuit obtenues dans les simulations sont montrées dans le tableau 25 ainsi :

Tableau 25

Valeurs du courant de défaut

\begin{tabular}{|c|c|}
\hline Compensation série & Courant de défaut monophasé A(rms) \\
\hline Non compensé & $700 \mathrm{~A}$ \\
\hline$X_{c=, 8 \Omega(\tau=33.75 \%)}$ & $985 \mathrm{~A}$ \\
\hline$X_{c}=, 13 \Omega(\tau=54.85 \%)$ & $1440 \mathrm{~A}$ \\
\hline$X_{C}=15.66 \Omega(\tau=66 \%)$ & $1650 \mathrm{~A}$ \\
\hline
\end{tabular}

Lorsque le réenclenchement de la ligne est effectué, on remarque la présence d'oscillations de grande amplitude de courant de la source, des courants statoriques des moteurs, ainsi que la tension en aval du condensateur série et la tension à la charge. Ces oscillations apparaissent lorsque le réseau est compensé à $\tau>54.85 \Omega$. On observe la présence d'oscillations du couple des groupes moteurs avec un pic négatif lorsque le réseau est compensé à $\tau=21.1$ et $\tau=54.85 \%$. L'amortissement des oscillations du couple mécanique est plus lent à chaque fois qu'on augmente le taux de compensation. Mais ils retrouvent leur valeur en régime permanent. Cela indique que le réseau se retrouve sous de bonnes conditions de stabilité. La perte de la stabilité est remarquable lorsqu'on compense le réseau à $\tau=66 \%\left(\mathrm{X}_{\mathrm{C}}=15.66 \Omega\right)$. La vitesse des moteurs est très basse et elle ne retrouve pas leur valeur en régime permanent. 


\subsubsection{Essai 6. Perturbation du réseau de distribution radial en présence d'un court circuit triphasé en amont du condensateur série à $t=0.5 \mathrm{~s}$}

L'objectif de cet essai est de simuler un court circuit triphasé en amont du condensateur série afin d'observer les effets sur les paramètres électriques du banc de condensateurs et des groupes moteurs branchés à la charge. Ce type de défaut est considéré comme une contingence extrême du fait qu'elle peut entraîner des forts courants qui produiront des valeurs de puissance de court-circuit trop élevées. Le modèle numérique utilisé pour simuler ce type de contingence extrême dans les réseaux électriques est montre à la figure 38 .

Dans le cas d'un défaut triphasé en amont du banc de condensateurs série, on observe à la figure 89 une réduction de la tension de la source qui est plus grande que la tension à la charge, figure 90. Le courant à source étant très élevé, figure 91. Cette élévation est aussi observée sur le courant à la charge, figures 91 . Dans les deux groupes moteurs le courant statorique a un pic négatif très élevé lors du court-circuit triphasé. Pendant cette contingence, les courants statoriques présentent des oscillations de grandes amplitudes surtout le groupe moteur M1. Pour un taux de compensation de $\tau=54.85 \%(X c=13 \Omega)$, l'amortissement des oscillations du courant devient plus rapide pour le groupe moteur M2 que pour le groupe moteur M1, figure 93.

Les courants de défaut aux différents taux de compensation série sont presque égaux du fait que le banc de condensateurs se trouve en aval du défaut triphasé. La valeur du courant est d'environ $900 \mathrm{~A}$ ( $\mathrm{rms}$ ). Lors du défaut triphasé, on observe la présence d'oscillations du courant de court-circuit. Ces oscillations sont produites par l'interaction du courant de la source et du courant généré par les moteurs qui fonctionnent comme des générateurs pendant leur décélération. On peut donc dire ici qu'il semble que la charge alimente le défaut. Cependant, l'effet n'est pas très important (pointes d'oscillation du courant de défaut entre $1280 \mathrm{~A}$ et $3700 \mathrm{~A} \mathrm{rms}$ ). Lors de l'élimination du défaut, le réseau retrouve son régime permanent relativement vite lorsqu'on compense le réseau à $\tau=33.76$ $\%(X c=8 \Omega)$. Par contre, lors du réenclenchement, une petite oscillation amortie est observée. Cette oscillation apparaît dans les courants des moteurs et de la source, ainsi que 
dans la tension en fin de ligne. Encore ici, on peut noter que ce type de perturbation pourrait éventuellement amorcer l'oscillation d'un réseau dont la résonance se manifeste à cette fréquence. En conclusion, il semble que ce type de défaut permet d'amorcer une résonance sous-synchrone du réseau. Par contre, pour ce type de défaut, la déformation de la tension est de plus courte durée que dans le cas du défaut en aval.
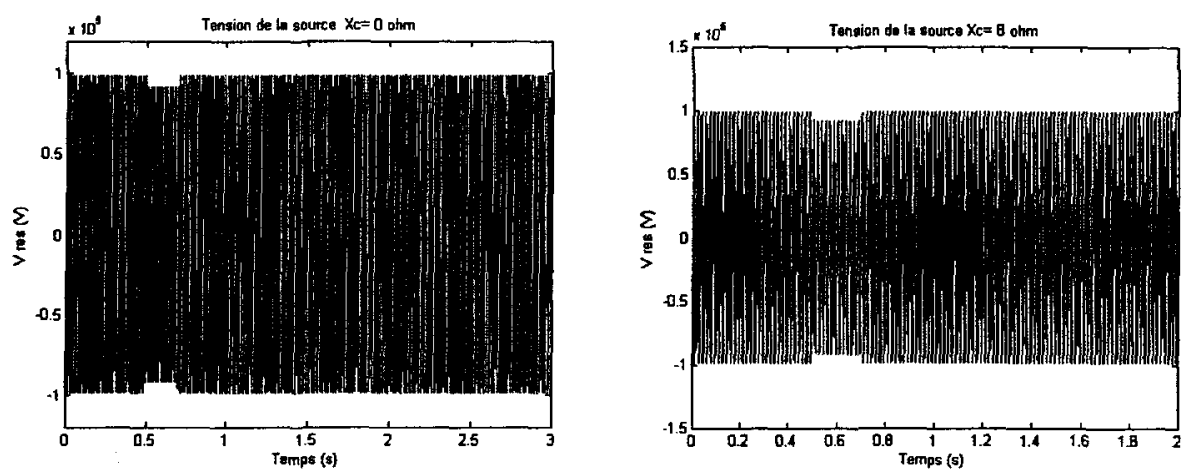

Figure 89 : Tension de la source à $\mathrm{Xc}=0$ et $8 \Omega$.
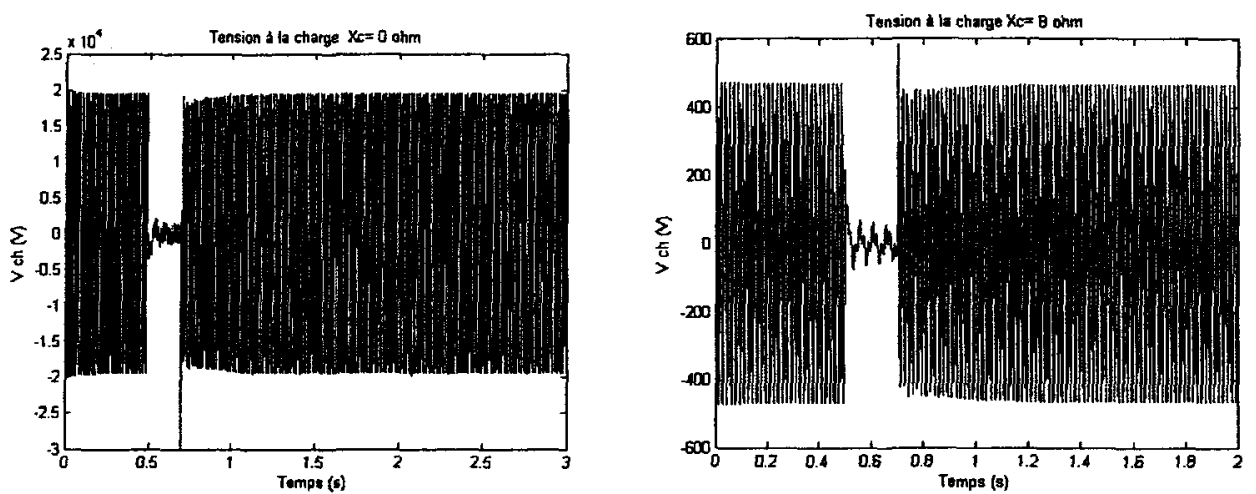

Figure $90:$ Tension à la charge à $\mathrm{Xc}=0$ et $8 \Omega$
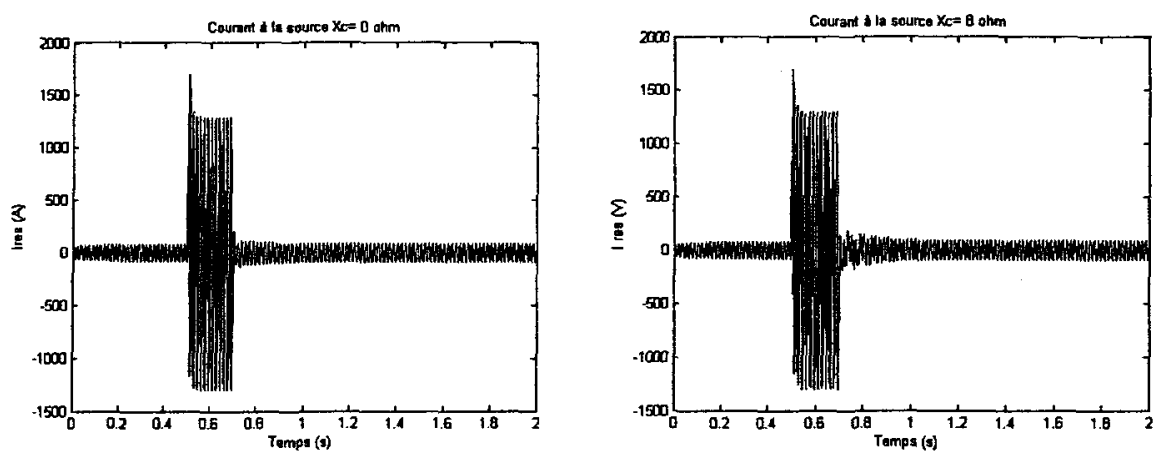

Figure 91 : Courant à la source à $X c=0$ et $8 \Omega$ 

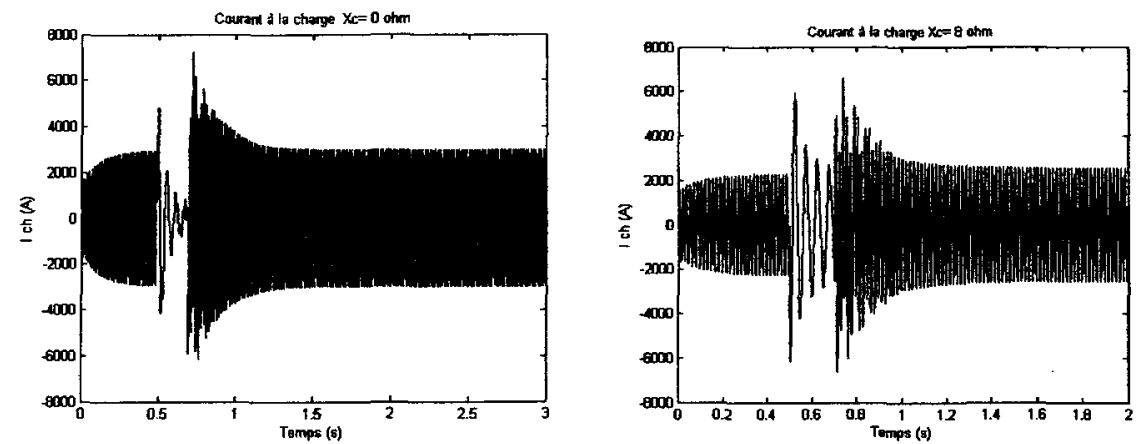

Figure 92 : Courant à la charge à $X c=0$ et $8 \Omega$
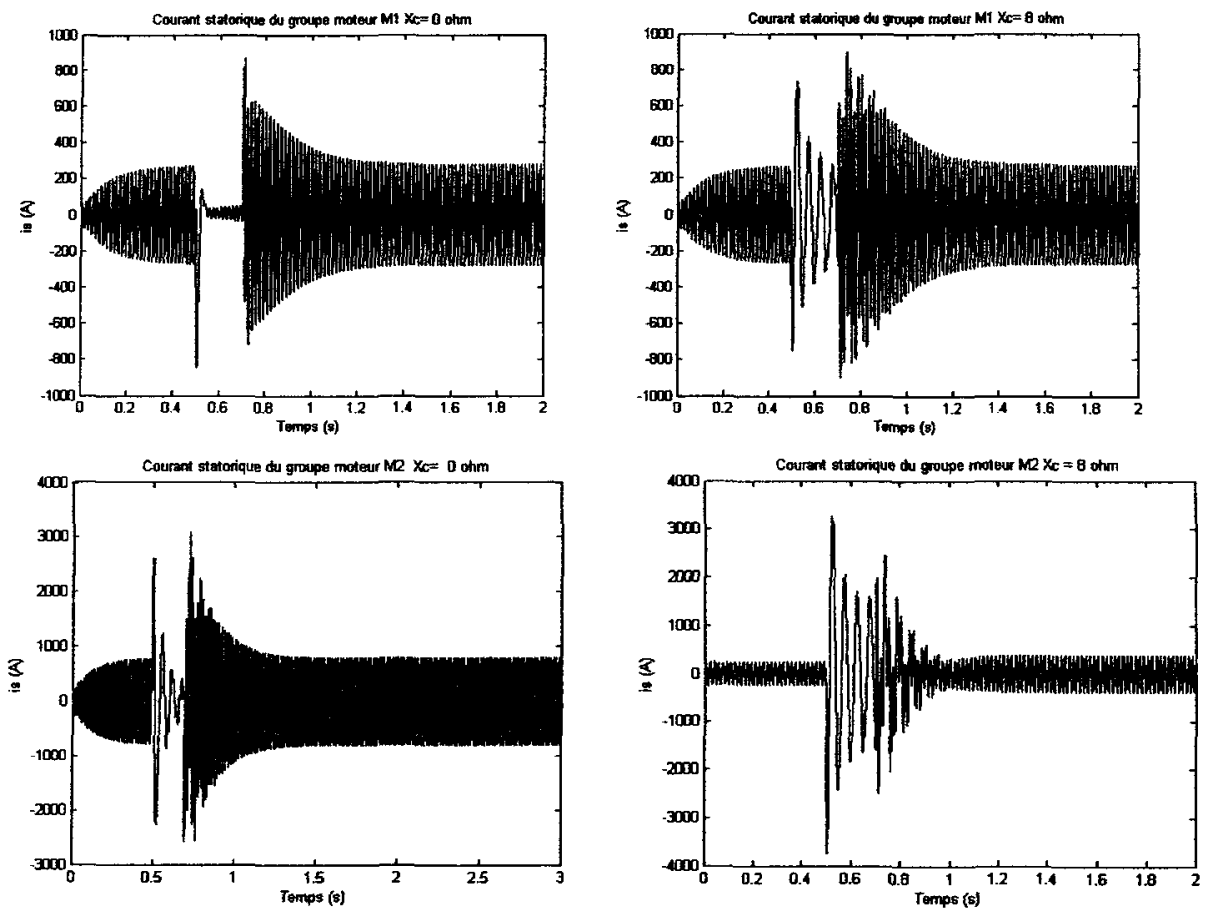

Figure 93 : Courant statorique des groupes moteurs M1 et M2 à $X c=0 \Omega$ et $X c=8 \Omega$

En ce qui concerne la vitesse de rotation, les groupes moteurs $\mathrm{M} 1$ et $\mathrm{M} 2$ présentent une réduction considérable des courants lors du défaut mais ceux-ci reviennent à leur valeur du régime permanent excepte celui du groupe moteur $M 2$ dont sa vitesse de rotation n'est pas la même après que le défaut soit éliminé, figure 94 . Le phénomène décrit précédemment se produit lorsqu'on a compensé le réseau à $\tau=54.85 \%(\mathrm{Xc}=$ $13 \Omega)$. Pour un taux de compensation série de $\tau=66 \%(\mathrm{Xc}=15.66 \Omega)$, les deux groupes moteurs montrent une réduction brusque de la vitesse qui se termine pour de petites oscillations en déstabilisant le système, figure 95 . 

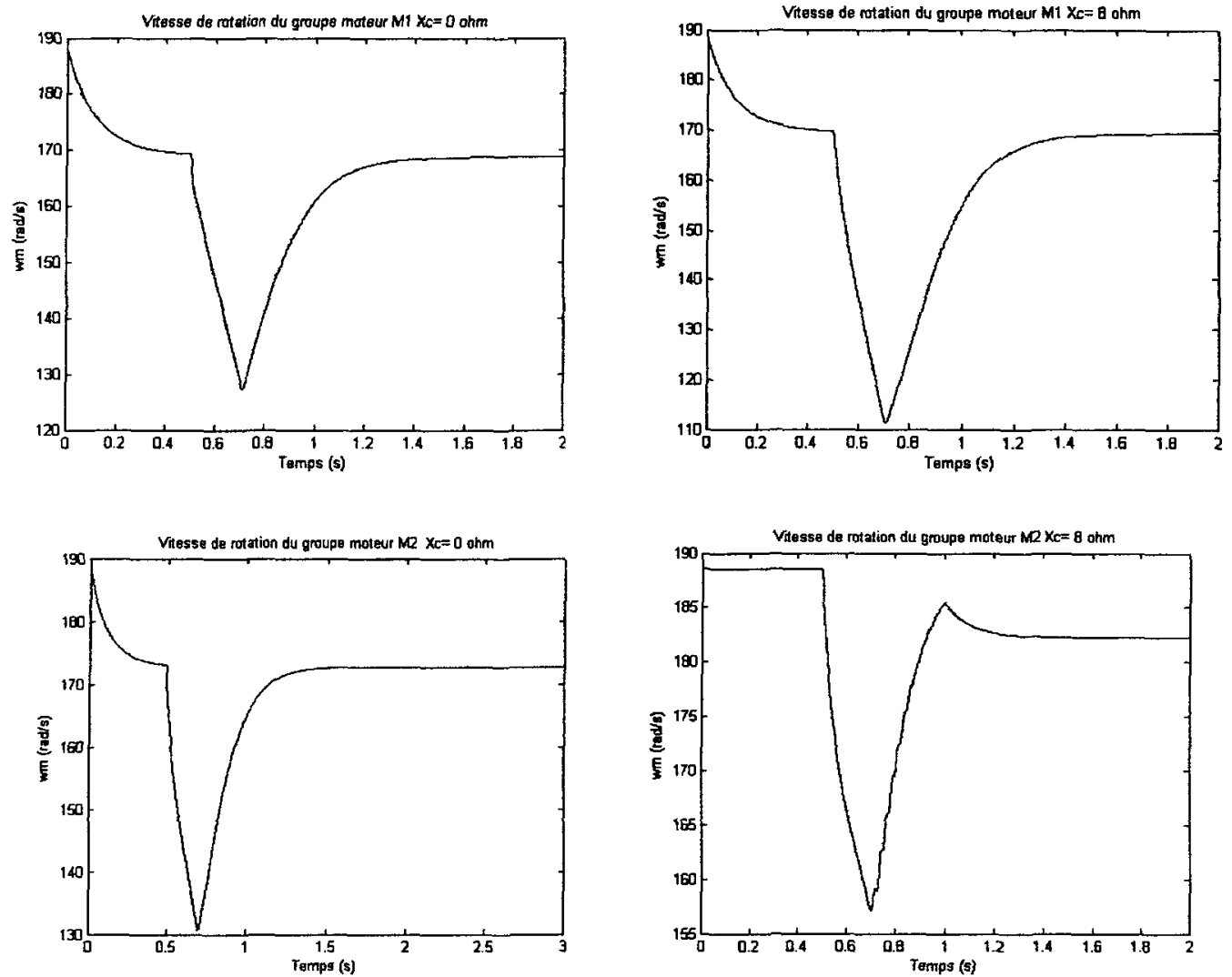

Figure 94 : Vitesse de rotation des groupes moteurs M1 et M2 à $X c=0$ et $8 \Omega$

Le couple mécanique des groupes moteurs se réduit instantanément en quelques cycles, le couple revient à la valeur en régime permanent après le réenclenchement de la ligne. Le groupe moteur M2 présente des oscillations de grande amplitude qui s'amortirent rapidement pour revenir en régime permanent, figure 96.
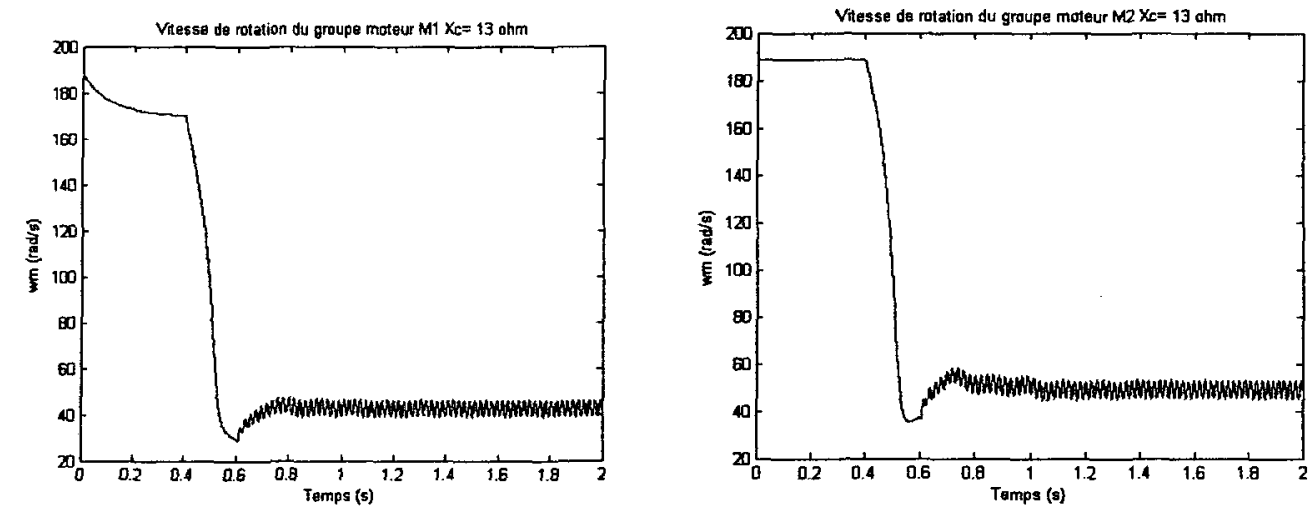

Figure 95 : Vitesse de rotation du groupe moteur M1 et M2 à $X c=13 \Omega$ 

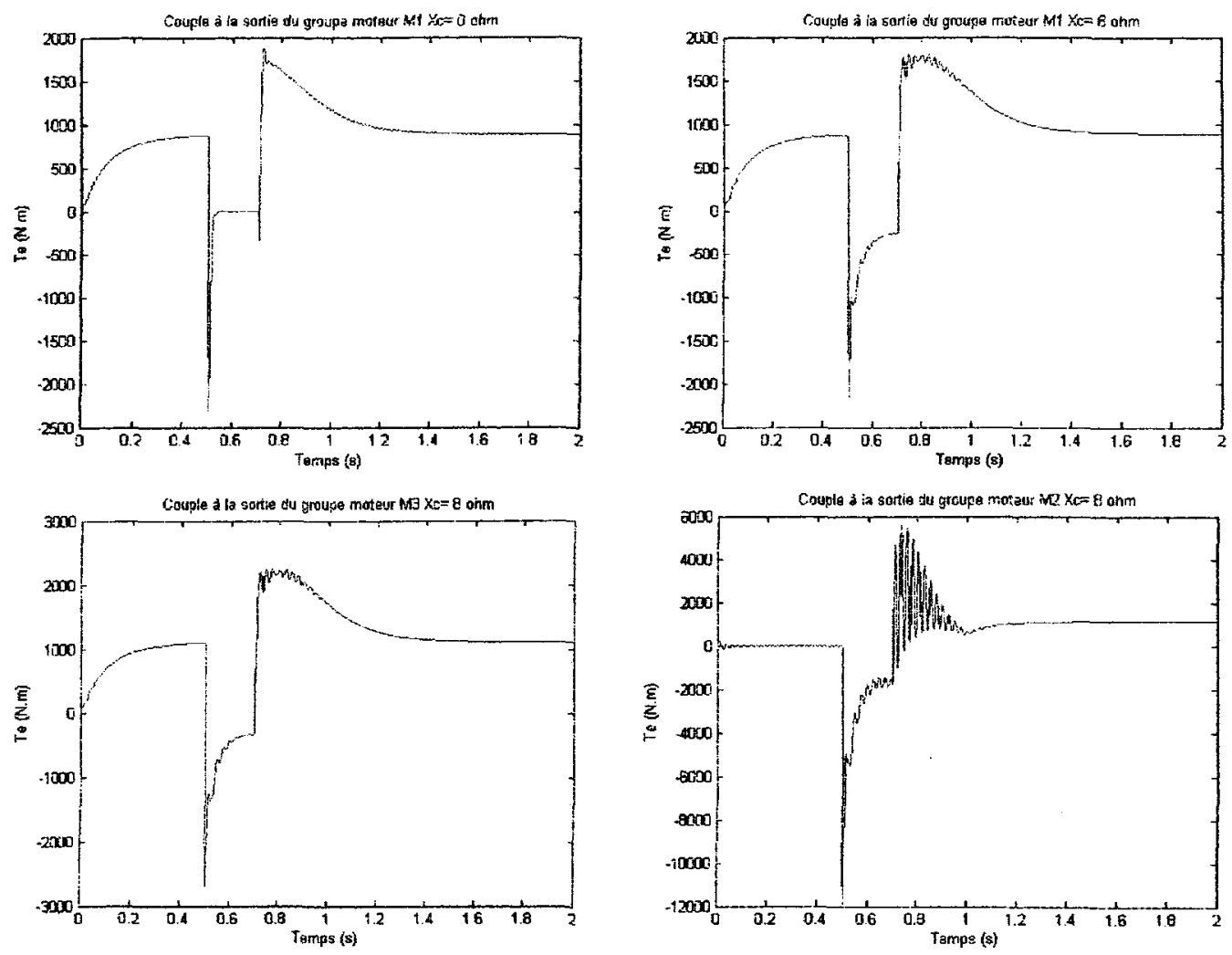

Figure 96 : Couple à la sortie du groupe moteur M1 et M2 à $X c=0 \Omega$ et $8 \Omega$

\subsubsection{Conclusions de l'essais du réseau de distribution radial en présence d'un court circuit triphasé en amont du condensateur série à $t=0.5 \mathrm{~s}$.}

On identifie des oscillations de grande amplitude lors du défaut. Ces oscillations affectent les courants des groupes de moteurs, le courant à la source, ainsi que la tension en aval du condensateur série.

On remarque que la valeur du courant de défaut devient plus grande à chaque fois qu'on augmente le taux de compensation. Le tableau 26 nous montre les valeurs du courant pour chaque valeur de compensation simulée : 
Tableau 26

Valeurs de courant de défaut

\begin{tabular}{|c|c|}
\hline Taux de compensation série & Courant de défaut triphasé A(rms) \\
\hline Non compensé & $1280 \mathrm{~A}$ \\
\hline $\mathrm{Xc}=, 8 \Omega(\tau=33.76 \%)$ & 1980 \\
\hline $\mathrm{Xc}=13 \Omega(\tau=54.85 \%)$ & $3700 \mathrm{~A}$ \\
\hline
\end{tabular}

Lorsque la ligne est réenclenchée, de forts courants sont demandés par les moteurs afin de retrouver leur valeur de régime permanent. Ceci produit une réduction de la tension à la charge ainsi qu'une diminution de la vitesse des groupes de moteurs qui se maintient dans les limites de stabilité pour un taux de compensation plus grand ou égal à $\tau=33.76 \%, X c=8 \Omega$. Le cas le plus critique est identifié dans le groupe moteur N.2. Ce groupe présente une forte réduction de la vitesse de rotation pour un taux de compensation plus grand que $33.76 \%$. Le même phénomène se produit lors de prédiction du couple des groupes moteurs qui présente des oscillations une fois le défaut éteint. Dans ce type de contingence, il est très dangereux de brancher toutes las charges lors du réenclenchement de la ligne afin d'éviter que les grandeurs électriques des moteurs fonctionnent hors limites de stabilité.

\subsubsection{Essai 6. Perturbation du réseau de distribution radial en présence d'un court-circuit triphasé en aval du condensateur série à $t=0.5 \mathrm{~s}$}

Cet essai aura comme objectif de simuler un court circuit triphasé en aval du condensateur série. Cette contingence est aussi considérée comme extrême du fait de la position du défaut par rapport au condensateur série. Lorsque le réseau est compensé, la réactance équivalente de la ligne devra supporter de forts courants et une puissance de court-circuit très élevée. Cette caractéristique de la ligne compensée ainsi que la performance des variables électriques des groupes moteurs sont observées dans les simulations. Le modèle numérique utilisé pour simuler ce type de contingence est montre à la figure 73. Ce type de défaut affecte fortement les conditions de stabilité dynamique. Pour un taux de compensation de $\tau=21.1 \%(X c=5 \Omega)$, l'amplitude du courant de la source devient élevée lorsque le court-circuit est produit sur la ligne de 
figure 99. Le réseau maintient la stabilité dynamique lors le court circuit. Cela peut être observé dans les figures 97 et 98 de la tension de la source et de la charge respectivement. Un grand pic négatif de tension d'un cycle est observé dans la tension à la charge après que le défaut soit éteint du fait du réenclenchement de la ligne, figure 100.
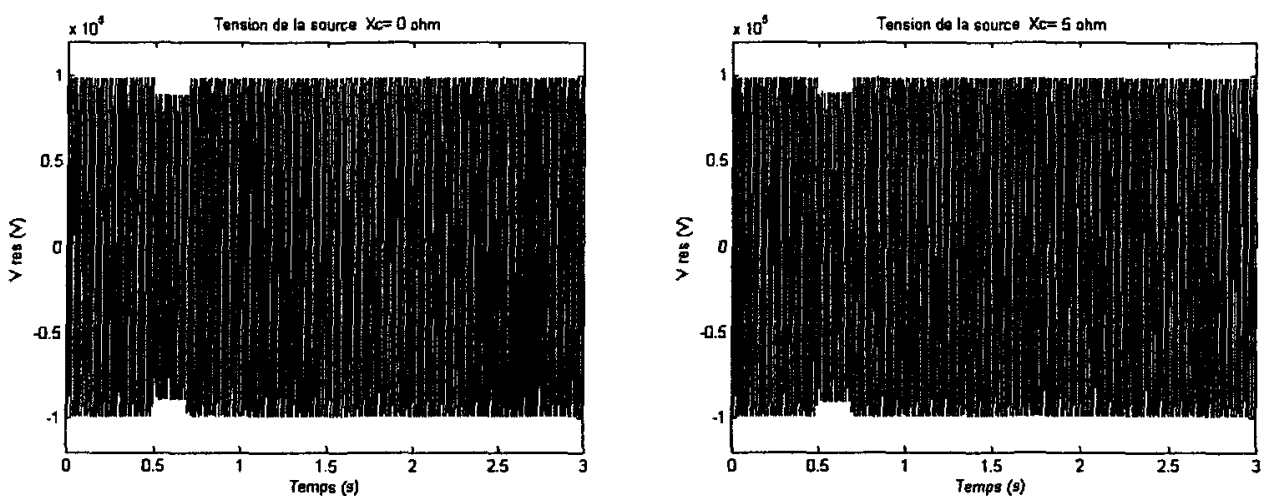

Figure 97 : Tension de la source à $X c=0 \Omega$ et $5 \Omega$
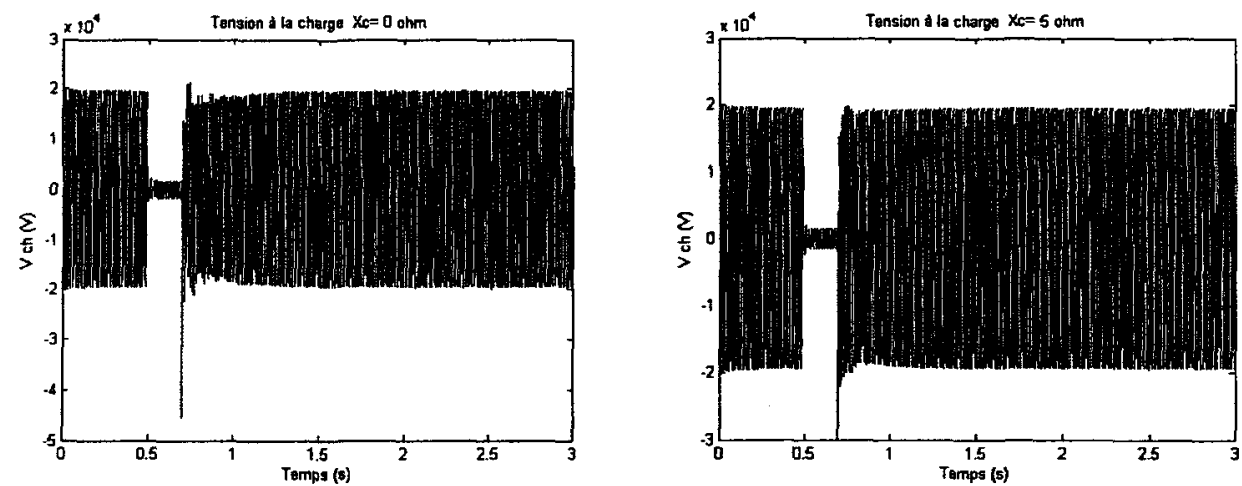

Figure 98 : Tension à la charge à $X c=0 \Omega$ et $5 \Omega$
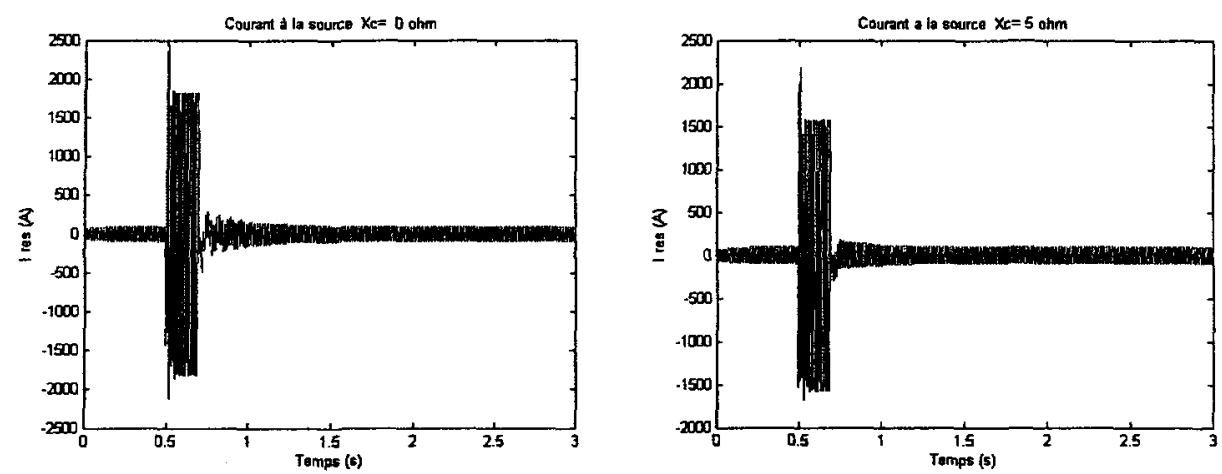

Figure 99 : Courant à la source à $X c=0 \Omega$ et $5 \Omega$. 

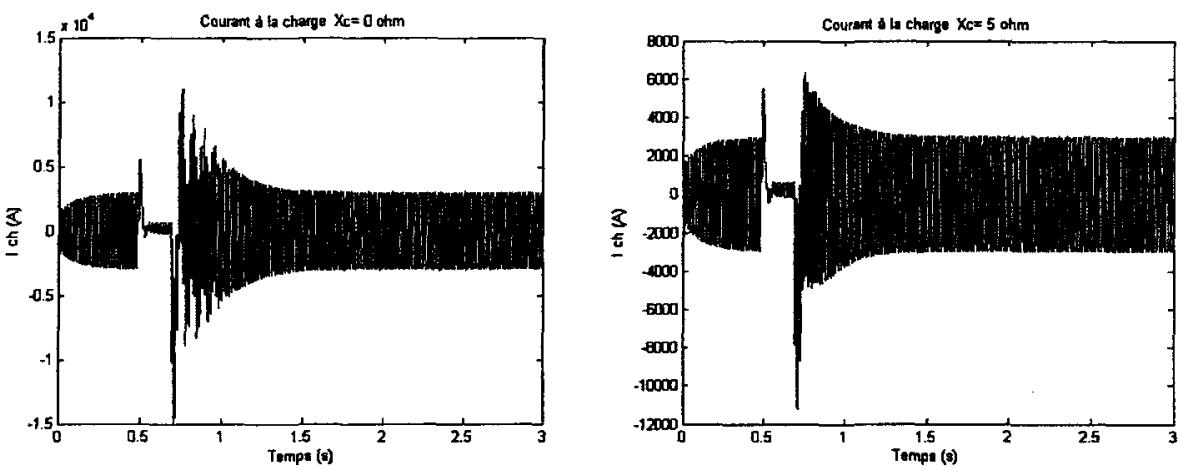

Figure 100 : Courant à la charge.

L'étude de stabilité dynamique développée dans les moteurs nous montre le fort effet de ce type de court circuit. Ensuite lorsque le défaut est supprimé, des oscillations de grandes amplitudes se présentent dans les courants statoriques. Mais ces oscillations sont réduites lorsque la ligne est compensée à $\tau=21.1 \%(X c=5 \Omega)$, figure 101 . On note que le pic négatif de courant à la charge est aussi observé dans les courants statoriques.
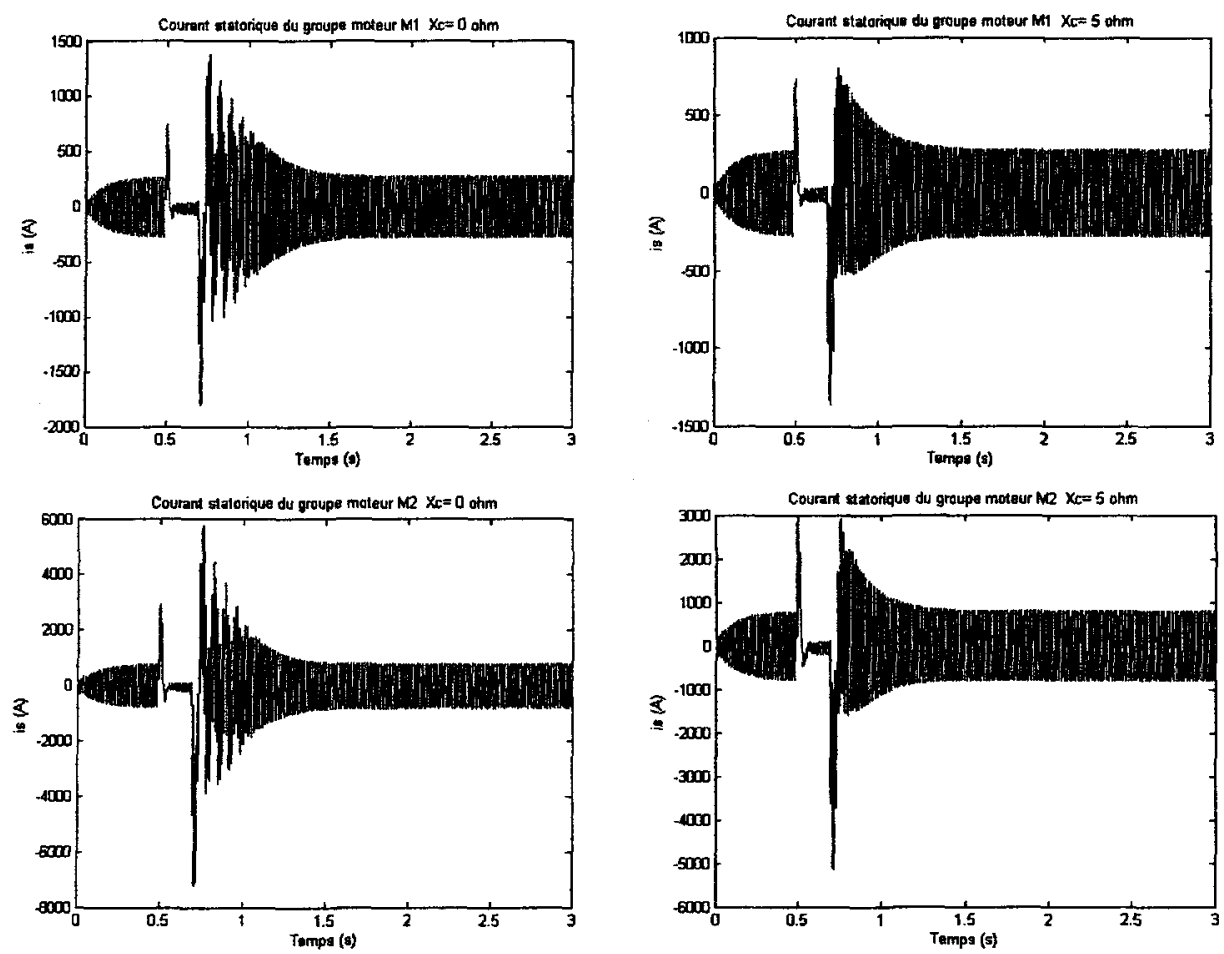

Figure 101 : Courant statorique des groupes moteurs M1 et M2 à $X c=0$ et $5 \Omega$ 
La vitesse de rotation des groupes moteurs diminue. Lorsque le réseau est compensée à $\tau=21.1 \%(X c=5 \Omega)$, la vitesse tombe presque instantanément à une valeur plus basse que pour la ligne non compensée. La compensation série améliore la performance dynamique des moteurs après le réenclenchement de la ligne. On observe que le temps de réponse des moteurs est plus rapide, figure 102.

Pour $\tau=54.85 \%(X c=13 \Omega)$, les groupes moteurs se trouvent hors des limites de stabilité dynamique. La vitesse ne revient pas à la valeur en régime permanent, après une grande oscillation; elle tombe à une valeur plus basse que la vitesse nominale après quelques secondes, figure 103.
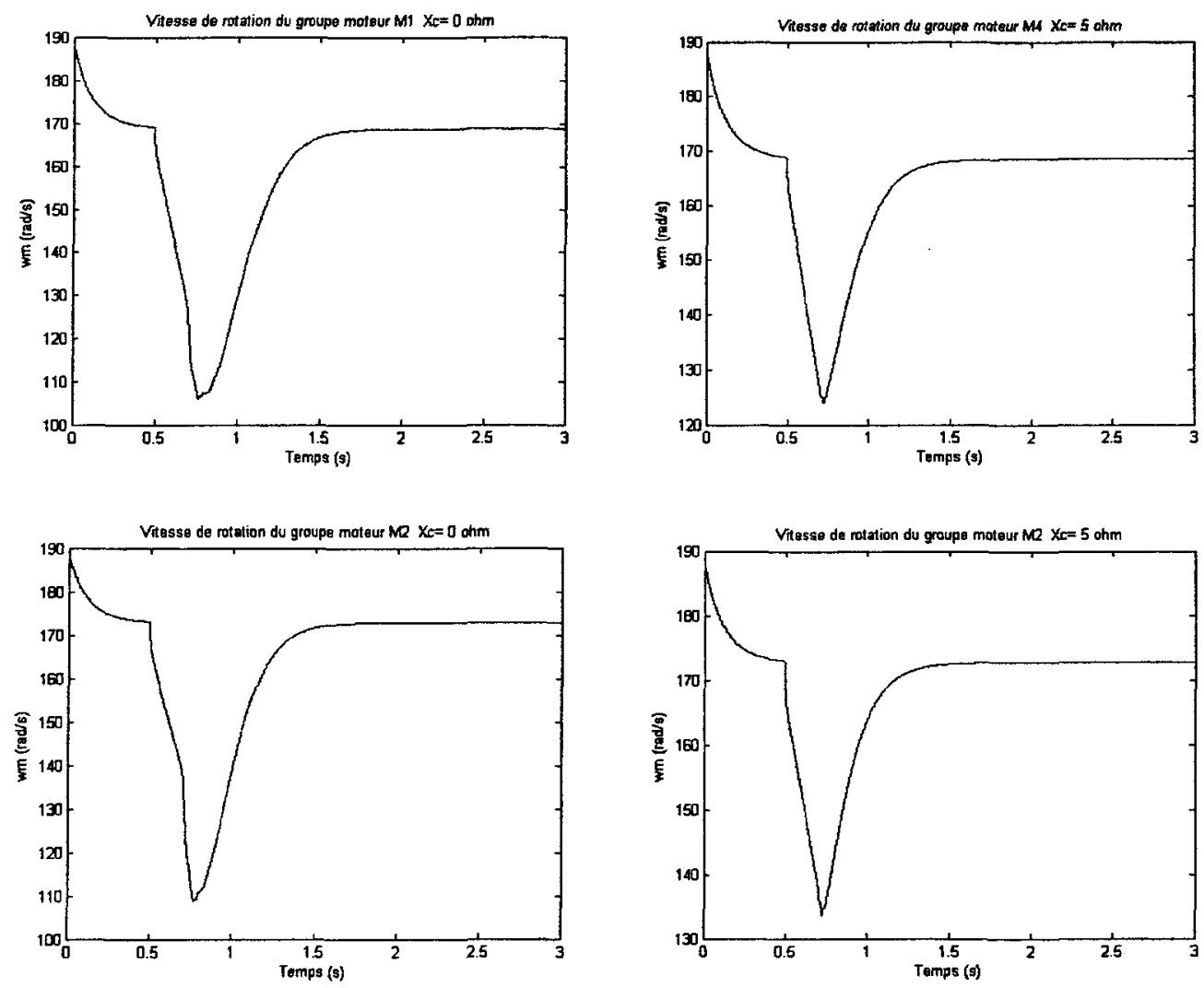

Figure 102 : Vitesse de rotation des groupes moteurs $M 1$ et $M 2$ à $X c=0 \Omega$ et $5 \Omega$ 

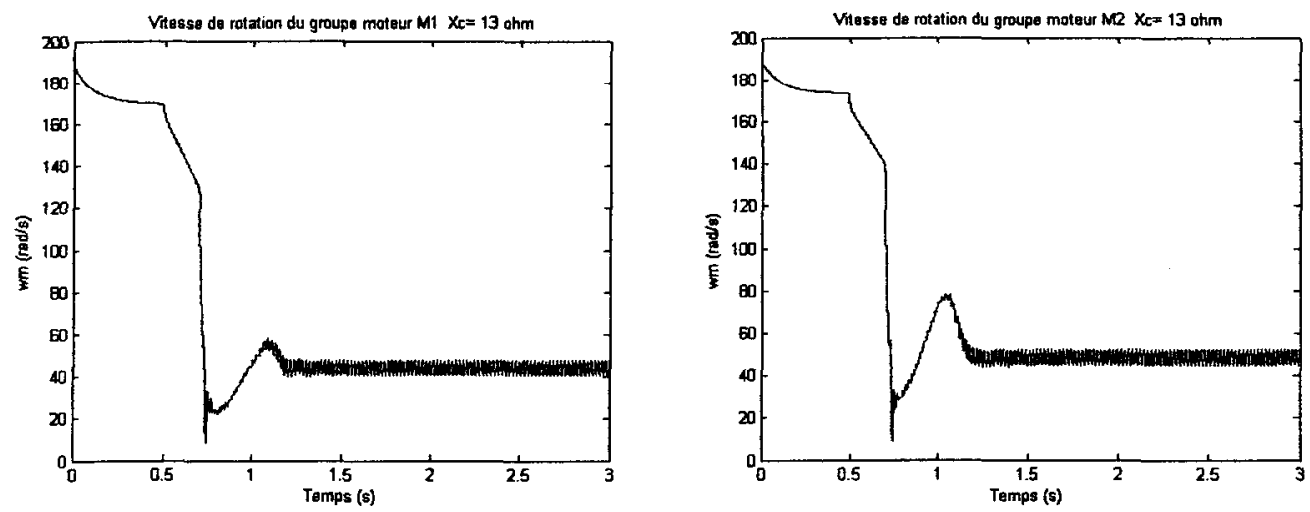

Figure 103 : Vitesse de rotation des groupes moteurs $X c=0$ et $13 \Omega$.

Le couple mécanique des groupes moteurs présente de fortes réductions à $t=0.5$ et $\mathrm{t}=0.7 \mathrm{sec}$. Après, il revient à la valeur en régime permanent lors du réenclenchement de la ligne. Des petites oscillations son observées sur le couple des groupe moteurs M1 et M2, mais elles sont amorties rapidement lorsqu'on compense le réseau à $\tau=21.1 \%, X c=5 \Omega$, figure 104. $\grave{A}$ partir de ce taux de compensation le couple devient instable avec des oscillations de grandes amplitudes. Ces oscillations conduiront à l'instabilité, figure 105.
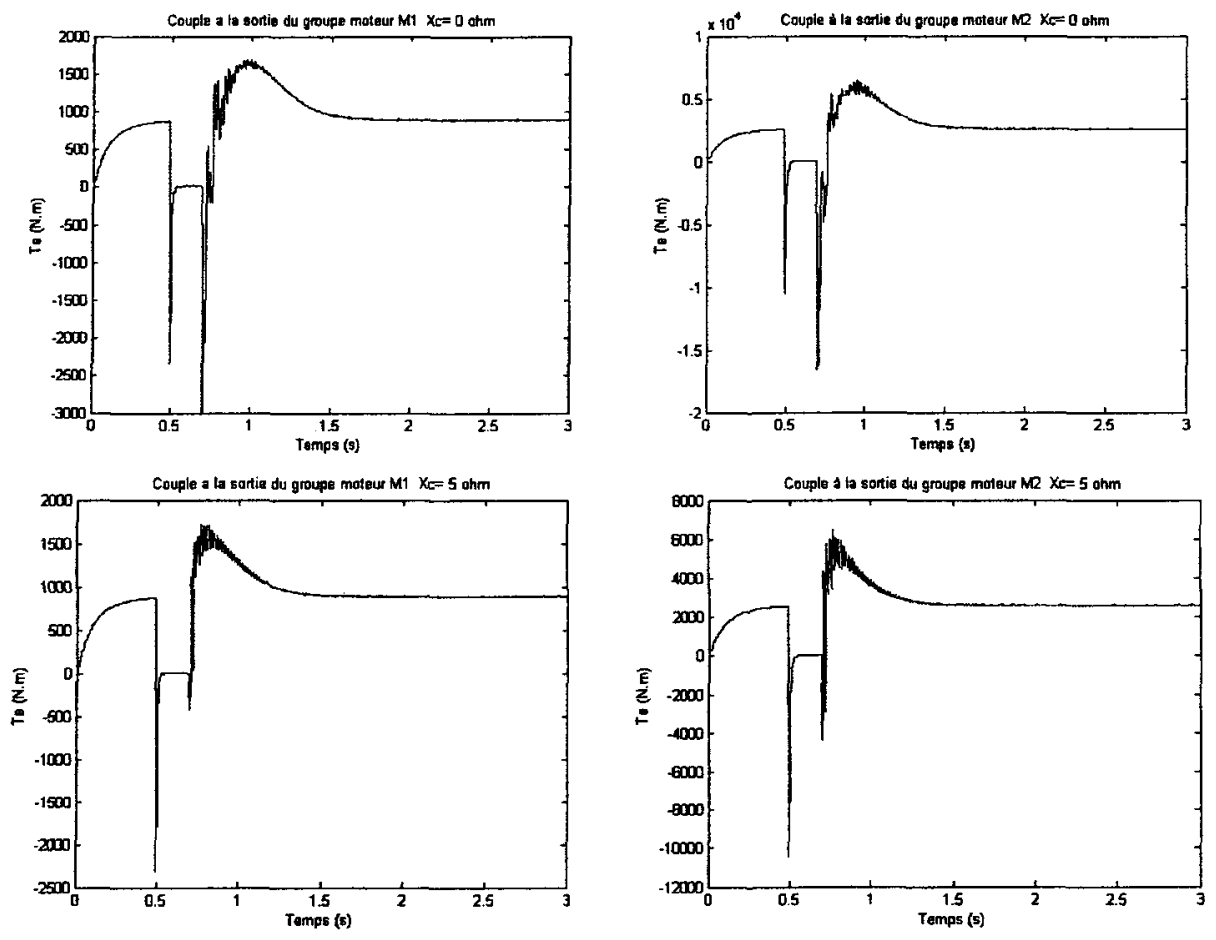

Figure 104 : Couple à la sortie des groupes moteurs M1 et M2 à $X c=0 \Omega$ et $5 \Omega$ 

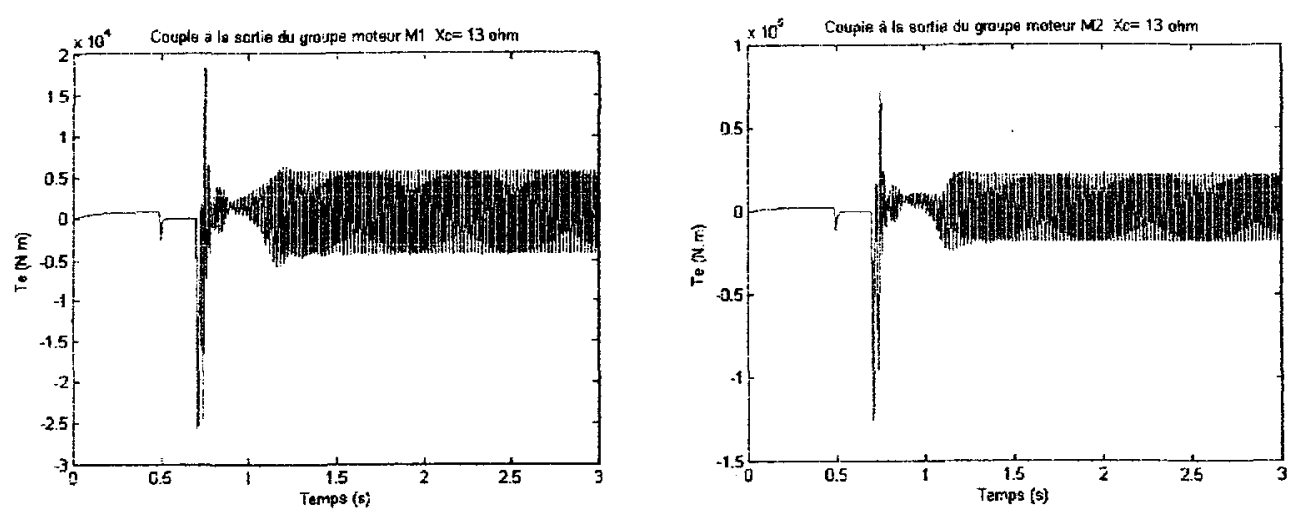

Figure 105 : Couple à la sortie des groupes moteurs $\mathrm{M} 1$ et $\mathrm{M} 2$ à $X c=13 \Omega$.

\subsubsection{Conclusions de l'essais du réseau de distribution radial en présence d'un court circuit triphasé en aval du condensateur série à $t=0.5 \mathrm{~s}$}

Les simulations effectuées nous ont permis d'identifier l'apparition d'une petite oscillation lors du réenclenchement. Cette oscillation apparaît sur les courants des groupes moteurs, sur le courant à la source ainsi que sur la tension en fin de ligne. Le court circuit triphasé est considéré comme une contingence extrême qui peut limiter l'application de la compensation série du réseau de distribution. Dans cet essai, la valeur du courant de défaut devient très grande lorsque on augmente le taux de compensation série. Ce courant dépend du courant de la source et des courants statoriques des groupes moteurs qui se comportent comme des générateurs lors des courts-circuits triphasés. On peut comparer dans le tableau 27 les valeurs du courant de défaut pour les valeurs de compensation étudiées :

Tableau 27

Valeurs du courant défaut

\begin{tabular}{|c|c|}
\hline & \\
Taux de compensation série & Courant de défaut triphasé A (rms) \\
\hline Non compensé & $1400 \mathrm{~A}$ \\
\hline$X c=, 5 \Omega(\tau=21.1 \%)$ & $1500 \mathrm{~A}$ \\
\hline$X c=, 13 \Omega(\tau=33.76 \%)$ & $1650 \mathrm{~A}$ \\
\hline
\end{tabular}

Des oscillations de grande amplitude se manifestent après que le défaut est éteint. Cette oscillation affecte la performance des courants statoriques des groupes des 
le courant à la source, ainsi que dans la tension en aval du condensateur série. La vitesse de rotation, par exemple, tombe aux valeurs plus basses que la vitesse nominale mais lorsque du réenclenchement se produit, les groupes moteurs reviennent rapidement à sa valeur en régime permanent.

\subsubsection{Essai 7. Performance du réseau de distribution radial due à la variation brusque de charge dans le groupe moteur $\mathrm{M} 2$ à $\mathrm{t}=0.6 \mathrm{sec}$.}

L'objectif est de simuler cette perturbation est d'observer l'effet sur le réseau et les groupes moteurs lorsque on applique un grand couple aux moteurs. Il s'agit de doubler le couple du groupe moteur M2 et d'évaluer la performance dynamique du réseau et des moteurs à différents taux de compensation série.

Dans cet essai, on a observé l'effet de cette perturbation sur la tension d'alimentation qui affecte grandement le couple mécanique des groupes moteurs. Le couple disponible est fonction du carré de la tension. Lorsque le réseau aune faible limite de transit (transfert de puissance), l'absorption du courant peut faire écraser la tension et de ce fait, affecter la caractéristique couple-vitesse des moteurs asynchrones.

On simule le réseau de distribution sans et avec la valeur optimal du taux de compensation série trouvé à partir des résultats analytiques. En ce qui concerne à la performance dynamique du réseau lors la variation brusque de charge, le courant à la source et à la charge grimpe en se stabilisant à $t=1.2 \mathrm{sec}$ où la grandeur du courant acquiert une nouvelle valeur en régime permanent pour tous les cas de compensation, figure 106 et 106. Le même effet se produit dans le groupe moteur M2 où l'élévation du courant statorique est plus remarquable du fait qu'on a varié son couple, figure 108. Quelques oscillations sont identifiées sur le courant statorique des deux groupes moteurs lorsque on compense le réseau à $\tau=54.85 \%(X c=13 \Omega)$. Le groupe moteur M1 a une petite variation du courant de à l'absorption du courant du groupe moteur M2 pour doubler le couple mécanique, figure 108 . 

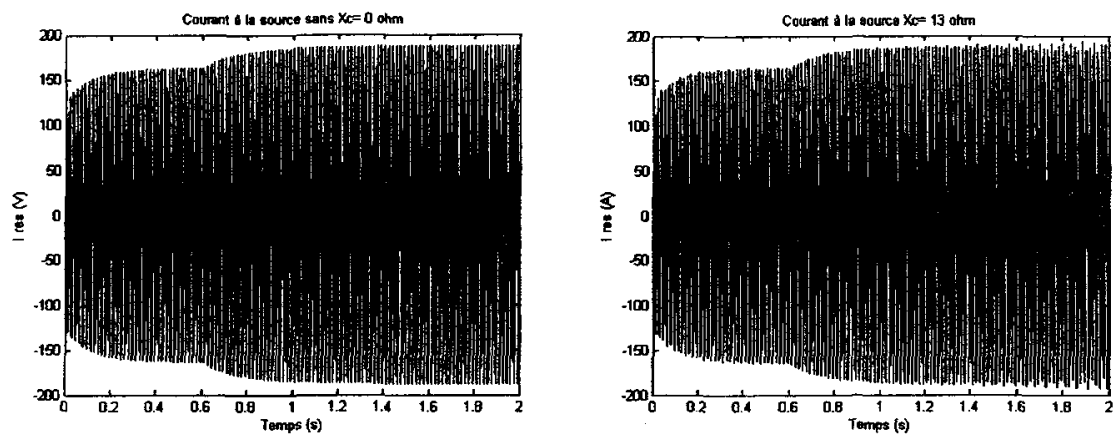

Figure 106 : Courant à la source
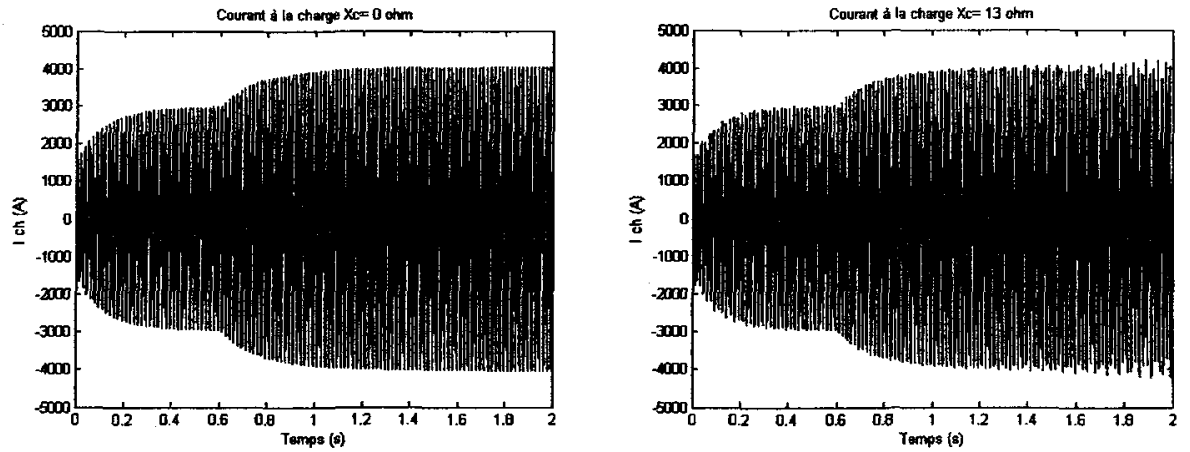

Figure 107 : Courant à la charge à $X c=0 \Omega$ et $13 \Omega$
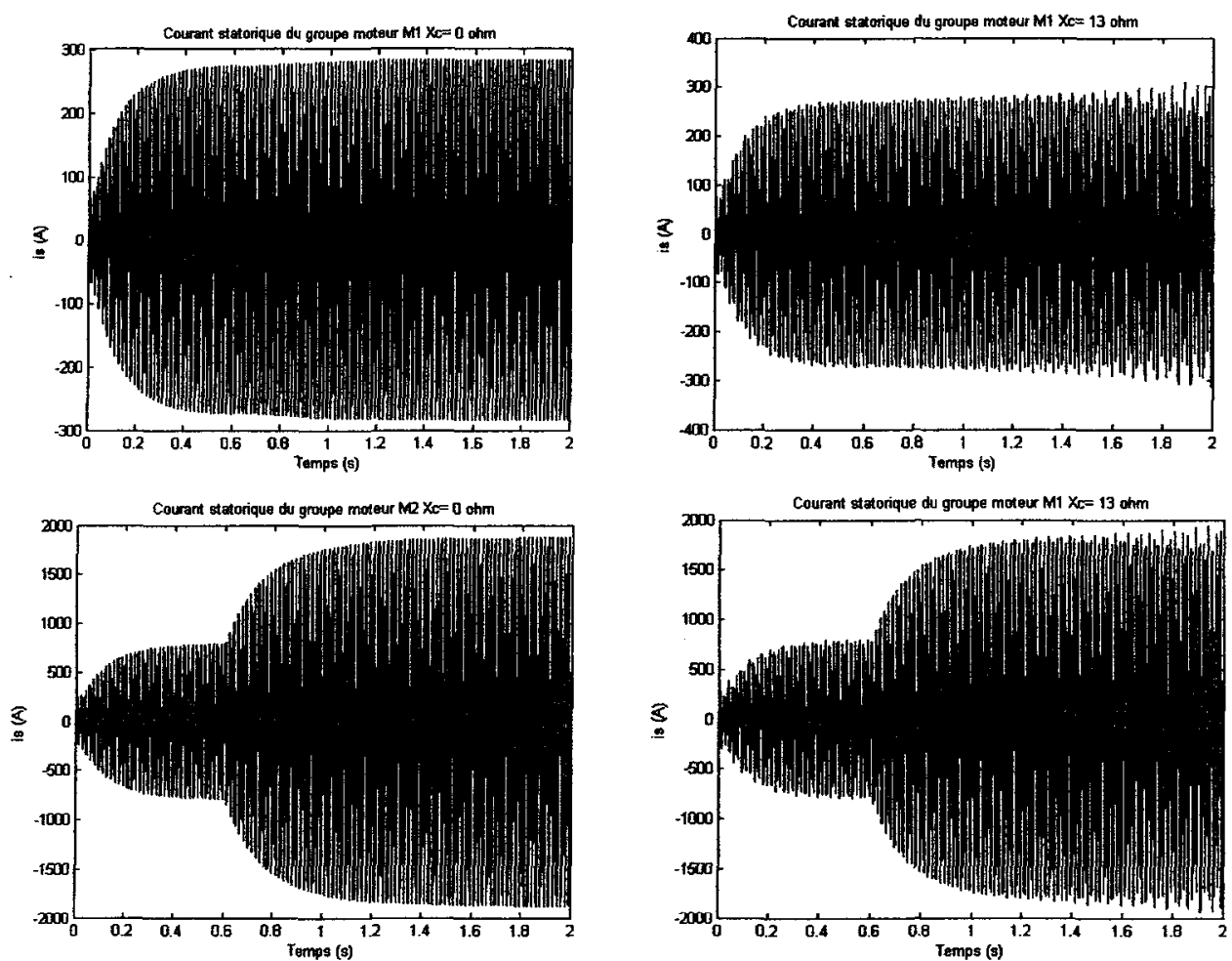

Figure 108 : Courant statorique du groupe moteur M1 et M2 à $X c=0 \Omega$ et $13 \Omega$ 
En ce qui concerne la vitesse de rotation, celle-ci se réduit dans les deux groupes des moteurs, étant donné la demande du courant et le couple appliqué sur le groupe moteur M2. L'observation précédemment décrite se produit pour les cas sans compensation et avec un taux de compensation de $\tau=54.85 \%(\mathrm{Xc}=13 \Omega)$, figure 108 . Pour le cas du réseau compensé à $\tau=66 \%(\mathrm{Xc}=15.66 \Omega)$, la vitesse de rotation des deux groupes moteurs tombe à une valeur très basse que sa valeur nominale en conduisant le réseau hors limites de stabilité dynamique, figure 109.
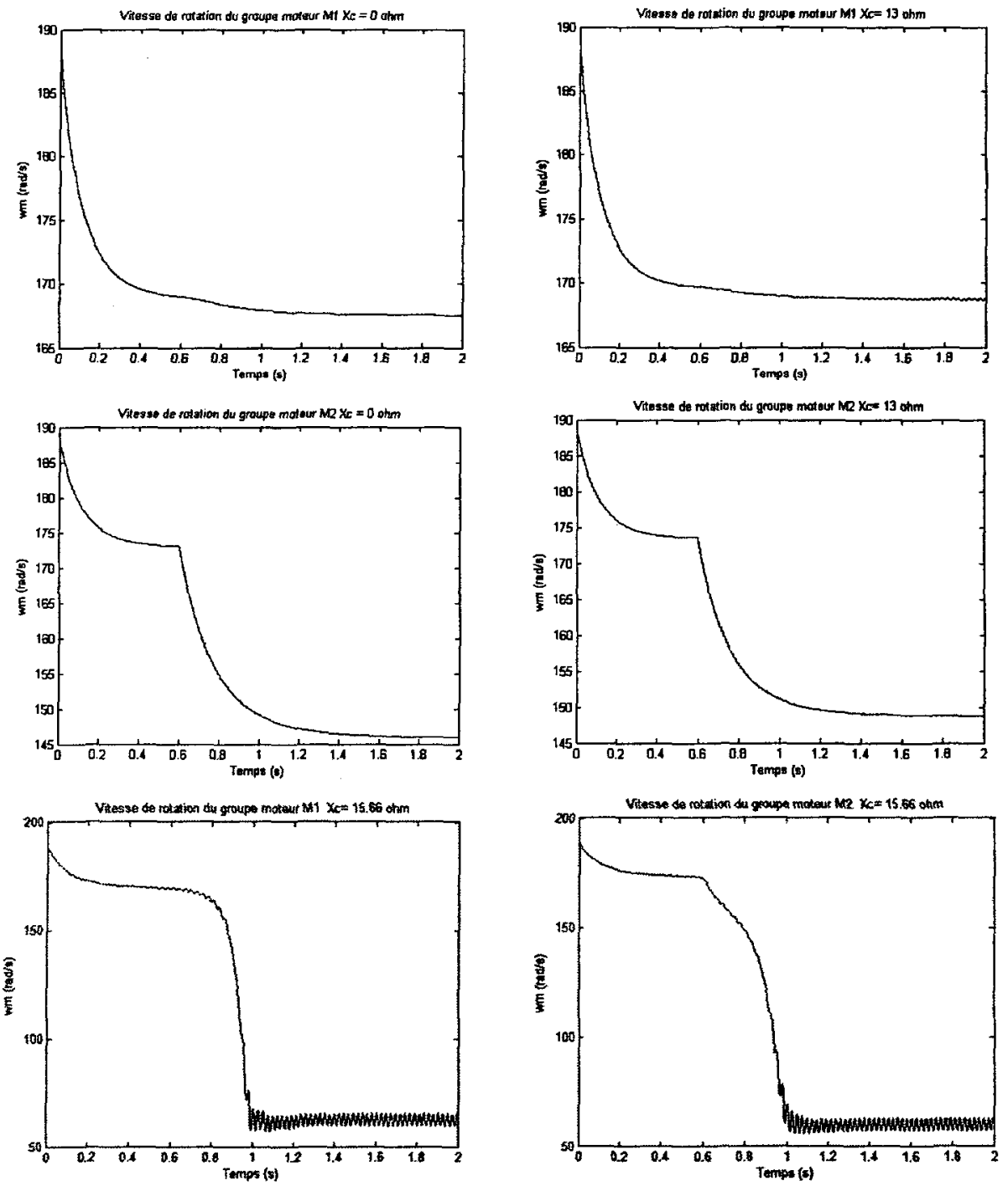

Figure 109 : Vitesse de rotation du groupe moteur M1 et M2 
Le couple mécanique du groupe moteur Ml est affecté par la variation du couple de groupe moteur M2. Lorsque on applique la compensation série dans le réseau, des oscillations du couple mécanique se manifestent en affectant la stabilité dynamique. Ces oscillations tendent à croître sans revenir à sa valeur en régime permanent, figure 110 et 111.
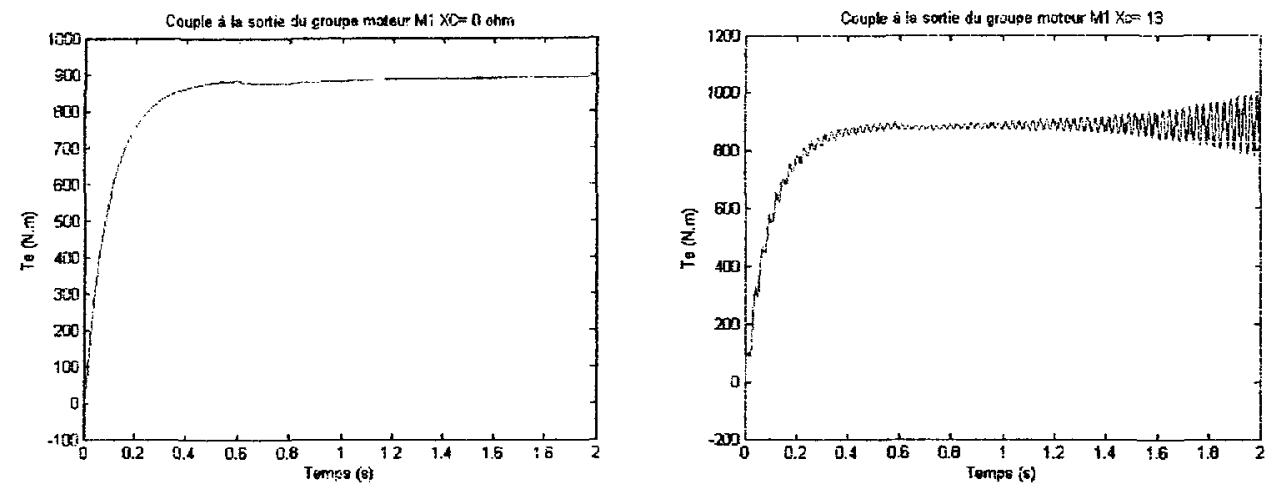

Figure 110 : Couple à la sortie du groupe moteur M1
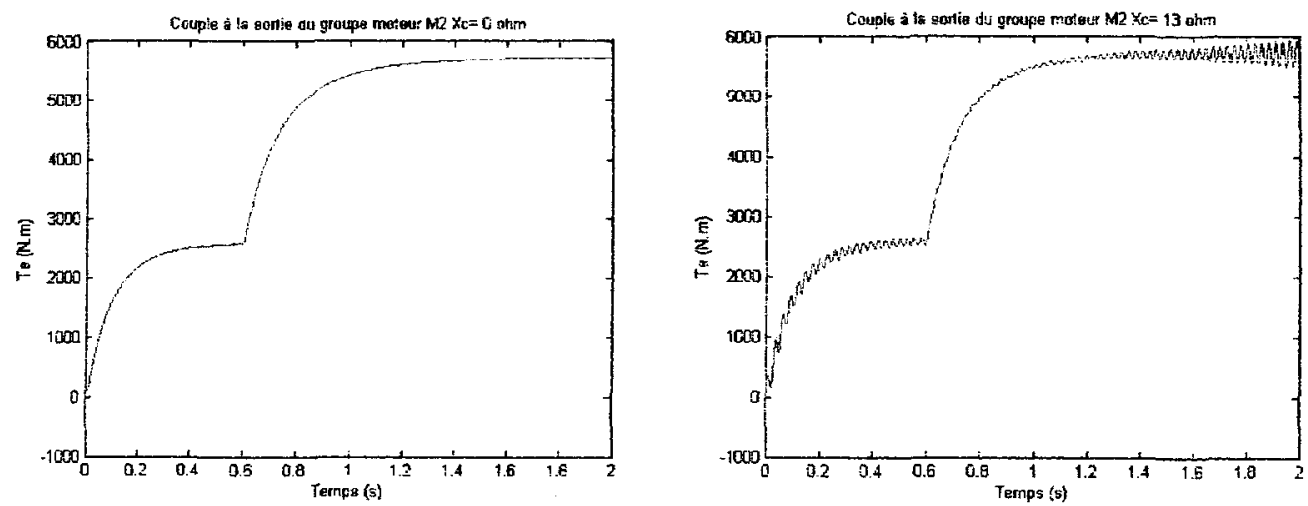

Figure 111 : Couple à la sortie du groupe moteur M2

\subsubsection{Conclusions de l'essai de variation brusque de la charge mécanique du groupe moteur N. 2}

L'analyse des résultats pour l'essai de variation brusque de la charge mécanique a été effectuée. On a fait plusieurs observations qui nous déterminent la limite de stabilité 
du réseau compensé par rapport aux perturbations sur les paramètres de moteurs asynchrones et dans le réseau de distribution. Les observations sont analysées comme suit :

Pour une compensation de $\tau=21.1 \%(X c=5 \Omega)$, la variation brusque de charge du groupe moteur M2 produit une élévation du courant dans la ligne étant donné l'absorption du courant au stator demandé afin de supporter la variation de couple. Nous remarquons une réduction de la vitesse du groupe moteur M2 ainsi qu'une petite variation de vitesse dans les autres moteurs qui ont été affectée par cette perturbation. Cela provoque une réduction du couple mécanique des autres moteurs. On observe que le réseau récupère la stabilité dynamique à $t=1.5$ seconds. La tension en aval du condensateur série présente une réduction sur la tension. Ici on peut noter l'effet instantané sur le condensateur dû aux changements provoqués pour la variation du couple mécanique. À partir du $\tau=54.85 \%((X c=13 \Omega)$, les performances des tensions et des courants autant de la ligne comme de la charge deviennent instables. Les oscillations du couple des moteurs sont remarquables lors du démarrage et postérieurement en régime permanente. Le cas le plus critique d'instabilité est observé pour $\tau=66 \%(X c=15.66 \Omega)$, où la compensation série provoque des perturbations extrêmes sur des tensions et des courants de la ligne, le condenseur série et la charge. De plus, nous remarquons la perte de la stabilité dynamique des groupes moteurs dont la vitesse oscille à $\mathrm{t}=1 \mathrm{sec}$.

\subsubsection{Essai 8. Démarrage à vide des moteurs asynchrones}

Dans cet essai, nous présentons les résultats de simulations du démarrage à vide des groupes moteurs M1 et M2. Le réseau de distribution est simulé sans la compensation et avec différents taux de compensation série afin d'analyser les performances des variables des moteurs telles que le courant statorique, la vitesse de rotation et le couple mécanique de chaque groupe moteur. On a remarqué de forts courants statoriques lors du démarrage avec un diminution de la tension à la charge, figure 112. Mais ces courants baissent considérablement lorsque les groupes moteurs ont 
que le couple de groupes moteurs est très distorsioné mais, se stabilise lorsque il s'approche à sa valeur en régime permanent. L'application de la compensation série à $\tau=54.85 \%(X c=13 \Omega)$ permet aux groupes moteurs de démarrer légèrement plus rapidement en améliorant la performance de la vitesse de rotation, figure 114 et le couple mécanique, figure 115. Le démarrage de groupes moteurs n'entraîne pas des oscillations de la tension au début de la ligne mais, en fin de la ligne; on peut observer que lors du démarrage, la tension augmente brusquement par rapport à la valeur en régime permanent, figure 112. L'application de la compensation permet d'avoir des valeurs de tensions plus hautes en fin de ligne, mais ne permet pas d'éviter l'augmentation de la tension. Puisque que les groupes moteurs démarrent simultanément, le réseau de distribution met plus temps afin de retrouver la valeur de la tension en régime permanent. En général, pour tous les cas de compensation, permettent d'améliorer la forme de la tension mais ne permet pas d'éliminer complètement l'effet de la perturbation à exception du taux de compensation de $\tau=54.85 \%$.
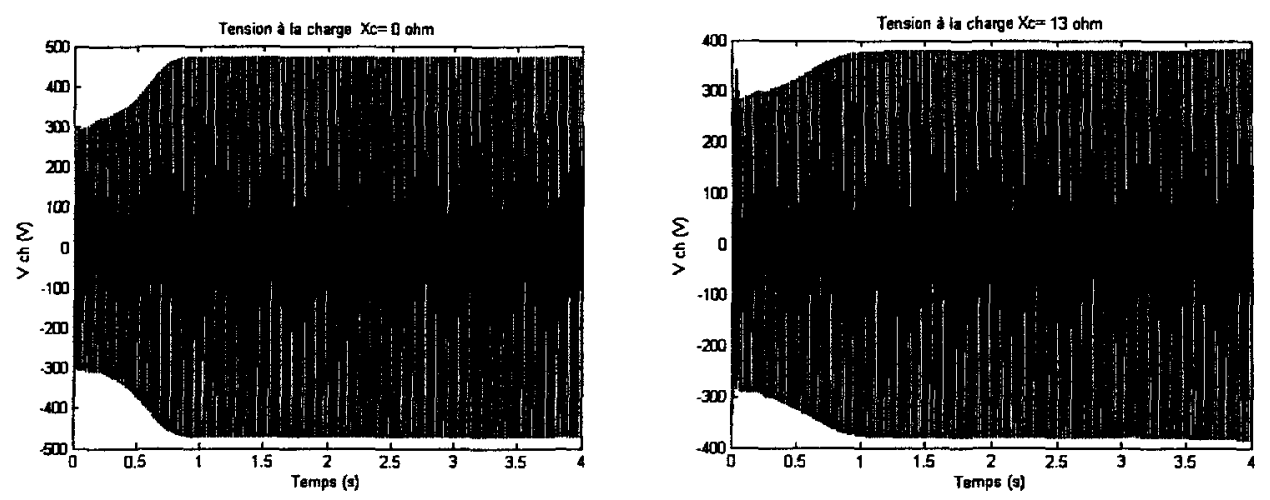

Figure 112: Tension à la charge à $\mathrm{Xc}=0$ et $13 \Omega$
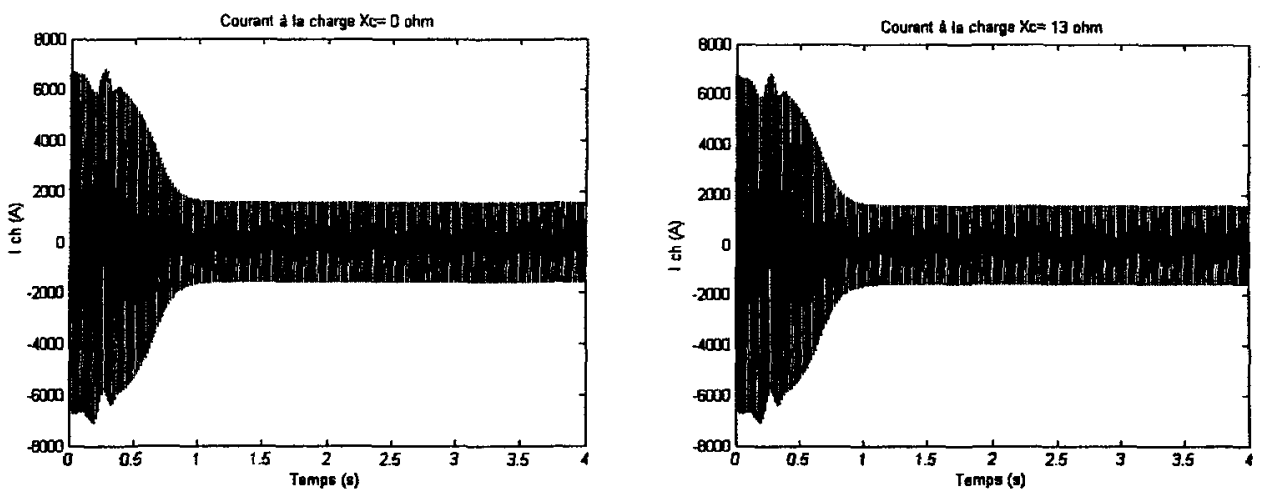

Figure 113 : Courant à la charge à $\mathrm{Xc}=0$ et $13 \Omega$ 

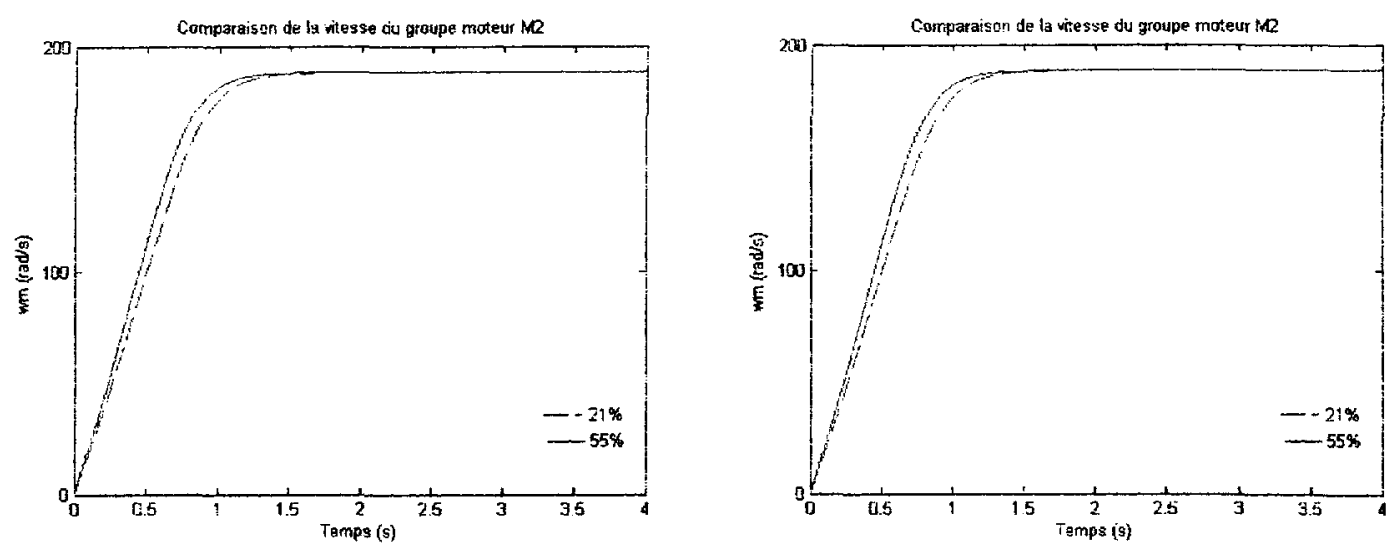

Figure 114 : Comparaison de la vitesse de rotation du groupe moteur M1 et M2 à Xc=0 et $13 \Omega$
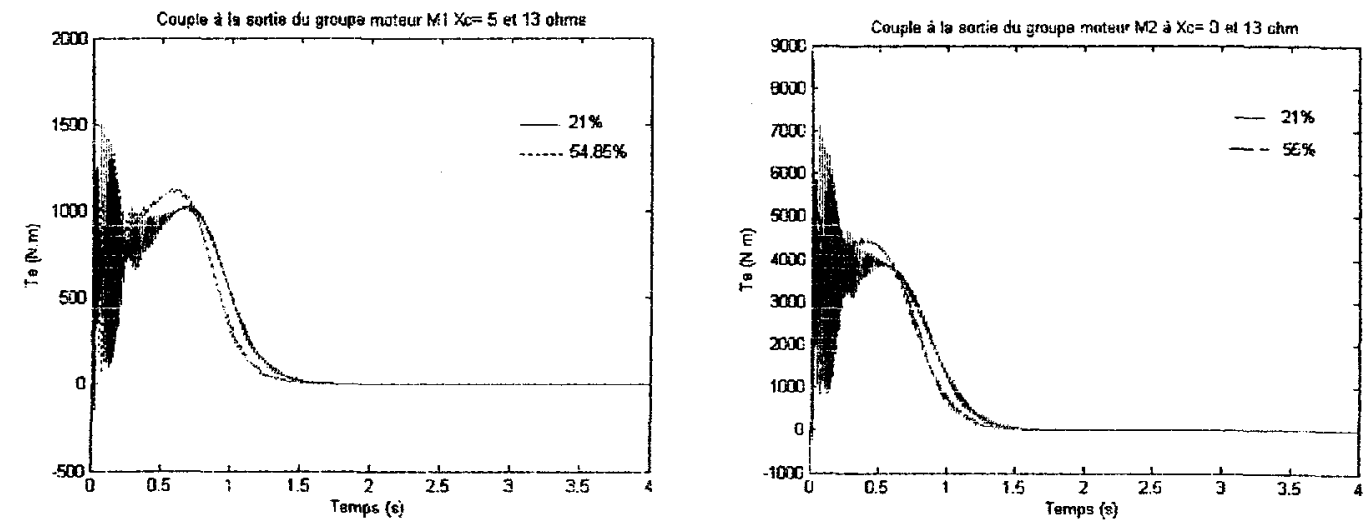

Figure 115 : Comparaison du couple à la sortie du groupe moteur M1 et M2 à Xc=0 et $13 \Omega$

\subsubsection{Conclusions de l'essai de démarrage à vide de moteurs asynchrones}

Il faut signaler que le modèle du moteur à induction utilisé pour faire l'essai de démarrage à vide, est non saturable. Par contre, aux différents taux de compensation série, de forts courants sont nécessaires au démarrage des groupes moteurs en affectant la tension à la charge. Cependant, ce courant se stabilise lorsque le régime permanent est atteint. En général, la compensation série permet d'améliorer la performance de la tension mais n'élimine pas totalement l'effet d'une perturbation majeure. Cependant, ce type de perturbation n'entraîne pas de résonance du réseau. 
L'ajoute de la compensation série améliore le temps de réponse du gros M2 et de petit groupe moteur M1, ainsi que la performance de la vitesse de rotation et les couples mécaniques fournis par les arbres des moteurs.

Le groupe moteur M2, qui est le plus robuste présente moins de distorsion lorsque le réseau est compensé à $\tau=54.85 \%(\mathrm{Xc}=13 \Omega)$. La même performance est observée avant que la tension arrive à sa valeur en régime permanent. 


\section{CONCLUSION GÉNÉRALE}

La méthode d'optimisation développée dans ce mémoire de maitrise intègre une formulation analytique des contraintes de stabilité et des simulations numériques suivant les contingences du réseau ont été effectuées sur Matlab/Sympowersys pour valider les résultats de l'optimisation. On a considéré les différents phénomènes qui affectent les réseaux de distribution radial, spécialement les lignes de grande distance et qui doivent transmettre de l'énergie aux charges résidentielles et industrielles.

Le développement du projet et les résultats obtenus ont été très important pour I'HydroQuebec du au fait que la méthode créé permet de calculer le degré de compensation série optimal sur les lignes de ditribution rurales de l'Abitibi Teminscamingue. Prochainement plusieurs sceries et mines seront construits dans la région. Ces charges sont loin du poste de distribution et pour donner un service de bonne qualité de l'énergie I'HydroQuebec devra compenser les lignes. La méthode proposée dans le projet sera utilisée pour obtenir la valeur optimale de compensation série et la position optimale sur la ligne.

D'ailleurs, Pour le premier essai, l'enclenchement de la ligne avec le transformateur à vide, on a observé que lorsqu'on augmente le degré de compensation série, l'amortissement du courant d'envahissement devient plus lent. Aussi, on a détecté la présence de fortes perturbations du courant et de la tension provoquées par des oscillations amorties.

Pour le cas des simulations de démarrage à vide de moteurs, on a observée que ce procédé demande de forts courants qui produisent une réduction de la tension à la fin de la ligne. Le temps mis par le moteur pour atteindre la valeur nominale de la vitesse de rotation est plus grand lorsqu'on augmente le taux de compensation série. Au niveau des 
oscillations de la tension on peut dire que pendant des fortes oscillations, la compensation n'est pas suffisante pour éviter la déformation de la tension, sauf pour la valeur du taux optimal de compensation.

En ce qui concerne l'essai de variation brusque de la charge mécanique, les moteurs présentent des oscillations sur les tensions et les courants ainsi que l'apparition des oscillations de vitesse du rotor en conduisant le groupe moteur à une valeur de vitesse plus basse que la vitesse nominale. Les phénomènes précédemment posés se produisent pour des valeurs de compensation plus grande que $\tau=55.85 \%$. Cela conduit le réseau sous des conditions d'instabilité. Pour des valeurs de compensation plus petite ou égal à $\tau=55.85 \%$, on remarque que la variation de la charge d'un gros groupe moteur affecte légèrement des grandeurs comme la vitesse et le couple d'autres moteurs.

Pour une contingence de court circuit monophasé, on a noté une légère oscillation lors de l'élimination du défaut en amont du condensateur série. Pour des taux de compensation de $\tau \leq 55.85 \%$, les groupes moteurs présentent une réduction dans leur vitesse qui revient à la valeur nominale une fois le défaut éteint. Pour des conditions de compensation $\tau \geq 55.85 \%$, les moteurs ne reviennent pas à leur valeur de vitesse nominale en évitant de maintenir la stabilité dynamique.

Pour des contingences extrêmes comme les défauts triphasés, on a détecté des oscillations lors du réenclenchement de la ligne. Dans ce cas, le taux de compensation pour lequel le réseau se trouve dans des conditions de stabilité est d'environ $\tau=33.76 \%$ $(\mathrm{Xc}=8 \Omega)$. Pour des valeurs de compensation de $\tau \geq 33.76 \%$, le réseau perdra la stabilité dû fait que la vitesse de rotation des moteurs présente des oscillations permanentes.

Les résultats obtenus par la méthode d'optimisation donne une valeur minimale qui est calculé à partir d'un procède mathématique basé sur la puissance de court circuit minimal que le réseau pourrait supporter. Par rapport à la valeur maximale de compensation, on a développé des simulations numériques pour évaluer la stabilité du réseau aux différents taux de compensation série. Cette analyse nous permet de conclure que la stabilité 
dynamique et transitoire du réseau est $\tau=55.85 \% \quad\left(\mathrm{Xc}_{\mathrm{c}}=13 \Omega\right)$. Toutefois, sous de conditions de défaut triphasé, le réseau trouve leur condition de stabilité lorsqu'on compense à $\tau=33.76 \%(\mathrm{Xc}=8 \Omega)$. Il faut signaler que le banc de condensateur série contient un dispositif de protection qui permet d'isoler les condensateurs lors des conditions de court circuit monophasé et triphasé. On a observé la performance de la tension à la charge pendant des contingences de cour circuit et de variation brusque de charge. Pour $\mathrm{Xc}=5$ et $13 \Omega$, le réseau éprouve une réduction brusque dans leur grandeur lorsque un court circuit se produit en amont du condensateur. Pour le cas de variation de charge, le profil de la tension à la charge est affecté dans la grandeur en présentant une réduction de tension.

On a également observe que la performance de la vitesse de rotation du groupe moteur de grande et petite demande de puissance. Pour des valeurs de réactance $\mathrm{Xc}=5$ et $13 \Omega$, les deux types de moteur réduisent la vitesse de rotation mais lorsque le défaut est éteint leur vitesse revient à la vitesse nominale en maintenant la stabilité en régime permanent. Pour l'essai de variation brusque de charge, le couple du groupe moteur M2 a été doublé pour cette raison, la vitesse se réduit brusquement grâce à l'effort qui doit faire le moteur pour maintenir le couple nominal. La variation de couple du groupe moteur M2 affecte directement la vitesse de rotation du groupe moteur M1.

Différents technologies pour éliminer la résonance sous-synchrone on été exposée dans la mémoire de maitrise ainsi qu'un protocole de démarrage séquentiel des plusieurs groupes de moteurs pour permettre une meilleur contrôle de la résonance sous-synchrone et une réduction de la demande de puissance des charges industrielles.

En ce qui concerne les perspectives du projet de maîtrise, la méthode d'optimisation peut être utilisée dans un réseau à plusieurs lignes de distribution ainsi qu'elle se pourra $s$ 'appliquer aux lignes de transmission de l'énergie. Une étude des pertes réactives dans le réseau sera nécessaire pour obtenir les pertes de la puissance réactive à chaque ligne. Cela permettra d'identifier les cas les plus critiques et ainsi appliquer la méthode d'optimisation pour obtenir la valeur de compensation série et la position. 


\section{REFERENCES}

Miske S.A. April 2001 "Considerations for the Application of Series Capacitors to Radial Power distribution Circuits". IEEE Transactions on Power Delivery.

René Wamkeue, Nahi Kandil, Jacques East, Yves Boisclair. 9-11 april 2003 Series Compensation For a Hydro-Quebec Long Distribution Line. ICREPQ'03 International Conference on Renewable Energies and Power Quality, Vigo, Espagne.

D.Povh, R. Mihalic, 1990."Enhacement of Transient Stability on AC Transmission by means of controlled Series and Parallel Compensation. Siemens AC.

R.A. Barr, D. Platt. January 1997. Use of a Saturing choke in the Series Capacitor Compensation of distribution Lines. IEE Proceeding Generation, Transmission and Distribution. Vol144, N.1.

F.J. Rossow 2001.“Compensator Rating for Radial Distribution Lines”. IEEE Transactions on Power Systems.

Dequang Gan, Robert J. Thomas, Ray D Zimmerman August 24-28. “A Transient Stability Constrained Optimal Power Flow. Bulk Power Dynamics and Control IVRestructuring". Greece.

A.A. Johnson 1984. "Application of Capacitors to Power Systems". Westing House HandBook.

Kaoru Inoue, Toshimitsu Ushio, Takashi Hikimira 1999. "bifurcation and Huning Phenomena of Rotating Speed in a Flexible Rotor System caused by Whirling Motion. International Journal of bifurcation and Chaos. Vol 9, N..8. 
Sebastiaio Oliveira., Istvan Gardos, august 1991. "Representation of Series Capacitors in Electrical Power System Stability Studies". IEEE Transactions on Power Systems, vol 6, N.3.

Damir Novosel, Arun Phadke, Murari Mohan Saha.. March 25-27 1997 . "Problems and Solutions for Microprocessor of Series Compensated". Publication N 434, IEE Conference in Power System Protection,

Jacques East janvier. 1997. Utilisation des condensateurs série sur le réseau de distribution. HydroQuebec. Rouyn Noranda..

René Wierda december. 1995. Cahier technique N. 176. Flicker Phenomenon. Merlin Gerin.

Benoitz de Mertz-Noblatz, Gérard JeanJean janvier. 1997. Cahier Technique N. 185. Stabilité dynamique des réseaux électriques industriels. Merlin Gerin.

Ayshan A. Mutlu, Elham B. Makran. 1998. "Analysis of Series Compensation From different aspects". IEEE Transactions on Power Systems.

N. Christi, Sadek Lützelberger. November. 1991. "System Studies and Basic Design for an Advanced Series Compensation Scheme (ASC)". IEE Int. Conference on Advances in Power System Control, Operation and Management, Honk Kong.

Xiao Wang, Shu-Zu Dai, Boon-Teck Ooi. 1991. A Series Reactance Compensator Based on Voltage-Source PWM Converter. IEEE Transactions on Power Systems.

Nadia Yousif, Majid Al-Dabbagh. May 2001. “ Subsynchronous Resonance Damping in Interconnected Power Systems. IEEE Transactions on Power Systems. 
Abdel-Aty Edris. February 1990. "Series Compensation Schemes Reducing the Potential of SSR". IEEE Transaction on Power Systems, vol 5.

R. Gagnon, P. Vairouge, G. Sybille. 1995. Étude de la Ferrorésonance dans un Réseau compensé Série. IEEE Transactions on Power Delivery.

Ronald A. Hedin, Stephen Weiss, Duane Torgeson, Eilts L. E. May 1995. "SSR Characteristics of Alternative Types of Series Compensation Schemes". IEEE Transactions on Power System, Vol 10. N.2.

Larry Morgan, James M.Barcus, Satoru Ihara. July 1993. "Distribution Series Capacitor with High Energy Varistor Protection”. IEEE.Transactions on Power Delivery. Vol 8, N.3.

S. sugimoto, J. Kida, H. Arita, C. Fukui, T. Yamagiwa. April 1996. Principle and Characteristics of a Fault Current Limiter with Series Compensation. IEEE Transactions on Power Delivery, Vol N. 2.

Jean-Christophe Culioli. 1994. Introduction à l'Optimisation. Ellipses. Paris France.

J. Duncan Glover, Mulukutla Sarma. 1994. Power System Analysis and Design. Second Edition. PWS Publishing Company.

John J. Grainger, William D. Stevenson Jr. 1996. Power Systems Analysis. McGrawHill, USA.

L.L. Grigsby. 2001 The Electric Power Engineering Handbook. IEEE Press.

Westinghouse. 1964 Electrical Transmission and Distribution References Book. Westinghouse Central Station Engineers, east Pittsburgh, Penn. 
Richard Marceau. 1993. Chapitre 6, Exploitation d'un réseau électrique. Département de génie électrique et génie informatique.

Hydroquebec. May 1981. Notions et Règles du phénomène de papillotement. Planification et conduite du réseau. Direction distribution.

Hydroquebec. 2000. MINIAQO-MINICHART- Explication du manuel de l'utilisateur de l'analyseur de la qualité de l'onde MiniAQO. Mario Tremblay.

CEI 61000-4-15. Novembre 1997. Compatibilité electromagnetique 9CEM0 Techniques d'essai et de mesure-Section 15: Flickemetre-Specifications fonctionelles et de la conception. 


\section{APPENDICES}

A. Données du réseau électrique de distribution radial et des groupes moteurs.

B. Normes des tensions recommandées pour les réseaux à courant alternatif de 0 à $1000 \mathrm{~V}$. Association canadienne de normalisation, CAN3-C235-83.......150

C. Calcul du transfert maximal de puissance. Jacques East, Ing. HydroQuébec......152

D. Paramètres d'équipement auxiliaire du banc de condensateur série................158

E. Étude de papillotement. Normes C.22.1 et C.22.2. Hydro- Québec.................162

F. Description de logiciel MINIAQO et méthode de mesure du phénomène de papillotement obtenu par Hydro-Québec dans la scierie contois...................166

G. Étude de réponse en fréquence. Power System Blockset/Simulink ${ }^{\circledR}$..............180

H. Programme développé dans le logiciel Matlab ${ }^{\circledR}$. Calcul du taux de compensation série optimal et sa position.....................................182

I. Profils de tension en amont et en aval du condensateur série...................185

J. Simulations effectuées dans Power System Blockset/Simulink ${ }^{\circledR}$.................187

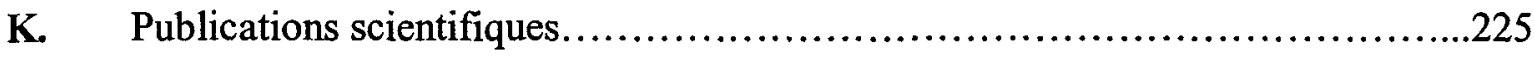




\section{APPENDICE A}

Données du réseau électrique de distribution de distribution radial et des groupes moteurs 


\section{Diagramme électrique du réseau de distribution radial.}

Transformateur distribution en parallel D-Y. $120 \mathrm{kV} / 26.4 \mathrm{kV}, 13.5 / 18 / 22.5 \mathrm{MVA}$

$\mathrm{R}_{\mathrm{tl}}=0.0185, \mathrm{X}_{\mathrm{tl}}=0.345$,

$\mathrm{Rm}=1150 \mathrm{pu}, \mathrm{Ym}=700 \mathrm{pu}$ at $\mathrm{V}_{1}=1.05 \mathrm{pu}$

Charge R, L: IMVA

Transformateur charge $\mathrm{Y}-\mathrm{Y}$ $25 \mathrm{kV} / 600 \mathrm{~V}, 833.33 \mathrm{KVA}$ $\mathrm{R}_{\mathrm{t} 2}=0.002 \mathrm{pu} \mathrm{X}_{\mathrm{t} 2}=0.08 \mathrm{pu}$

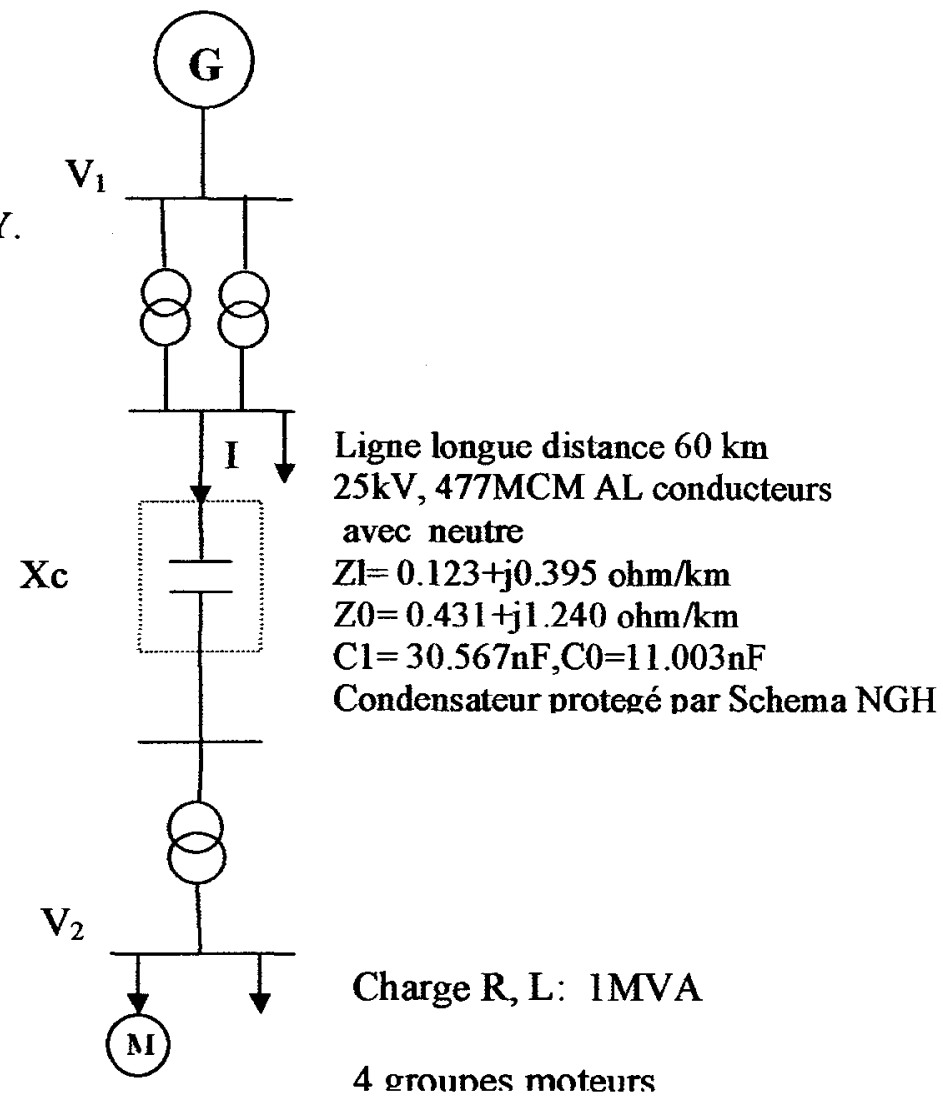

Figure A.1 Diagramme unifilaire du réseau de distribution radial 


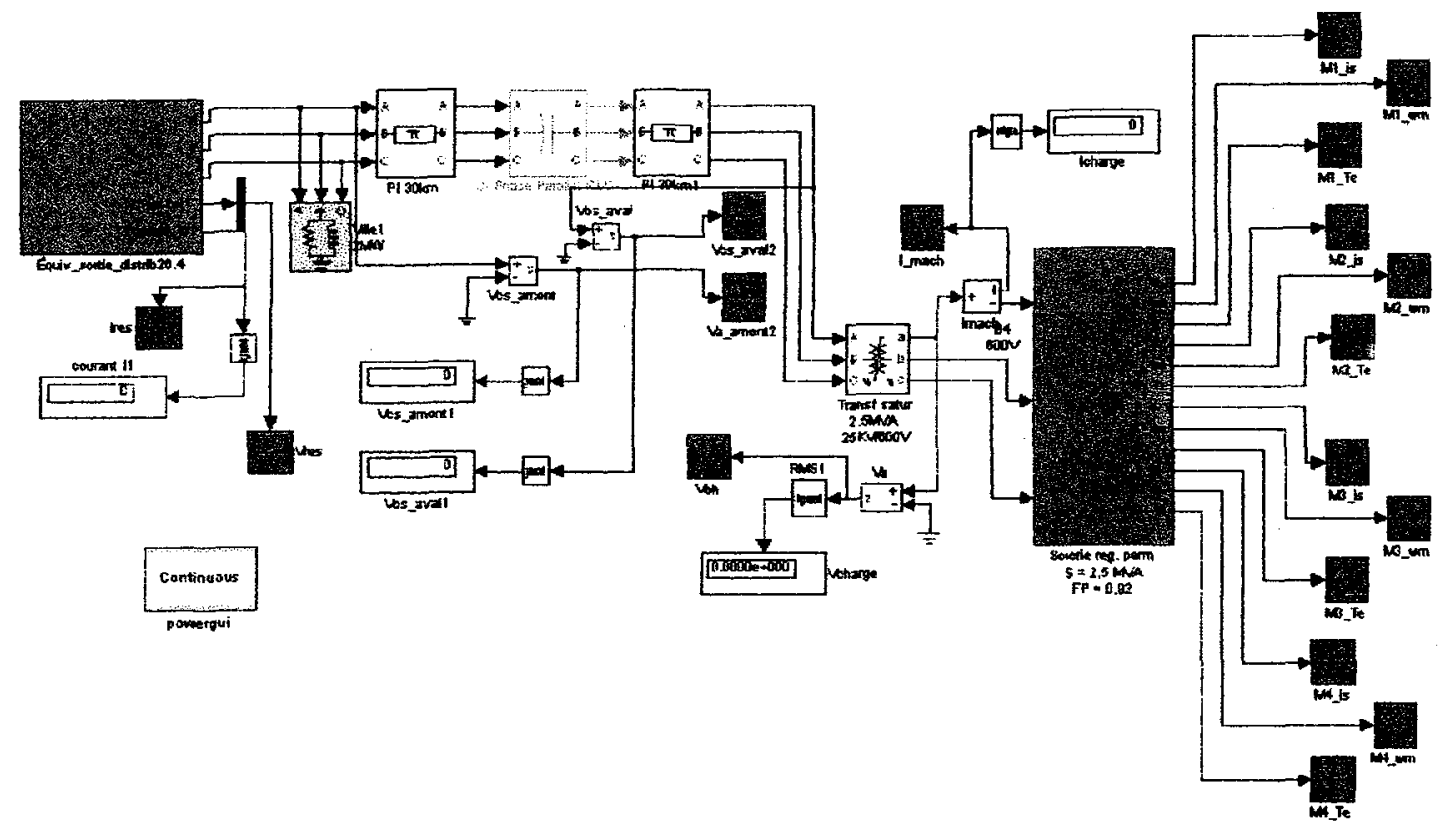

Figure A.2 Diagramme équivalent du réseau de distribution en SimPowerSystems-Simulink

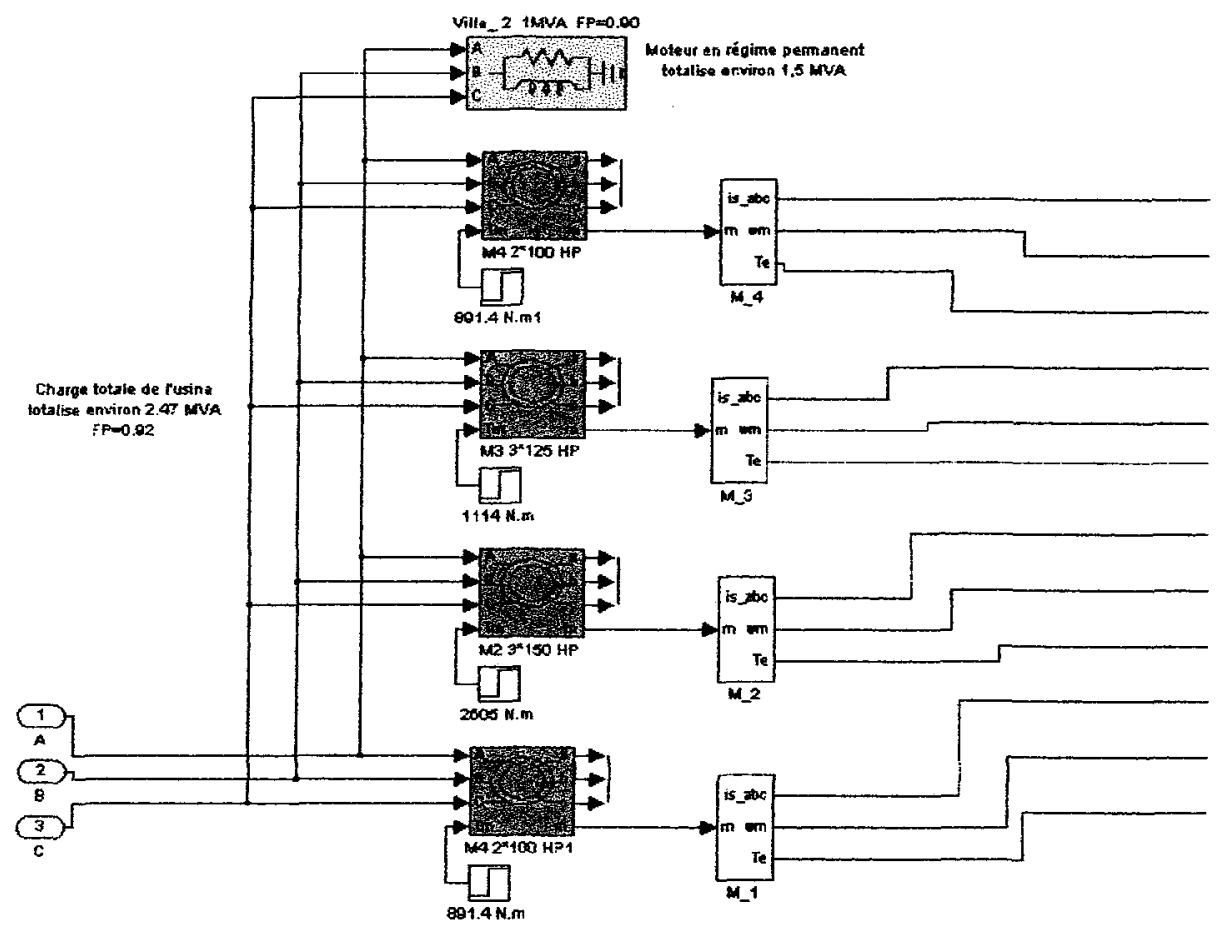

Figure A.3 Modèle numérique Matlab/Simulink du bloc des groupes moteurs M1, M2, M3 et M4 


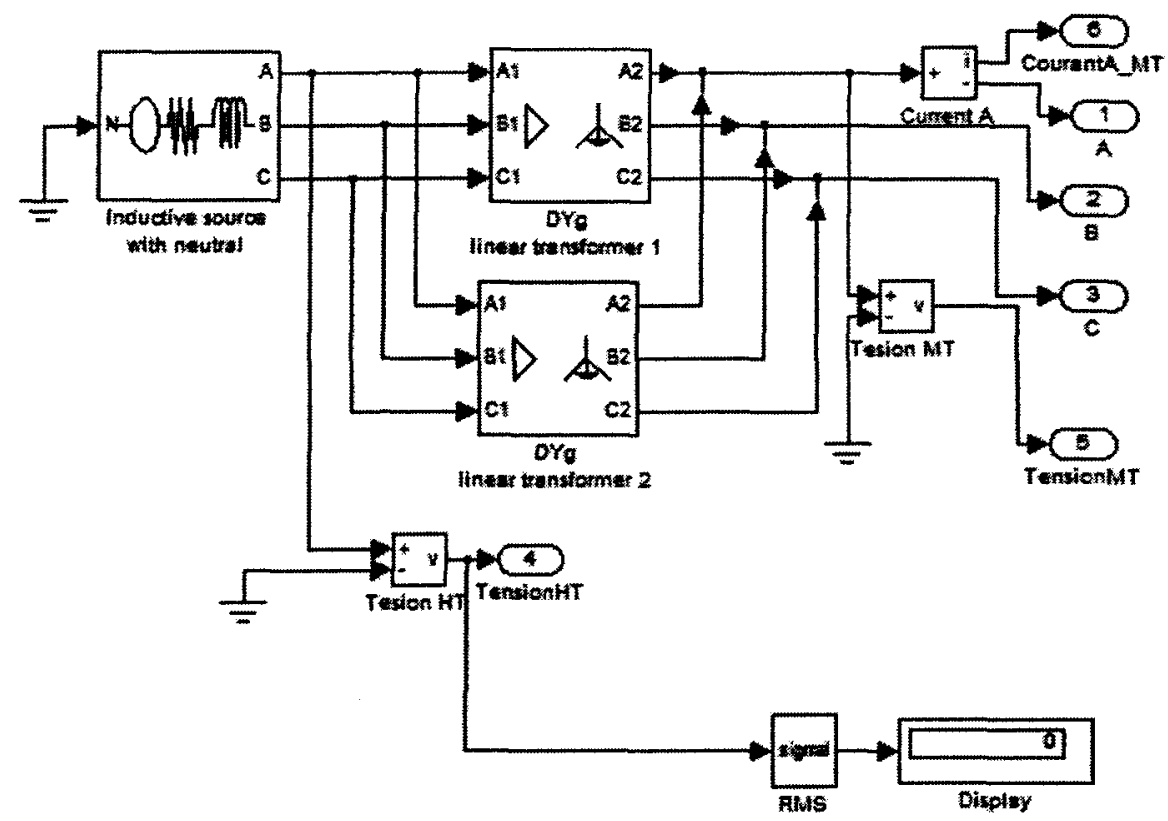

Figure A.4 Modèle numérique Matlab/Simulink du bloc de la source en SimPowerSystems 


\section{Tableau A.1}

Paramètres de la source équivalent thevenin

Circuit équivalente thevenin

\begin{tabular}{|l|c|c|}
\hline \multicolumn{2}{|l|}{ p.u. } & réel \\
\hline Tension & 1,05 & $126 \mathrm{kV}$ \\
\hline Impédance & $0,03+\mathrm{j} 0,2$ & $4,32 \mathrm{ohm}$ \\
& & $76,39 \mathrm{mH}$ \\
\hline
\end{tabular}

p.u. sur base 100MVA, $120 \mathrm{kV}$

Tableau A.2

Paramètres des transformateurs de distribution :

\begin{tabular}{|l|c|}
\hline \multicolumn{1}{|c|}{ Paramètre } & Valeur \\
\hline Capacité du transformateur (MVA) & $15,5 / 18 / 22,5$ \\
\hline Z1(p.u.) sur base 100 MVA & $0,037+j 0,69$ \\
\hline Z0(p.u.) sur base 100 MVA & $0,037+j 0,69$ \\
\hline Admittances à 1.0 p.u. de Vnom sur base 100 MVA & 0,001 p.u. \\
\hline Admittance à 1.05 p.u. de Vnom sur base 100 MVA & 0,0014 p.u. \\
\hline
\end{tabular}

\section{Impedance et admitance du transformateur en p.u.}

$$
\mathrm{Z}_{\mathrm{pu}(22.5 \mathrm{MVA})}=\mathrm{Z}_{\mathrm{pu}} \cdot \frac{22.5 M V A}{100 M V A} \quad \text { (1) } \quad \mathrm{Y}_{\mathrm{pu}(22.5 \mathrm{MVA})}=\mathrm{Y}_{\mathrm{pu}} \cdot \frac{100 M V A}{22.5 M V A}
$$

Tableau A.3

Modèle PI d'une ligne de distribution

\begin{tabular}{|l|c|}
\hline \multicolumn{1}{|c|}{ Paramètre } & Valeur \\
\hline Longueur & $60 \mathrm{kms}$ \\
\hline Type de conducteur & $\begin{array}{c}\text { Phase }: 477 \mathrm{AL} \\
\text { Neutre }: 2 / 0 \mathrm{ACSR}\end{array}$ \\
\hline $\mathrm{Z1}$ (courant faible) & $0.123+\mathrm{j} 395 \Omega$ \\
\hline Z0 (courant faible) & $0.431+\mathrm{j} 1.24 \Omega$ \\
\hline Z1 (courant fort) & $0.134+\mathrm{j} 0.395 \Omega$ \\
\hline Z0 (courant fort) & $0.443+\mathrm{j} 1.240 \Omega$ \\
\hline
\end{tabular}


Ligne de distribution

$$
\mathrm{Z}=\mathrm{R}+\mathrm{j} \mathrm{X}_{\mathrm{L}}=[0.123+\mathrm{j} 0.395 \Omega / \mathrm{km}] .60 \mathrm{kms}=7.38+\mathrm{j} 23.7 \Omega
$$

\section{Tableau A.4}

Paramètres de la ligne de distribution

\begin{tabular}{|l|c|}
\hline Paramètre & Valeur \\
\hline Longueur & $60 \mathrm{kms}$ \\
\hline Fréquence & $60 \mathrm{~Hz}$ \\
\hline R1 (ohm/km) & 0,123 \\
\hline R0 (ohm $/ \mathrm{km})$ & 0,431 \\
\hline L1 $(\mathrm{h} / \mathrm{km})$ & $1,05 \mathrm{E}-03$ \\
\hline LO $(\mathrm{ohm} / \mathrm{km})$ & $3,29 \mathrm{E}-03$ \\
\hline$C 1(\mathrm{~F} / \mathrm{km})$ & $3,06 \mathrm{E}-08$ \\
\hline$C 0(\mathrm{~F} / \mathrm{km})$ & $1,10 E-08$ \\
\hline
\end{tabular}

Paramètres modèle PI de la ligne

\section{Condensateur série}

$$
\mathrm{C}=\frac{1}{2 \pi \cdot f \cdot X_{C}}
$$


Transformateur à la charge

Tableau A.5

Paramètres du transformateur saturable

\begin{tabular}{|c|c|}
\hline Paramètre & Valeur \\
\hline Puissance apparente triphasée & 833,33KVA \\
\hline Fréquence & $60 \mathrm{~Hz}$ \\
\hline Primaire & 0,123 \\
\hline Tension ligne ligne & 0,431 \\
\hline Rp.u. & $1,05 \mathrm{E}-03$ \\
\hline Xp.u. & $3,29 \mathrm{E}-03$ \\
\hline Secondaire & $3,06 \mathrm{E}-08$ \\
\hline Tension ligne ligne & $1,10 \mathrm{E}-08$ \\
\hline R p.u. & $64,00 \mathrm{E}-03$ \\
\hline xp.u. & $1,00 E-03$ \\
\hline \multicolumn{2}{|l|}{ Banc de magnétisation } \\
\hline Rm p.u. & $2,70 \mathrm{E}+02$ \\
\hline $\begin{array}{l}\text { Xm p.u. } \\
\text { (courbe de magnétisation } \\
\text { [I p.u. Flux p.u.] }\end{array}$ & 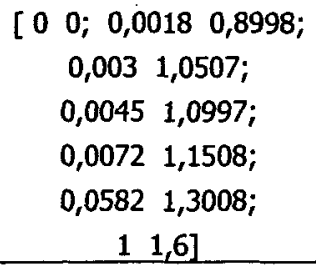 \\
\hline
\end{tabular}

Tableau A.6

Données plaque signalétique du transformateur de distribution

\begin{tabular}{|c|c|c|c|c|}
\hline \multicolumn{2}{|c|}{ PUISSANCE } & $2500 \mathrm{KVA}$ & & \\
\hline \multicolumn{2}{|l|}{ PHASE } & 3 & & \\
\hline \multicolumn{2}{|c|}{ FREQUENCE } & $60 \mathrm{~Hz}$ & & \\
\hline \multicolumn{2}{|c|}{ IMPEDANCE À $85 \mathrm{c}$} & 4,9 & & \\
\hline \multicolumn{2}{|c|}{ NIVEAU D'ISOLEMENT } & $425 \mathrm{kV}$ & & \\
\hline \multicolumn{2}{|c|}{ Haute Tension } & $24940 \mathrm{~V} / 14400 \mathrm{~V}$ & & \\
\hline \multicolumn{2}{|c|}{ Basse Tension } & $600 \mathrm{~V} Y / 347 \mathrm{~V}$ & & \\
\hline \multicolumn{2}{|c|}{ PRESSION POS. } & $69 \mathrm{Kpa}$ & & \\
\hline \multicolumn{5}{|c|}{ ATTENTION: SP(OPERER HOR TENSION SEUL) } \\
\hline $105 \%$ & $102,50 \%$ & $100 \%$ & $97,50 \%$ & $95 \%$ \\
\hline A & $\mathrm{B}$ & $\mathrm{C}$ & D & $E$ \\
\hline \multirow{3}{*}{$\begin{array}{l}\text { FUSIBLE } \\
\text { LIMITEUR } \\
\text { (FLC) }\end{array}$} & FABRIQUE & General Elect. & & \\
\hline & TENSION & $15.5 \mathrm{kV}$ & & \\
\hline & AMPERES & $125 \mathrm{~A}$ & & \\
\hline
\end{tabular}


Tableau A.7

Calcule de la résistance des enroulements

\begin{tabular}{|c|c|c|c|c|c|}
\hline \multicolumn{2}{|c|}{ METHODE D'ESSAI } & ENROULEMENT & $\mathrm{H} 1-\mathrm{HO}$ & $\mathrm{H} 2-\mathrm{HO}$ & $\mathrm{H} 3-\mathrm{HO}$ \\
\hline \multirow{7}{*}{\multicolumn{2}{|c|}{ Multi Amp. 830280}} & 26187 & 0,534 & 0,536 & 0,535 \\
\hline & & 25563 & 0,523 & 0,525 & 0,522 \\
\hline & & 24940 & 0,512 & 0,512 & 0,511 \\
\hline & & 24316 & 0,498 & 0,499 & 0,498 \\
\hline & & 23693 & 0,487 & 0,485 & 0,486 \\
\hline & & & $\times 1-\times 0$ & $\times 2-x 0$ & $\times 3-\times 0$ \\
\hline & & 600 & 0,000252 & 0,000241 & 0,000236 \\
\hline \multicolumn{6}{|l|}{ Rapport } \\
\hline \multicolumn{2}{|c|}{ CALCULE } & \multicolumn{4}{|c|}{ MESURES } \\
\hline Haute tension & Basse tension & rapport & $\begin{array}{c}\mathrm{H} 1 \mathrm{H} 0-\mathrm{X} 1 \\
\times 0 \\
\end{array}$ & $\begin{array}{c}\mathrm{H} 2 \mathrm{H} 0-\mathrm{X} 2 \\
\times 0 \\
\end{array}$ & $\begin{array}{c}\mathrm{H} 3 \mathrm{HO}-\mathrm{X} 3 \\
\times 0 \\
\end{array}$ \\
\hline 26187 & 600 & 43,645 & 43,604 & 43,608 & 43,608 \\
\hline 25563 & 600 & 42,605 & 42,6 & 42,603 & 42,603 \\
\hline 24940 & 600 & 41,566 & 41,598 & 41,607 & 41,607 \\
\hline 24316 & 600 & 40,529 & 40,501 & 40,506 & 40,506 \\
\hline 23693 & 600 & 39,488 & 39,497 & 39,502 & 30,506 \\
\hline
\end{tabular}

Tableau A.8

Courant d'excitation et pertes à vide

\begin{tabular}{|c|c|c|c|c|c|c|c|}
\hline \multirow{2}{*}{ APPLIQUÉ } & \multicolumn{2}{|c|}{ TENSION } & COURANT & \multicolumn{2}{c|}{ PERTES DANS LE NOYAU EN WATTS } \\
\cline { 2 - 4 } & Mult & Total & Total & W1 & W2 & W3 & $\begin{array}{c}\text { Tension } \\
\text { primaire RMS }\end{array}$ \\
\hline X1-X2 & RMS & Moyen & RMS & (W) & & (VAR) & (VR) \\
\hline 86,75 & 86 & 86 & 1,003 & 200 & & 170 & 358 \\
\hline 260,25 & 260 & 261 & 2,508 & 1660 & & 1060 & 10880 \\
\hline 312,3 & 312 & 312 & 3,344 & 2490 & & 1910 & 13180 \\
\hline 347 & 347 & 347 & 5,165 & 3310 & & 4230 & 14643 \\
\hline 364,35 & 364 & 363 & 8,584 & 3900 & & 8500 & 15320 \\
\hline 381,7 & 381 & 379 & 20,95 & 4900 & & 23000 & 16090 \\
\hline 399,05 & 399 & 393 & 53,11 & 6700 & & 62200 & 16806 \\
\hline
\end{tabular}


Tableau A.9

Paramètres électriques des groupes moteurs M1, M2, M3 ET M4

\begin{tabular}{|c|c|c|c|c|}
\hline & $\begin{array}{c}\text { Groupe } \\
\text { Moteur 1 } \\
2 \times 200 \mathrm{HP}\end{array}$ & $\begin{array}{c}\text { Groupe } \\
\text { Moteur 2 } \\
3 \times 150 \mathrm{HP}\end{array}$ & $\begin{array}{c}\text { Groupe } \\
\text { Moteur 3 } \\
3 \times 125 \mathrm{HP}\end{array}$ & $\begin{array}{c}\text { Groupe } \\
\text { Moteur 4 } \\
3 \times 200 \mathrm{HP}\end{array}$ \\
\hline Puissance (HP) & $200 \mathrm{HP}$ & $450 \mathrm{HP}$ & $375 \mathrm{HP}$ & $200 \mathrm{HP}$ \\
\hline $\mathrm{Te}(\mathrm{N} . \mathrm{m})$ & 891.7 & 2605 & 1114 & 891.7 \\
\hline $\mathrm{Rs}(\Omega)$ & 0.08675 & 0.0214 & 0.0694 & 0.08675 \\
\hline $\mathrm{X}_{\mathrm{ls}}(\Omega)$ & 0.2 & 0.0284 & 0.16 & 0.2 \\
\hline $\mathrm{X}_{\mathrm{m}}(\Omega)$ & 6.795 & 1.938 & 5.435 & 6.795 \\
\hline $\mathrm{X}_{\mathrm{lr}}(\Omega)$ & 0.0685 & 0.0277 & 0.0548 & 0.0685 \\
\hline $\mathrm{R}_{\mathrm{r}^{\prime}}(\Omega)$ & 0.1645 & 0.04815 & 0.1316 & 0.1645 \\
\hline $\mathrm{J}\left(\mathrm{kg} \cdot \mathrm{m}^{2}\right)$ & 4.93 & 18.6 & 6.16 & 4.93 \\
\hline Paire de pôles & 2 & 2 & 2 & 2 \\
\hline Friction & 0 & 0 & 0 & 0 \\
\hline
\end{tabular}




\section{APPENDICE B}

Normes des tensions recommandées pour les réseaux à courant alternatif de 0 à $1000 \mathrm{~V}$. Association canadienne de normalisation, CAN3-C235-83 
Tableau A. 10

Limites recommandées pour les variations de tension aux points d'utilisation pour des circuits allant jusqu'à $1000 \mathrm{~V}$

\begin{tabular}{|c|c|c|c|c|}
\hline \multirow{3}{*}{$\begin{array}{c}\text { Tensions } \\
\text { nominales }\end{array}$} & \multicolumn{4}{|c|}{$\begin{array}{l}\text { Limites de variations de tension } \\
\text { applicables au point d'utilisation }\end{array}$} \\
\hline & \multicolumn{4}{|c|}{ Conditions marginales d'explotation } \\
\hline & & \multicolumn{2}{|c|}{ Conditions normales d'explotation } & \\
\hline $\begin{array}{c}\text { Monophasé } \\
120 / 240 \\
240 \\
480 \\
600\end{array}$ & $\begin{array}{c}104 / 208 \\
208 \\
416 \\
520\end{array}$ & $\begin{array}{c}108 / 216 \\
216 \\
432 \\
540\end{array}$ & $\begin{array}{c}125 / 250 \\
250 \\
500 \\
625\end{array}$ & $\begin{array}{c}127 / 254 \\
254 \\
508 \\
635\end{array}$ \\
\hline Triphasé & & & & \\
\hline $\begin{array}{l}4 \text { conducteurs } \\
120 / 208 Y\end{array}$ & $108 / 187$ & $110 / 190$ & $125 / 216$ & $127 / 220$ \\
\hline $240 / 416 Y$ & $216 / 374$ & $220 / 380$ & $250 / 432$ & $254 / 440$ \\
\hline $277 / 480 Y$ & $240 / 416$ & $250 / 432$ & $288 / 500$ & $293 / 508$ \\
\hline $\begin{array}{l}347 / 600 Y \\
\text { Triphasé }\end{array}$ & $300 / 520$ & $312 / 540$ & $360 / 625$ & $367 / 635$ \\
\hline 3 conducteurs & & & & \\
\hline 240 & 208 & 216 & 250 & 254 \\
\hline 480 & 416 & 432 & 500 & 508 \\
\hline 600 & 520 & 540 & 625 & 635 \\
\hline
\end{tabular}




\section{APPENDICE C}

Calcul du transfert maximal de puissance. Jacques East, Ing. HydroQuébec. 
a) Contrainte de stabilité : Transfert maximal de puissance

La contrainte sur la puissance réactive $Q_{1}$ est le facteur de puissance à la charge :

$$
\begin{gathered}
k=\cos ^{-1} F P \\
Q_{1}=P_{1} \tan k
\end{gathered}
$$

Le courant sur la ligne $I$ est utilisé pour calculer la puissance active et réactive :

$$
\begin{gathered}
I=\frac{V_{1}-V_{2}}{Z} \\
P+\mathrm{j} Q=V . I \cos \phi+\mathrm{j} V \sin \phi \quad\left(V \text { est la tension entre } V_{1} \text { et } V_{2}\right)
\end{gathered}
$$

Alors on utilise les équations des tensions de la source et de la charge (9) et (10) dans l'équation (11) pour trouver (13).

$$
\begin{gathered}
V_{1}=\overline{V_{1}}(\cos \delta+j \sin \delta) \\
V_{2}=\overline{V_{2}}(\cos \theta+j \sin \delta) \\
P_{1}+\mathrm{j} Q_{1}=\frac{-V_{2} \cdot V_{2}+V_{1} \cdot V_{2} \cdot e^{-j \theta}}{Z} \\
P_{1}+\mathrm{j} Q_{1}=\frac{V_{1} \cdot V_{2} \cdot e^{-j \theta}}{Z}-\frac{V_{2}^{2}}{Z} \\
P_{1}+\mathrm{j} Q_{1}=\frac{V_{1} \cdot V_{2} \cdot \cos (\delta-\theta)}{Z}-\frac{V_{2}^{2} \cdot \cos (\theta-\theta)}{Z}
\end{gathered}
$$


En considérant l'équation (6), nous obtenons les équations de $P_{1}$ et $Q_{1}$ en développant l'équation (13) comme suit :

$$
\begin{gathered}
P_{I}=\frac{V_{1} V_{2}}{Z} \cos (\delta-\theta)-\frac{V_{2}{ }^{2}}{Z} \cos \delta \\
P_{1} \tan k=\frac{V_{1} V_{2}}{Z} \sin (\delta-\theta)-\frac{V_{2}^{2}}{Z} \sin \delta
\end{gathered}
$$

En partant de la relation trigonométrique $\cos ^{2} x+\sin ^{2} x=1$, on formule les équations $P_{1}$ et $P_{2}$ en considérant que $P_{1}$ devient comme la puissance maximale $P_{\max }$ et en éliminant l'angle $\theta$ on obtient l'expression résultant suivante:

$$
\left(P_{\max }+\frac{V_{2}^{2}}{Z} \cos \delta\right)^{2}+\left(P_{\max } \tan x+\frac{V_{2}^{2}}{Z} \sin \delta\right)^{2}=\left(\frac{V_{1} V_{2}}{Z}\right)^{2}
$$

En développant,

$P_{\max }{ }^{2}+2 \frac{P_{\max } V_{2}^{2}}{Z} \cos \delta+\frac{V_{2}^{4} \cos ^{2} \delta}{Z^{2}}+P_{\max }{ }^{2} \tan ^{2} x+2 \frac{P_{\max } \tan x V_{2}^{2} \sin \delta}{Z}+\frac{V_{2}^{4} \sin ^{2} \delta}{Z^{2}}=\frac{V_{1} V_{2}}{Z^{2}}$

Pour simplifier l'expression ci-dessus, on utilise l'identité trigonométrique

$$
\begin{gathered}
\sec ^{2} k=I+\tan ^{2} k \\
P_{\max } \sec ^{2} x+P_{\max }\left[\frac{2 V_{2}(\cos \delta+\tan x \sin \delta)}{Z}\right]+\left[\frac{V_{2}^{4}-V_{1}^{2} V_{2}^{2}}{Z^{2}}\right]=0
\end{gathered}
$$

Alors la formulation résultant est une expression quadratique que peut se résoudre comme une équation quadratique de deuxième ordre en utilisant l'expression (20) : 


$$
\begin{gathered}
P_{\max }=\frac{-b \pm \sqrt{b^{2}-4 a c}}{2 a} \\
\mathrm{P}_{\max }=\frac{\frac{-2 V_{2}^{2}(\cos \delta+\tan x \sin \delta)}{Z} \pm \sqrt{\frac{4 V_{2}^{4}(\cos \delta+\tan x \sin \delta)^{2}}{Z^{2}}-\frac{4 \cos ^{2} x\left(V_{2}{ }^{4}-V_{1}{ }^{2} V_{2}{ }^{2}\right)}{Z^{2}}}}{2 \sec ^{2} x}
\end{gathered}
$$

L'identité trigonométrique $\sec ^{2} \mathrm{x}=\frac{1}{\cos ^{2} x}$ réduit l'expression de $P_{m a x}$ comme l'équation (22) :

$$
\begin{gathered}
=\frac{\cos ^{2} x\left(-V_{2}^{2}(\cos \delta-\tan x \sin \delta) \pm \sqrt{V_{2}^{{ }^{2}}(\cos \delta+\tan x \sin \delta)^{2}-\sec ^{2} x\left(V_{2}^{4}-V_{1}^{2} V_{2}^{2}\right)}\right)}{Z} \\
P_{\max }=\frac{\left.\cos ^{2} x\left(-V_{2}^{2}(\cos \delta-\tan x \sin \delta)\right) \pm \cos x \sqrt{r_{2}^{2}(\cos \delta \cos x+\tan x \cos x \sin \delta)^{2}-\left(V_{2}^{4}-V_{1}^{2} V_{2}^{2}\right)}\right)}{Z}
\end{gathered}
$$

En développant $P_{\max }$ et en considérant que tan $k=\frac{\sin k}{\cos k}$, l'équation devient,

$$
P_{\max }=\frac{\left.\cos x\left(V_{2}^{2}(\cos \delta \cos x-\tan x \cos x \sin \delta)\right) \pm \cos x \sqrt{V_{2}^{2}(\cos \delta \cos x+\tan x \cos x \sin \delta)^{2}-\left(V_{2}^{4}-V_{1}^{2} V_{2}^{2}\right)}\right)}{Z}
$$

L'équation de $P_{\max }$ devient,

$$
P_{\max }=\frac{-V_{2}(\cos \delta \cos x+\sin x \sin \delta) \cos x \pm \cos r \sqrt{V_{2}^{4}(\cos \delta \cos x+\sin x \sin \delta)^{2}-\left(V_{2}^{4}-V_{1}^{2} V_{2}^{2}\right)}}{Z}
$$

En simplifiant $\mathrm{V}_{2}^{4}$ dans la racine, 


$$
P_{\max }=\frac{-V_{2} \cos (\delta-\theta) \cos x \pm \sqrt{V_{2}^{4} \cos ^{2}(\delta-\theta)-\left(V_{2}^{4}-V_{1}^{2} V_{2}^{2}\right)}}{Z}
$$

En utilisant dans le racine l'expression trigonométrique,

$$
\begin{gathered}
\sin ^{2}(\delta-\theta)=1-\cos ^{2}(\delta-\theta) \\
P_{\max }=\frac{-V_{2}^{2} \cos (\delta-\theta) \cos x \pm \cos x \sqrt{V_{1}^{2} V_{2}^{2}-V_{2}^{4} \sin ^{2}(\delta-x)}}{Z}
\end{gathered}
$$

En développant l'équation précédente au moyen de la suivante formulation :

Donnes du réseau :

FP: 0.92

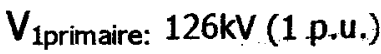

$V_{1 \text { secondaire: } 26 \mathrm{kV}}$

$$
\begin{gathered}
X c 2: 0,1,2, \ldots, 17 . \\
T(X C 2):=\operatorname{atan}\left(\frac{X-X c_{2}}{R_{L}}\right) \\
Z t(X c 2):=\sqrt{R_{L}^{2}+\left(X_{L}-X c_{2}\right)^{2}} \\
A p=1+(\tan k)^{2} \\
A p(X c 2):=\frac{2 \cdot V_{2}^{2} \cdot\left(\cos \left(t\left(X c_{2}\right)\right)+\tan (k) \cdot \sin \left(t\left(X c_{2}\right)\right)\right)}{Z t\left(X c_{2}\right)} \\
C p\left(X c_{2}\right):=\frac{V_{2}^{4}-V_{1}^{2} V_{2}^{2}}{Z t\left(X c_{2}\right)^{2}}
\end{gathered}
$$




$$
P_{\max }=\frac{-B p\left(X c_{2}\right)+\sqrt{B p\left(X c_{2}\right)^{2}-4 \cdot A p \cdot C p\left(X c_{2}\right)}}{2 \cdot A p}
$$

Conclusion de ce critère:

- La puissance maximal transitée avec la valeur minimal du taux de compensation minimal acceptable dans la méthode d'optimisation est de $3.08 \mathrm{MW}$.

- La puissance maximal qui peut être transitée avec une réactance de compensation $\mathrm{Xc}=15 \Omega(63.3 \%)$ est de $3.87 \mathrm{MW}$.

b) Transfert de puissance active par rapport à la ligne de transport

Donnes du réseau :

FP: 0.92

$V_{\text {1primaire: }} 126 \mathrm{kV}$ (1 p.u.)

$V_{1 \text { secondaire: } 26.4 \mathrm{kV}}$

Valeurs de réactance capacitive: Longueur de la ligne :

$X c 2: 0,8,14,17 \Omega$

$\mathrm{L}: 10,20,30,40,50,60 \mathrm{kms}$

Valuers de transfert de puissance par rapport à la longueur de la ligne:

Po : Transfert de puissance active sans compensation série. Po : $2.3 \mathrm{MW}$

$P_{1}$ : Transfert de puissance active à $\mathrm{XC}=8 \Omega ; \tau=33.76 \% ; P_{1}: 3.08 \mathrm{MW}$

$P_{2}:$ Transfert de puissance active à $\mathrm{XC}=14 \Omega ; \tau=59.07 \% ; P_{2}: 3.29 \mathrm{MW}$

$P_{3}:$ Transfert de puissance active à $\mathrm{Xc}=17 \Omega ; \tau=71.73 \% ; P_{3}: 3.87 \mathrm{MW}$

$$
P o<P_{1}<P_{2}<P_{3}
$$

$$
2.3 \mathrm{MW}<3.08 \mathrm{MW}<3.29 \mathrm{MW}<3.87 \mathrm{MW}
$$




\section{APPENDICE D}

Paramètres d'équipement auxiliaire du banc de condensateur série 


\section{Disjoncteur}

Tableau A.11

Données techniques du disjoncteur du banc de condensateurs série

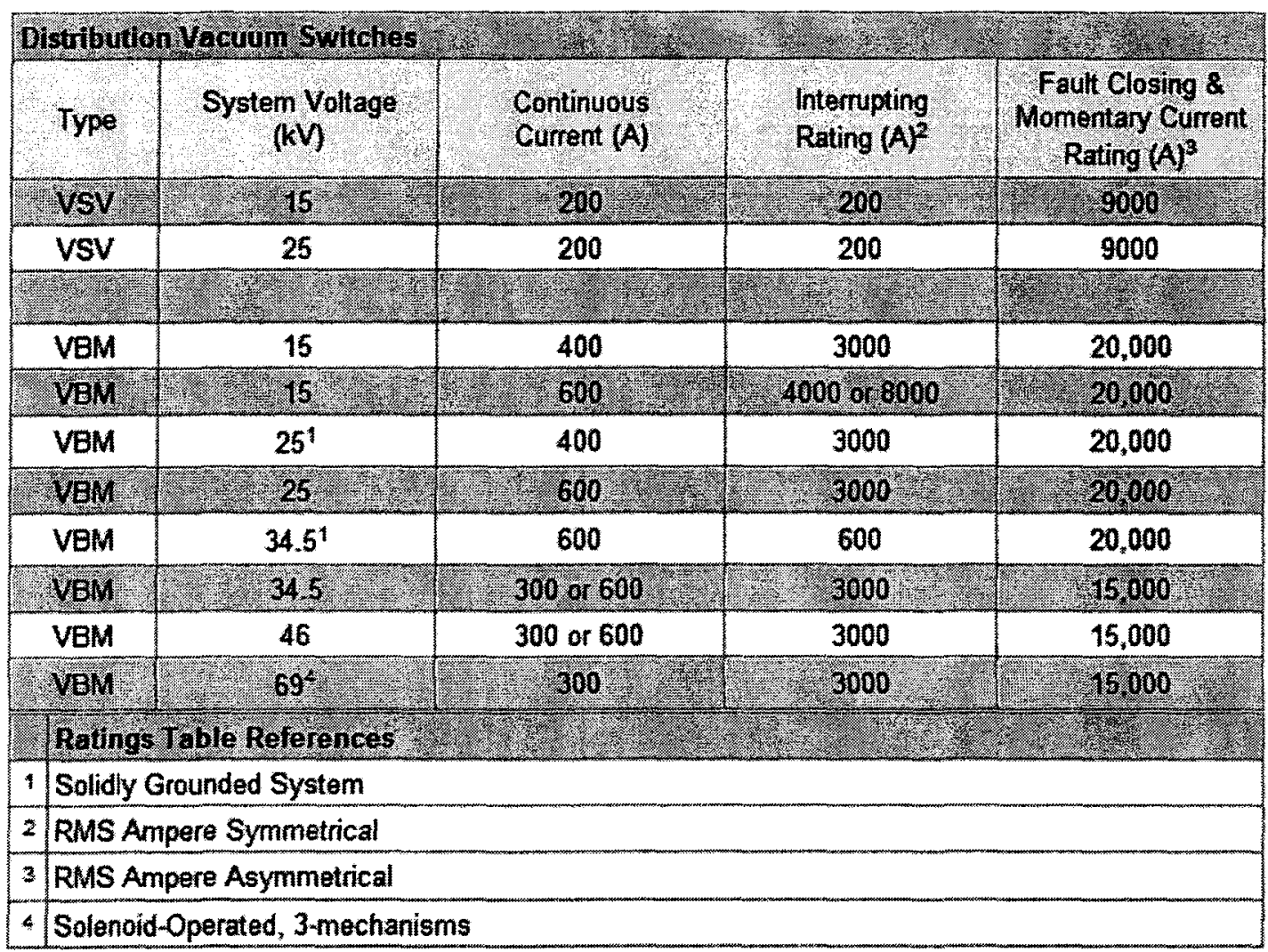
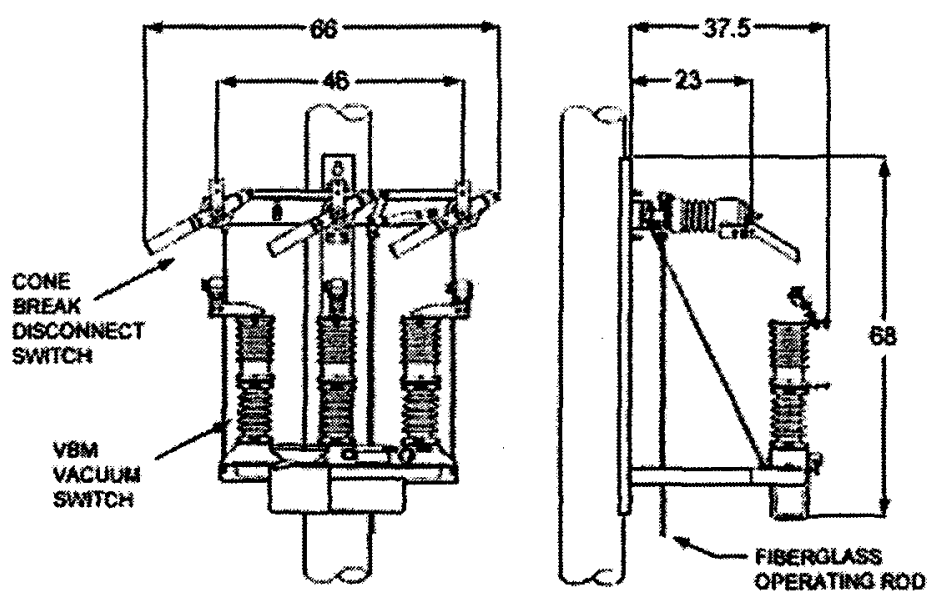

Figure A.5 Vue frontale et latérale du disjoncteur 


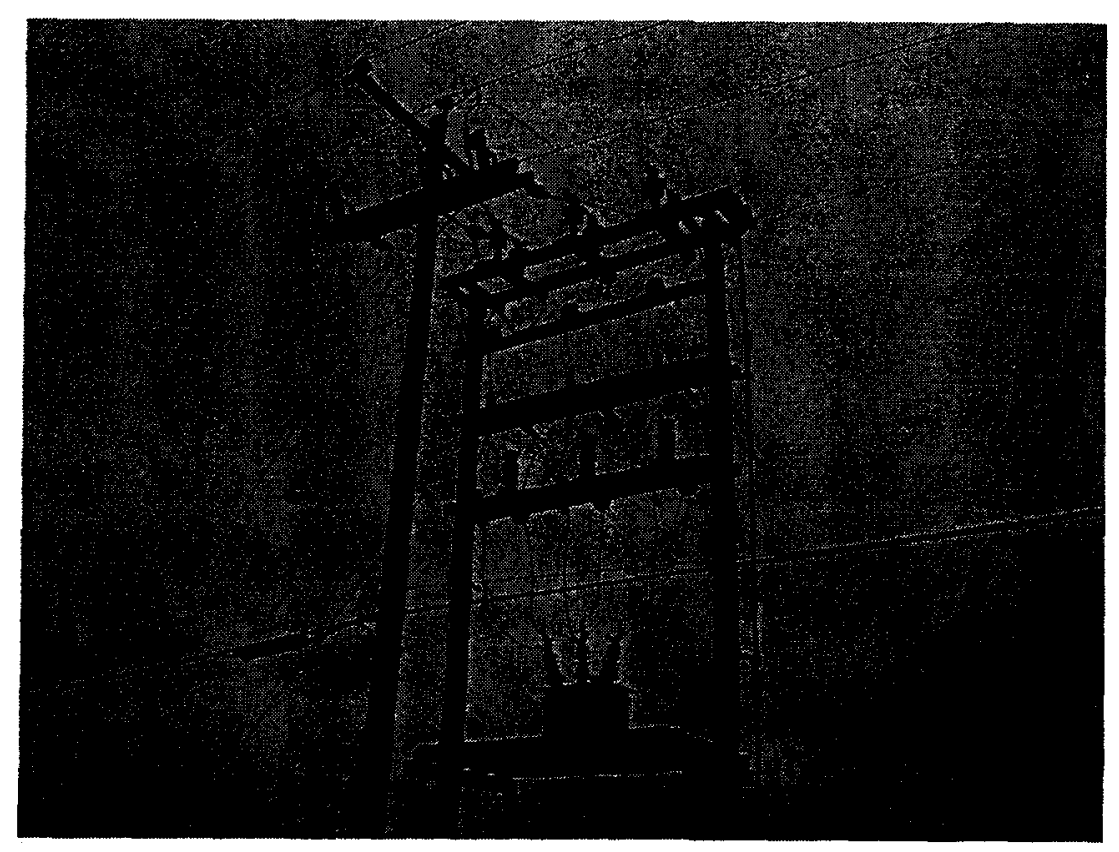

Figure A.6 Vue frontale du disjoncteur triphasé installé sur la ligne de distribution compensé avec du banc de condensateur série (poste Hydro-Quebec).

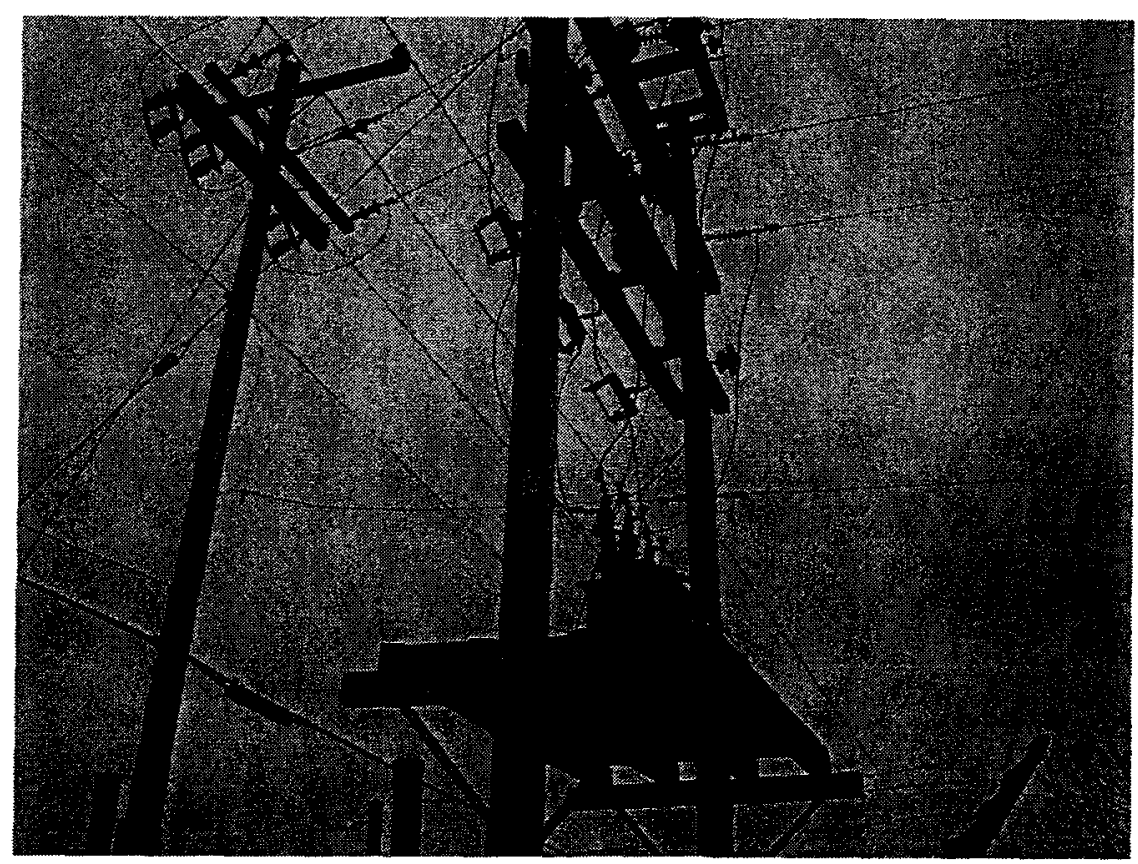

Figure A.7 Vue latérale du disjoncteur triphasé installé sur la ligne de distribution compensé avec du banc de condensateur série (poste Hydro-Québec) 


\section{Banc de condensateurs série}

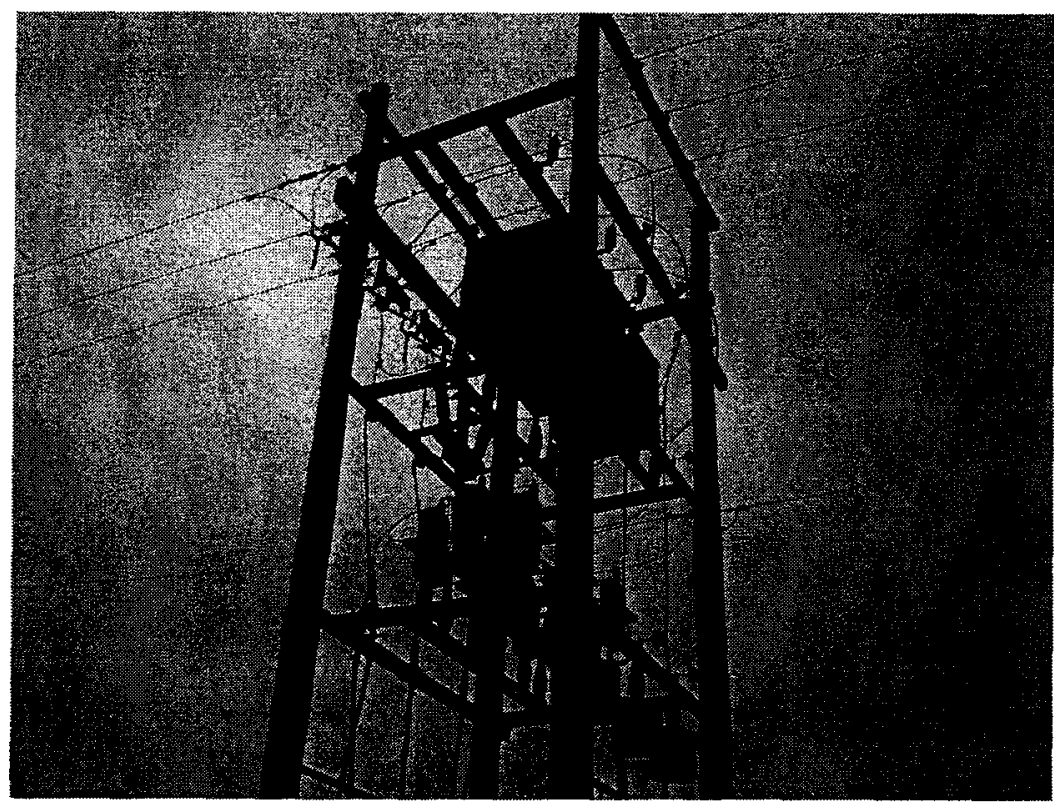

Figure A. 8 Vue latérale du banc de condensateurs série et les résistances antirésonance sous-synchrone

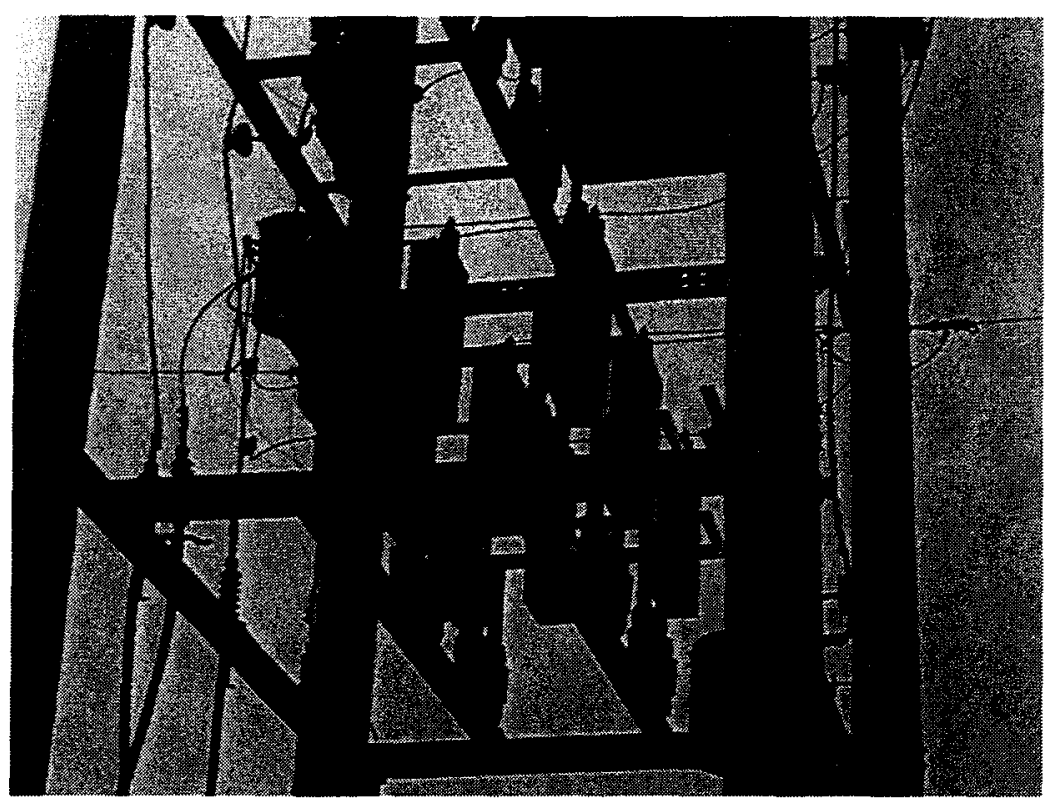

Figure A.9 Vue latérale du banc de condensateurs série 
Équipements auxiliaires du banc de condensateurs série

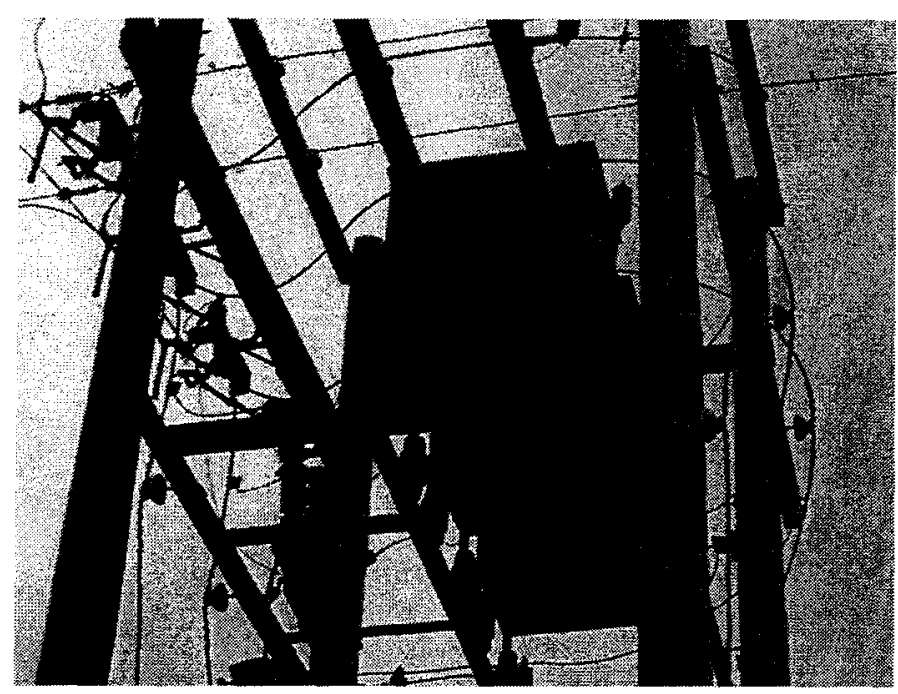

Figure A.10 Résistances en parallèle du banc de condensateurs série

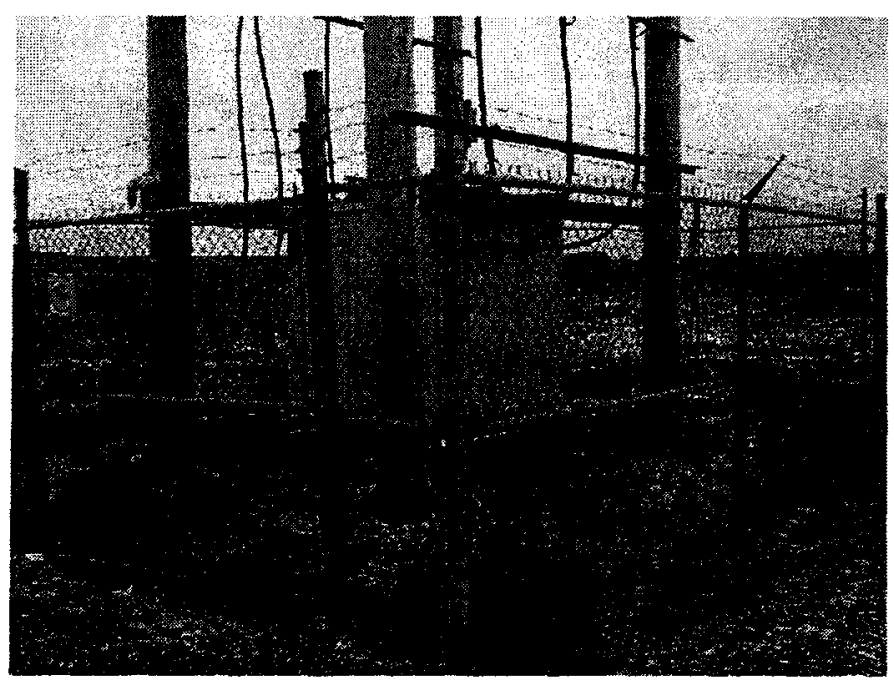

Figure A.11 Équipement de commande du banc de condensateurs série triphasé 
APPENDICE E

Étude de papillotement. Normes C.22.1 et C.22.2. Hydro-Québec 
NORME C.22.1

ma1 1981

page 4 de 4

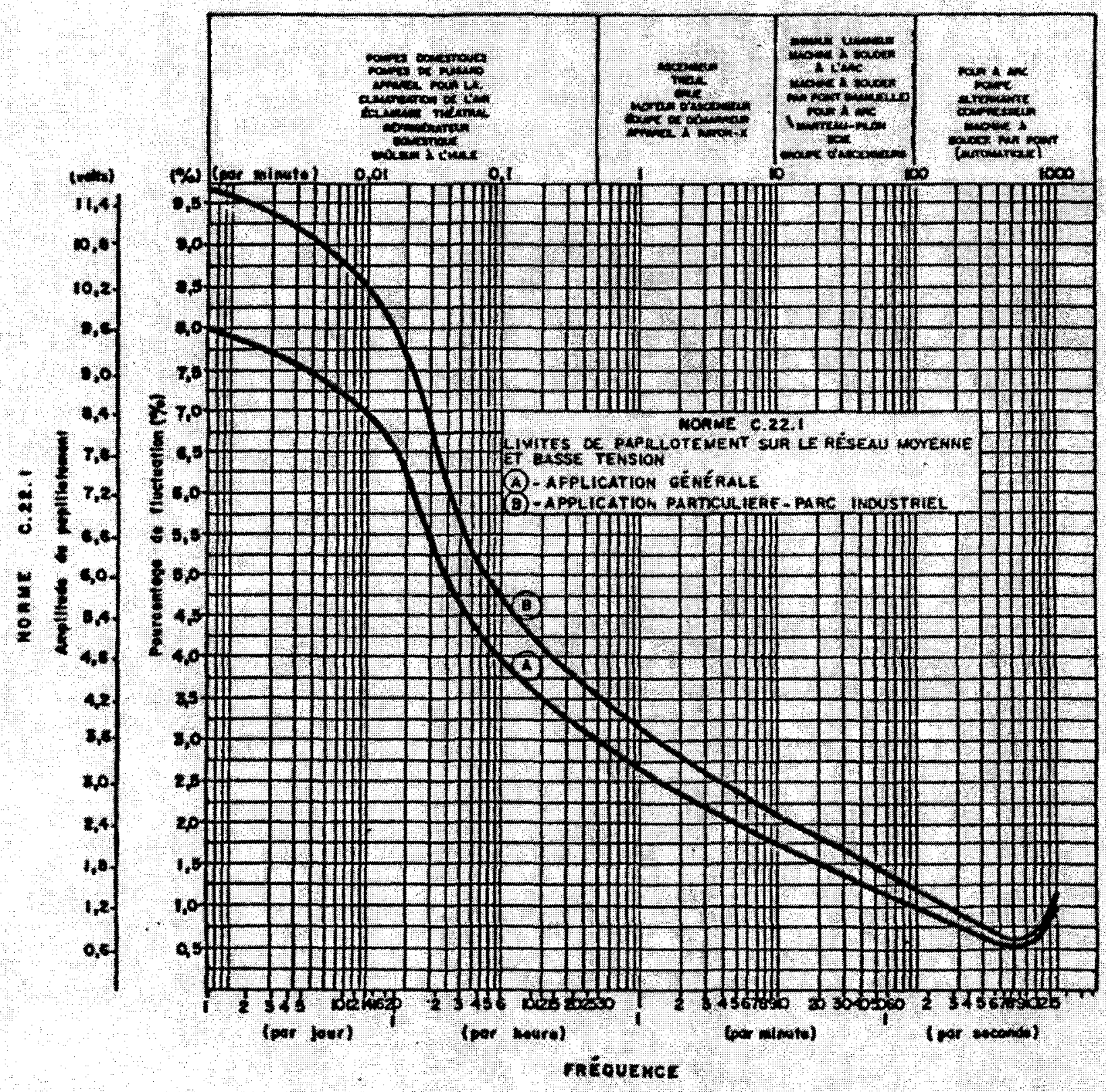

Figure A.12 Norme de l'étude papillotement C22.1 
NORME C.22.2

Figure A.13 Norme de l'étude de papillotement C.22.2

ma1 1981

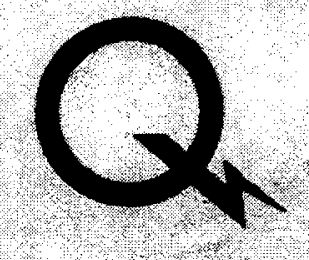

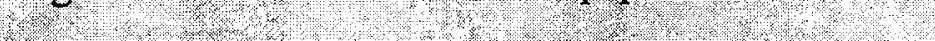
pege 5 de 5

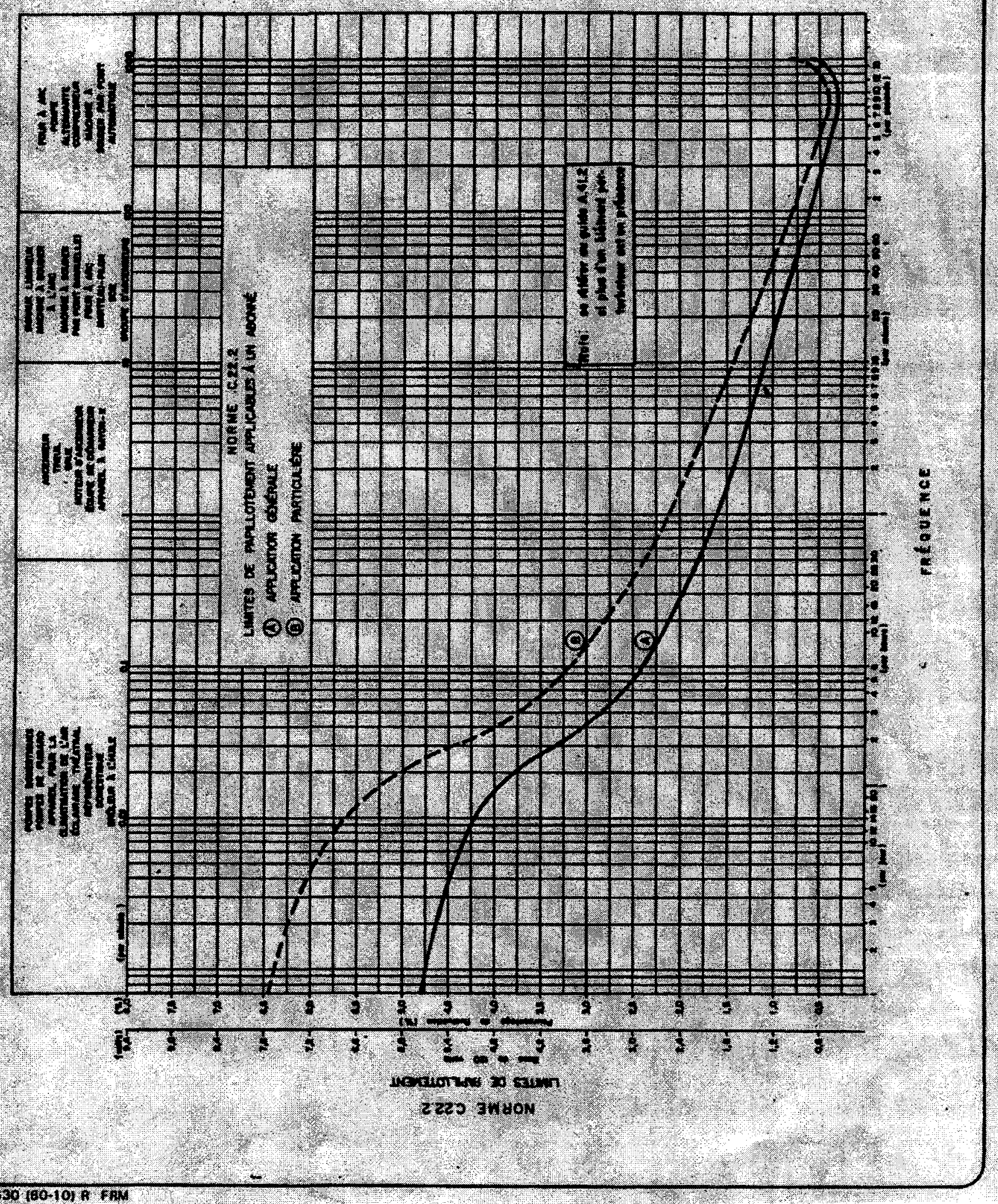




\section{APPENDICE F}

Description de logiciel MINIAQO et méthode de mesures du phénomène de papillotement obtenu par Hydro-Québec dans la scierie Contois. 


\section{Description de logiciel MINIAQO}

Ce logiciel consiste à analyser la qualité de l'onde de différents paramètres du réseau électrique. MINIAQO est composé de trois logiciels MINIAQO, WINAQO et MINICHART. Les logiciels MINIAQO et WINAQO sont utilisés pour mesurer les facteurs de qualité qui caractérisent la qualité de l'onde sur un lieu donné du réseau de distribution. La présentation des résultats dans ces logiciels est basée sur la statistique et les tableaux d'occurrences. Aussi quelques graphiques obtenues sont disponibles sous forme de pires cas. Il est important de signaler que le taux d'échantillonnage devient comme la différence principale entre le logiciel MINIAQO et MINICHART. Le logiciel MINIAQO utilise un circuit de phase à verrouillage de phase pour avoir un nombre de points fixe par cycle, pour effectuer une transformer de Fourier rapide. Mais dans certains cas, ce mode d'échantillonnage cause des déformations du signal analysé. Par exemple, lorsque il se produit une variation de la fréquence ou que le circuit de verrouillage de phase se désynchronise.

Par le cas du logiciel MINICHART, celui utilise un taux d'échantillonnage fixe en permettant d'avoir une représentation juste du signal en tout temps. En plus ce échantillonnage fixé permet un marquage temporel exact et aussi précis que le l'horloge de la carte de conversion numérique qui équipe le Mini-AQO.

Le papillotement est une des éléments mesurés pour les techniciens de l'Hydroquebec sur la scierie Contois. La sélection de l'enregistrement du papillotement permet d'établir clairement la performance de la tension aux variations de charges. La fréquence d'enregistrement n'est pas programmable et est fixé à 10 minutes. La sélection des calcules est divisée en deux parties. La première correspond au calcul du papillotement conventionnel Pst (courte durée short time) et Plt (long durée long time) [CEI 61000-4-15, 1997]. 


\section{Méthode de mesure}

La méthode de mesure doit être représentative de l'ennui qu'elle représente et tenir compte des mécanismes de la vision. À cet effet, le papillotement doit être évalué pendant une période de temps suffisamment long. Due que le papillotement est provoqué uniquement par certaines charges, son niveau instantané peut varier considérablement et de manière imprévisible pendant cette période. Un intervalle de 10 minutes a été établi comme la période correcte pour évaluer ce qui on appelle "papillotement de courte durée », ou Pst. Cet intervalle est assez long pour éviter de donner trop d'importance aux variations de tension isolées, ainsi que pour permettre qu'une personne ne remarque pas la perturbation et sa persistance. Parallèlement, il est trop court pour permettre de caractériser de manière fiable un équipement perturbateur avec un long cycle de fonctionnement. La période de 10 minutes sur laquelle est basée l'évaluation de la sévérité du papillotement de courte durée est valable pour l'estimation des perturbations causées par les sources individuelles comme par exemple ce qui est lamineuses, les bombes de chaleur ou les équipements électroménagers. C'est le cas où l'effet combiné de plusieurs charges perturbatrices fonctionnent de manière aléatoire (comme par exemple les arcs de soudure ou les moteurs) ou quand il s'agira de sources de papillotement de cycle de fonctionnement long ou variable (fours électriques d'arc), il est nécessaire d'évaluer la perturbation crée pendant une période de temps plus long. La durée de mesure est définie dans 2 heures, durée considérée comme appropriée au cycle de fonctionnement de la charge ou durée pendant laquelle un observateur peut être sensible au papillotement de longue durée. Le calcul du papillotement de longue durée, ou de Plt sera effectué à partir des valeurs de courte durée.

L'analyse du contenu spectral du papillotement correspond à la deuxième partie. Cette analyse permet de déterminer la fréquence des phénomènes qui provoqueront le papillotement et leurs respectives amplitudes. Il faut signaleur que la somme des raies spectrales obtenues de cette analyse correspond à la valeur du papillotement obtenu 
pendant la même période de 10 minutes (Pst). La sévérité d'un papillotement de longue durée Plt est déduit du Pst par l'équation (25) :

$$
P l t=\sqrt[3]{\sum_{i=1}^{N} P s t_{i}^{3}}
$$

Où $P_{s t_{i}}(\mathrm{i}=1,2,3, \ldots)$ sont les valeurs consécutives de sévérité de Pst obtenues. La Plt doit être déduite des valeurs $P s t$ sur une durée appropriée liée au cycle de fonctionnement de la charge, ou sur une période pendant laquelle un observateur peut être sensible au papillotement, par exemple quelques heures (normalement 2 heures).

L'ordre de grandeur de la limite tolérable est : $P l t=0,74$. Le paramètre $P s t$ global, dû à différents pollueurs raccordés à un même réseau, et évalué en un point donné, est calculé selon la règle de sommation $(26)$ :

$$
P_{s t}=\sqrt[m]{\sum P s t_{i}^{m}}
$$

Habituellement $m=3$, mais d'autres valeurs peuvent être utilisées.

D'ailleurs, dans le logiciel, la sélection du type de lampe précise le choix d'éclairage à analyser. Par exemple par les clients résidentiels qui observent que ses lumières fluorescentes clignotent, il faut choisir dans le logiciel la lumière de $120 \mathrm{~V}$ fluorescente.

Les résultats obtenues par MINICHART sont analysé à travers des graphiques en représentant la performance de paramètres tels que :

- Profile de tension TN des phase A, B, et C .

- Profile de tension efficace TE de phases A, B et C.

- Distorsion de la tension DI de phases A, B et C.

- Pire cas de creux de tension efficace de phases A, B et C.

- Papillotement instantané de la phase A. 
Analyse de tensions de phase obtenus dans les essais

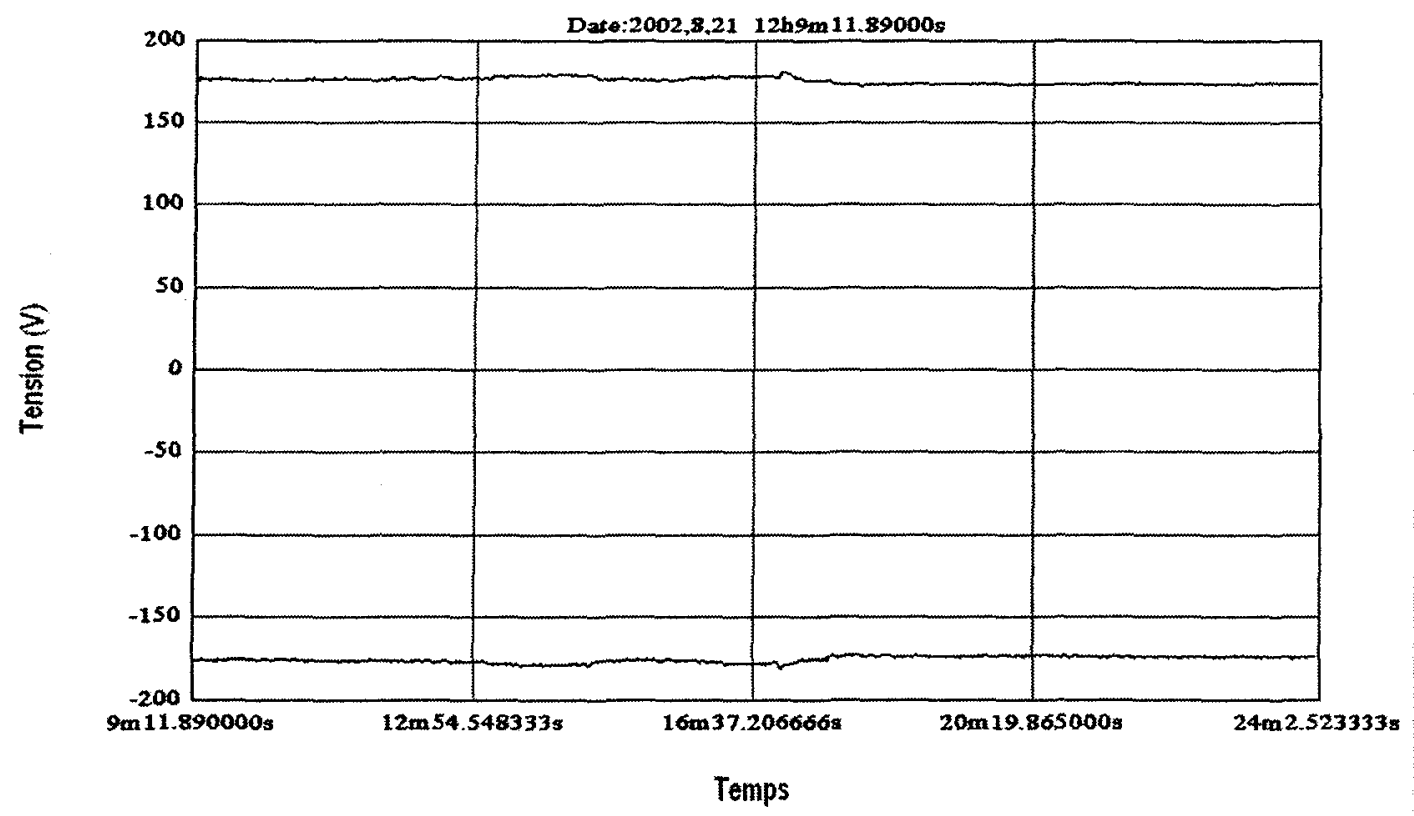

Figure A.14 Profile de tension phase A

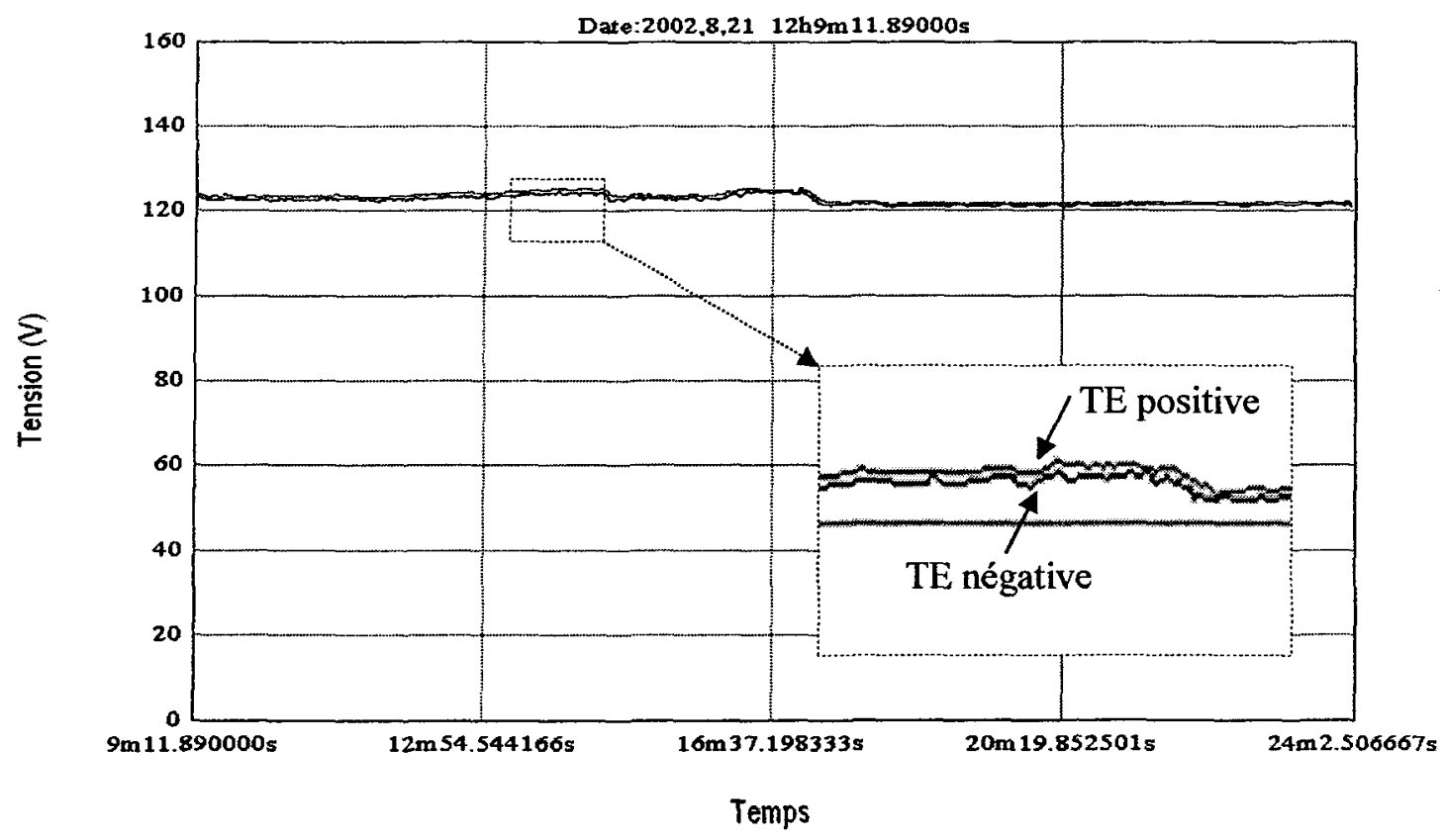

Figure A.15: Tension efficace phase A 


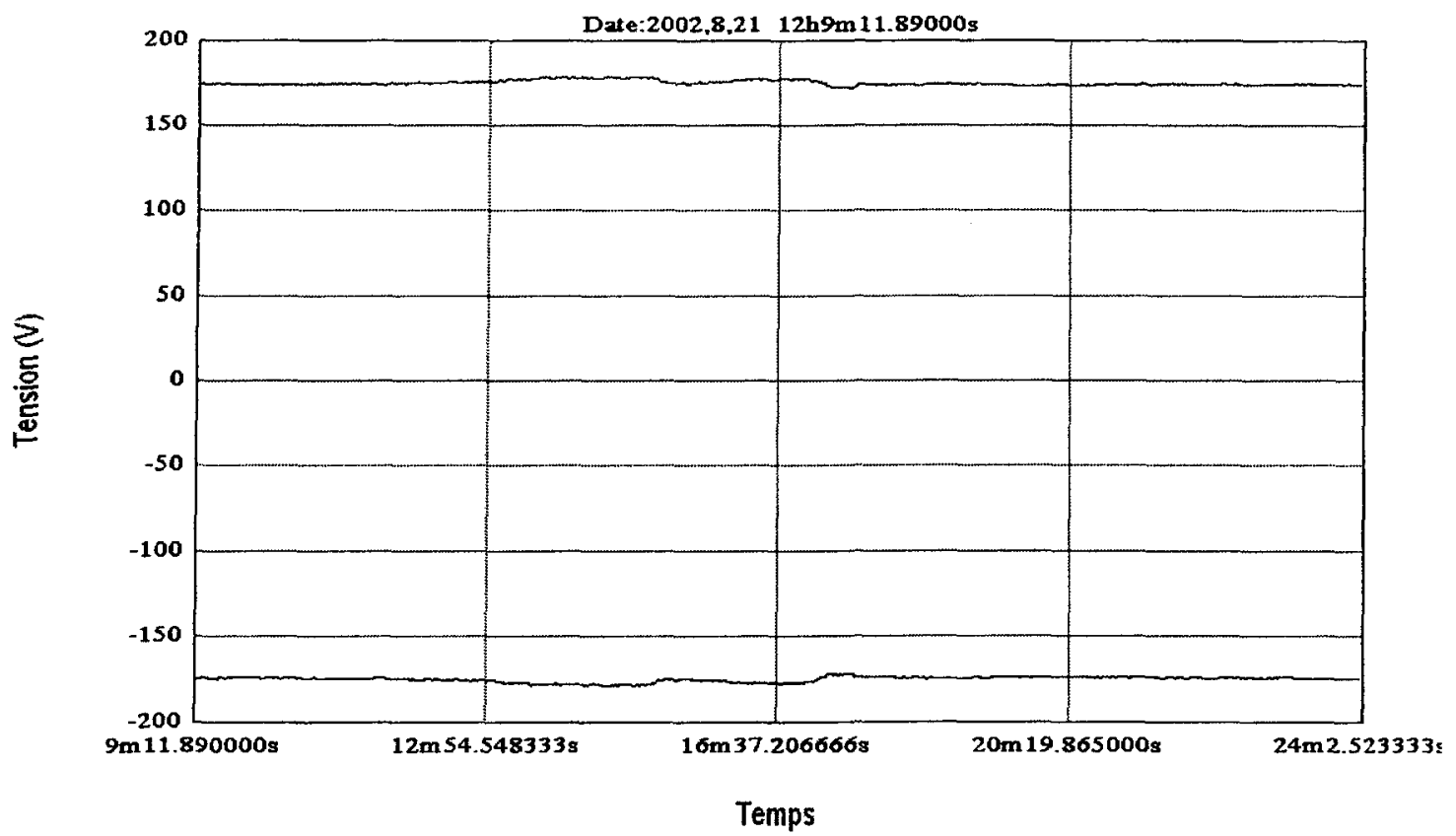

Figure A.16 : Profil de tension phase B

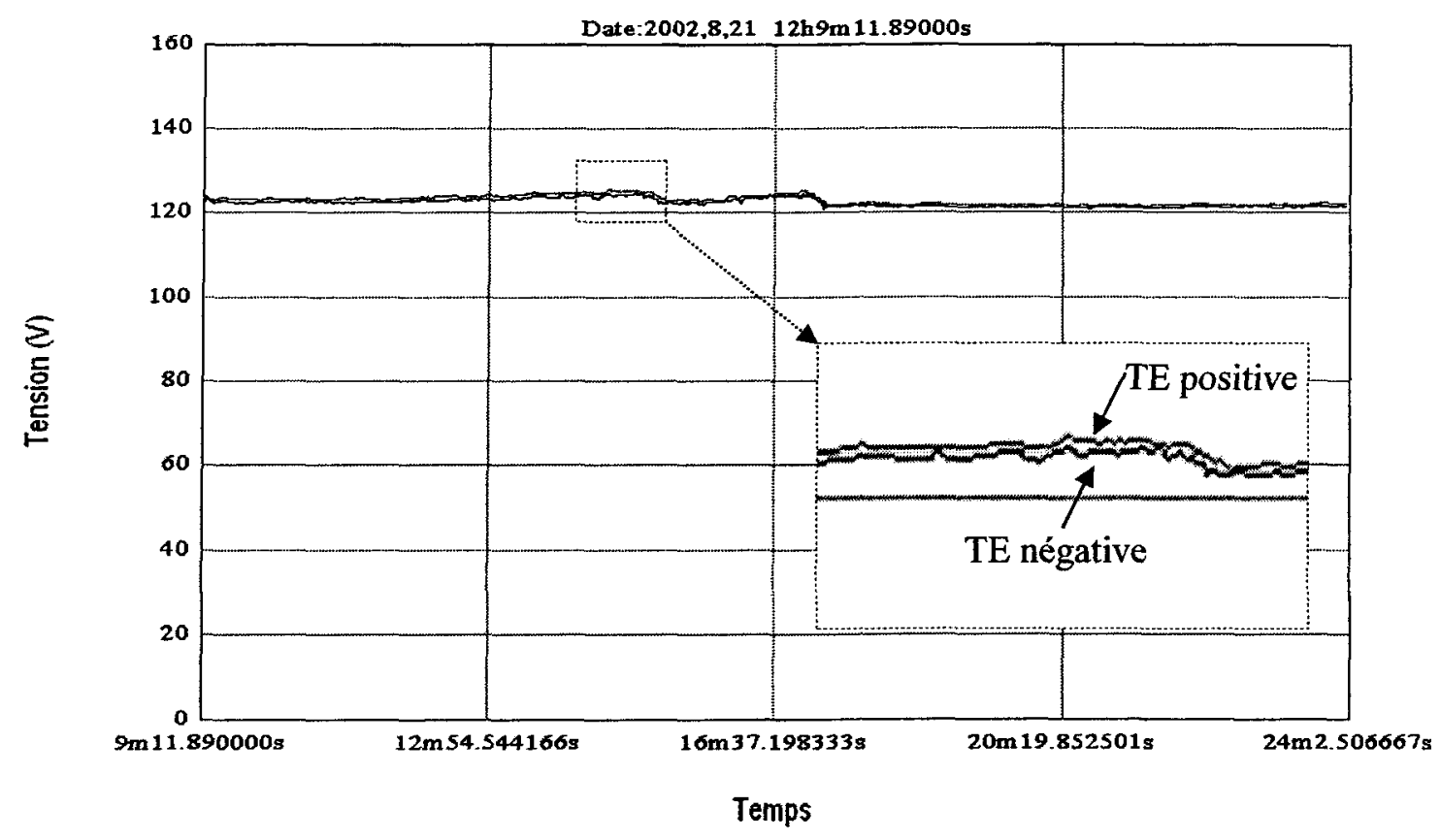

Figure A.17 : Tension efficace phase B 


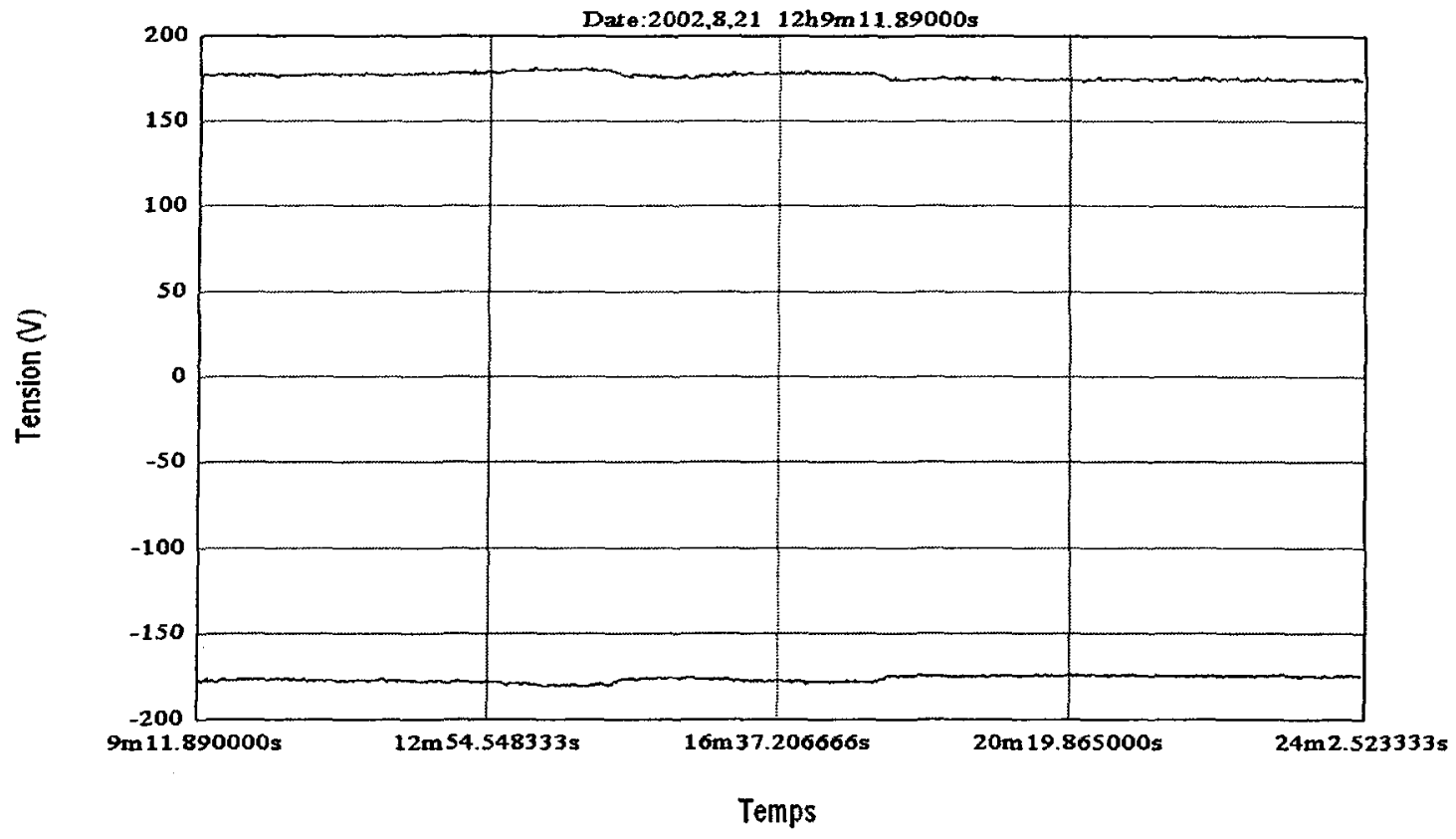

Figure A.18 : Profil de tension phase C

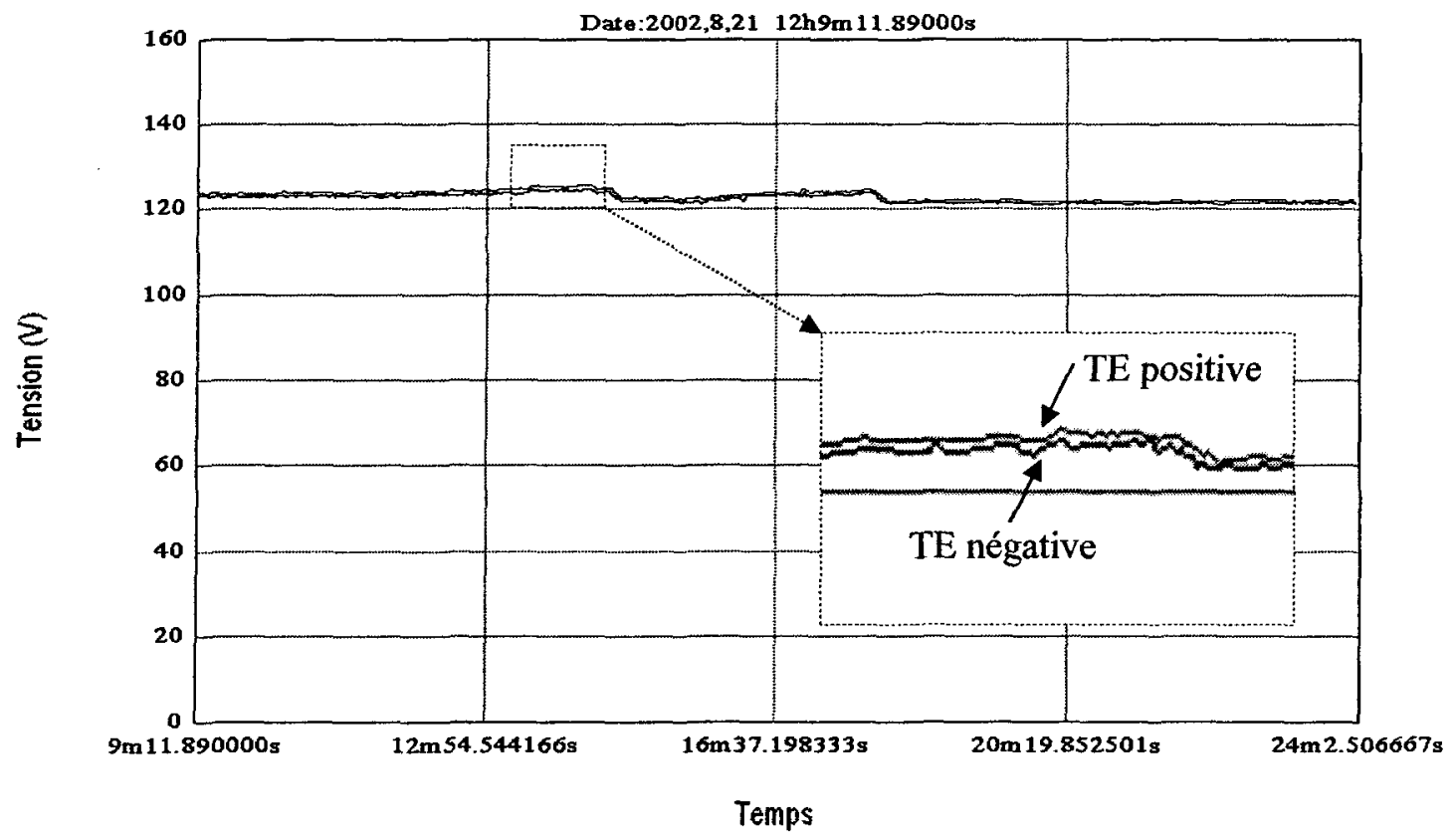

Figure A.19: Tension efficace phase C 
On mesure la tension nominale $T N$ à la phase $\mathrm{A}, \mathrm{B}$ et $\mathrm{C}$ à partir de $\mathrm{t}=9 \mathrm{~min} 12 \mathrm{sec}$, jusqu'à $t=24 \mathrm{~min}$. $2.5 \mathrm{sec}$, figure . La tension maintient sa valeur près de la valeur nominale mais elle n'est pas constante. On observe la présence de deux fluctuations importantes sur les profils de tension efficace des trois phases au point de raccordement à environ $\mathrm{t}=14 \mathrm{~min}$ et $\mathrm{t}=17 \mathrm{~min}$. Les tensions nominales sont observées aux figures $3,5,7$ et les tensions efficaces aux figures 4,6 et 8 .

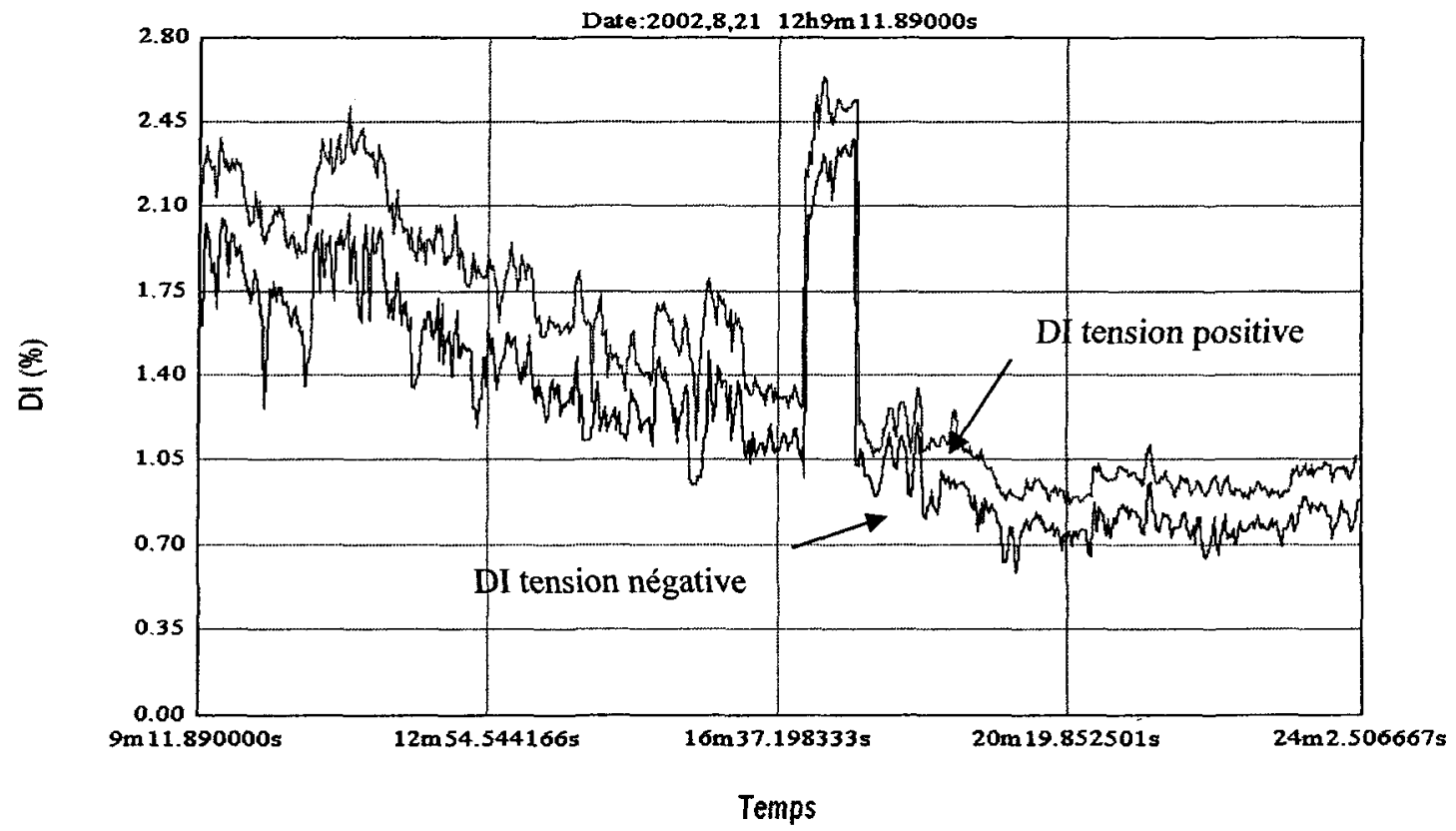

Figure A.20 : Distorsion de la tension phase A 


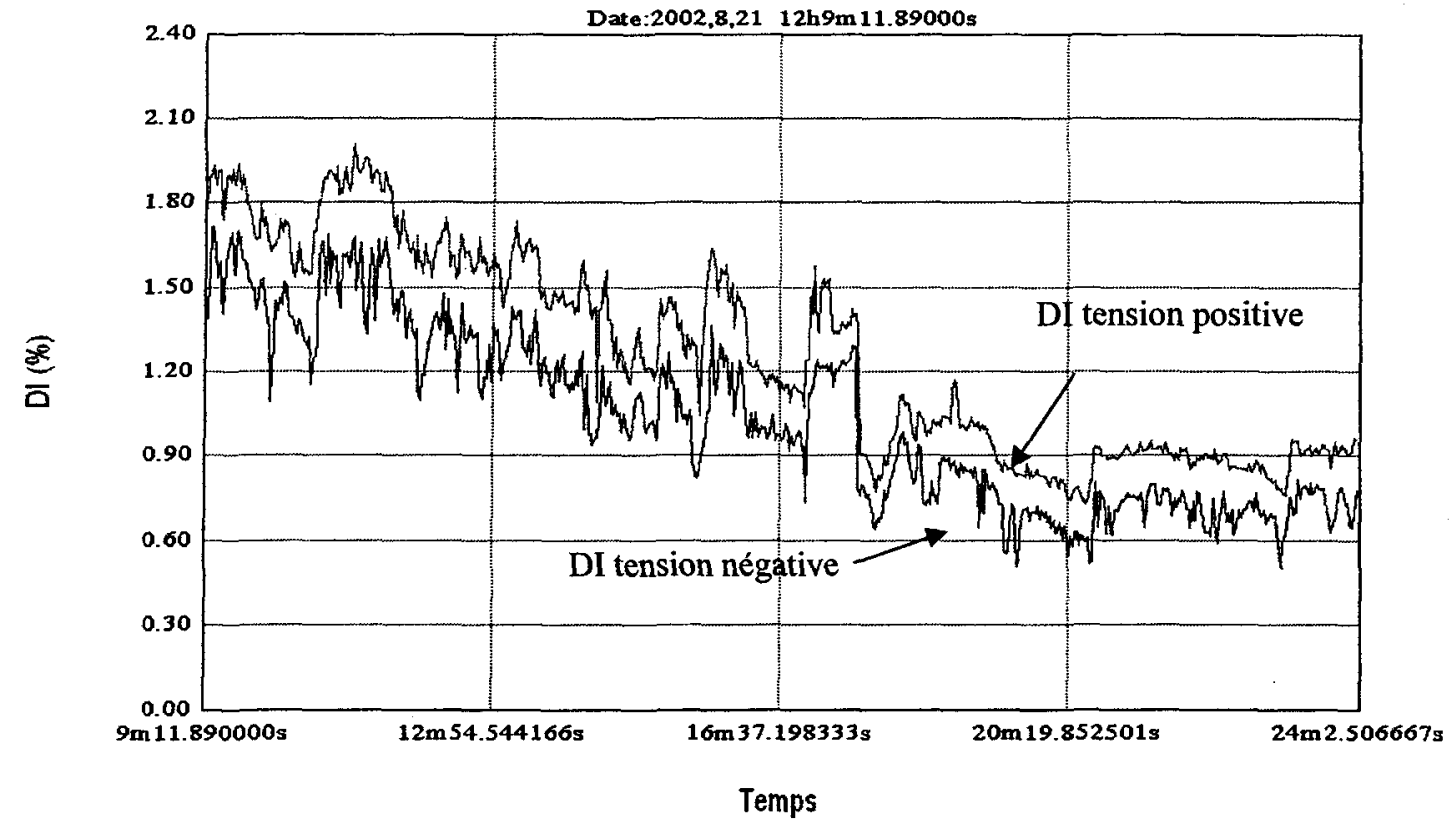

Figure A.21 : Distorsion de la tension phase B

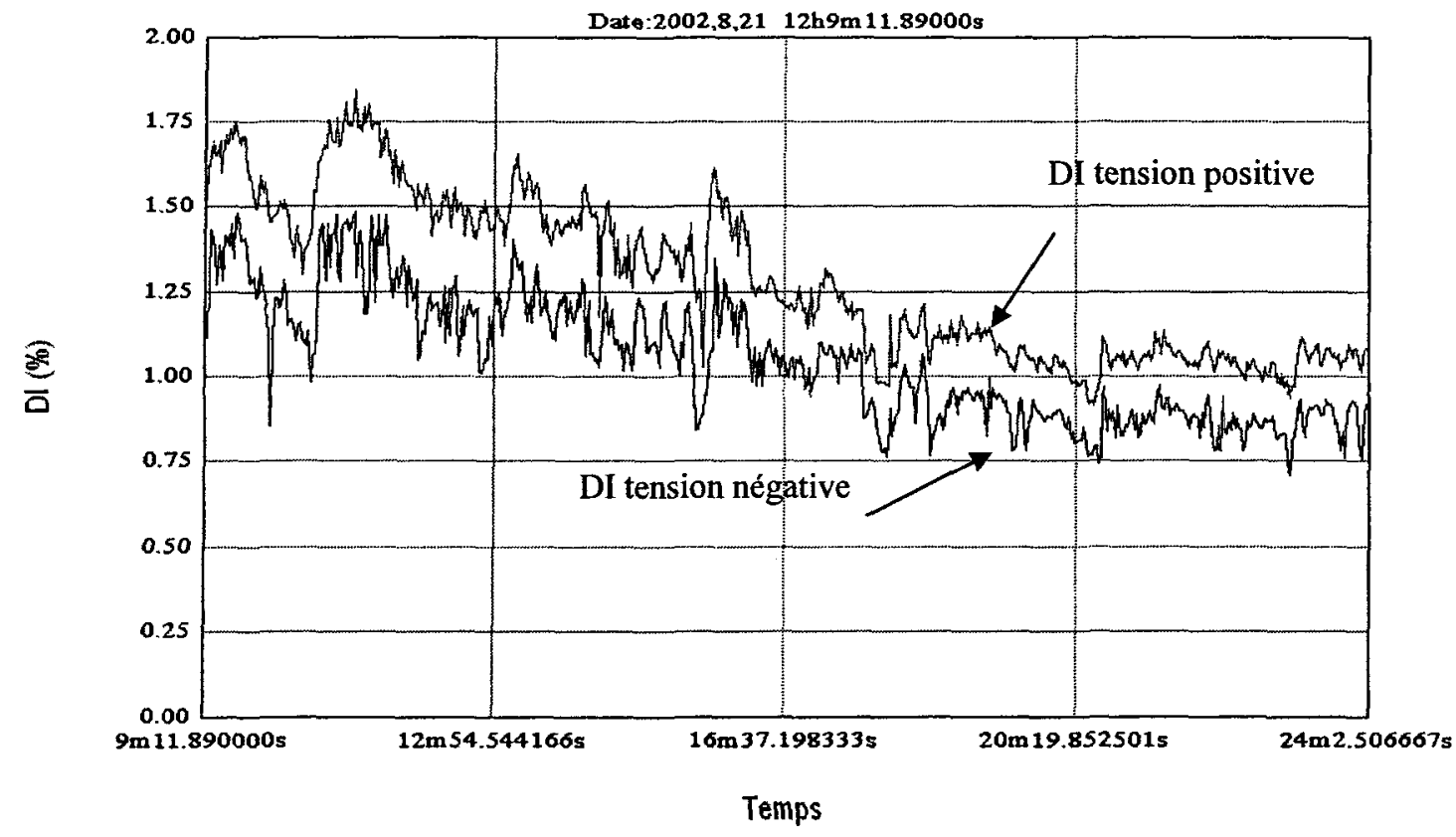

Figure A.22 : Distorsion de la tension phase C 
La distorsion de tension DI a été obtenue par le même période de mesure que par les profils de tensions de phase, figures 9,10 et 11 . On remarque des variations brusques du taux de distorsion de la tension dont la performance oscille entre $1.76 \%$ et $0.75 \%$. On observe à $\mathrm{t}=16 \mathrm{~min}$ et $17 \mathrm{sec}$, il se produit une élévation très élevée du taux de distorsion, plus notable à la phase $\mathrm{A}$, lorsque un pic de tension se produit au point de raccordement. $\mathrm{Ce}$ pic de tension peut être provoqué par un pic du courant important entraîné par l'utilisation d'un élément perturbateur dans ce moment.

À partir d'environ $t=26$ min et $20 \mathrm{sec}$, le taux de distorsion oscille entre $1.25 \%$ et $0.75 \%$. Cela nous indique qu'il y a une réduction de la distorsion de la tension.

La distorsion de la tension négative au point de raccordement est plus basse que pour la distorsion de tension positive en toutes les phases.

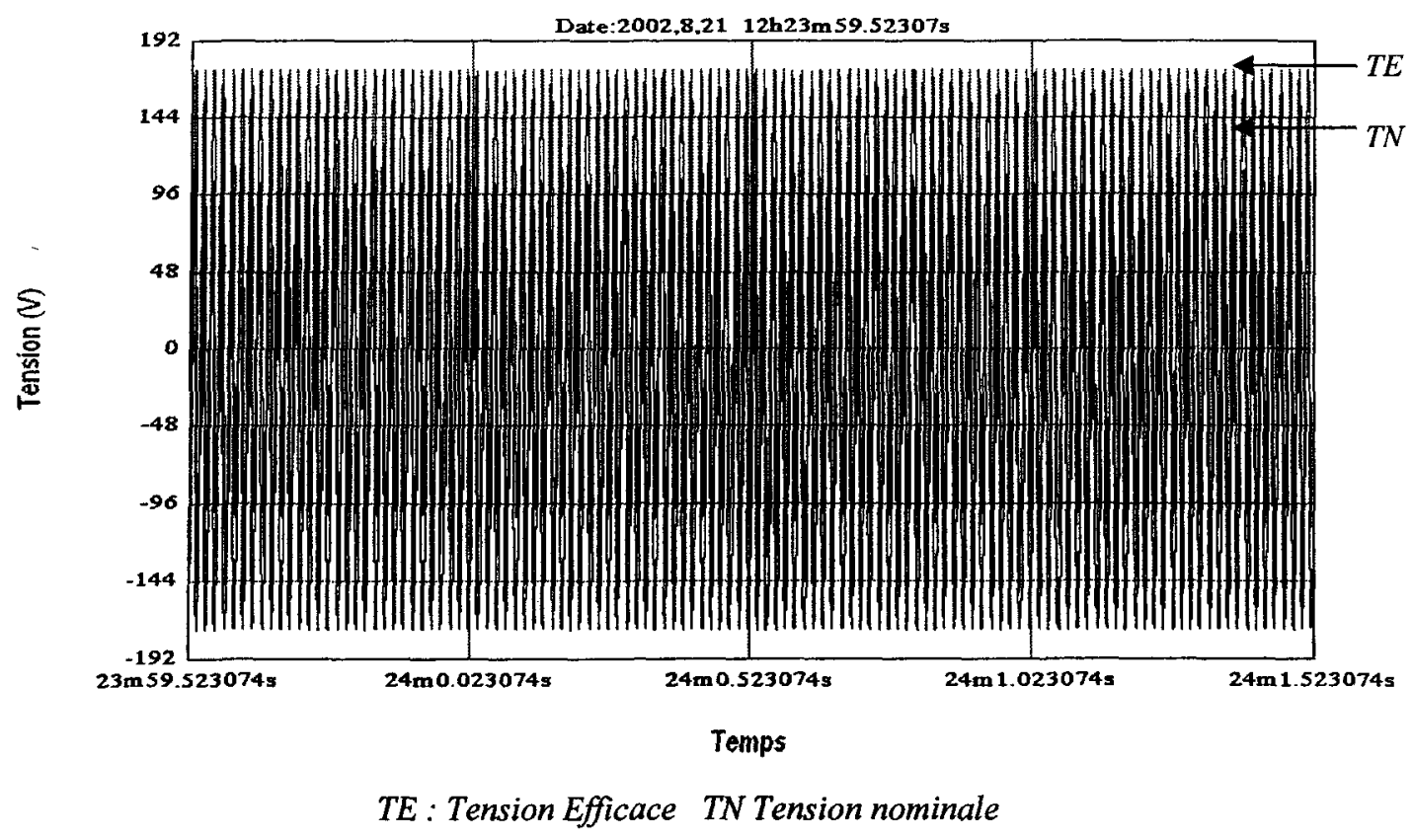

Figure A.23 : Pire cas creux de tension efficace phase A 


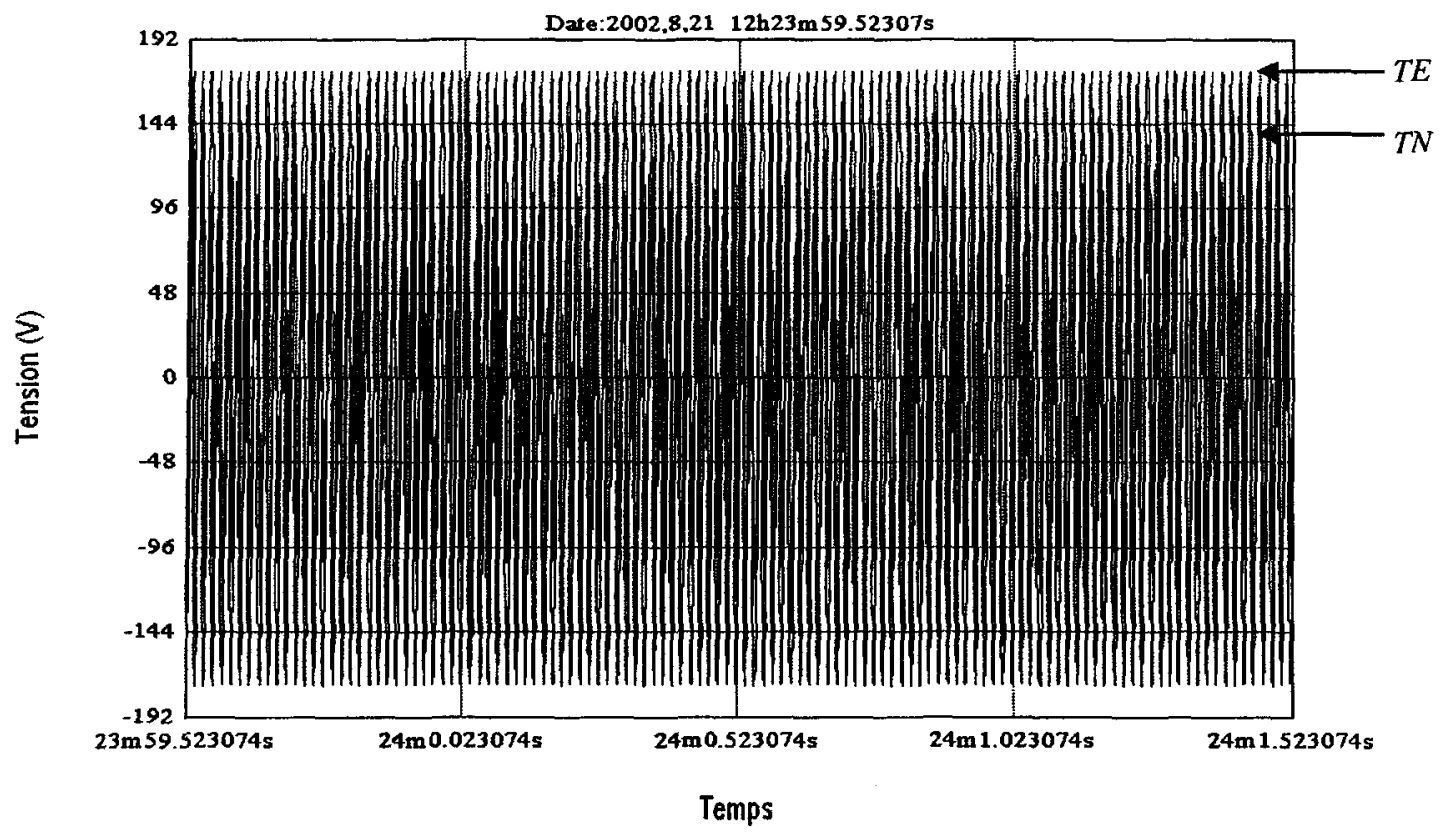

TE : Tension Efficace TN Tension nominale

Figure A.24 : Pire cas de creux de tension efficace phase B

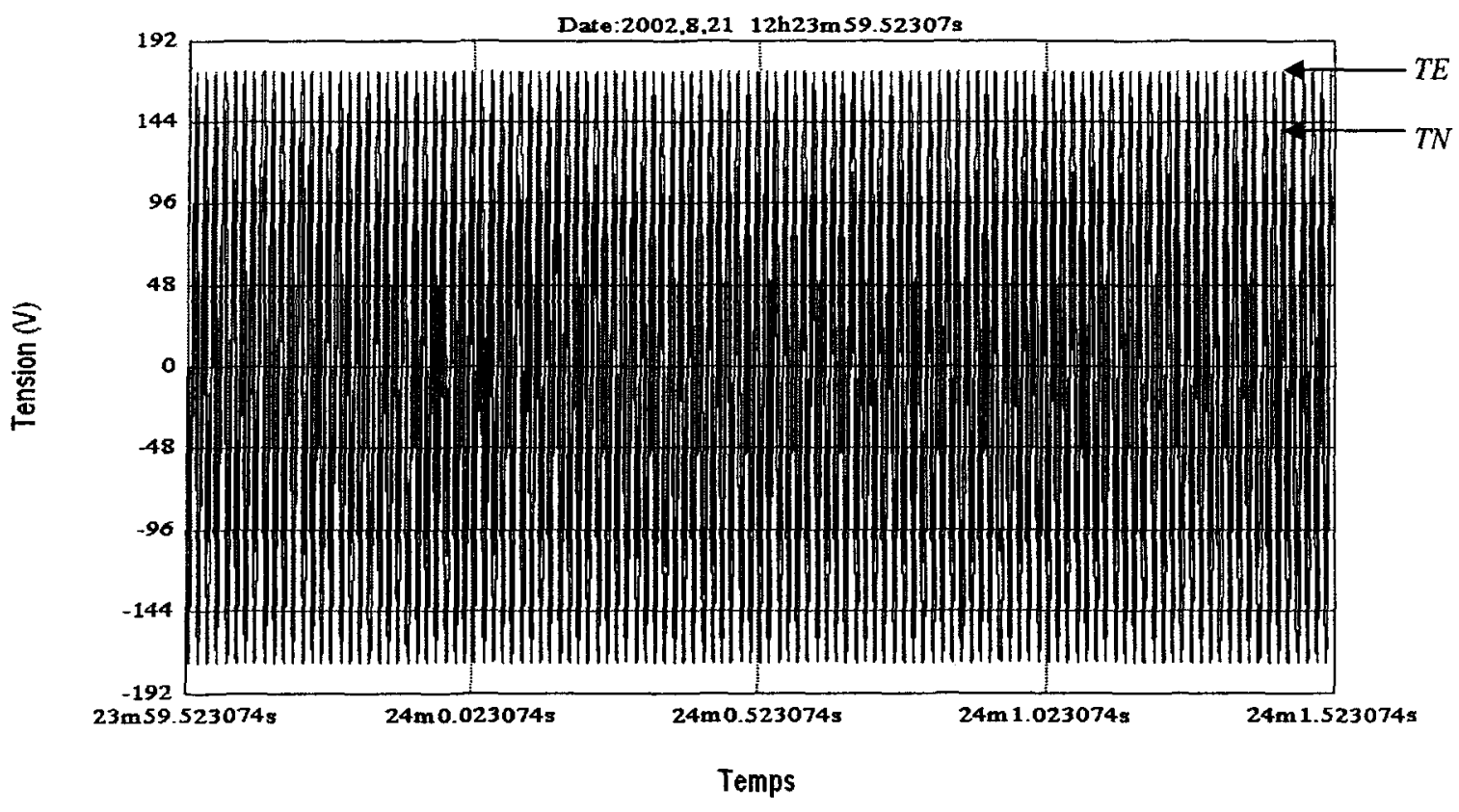

TE : Tension Efficace TN Tension nominale

Figure A.25: Pire cas de creux de tension efficace phase $\mathrm{C}$ 
Le logiciel MINICHART a choisi le pire cas de creux de tension efficace TE des phases $\mathrm{A}, \mathrm{B}$ et $\mathrm{C}$ au point de raccordement. On observe aux figures 12,13 et 14 , que le pire cas oscille trouve entre $t=23 \mathrm{~min} 59.52 \mathrm{sec}$ et $t=24 \mathrm{~min} 1.52 \mathrm{sec}$. Le creux de tension TN est plus remarquable à la phase $\mathrm{C}$ où on observe des variations rapides de la tension dont les pics deviennent plus grands que $144 \mathrm{~V}$. 


\section{Analyse de papillotement par phase obtenus dans les essais}

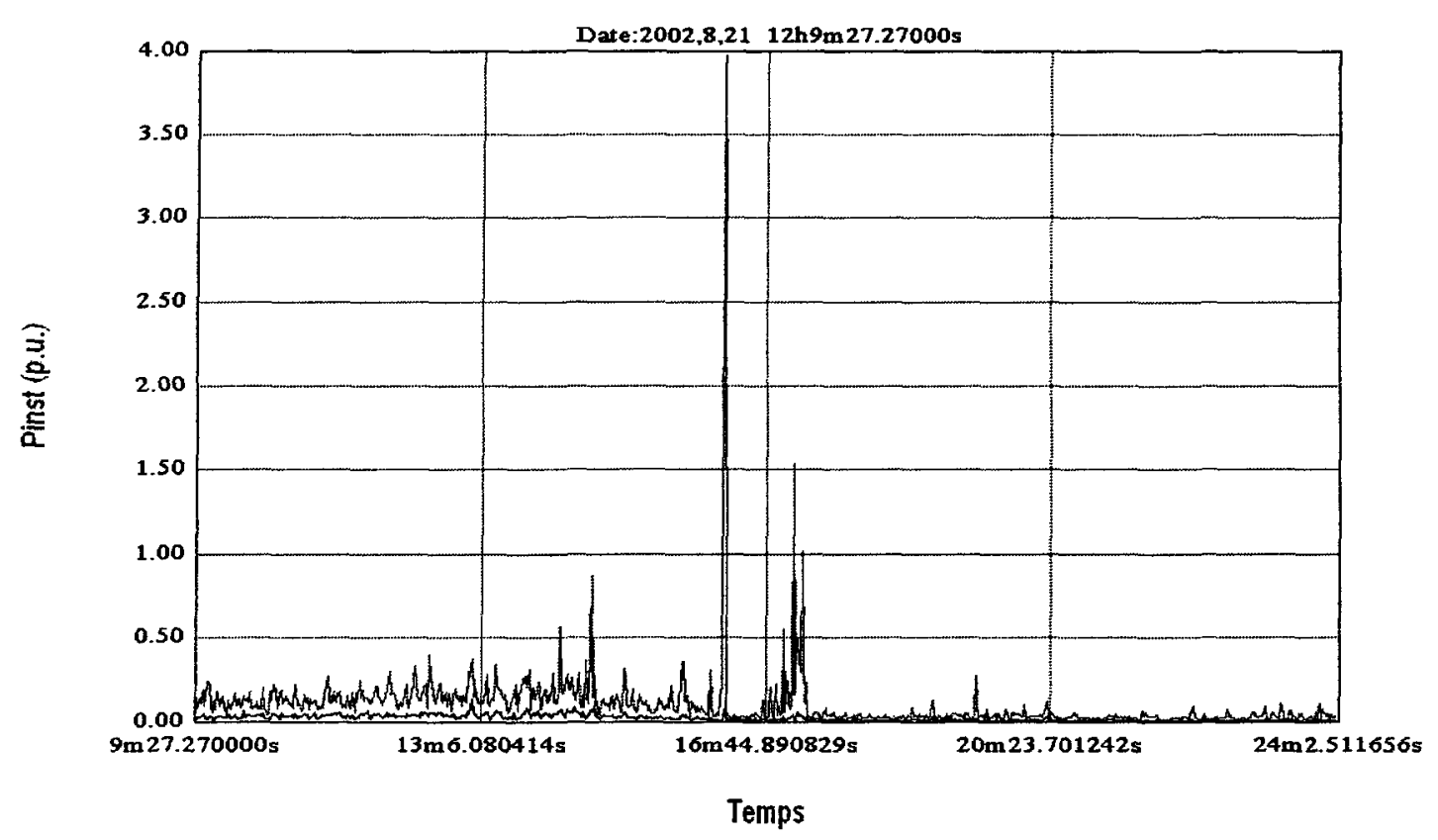

Figure A26 : Papillotement instantané de la phase A

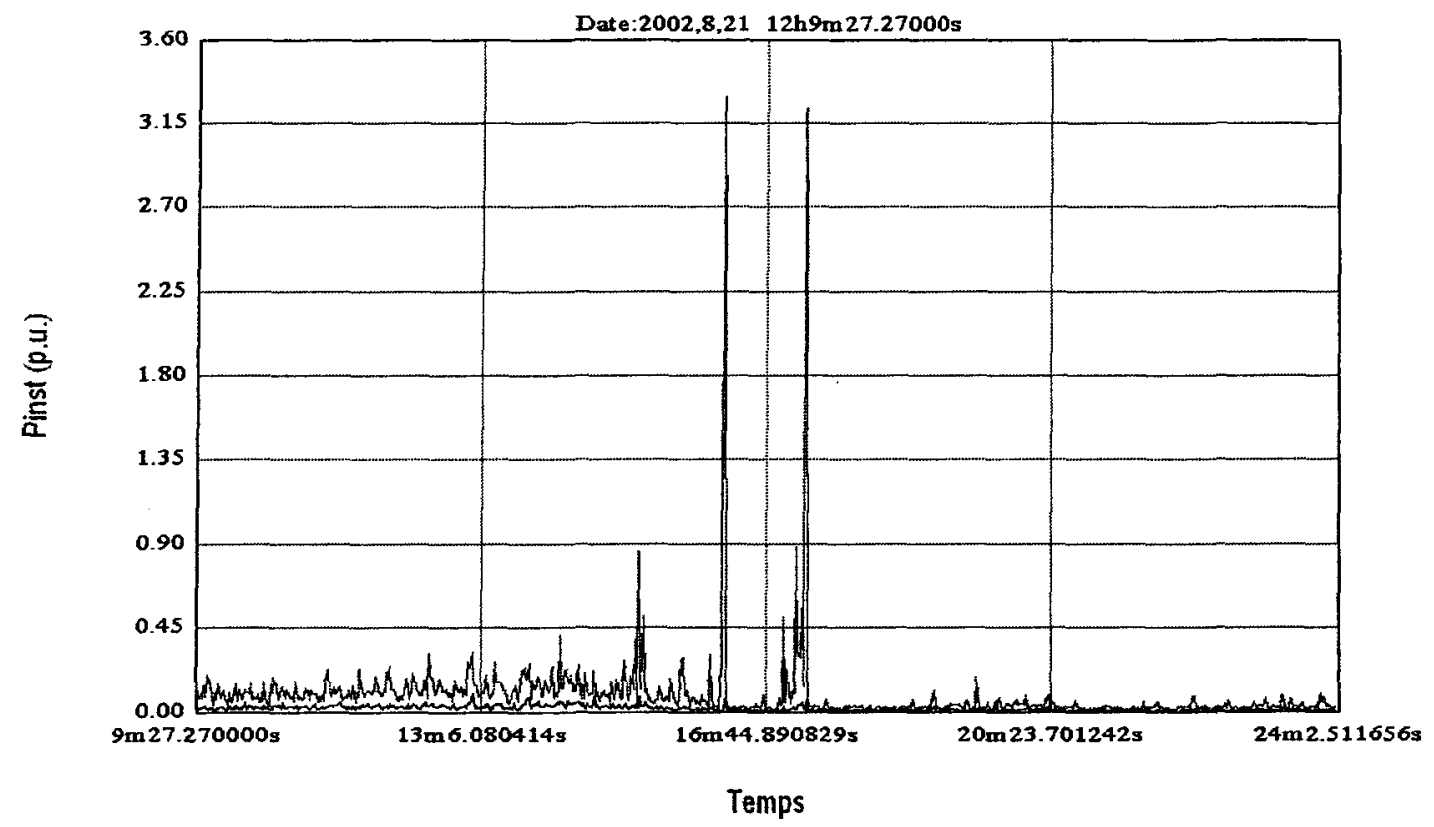

Figure A.27 : Papillotement instantané de la phase B 


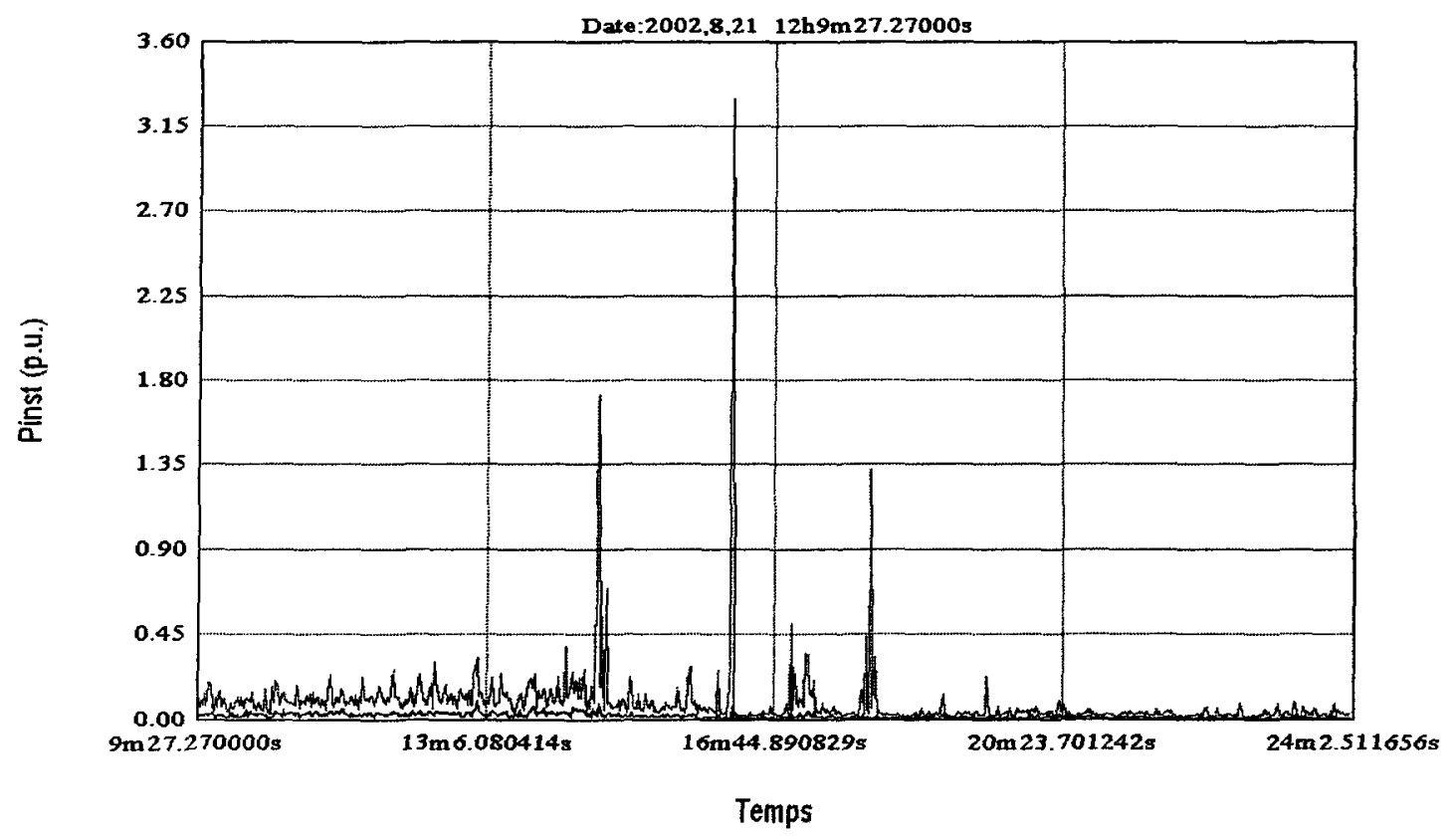

Figure A.28 : Papillotement instantané de la phase $\mathrm{C}$

Les figures A.15, A.16 et A.17 montrent l'enregistrement qui correspond à la sortie du calcul du papillotement tel que décrit dans la norme CEI 61000-4-15. Dans ce calcul, il faut tenir compte que il n'existe pas une relation directe entre l'amplitude du papillotement instantané et celui du Pst. Le Pst est le résultat de l'action combiné de l'amplitude et de la fréquence du papillotement instantané (Tremblay, 2000).

On observe trois instants du temps où le papillotement présente une grande amplitude. Le premier à environ $t_{1}=15 \mathrm{~min}, t_{2}=16$ min et $t_{3}=18 \mathrm{~min}$. Le Pinst à $t_{1}$ montre la valeur la plus petite dans la phase $\mathrm{C}$ dont la valeur maximal est de environ Pinst $=1.8$ p.u. Aux figures A.15, A.16 et A.17, le papillotement Pst à $t_{2}$ est le plus grande à environ 4 p.u. à la phase $\mathrm{A}$ et 3.20 p.u.à la phase $\mathrm{B}$ et $\mathrm{C}$. Pour $\mathrm{t}_{3}$, le papillotement instantané présente une grande amplitude à la phase $\mathrm{B}$ à $3.20 \mathrm{p}$.u ainsi que du Pinst à $\mathrm{t}_{2}$. Pour les phases $\mathrm{A}$ et $\mathrm{C}, \mathrm{le}$ papillotement instantané aura une valeur de 1.50 p.u. et 1.35 p.u. respectivement. 
APPENDICE G

Étude de réponse en fréquence. SimPowerSystems-Simulink ${ }^{\circledR}$ 
Tableau A.12

\begin{tabular}{|c|c|c|c|c|c|c|c|}
\hline & \multicolumn{2}{|c|}{ Début de la ligne } & \multicolumn{2}{|c|}{ Fin de la ligne } & \multicolumn{2}{|c|}{ Charge } \\
\hline & & $\begin{array}{c}\text { Fréquence } \\
(\mathbf{H z})\end{array}$ & $\begin{array}{c}\text { Impédance } \\
\text { (ohm) }\end{array}$ & $\begin{array}{c}\text { Fréquence } \\
(\mathbf{H z})\end{array}$ & $\begin{array}{c}\text { Impédance } \\
\text { (ohm) }\end{array}$ & $\begin{array}{c}\text { Fréquence } \\
(\mathbf{H z})\end{array}$ & $\begin{array}{c}\text { Impédance } \\
\text { (ohm) }\end{array}$ \\
\hline \multicolumn{2}{|c|}{ Non-compensé } & $60 \mathrm{~Hz}$ & 4,465 & $60 \mathrm{~Hz}$ & 27,22 & $60 \mathrm{~Hz}$ & 0,0342 \\
\hline$X_{c}=5$ & $29.54 \%$ & $\begin{array}{l}60 \mathrm{~Hz} \\
800 \mathrm{~Hz}\end{array}$ & $\begin{array}{c}4,466 \\
100 \\
\end{array}$ & $\begin{array}{c}6 \mathrm{~Hz} \\
740 \mathrm{~Hz}\end{array}$ & $\begin{array}{c}193 \\
1055 \\
\end{array}$ & $7 \mathrm{~Hz}$ & 0,1 \\
\hline$X_{c}=8$ & $33,76 \%$ & $\begin{array}{l}60 \mathrm{~Hz} \\
720 \mathrm{~Hz} \\
\end{array}$ & $\begin{array}{c}4,466 \\
100 \\
\end{array}$ & $\begin{array}{c}6 \mathrm{~Hz} \\
740 \mathrm{~Hz}\end{array}$ & $\begin{array}{c}195 \\
1068\end{array}$ & $6 \mathrm{~Hz}$ & 0,101 \\
\hline$X_{c}=9$ & $37,97 \%$ & $\begin{array}{c}60 \mathrm{~Hz} \\
720 \mathrm{~Hz}\end{array}$ & $\begin{array}{c}4,466 \\
100 \\
\end{array}$ & $\begin{array}{c}6 \mathrm{~Hz} \\
740 \mathrm{~Hz}\end{array}$ & $\begin{array}{c}193,5 \\
1068 \\
\end{array}$ & $6 \mathrm{~Hz}$ & 0,093 \\
\hline$X_{c}=10$ & $42,19 \%$ & $\begin{array}{r}60 \mathrm{~Hz} \\
745 \mathrm{~Hz}\end{array}$ & $\begin{array}{c}4,466 \\
1071 \\
\end{array}$ & $\begin{array}{c}8 \mathrm{~Hz} \\
740 \mathrm{~Hz}\end{array}$ & $\begin{array}{r}225 \\
1070 \\
\end{array}$ & $7 \mathrm{~Hz}$ & 0,115 \\
\hline$X_{c}=11$ & $46,41 \%$ & $\begin{array}{l}60 \mathrm{~Hz} \\
745 \mathrm{~Hz}\end{array}$ & $\begin{array}{c}4,466 \\
108 \\
\end{array}$ & $\begin{array}{c}8 \mathrm{~Hz} \\
763 \mathrm{~Hz}\end{array}$ & $\begin{array}{r}205 \\
1130 \\
\end{array}$ & $8 \mathrm{~Hz}$ & 0,104 \\
\hline$X_{c}=12$ & $50,63 \%$ & $\begin{array}{r}60 \mathrm{~Hz} \\
722 \mathrm{~Hz}\end{array}$ & $\begin{array}{l}4,466 \\
100,2 \\
\end{array}$ & $\begin{array}{c}8 \mathrm{~Hz} \\
740 \mathrm{~Hz}\end{array}$ & $\begin{array}{c}225 \\
1070 \\
\end{array}$ & $7 \mathrm{~Hz}$ & 0,115 \\
\hline $\begin{array}{l}X_{c}=13 \\
f\end{array}$ & $54,85 \%$ & $\begin{array}{c}60 \mathrm{~Hz} \\
744 \mathrm{~Hz}\end{array}$ & $\begin{array}{c}4,466 \\
107,25\end{array}$ & $\begin{array}{c}8 \mathrm{~Hz} \\
763 \mathrm{~Hz}\end{array}$ & $\begin{array}{c}234 \\
1130\end{array}$ & $7 \mathrm{~Hz}$ & 0,12 \\
\hline$X_{c}=14$ & $59,07 \%$ & $\begin{array}{r}60 \mathrm{~Hz} \\
722 \mathrm{~Hz}\end{array}$ & $\begin{array}{l}4,466 \\
100,5 \\
\end{array}$ & $\begin{array}{r}8 \mathrm{~Hz} \\
763 \mathrm{~Hz} \\
\end{array}$ & $\begin{array}{c}231 \\
1130 \\
\end{array}$ & $8 \mathrm{~Hz}$ & 0,12 \\
\hline$X c=15$ & $63,29 \%$ & $\begin{array}{r}60 \mathrm{~Hz} \\
722 \mathrm{~Hz}\end{array}$ & $\begin{array}{l}4,466 \\
100,2 \\
\end{array}$ & $\begin{array}{c}8 \mathrm{~Hz} \\
763 \mathrm{~Hz}\end{array}$ & $\begin{array}{r}225 \\
1130 \\
\end{array}$ & $8 \mathrm{~Hz}$ & 0,116 \\
\hline$X c=16$ & $67,51 \%$ & $\begin{array}{c}60 \mathrm{~Hz} \\
722 \mathrm{~Hz}\end{array}$ & $\begin{array}{l}4,466 \\
100,5\end{array}$ & $\begin{array}{c}9 \mathrm{~Hz} \\
763 \mathrm{~Hz}\end{array}$ & $\begin{array}{c}213 \\
1130 \\
\end{array}$ & $8 \mathrm{~Hz}$ & 0,112 \\
\hline$X_{c}=17$ & $71,73 \%$ & $\begin{array}{c}60 \mathrm{~Hz} \\
722,5 \mathrm{~Hz}\end{array}$ & $\begin{array}{c}4,466 \\
100,28\end{array}$ & $\begin{array}{c}10 \mathrm{~Hz} \\
763 \mathrm{~Hz}\end{array}$ & $\begin{array}{l}228,5 \\
1130 \\
\end{array}$ & $10 \mathrm{~Hz}$ & 0,1157 \\
\hline
\end{tabular}




\section{ANNEXE H}

Programme développé dans le logiciel Matlab ${ }^{\circledR}$.

Calcul du taux de compensation série optimal et sa position. 
$\% \quad$ PROJET DE MAITRISE EN INGENIERIE- UNIVERSITE DU QUEBEC EN ABITIBI TEMINSCAMINGUE- DEPARTEMENT DES SCIENCES APPLIQUÉES

$\%$

$\%$ Nom : Fernandez Ramirez

$\%$ Prenom : Eder Ernesto

$\%$ Directeur : René Wamkeue,Ing. $\mathrm{PhD}$.

$\%$ Co-directeur: Nahi Kandil,Ing. PhD.

$\%$ Date : Janvier 2005

$\%$ Fichier : Programme calcule des éléments de la méthode d'optimisation

$\% \quad$ du taux de compensation série d'une ligne de distribution

clear all

\%Entre des paramètres électriques de la ligne de distribution et compensation série

$\mathrm{L}=$ input(' Entrez la distance total de la ligne de distribution (Kms): ')

$\mathrm{RK}=$ input(' Entrez la valeur de la résistance de la ligne $\mathrm{R}(\mathrm{ohm} / \mathrm{Km}):$ ')

$\mathrm{XC}=$ input(' Entrez la valeur de XC: ')

$\mathrm{XL1}=$ input(' Entrez la valeur de la réactance inductive de la ligne $\mathrm{XL}(\mathrm{ohm} / \mathrm{Km}):$ ')

L1=input(' Entrez la position du banc de condensateurs série $\mathrm{Xc}(\mathrm{Kms})$ : ')

$\%$ Contrainte Inv Icc

if $((\mathrm{L} 1 *(\mathrm{XL} 1))<\mathrm{XC})$

disp('la valeur de réactance capacitive Xc peut produire une inversion du courant Icc') else

$\%$ Contraintes hunting

if $\left(\left(\left(\mathrm{RK}^{*} \mathrm{~L}\right) /((\mathrm{XL} 1 * \mathrm{~L})-\mathrm{XC})\right)>1\right)$

disp('La valeur de Xc peut produire des oscillations de vitesse de rotation de moteur');

else

\section{L2=L-L1}

open('SCOM.mdl')

sim('SCOM.mdl')

$\operatorname{display}\left(\mathrm{V}_{-} 1\left(\right.\right.$ length $\left.\left.\left(\mathrm{V}_{-} 1\right)\right)\right)$;

display(V_2(length(V_2)));

display(V_aval(length(V_aval)));

$\%$ Imprimer les valeurs de variation de tension en aval du condensateur et a la charge $\mathrm{A}=\left[\mathrm{V} \_1 ; \mathrm{V} \_\right.$aval; $\left.\mathrm{V}_{-} 2\right]$;

$\mathrm{V} 1=\overline{\mathrm{A}}(1, ;)$ 
Vaval $=\mathrm{A}(2,:)$

$\mathrm{V} 2=\mathrm{A}(3,:)$

$\%$ Calcul de la transfert de puissance

$$
\mathrm{P}=\left(\left((\mathrm{V} 1 * \mathrm{~V} 2)-\left(\mathrm{V} 2^{\wedge} 2\right)^{*} \cos \left(\left(\mathrm{RK}^{*} \mathrm{~L}\right) /\left(\mathrm{XL} 1^{*} \mathrm{~L}\right)-\mathrm{XC}\right)\right) /\left(\left(\mathrm{XL} 1^{*} \mathrm{~L}\right)-\mathrm{XC}\right)\right)
$$

$\%$ Calcule de la variation de tension a la charge $\mathrm{dV}=\mathrm{V} 1-\mathrm{V} 2$;

disp(' La variation de tension a la charge est: ') $\operatorname{disp}(\mathrm{dV})$

$\%$ Calcule de la variation de tension en aval du condensateur série

disp('Calcule de la variation de tension en aval du banc de condensateur série') $\mathrm{dVXc}=\mathrm{V} 1-$ Vaval;

disp('La variation de tension en aval du condensateur est: ') $\operatorname{disp}(\mathrm{dVXc})$

end

end 


\section{APPENDICE I}

Profils de tension en amont et en aval du banc de condensateur série 


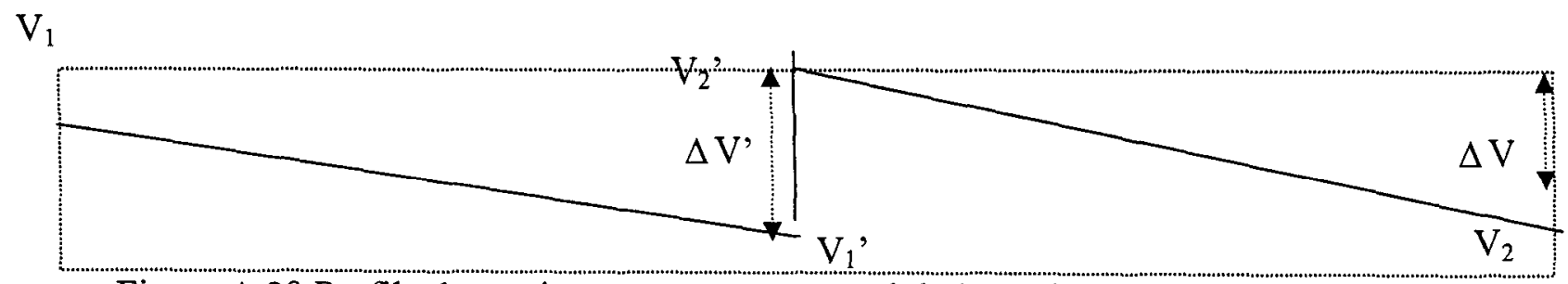

Figure A.29 Profile de tension en amont et en aval du banc de condensateurs série

Tableau A.13

Profile de tension en amont et en aval du banc de condensateurs série

\begin{tabular}{|c|c|c|c|c|c|c|c|c|c|c|c|c|c|c|c|c|}
\hline \multicolumn{2}{|c|}{ Ohms $(\Omega)$} & \multicolumn{3}{|c|}{$10 \mathrm{Km}$} & \multicolumn{3}{|c|}{$20 \mathrm{Km}$} & \multicolumn{3}{|c|}{$30 \mathrm{Km}$} & \multicolumn{3}{|c|}{$40 \mathrm{Km}$} & \multicolumn{3}{|c|}{$50 \mathrm{Km}$} \\
\hline \multirow[t]{2}{*}{5} & $V_{2}$ & 24,86 & $\mathrm{dv}$ & 0.93 & 24,67 & $d V$ & 0,74 & 24.48 & $\mathrm{dV}^{\prime}$ & 0.55 & 24,27 & $\mathrm{dV}^{\prime}$ & 0,34 & 24,06 & $d V^{\prime}$ & 0,13 \\
\hline & $v_{2}$ & 23,96 & $d V$ & 1,04 & 23,79 & $\mathrm{dV}$ & 1,21 & 23,78 & $d V$ & 1,22 & 23,8 & $\mathrm{dV}$ & 1,2 & 23,82 & $\mathrm{dV}$ & 1,18 \\
\hline \multirow[t]{2}{*}{7} & $v_{2}$ & 24,91 & $\mathrm{dV}$ & 0,98 & 24.74 & $\mathrm{dV}$ & 0,81 & 24,55 & dV & 0.62 & 24,34 & $d v$ & 0,41 & 24,1 & $d V$ & 0,17 \\
\hline & V2 & 23,82 & $\mathrm{dV}$ & 1,18 & 23,84 & $\mathrm{dV}$ & 1,16 & 23,86 & $d V$ & 1,14 & 23,87 & $d V$ & 1,13 & 23,89 & $d V$ & 1,11 \\
\hline \multirow[t]{2}{*}{9} & $v_{2}$ & 24,96 & $\mathrm{dV}$ & 1,03 & 24,79 & $d V^{\prime}$ & 0,86 & 24,6 & $\mathrm{dy}$ & 0.67 & 24.44 in & $\mathrm{dV}$ & 0,48 & 24,2 & $\mathrm{dV}$ & 0,27 \\
\hline & v2 & 24,08 & $\mathrm{dV}$ & 0,92 & 23,89 & $\mathrm{dV}$ & 1,11 & 23,93 & $\mathrm{dV}$ & 1,07 & 23,94 & $d V$ & 1,06 & 23,95 & $d V$ & 1,05 \\
\hline \multirow[t]{2}{*}{11} & V $2^{\prime}$ & 25,01 & $\mathrm{dV}$ & 1,08 & 24,86 & $\mathrm{dV}$ & 0,93 & 24,67 & $\mathrm{dV}$ & 0,74 & 24,48 & $\mathrm{dV}^{\prime}$ & 0,55 & 24,27 & $\mathrm{dV}$ & 0,34 \\
\hline & v2 & 23,94 & $d V$ & 1,06 & 23,99 & $d V$ & 1,01 & 23,98 & $d V$ & 1,02 & 24,01 & $d V$ & 0,99 & 24,04 & $d V$ & 0,96 \\
\hline \multirow[t]{2}{*}{13} & v2 & 25,07 & $\mathrm{dV}$ & 1,14 & 24,89 & $\mathrm{dV}$ & 0,96 & 24,72 & $\mathrm{dV}$ & 0,79 & 24,55 & $\mathrm{dv}^{\prime}$ & 0,62 & 24,34 & $\mathrm{dV}$ & 0,41 \\
\hline & v2 & 23,98 & $d V$ & 1,02 & 24,01 & $\mathrm{dV}$ & 0,99 & 24,04 & $\mathrm{dV}$ & 0,96 & 24,08 & $\mathrm{dV}$ & 0,92 & 24,11 & $d V$ & 0,89 \\
\hline 15 & $v_{2}$ & 25,17 & $\mathrm{dV}$ & 1,24 & 24,89 & $\mathrm{dV}$ & 0,96 & 24,83 & $\mathrm{av}^{\prime}$ & 0.9 & 24,58 & dv' & 0,65 & 24,41 & $d V^{v}$ & 0,48 \\
\hline
\end{tabular}




\section{APPENDICE J}

Simulations effectuées dans SimPowersystems-Simulink ${ }^{\circledR}$ 
Simulations de l'essai d'un court circuit monophasé en amont du condensateur série à $t=0.5 \mathrm{~s}$

\section{Réseau de distribution radial sans compensation}
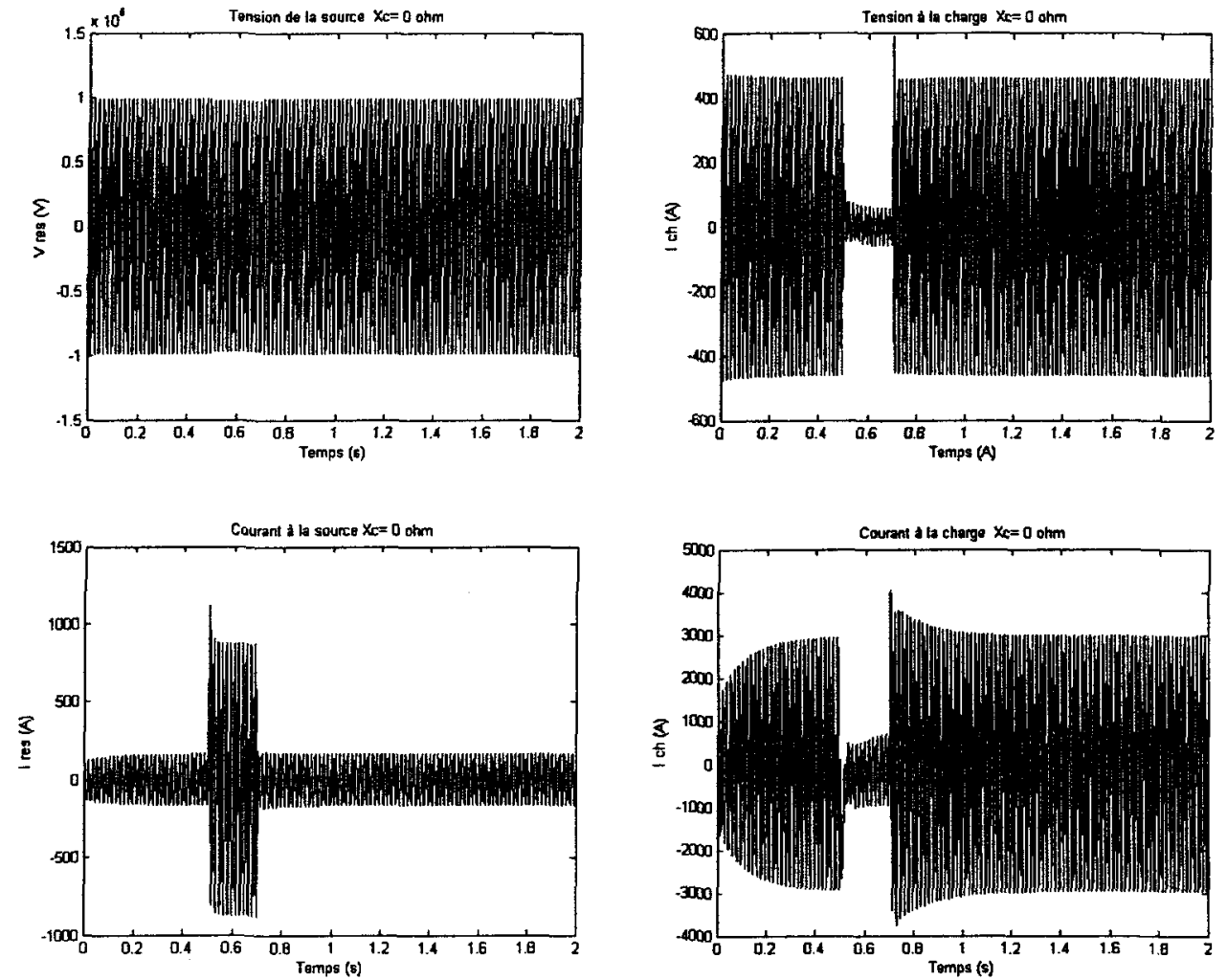

Figure A.30 : Tension et courant à la source et à la charge

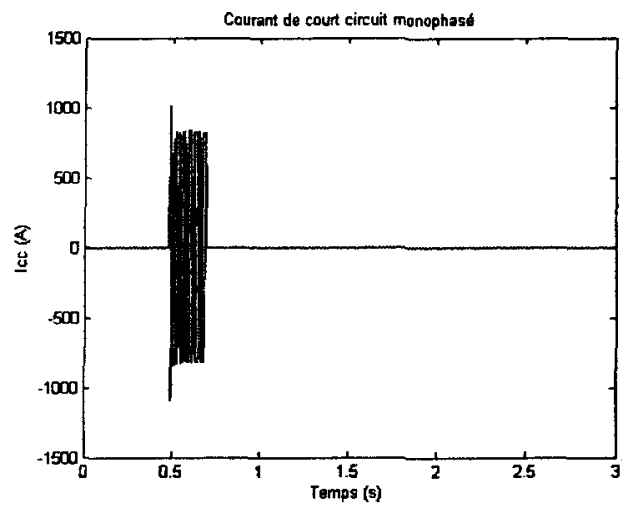

Figure A.31 : Courant de court circuit monophasé 

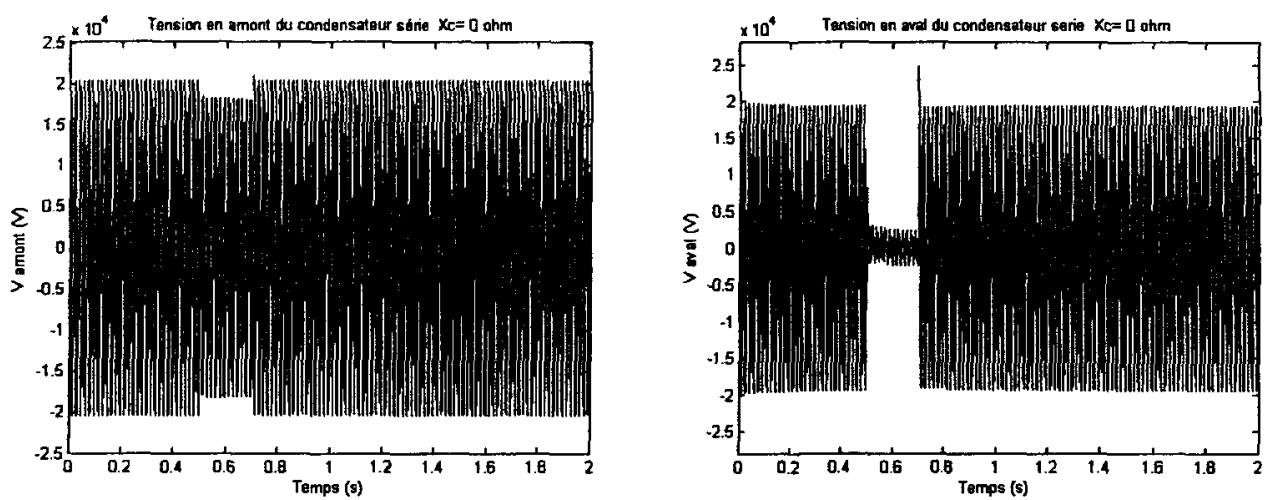

Figure A.32 : Tension en amont et en aval du condensateur série
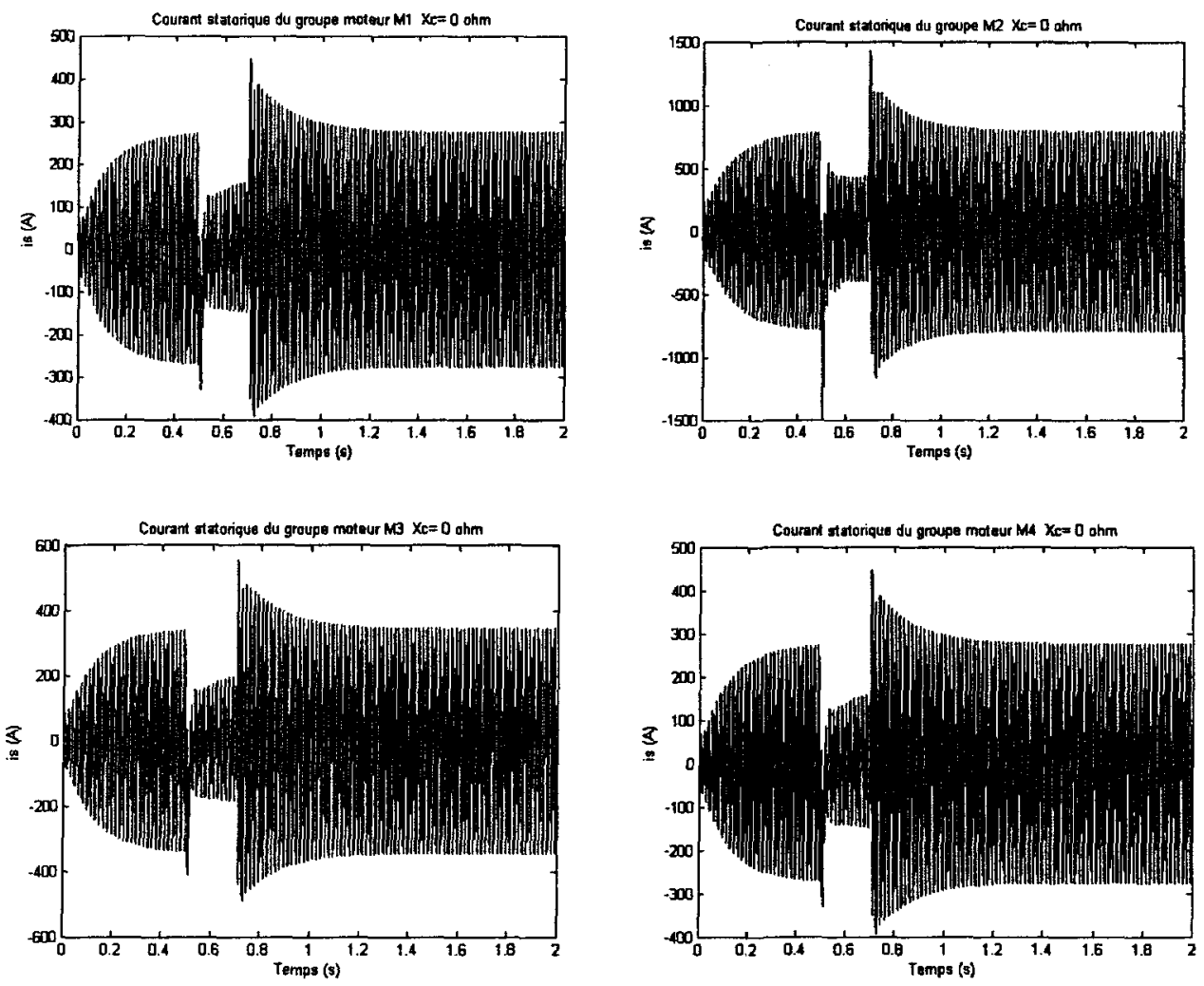

Figure A.33 : Courant statorique des groupes de moteurs asynchrones 

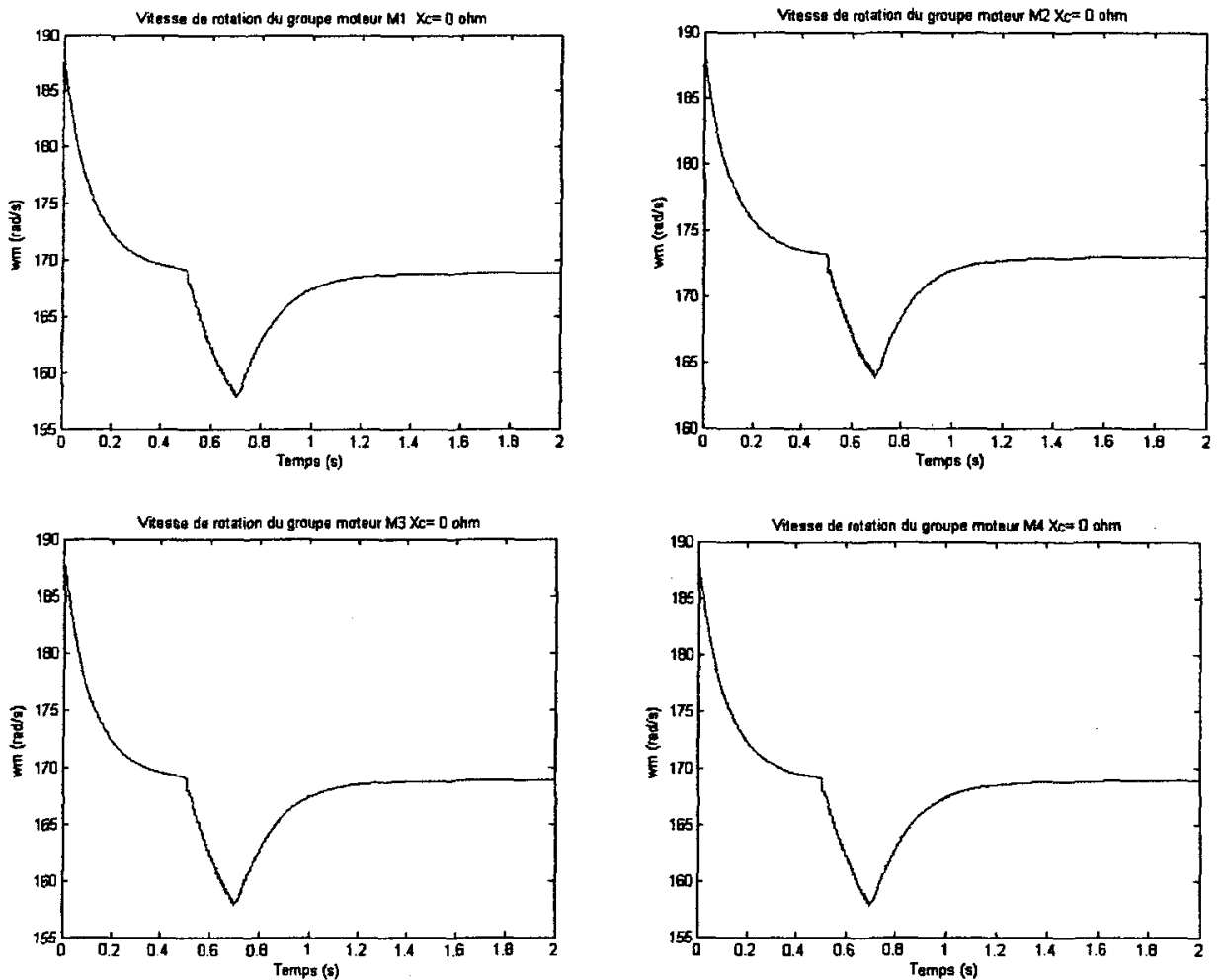

Figure A.34 : Vitesse de rotation des groupes moteurs
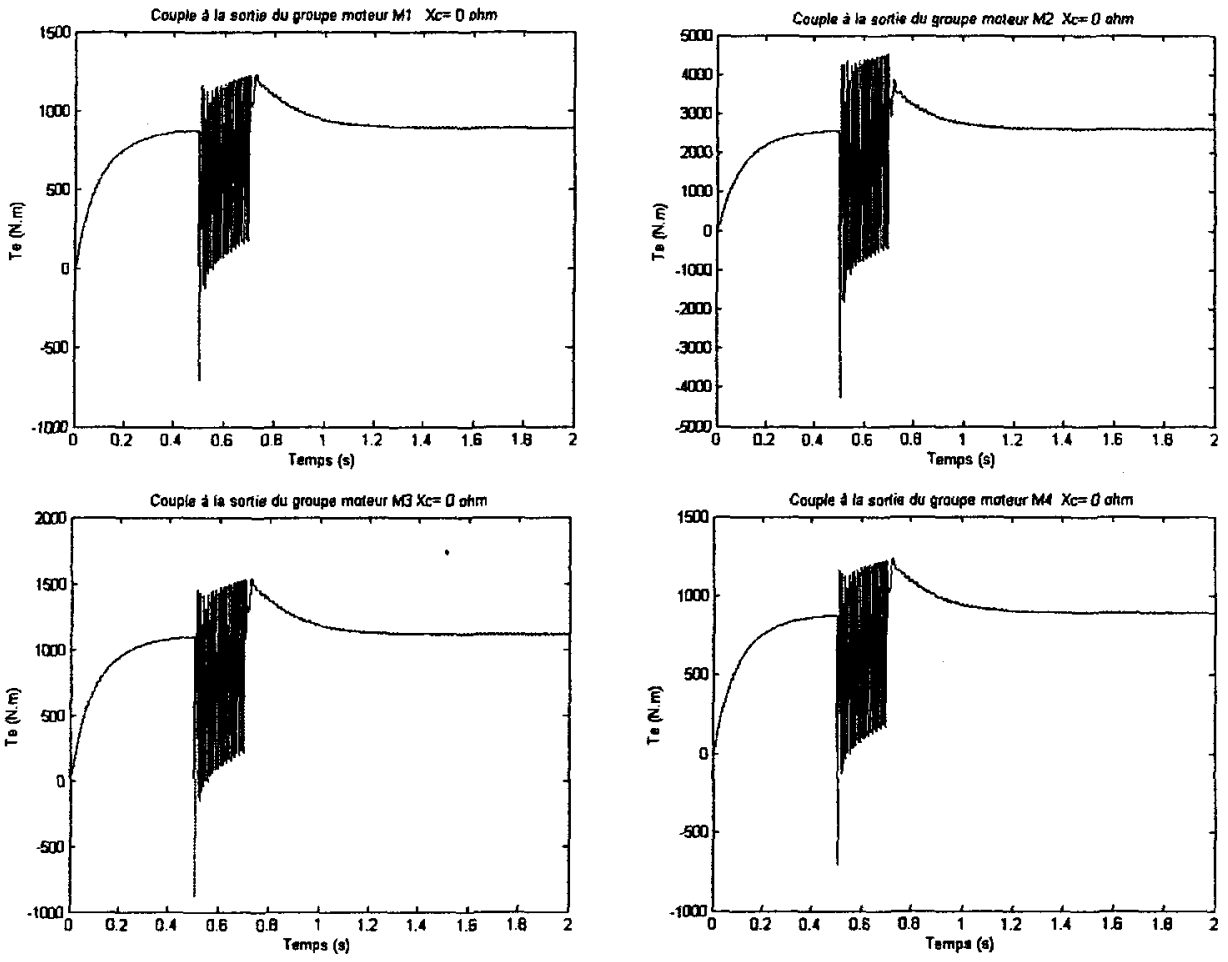

Figure A.35 : Couple à la sortie des groupes moteurs 
Réseau de distribution radial à $\tau=54.85 \% \mathrm{Xc}=13 \Omega$
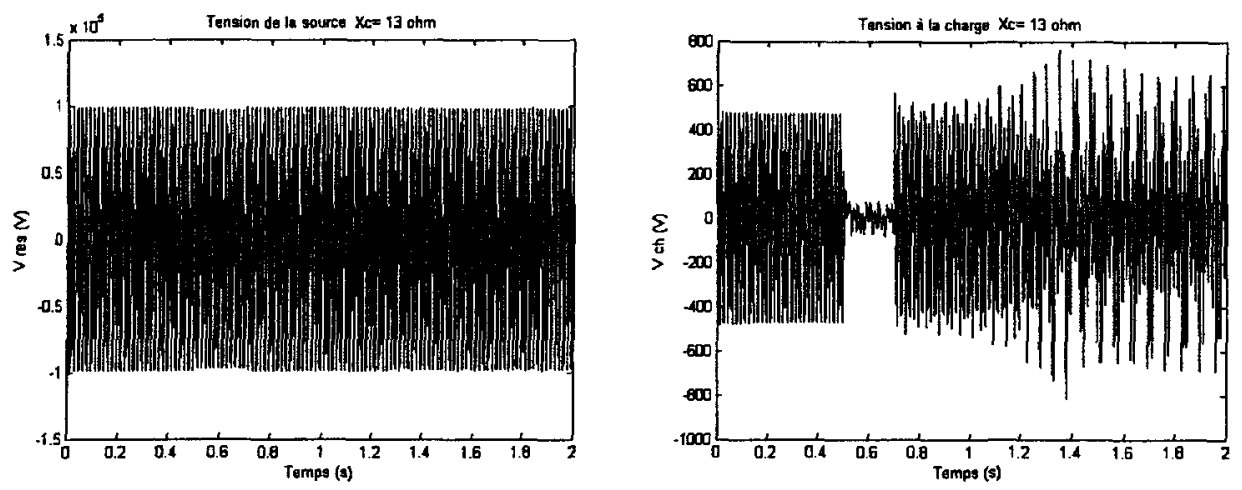

Figure A.36 : Tension de la source et à la charge
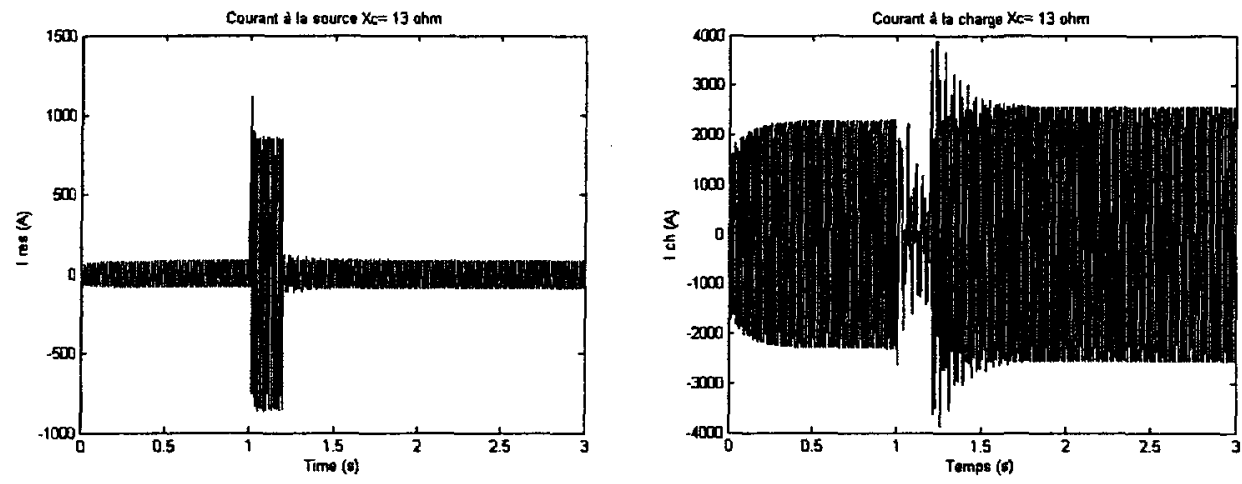

Figure A.37 : Courant à la source et à la charge
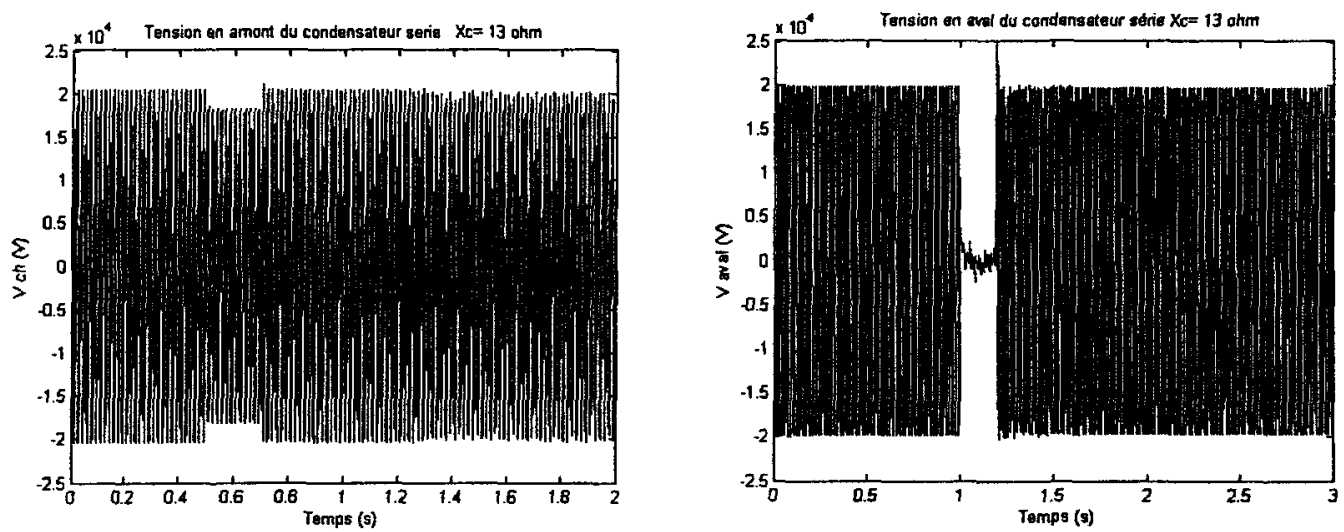

Figure A.38 : Tension en amont et en aval du condensateur série 

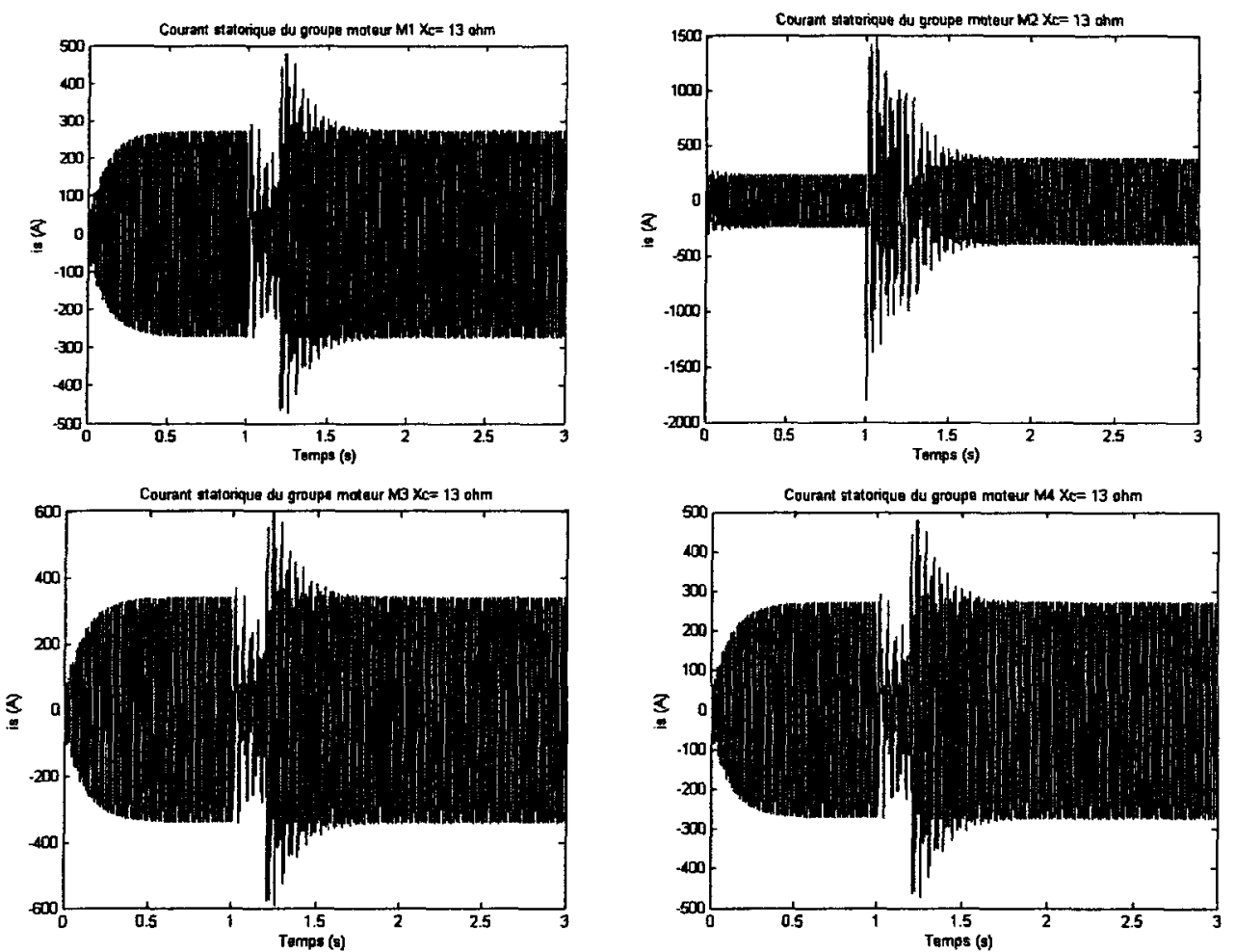

Figure A.39 : Courants statoriques des groupes moteurs
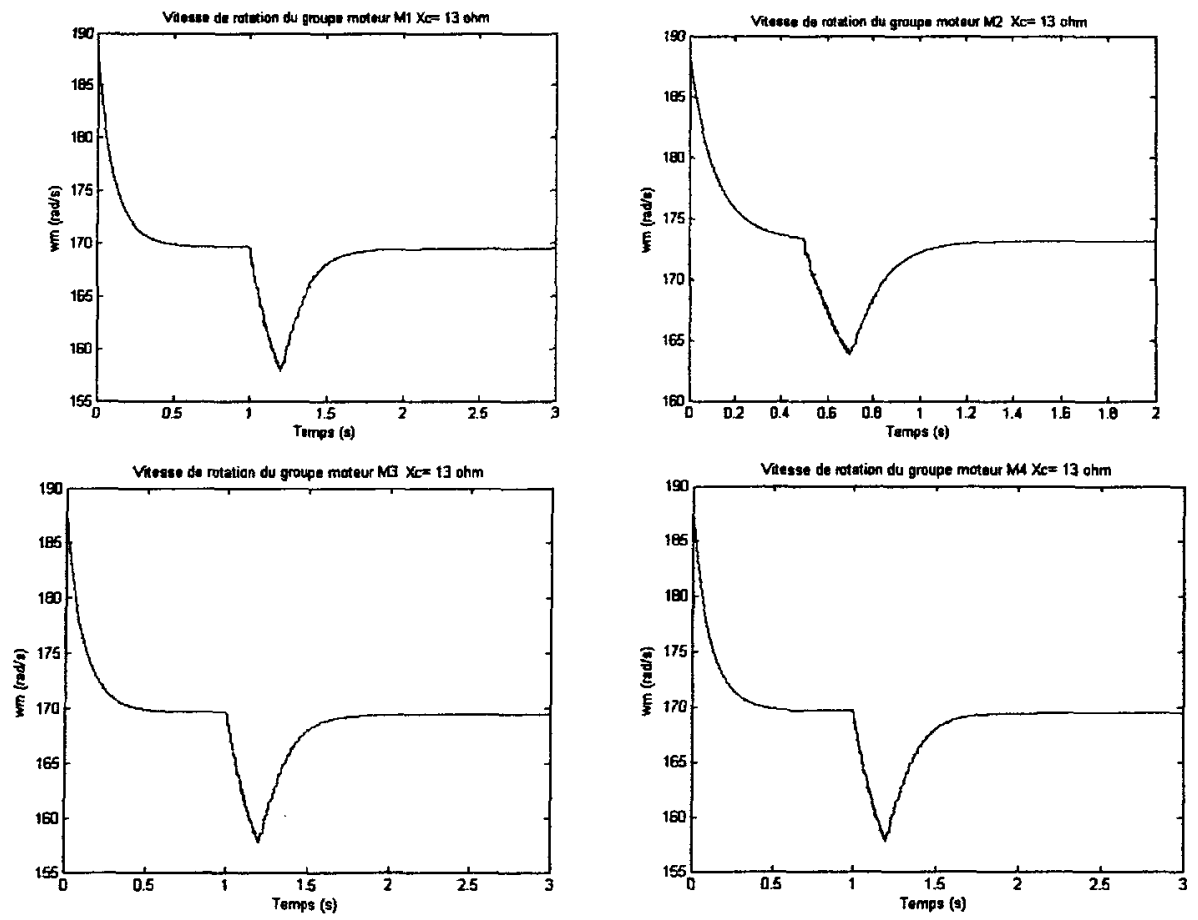

Figure A.40 : Vitesse de rotation des groupes moteurs 

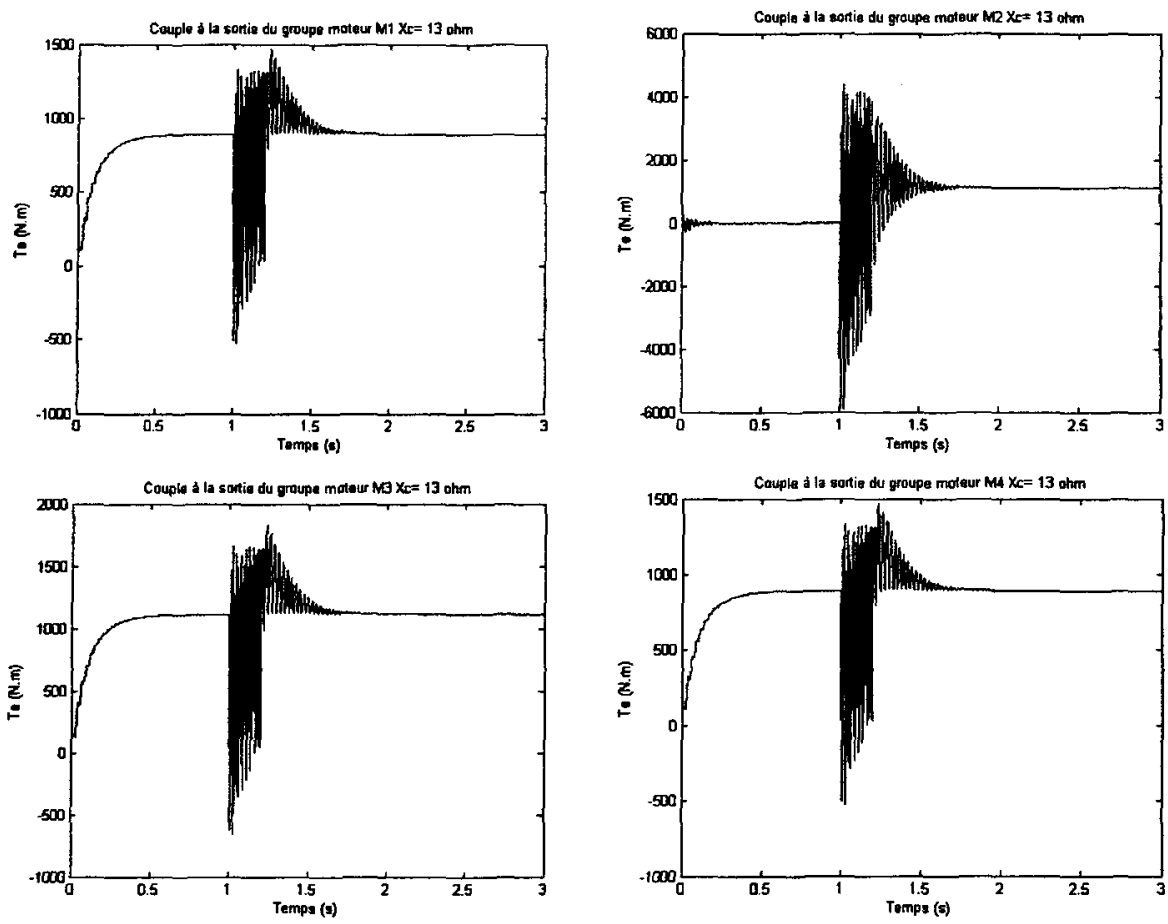

Figure A.41: Couple à la sortie des groupes moteurs

Réseau de distribution radial à $\tau=66 \% \mathrm{Xc}=15.66 \Omega$
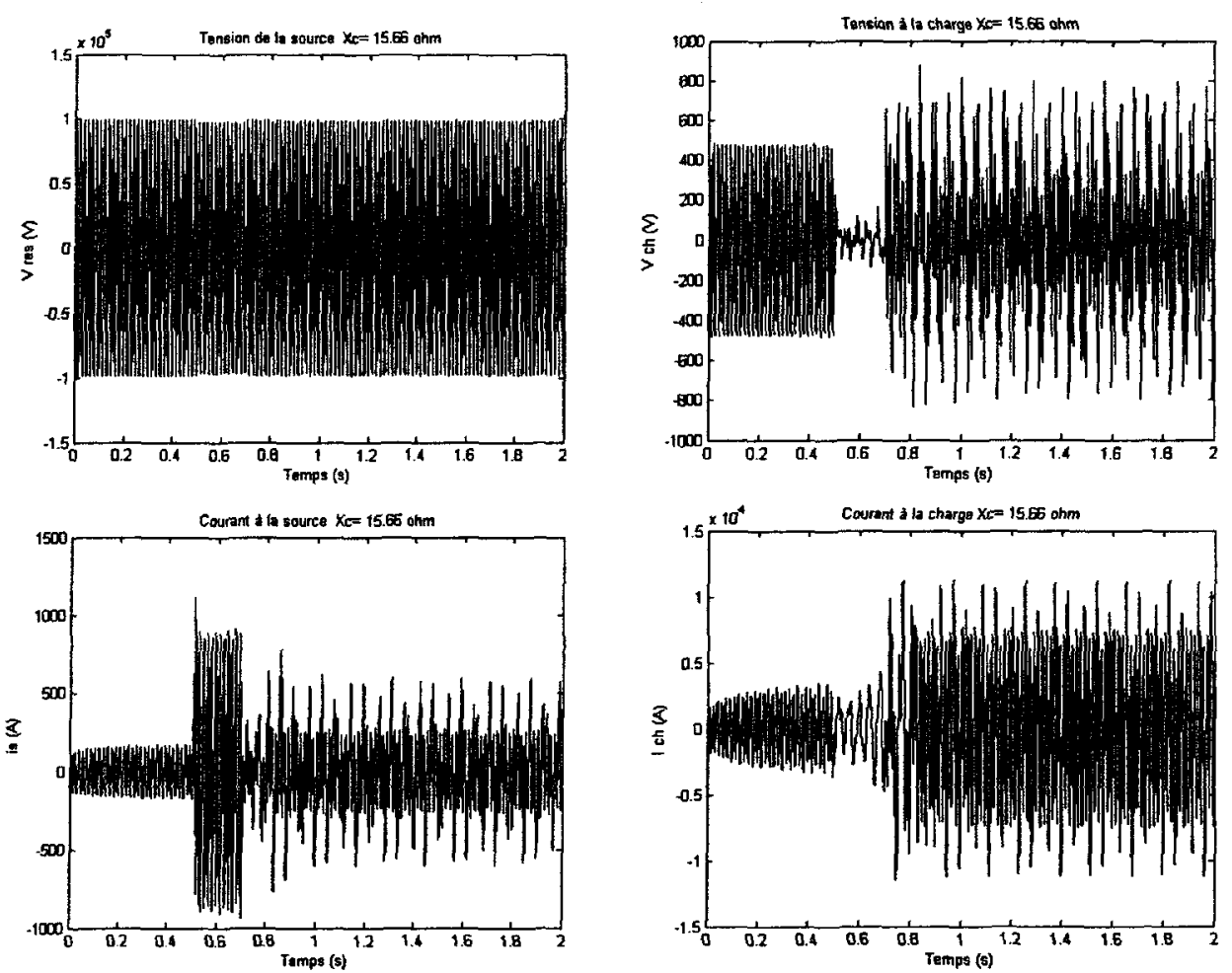

Figure A.42 : Courant et courant à la source et à la charge 

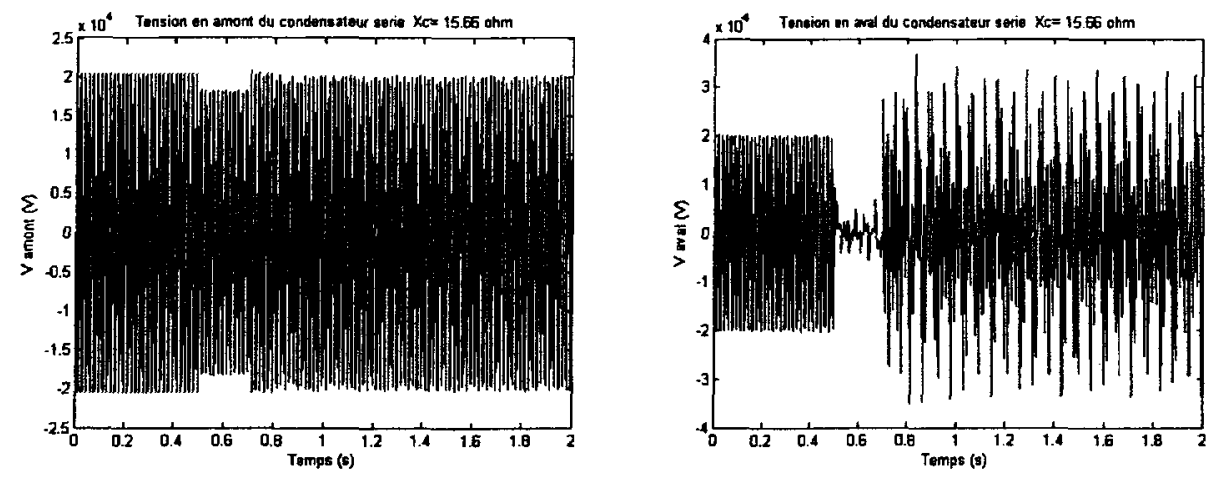

Figure A.43 : Tension en amont et en aval du condensateur série
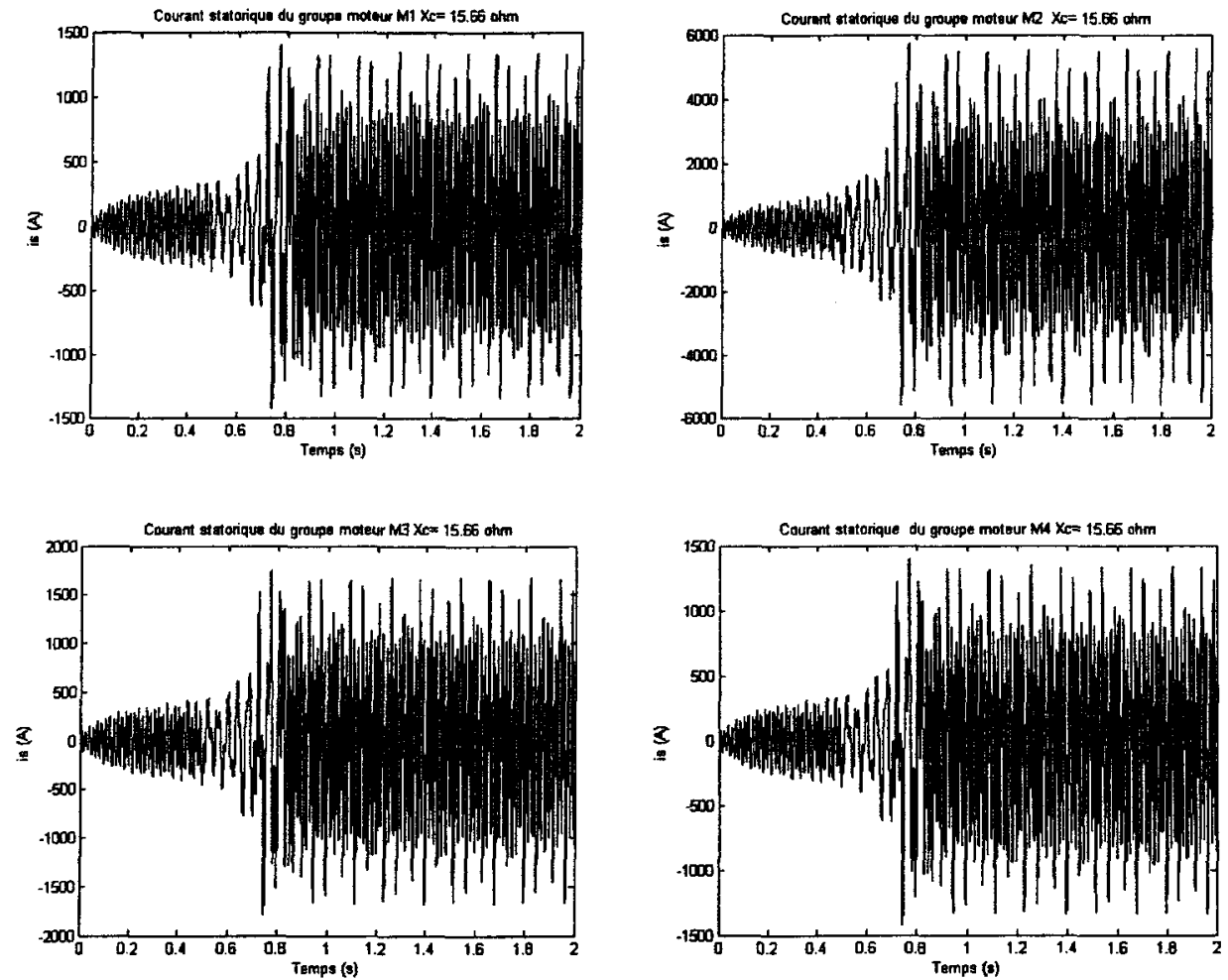

Figure A.44 : Courants statoriques des groupes moteurs 

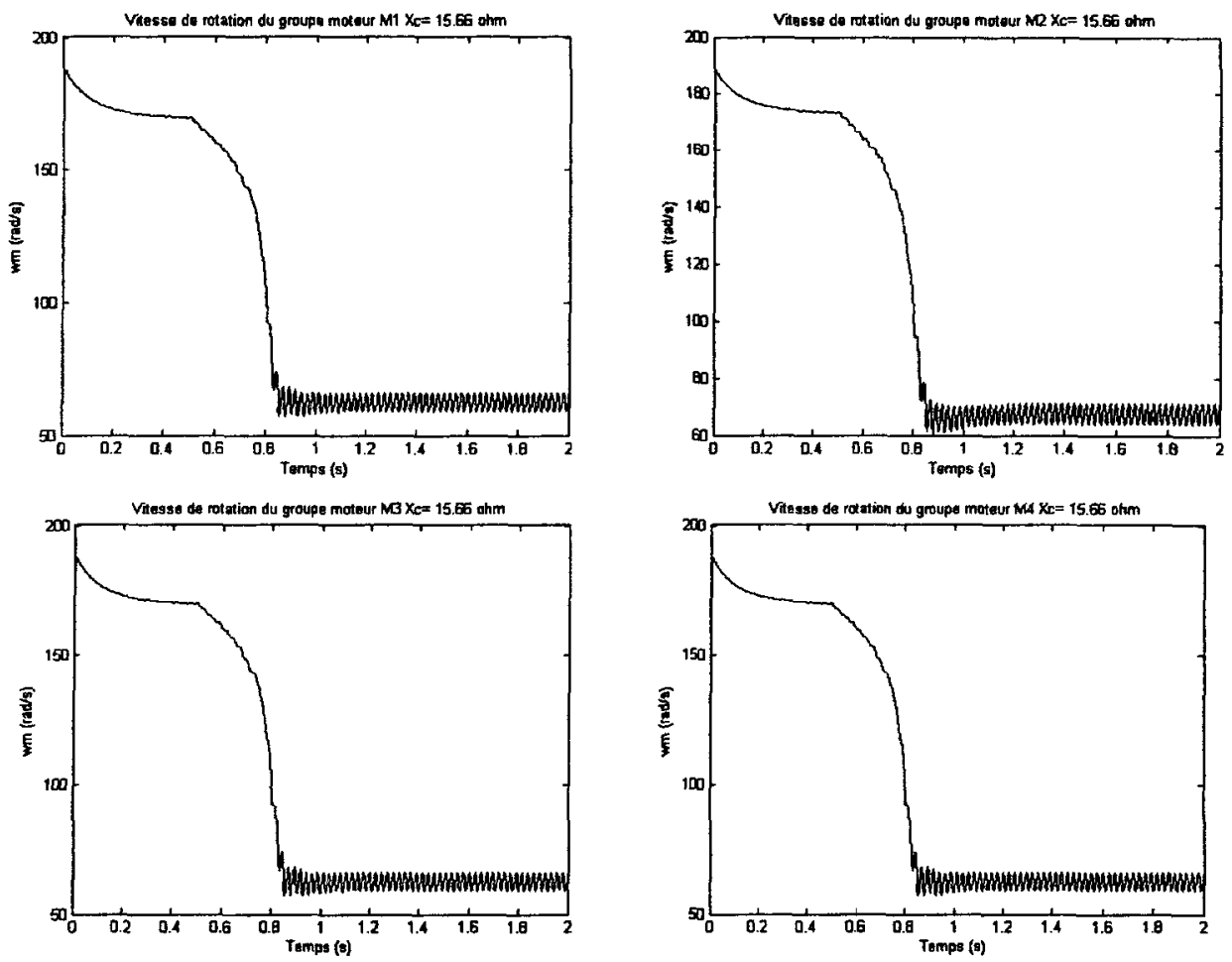

Figure A.45 : Vitesse de rotation des groupes moteurs
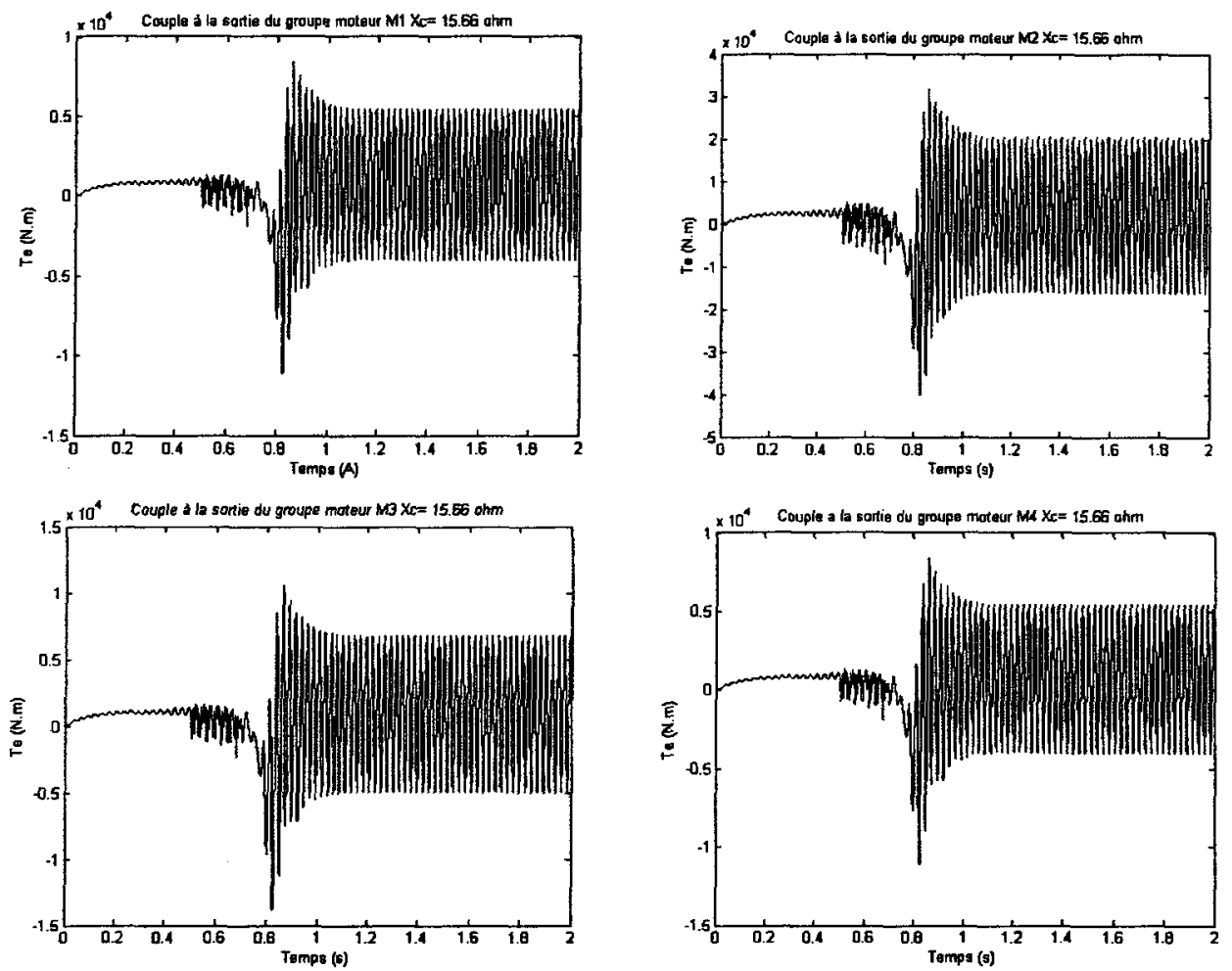

Figure A.46 : Couple à la sortie des groupes moteurs 
Simulations de l'essai d'un court circuit triphasé en amont du condensateur série à $t=0.5 \mathrm{~s}$

Réseau de distribution radial sans compensation série
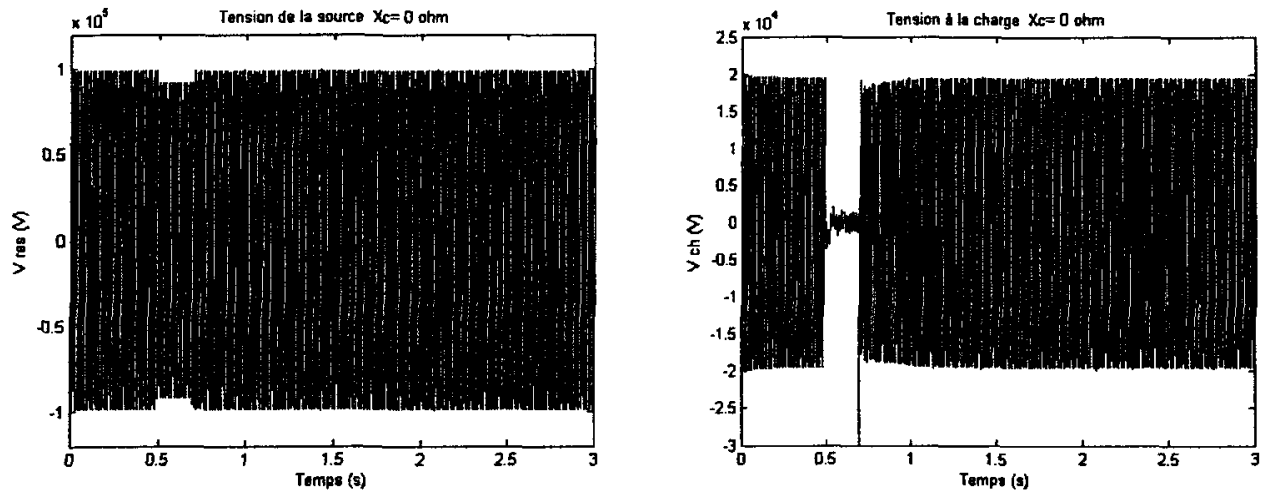

Figure A.47 : Tension de la source et à la charge
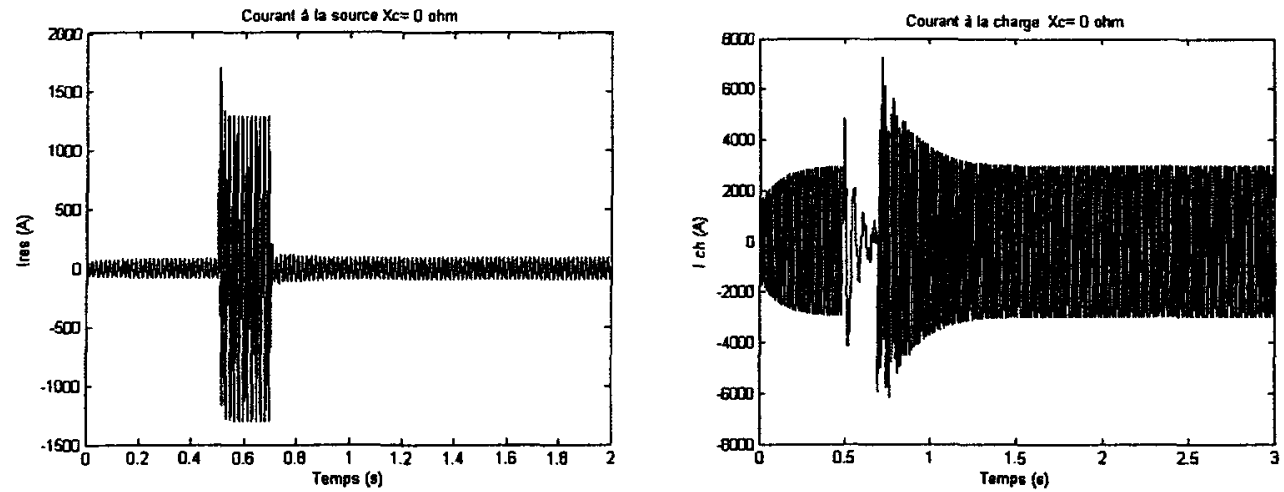

Figure A.48 : Courant à la source et à la charge
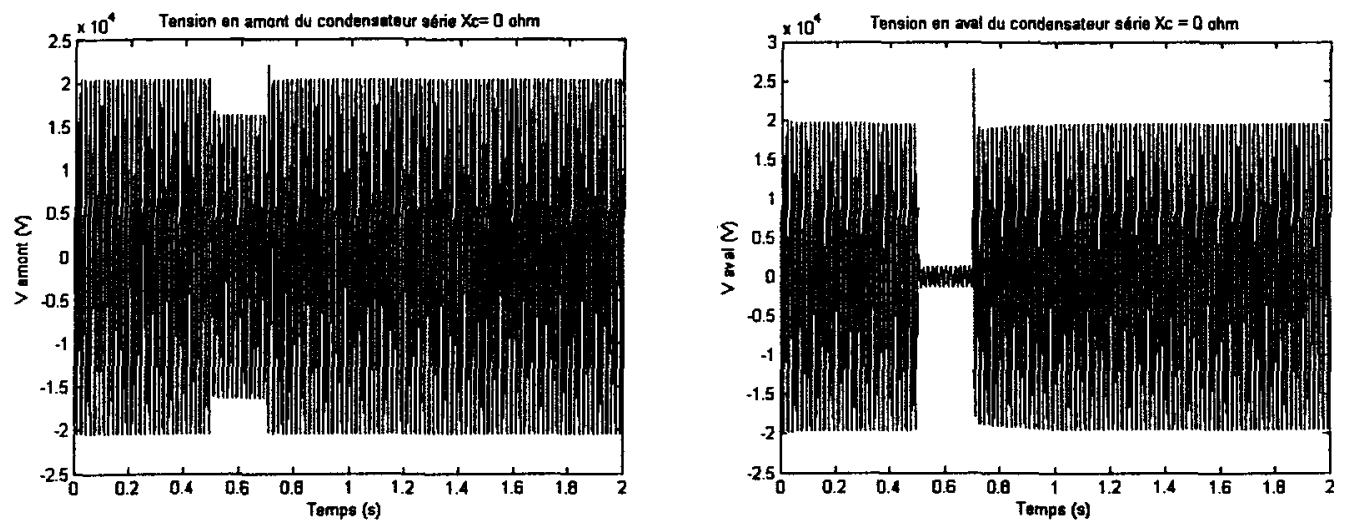

Figure A.49: Tension en amont et en aval du condensateur série 

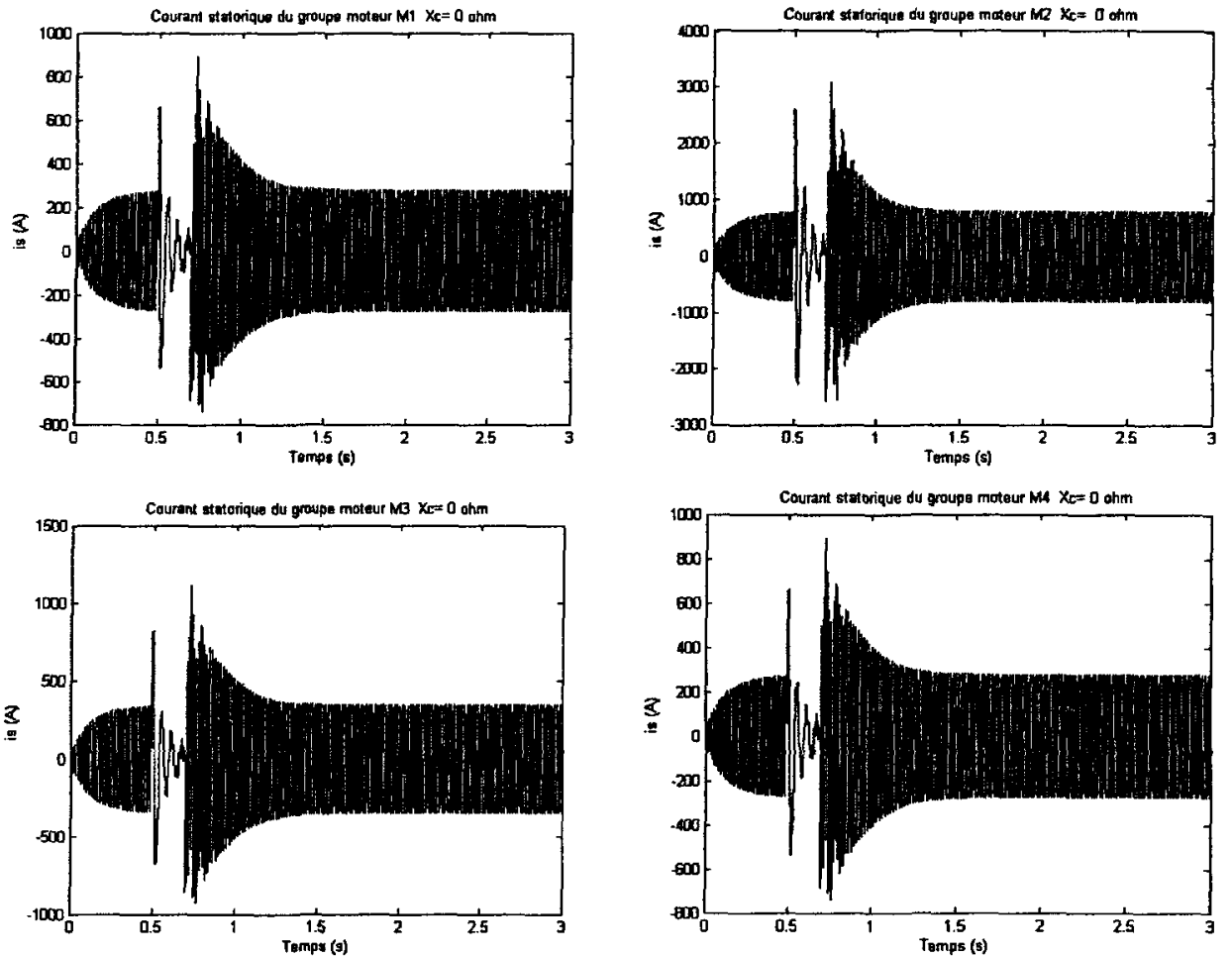

Figure A.50 : Courants statoriques des groupes moteurs
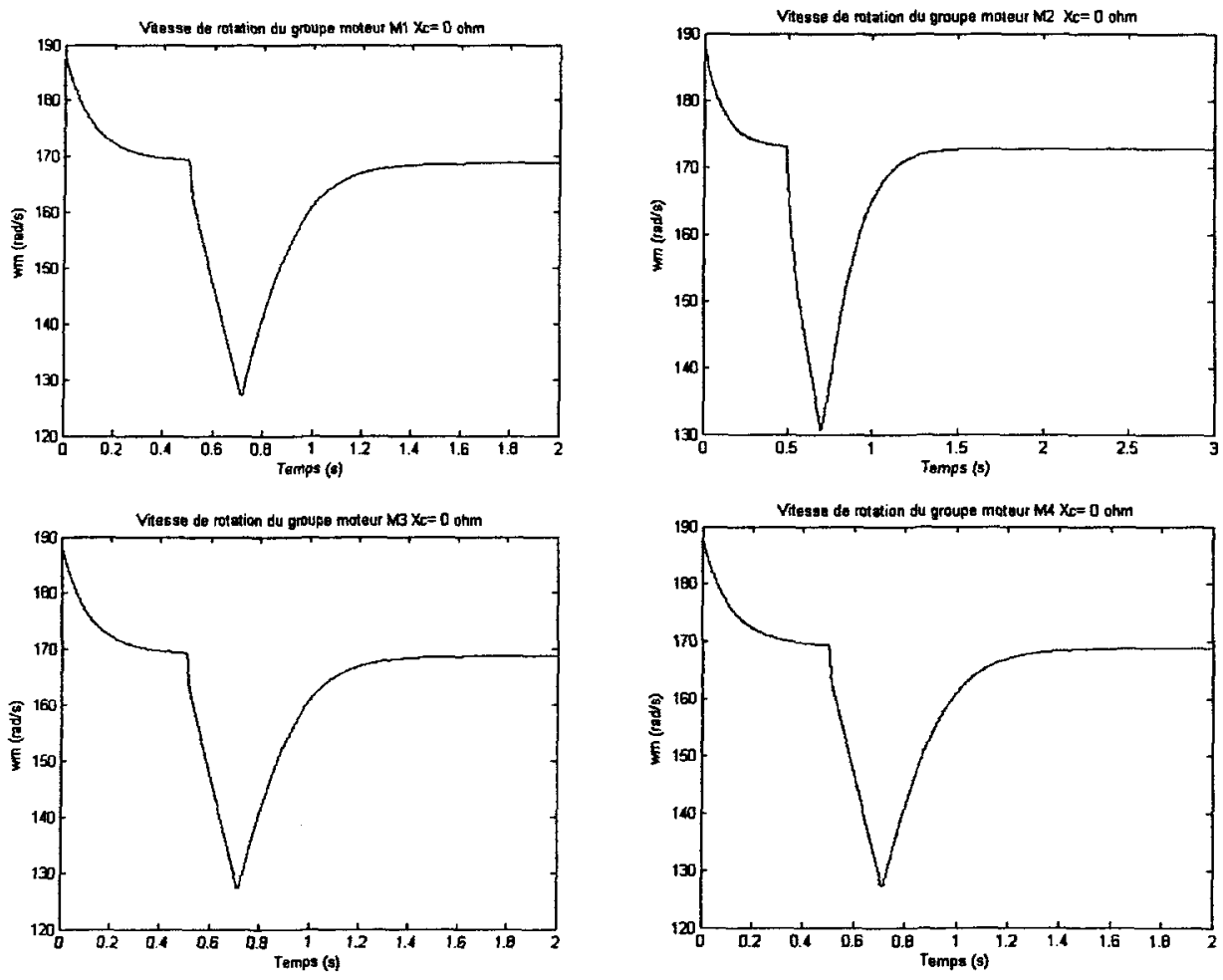

Figure A.51 : Vitesse de rotation des groupes moteurs 

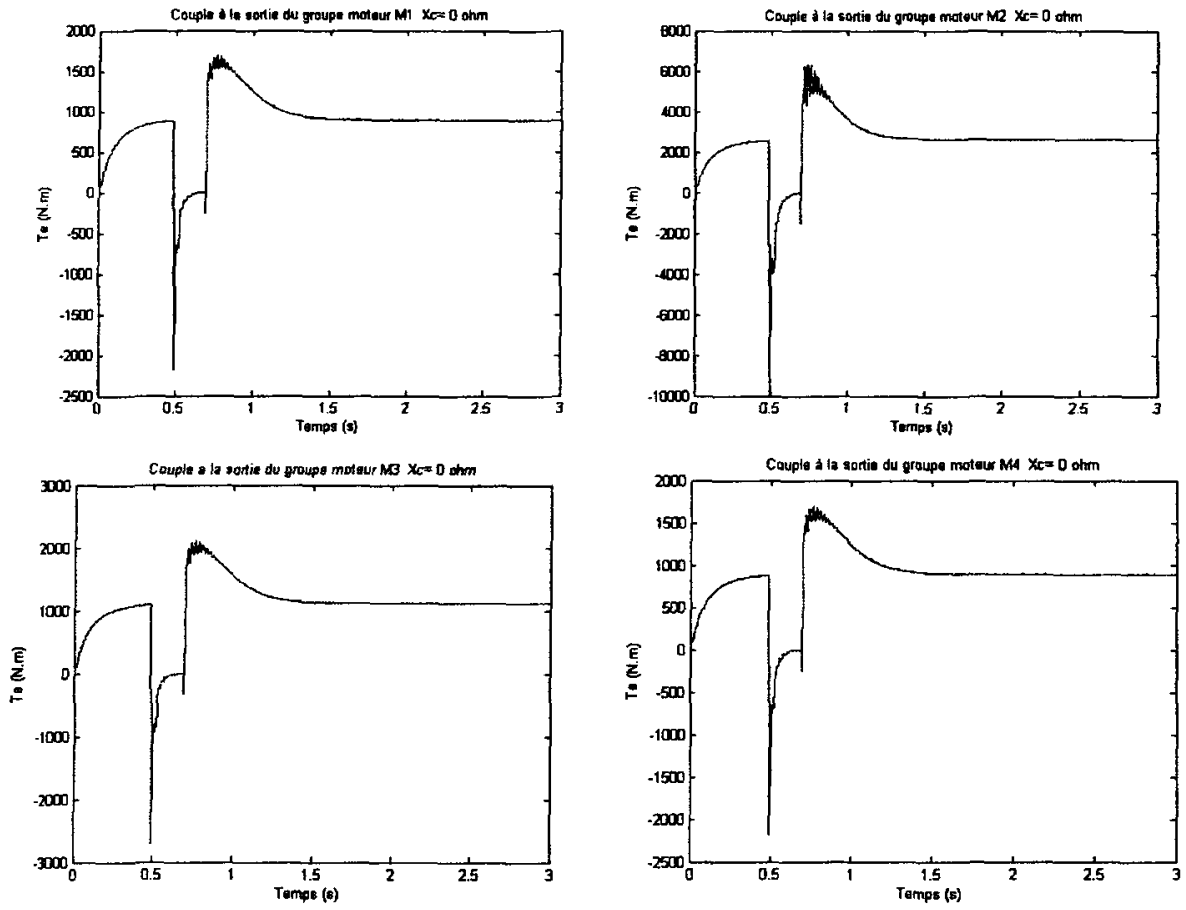

Figure A.52 : Couple à la sortie des groupes moteurs

\section{Réseau de distribution radial à $\tau=33.75 \% \mathrm{Xc}=8 \mathrm{ohm}$}
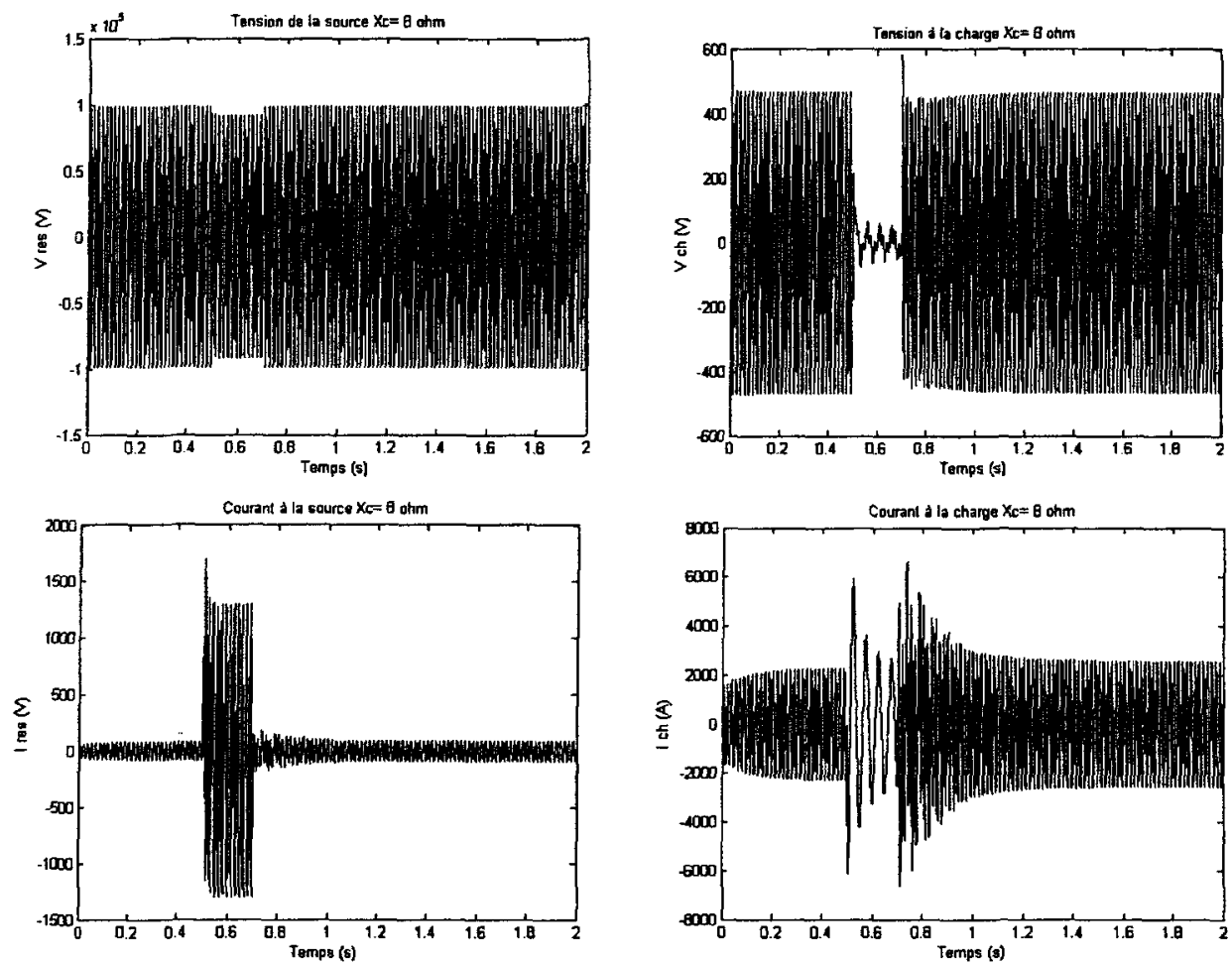

Figure A.53 : Courant et courant à la source et à la charge 

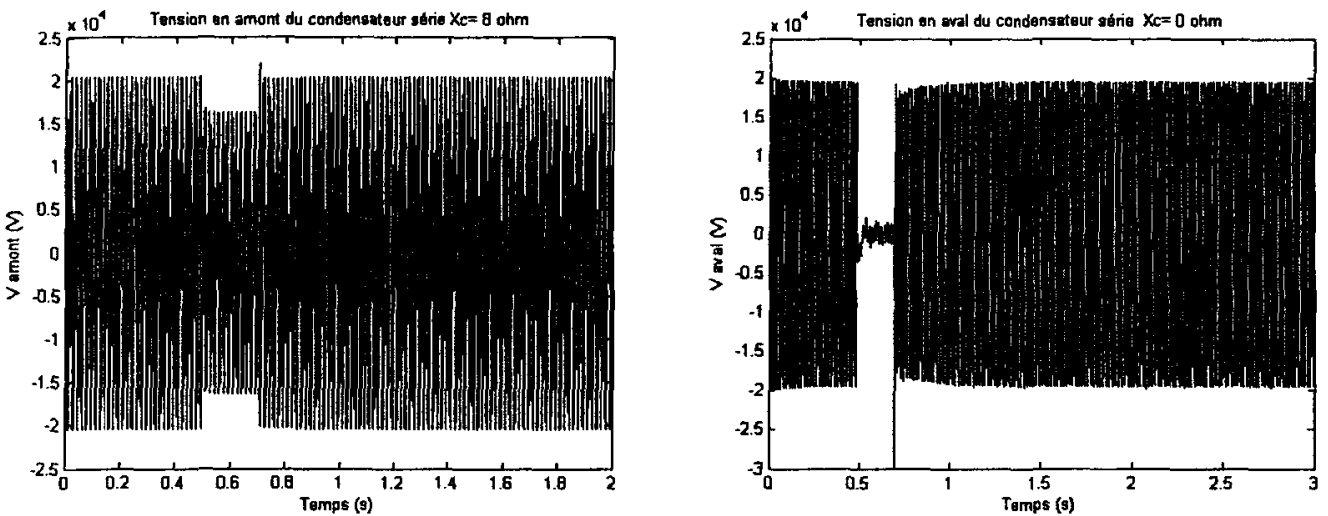

Figure A.54 : Tension en amont et en aval du condensateur série
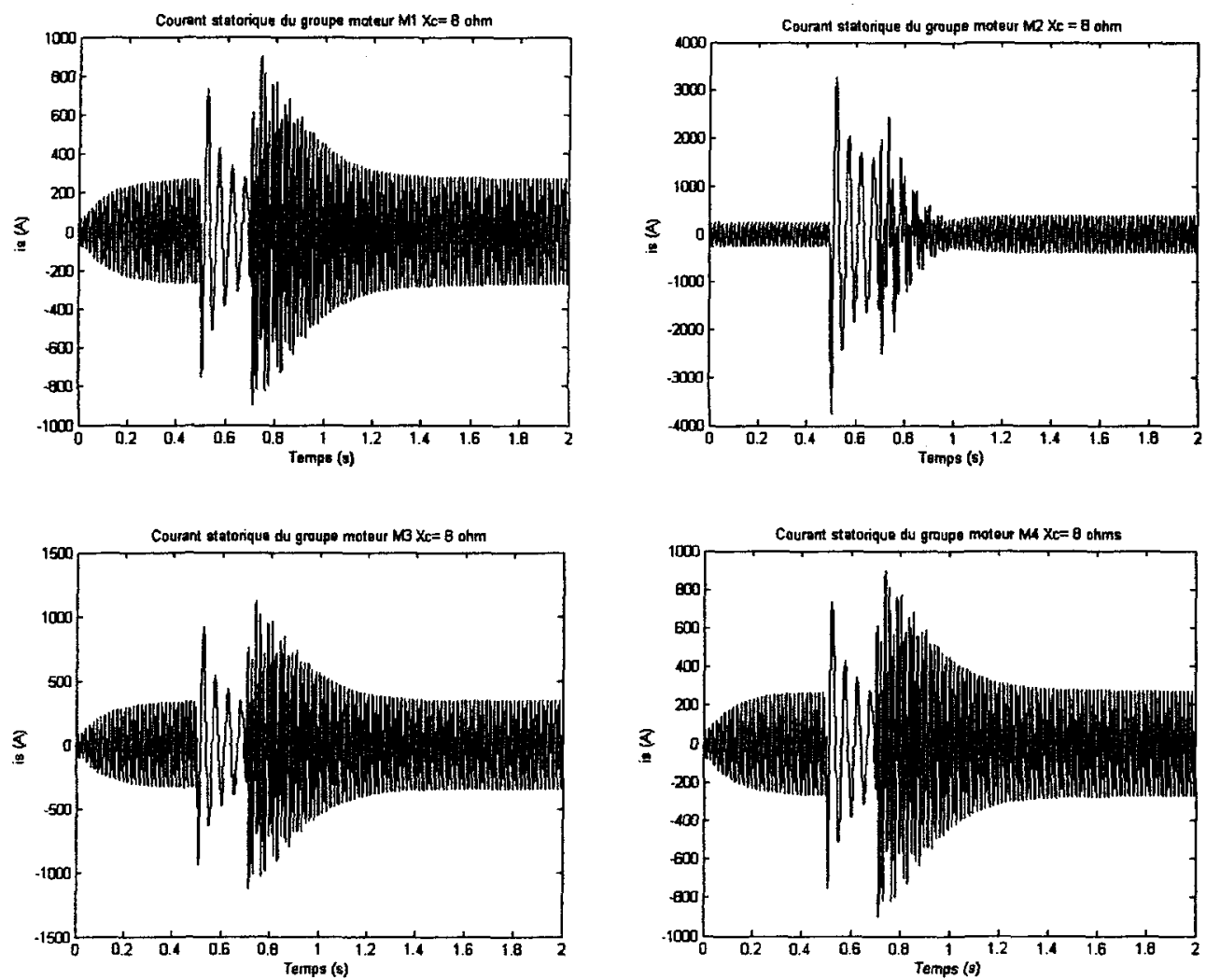

Figure A.55 : Courants statoriques des groupes moteurs 

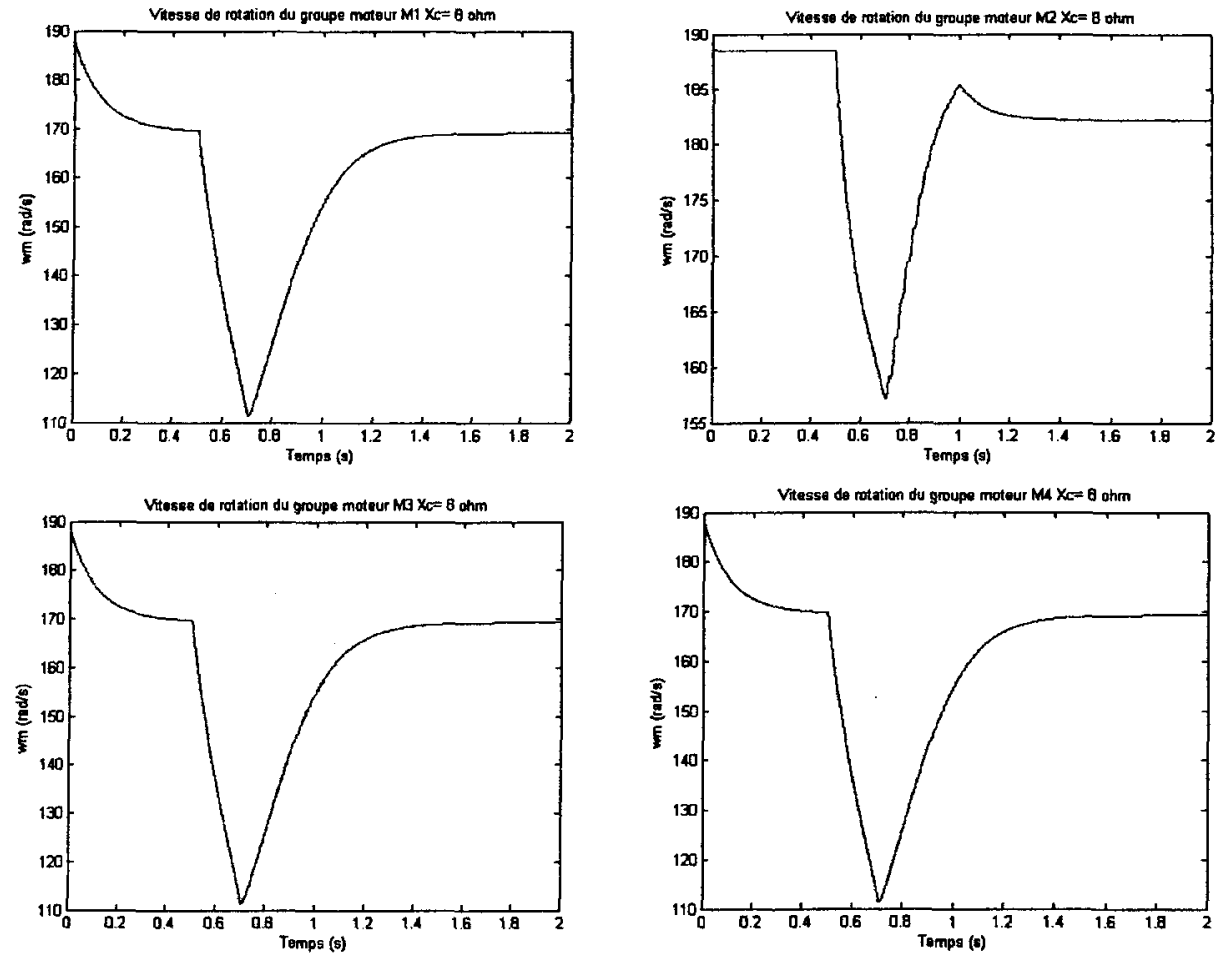

Figure A.56 : Vitesse de rotation des groupes moteurs
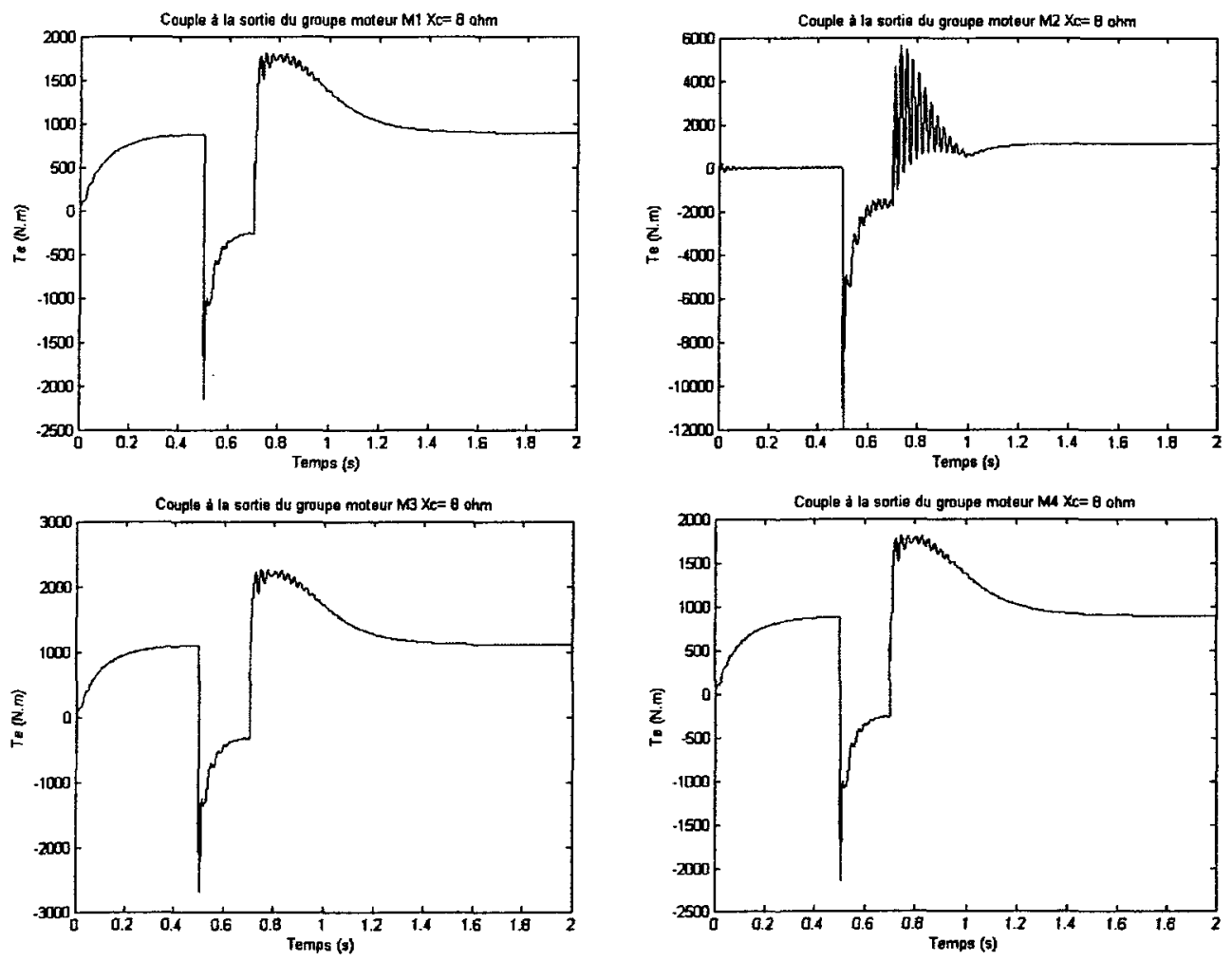

Figure A.57 : Couple à la sortie des groupes moteurs 
Réseau de distribution radial à $\tau=54.85 \% \mathrm{Xc}=13 \mathrm{ohm}$
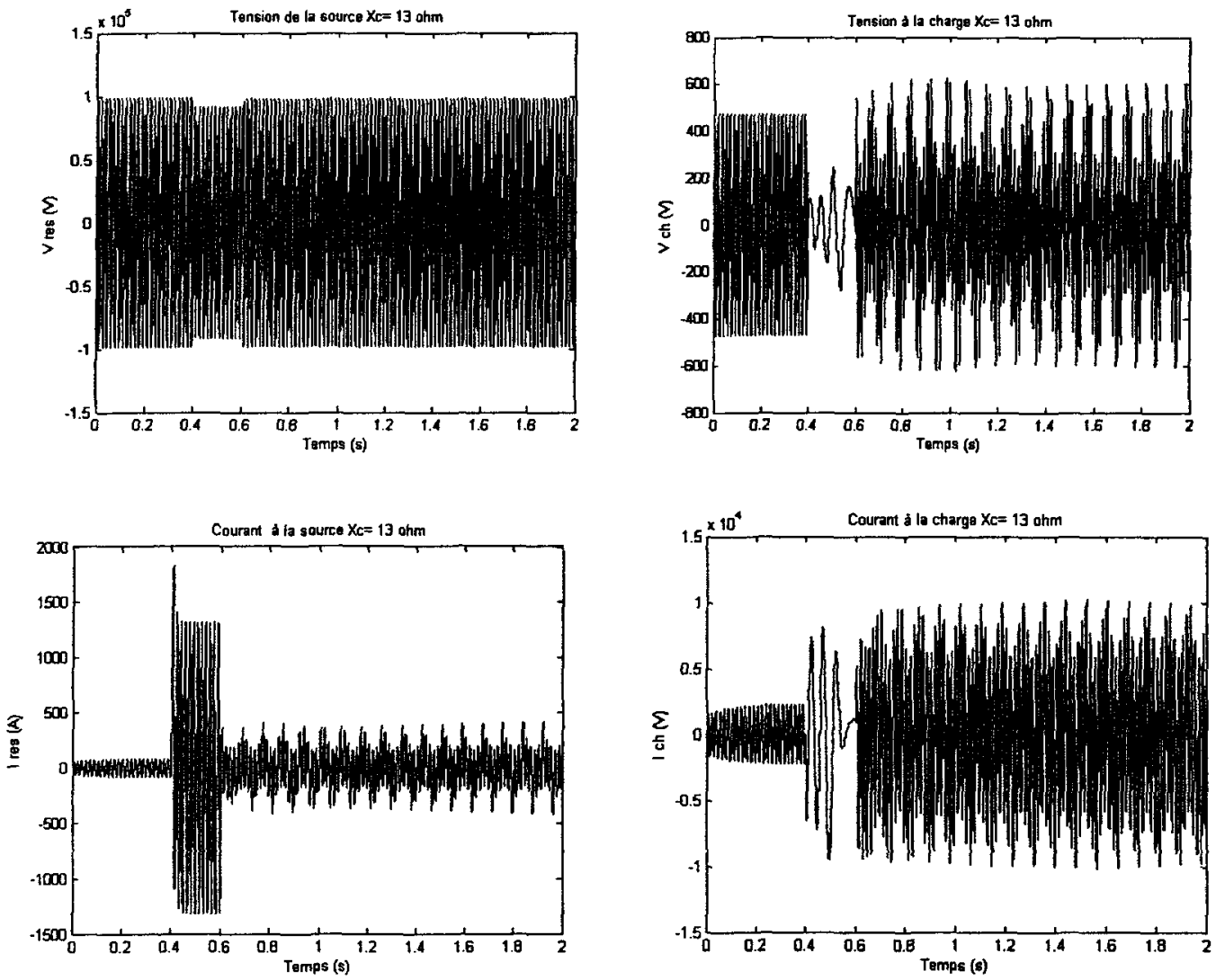

Figure A.58 : Tension et courant à la source et à la charge
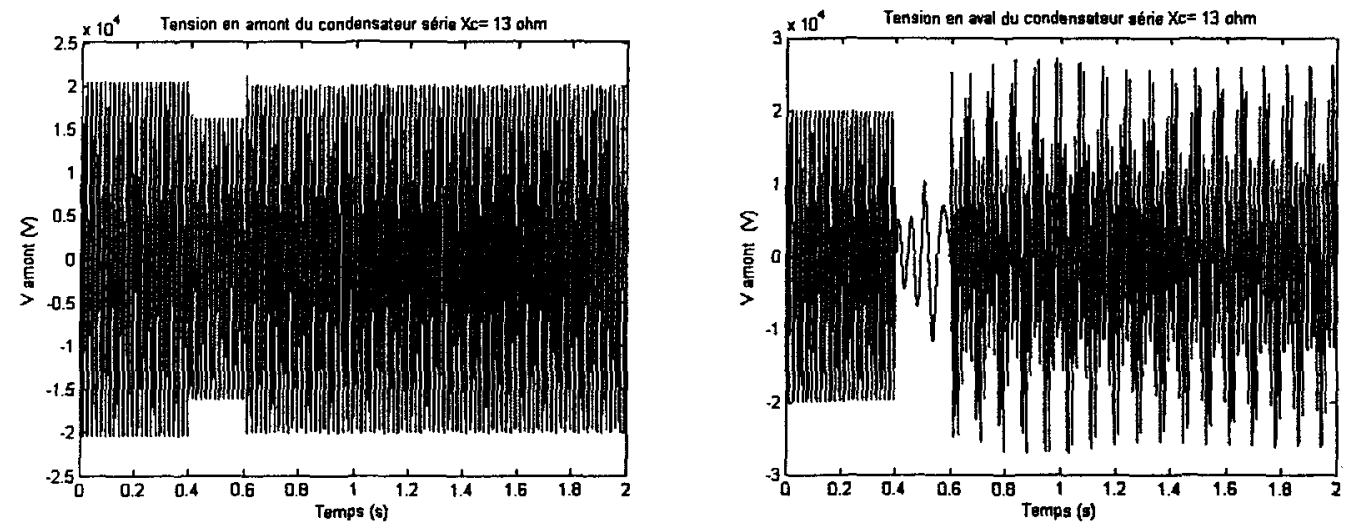

Figure A.59 : Tension en amont et en aval du condensateur série 

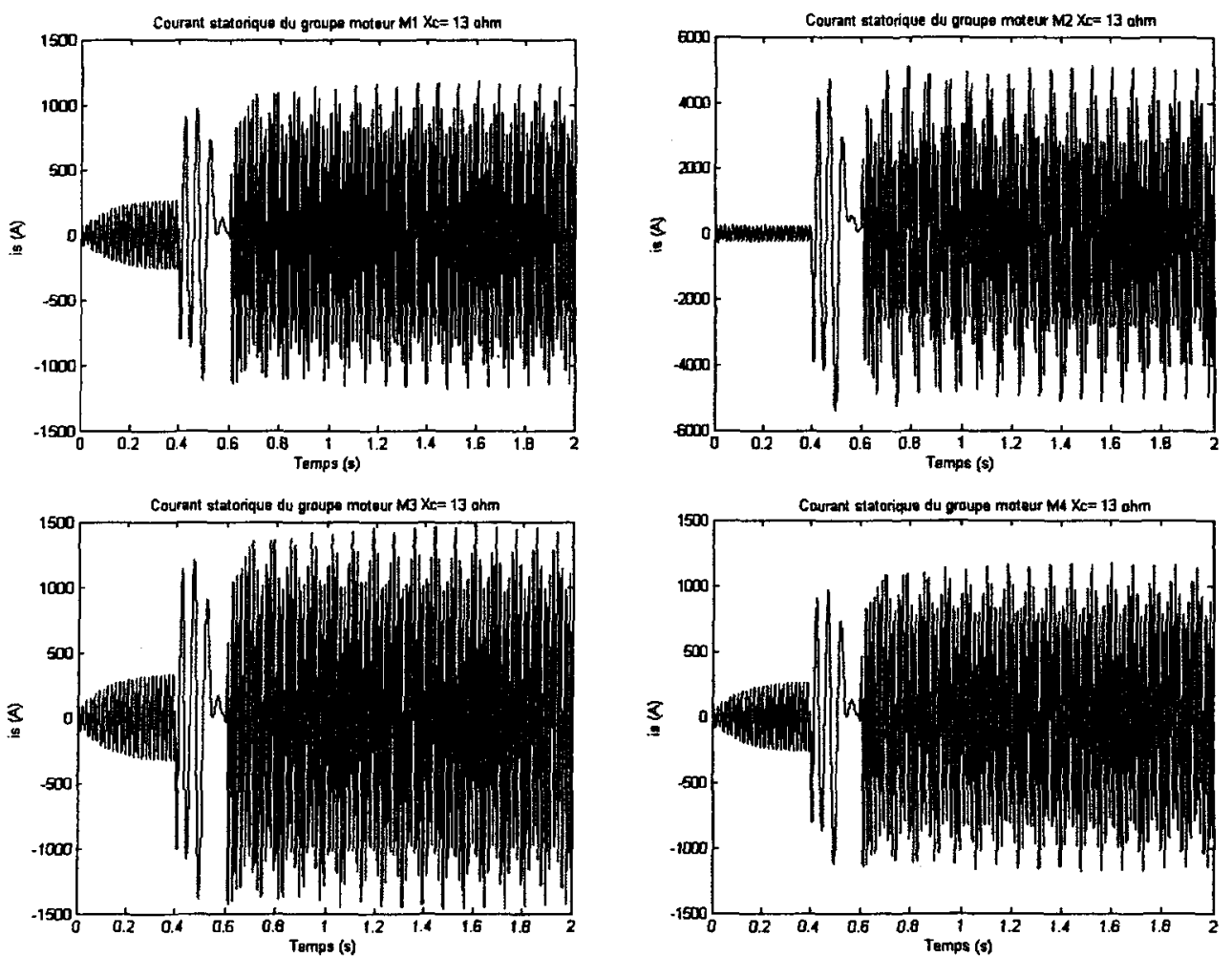

Figure A.60 : Courants statoriques des groupes moteurs
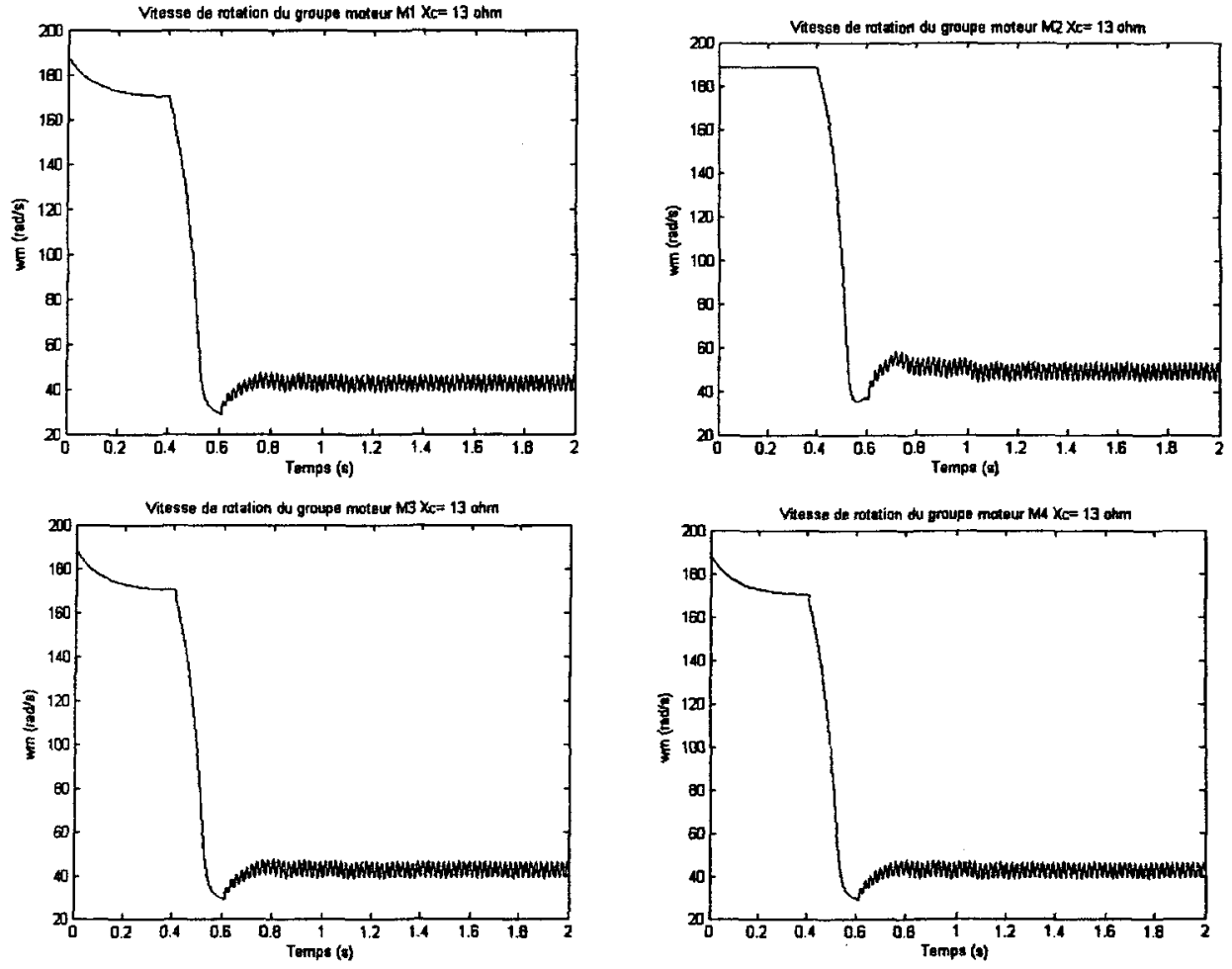

Figure A.61 : Vitesse de rotation des groupes moteurs 

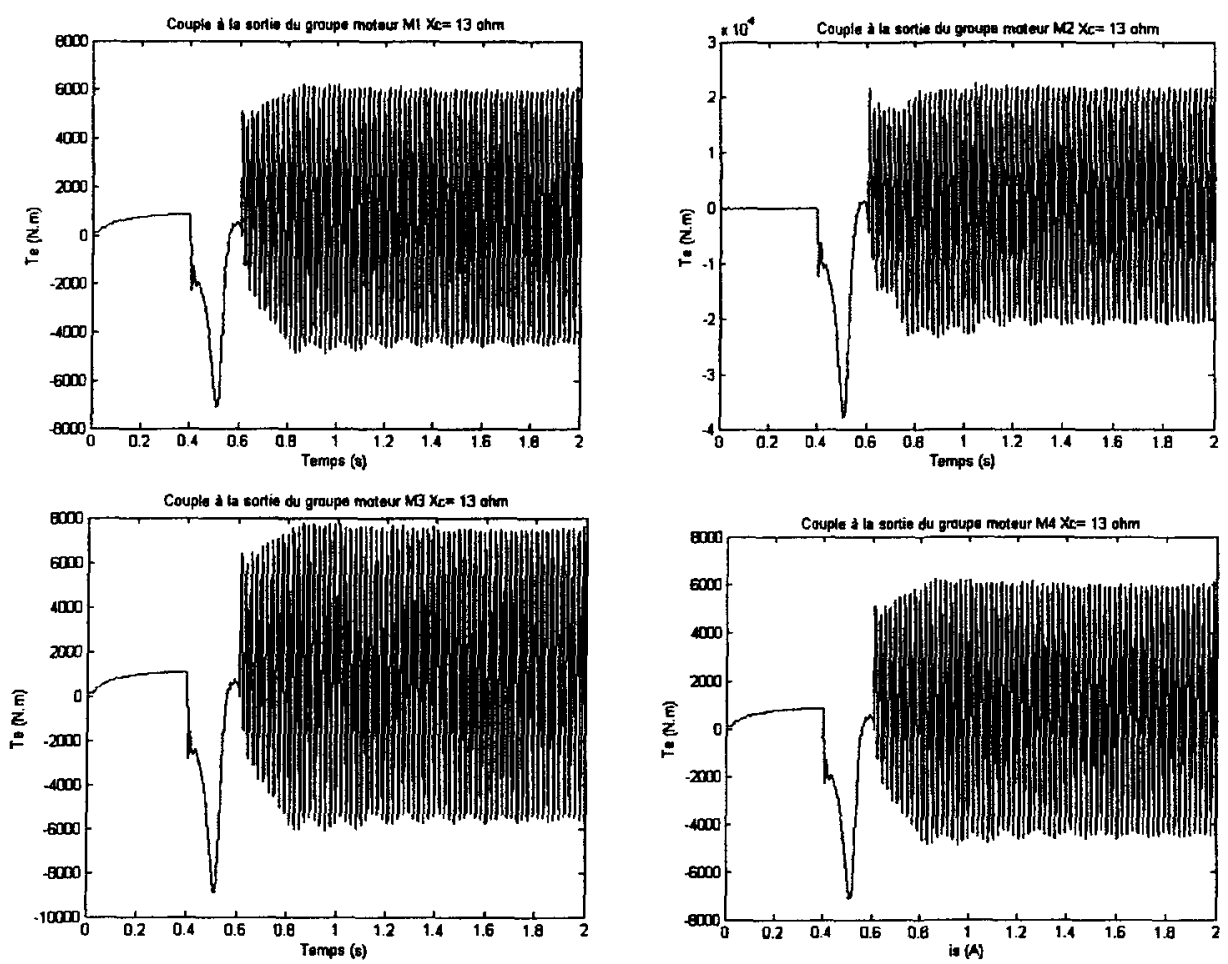

Figure A.62 : Couple à la sortie des groupes moteurs

Simulation de l'essai d'un court circuit monophasé en aval du condensateur série à $t=0.5 \mathrm{~s}$ Réseau de distribution radial sans compensation série
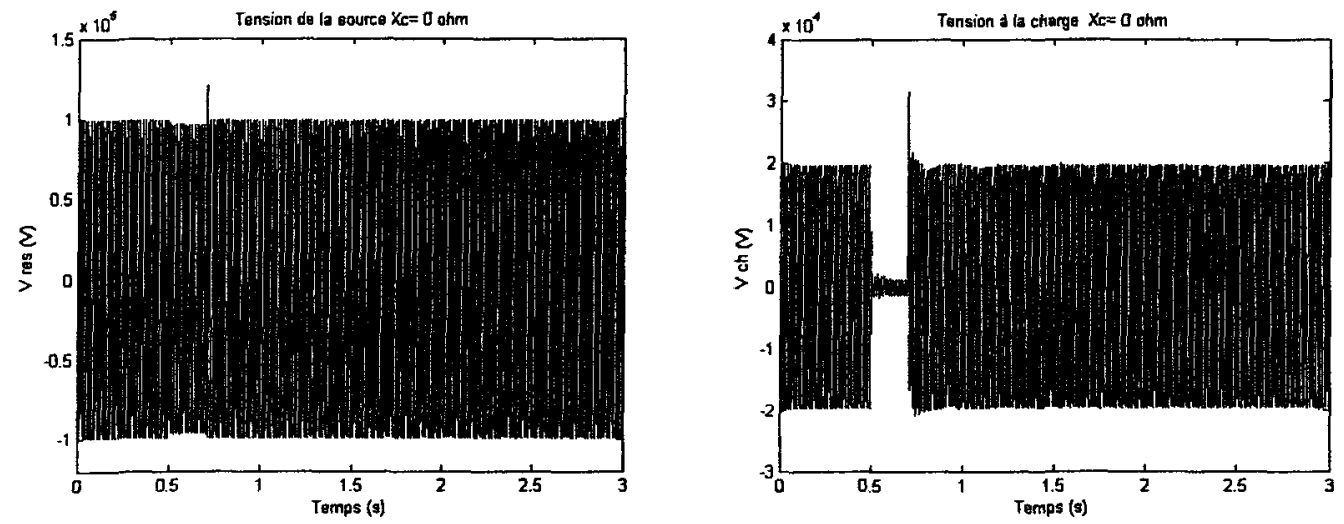

Figure A.63 : Tension de la source et à la charge 

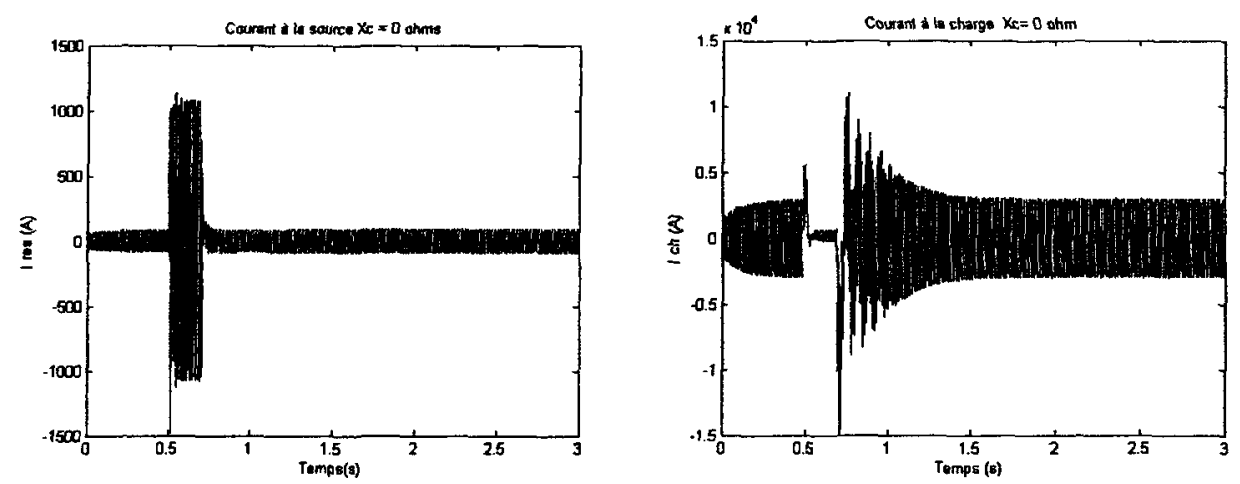

Figure A.64 : Courant à la source et à la charge
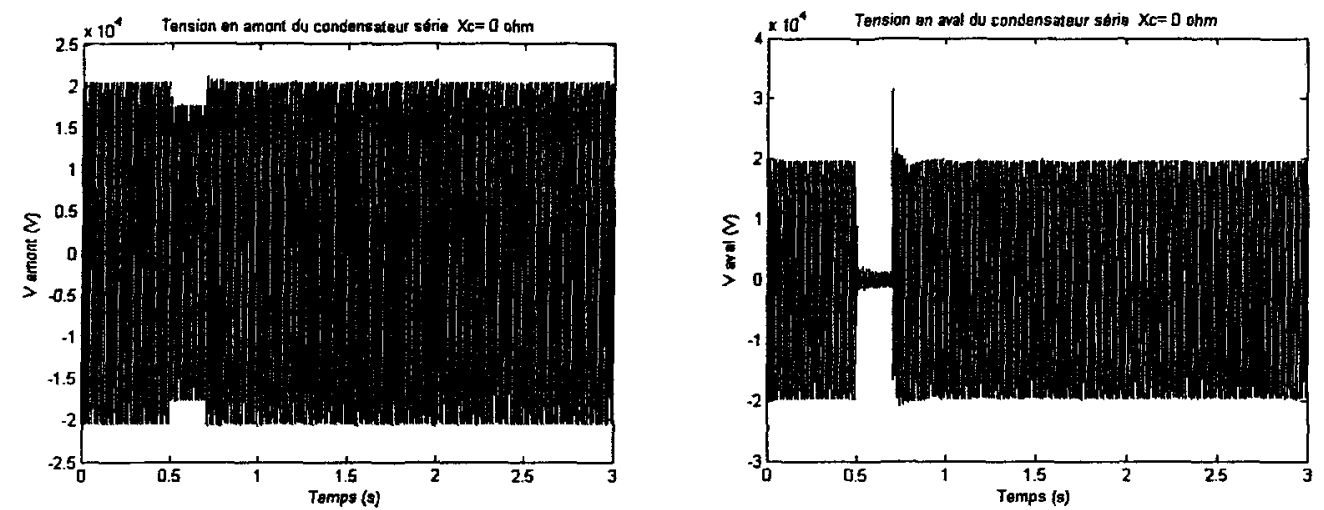

Figure A.65 : Tension en amont et en aval du condensateur série
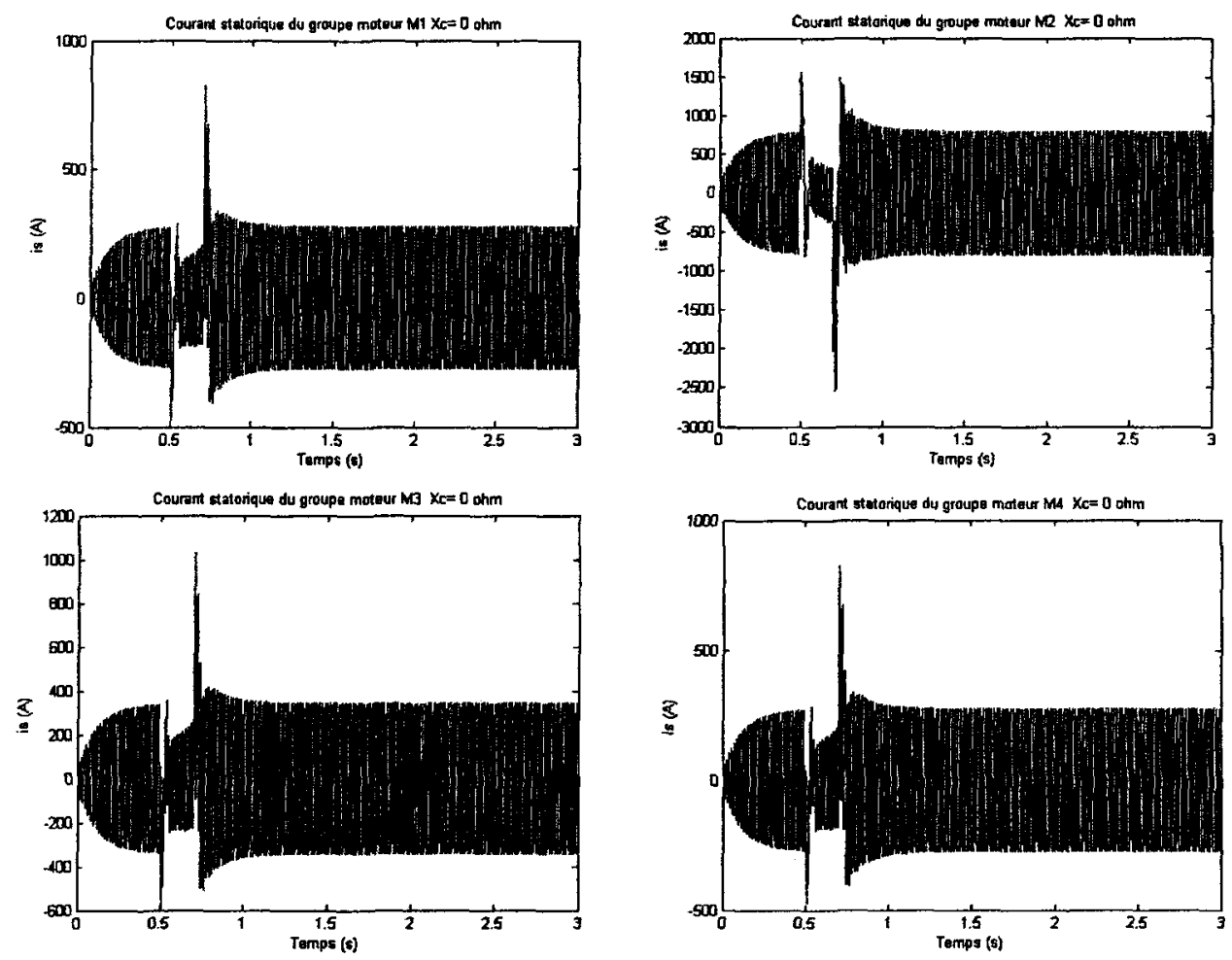

Figure A.66 : Courants statoriques des groupes moteurs 

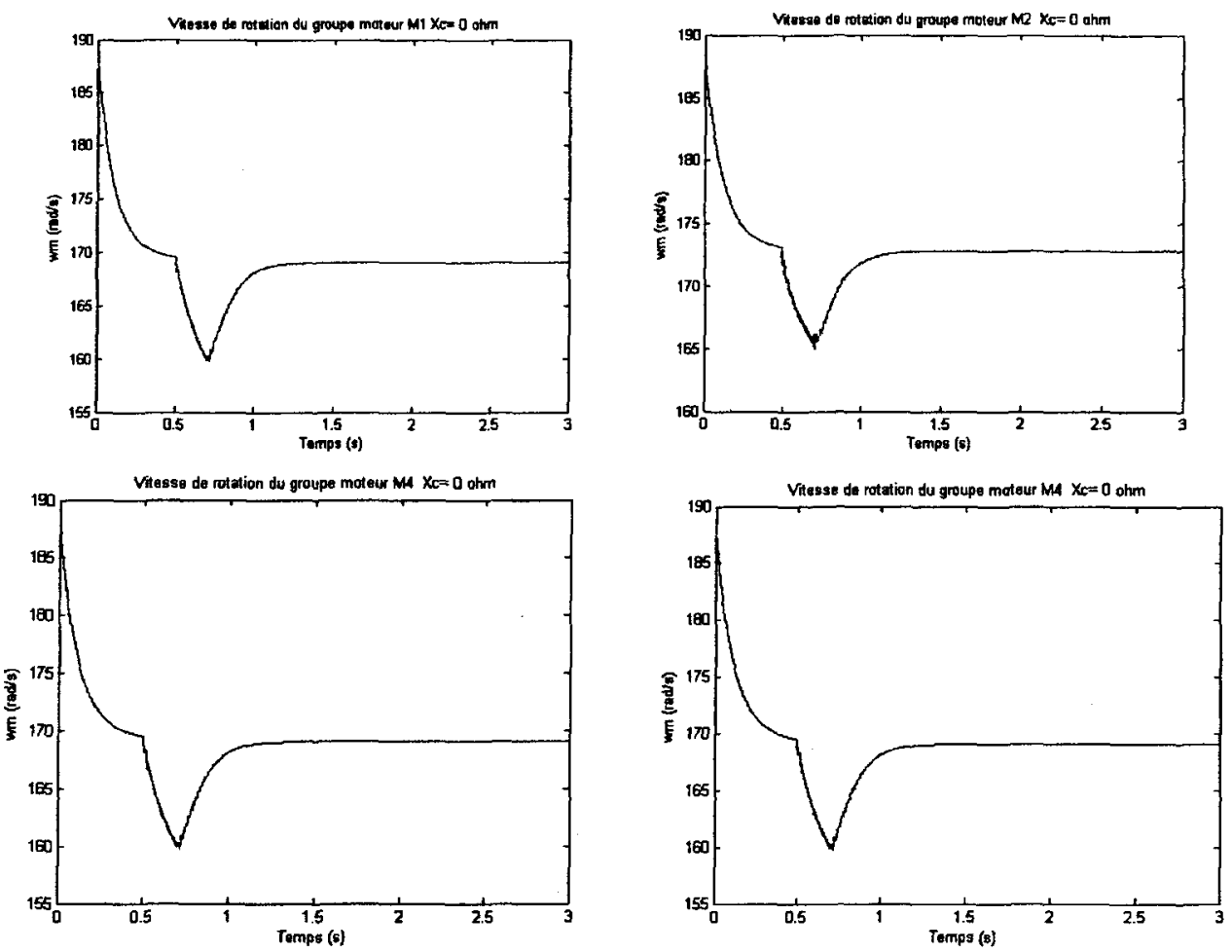

Figure A.67 : Vitesse de rotation des groupes moteurs
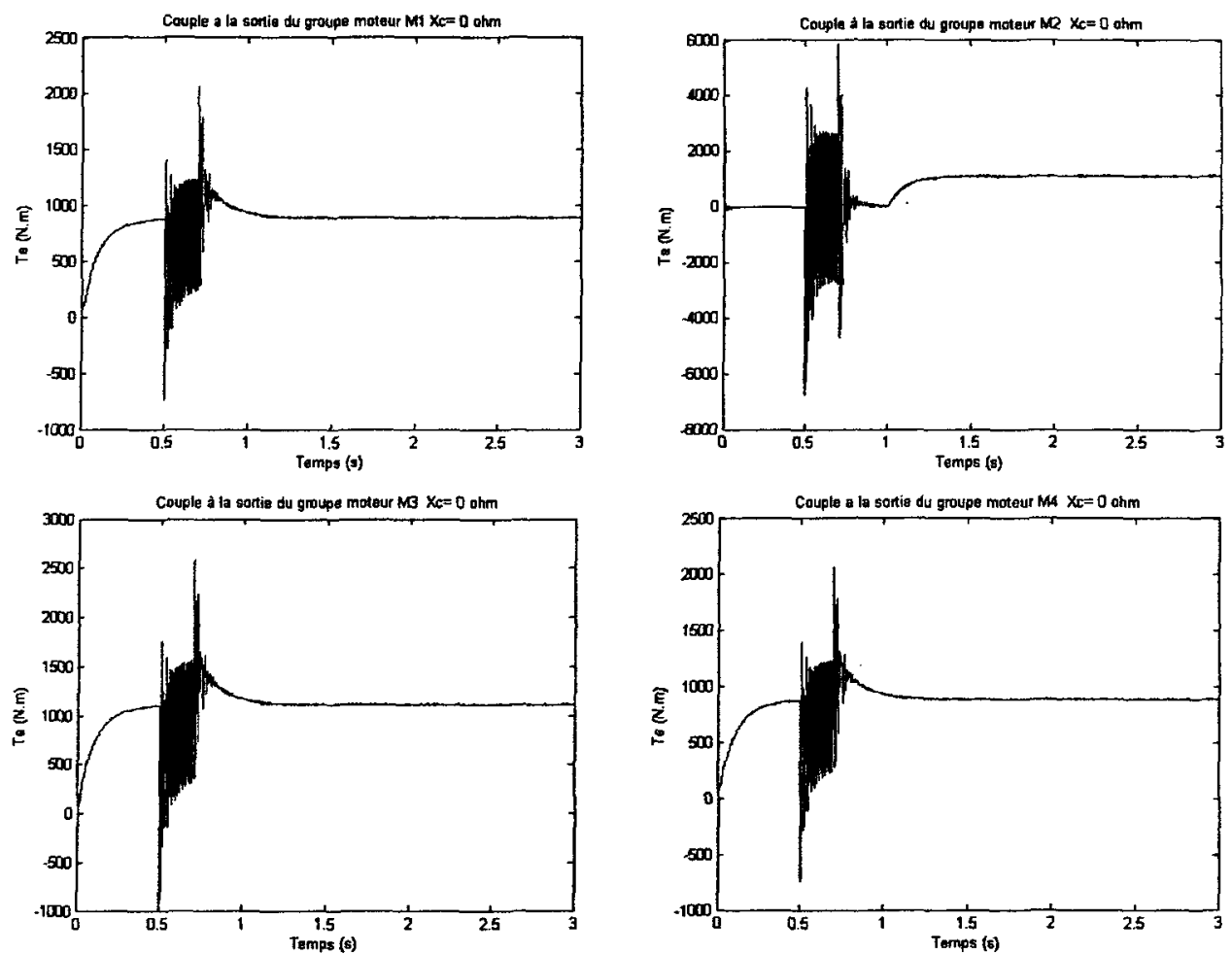

Figure A.68 : Couple à la sortie des groupes moteurs 
Réseau de distribution radial à $\tau=54.85 \% \mathrm{Xc}=13 \Omega$
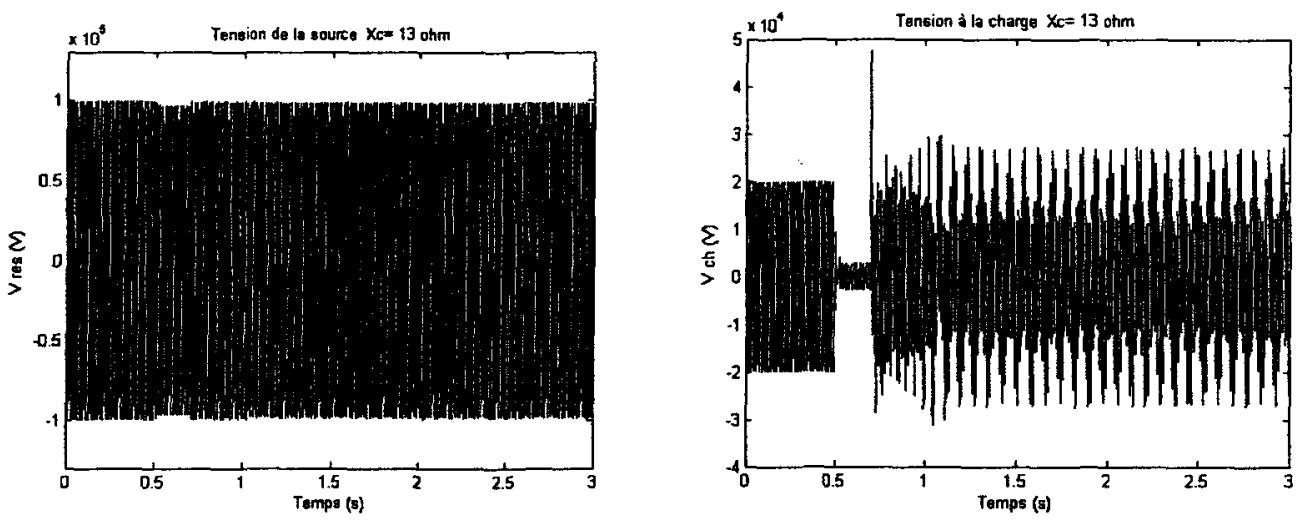

Figure A.69 : Tension de la source et à la charge
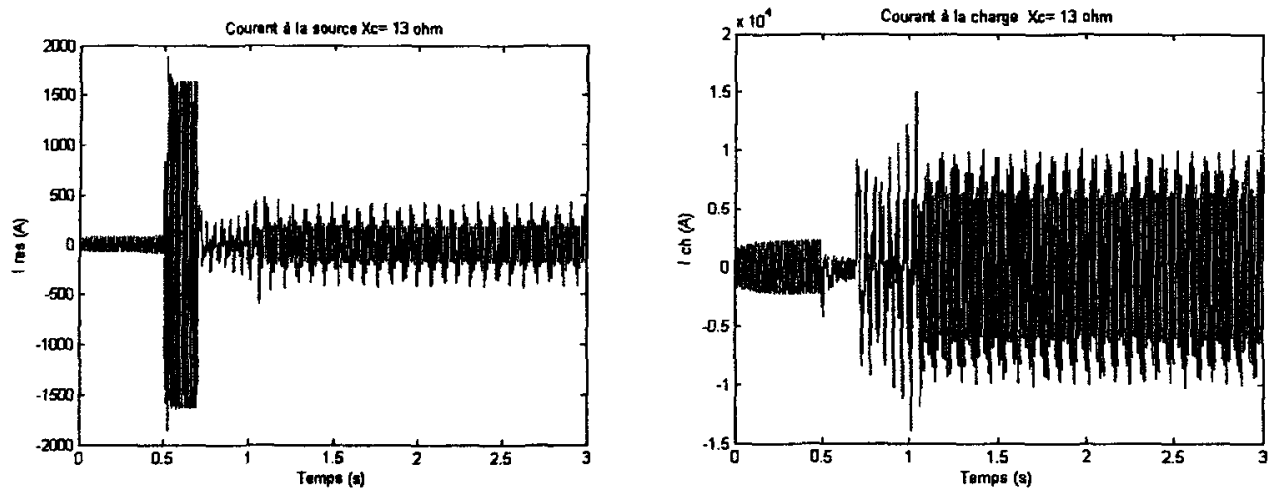

Figure A.70 : Courant à la source et à la charge
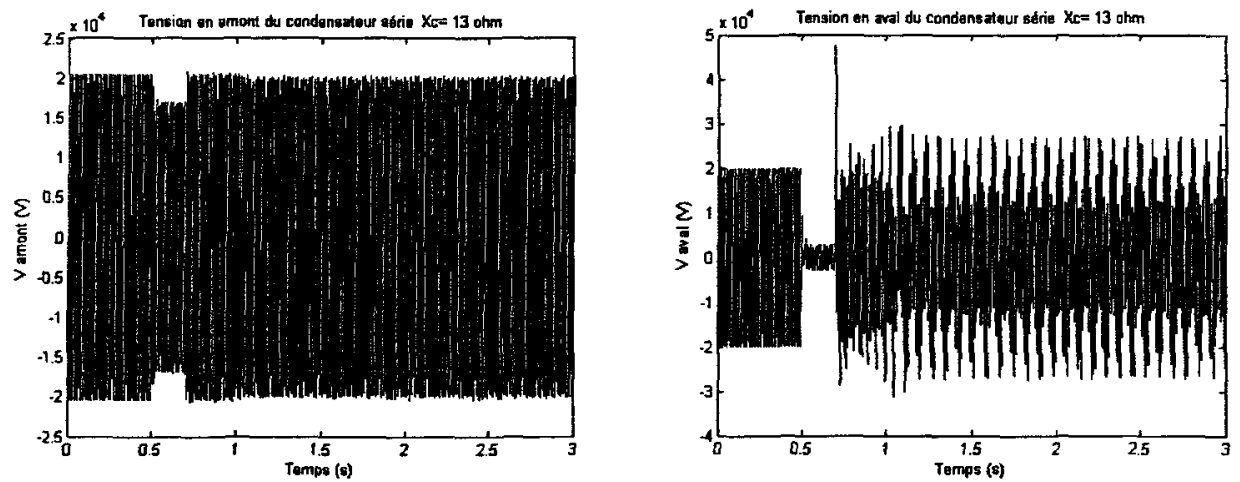

Figure A.71 : Tension en amont et en aval du condensateur série 

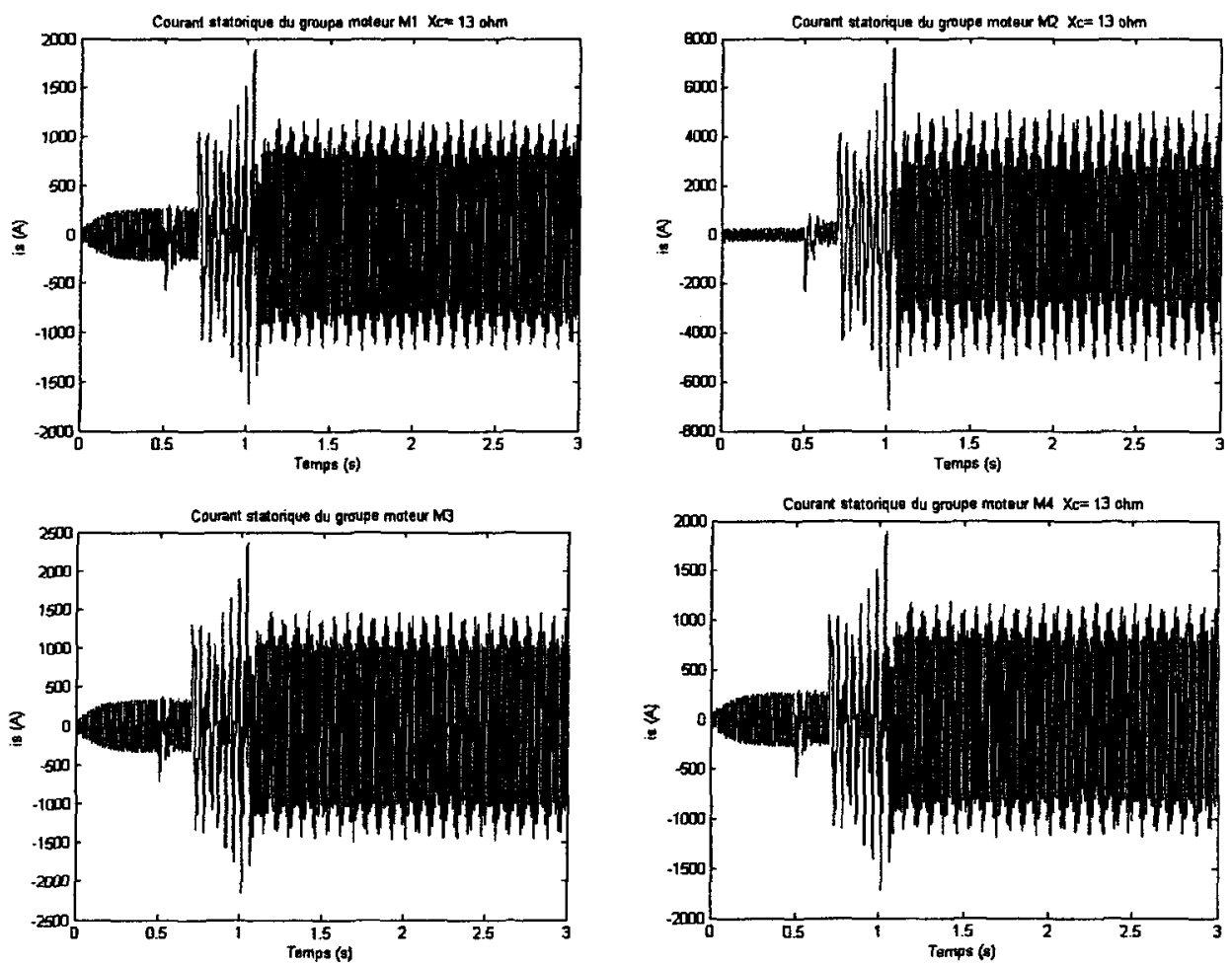

Figure A.72 : Courants statoriques des groupes moteurs
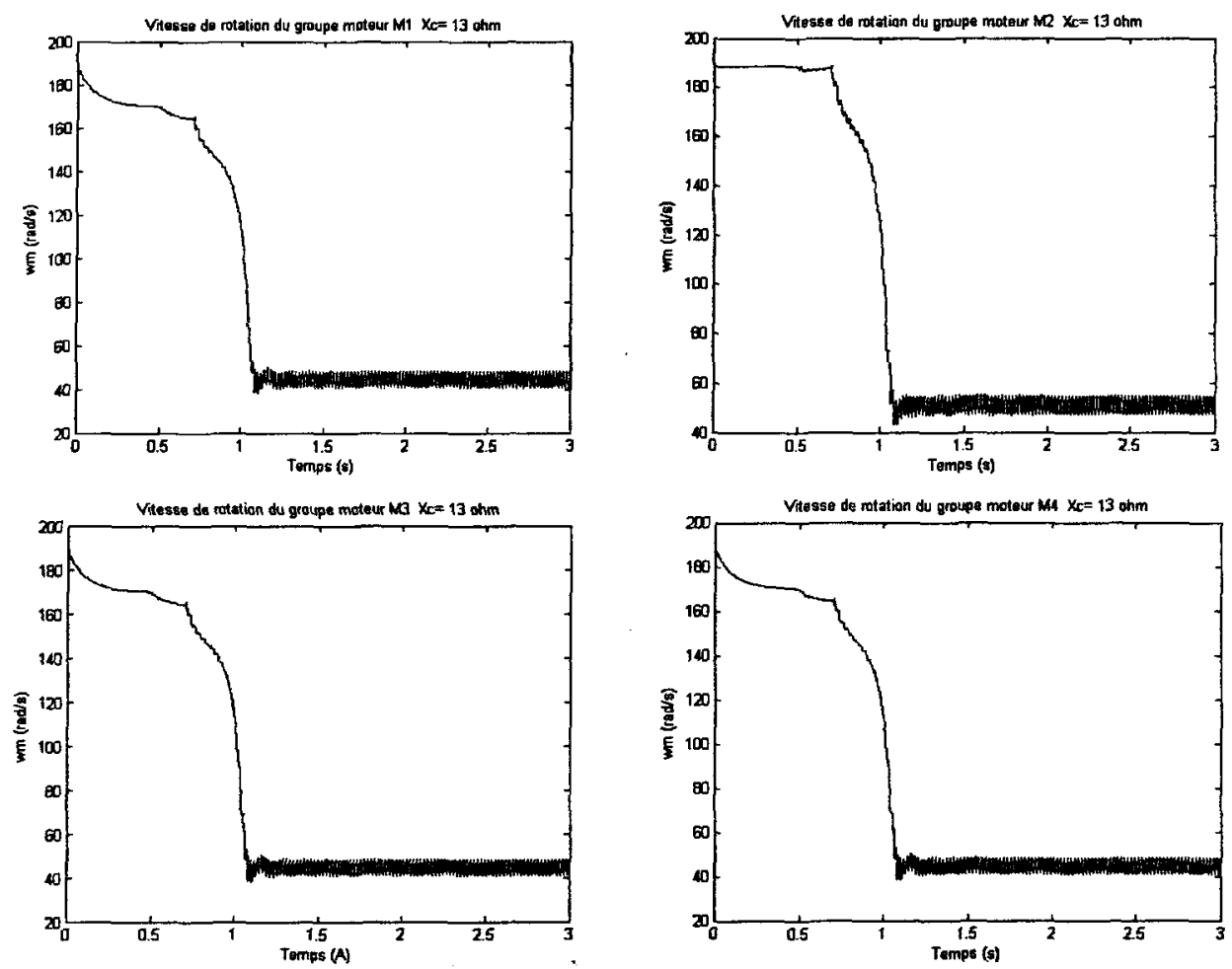

Figure A.73 : Vitesse de rotation des groupes moteurs 

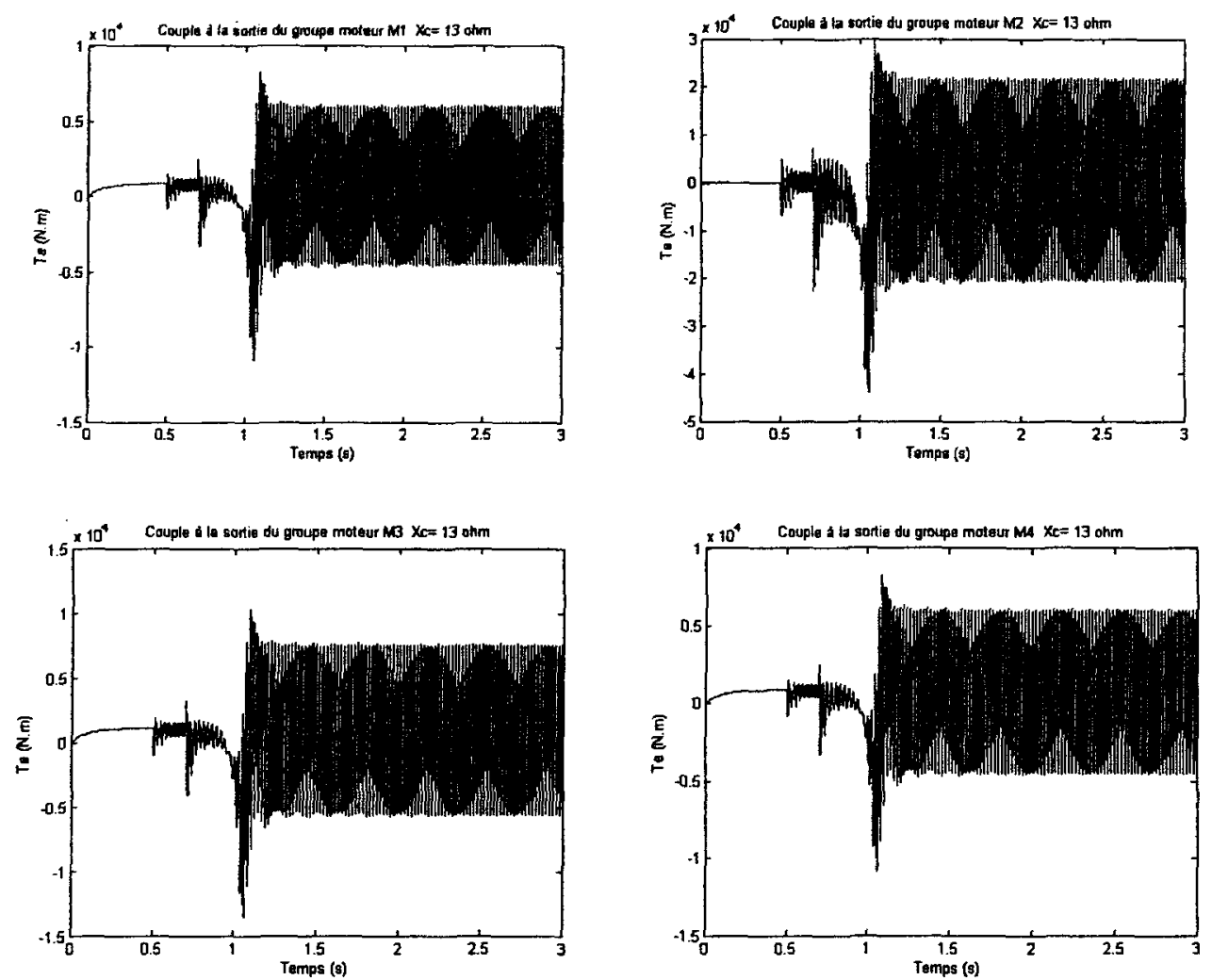

Figure A.74 : Couple à la sortie des groupes moteurs

Simulation de l'essai d'un court circuit triphasé en aval du condensateur série à $t=0.5 \mathrm{~s}$

Réseau de distribution radial sans compensation série
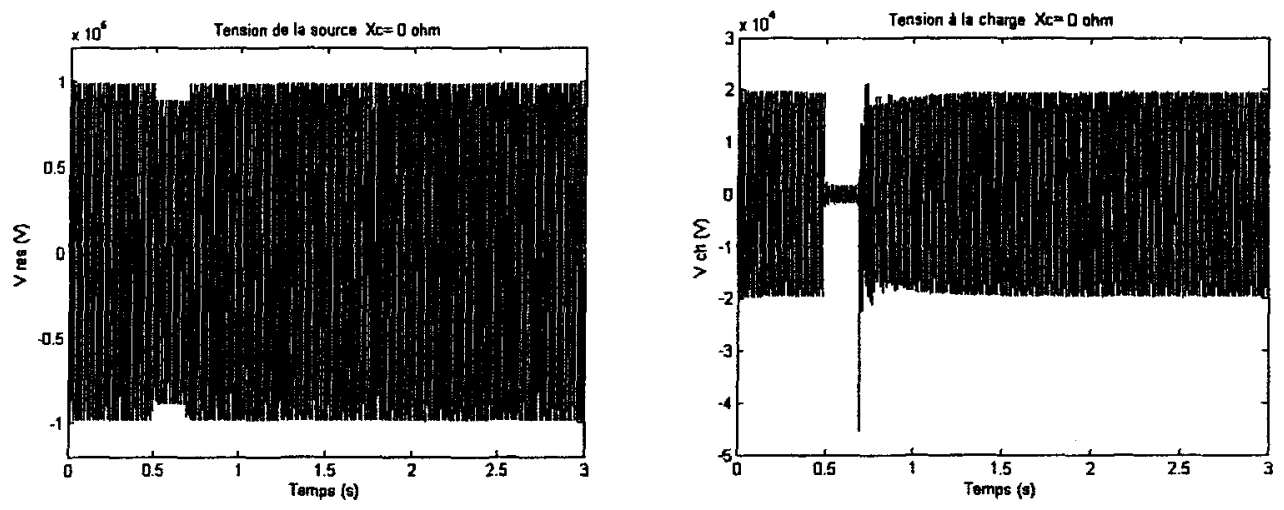

Figure A.75 : Tension de la source et à la charge 

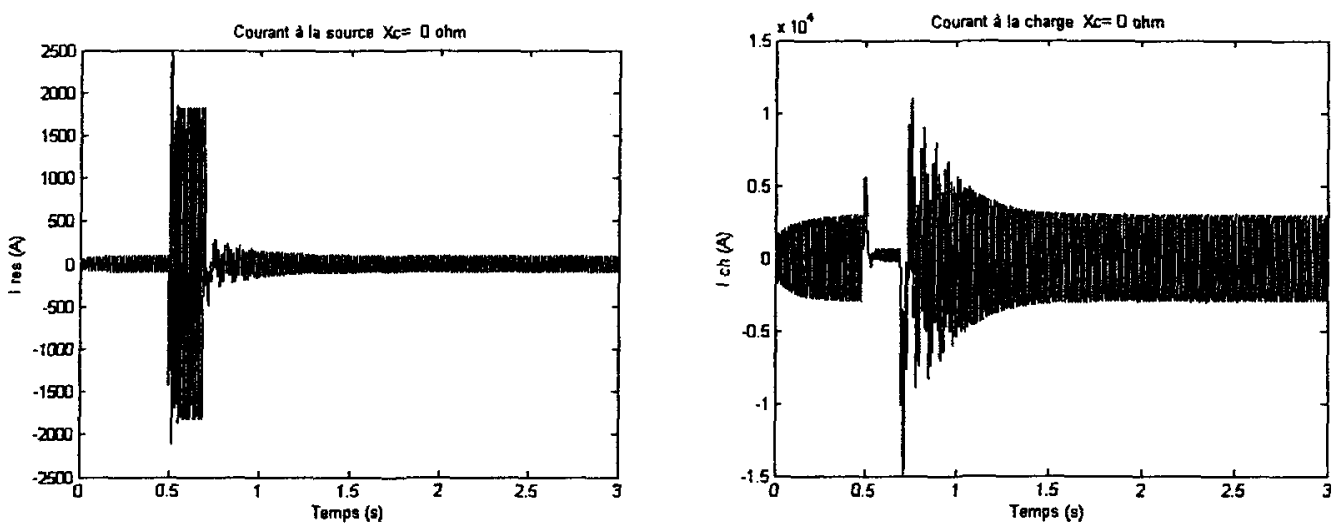

Figure A.76: Courant à la source et à la charge
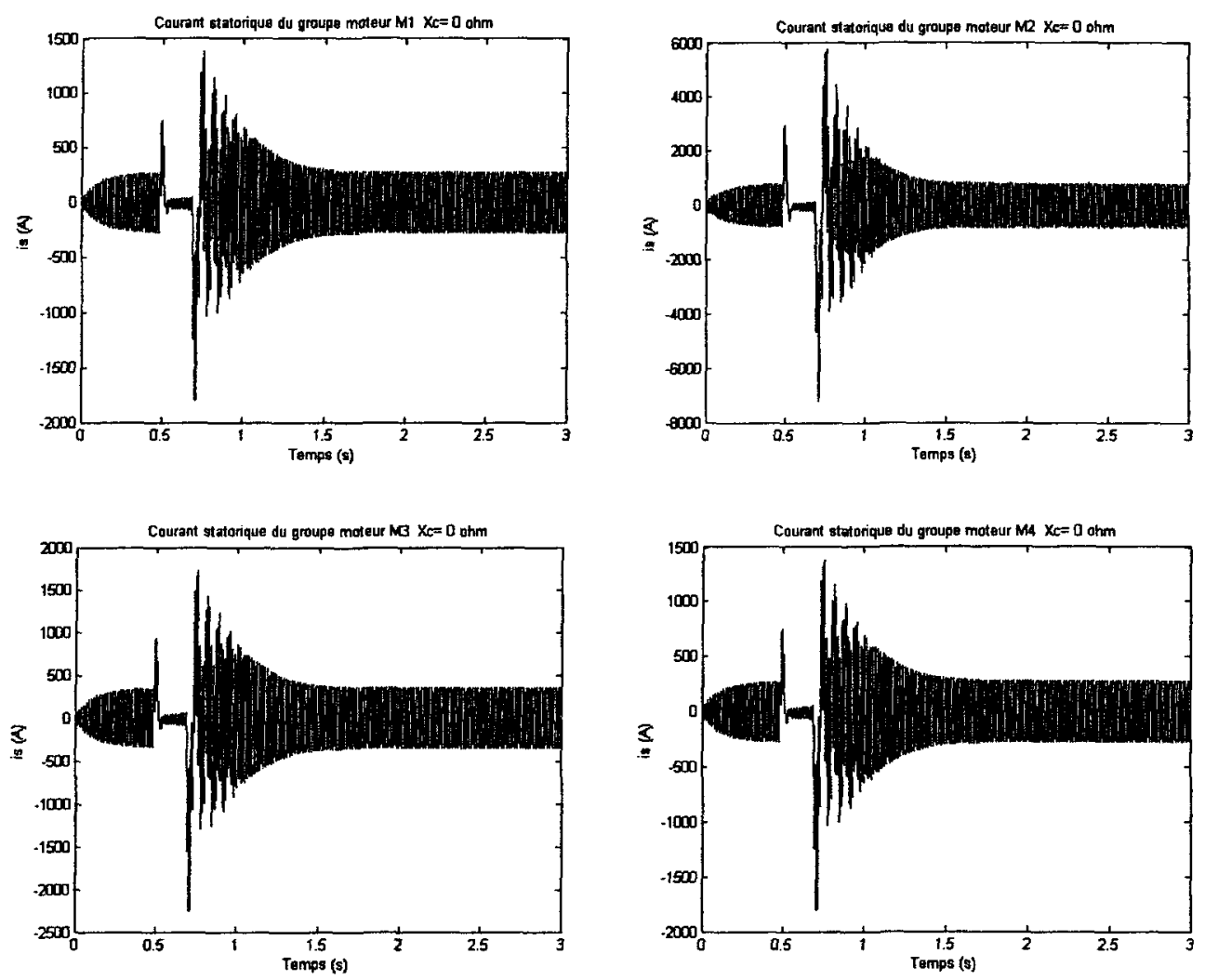

Figure A.77 : Courants statoriques des groupes moteurs 

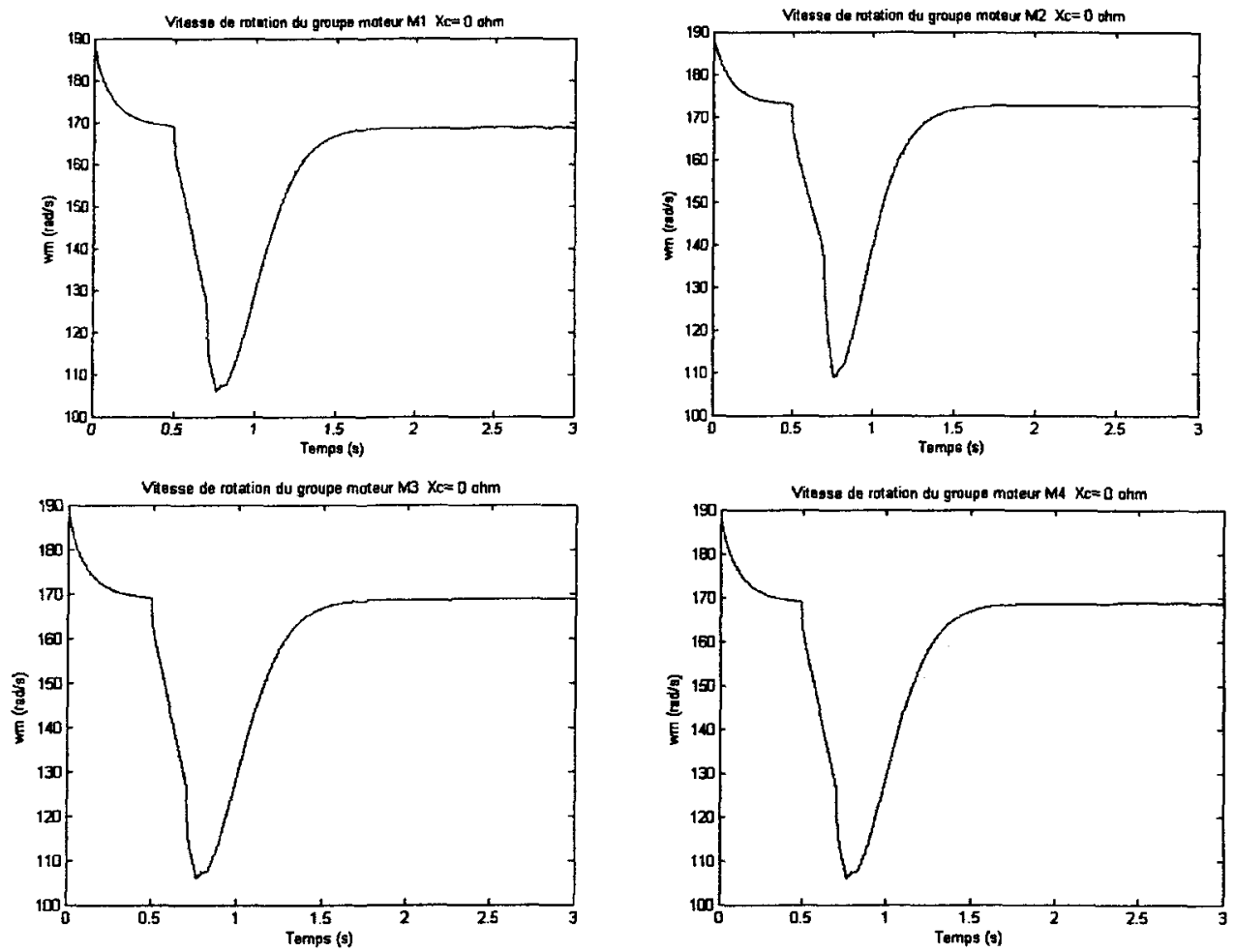

Figure A.78 : Vitesse de rotation des groupes moteurs
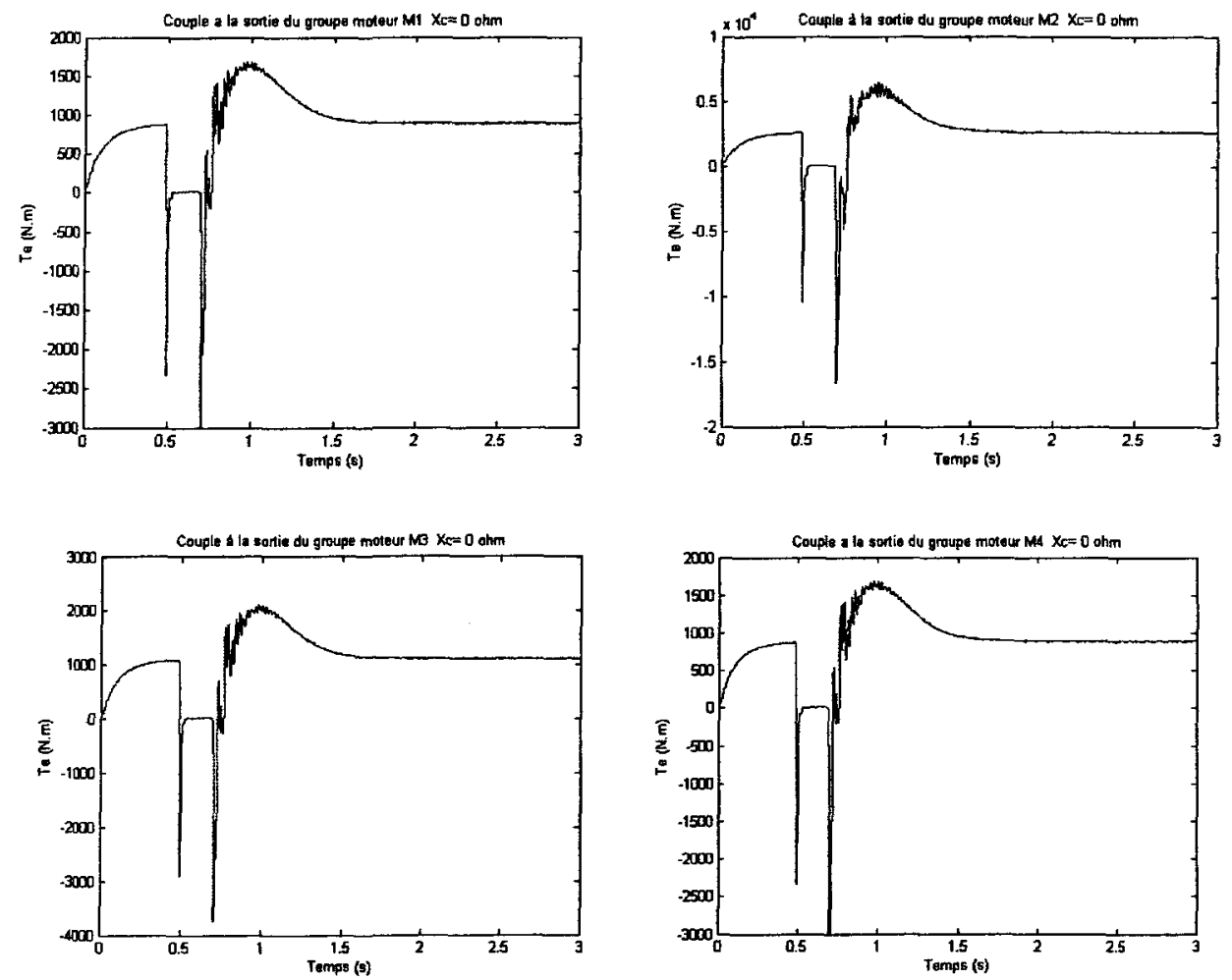

Figure A.79 : Couple à la sortie des groupes moteurs 
Réseau de distribution radial à $\tau=\mathbf{5 4 . 8 5} \%$
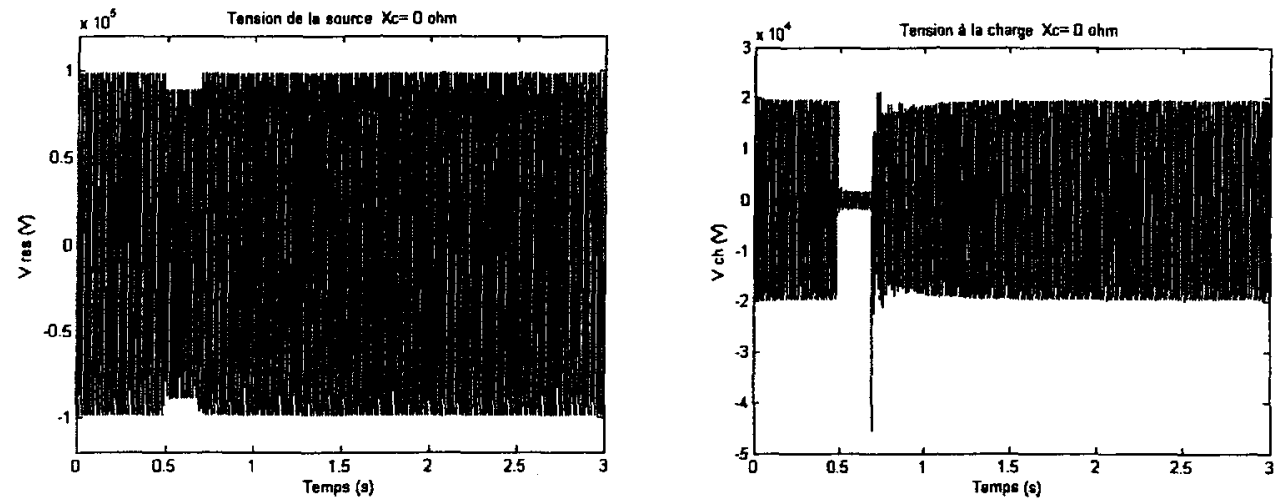

Figure A.80 : Tension de la source et à la charge
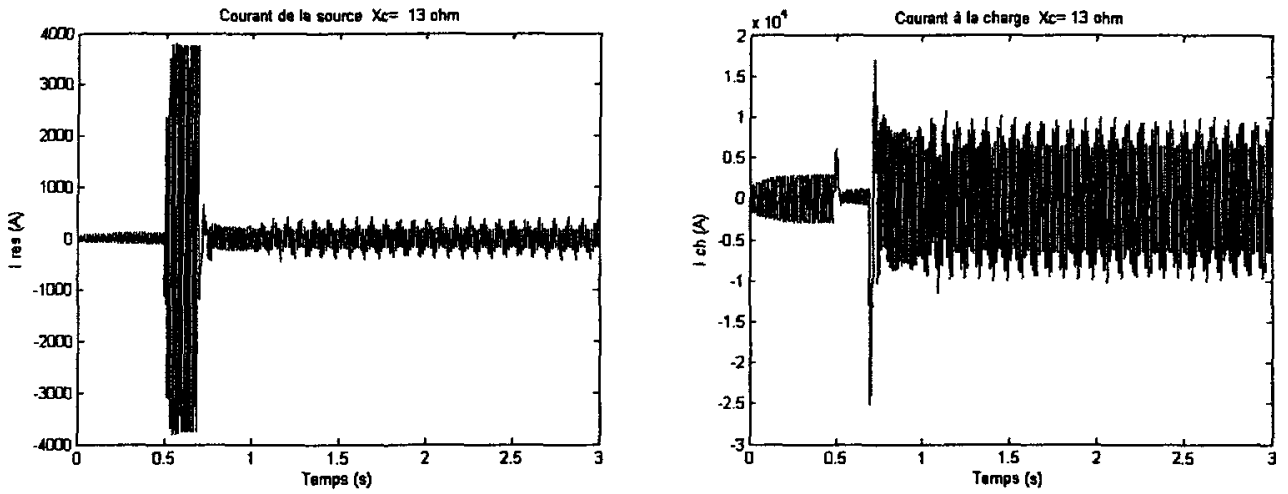

Figure A.81 : Courant à la source et à la charge
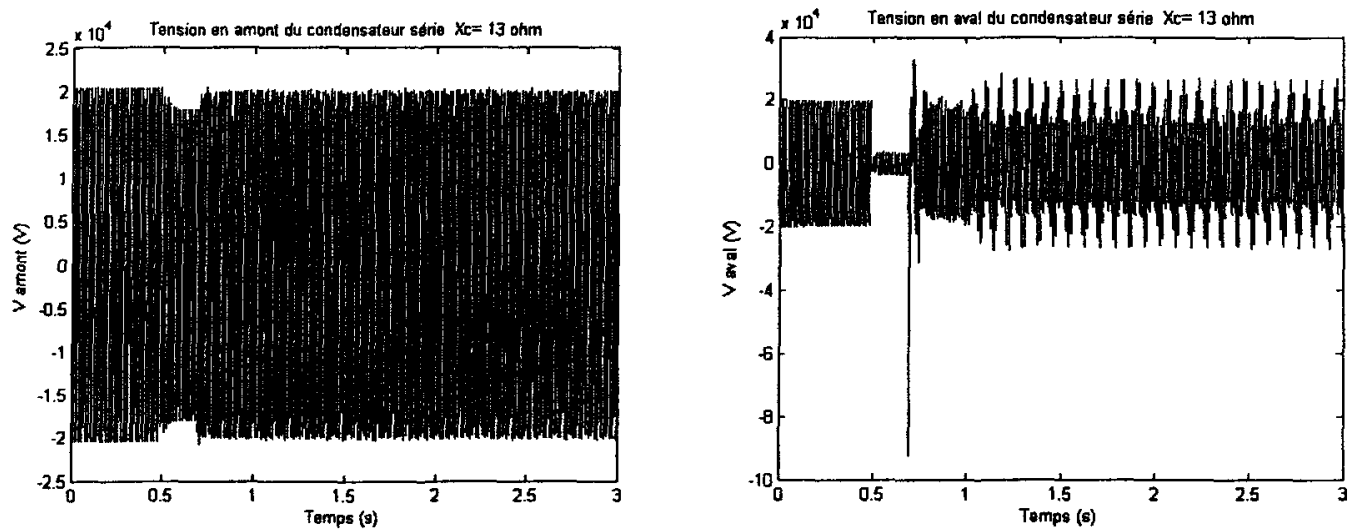

Figure A.82: Tension en amont et en aval du condensateur série 

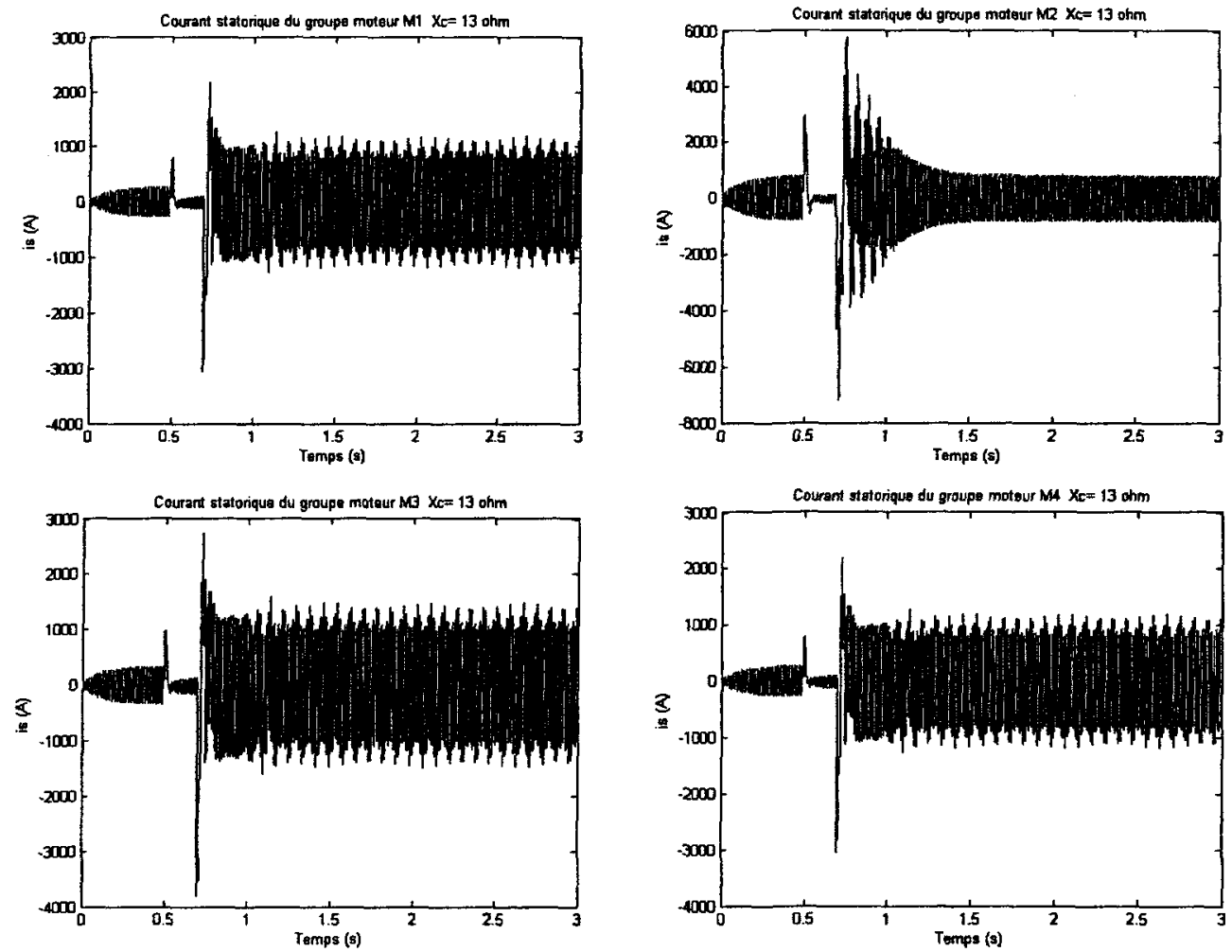

Figure A.83 : Courant au stator pour les groupes de moteurs asynchrones
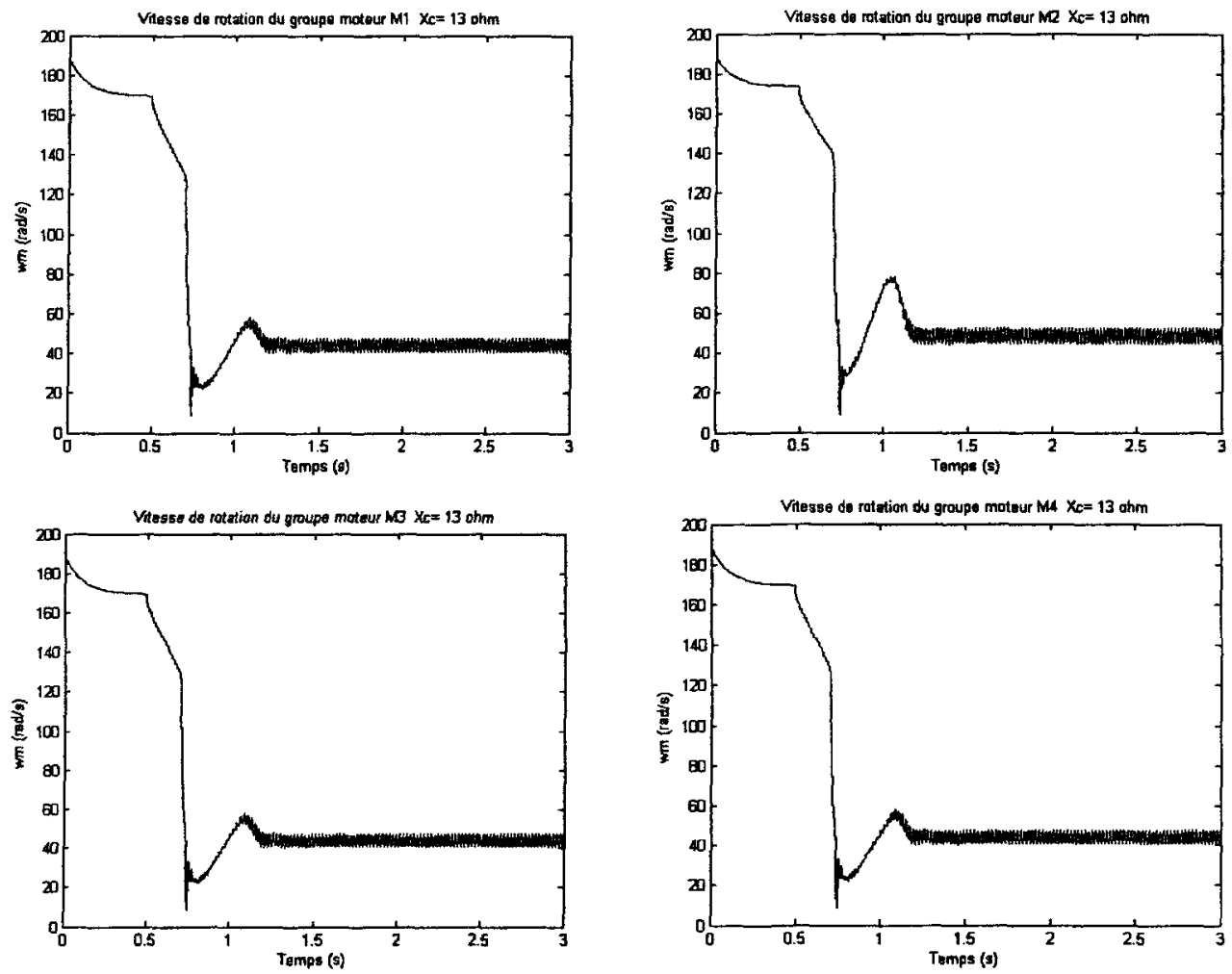

Figure A.84 : Vitesse de rotation des groupes moteurs 

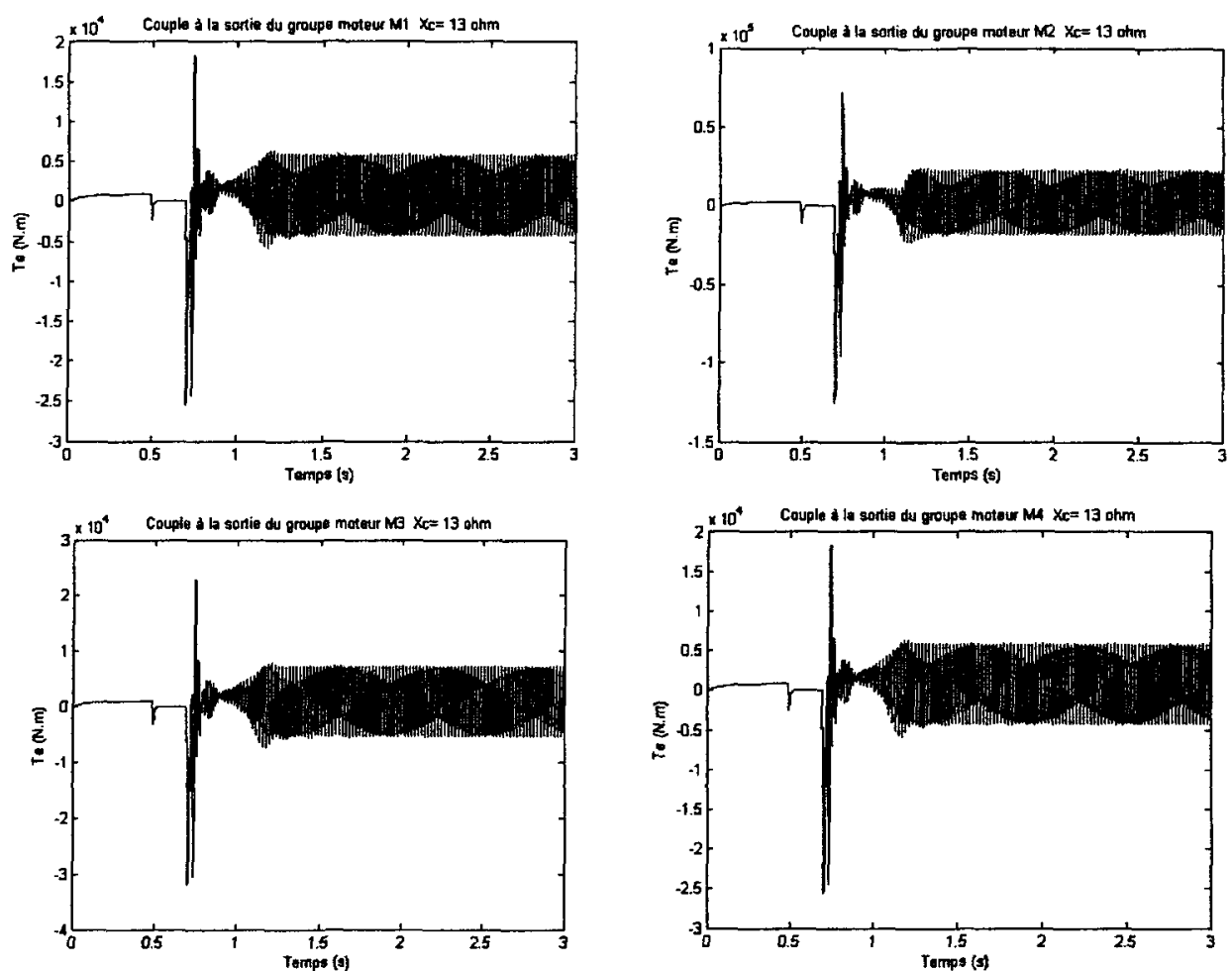

Figure A.85 : Couple à la sortie des groupes moteurs

Simulation de l'essai de la variation brusque de charge dans le groupe moteur N. 2 à $t=2$ sec.

Réseau de distribution radial sans compensation série
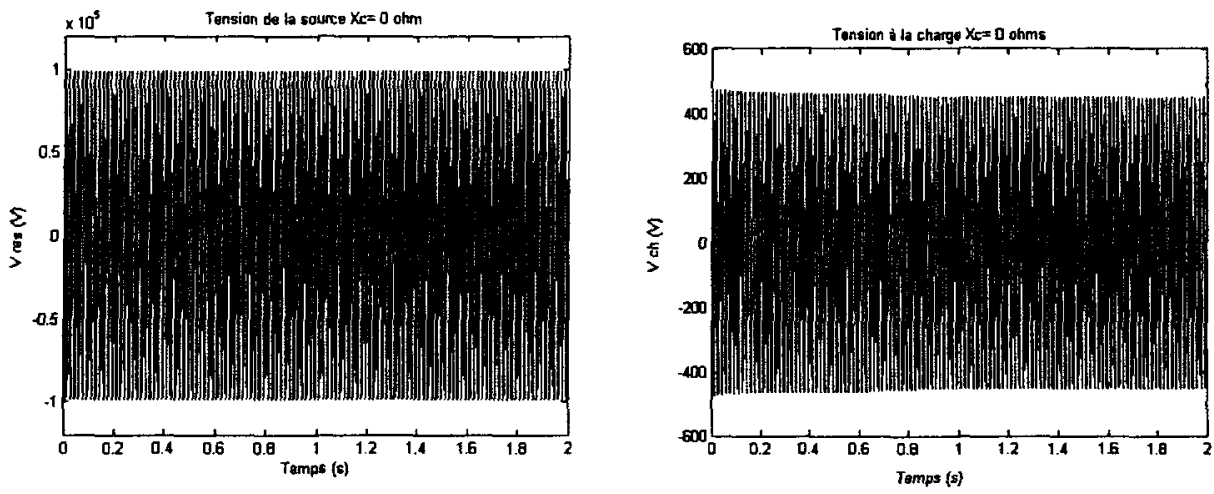

Figure A.86: Tension de la source et à la charge 

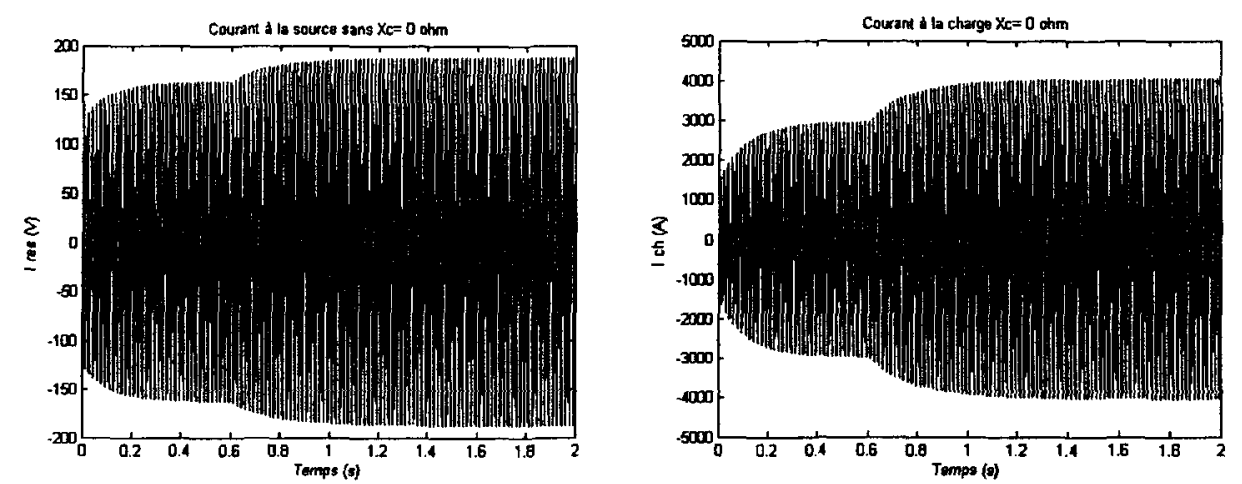

Figure A.87 : Courant à la source et à la charge
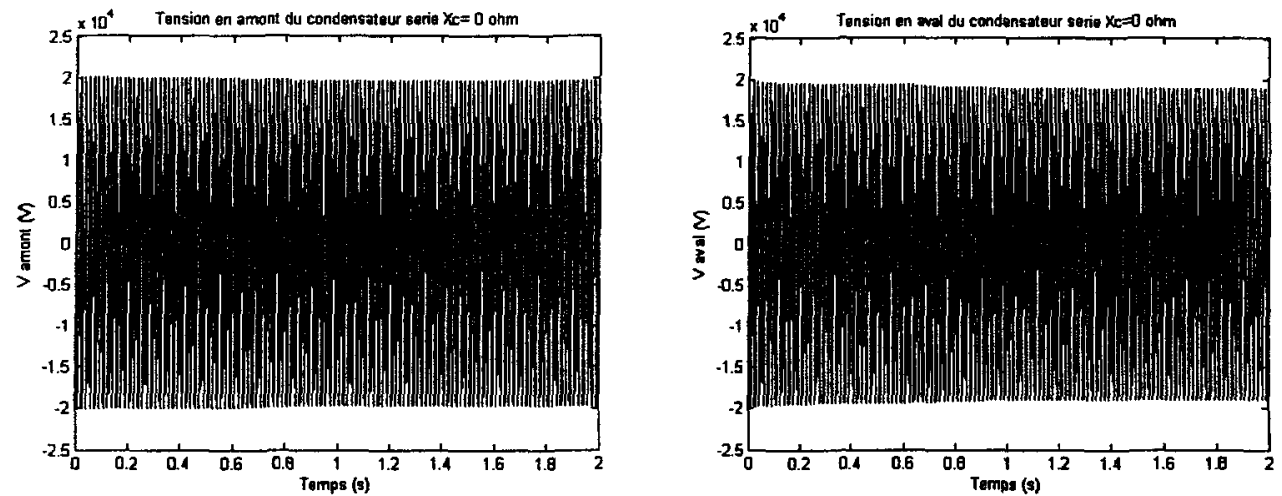

Figure A.88 : Tension en amont et en aval du condensateur série
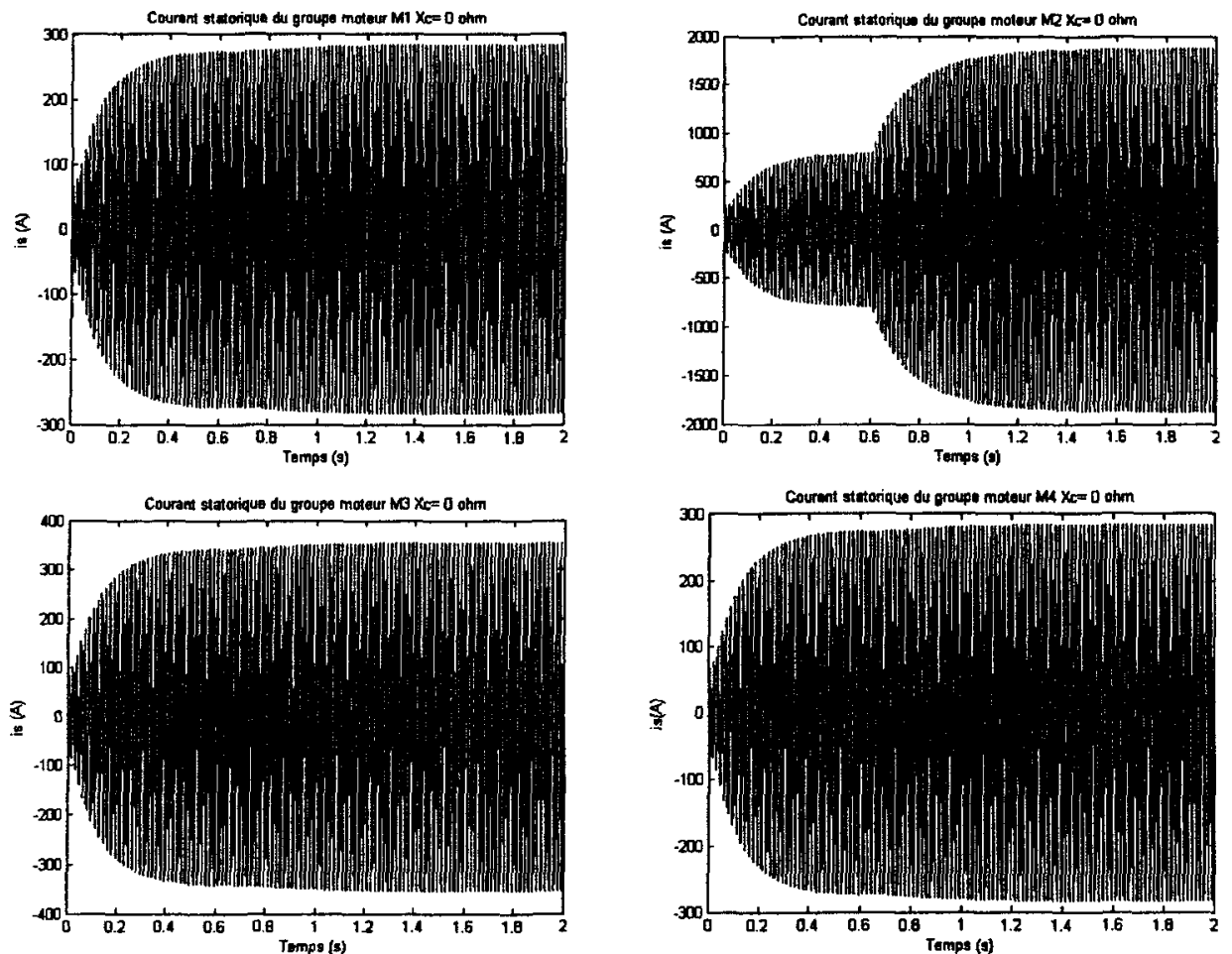

Figure A.89 : Courants statoriques des groupes moteurs 

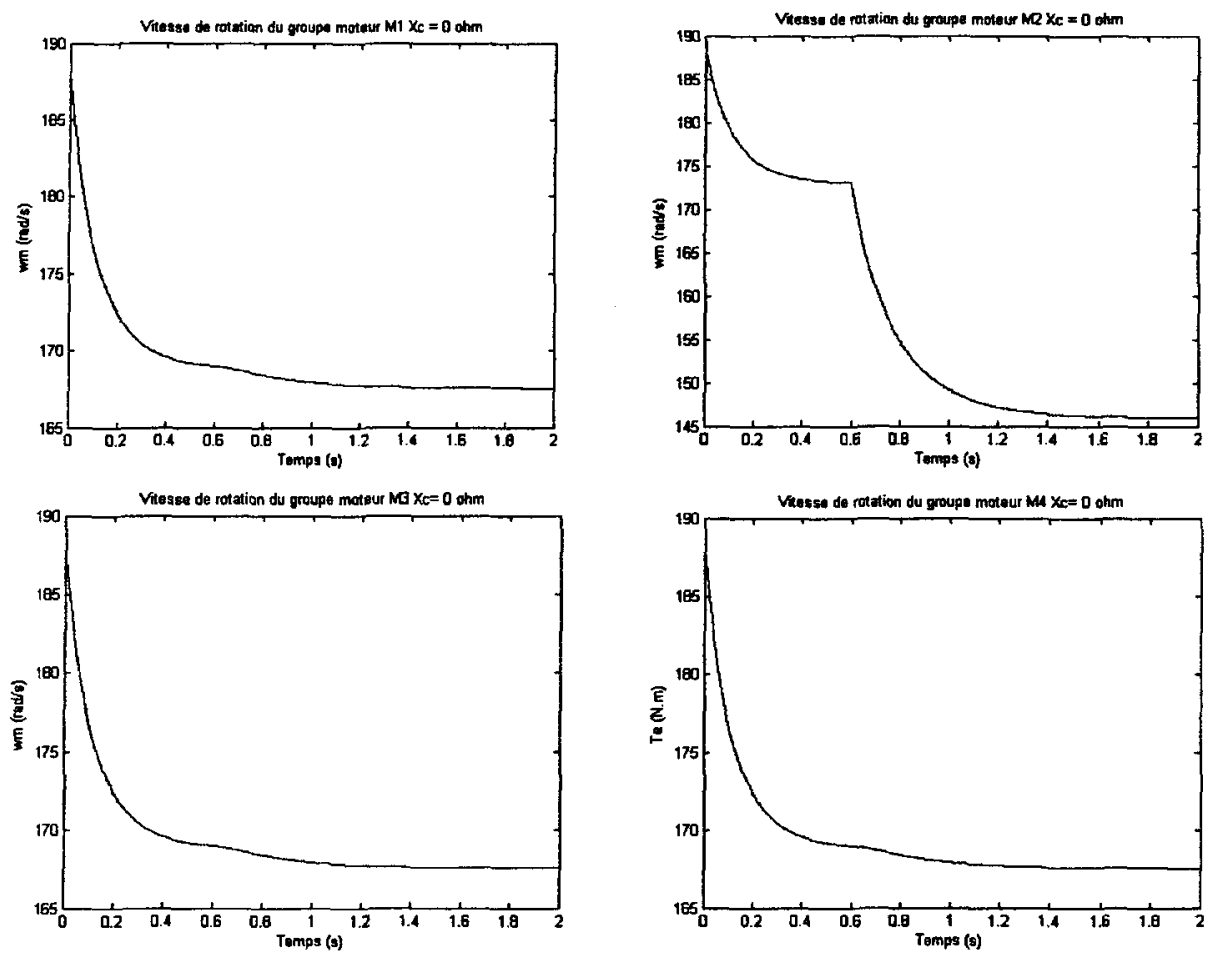

Figure A.90 : Vitesse de rotation des groupes moteurs
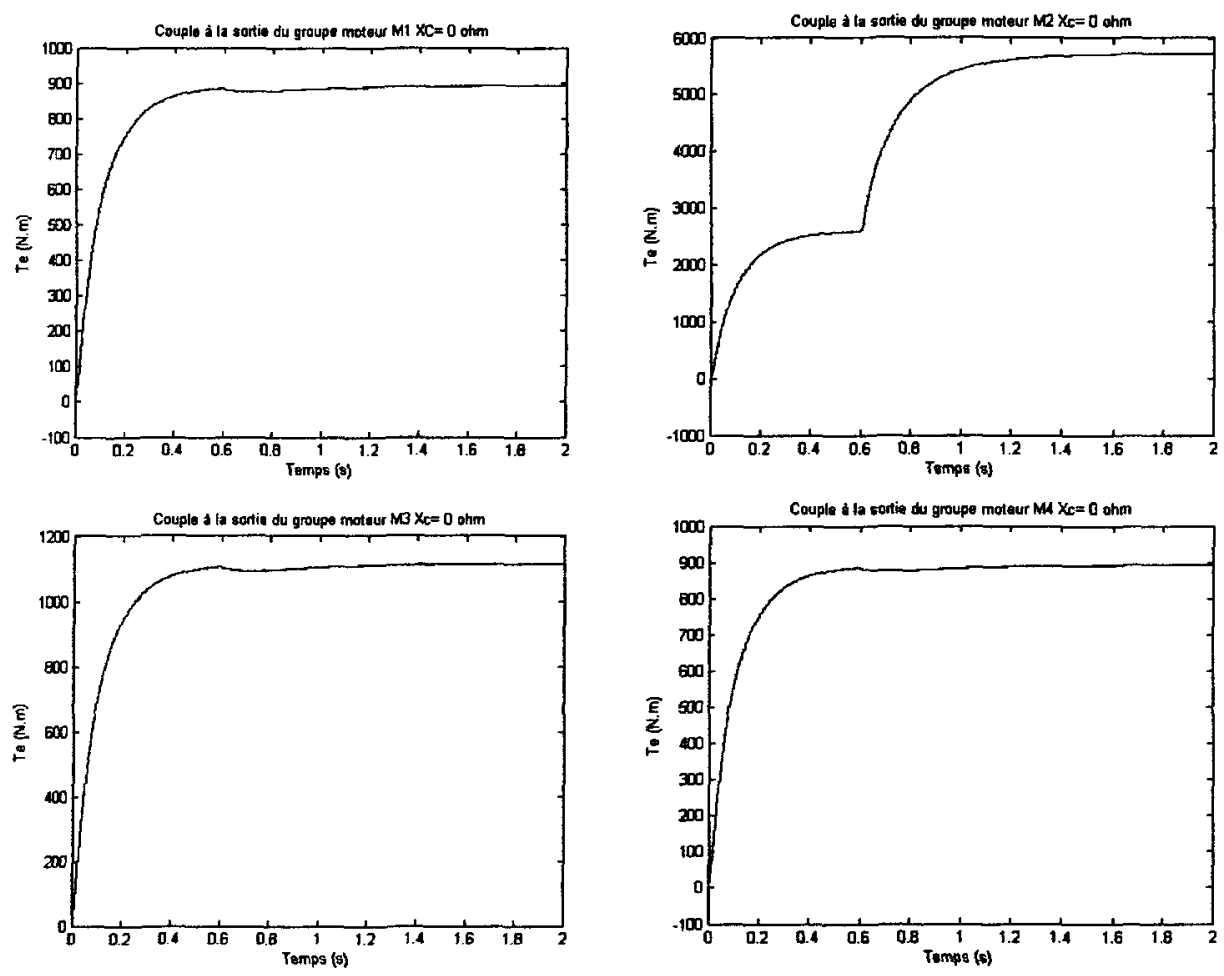

Figure A.91 : Couple à la sortie des groupes moteurs 
Réseau de distribution radial à $\tau=\mathbf{5 4 . 8 5} \%$
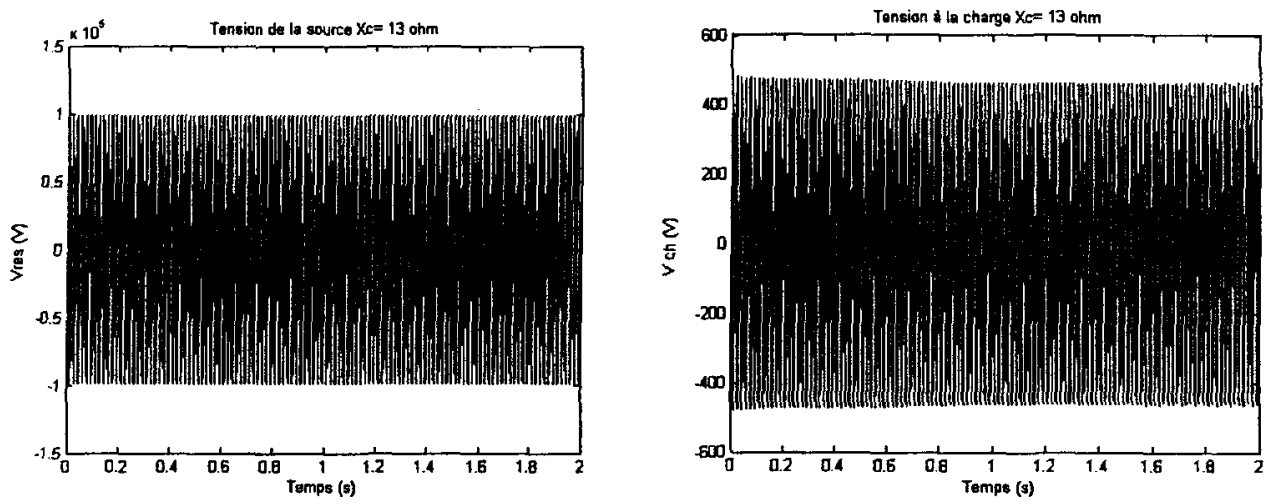

Figure A.92: Tension de la source et à la charge
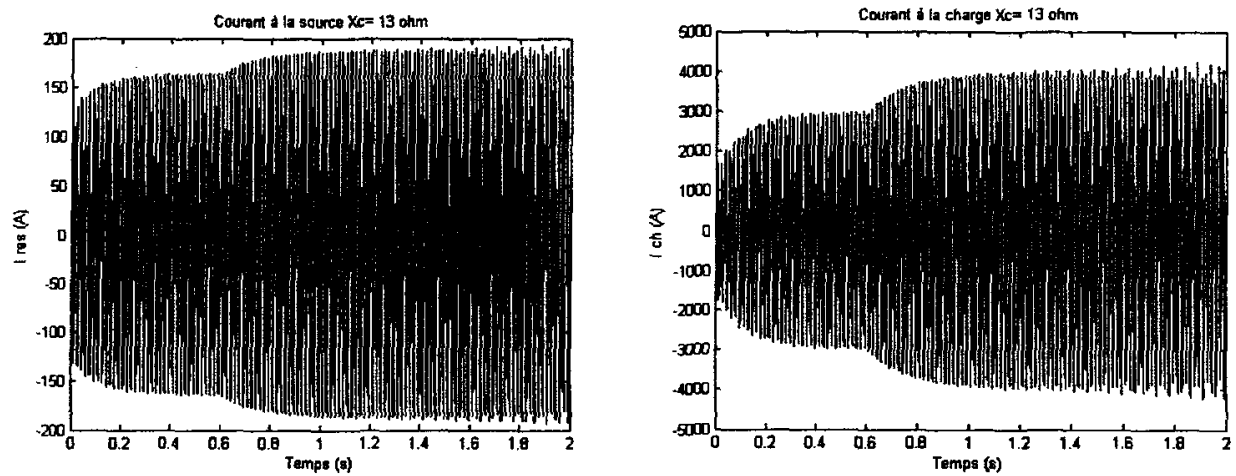

Figure A.93: Courant à la source et à la charge
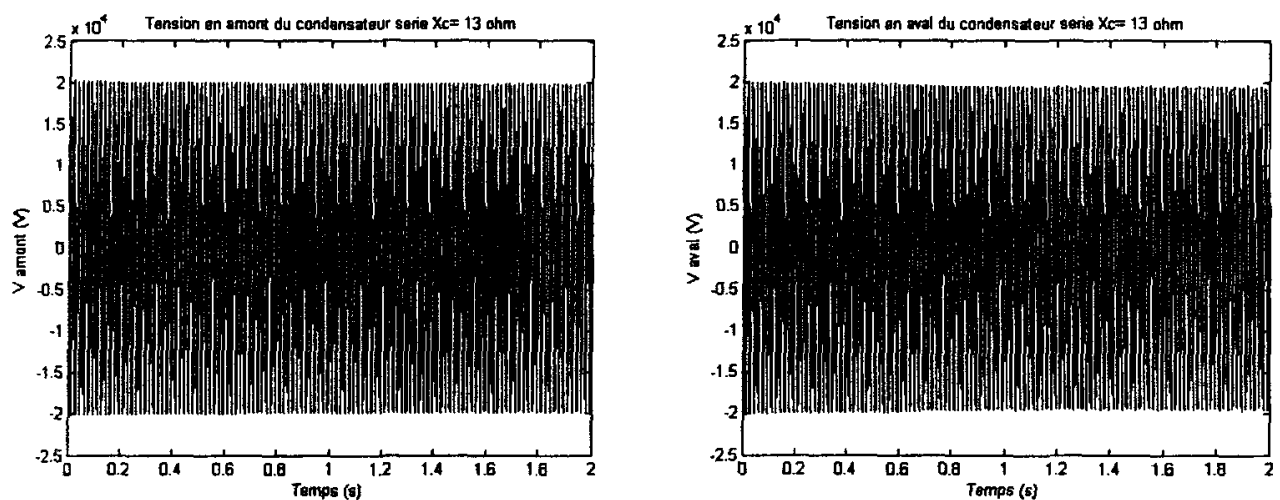

Figure A.94 : Tension en amont et en aval du condensateur série 

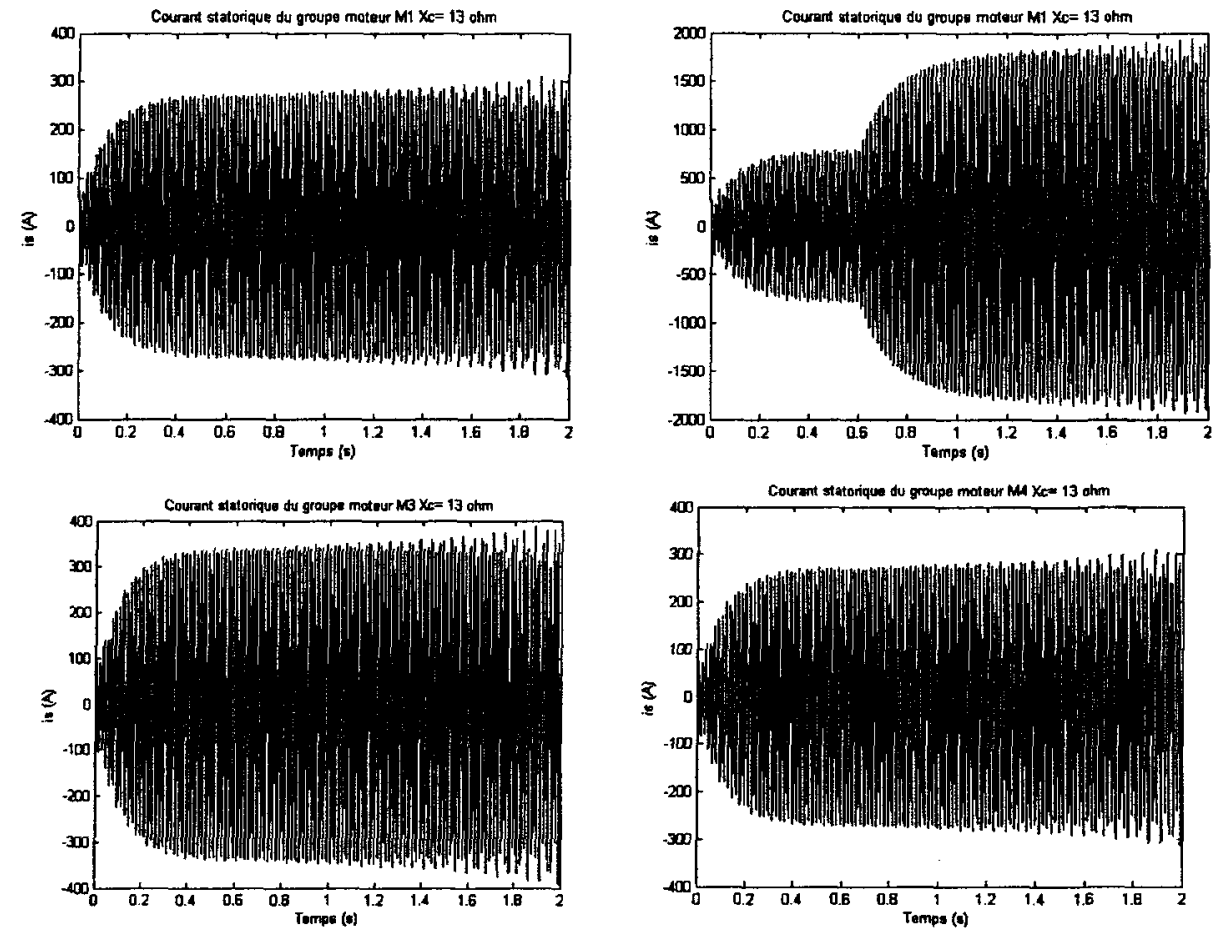

Figure A.95 : Courants statoriques des groupes moteurs
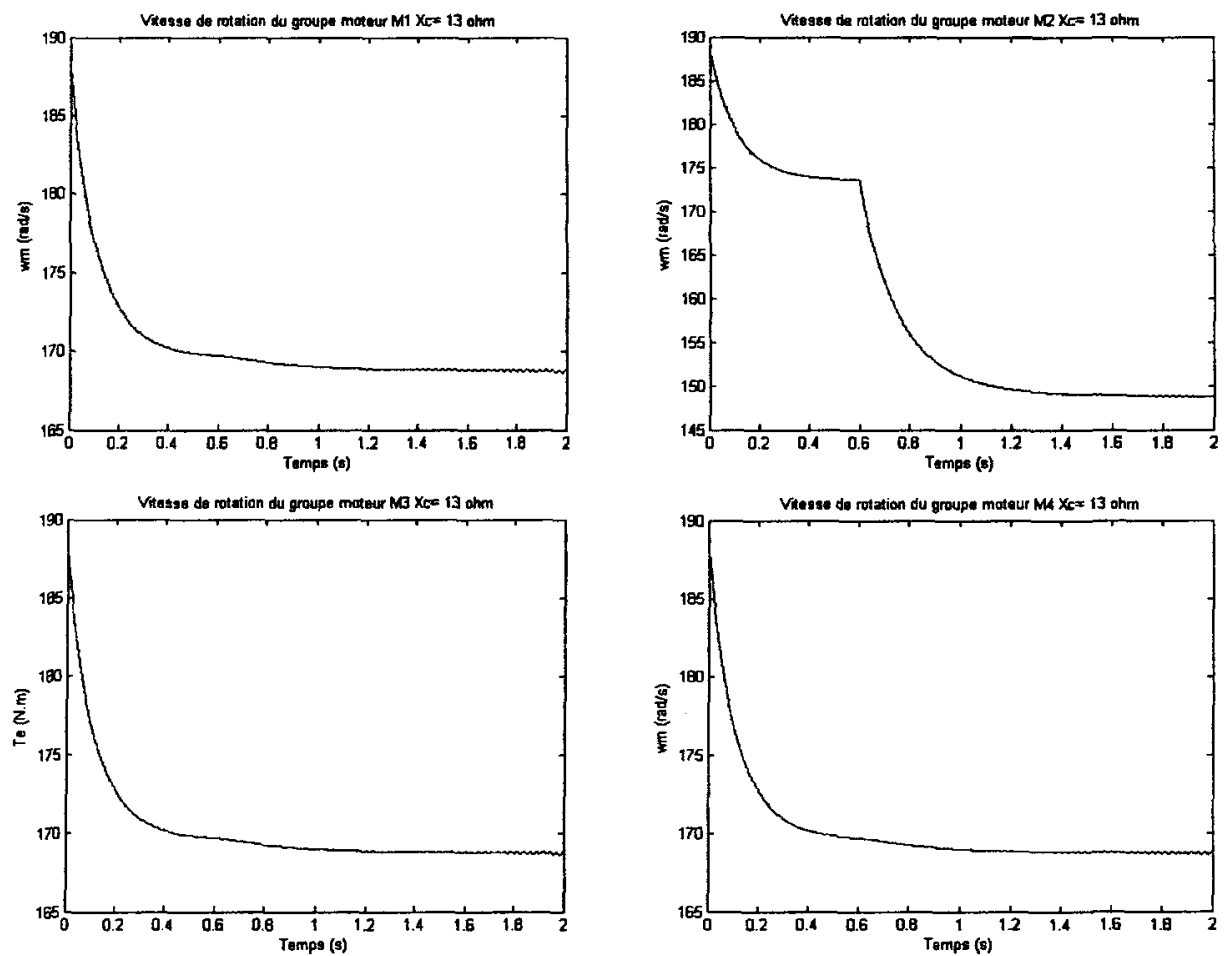

Figure A.96 : Vitesse de rotation des groupes moteurs 

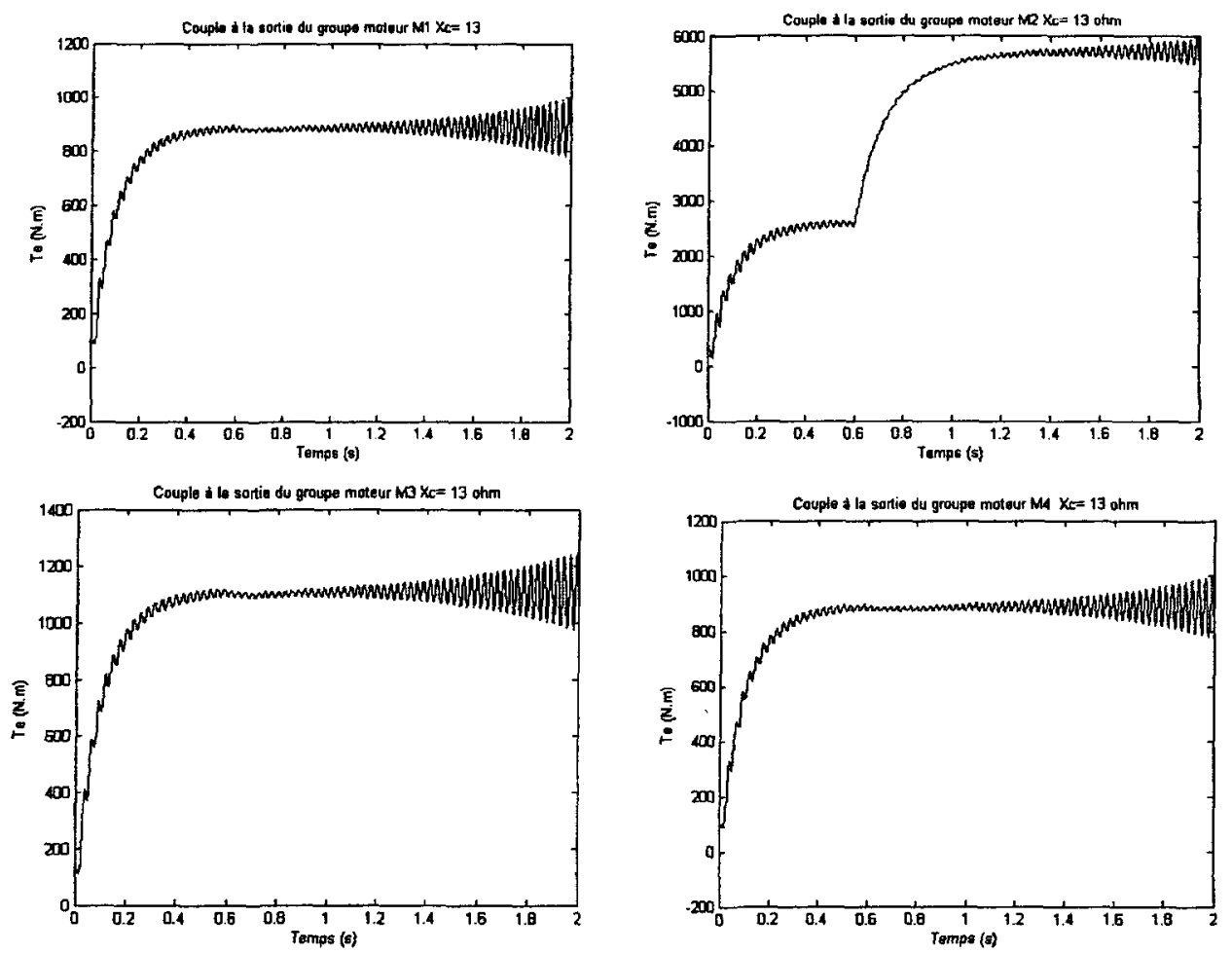

Figure A.97 : Couple à la sortie des groupes moteurs

Simulation de l'essai de démarrage à vide des moteurs asynchrones

\section{Réseau de distribution radial sans compensation série}

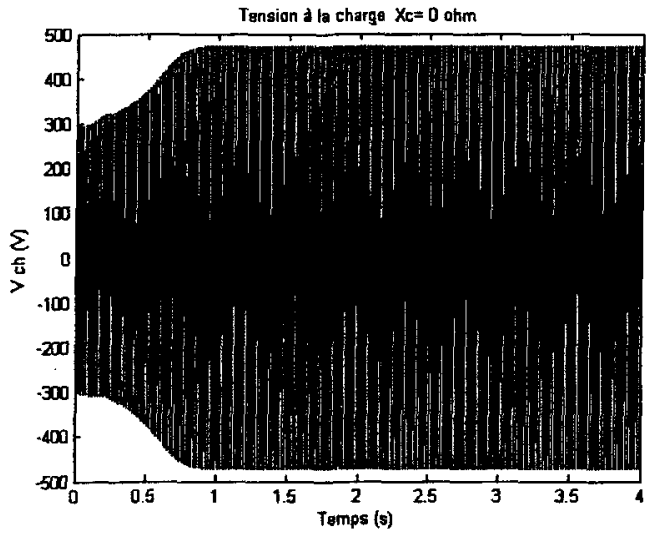

Figure A.98 : Tension à la charge

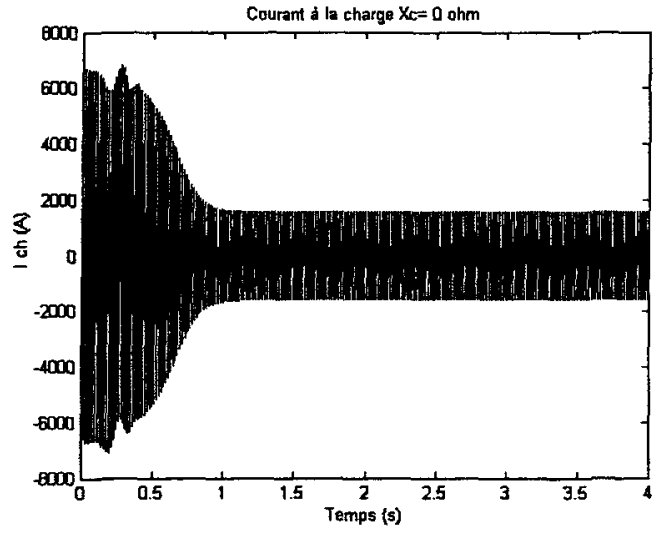

Figure A.99 : Courant à la charge 

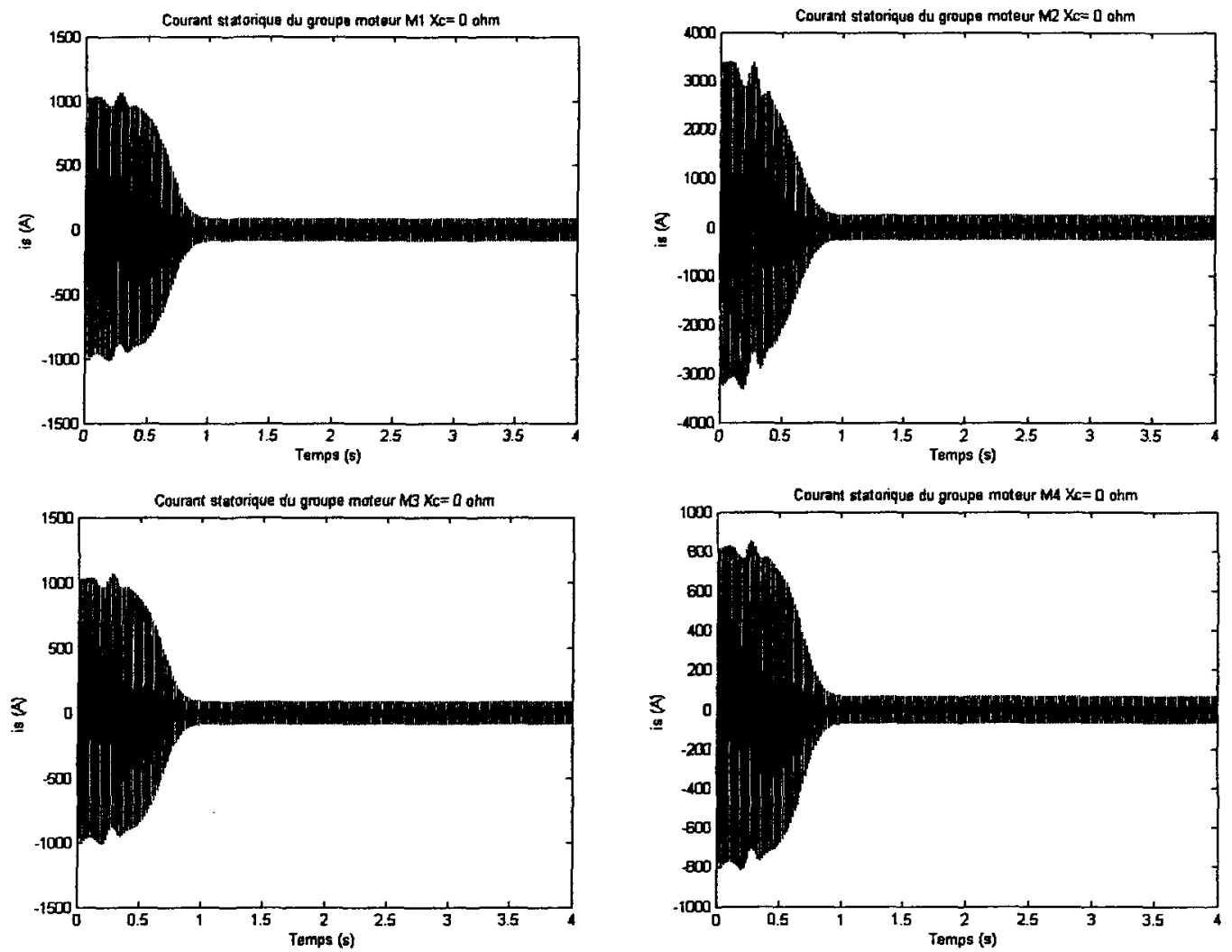

Figure A.100: Courants statoriques des groupes moteurs
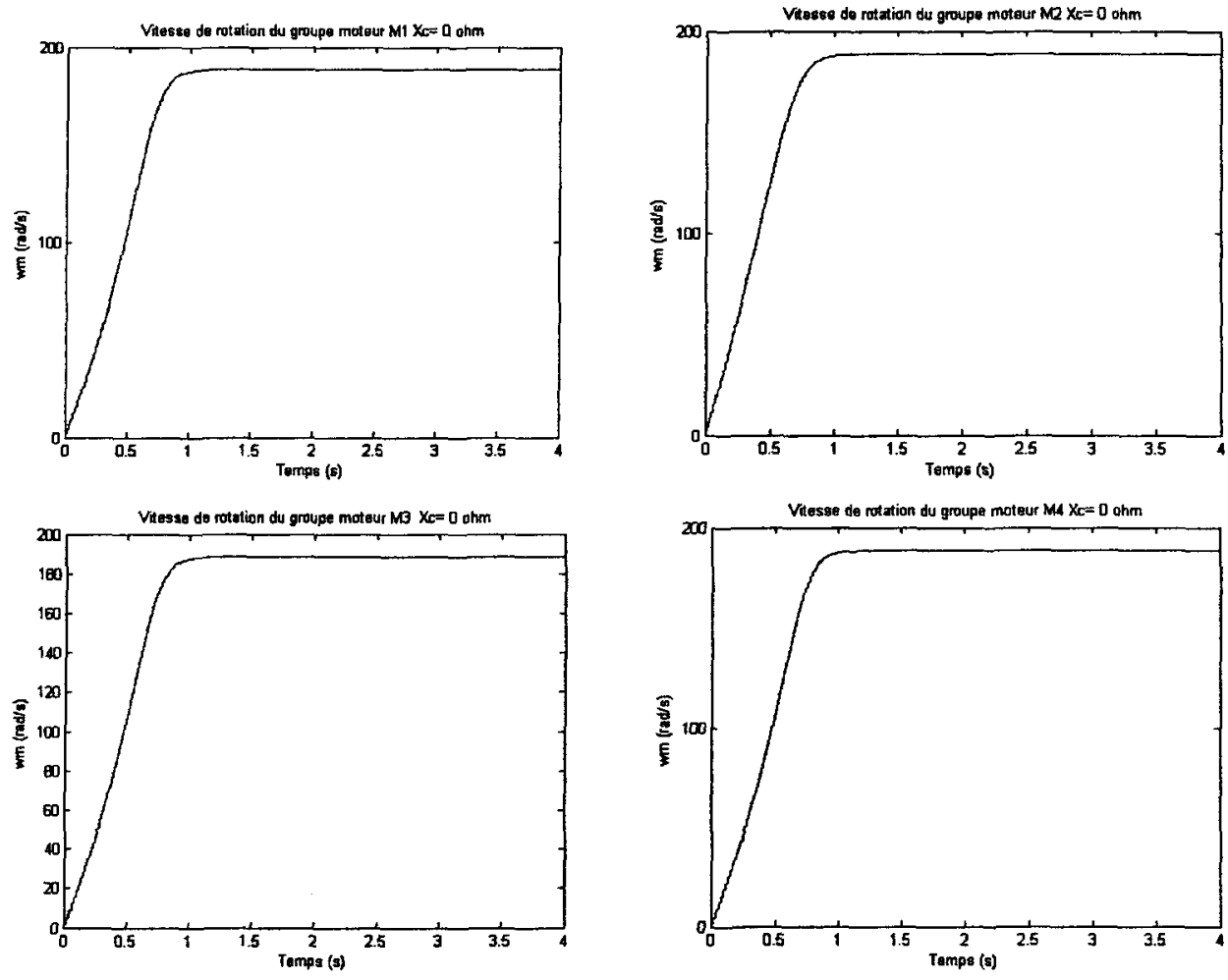

Figure A.101 : Vitesse de rotation des groupes moteurs 

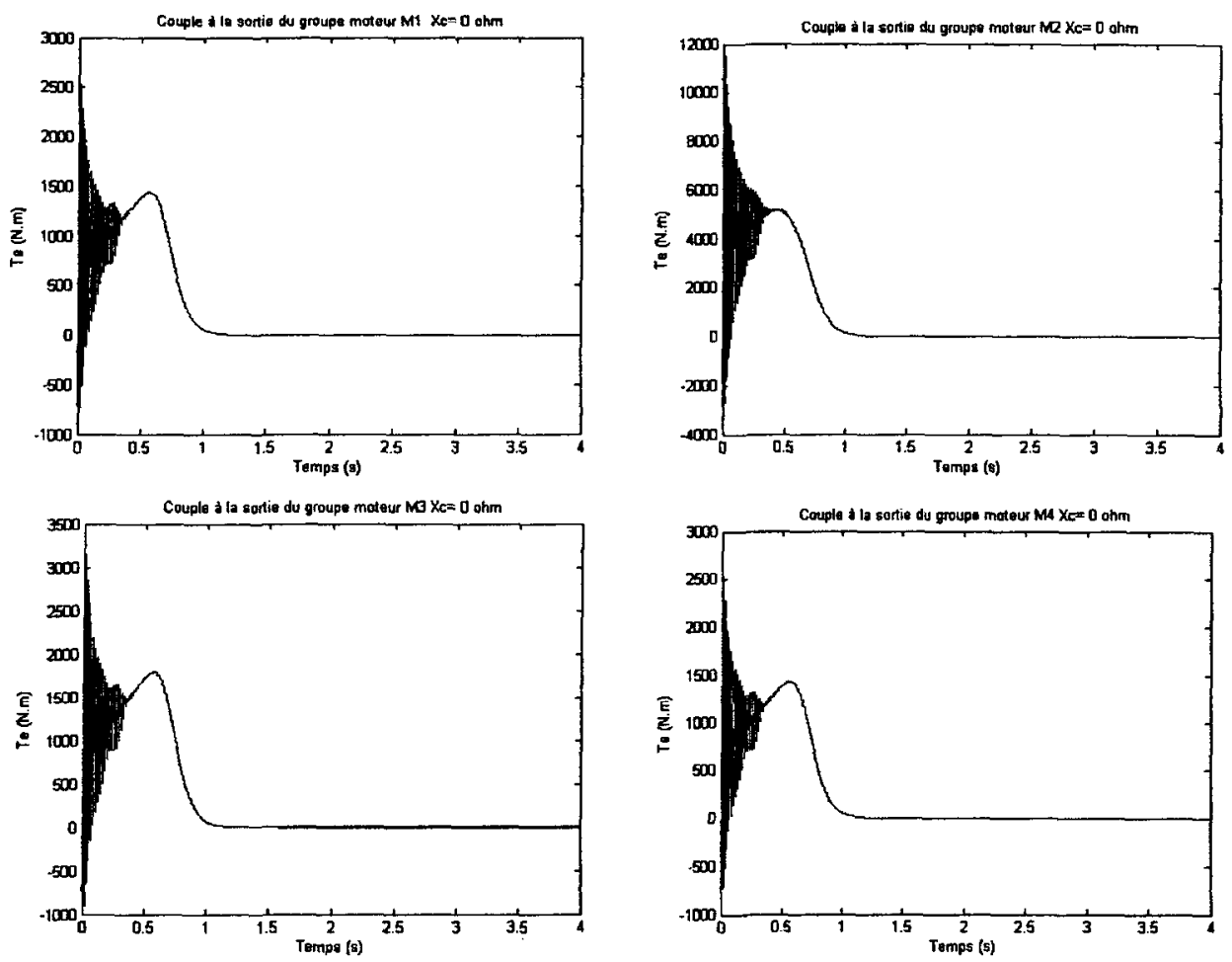

Figure A.102 : Couple à la sortie des groupes moteurs

Réseau de distribution radial à $\tau=\mathbf{5 4 . 8 5} \%$

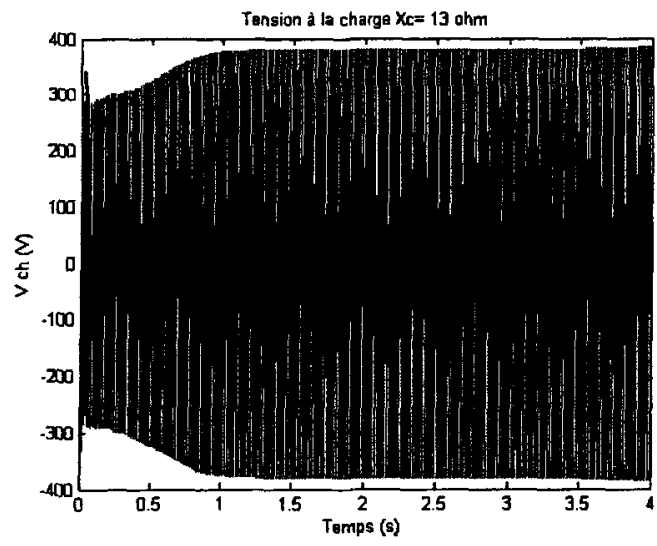

Figure A.103 : Tension à la charge

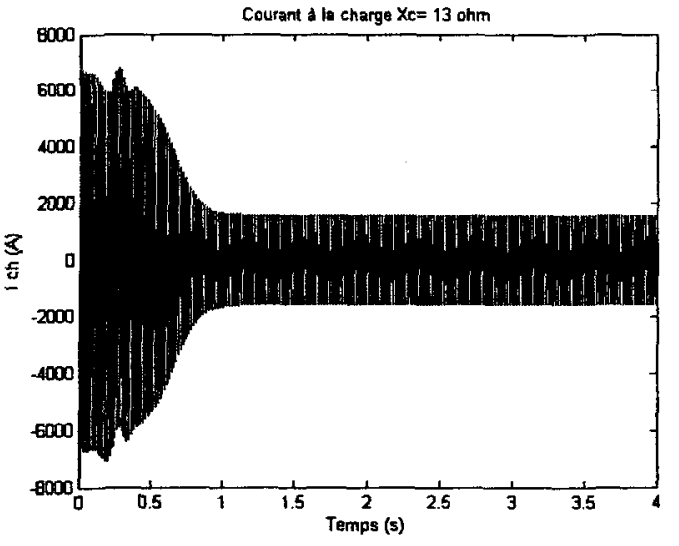

Figure A.104 : Courant à la charge 

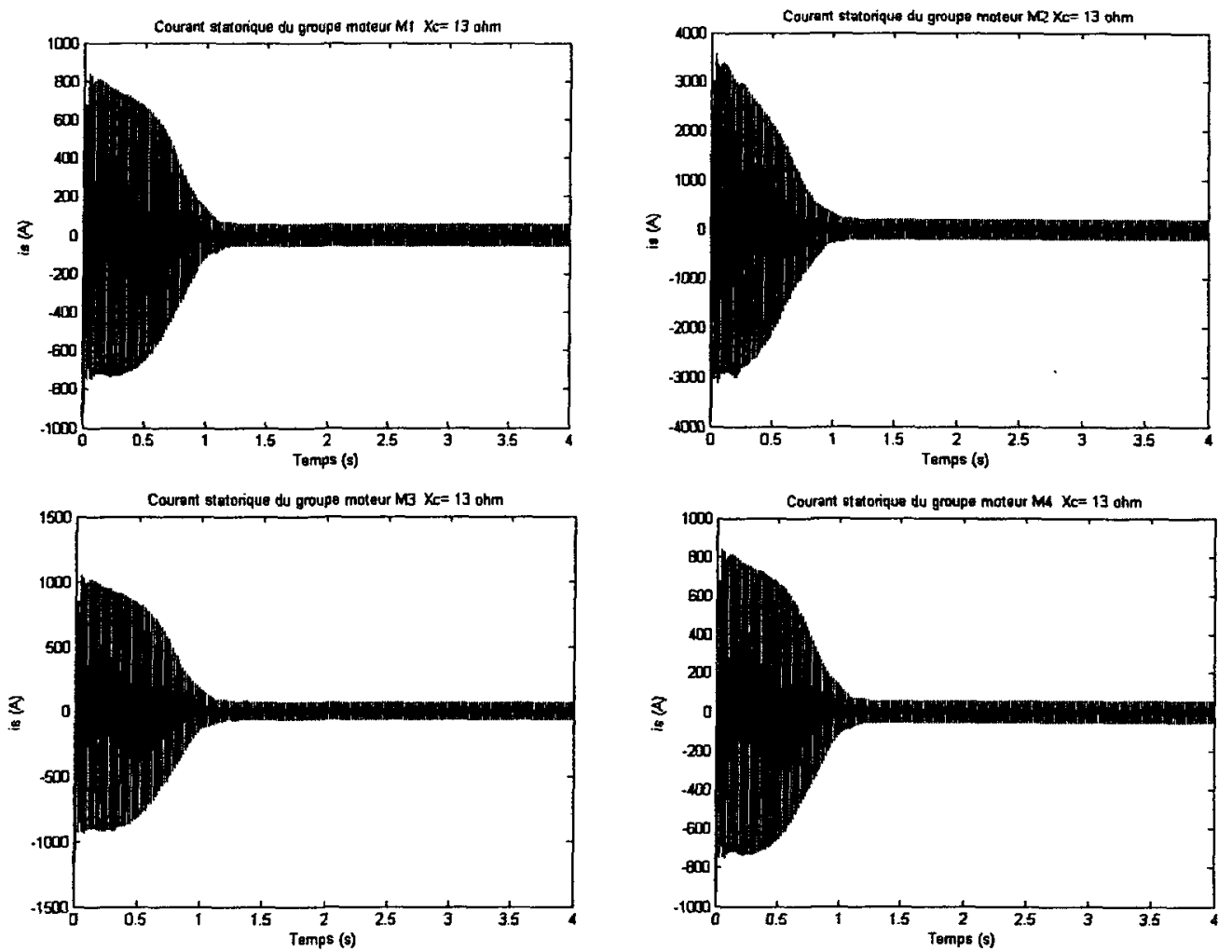

Figure A.105: Courants statoriques des groupes moteurs
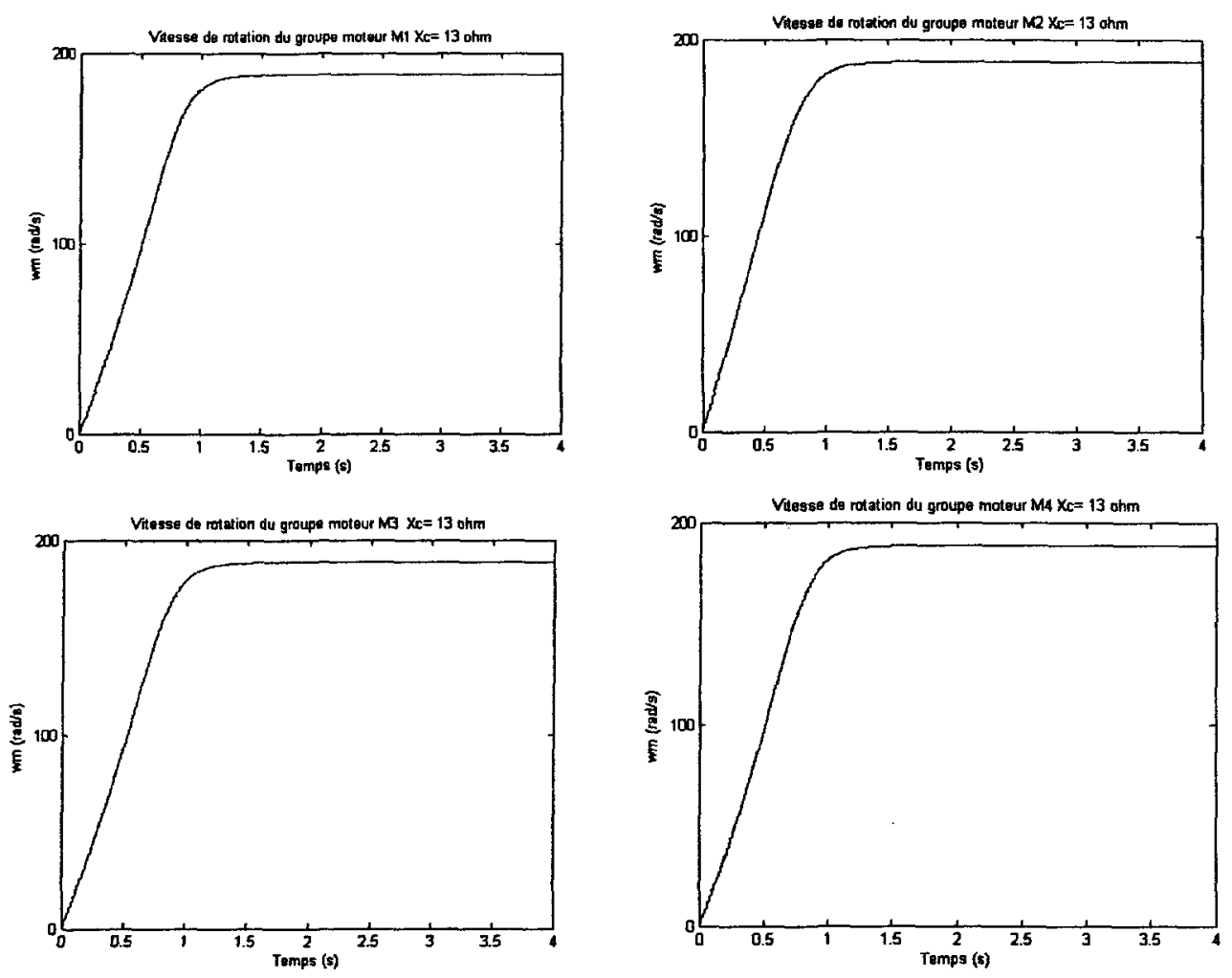

Figure A.106 : Vitesse de rotation des groupes moteurs 

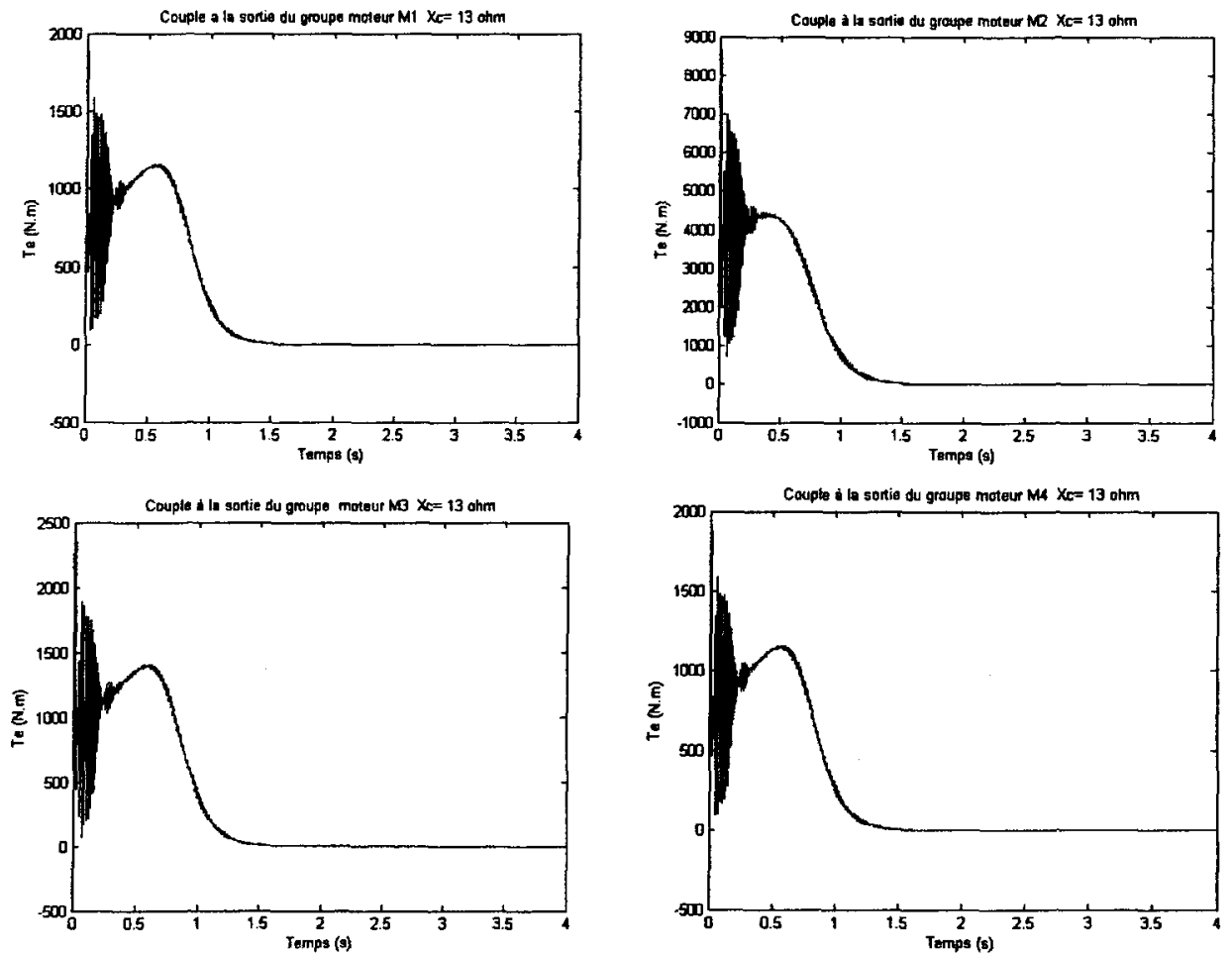

Figure A.107 : Couple à la sortie des groupes moteurs

Réseau de distribution radial à $\tau=66 \%$
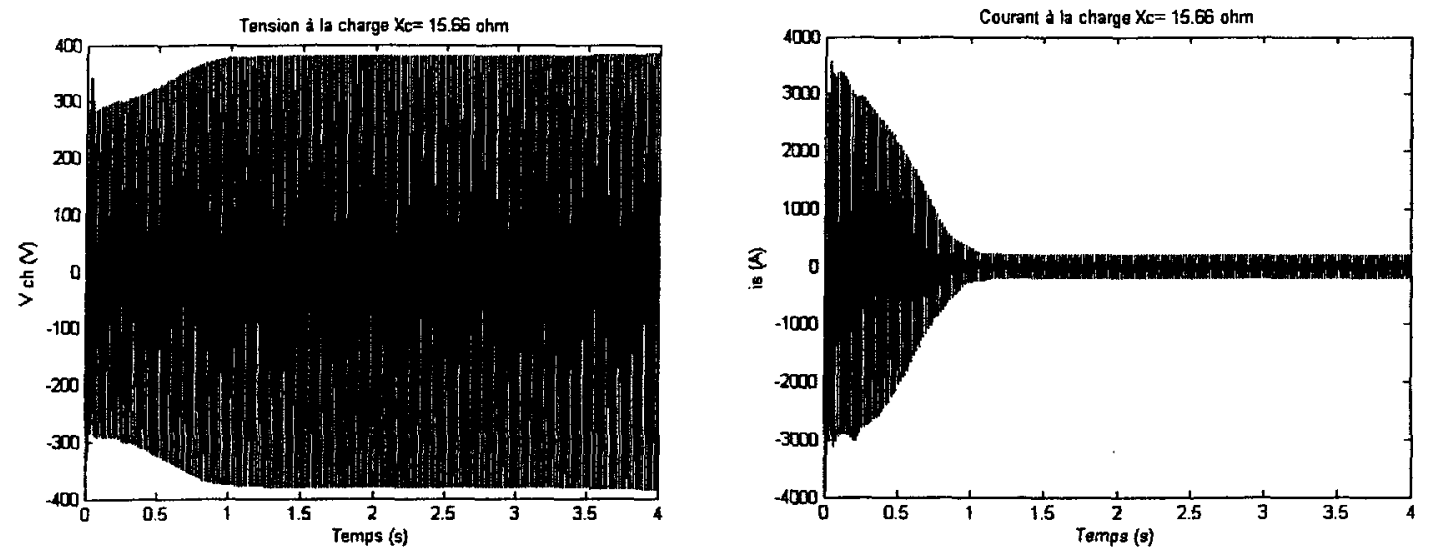

Figure A.108: Tension à la charge Figure 109 : Courant à la charge 

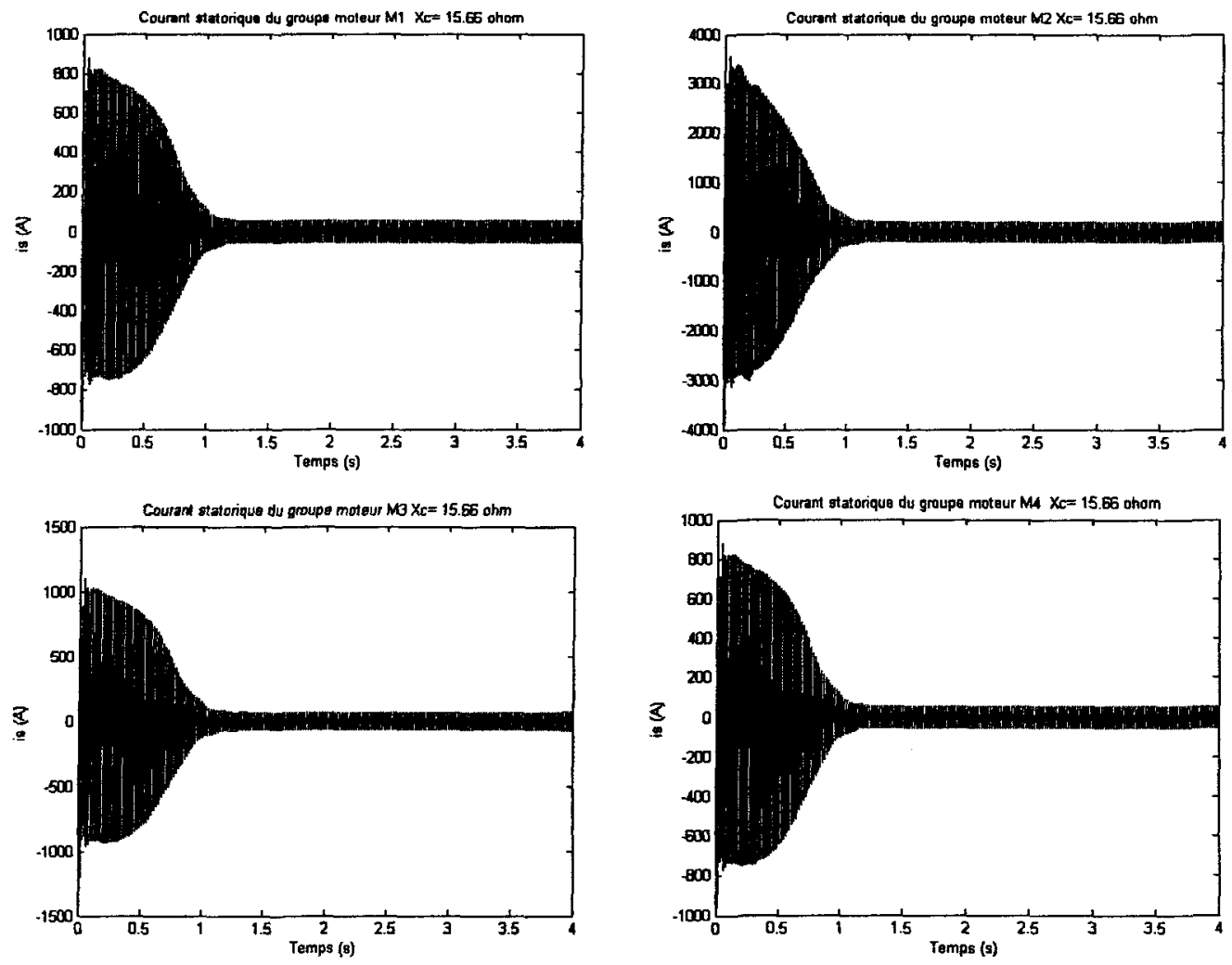

Figure A.110: Courants statoriques des groupes moteurs
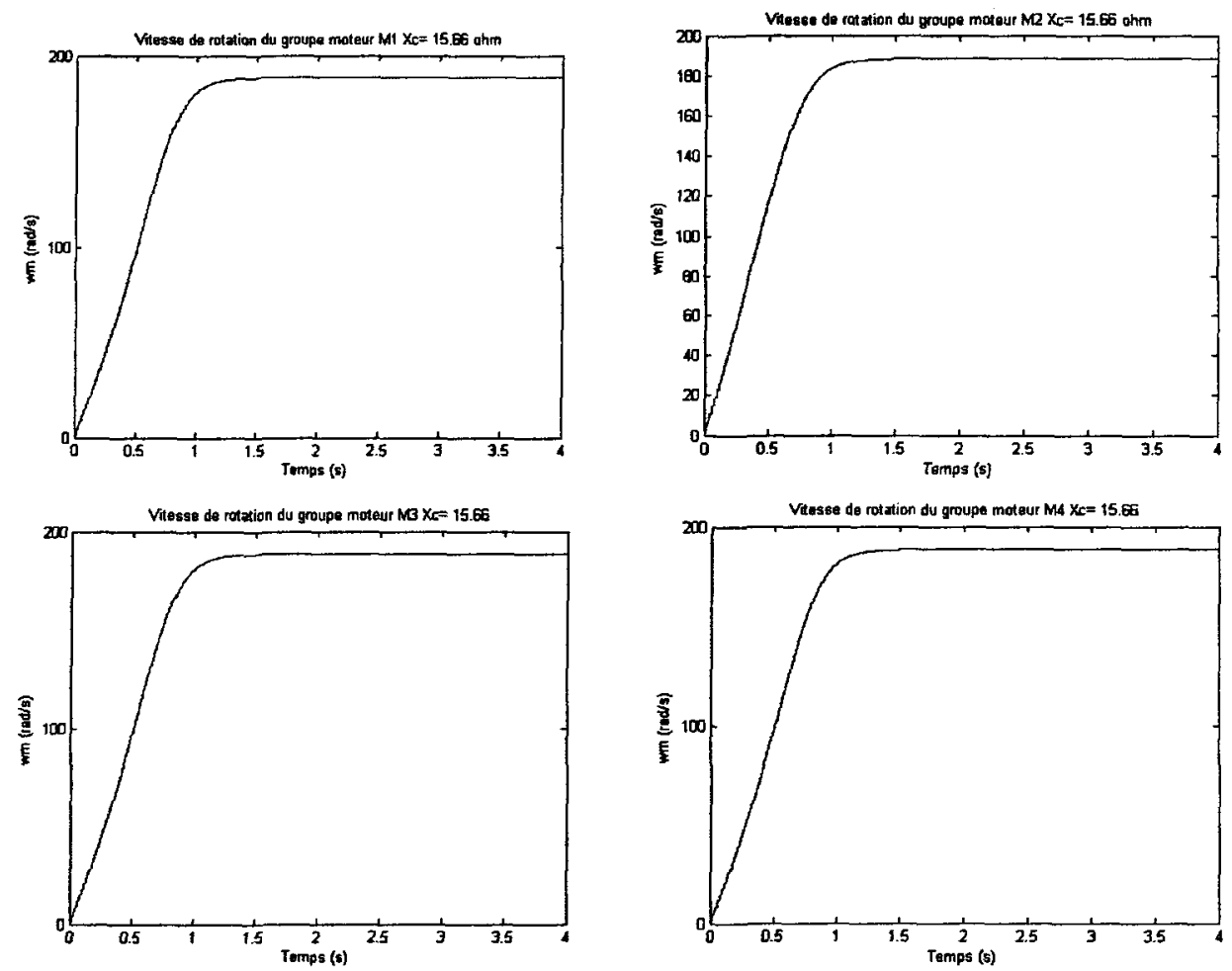

Figure A.111 : Vitesse de rotation des groupes moteurs 

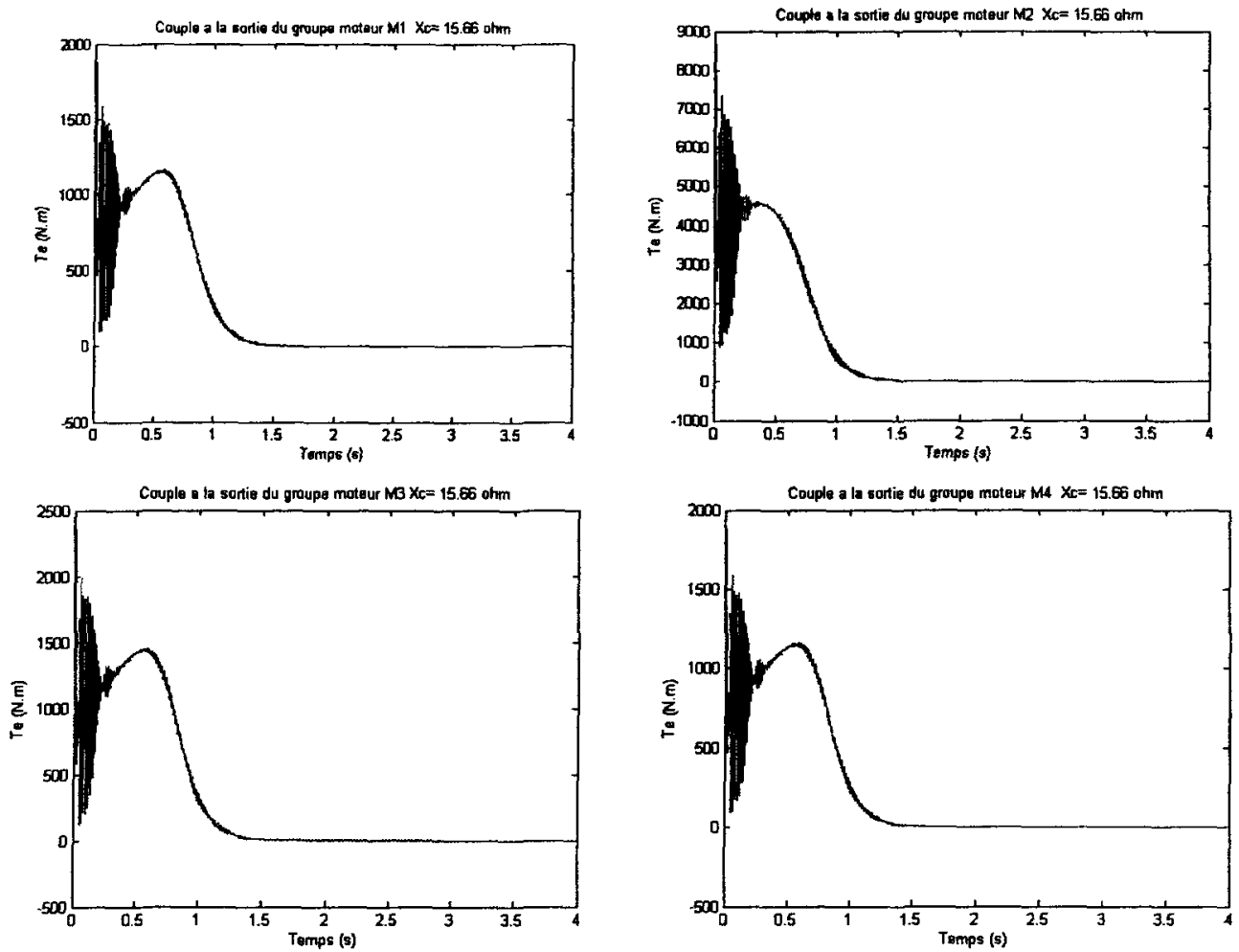

Figure A.112 : Couple à la sortie des groupes moteurs 


\section{APPENDICE K}

Publications scientifiques 


\title{
SERIES COMPENSATION OPTIMISATION METHOD «SCOM » FOR THE LONG DISTRIBUTION LINES.
}

\author{
René Wamkeue \\ Eder Fernandez R. \\ Nahi Kandil
Département des sciences appliquées, University of Quebec in Abitibi Temiscamingue 445 blvd de l'université, Rouyn Noranda, QC, J9X5E4
rene.wamkeue@uqat.ca

\begin{abstract}
In this paper, we develop an optimization method to determine the optimal series compensation ratio for a distribution network. The objective function consists of minimizing the voltage variation under several stability constraints. An initial power flow solution is used to evaluate the problem constraints formulated via algebraic equations and inequalities. The validation and effectiveness of the optimization method have been demonstrated by means of simulation analysis using PSB Power System Blockset Program in conjunction with Simulink.
\end{abstract}

Keywords: Power flow, reactive power, Hunting, Series Compensation, Flicker, SSR and Simulation.

\section{INTRODUCTION}

In recent years the constant growth of the residential and industrials loads forced the companies to undertake new projects in the construction of the electrical supply networks and important investments in research for new technologies to improve the performance of the existing distribution networks. Several power quality issues such as poor voltage regulation, voltage flicker, reduction of power flow, harmonics distortion and the strong loads variations produced by motors starting which is the one of the most important aspects that leading the distribution network to instability $[1,2]$.

In the North of Québec, Canada, the distribution network of Hydro-Quebec has to supply rural areas characterized by the long distance lines. In the operating hours at full load starting of motors, crushers, winches, etc, produces a strong voltage drop. Series compensation have been selected as an appropriate technology to give and to avoid the perturbations caused by industrial loads such as mines and sawmills connected by long distance feeders. [3,4,7]. A principal disadvantage of series compensation is the occurrence of sub-synchronous resonance which are torsional stresses on the turbine-generator shaft. But many technologies are available today to solve this problem $[10,11,12]$. In this paper, we develop an optimization method to compute the suitable series compensation degree under various network constraints evaluated using initial power flow solution. The effectiveness of the proposed network analysis is confirmed through simulations.

\section{SYSTEM DESCRIPTION}

The investigated system configuration investigated is shown in Fig. 1. It consists of two subsystems interconnected by a distribution line.

Subsystem A

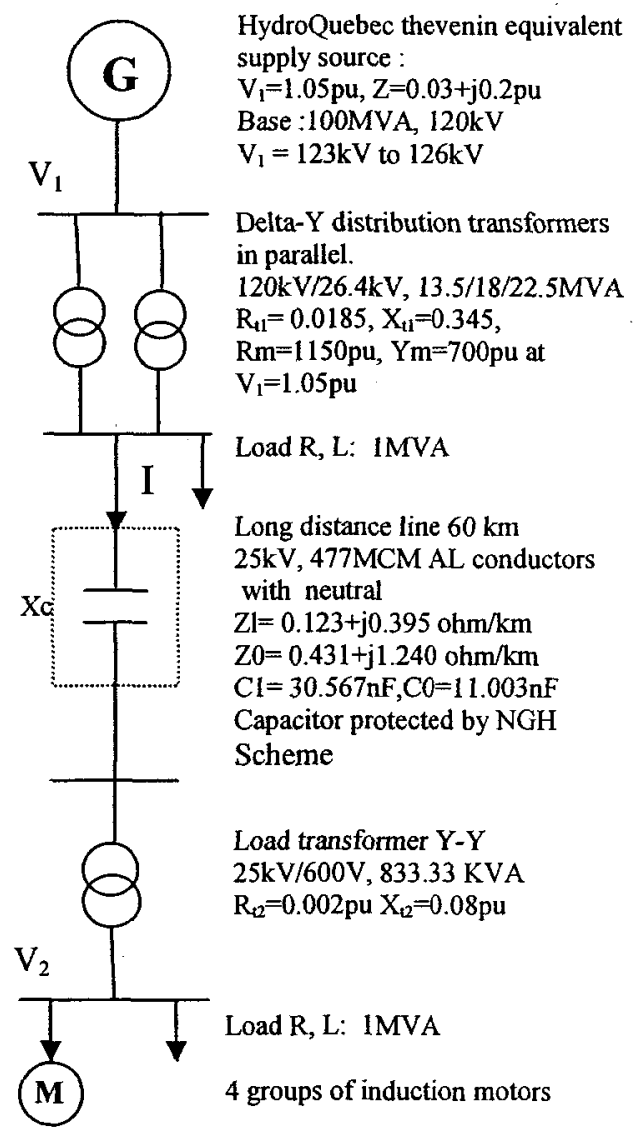

Subsystem B

Fig. 1 System configuration 
The operating frequency of the system is $60 \mathrm{~Hz}$. At $25 \mathrm{kV}$ voltage level, the power is transmitted from subsystem $A$ to $B$ via distribution line compensated by a series capacitor. The subsystem $A$ is represented by a thevenin equivalent supply source and a load R,L of 1 MVA. The subsystem $B$ by a load RL of 1 MVA and 4 groups of asynchronous motors at load, table 1.

Table1. Parameter of the motors groups

\begin{tabular}{|l|c|c|c|c|}
\hline Parameter & $\begin{array}{l}\text { Group 1 } \\
\text { 2 motors }\end{array}$ & $\begin{array}{l}\text { Group 2 } \\
\text { 3 motors }\end{array}$ & $\begin{array}{l}\text { Group3 } \\
\text { motors }\end{array}$ & $\begin{array}{l}\text { Group 4 } \\
\text { 2 motors }\end{array}$ \\
\hline Power HP & 400 & 450 & 375 & 400 \\
\hline$R s(\Omega)$ & 0.0868 & 0.0694 & 0.0214 & 0.08675 \\
\hline$X l s(\Omega)$ & 0.2 & 0.16 & 0.0284 & 0.2 \\
\hline$X m(\Omega)$ & 6.795 & 5.435 & 1.938 & 6.795 \\
\hline$X l r(\Omega)$ & 0.0685 & 0.0548 & 0.0277 & 0.0685 \\
\hline$R r^{\prime}$ & 0.1645 & 0.1316 & 0.0482 & 0.1645 \\
\hline$J K g \cdot m^{2}$ & 4.93 & 6.16 & 18.6 & 4.93 \\
\hline Torque Nm & 891.4 & 2605 & 1114 & 891.4 \\
\hline
\end{tabular}

\section{OPTIMIZATION METHOD FORMULATION}

The optimization method developed in this paper is formulated such as given in [3]:

\subsection{Objective Function}

The objective function depends on the circuit parameters as the Voltage magnitude $V_{2}$, the load power factor $(\cos \theta)$, the load magnitude $\mathrm{P}$ and $\mathrm{Q}$, the inductive reactance of the feeder $X_{L}$ and capacitive reactance of series capacitor Xc [1]. The objective function can be minimized increasing the voltage profile at load $V_{2}$ and respecting all the stability constraints. Load can change from hour to hour, at strong load variations and second to second when motor are started. In this case, series capacitor will improve instantaneously the voltage quality at the loads downstream from the series capacitor $[1,2]$. According to equations (1) and (2) the demanded reactive and active power can be expressed as follows:

Active power : $P=V_{2} \cdot I \cdot \cos \theta$

Reactive Power : $Q=V_{2} \cdot I \cdot \sin \theta$

Using the equations (1) and (2), we obtain (3) to find (4). The criterion based on minimizing the voltage variation can be built using (4) as the objective function of the optimization process. This equation introduces the series compensation reactance Xc [3]:

$$
\begin{aligned}
& \Delta V \equiv V_{1}-V_{2} \cong R \cdot I \cdot \cos (\delta-\theta)+X_{L} . I \sin (\delta-\theta) \\
& \Delta V \cong \frac{P \cdot R+Q \cdot\left(X_{L}-X c\right)}{V_{2}}=f(X c) \\
& \min f\left(X_{c}\right)=\Delta V \\
& \text { h1: } 0 \leq \frac{X c}{X_{L}} \leq 100 \% \quad \text { h2: } \quad P_{o} \leq P_{N} \\
& \text { h3: } 0 \leq \frac{R}{X} \leq 1 \quad \mathrm{~h}_{4}: \quad X c \leq x \cdot X_{L} \\
& \text { h5: } K V A_{C c} \geq \frac{\alpha .100}{0.5} \quad \text { h6: } Q_{L} \leq Q_{L o} \\
& \text { h7: } 0 \leq x \leq 60 \mathrm{Km} \quad \text { h8: } f=60 \mathrm{~Hz}
\end{aligned}
$$

Where $h$ is a set of eight network stability constraints defined as $\mathrm{h} 1$ series compensation degree and h2 maximum power transfer (6); h3 hunting phenomenon and h4 short circuit inversion current (7); h5 flicker voltage and h6 reactive power losses (8); h7 optimal series capacitor location and h8 Subsynchronous resonance (9). Those network stability constraints are defined in the next section.

\subsection{Network Stability constraints}

\subsubsection{Series Compensation degree h1}

The degree of compensation is the ratio between the capacitive reactance of series capacitors and the inductive reactance of the distribution line. By degrees higher than $100 \%$, it is said that the distribution network is overcompensated and the ferroresonance phenomenon is caused [1]. Series compensation degree is defined by $(10)$ :

$$
\tau=\frac{X c}{X_{L}}
$$

This constraint determines that $\tau=100 \%$ is the upper bound of the optimization method.

\subsubsection{Maximum Power Transfer h2}

It is well known that series capacitors improve stability bounds and increase the power transfer capabilities. Depending on the series compensation degree, the transfer impedance can be reduced increasing the transmitted power limits[4,5]. Typically 
in the power flow analysis of transmission systems, the line resistance is neglected. For distribution networks the resistance must be included since its effects are relatively high $[1,4]$. An accurate mathematical calculation of the transmitted power is developed. The following equations (11) and (12) are used to develop this constraint [5]:

$\mathrm{P}=\frac{V_{1} V_{2}}{Z l} \cos (\delta-\theta)-\frac{V_{2}}{Z l} \cos \delta$

$\mathrm{Q}=\frac{V_{1} V_{2}}{Z l} \sin (\delta-\theta)-\frac{V_{2}^{2}}{Z l} \sin \delta$

$\kappa=\cos ^{-1} F P ; Q=P \tan \kappa$

Solving equations (11), (12) and (13), the equation (14) is obtained to compute $P$ as follows:

$$
P^{2} \sec ^{2} \kappa+P\left[\frac{2 V_{2}(\cos \delta+\tan \kappa \sin \delta)}{Z l}\right]+\left[\frac{V_{2}^{4}-V_{1}^{2} V_{2}^{2}}{Z l^{2}}\right]=0
$$

Solving the equation (14) and knowing that the equivalent reactance $Z l=R+j\left(X_{L}-X_{C}\right)$, the transmitted power is equal to (15):

$P=\frac{-V_{2}^{2} \cos (\delta-\theta) \cos \kappa \pm \cos k \sqrt{V_{2}^{4} \cos ^{2}(\delta-\theta)-\left(V_{2}^{4}-V_{1}^{2} V_{2}^{2}\right)}}{Z l}$

Four different series compensation degrees have been computed to show the transmitted power performance along the line (see fig. 2 and table 2).

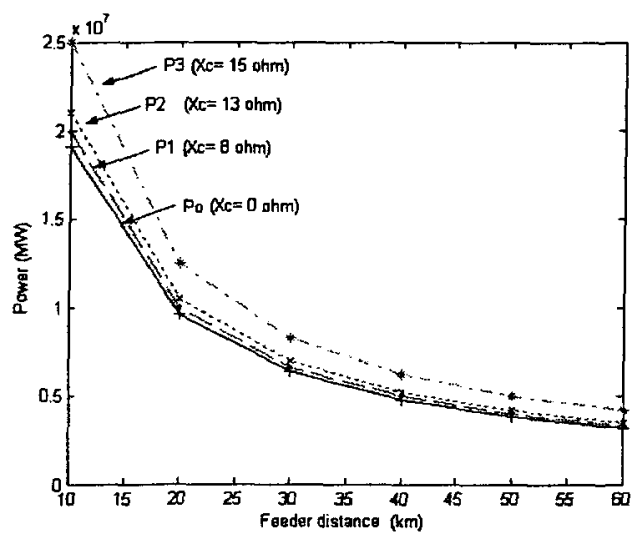

Fig. 2 Performance of power flow along the line

Table 2. Maximum power transfer

\begin{tabular}{|l|c|c|c|}
\hline Power & $\mathrm{MW}$ & $X c(\Omega)$ & $\tau(\%)$ \\
\hline$P_{0}$ & 2.5 & 0 & 0 \\
\hline$P_{1}$ & 3.08 & 8 & 33.76 \\
\hline$P_{2}$ & 3.29 & 13 & 55 \\
\hline$P_{3}$ & 3.87 & 15 & 63.3 \\
\hline
\end{tabular}

This constraint can be represented as an inequality (16):

$P_{o} \leq P_{N}$

Where the initial value of transmitted power is $P_{o}$, and $P_{N}$ is the new power magnitude after applying different series compensation degrees [3].

\subsubsection{Motors Hunting Phenomenon h3}

Hunting is a disturbance of a lightly-loaded synchronous motor caused by switching of the power circuits and changes in load or excitation current of the motors [4]. But this phenomena is not limited to synchronous motors, in induction motors driving reciprocating loads, the application of series capacitors can cause hunting. The hunting can be predicted by the ratio of the dissipation energy of the feeder resistance to the energy storage capacity of the total feeder reactance (including the series capacitor). The distribution networks supplying rural areas present a relatively high $R / X$ ratio of the long distance distribution cables [4]. The expression can be written as an inequality (17) as follows:

$0 \leq\left[\tan (\angle Z l)=\frac{R}{X_{L}-X_{C}}\right] \leq 1$

To develop this constraint, it is necessary to evaluate this phenomenon using the numerical simulation to detect the oscillations of the mechanical speed (see figs 12 and 13). To avoid the hunting phenomena, $\tau$ can be between 0 and $66 \%(\mathrm{Xc}=15.7 \Omega)$. For the investigated distribution network fig. 1, the upper bound to avoid the hunting is $55 \% \quad(\mathrm{X} c=13 \Omega)$.

\subsubsection{Short Circuit Current Inversion h4}

Current Inversion is caused for a short circuit fault when the inductive reactance is less than the capacitive reactance of the series capacitor and the relay location is capacitive with respect to the source voltage due the capacitive magnitude in the fault loop [6]. The constraint is determined as the expression (18). Equivalent reactance of the line was founded capacitive to values bigger than $\tau=55 \%(\mathrm{Xc}=13 \Omega)$.

$X c<X_{L}$ and $X C>X_{L}-R$ 
Fig. 3 show an example of short circuit inversion when the equivalent reactance is negative.

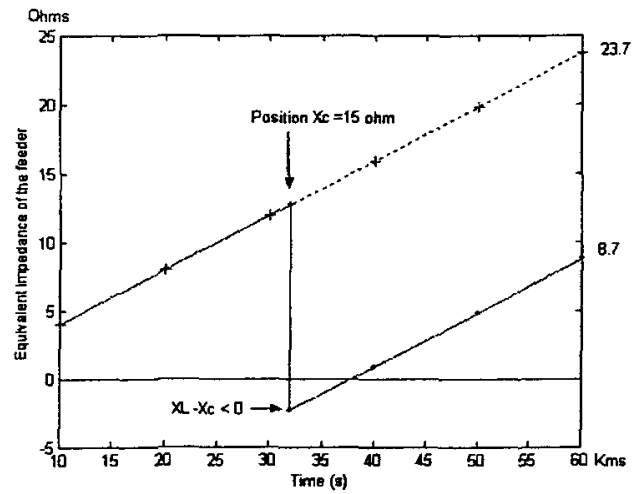

Fig. 3 Performance of the equivalent reactance

\subsubsection{Flicker Voltage h5}

The flicker is associated with an excessive voltage drop along the feeder reactance caused by a momentarily large current with an inherently low power flow. This phenomenon is usually detected when large motors start at the end of a feeder [4].The procedure to quantify and develop this constraint begins calculating the flicker voltage using the equation (19) [7]:

$$
\Delta V \%=\frac{K V A s t a r t}{K V A_{C C}} * 100
$$

The flicker voltage to the industrial costumers is about $0.5 \%$ at a frequency of $8 \mathrm{~Hz}$ [7]. The demanded power at load can be solved using the starting $I d$ and nominal current $I n$, the performance $\eta$ and the starting class of motor $\beta$, equation (20).

$$
\text { KVAstart }=\frac{H P \cdot 0.746 \cdot(I d / I n) \cdot \beta}{\eta \cdot P F}
$$

The flicker frequency $g[\mathrm{f}(\mathrm{i})]$ can be computed using (fig 4). Flicker voltage is included in equation (19).

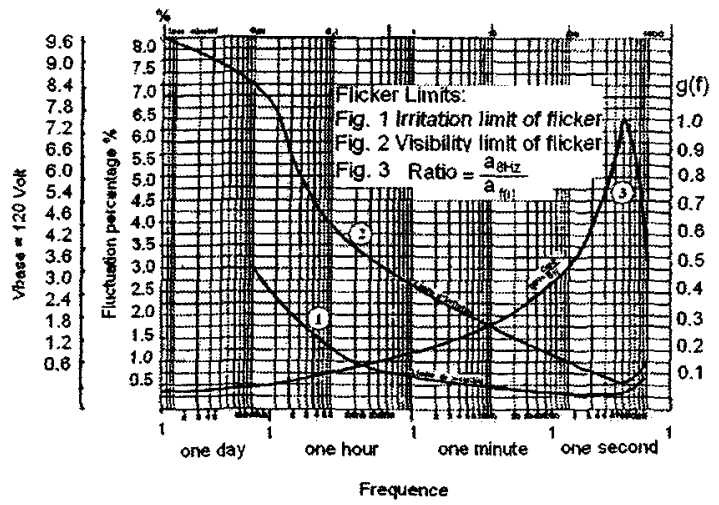

Fig. 4 Flicker Limits
The $\mathrm{g}[\mathrm{f}(\mathrm{i})]$ is obtained from residential costumers in the periods of 1 hour, 1 minute and 1 second to build the curve 3 (fig. 4). Now, we calculate the $\alpha$ at each motor (21) and the $\alpha$ total, as equation (22) [7].

$$
\begin{aligned}
& \alpha_{i}=\frac{H P \cdot 0.746 W \cdot \frac{I d}{I n} \cdot \beta}{\eta \cdot F P} \cdot g[f(i)] \\
& \alpha_{8 H z}(\mathrm{t})=\sqrt{\sum_{i} a_{8}{ }^{2}(t)}
\end{aligned}
$$

Solving (21) the minimal short circuit power at load to avoid the flicker is computed using (23) as follows:

$$
K V A_{C C} \geq \frac{\alpha .100}{0.5}
$$

Now we compare the value calculated with (23) and the short circuit power from (24) to determine the minimal series compensation degree in which the short circuit power is minimal and the flicker is reduced.

$K V A c c=\frac{V_{2} / \sqrt{3}}{\sqrt{(R s+R t+R)^{2}+\left(X s+X_{1}+X_{L}-X c\right)^{2}}}$

Where $R s+j X s$ is the equivalent impedance of the source and $X t$ the transformer impedance. This constraint allows to determine the minimal $K V A_{C C}$, that is equal to $14.6 \mathrm{MVA}$ with $\tau=21 \%(\mathrm{Xc}=5 \Omega)$.

\subsubsection{Reactive Power Losses h6}

Reactive power maintains the voltage to deliver active power through distribution lines. Series capacitors increase the reactive power and reduce the losses. A reactive power study is developed with different series compensation in the distribution network fig 1 [8]. Reactive and active power can be using (25) to develop the equation (26) as follows:

Knowing that: $V=V_{1}^{2}+V_{2}^{2}-2 V_{1} V_{2} \cos \delta$

$P+j Q=\stackrel{2}{I}(R+j X)=V^{2}\left[\frac{R}{R^{2}+X^{2}}\right]+V^{2} \mathrm{j}\left[\frac{X}{R^{2}+X^{2}}\right]$

This constraint can be written as inequality (27):

$$
Q_{L} \leq Q_{L o}
$$

Where the initial values of reactive power losses are $Q_{L O}$, and $Q_{L}$ the new values after the application of different series compensation degrees.[3]. 


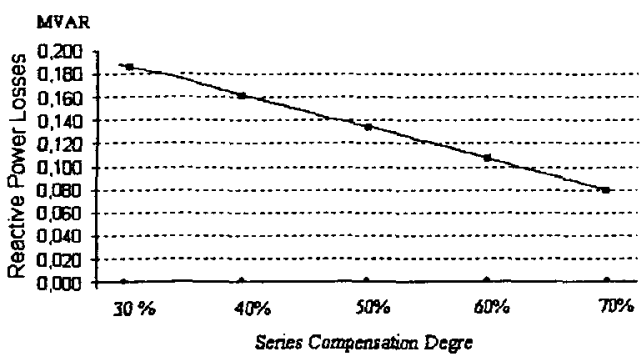

Fig.5 Reactive power losses

The fig. 5 shows that effectively using series capacitor, the reactive power losses will be reduced increasing the receiving end voltage.

\subsubsection{Optimal Series Capacitor Location h7}

In this section a mathematical method has been developed based on the voltage variations at each side of series capacitor and load downstream. (see Fig. 6)[1,7].

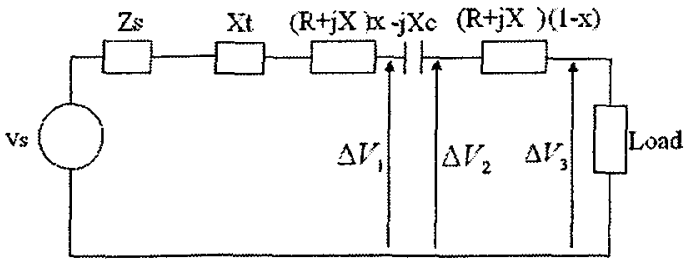

Fig.6 Single-line diagram of a radial circuit.

The circuit voltages upstream (28), downstream from the capacitor (29) and the load voltage (30) can be calculated using fig. 6 , as follows:

$$
\begin{aligned}
V_{1} & =\frac{(1-x) Z l-j X c+Z c}{Z s+Z l-j X c+Z c} V s \\
V_{2} & =\frac{(1-x) Z l+Z c}{Z s+Z l-j X c+Z c} V s \\
V_{3} & =\frac{Z c}{Z s+Z l-j X c+Z c} V s
\end{aligned}
$$

According to fig. 6, the expressions (31) determine the performance of series capacitor:

$$
\Delta V_{1} \% \leq \Delta V_{2} \% \text { and }\left|\Delta V_{3} \%\right| \leq\left|\Delta V_{2} \%\right|
$$

Solving (31), the resulting expression is developed in equation (32):

$$
|(1-x) Z l-j X c+Z c| \geq|Z c|
$$

Replacing the equivalent reactance of the line $\mathrm{Zl}$, the equation (32) results in the equation (33):

$$
(I-x)\left(R+X_{L}\right)-X c \geq 0
$$

Solving the equations (33), the optimal series capacitors position $x$ is computed as the equation (34), considering the resistance of the line as follows:

$$
x \leq \frac{R+X_{L}-X c}{R+X_{L}} \quad \text { to } X c<X_{L}
$$

\subsubsection{Sub-Synchronous Resonance SSR h8}

The distribution network investigated is equipped with a thyristor control reactor connected in parallel in order to protect series capacitor against SSR, fig 7. It is based on the scheme developed by Narian G. Hingorani known as NGH-Scheme $[9,10,11]$. The protection consists of a damping scheme that measure the half-cycle of series capacitor voltage and if this half-cycle period exceeds a present period, the energy of the capacitor will be dissipated into a resistor shunting the series capacitor through two anti-parallel thyristor damping of the SSR.[9].

Using the steady state frequency response, we can identify a SSR about $7 \mathrm{~Hz}$ ( $\tau=55 \%$ ), fig.8, removed it using NGH scheme (fig.7).

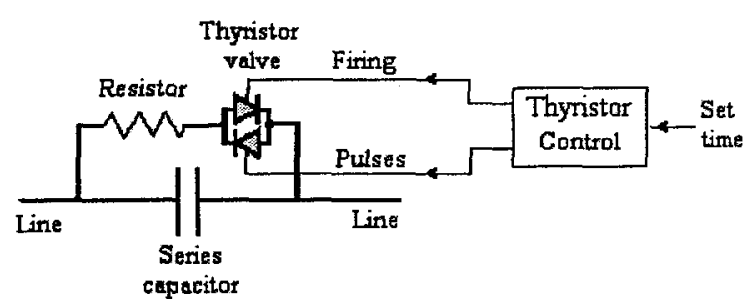

Fig. 7 NGH SSR Damping device

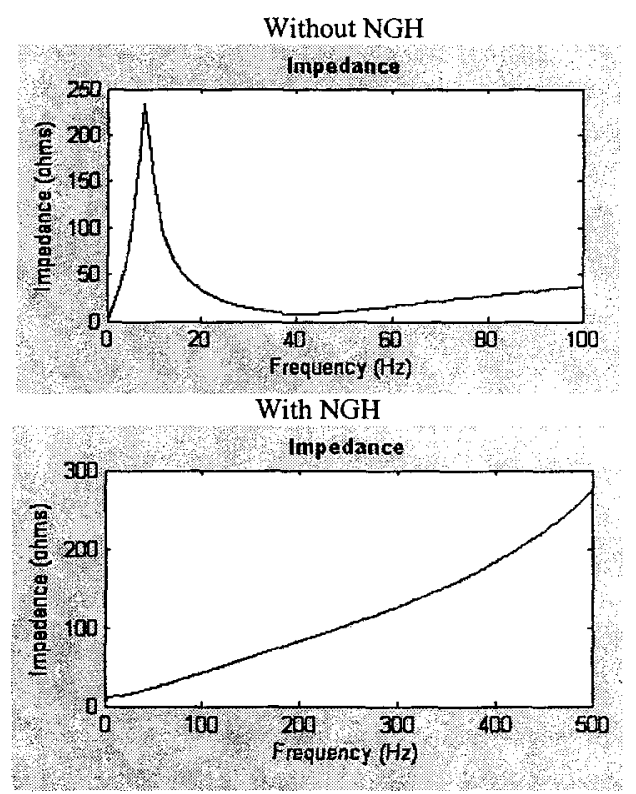

Fig.8 Elimination of SSR using NGH scheme for $\mathrm{Xc}=13 \Omega$ 


\section{OPTIMIZATION PROCEDURE}

Step 1.Identification and definition of the stability constraints and objective Function.

Step 3 Power Flow Solution.

Step 4 Verifying of the constraints and the objective function to find the SCOR.

Step 5 Condition N.1: $X_{C}<X_{L}$ must be accomplished Step 6 Data Base of $X_{C}$

Step 7 Condition N.2: Selection of the $X_{C}$ optimal

Step 6 Results of the optimization method and simulation of typical tests

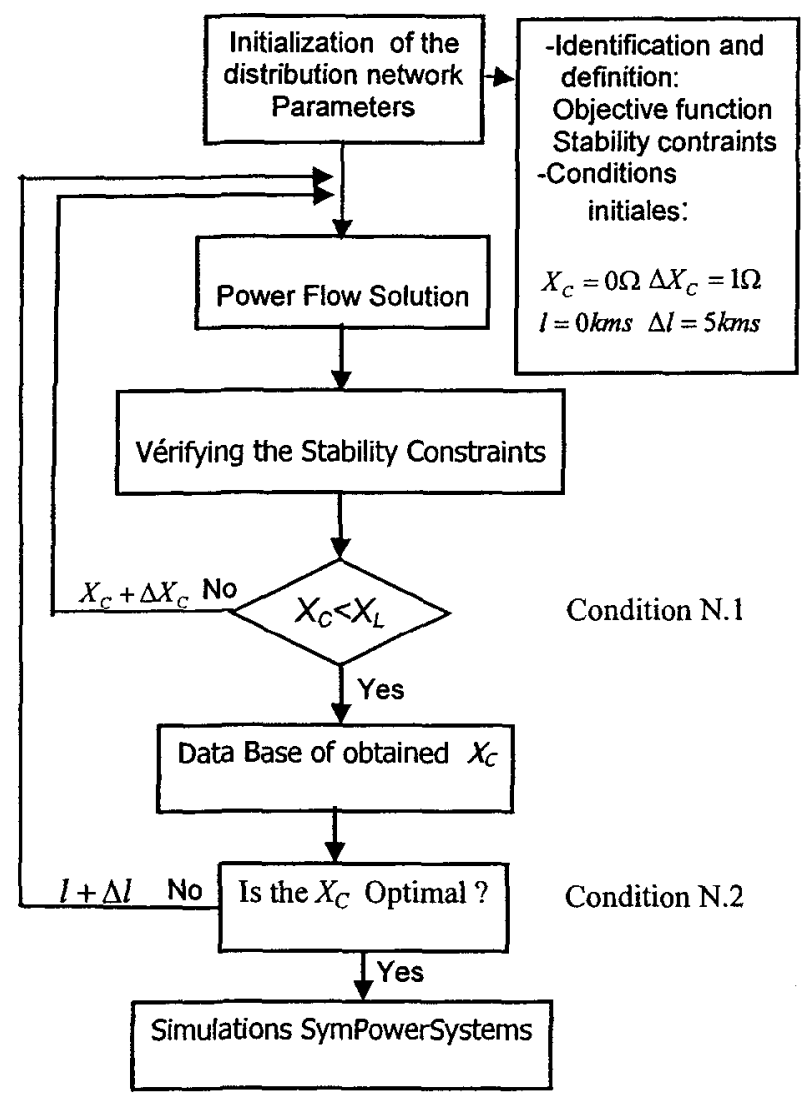

Fig.9 Optimization method

\section{OBTAINED RESULTS: THE SERIES COMPENSATION REGION "SCOR" \\ OPTIMIZATION}

\subsection{THE SCOR:}

The performance of the optimization method can be observed using an operation region modeling based on the lower and upper bounds of the stability constraints. The objective function is included on this region to evidence the minimization. As shown in fig. 10, the constraints about the maximum power flow and reactive power losses have been accomplished. The constraint h3 about Hunting phenomenon reduces the SCOR to $\tau=66 \%$. The constraint h4 limits the SCOR to $\tau=55 \%(\mathrm{Xc}=13 \Omega)$, and the equivalent reactance of the line is positive. The constraint $\mathrm{h} 5$, flicker voltage, define the minimal $\tau$ in which this phenomenon is avoided. Fig. 10 resumes the previous network stability constraints as follows:

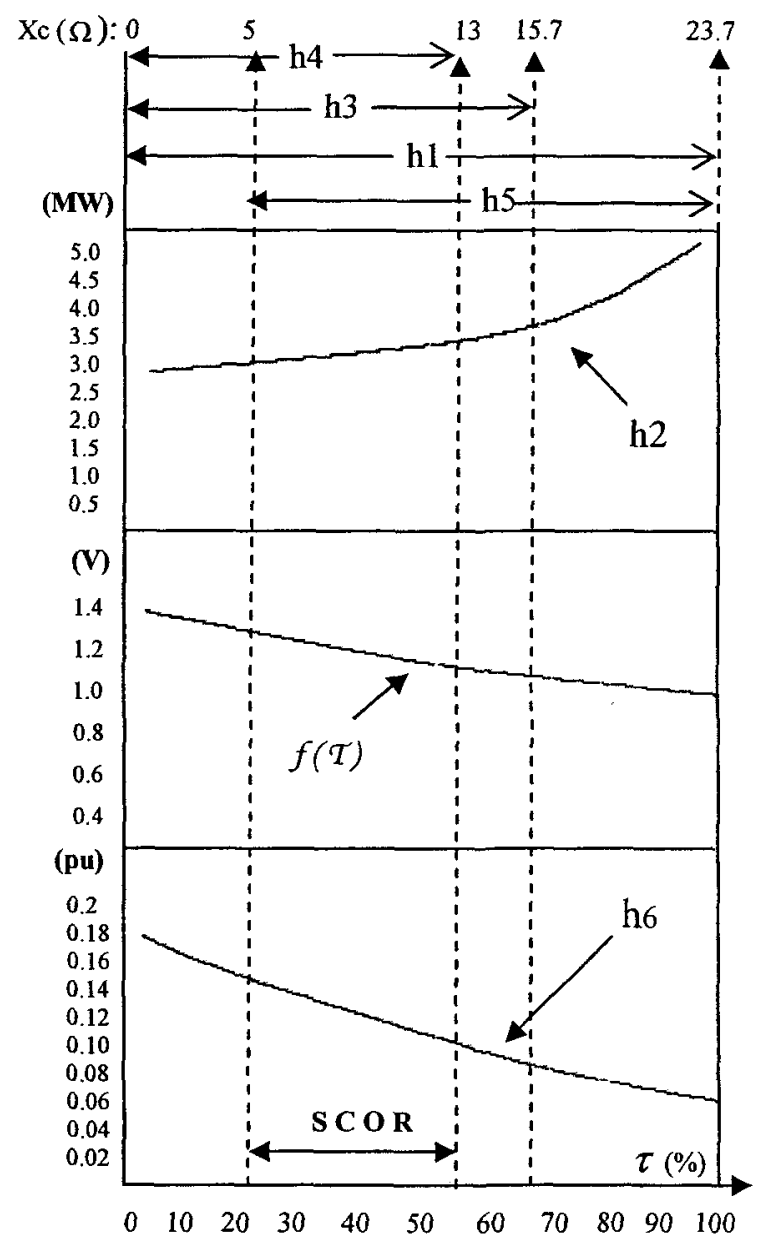

Fig.10 Series Compensation Optimization Region SCOR

\subsection{NETWORK PERFORMANCE PREDICTION}

The simulations were developed considering the analytical results obtained by means of the Series Compensation Operation Region, SCOR. The electrical torque and the mechanical speed of the motors group 2 is simulated at different conditions to observe its performance during the typical perturbations produced in the distribution network. Two scenarios have been evaluated considering the lower and upper bound of the SCOR. Hunting phenomenon also was detected during the perturbations. The first scenario is a single phase to ground short circuit fault, fig. 11, occurs on phase A between the transformer $\mathrm{X}_{\mathrm{t} 1}$ and the series capacitor at 
$t=1 \mathrm{~s}$. The series compensation increases the fault current flow at $750 \mathrm{~A}(\tau=55 \%)$, due to the reduction of the reactance from the source to the location of the fault. The strong reduction in the mechanical speed is observed during the fault. The performance of the mechanical speed for $\tau=55 \%$ is better than $\tau=21 \%$ (fig. 12). The elevation of the magnitudes and the duration of the oscillations in the electrical torque are observed when $\tau \geq 21 \%$ (fig. 12). For the second scenario, a sudden change of mechanical torque is applied in motors group 2 at $t=3 \mathrm{~s}$. The mechanical speed is reduced strongly when the torque is doubled. In this perturbation the performance of $\tau=55 \%$ is observed (figs. 12 and 13). The instantaneous return to the steady state of the load voltage is also observed after the fault conditions, fig. 14.

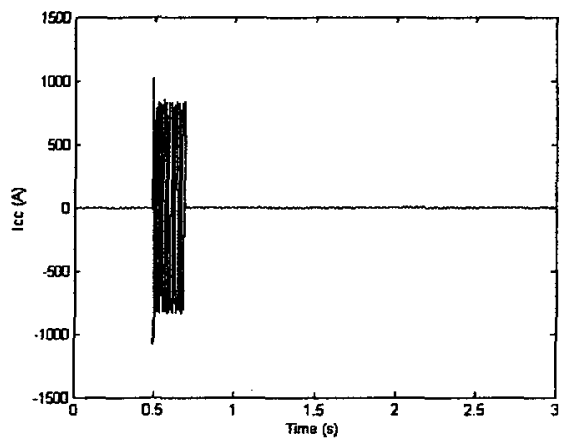

Fig. 11 Short circuit current at $\tau=55 \%(\mathrm{Xc}=13 \Omega)$

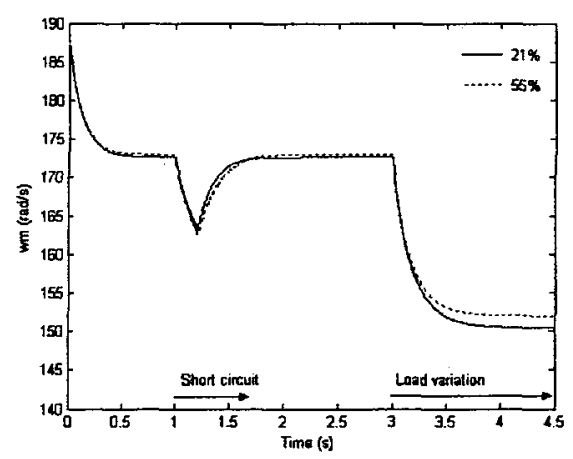

Fig.12 Mechanical speed of heaviest motors (group 2)

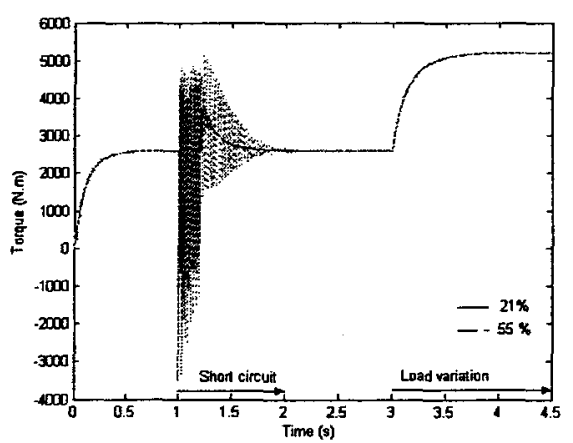

Fig.13 Electrical torque of heaviest motors (group 2)

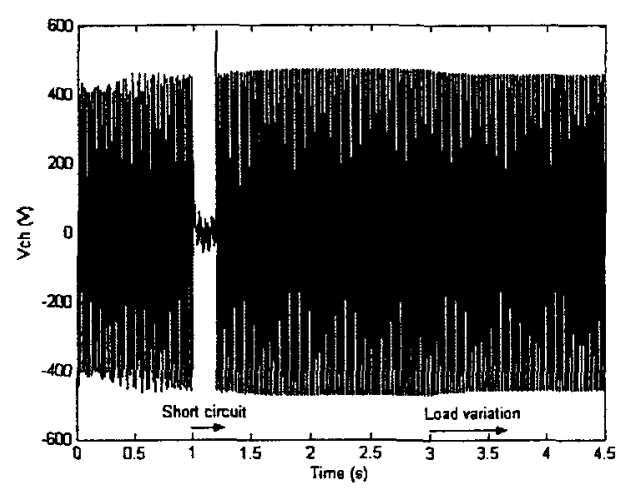

Fig. 14 Load voltage during short circuit and strong load variation at $t=3 \mathrm{~s}$.

As shown in figs. 15 and 16, mechanical speed is reduced in both scenarios but the steady state conditions are maintained when we compensate at $\tau=55 \%(\mathrm{Xc}=13 \Omega)$. After the perturbations, mechanical speed oscillates producing the hunting to $\tau>55 \%$.

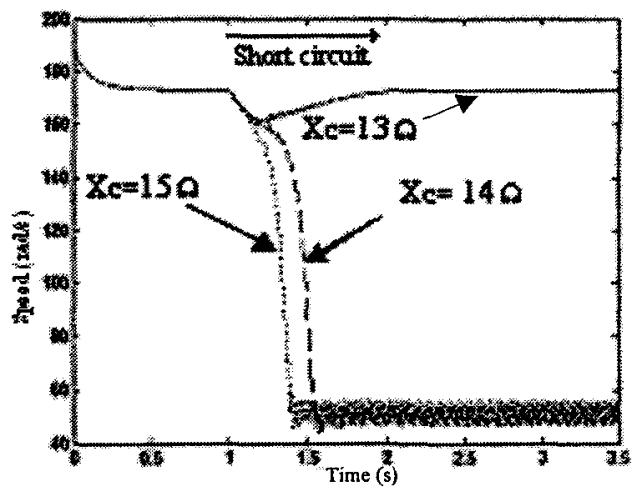

Fig.15 Mechanical speed of heaviest motors (group 2) during a short circuit at $\mathrm{t}=1 \mathrm{~s}$.

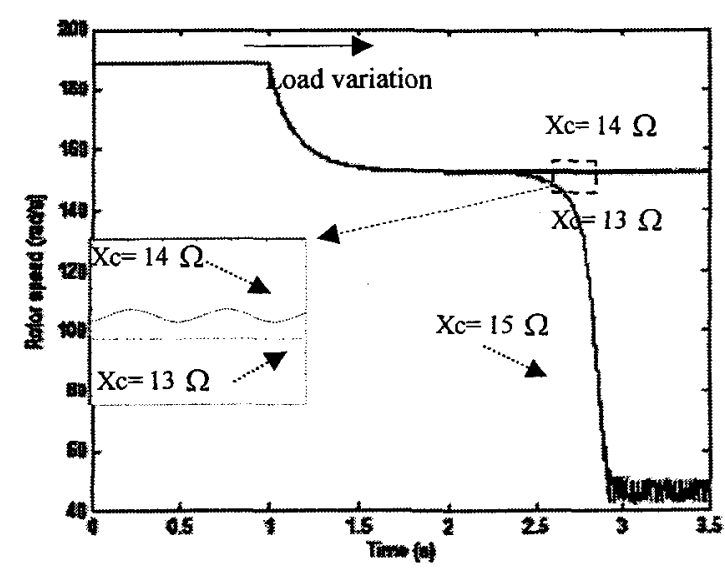

Fig.16 Mechanical speed of heaviest motors (group 2) during a strong load variation at $\mathrm{t}=1 \mathrm{~s}$. 
In fig. 17 (a), during the strong load, the load current maintains its stability. In fig. 17 (b), load current experiments several oscillations when the series compensation is increased at $\tau>55 \%(\mathrm{Xc}=13 \Omega)$. Those perturbation s leading the current to instability conditions.

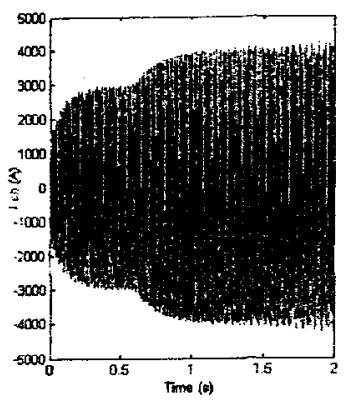

(a)

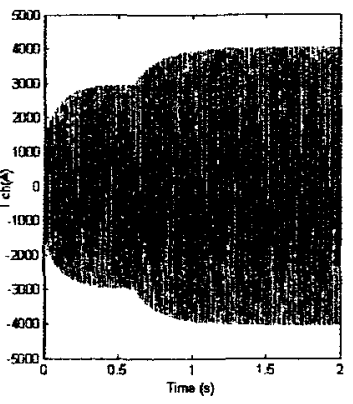

(b)
Fig. 17 Load current during a strong load variation

$$
\text { at } t=1 \mathrm{~s}
$$

\section{CONCLUSIONS}

In this paper, a new optimization method is proposed to find the optimal series compensation degree and the suitable location of series capacitor along the feeder. The modeling of the SCOR is a strategy to visualize the performance of the series compensation degree in a distribution network. In addition, the instantaneous effect of series capacitor has been simulated during start-up transient and steady state operation. For the case of investigated distribution network, Hunting was the constraint to determine the upper bound of SCOR, $\tau=55 \%(\mathrm{Xc}=13 \Omega)$. The flicker voltage determined the minimal value to compensate the distribution network, $\tau \geq 21 \%$. The series compensation degree $\mathrm{X}_{\mathrm{c}}=13 \Omega$ can be implemented to $35 \mathrm{Kms}$ from the source, reducing $\Delta V$ to $15 \mathrm{~V}(600 \mathrm{~V}$ ) (table 3 ) or to $605 \mathrm{~V}(25 \mathrm{kV})$, increasing the voltage load and maintaining the distribution network in stability conditions.

Table 3. Voltage at load bus $\mathrm{V}_{2}$

\begin{tabular}{|c|c|c|}
\hline $\begin{array}{c}\text { Series compensation } \\
\text { ratio }\end{array}$ & $\begin{array}{c}\text { Low voltage } \\
(600 \mathrm{~V})\end{array}$ & $\begin{array}{c}\text { High voltage } \\
(25 \mathrm{kV})\end{array}$ \\
\hline $0 \%(\mathrm{Xc}=0 \Omega)$ & $577.6 \mathrm{~V}$ & $24.03 \mathrm{kV}$ \\
\hline $21 \%(\mathrm{Xc}=5 \Omega)$ & $582.4 \mathrm{~V}$ & $24.23 \mathrm{kV}$ \\
\hline $55 \%(\mathrm{Xc}=13 \Omega)$ & $584.6 \mathrm{~V}$ & $24.32 \mathrm{kV}$ \\
\hline
\end{tabular}

\section{ACKNOWLEDGMENTS}

We would like to thank to Mr Jacques East for his valuable help during the development of the research.

\section{REFERENCES}

[1] Miske S.A., "Considerations of the Application of Series Capacitors to Radial Power distribution Circuits". IEEE Transactions on Power Delivery, April 2001.

[2] René Wamkeue, Nahi Kandil, Jacques East, Yves Boisclair. Series Compensation For a Hydro-Quebec Long Distribution Line. ICREPQ'03 International Conference on Renewable Energies and Power Quality, Vigo, Espagne, 9-11 april, 2003.

[3] Dequang Gan, Robert J. Thomas, Ray D Zimmerman. "A Tran sient Stability Constrained Optimal Power Flow. Bulk Power Dymanics and Control IV- Restructuring", August 24-28, Greece.

[4] A.A. Johnson. "Application of Capacitors to Power Systems". Westing Hou se HandBook.1984.

[5]Sebastiaio Oliveira., Istvan Gardos, "Representation of Series Capacitors in Electrical Power System Stability Studies". IEEE Transactions on Power Systems, vol 6 , N.3, August 1991.

[6] Damir Novosel, Arun Phadke, Murari Mohan Saha. "Problems and Solutions for Microprocessor of Series Compensated". Publication N 434, IEE Conference in Power System Protection, 25-27 $7^{\text {th }}$ march 1997.

[7] Jacques East. Utilisation des condensateurs série sur le réseau de distribution. HydroQuebec. Rouyn Noranda. Jan vier 1997.

[8] Ayshan A. Mutlu, Elham B. Makram. "Analysis of Series Compen sation From different aspects". IEEE 1998.

[9]N. Christi, Sadek Lützelberger, "System Studies and Basic Design for an Advanced Series Compensation Scheme (ASC)". IEE Int. Conference on Advances in Power System Control, Operation and Management November 1991, Honk Kong.

[10]Abdel-Aty Edris, "Series Compensation Schemes Reducing the Potential of SSR". IEEE Transaction on Power Systems, vol 5, February 1990.

[11]Ronald A. Hedin, Stephen Weiss, Duane Torgeson, Eilts L. E."SSR Characteristics of Altemative Types of Series Compensation Schemes". IEEE Transactions on Power System, vol 10. N.2, may 1995. 